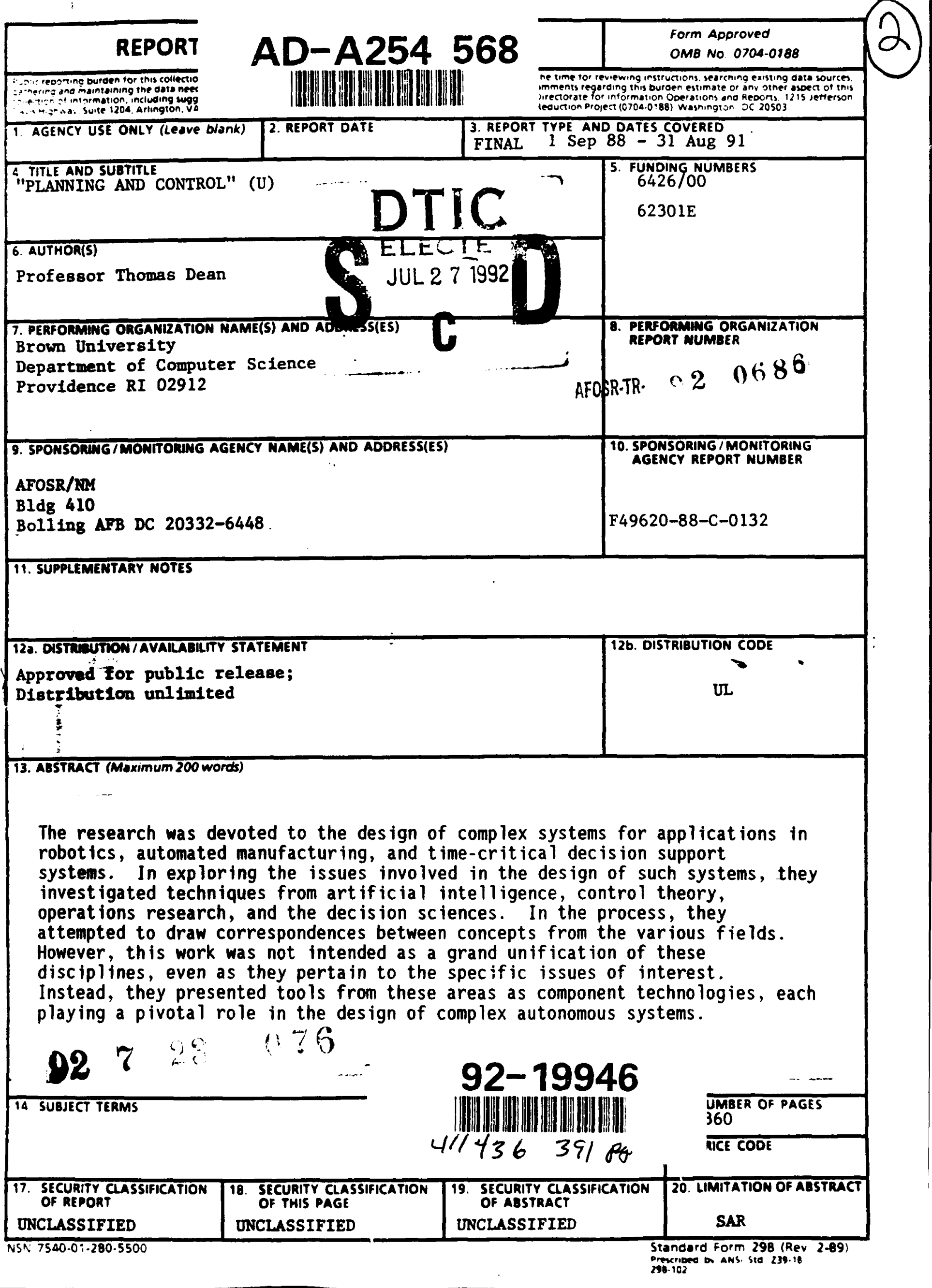




\section{DISCAIND NOTED}

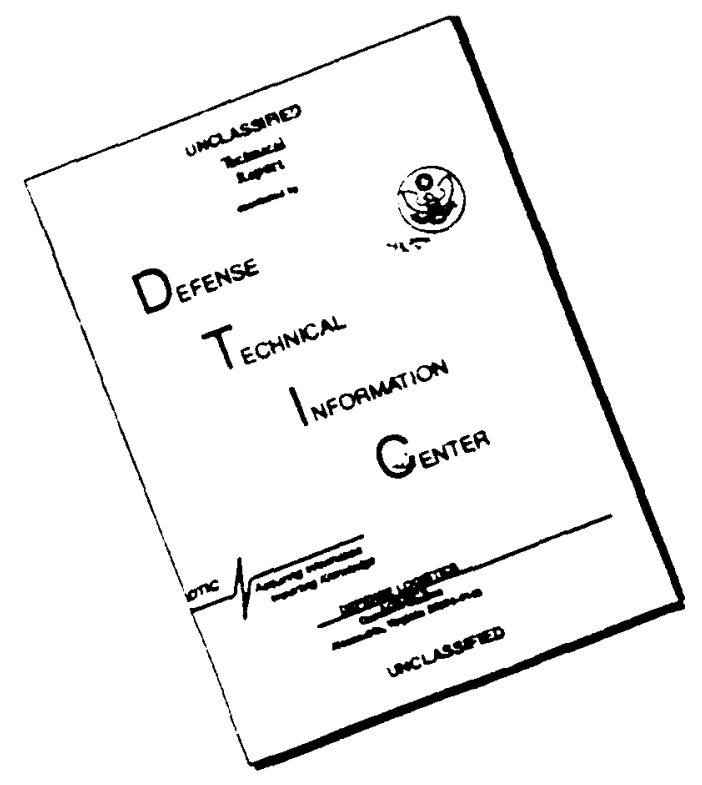

THIS DOCUMENT IS BEST

QUALITY AVAILABLE. THE COPY

FURNISHED TO DTIC CONTAINED

A SIGNIFICANT NUMBER OF

PAGES WHICH DO NOT

REPRODUCE LEGIBLY. 


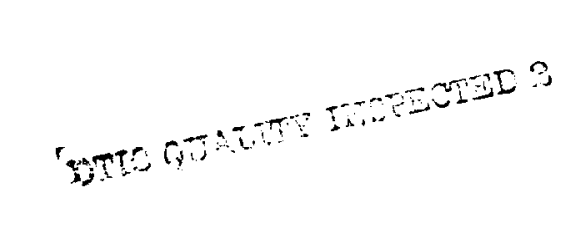

Final Report

ARPA Order: 6426

Program Code: 8E20

Contractor: Brown University

Effective Date: September 1, 1988

Expiration Date: August 31, 1991

Amount: $\$ 440,000$

Contract Number: F49620-88-C-0132

Principal Investigator: Thomas Dean (401) 863-7645

Program Manager: Abraham Waksman (202) 767-5028

Title: Coordinating Planning and Control

\section{Sponsored by}

Defense Advanced Research Projects Agency

DARPA Order No. 6426

Monitored by AFOSR Under

Contract No. F49620-88-C-0132 


\title{
Planning and Control
}

\author{
Thomas L. Dean
}

Brown University

Michael P. Wellman

USAF Wright Laboratory

Morgan Kaufmann Publishers, Inc. 


\section{Preface}

This book is devoted to the design of complex systems for applications in robotics, automated manufacturing, and time-critical decision support systems. In exploring the issues involved in the design of such systems, we investigate techniques from artificial intelligence, control theory, operations research, and the decision sciences. In the process, we attempt to draw correspondences between concepts from the various fields. However, this work is not intended as a grand unification of these disciplines, even as they pertain to the specific issues of interest. Instead, we present tools from these areas as component technologies, each playing a pivotal role in the design of complex autonomous systems.

In our attempt to draw a coherent picture of the broad range of problems and techniques considered here, we rely on the central themes of observation, prediction, and computation. In an uncertain environment, we must employ observation to augment our incomplete knowledge with evidence from the senses. We invoke prediction to extrapolate from our knowledge and observations the effects of our actions over time. Revising and making effective use of our knowledge requires computation to translate models and observations to meaningful action. The design of a system to control complex processes consists largely of strategies for deciding dynamically what and how to observe, predict, and compute.

In the 1980s, the traditional view of planning as offline computation r:lying on precise models and perfect information was challenged by research in artificial intelligence on robotic control systems embedded in complex environments. The challenge was met with proposals for reactive systems: systems designed to respond directly to perceived conditions in situations where there is little or no time to deliberate on how best to act. One disconcerting aspect of the focus on reactive systems was that it diverted effort from planning: predicting possible futures and formulating plans of action that take into account those possibilities. As research progressed, it became 
apparent that there was significant overlap between the work on reactive systems and the work in control theory. This book connects traditional research in planning with the constraints governing embedded systems, by reformulating the process of planning in terms of control.

Viewed from a control perspective, reactive systems embody particular strategies for controlling processes. In order to evaluate reactive.systems, we have to analyze the connection between such strategies and the physical systems they seek to control. The tools required to perform such analyses are readily available from control theory, computer science, and artificial intelligence. This book focuses on the issues involved in modeling processes and generating sequences of commands in a timely manner. The practice of constructing formal models of physical systems and then using those models to develop programs to control processes is examined in some depth.

This book is intended for graduate and advanced undergraduate students in computer science and engineering. It is meant for students trying to orient themselves with respect to the many disciplines that have something significant to say about planning and control for applications in robotics and automation. The material in this book is suitable for a one-semester course offered to graduate and advanced undergraduate students. Given that the material covers a range of disciplines, we assume a somewhat varied background.

From computer science, we assume some familiarity with the theory of computation [12] and basic complexity theory [8]. Pidgin ALGoL [1] and Edinburgh PROLOG [5] are employed in describing algorithms. Some background in logic [14] and its application in artificial intelligence are also expected $[4,15]$. Elementary probability theory plays a role in the chapters on uncertainty and stochastic modeling $[11,13]$. While no background in control theory is required, we assume some familiarity with linear algebra and elementary differential equations [17]. We refer occasionally to standard techniques in robotics and machine vision, but no detailed knowledge is assumed. References, both general and specific, are provided at the end of each chapter, so that readers can fill in any missing background knowledge.

The book introduces advanced techniques that derive from work in a number of disciplines. The exposition of these techniques is largely selfcontained, with pointers to more detailed treatments. In particular, the text explores the use of default reasoning [9] and temporal logics [18] in modeling processes, a framework for integrating techniques from control theory $[6,10]$ into a theory of planning, and several methods for coping with uncertainty derived from work in artificial intelligence [16], control theory [2], and deci- 
sion analysis [3]. The phrase "Intelligent Control" was coined by Fu [7] to describe the field corresponding to the intersection of artificial intelligence and automatic control. Our interests in this book often coincide with those of the intelligent control community, and, where appropriate, we provide pointers to this literature.

The original idea for this book came from a course on robot problemsolving taught by Tom Dean at Brown University. In the Spring of 1989, Dean began work on a textbook based on his lecture notes for this course. Mike Wellman joined the project in the Fall of 1990 . The collaboration has worked out well, and we expect to continue working together on future projects.

We consider this book as a tentative first step towards an integrated view of planning and control. We expect that the ideas presented herein will undergo major revision as the field proceeds to define itself. There were times when we began exploring details that threatened to delay the book by months if not years. Our editors, colleagues, and students persuaded us, however, that it was more important to publish a first approximation to the theory we were seeking in order to enlist the combined efforts of the rest of the research community. In the end, we were content to provide a rather high-level travel guide to exploring the territory. It is our hope and expectation that this book will be rewritten every three or four years for the foreseeable future; not necessarily by us, but by our students and colleagues in a variety of disciplines.

\section{Acknowledgments}

We would like to thank Steve Cross, Bob Simpson, and Rand Waltzman at DARPA, Abe Waksman at AFOSR, Nort Fowler at Rome Laboratory, Sanjaya Addanki at IBM, and Ken Laws at NSF for their support of the research that went into making this book possible.

A large portion of this book was completed while Tom Dean was spending his sabbatical leave at Stanford University. Jean-Claude Latombe and Yoav Shoham at Stanford provided a stimulating working environment that significantly influenced the final content of the book. Wonyun Choi-a graduate student at Stanford-and Hideki Isozaki-a visiting scholar from Japanhelped with some of the examples. Obtaining the early (junior) sabbatical leave would not have been possible without the support of Eugene Charniak and John Savage from Brown. 
During the two years of preparation, we received encouragement and feedback from several people. Nils Nilsson of Stanford was enthusiastic about the book from its conception. We received useful feedback from Chris Brown at Rochester, Jon Doyle at MIT, Greg Hager and Dan Koditschek at Yale, Peter Ramadge and Elisha Sacks at Princeton, Stuart Russell at Berkeley, Reid Simmons at Carnegie-Mellon, Rich Sutton at GTE Laboratories, and Bill Wolovich at Brown. Many Brown students-Ken Basye, Mark Boddy, Ted Camus, Robert Chekaluk, Seungseok Hyun, Jak Kirman, Keiji Kanazawa, Jin Joo Lee, Moises Lejter, Neal Lesh, Oded Maron, Linda Nunez-Mensinger, Margaret Randazza, John Shewchuk, and Tu-Hsin Tsaicontributed to the research that went into this project. We are especially grateful to Neal Lesh and Oded Maron, who wrote the code for most of the examples used in the text. Finally, we wish to thank Mike Morgan for obtaining reviews in a timely manner, urging us to focus the book, and generally remaining enthusiastic and supportive throughout the whole project.

\section{References}

[1] Alfred V. Aho, John E. Hopcroft, and Jeffrey D. Ullman. The Design and Analysis of Computer Algorithms. Addison-Wesley, Reading, Massachusetts, 1974.

[2] M. J. Ashworth. Feedback Design of Systems with Significant Uncertainty. John Wiley and Sons, New York, 1982.

[3] V. Barnett. Comparative Statistical Inference. John Wiley and Sons, New York, 1982.

[4] Eugene Charniak and Drew V. McDermott. Introduction to Artificial Intelligence. Addison-Wesley, Reading, Massachusetts, 1985.

[5] W. F. Clocksin and C. S. Mellish. Programming in Prolog. SpringerVerlag, New York, 1984.

[6] Richard C. Dorf. Modern Control Systems. Addison-Wesley, Reading, Massachusetts, 1989.

[7] K. S. Fu. Learning control systems and intelligent control systems: An intersection of artificial intelligence and automatic control. IEEE Transactions on Automatic Control, 16(1):70-72, 1971. 
[8] Michael R. Garey and David S. Johnson. Computers and Intractibility: A Guide to the Theory of NP-Completeness. W. H. Freeman and Company, New York, 1979.

[9] Michael R. Genesereth and Nils I. Nilsson. Logical Foundations of Artificial Intelligence. Morgan-Kaufmann, Los Altos, California, 1987.

[10] Francis J. Hale. Introduction to Control System Analysis and Design. Prentice-Hall, Englewood Cliffs, New Jersey, 1973.

[11] Paul G. Hoel, Sidney C. Port, and Charles J. Stone. Introduction to Probability Theory. Houghton Mifflin, Boston, Massachusetts, 1971.

[12] Harry R. Lewis and Christos H. Papadimitriou. Elements of the Theory of Computation. Prentice-Hall, Englewood Cliffs, New Jersey, 1981.

[13] D. V. Lindley. Introduction to Probability and Statistics. Cambridge University Press, 1980.

[14] Elliot Mendelson. Introduction to Mathematical Logic. D. Van Nostrand, New York, 1979.

[15] Nils J. Nilsson. Principles of Artificial Intelligence. Tioga Publishing Company, Palo Alto, California, 1980.

[16] Judea Pearl. Probabilistic Reasoning in Intelligent Systems: Networks of Plausible Inference. Morgan-Kaufmann, Los Altos, California, 1988.

[17] Albert L. Rabenstein. Elementary Differential Equations with Linear Algebra. Academic Press, New York, 1975.

[18] Yoav Shoham. Reasoning About Change: Time and Causation from the Standpoint of Artificial Intelligence. MIT Press, Cambridge, Massachusetts, 1988. 


\section{Chapter 1}

\section{Introduction}

It is late and you are returning home after shopping at the grocery store. You thread your car through the narrow streets of your neighborhood. and maneuver carefully into a parking place barely large enough to accommolate your vehicle. You gather up the groceries. walk up the steps to your apartment. and grope your way down the hall trying to feel the light switch so you can find the right kev. After setting the groreries on the kitchen table. you put some leftovers in the oven, and step into the bathroom to start running a hot bath. Returning to the kitchen, you begin putting the groceries array. About midray through shelving the groceries. you return to the bathroom and adjust the faucets to ensure a comfortable temperature for your bath. When you return to the kitchen. you turn the oven down before finishing with the groceries.

Parking a car. carrying groceries. heating food, and running a warm bath are all examples of controlling processes. Quite oftell, we are engaged in controlling several processes sinultaneously, as in the case of running a bath and heating leftovers. There are some processes that we have considerable control over. such as those having to do with the movement of our arms and legs. and other processes that we have very little control over, such as the process governing how many people in an apartment building are using the hot water at any given moment. There are limits. however. even to our control over our arms and legs. The arnis and legs in conjunction with neural circuits in the spinal cord respond to stimuli without conscious effort: the arm jerks the hand back from a hot surface. the legs move involuntarily to save us from falling if we stumble. Many of the processes that we are user to

\footnotetext{
${ }^{\circ}$ C) 1990 Thoulas Dean. All rights reserved.
} 
dealing with on a day-to-day basis ( $\epsilon . g .$. the weather) are completely outside of our control. We learn how to influeuce those processes'we can exert some $t a .+1$. control over. and adapt our behavior to cope with those ve caunot.

This ograth is concerned with the design of programs that coutrol the behavior of physical processes. Intuitively, a process is just a series of changes in the state of the world. Controlling a process consists of making certain changes in the state of the world in order to determine u'hat additional changes in the state of the world will occur and when. We distinguish between the controller. a device that includes hardware to run a control program. and the controlled process, ofteu another device or group of devices whose behavior the controller is seeking to influence. In control theory, the coutrolled process is refe: red to as the plant. In robotics. the controlled process inight correspond to certain mechanical componenis of the robot such as a manipulator or a drive mechanusm. or it might corres?ond to the enviroument in which the robot is meant to function. The controller exerts control over the controlled process and monitors its progress through the use of auxiliary interface de vices. Generally, these devices correspond to sensors and robotic mauipulators, but there are other sorts of interfaces. For instance. the desiguer of a special-purpose microprocessor may view the wicroprocessor as the controller and its inpul and output ports as inturface devices.

The distinction between controller and controlled process is quite uatural from an engineer's point of view; the controller is a device that the engineer designs and builds. It is important to keep in mind. however, that the controller is itself a process. Both the controller and the coutrolled process operate in the same spatial and temporal context: both are embedded in a larger process. The study oi control is the study of the relationship between controlling and controlled processes. This relationship is central to our investigations.

In order to coutrol the behavior of a process. it is often useful to have sone infornaticn concerwing its current stale. This information can be obtained in two different ways: you can observe the state directly. or you can predict in from information about earlier states. In order to predict the current stale of a process from its past states, it is necessary to have a model of that process. A model is a description of a process used to derive information about present and future states of the process given infornation about its curreut and past states.

If you see a projectile hurtling toward you, then you might predict that the projectile will bit you if you remain in your current position. and you 
might use the prediction as a justification for your ducking. If you know that there is a protective barrier between you and the projectile. or you know that the projectile is tethered on a short string, then you can save yourself the trouble of ducking. Determining low to act to satisfy certain goals based upon predictions of possible future states is what is generally referred tc as planning. There are situations. however. in which making careful predictions is either unnecessary, impractical. or impossible.

When you place leftovers in an oven set at a certain temperature. you employ a very simple model to predict when those leftovers will we ready to eat. You could place a temperature sensor in the left . iers. and continually check the sensor until it reached a preset value. This is what is referred to as monitoring a process. Civen the predictability of most ovens. it is hardly necessary to monitor the warming of leftovers. There are processes that are so unpredictable that they warrant constant monitoring (e.g.. air traffic over a metropolitan area). The decision of whether to monitor or predict the behavior of a process is a complex one involving subtle tradeoffs. Deploving sensors for monitoring can be expensive in that the sensors may not be available to monitor other processes. 'l'here are also often significant computational costs associated with both monitoring and prediction. The study of control is intimately tied up with utilizing scarce resources corresponding to sensors, nlanipulators, and associated romputing machinery. Planning provides a fraulework for reasoning about tradeoffs and directly addresses the problem of resource utilization. This mowezmph explores control from the perspective of planning, and planning from the perspective of control. The idea being that the two are intimately related but emplasize different aspects of the same problem.

In the rest of this cliapter, we explore the notion of control and how it relates to planuing in sontewhat more detail. Our discussion will revolve around the idea of morleling processes and using models to direct control.

\subsection{Controlling Processes}

So far, wie have talked about processes as though they actually exist in the world, whereas, in point of fact, they exist in our heads for the 'urpose of explaining our observations of physical phenomema. A process is an abstract description of physical phenonena. Such a description makes use of some vocabulary for speaking about the state of the world. For iustance, we may want to speak about the position $(x, y$, and $\Sigma$ courdinates $)$ of a robot with 




Figure 1.1: A simple control problem

respect to some frame of reference. or the charge ( $c$ measured in ampere hours) on a battery used to power the rohot. Variables such as $x, y, z$, and $c$ are referred to as statc tariables. We assume that the state of the world can be accurately described in terms of some number of state variables. Of course. the notion of accuracy hos to be defined with respect to a particular task. Which brings us to an important question. Why do we want to describe the state of the world at all?

Presumably, we are interested in controlling (i.e.. influencing the value of) certain state variables. We are interested in other state variables insofar as they provide us with information that enables us to exercise better control. . In example should make the discussion more concrete.

Figure 1.1 depicts a cylindrical tank containing fluid with one pipe leading in and one pipe leading out. There is a rotary valve mounted on each pipe that restricts the flow of fluid through the pipe. The position, $\theta$, of the valve leading in detcrmines how much fluid flows into the tank. In this example, we are interested in maintaining the height, $h$, of the fluid in the tank as close as possible to some preset value, say 3 meters, referred to as the target value. We will assume that the valve mounted on the pipe leading out is locked in position.

The process that we are interested in controlling can be described by the two functions of time, $\theta(t)$ and $h(t)$, corresponding to the two state variables. $\theta$ and $h$. As far as we are concerned, the state of the world at a particular tinie $t$ is determined by $\theta(t)$. and $h(t)$. We can predict future states of the process from past states if we have an appropriate inodel. For the process 


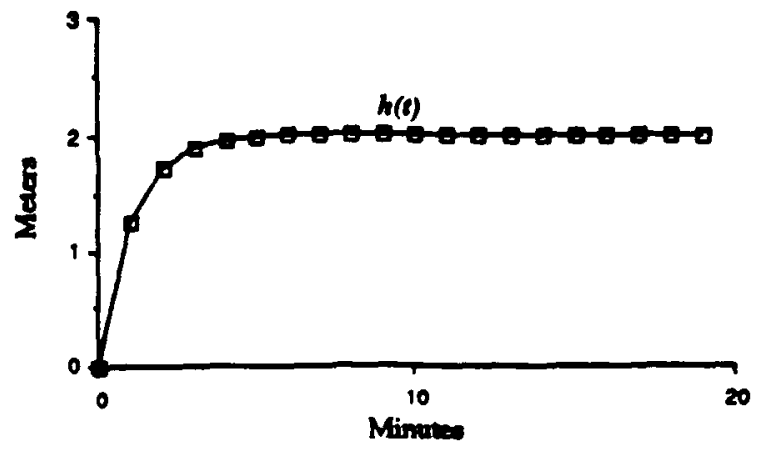

Figure 1.2: Change in fluid height for a constant valve position of $10^{\circ}$

described above. a simple first-order differential equation provides a suitable model.

where $K_{\text {in }}$ is the flow constant in rubic meters per degree minute for the

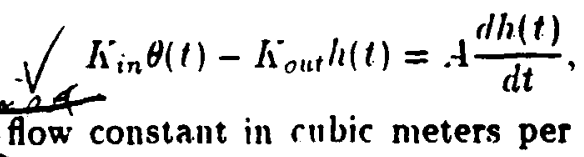
valve governing flow through the input pipe. $K_{\text {out }}$ is the flow constant in square meters per minute for the output pipe. and $A$ is the surface area of the tank. By solving this equation. we can predict the state of the process at time $t$, given information about the state of the process at some earlier time $t_{0}$. The solution to the above differential equation is

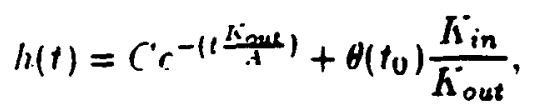

where $C$ is obtained from the initial conditions as.

$$
C=h\left(t_{0}\right)-\frac{\Lambda_{\text {in }}}{\Gamma_{\text {out }}} \theta\left(t_{0}\right) \text {. }
$$

Figure 1.2 shows the predictions made by the above model for a constant valve position of $10^{\circ}$, where $\frac{K_{\text {in }}}{K_{\text {sut }}}=0.2$ meters/degree. $\frac{K_{\text {ouk }}}{A}=1$ minute. and the tank is initially empty. Note that if we are aware of changes in the variable $\theta$, we can use this information and our model to make predictions about changes in the variable $h$. Given a sequence of changes in $\theta$. we can evaluate the effectiveness of that sequence using the predicted changes in $h$ and some set of criteria for effective control (e.g.. how rapidly $h$ converges to the target value).

We still need to specify how the controller seuses the world and how it might act to control the height of the fluid in the tank. Figure 1.3 depicts the two sensors used by the controller: one that provides information about $h$. and a second that provides iufornation about $\theta$. In addition. we will assume that the controller can influence $\theta$ by issuing one of two conmands: 

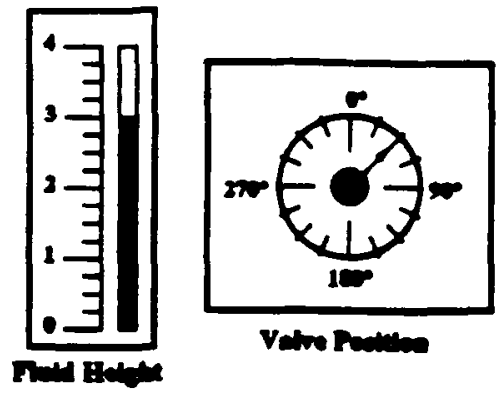

Vare romenge

Figure 1.3: Sensors for controlling processes

\begin{tabular}{|c|c|c|c|c|c|c|}
\hline & $0-10^{\circ}$ & $10-15^{\circ}$ & $15-20^{\circ}$ & $20-30^{\circ}$ & $30-60^{\circ}$ & $60-180^{\circ}$ \\
\hline $0.00-1.50 \mathrm{~m}$ & 1 & 1 & 1 & 1 & 1 & 0 \\
\hline $1.50-2.50 \mathrm{~m}$ & 1 & 1 & 1 & 1 & $n$ & -1 \\
\hline $2.50-2.80 \mathrm{~m}$ & 1 & 1 & 1 & 0 & -1 & -1 \\
\hline $2.80-3.00 \mathrm{~m}$ & 1 & 0 & -1 & -1 & -1 & -1 \\
\hline $3.00-3.20 \mathrm{~m}$ & -1 & -1 & -1 & -1 & -1 & -1 \\
\hline $3.20-4.00 \mathrm{~m}$ & -1 & -1 & -1 & -1 & -1 & -1 \\
\hline
\end{tabular}

Table 1.1: Table used by the function table lookup

turn right or turn_left. The first turns the valve mounted on the pipe leading into the tank $5^{\circ}$ in a clockwise direction. and the second turns the same valve $5^{\circ}$ in a counter-clockwise direction. For the time being, we will assume that the changes initiated by these two commands happen nearly instantaneously (i.e.. if a turnsight command is issued at time $t$. then $\theta(t+\epsilon)=\theta(t)+5$, where $\epsilon$ is negligible).

Now de can predict future states of the process, but how do we control the process? Perhaps the simplest way is just to experiment and see what works. Suppose that we have done just that, and we have compiled a table that tells us exactly what action to take in every situation. Such a table is shown in Table 1.1. Recall that the task of the controller is to restore the height of the fluid in the tank to the target value of 3 meters. Given information about the current Muid height and valve position, Table 1.1 indicates 1 if the correct action is turn right, -1 if the correct action is turn_loft, and 0 if the correct action is not to do anything at all. Using this table, we define a simple control algorithm as follows: 


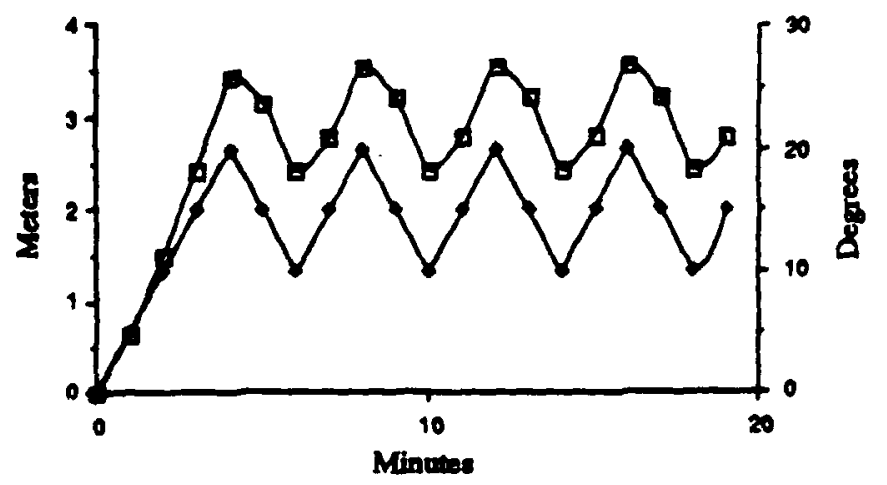

Figure 1.4: The controller's beliavior with a 1 minute sample period

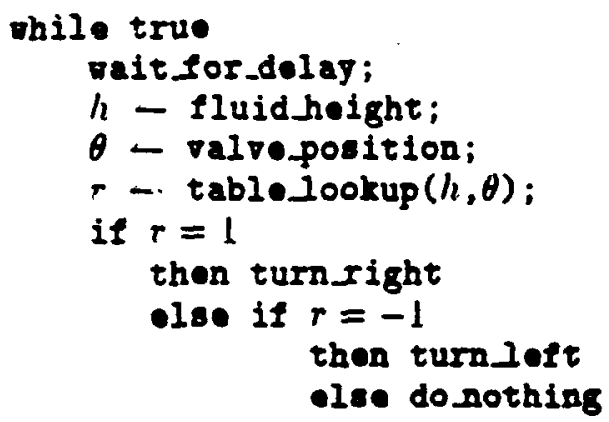

where fluid hoight and valve position read the corresponding sensors, and table-1ookup extracts the appropriate value from the table in Table 1.1 using indices computed from the sensor readings. The procedure vait_for_dolay causes the controller to pause for a fixed interval of time referred to as the sample period. Figure 1.4 describes the changes in $h$ and $\theta$. with $\theta$ controlled by the algorithm described above. the sample period set to 1 minute, and the other variables as set for Figure 1.2.

As an alternative to experimenting in the real world. we could use the model described earlier to experiuient with various control strategies for responding to information returned by the sensors. These model-based experiments could then be used to compile a table very much like the one shown in Table 1.1. If the model is reasonably accurate. then the resulting table should look very much like the one developed from experimenting in the real world. Of course. not only do we need an accurate model of the controlied process, but we also need an accurate model of the controller in order to compile an accurate table of responses. So far. we have neglected discusaing the controller at all.

In the preceding discussion. we made a mumber of assumptions (e.g.. the valve restricting the output pipe is fixed, and changes initiated by controller commands are nearly instantaneous). Now it is time to review some 


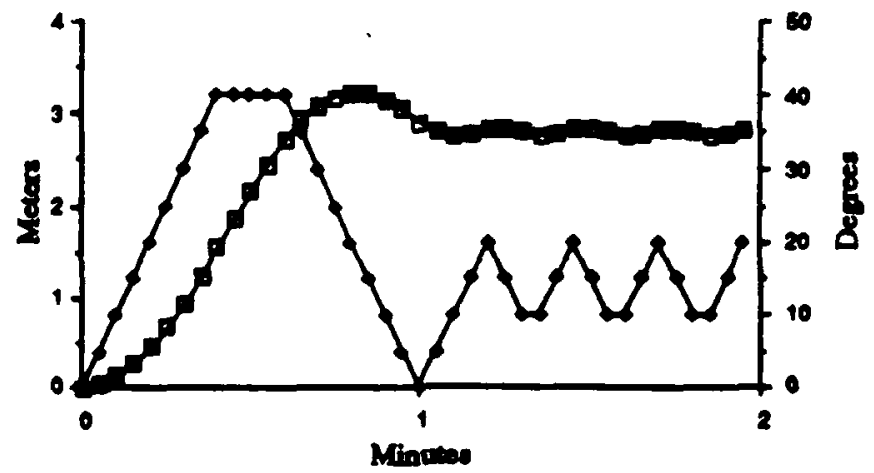

Figure 1.5: The coutroller's behavior with a 1 second sample period

of those assumptions, and bring to light a number of additional assumptions that were inplicit in our discussion of controliers and their response characteristics.

To begin with, we reconsider the role of the sample period in our simple control algorithn. In the description of the algorithm's performance in Figure 1.4, we mentioned that the sauple period was set to 1 nuuute. What if instead we set the sample period to 1 second? 'Vell. for one thing, we would get markedly improved performance, in the sense that the controller would appear to rapidly converge on the target value. Figure 1.5 shows how the controller would respond given a 1 second sample period, assuming that the changes initiated by the commands turn right and turn_loft occur nearly instantaneously. When we are talking about conmands issued every ininute, the consequences of such an assumption may be minor, bnt, if we are talking about commands issued every secoud, we may be making unrealistic Tassumptions about the hardware available for carrying such commands. The magnitude of the controller's response is governed by the controller's gain (a measure of how fast a controlled variable caul change). Generally speaking. the higher the gain, the more massive the controller, the more power it is likely to consume, and the more costly it will be to purchase. Our (implicit) model of the mechanical system for changing the position of the valie is inadequate for a careful analysis of the overall control system.

Another related aspect of the controller's performance that we failed to account for concerus the procedures and how quickly they run on some particular computing hardware. How long does it take to read a sensor? How long does it lake to perform all of the auxiliary computatious reyuired in the control algorithm? Evell table lookup takes time (6.g., time to page 
the table into memory from disk and compute the indices). Procedures may invoke additional processes whose offects may not be inmediately apparent (e.g., the procedure corresponding to turn $x$ ight may take only a few micro seconds to return. bitt the servo mechanism responsible for actually turning the valve may take several seconds. to carry out the command). Suppose that the controller issues the three commands. turn_left. turn loft. and turn_ight. in quick succession. Does the second turn left command get canceled out by the following turn right command, or does the controller swing a full $10^{\circ}$ in a clockwise direction before swinging back $5^{\circ}$ in a counterclockwise direction?

Desiguing good models to capture real-world phenomena can be quite complex. A process model is an abstraction: an idealization appropriate in only a limited context. In the model for a tank filling, we failed to account for evaporation, condensation. malfunctioning valves, other agents adding to or removing from the tank in unpredictable ways. and any of a number of other factors. Correcting for such factors is not simply a matter of providing a more accurate nodel or better servo mechanisms: hardware lias its linitations and, generally speaking, better models take longer to compute and rely upon more detailed information.

Fortunately, lack of precision in the model can be offset somewhat by relying upon the model only for short-term predictions. Feedback through frequent sensing can serve to correct for errors in long term predictions introduced by imperfect or faulty hardware. Sensing and feedback do not. lowever. obviate the need to take long-term predictions into account. If you expect to be traveling to a foreign country in the next two weeks. you had better check that your passport is in order today; you risk ruining your travel plans by waiting nntil the last minute.

Another thing to note is that not all predictions are equally nseful. It is not necessary-and generally not possible-to predict every consequence of the events that you observe. A little rain may slightly increase the height of fluid in the tank, but the effect is negligible given the flow through the input and output pipes. On the other hand, predicting that someone is about to close the valve mounted on the output pipe could significantly change the optimal strategy for controlling the input valve. As we will see in Chapter

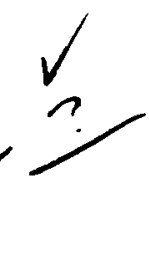
oit to be dificult. 


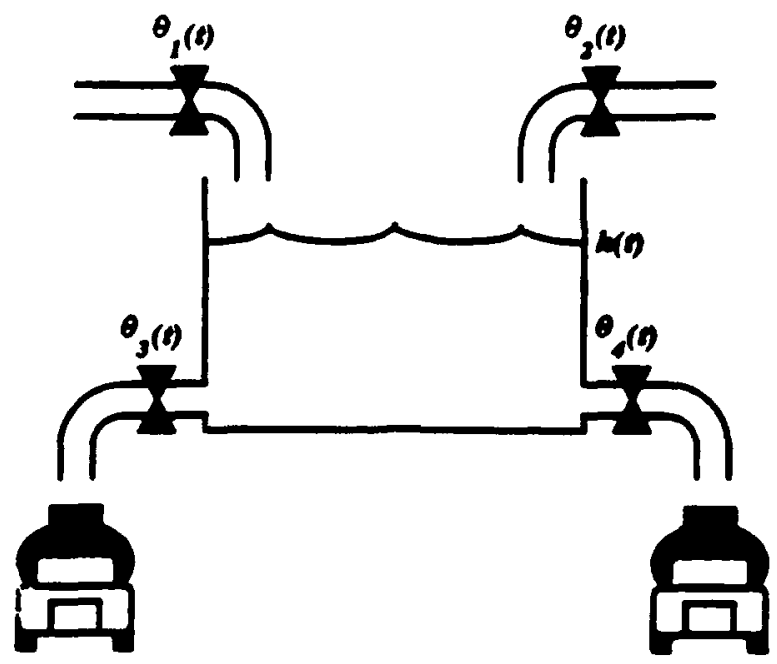

Figure 1.6: A more complex control problem

\subsection{Planning}

The problem describerl in Figure 1.1 is rather simple, and it is not difficult to design effective control systems for solving such a problenn. Consider what happens when the control problem gets more complicated: several variables to control. other agents to contend with, and sque-degree of uncertainty about the future. In the situation depicted in Figure 1.6, there are two pipes leading into and two pipes leading out of a tank similar to the one shown in Figure 1.1. Each of the four valves can be manipulated by a separate dedicated servo motor. In Chapter 5. we will consider a variant of this problem in which there is only one servo motor that can be positioned 80 as to control any one of the four valves. In anticipation of this complication requiring that the controller be mubile, we will refer to the controller as the molot.

Now we have to specify what it is that the robot is supposed to do. Figure 1.6 shows a tanker truck positioned under each of the two pipes leading oat of the tank. We will assume that at any given time there are zero or more tanker trucks waiting in a queue to be filled up. In addition to controlling the valves on the pipes leading into and out of the tank. the robot can command a truck waiting in the queue to position itself under one of the two pipes leading out of the tank. The two pipes whose valves are 
labeled $\theta_{1}$ and $\theta_{2}$ carry two different chemicals. The control task involves filling egctr tanker truck with a mixture containing approximately equal proportions of the two chennicals. We will assume that mixing occurs in the tank automatically and instantaneously. Any chenical mixture that flows over the top of the tank is lost and cannot be recovered. The exact proportion of the two chemicals puluped into a tanker truck is not critical. but. if the proportions of the two chemicals in a given truck differ by more than $10 \%$. the contents of the truck will have to be dumped. The robot gets paid for each truck completely filled with an acceptable mixture, and the robot is charged for any chemicals that flow throngh the two pipes leading into the tank. T'he robot's task is to maximize its net income.

Maintaining an acceptable mixture is simple if the robot has a separate servo directly controlling $\theta_{1}$ and $\theta_{2}$, and the valies have identical flow characteristics; the robot just adjusts the two valves in exactly the same way to guarantee equal proportions of each rhemical. The robot can keep the height of the fluid in the tank at. any level it chooses, but the higher the level is the faster the nuxture will How through the pipes leading out of the lank. and the faster the robol's earnings will accrue. Or course, there is some risk of spilling fluid if the height is kept too near the top and one of the output valves is suddenly closed, but we will assume that the robot has complete control over all four valves and knows the exact capacity of each truck waiting 10 be filled.

If we ignore the added task of positioning trucks, the problem of Figure 1.6 is really no mure complicaled to solve than the problem of controlling a single valve. We could construct a table such as that sliown in Table 1.1. or we could derive a fairly simple algorithm to compute the values stored in such a table. Inplementing the controller using table lookup is probably not a good idea given'the size of the necessary table-the table would have six dimensions (or indices) corresponding to the six state variables: $h . \theta_{1}$, $\theta_{2}, \theta_{3}, \theta_{4}$, and the capacity of the next tauker truck waiting in the queue.

Suppose that the robot knows that a tanker truck is within a cubic meter of being completely filled. Using this information, the robot can determine exactly when the valve to the pipe being used to fill the truck should be compintely closed. In fact, if there is only one truck to be filled. as soon as the truck is positioned under one of the two pipes leading out of the tank, the controller can use its model of the system of pipes and valves to deternuine the complete sequence of valve mauipulations required to fill the truck as quickly as possible. This idea of using a model to formulate sequences of actions is central to planning. In the following, we will examine some of the 


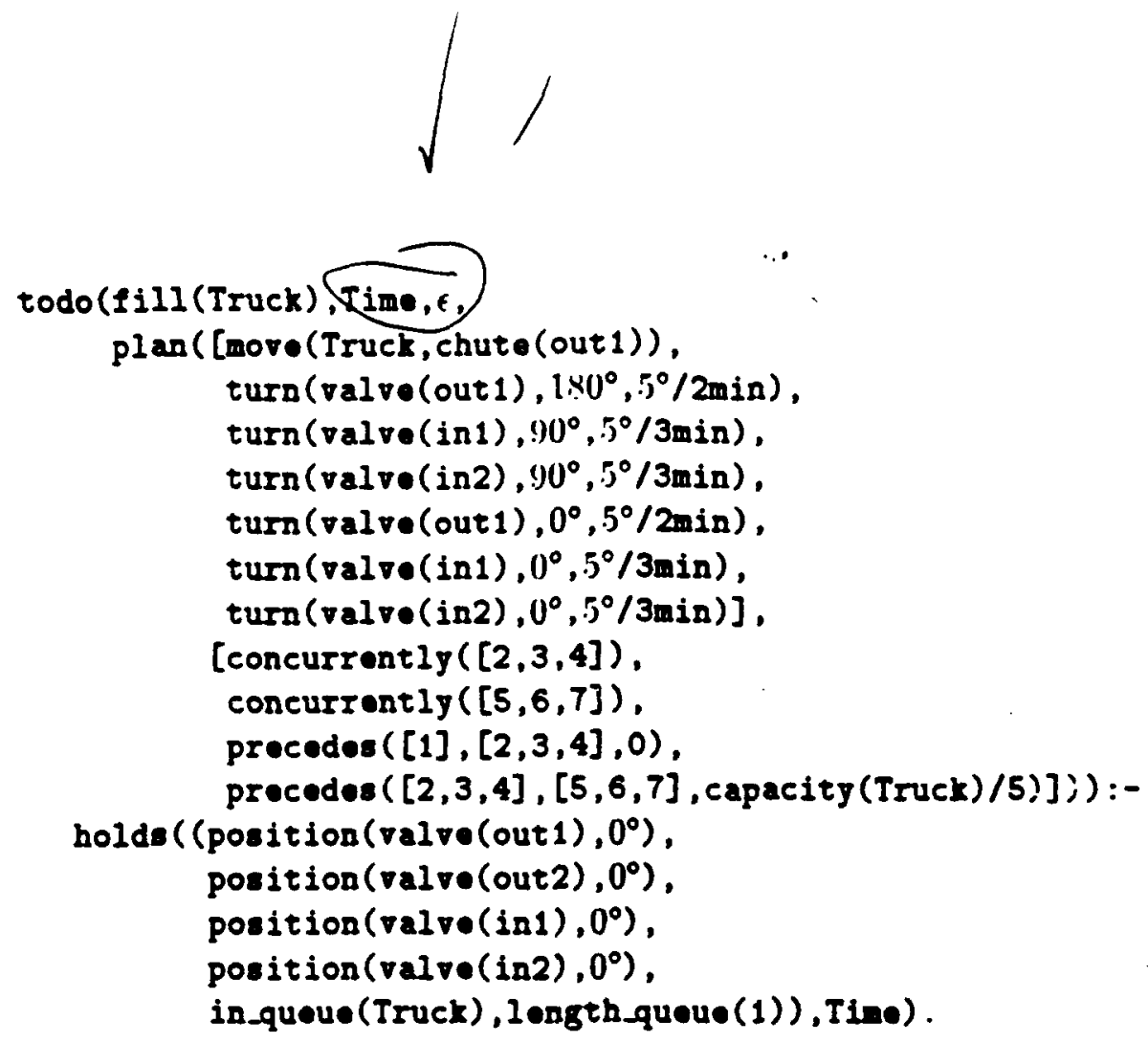

Figure 1.ĩ: Plan for filling a single truck

advantages and disadvantages of using such a technique. We begin with the advantages.

One can easily imagine a situation in which the robot cloes uot have inmediate access to infornation concerning all of the state variables. For instance. the robot might actually have to do some work to check on the height of fluid in the tank or the position of one of the valves. Rather than constantly perform the work necessary to consult the sensors, the robot can rely upon the model to generate an entire sequence of valve manipulations ill advance. We will not discuss how sequences of actions are proposed until Chapter 5; for now, just assume that there is an oracle that produces caudidate sequences when asked. The model comes into play when the robot wishes to compare different sequences in choosing the best one. The basic ides b quite simple. Given a sequence of actions, the robot uses the model to simulate the future as it would occur if the actions were carried out. The simulation if edls $_{h}$ the robof intormation about how long a particular tanker truck will take to fill and whether or not there is any danger of spilling chenucals using the proposed sequence of actions. This information can 
then be used to snggest nodifications to the proposed sequence of actions. or to compare the prop-ied sequence with alternative sequences.

It is also possible to simply store an often used sequence of actions. and inclex it in such a wav that it can be easily retrieved when applicable. This is analogous to the metlood discussed in the previous section for storing responses in tables. For instance. the robot will frequently find itself in the situation where all of the valies are closed. the tank is full. and a truck suddenly appears in the queue. Rather than derive an effective sequence of actions every time it is needed. the robot might store a description of such a sequence of actions-referred to as a plan-indexed so that it can be easily retrieved when needed. Figure $1 . \bar{i}$ shows a rule for retrieving such a plan. The notation is that of PROLOG. but understanding PROLOG is not necessary for our current discnssion.

The rule in Figure 1.i states that. if all of the valves are closed and there is exactly one Truck in the queue at Timo. then plan(stops, Constrainte) is a plan for filling the truck starting at Iinot $\epsilon$, where the Stope consist of seven commands numbered $1-i$, and the Constraints determine the order in which those commands are to be carried out. Issuing a command of the form turn (Valve, Angle, Rate) tells the hardware to turn the Valve to the indicated angle (in degrees) at the specified Rate (in degrces per minute). A constraint of the form concurrently (Stops) specifies that the Stops (indicated hy their order in the list of plan stepa) should begin at the same tine and run in parallel. A constraint of the form procedes (FiratStops, NextStope, $J$ ) specifies that the FirstStope should precede the NextStopa with a delay of $\Delta$ separating the last step to finish in FirstStops from the first step to begin in MoxtStops.

If the computatious required to derive what to do when a truck suddenly appears in the queue are coinplex. then having a response stored away for easy retrieval may reduce the amount of time trucks have to wait in the queve. Plans such as the one shown Figure 1.7 can be generated off line and evaluated using a model; complex plans for novel sitnations can also be constructed on line from simpler plans and evaluated using a model to ensure success. Thin idea of construrting complex plans from simpler ones is integral to nost theories of planuing, and we will examine it in greater detail in Chapter 5.

There are also potential disadrantages in generating sequences of actions. The most obvious disadvantage is that the model may be inaccurate. and the sequence of actions will fail to have the desired effect. Uniess the controller is really convinced of the accuracy of its model. it will want check that the 
plan is proceeding according to expectations. This cherking is referred to as monitoring the execution of a plan. aind may involve a considerable amount of effort. If problems are detected. it may be necessary to stop the secquence of actions specified in a plan in order 10 formulate a new plan or modify steps in the original one. By relying less upon the model, and more upon feedback from sensors, the controller will often save itself a lot of work in generating sequences of steps that are never carried out.

Still, in determining what to do now. it is not as though you can always ignore thinking abont what you will do next. Once the controller predicts when a truck will be full. it has to determine what steps are necessary to ensure that the truck's tank does not overflow. It is not enough to say "start closing the valve." Deternining when to start closing the valve and how quickly requires anticipating the entire sequence of steps. Keep in mind that a controller ouly has limited control over its enviroument: if a valve restricting the flow of fluid into a given truck is wide open. and the truck is nearly full, then the controller will not be able to avoid spilling some fluid. The real issue is not whether or not to plan-planning is an integral part of control-but in what detail to plan. If planning were inexpensive, we would not have to worry about this issue: a controller would always formulate the most detailed pian possible. and there wontd be no loss if the detailed sequence of steps was not carried out. I'nfortunately, planning can be very expensive.

While the problem of Figure 1.6 is a relatively easy one. there are simple modifications that can serve to fundamentally change the problem. Suppose, for instance. that the robot is charged a tax for the time a truck waits between entering the queue and being successfully filled (we will allow the robot to turn away trucks before admitting them to the queue). Now, in addition to its other concerns. the robot lias to try to minimize the time trucks spend waiting.

If the robot maximizes the flow of properly mixed chemicals from the tank, and makes sure that full trucks are moved out as quickly as possible and replaced by enpty trucks. the only other variable to control is which truck should be filled next. Assuming that the tax is computed as a linear function of the time a truck spends waiting, capacity is the critical factor infineacing the choice of next truck. Suppose that the capacity of a truck is an integer-valued quantity. For a given queue of trucks waiting to be filled, the robot will want $t$ issign each truck to one of the two pipes leading out of the tank so as to minimize the amount of time that either one of the two pipes is idle (see Figure 1.8). 


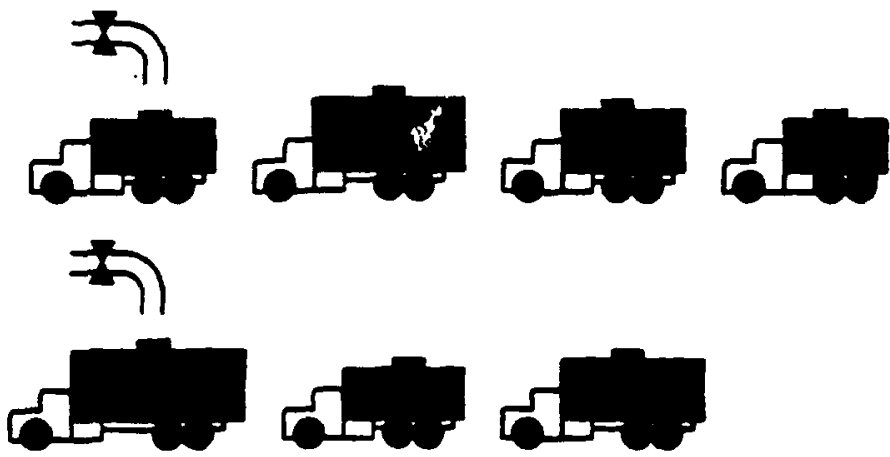

Figure 1.8: Scheduling tanker trucks of varying capacities

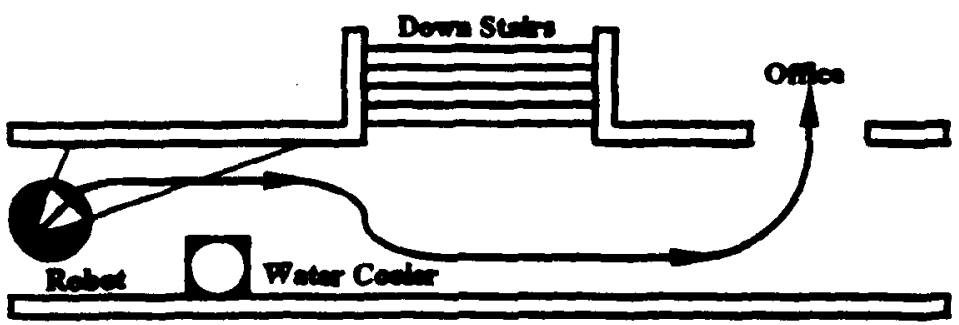

Figure 1.9: A robot navigation problem

Even if we allow that the trucks be instautaneously positioned and the valves instantaneousiy opened and closed, the problem of assigning the trucks so as to minimize idle time is computationally complex. The problem of deternining the optimal assigument of trucks is equivalent to dividing a set of $n$ integers (the capacities of the trucks) into two sets (trucks to be filled from the first pipe and trucks to be filled from the second pipe) so as to suminize absolute value of the lifference (time either of the two pipes is idle) of the sum of the integers in the lirst set (the time the first pipe is being utilized) and the sum of the integers in the second set (the time the secoud pipe is being utilized). This problem is referred to as the pertition problem [3], aud is known to be in the class of NP-complete problens (i.e.. the best known algorilhus for solving these problems have running times that are at least exponential in the size of their input-the number of trucks in the queue in our case).

For the particular NP-complete problem described above. there are good approximate solutious that run in polynomial time. If $n$ is small. it might even be feasible to use an algorithm that computes the exact solution and 
runs in exponential tine. There is a tradeof involving the time $s$,ent in deliberation and the time saved by compuling a better answer. While the robot is deliberating about how to fill the trucks. the trucks are waiting in the queve. and the robot is losing money.

It mav not seem critical that our robot takes a little extra time in filling the tanker trucks. A simple first-in-first-out stralegy for choosing the next truck to fill mav prove to be quite effective. There are. however. occasions in which there is more at risk than just a little higher income. Figure 1.90 shows a robot with a single seusor trying to navigate a hallway. In order to avoid hitting the water cooler, the robot has to look to the right; in order to avoid falling down the stairs, the robot has to look to the left. Whether or not the robot can successfully deploy its sensor : I avoid both obstacles depends a lot on how fast the robot is moving and how fast the robot can reorient its sensor and interpret the returned data. The designer could take a conservative approach and limit the maximum speed at which the iubot can travel so as to eusure the robot's safety, but auch a measure is likely to degrade performance significantly. It would be better if the robot could sonehow analyze each siluation in which it finds itself, weigh its options, and choose the option determined to be best.

The designer of control algorithms has to contend with the inherent linitations of computing hardware and software. There are times when even the simplest algorithms turu out to take too long. For instance. suppose that you wish to track a projectile. and suppose that you have a sensor that returns information concerning the current location of the projectile. By the time you get around to processing the sensor information. it may be out of date. so you will want to label the sensor information with the time that the data was gathered. The obvious thing to do if to label the sensor data using the compnter system s on-board clock. The problem is that reading the clock requires loading a procedure into memory, invoking the procedure, and waiting for it to return an answer; all of which takes time. and. more importantly. differeut amounts of time depending upon how memory is configured. whether or not the procedure has been invoked recently. and any number of other factors. This differential in how long the procadure takes to return an answer can adversely affect the usefulness of the labeled sensor data. For a legged robot trying to walk [6, 2]. it can mean the difference between falling or not: for a tennis playing robot [1]. it can mean the difference between winning a match or not. 


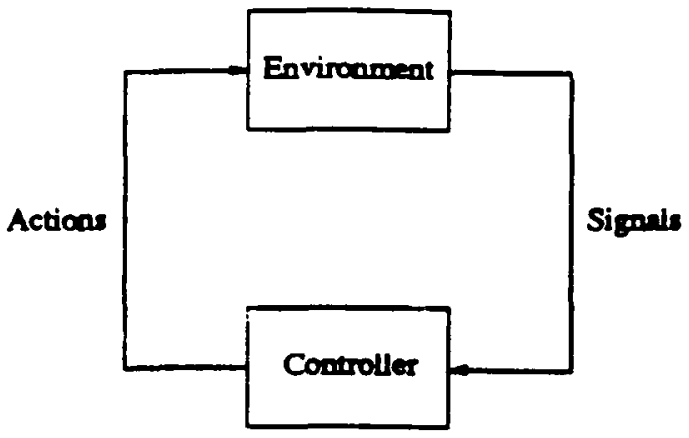

Figure 1.10: A machine coupled to its environment

\subsection{Dynamical Systems}

Let us return to the question of what it means to control something, and try to answer this question from the perspective of control theory. We begin by providing a general description of a coutroller coupled to an environment and given some task to achieve.

The controller is represented as a deterministic automaton that takes as input a signal and outputs some action. The environment can be viewed as another automaton that takes as input the controller's action and generates a signal to serve as the controller's next input. The controller is sajd to be coupled to its environment: the controller and its environment trading blows in a continuous cycle of interaction. Figure 1.10 (after Hosensebein [i]) illustrates this cycle of interaction.

In the following. we describe the interaction between the controlier and its environment in terms of a mathematical model called a dynamical system. Since we are interested in the behavior of the system over time, we introduce a set of time points or ins'nnts, $T$. At any given instant. the snvironment can be in any one of a large number of possible states. This set of stales, $X$, is called the state spxice of the dynamical system.

The controller generally cannot perceive the state of the environment at any given instant. and so we introdnce a set of outputs. $Y$. corresponding to what the controller perceives of the state of the environment. Finally, we represent the actions of the controller in terms of a set of inputs to the environment. $l$. Notice that the the terms "input" and "output" assume the perspective of the environment and not the controller: this is a standard convention in control theory, and we adopt it throughout this book. 
(Inless further qualified (e.g.. "The oulput of the controller"), the terms "input" and "output." refer to. respectively, the input to and output from the ellvironment.

Next. we introduce temporally indexed variables to represent the state, $x(t)$, input, $u(t)$, and output. $y(t)$. at any given point in time. $t$. We refer to the different ways in which the state. input. and output can evolve over time as histories, time lines, or. in the parlance of control theory, trajectories. The set of all possible state histories or state-space tiajectories is defined as a setof mappings from time points $j$ states.

$$
H_{x} \triangleq\left\{h_{x}: T-X\right\}
$$

Similarly, we can define the set of output historiss.

$$
H_{Y} \triangleq\left\{h_{Y}: T-Y\right\} \text {. }
$$

We generally restrict the set of state histc. ies $L$ y requiring that the evolution of the system state obey certain laws. 'l'hese laws governing the behavior of the environment are uften oferred to as the system state equation(s). We represent thestäte equation by a function that maps states and inputs to states,

$$
4
$$

$$
x(.+1)=f(x(t), u(t)) \text {. }
$$

Here we employ a difference equativi, but. we night have used a system of differential equations. a finite-state automaton, a stochastic process. or a set of axioms ia a suitable ' igic. The choice of representation will depend on the structure of tine (c.y., integers or the real numbers), the nature of the physical processes we are trying to inodel, and our own preferences.

Since the controller cannot dirertly perceive the state of the ellvironment, we also restrict the set of output histories by defining an out.put function that maps states to outputs corresponding to the signals received by the controller's sensors,

$$
y(t)=g(x(t))
$$

This signal invariably contains less infornation than we would like. and. in most cases, it is noisy and difficult to interpret. ${ }^{1}$

\footnotetext{
'It is the oncertainty resulting from this noisy signal and the fact that information about the state of the euvirunneut is [reyuently delayed in procesoing that give rise to the need for a systematic treatmenl of control [5].
} 
So far. we have said nothing about the role of the controller. As with states and outputs. we can define a set of input histories desc ibing the erolution of the actions taken by the controller over time.

$$
H_{l^{\prime}} \triangleq\left\{h_{l^{\prime}}: T-l\right\}^{\prime}
$$

We restrict input histories according to the hardware and software available to build controllers. We describe the set of possible controllers in terms of functions from the set of sequences of outputs. denoted $I *$. to inputs.

$$
P \triangleq\left\{p: I^{-U}-U\right. \text {. }
$$

These functions are called cald control laus or policies. In the simplest case. the output function. $g$, is just the identity function. only the last state is relevant to the decision regarding what action to take. and the set of policies is defined as

$$
P \triangleq\left\{p: \mathrm{I}-L^{i}\right\}
$$

Now we need some objective for the controller to pursue. We begin with a rather ideal objective and define the controller's task. $K$. as a relation on the cross-product space of input/output pairs,

$$
\text { IICI } \times U \text {. }
$$

Actually specifying $\Pi$ can be quite difficult given that $\Pi$ indicates exactly what the controller is to do in every possible circumstance.

It may seem more nalural to think of a task specified in terms of the best action for a given state,

$$
I \subset I \times H
$$

Int uitively, we ought to be able to state the task independent of the particular signals received by the controller. Recall. however, that as far as the controller is concerned. the set of states collapses into a set of equivalence claseses determined by the controller's ability to perceive its environment.

Defining a task is a direct method of specifying the desired behavior of a controller. Less direct methods involve somehow specifying restrictions on the state histories of the dynamical system. For instance, we might define a goal as a subset of the set of state histories.

$$
\text { Cic } I x \text {. }
$$




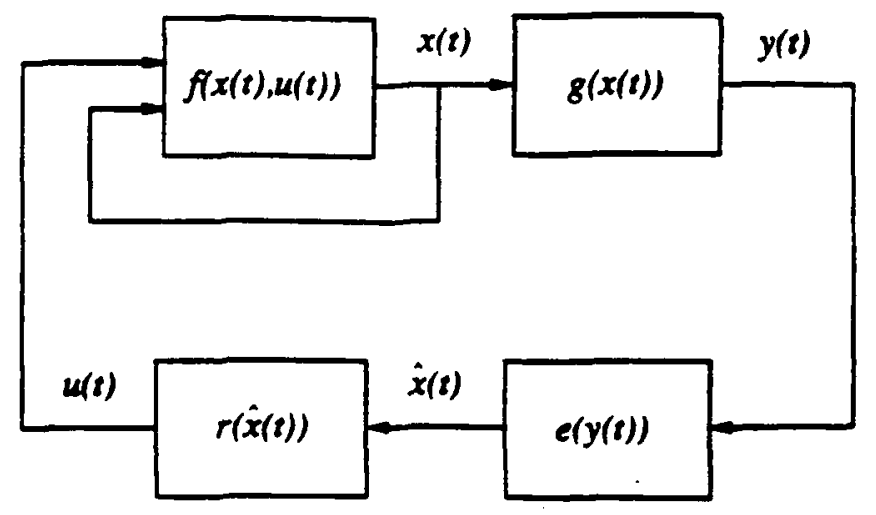

Figure 1.11: A dynamical system

In this case, we wish to find a policy, $p \in P$. such that a controller following $p$ restricts the behavior of the dynamical system to $G$. Such a policy is said to achicve $G$, and the solution is referred to as a satisficing solution.

Alternatively, we might define a value function,

$$
\mathrm{r}: H_{\mathrm{X}}-\mathbf{R} \text {. }
$$

that allows us to compare different state histories. In this case. we wish to find a policy, $p \in P$. such that a controller following $p$ forces the the state of the dynamical svstem to evolve according to a history that is maximal with respect to $V$. Such a policy is said to maximize $V$. and the solution is referred to as an optimizing solution. We ill refer to the problem of finding a policy to achieve a goal or maximize a value function as the control problem.

By providing the controller with a computational model of how certain properties of the environinent change over time. we can program the conI roller to extrapolate from a set of signals to predict what will happen with regard to those properties. A controller equipped with such a model can reasou about the consequences of its own actions and those of other processes. It is this aspect of reasoning about change over time that is mostly closely aseociated with the work in planning. The results of the reasoning are used to coastruct a plan or special-purpose policy to direct the controller's behavior. It is not required. however, that the reasoning be performed by the controller at the time the actions are being executed. The reasoning might be performed at some earlier time and the decisions as to what actions to take compiled into a compact program realizing a particular policy. 
As with most complex problems. it is useful to deconpose the control problem into componeut problems. For instance. the control problem is often decomposed into the state-estimation or obserintion problen and the inputregulation problem. The observation problem is concerned with recovering the system state from the system output. In the simplest case. designing a slate estimator or observer consists of choosing a function from the set.

$$
E \triangleq\{c: Y-X\}
$$

The output of the observer at time, $t$, is denoted, $\hat{x}$, indicating that it is an estimale. Similarly, designing a regulalor consists of choosing a $\sigma$ function from the set.

$$
R \triangleq\{r: \mathrm{X}-[:\} \text {. }
$$

Figure 1.11 shows a block diagran illustrating the various components of a dyuamical system and controller.

A good deal of the work in planuing implicitly assumes that the observation problem can be solved. and focuses on the input-regulation part of the coutrol problem. But planning need not. indeed should not. be conceived of so narrowly. As we will see. in niany problems. the state-estimation and input-regulation problems interact in a complex manner.

There are cases in which we can tackle the control problem by considering the state-estimation and input-regulation problems independently. In the case of linear dynamical systems corrupted by Gaussian noise and subject to quadratic performance criteria. the two problems are said to be scparable. and the dynamical systems are said to satiofy the separation property.

What this means in practice is that one engineer can go off and design an observer that is optimal by some established criterion (e.g.. produces an estimate minimizing the expectation of error). Then another engineer can independently design a regulator that is optimal with regard to a second criterion (e.g.. optimizes a particular value function over state histories). Separability guarantees that, when the observer and regulator are coupled together. the resulting controller will be optimal with regard to the stated criteria. This means that the actions taken by the regulator have no adverse affect on the ability of the observer to recover the system state. Conversely, the particular neasurements taken by the observer have no adverse affect on the ability of the regulator to control the system state.

Note that separability does not hold in general. Consider. for example, what separability would mean for a medical diagnosis and treatment problem. If the problem were separable. then we would not consider the cost of 


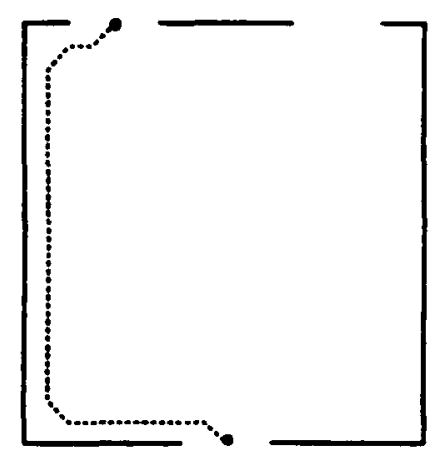

i.

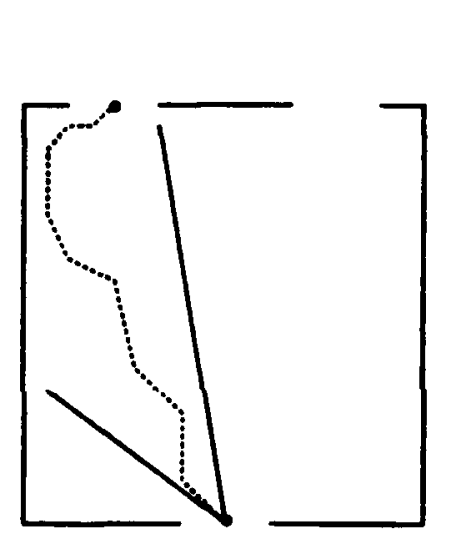

iii

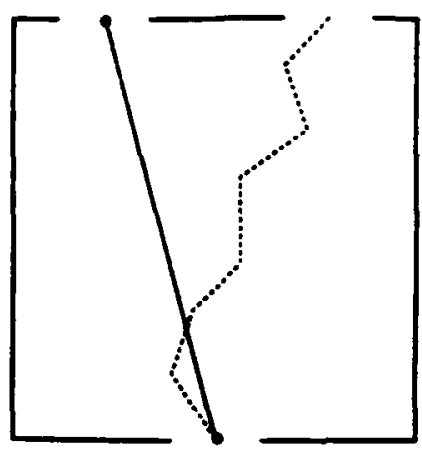

ii.

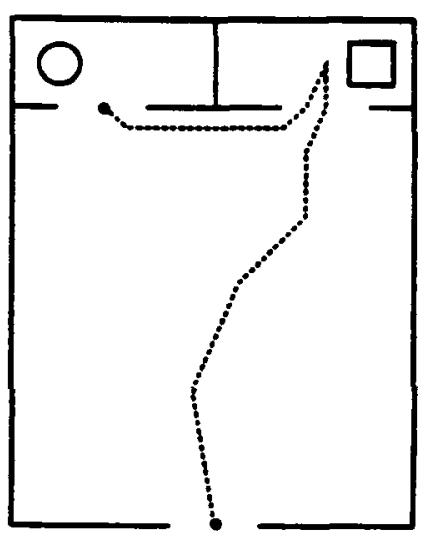

iv.

Figure 1.12: Interactions between observation and regulation

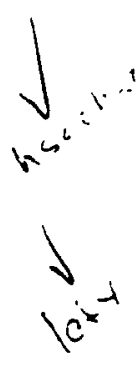

perforning tests when geuerating a diagnosis. In particular, there would be no reason to avoid eviscerating the patient in order to determine cause of the symptoms.

As another example. consider the task of a robot navigating in an office environment. Suppose that the robot is required to cross the room shown in Figure 1.12.j. The robot is to enter by the door shown at the bottom of the figure and leave by the door ou the at the top of the figure. Unfortunately, the robot's seusors do not provide accurate information regarding the robot's position and orientation. If the robot remains close to walls and it knows its initial positiou. it can generally do a good job of keeping track of its position with respect to the room. If, however, the robot roams off 
into the middle of the room. then it is likely to lose track of its position. In particular. if the robot tries to take the direct path rather than the wallhugging path as shown in Figure 1.12.ii. then it may very well exit by the wrong door. It is clear in this case that ubservation and regulation interact strongly.

Planning can play an important role in problens for which the separation property does not hoid. By using appropriate models. the controller can reason about the consequences of performing procedures given certair informational states, and. if necessary, design policies that result in the controller obtaining additional information. In Figure 1.12.iii, the controller, possessing a model of the robot s possible movenent errors, designs the following plan. While positioned near the door. the controller aims the robot so that by atlempting to drive straight it will either go through the door or arrive at a wall at which point it can move to the right hugging the wall to exit by the correct door. This plan is guaranteed to succeed assuming that the controller has an accurate model for movement errors. and will always be better than liugging the wall from very start.

In Figure 1.12.iv, the controller uses a somewhat different strategy. In this case. the controller directs the robot to head straight for the door on the left. The robot exits by the first loor it finds, but we assume that the robot can someliow distinguish between the offices that the two doors lead to. If the robot perceives that it is in the wrong office. then it exits the office, using the wall-hugging strategy to find the office next door.

The main point of this discussion is that as far as we are concerned the planning problem and the control problem are the same problem. In the rest of the book, we continue to talk about planning and control separately as a means of emphasizing particular issues or techniques closely associated with one or the other of the corresponding academic and engineering disciplines.

\subsection{Embedded Systems}

The primary computational task of a rohot controller is to make decisions conceraing what to do next. What to do next is generally thought of in terms of what actuator command to issue next, but there are often other deciajons to be made concerning what computations are to be performed and when. Robot decisions are made with regard to certain desirable behaviors (r.g., avoid running into obstacles, or avoid spilling expensive chemicals). These behaviors and the environnent in which they are to be achiever de- 


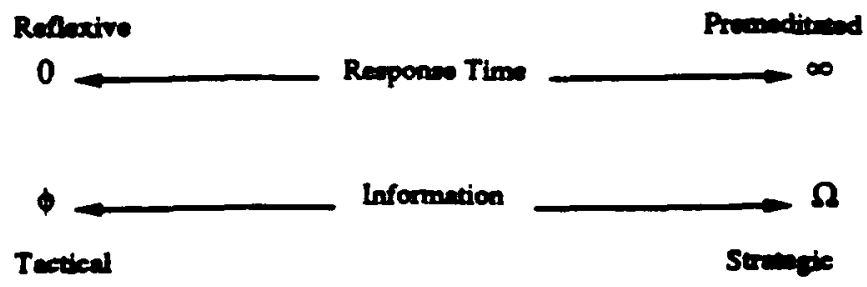

Figure 1.13: Two dimensions of control

termine how the rorresponding decision processes are to be implemented. As mentioned in the beginning of this cliapter, it is often convenient to distinguish between the controlle: and the controlled process. We can think about what we would like a cinil roller to do. but. when it comes down to building a controller, we have to comnit to specific hardware and software, and this commitment will determine what decisions the controller is capable of making. The controller is said to be rmbedded in its environment. To analyze a controller, we have to be able to relate the state of the controller and the state of the processes the controller is seeking to control. How well a controller can cope with a giver: environment will depend upon the amount of time between sensing a situation and being required to respond to that situation. and the availability and volatility of the information potentially "sefnl in deciding how to respond. These fartors snggest two dimensions useful in categorizing control problems and their solutions (see Figure 1.13). The less information available and the less time the robot has to process that information. the less likely that the robot's response will account for the possible consequences of its actions. The more information arailable and the more time. that the robot has, to reflect on it, the more likely that the robot will be able to generate a response thiat avoids nnpleasant consequences and takes advantage of pleasant ones. These dimensions are quite different from those used to categorize prohlems and solutions in inost areas of computer scienre.

Computer science concerns itself primarily with off-line computing tasks (i.e.. date processing tasks). There are two distinct criteria for such tasks: correctness and speed. Most computing tasks in robotics are concerned with controlling processes, and, in particular, controlling processes indirectly and in real time. The notion of correctness in the traditional framework assumes soune absolute standard that abstracts away from time. What a control algorithm should compute depends upon the sorts of procensen it attempt.s 
to control and the information about those processes it can extract from the environment.

Suppose that a controller generates a sequence of actuator commands that would have enabled the robot to perform a complex maneuver had they been generated a few seconds earlier. As it is, however, the robot fails to perform the inaneuver and tumbles down five flights of stairs. At first blush. it would appear that the controller has failed in its assigned task. but we may be taking too narrow a view. l'erhaps the robot was usinf most of its available computational resuurces to figure out how to disarm its malfunctioning nuclear self-destruct unit: a task that it did manage to carry out successfully.

The problem faced by a robot controller is essentially that of optimizing

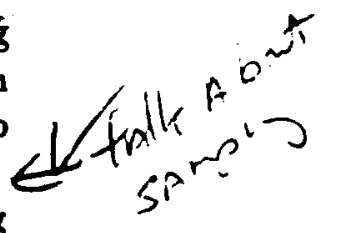
a large number of factors ( $\epsilon . g$. . time. monev, nechanical wear) simultaneously. In order to make such optimizations. a controller has to !'uild up a representation of a complex siluation (e.g., one spread out in time and space) and thell decide what to do by taking into account how the various pieces of the picture are predicled to interact with one another. For the uptimizations to be elfective. however, the robul must respond in a timely manner. It would be nice to prove that a given controller satisfied some specified criterion for correct behavior. Unlortunately, for most interesting applications in robotics, such a proof would be prohibitively complex.

Most existing planning systems tend to be far too committed to the plans they formulate and tend to rely heavily on models of the environment and sul enough on the environment itself [4]. Such systems do not tailor their decision making to the siluation at hand. Given the same abstract task to achieve. these systems will perform the same computations no matter how much time and information is available. They cannot deternine when further planning is futile, and they do uot liave the eapability to consider alteruative stralegies when pressed for time.

Most existing control systems tend to take a rather narrow view of the world and the processes that they seek to control. As long as the world sub. scribes to the controller's model. these systems behave elfectively. Sooner or later. however, unanticipated influences intrude to render the model's predictions inaccurate, resulting in undesirable, and sometimes disastrons, conequences. Building a more complicaled model is not always the solution. A complicated model mav require more time to compute, thereby reducing the system's response time. All alternative to building a more complicaled model is to employ several simple models, each one tuned to a different range of situations. The controller then tries to determine which simple 
model applies. and changes the model when circumstances dictate. In some sense. this multi-model controller is emploving a more complicated model. but it is a model that-at least inuplicitly-takes into account the computational capabilities of the underlying hardware and the anticipated behavior of the processes being controlled. C'lapter 8 develops a framework for taking such considerations into account explicitly. in order to dynamically allocate computational resources to suit a given situation.

In subsequeut chapters. we will explore a number of methods for con-. structing and evaluating models of complex systems. We will consider how models are used to control processes. and what sort of tradeoffs have to be made in building effective control systems. The discussion covers both theoretical and practical considerations. The former due to our need to justify design decisions in terms of acceptable mathematical foundations. The latter due to our primary motivation in terms of programming robots to perform useful work. We begin by discussing the theoretical foundations for inodeling processes. 


\section{Bibliography}

[1] Andersson, Russell L.. A Rohnt Ping-Pong Plnyer: Experiment in RealTime Intelligent ('ontibl. ( MIIT P'ress. ('ambridge, Massachusetts. 1988).

[2] Donner. Marc D.. Rral-Time C'ontml of llalking, (Birkhauser. Boston. Massachusetts. 198i).

[3] Garev. Michael R. and Johnson. David S.. Computing and Intructibility: A Guide to the Theory of NP.Completeness. (W. H. Freeman and ('ompany, New York. 1979).

[4] Cieorgeff. Michael !', Planning. Lranh. J.F. (Ed.), Annual Revieu' of Computer Scienre. lolume 2. (Anuual Review lnc. 1987).

[5] lioditschek. D.. Robot ('ontrol Systenıs. Shapiro. Stuart. (Ed.). Encyclopertia of Artificial Intelligrner. (John Wiley and Sons. New York. 198T). 902-92.3.

[6] Raibert. Marc IL.. Legged Robsts That Balance. (MIT Press. Cambridge. Massachusetts. 1986).

[i] Rosenschein. Stan. Formal Theories of Knotrledge in Al and Robotics. Technical Report (SLl-si-S4. ('enter for the Study of Language and Information, 198\%. 


\section{Chapter 2}

\section{Dynamical Systems}

For our purposes. a pmress model is a device that. given certain information about the state of a physical system. enables us to determine certain other information about that system. The device usually includes some mathematical characterization of the system s properties and how they relate to one another. It also includes some sort of a calculus whereby an engincer or a machine can compnte the predictions of the model given some initial conditions.

Process models are used hy engineers to resign control sustems. In soine cases. the process model is used only to evaluate a given controller. In other cases. the process model becones an integral part of the control system. In this chapter. we consider a few of the large number of process modeling techniques available to the engineer. and develop some notation for describing process models that will be used in subsequent chapters.

\subsection{Constructing Physical Models}

To construrt a morlel for a process, we liave to identify those properties of the world that determine the behavior of the process. First. there are those properties that prompted our int -est in the process to begin with. In the cace of the tank-filling process described in Chapter 1. we are primarily interested in the height of the Huid in the tank. Second, there are those properties that atfect the properties that we are interested in. In order to account for the level of fluid in the tank. we have to know the dimensions of lhe tank. the flow characteristics of the input and output pipes, and the

\footnotetext{
${ }^{\circ}$ (C) 1990 Thoma Deall. All righin recerved.
} 
I: sition of the valves. It is easy 10 minderestimate the difficulty of this part of the modeling task.

Textbooks typically just give the student the set of physical properties that he or she needs to be concerned with. There is an implicit assumption that these are all and ouly the properties that need to be considered. How do we know that the temperature of the lluid does not alfect the height of the Iluid in the tank? Well. of course. we don i know this. The temperature nay affect the fluid height by changing the rate at which the fuid evaporates: however. given that the temperature does not vary substantially, the effect of temperature on fluid height is negligible.

Almost any property of the world cun have an impact on the level of the fluid in the tank: agricultural trends affect global weather patterns that affect local tewperature and humidity that ultimately affect fluid height. The predictions wade by a particular model are likely to be accurate only if certain assumptious hold. Whether or not to account for a given property of the world in a particular model depends on a number of factors: the maguitude of the effect (i.e.. does it result in substantial changes in the properties of interest), the probability of the effect (i.e.. do the changes occur with high frequency), and the complexity of the model (i.e.. what additional compulations are required to account for the property in the model).

This last is particularly inportant. and. yet. it is often overlooked in evaluating a nodel. There is often some utility in getting an answer to a question quickly. If this were not the case. you would always want the model that makes the most accurate predictious possible. Given that time has to be t.aken into account. there is a tradeoff to be made regarding the accuracy of the model and the time that it takes to compute its predictions.

The following sections describe some basic methods for modeling physical processes in coutrol theory. Section 2.2 cousiders the use of the differential and integral calculus for modeling processes and analyzing the behavior of control systems. focussing on ideas from classical control theory. Section $\mathbf{2 . 3}$ considers the general problem of modeling dynamical systems and introduces idea from linear system theory. drawing upon results frow modern control theory. 


\subsection{Mathematical Modeling in Control Theory}

Much of control theory depends on the use of mathematical models based on the techniques of the integral and differential calculus. These techniques enable the control theorist to model a wide variety of nechanical. electrical. fluid. and thermodynamic systcms. By modeling both the controlling process and the process being controlled as a set of differential equations? the control theorist is able to anadyze behavior of the combined system. and predict the performance characteristics of the controlling process ( $\epsilon . g .$. how fast the system responds to a disturbance or change in input). In this section, Ne summarize some of the issues involved in modeling physical systems using the techniques of control theory.

Anvone who lias taken a course in differential equations or advanced ralculus has seen numerous examples of mathematical models of physical systems. Most introductory texts on the differential calculus incinde idealized models of population growth. the decay of radioactive materials. and the fluctuation in prices as a function of supply and demand. If you took a. physics course. you were early on exposed to Newton's laws of motion. Newton's second law of motion states that the product of a body's mass and the acceleration of its center of mass is proportional to the force acting on the body. Let $x$ be a function that depends on $t$ and denotes the position of the center of mass of the object as measured from some fixed point along a vertical line. Let $M$ be the mass of the object. and $\mathcal{F}$ be the force acting on the object in the direction of travel. The following differential equation

$$
M \frac{d^{2} x}{d t^{2}}=F
$$

is called the equation of motion of the body.' If we know something about the forces acting on the body. then we can use this equation to make predictions about the motion of the body.

If $x$ is the directed distance upward of the object as measured from the surface of the earth. and "o is the object's initial velocity, then. assuming that the only force acting on the object is gravity, Equation 2.1 becomes

$$
M \frac{d^{2} x}{d t^{2}}=-M g
$$

\footnotetext{
'To simplify the discunoson. we implicitly alnpt the standard ayatem of unitn for mensuring mase. diatance, and tinie so that lie constaut of proportionality is one.
} 
where is $g$ is the acceleration due in gravity near he surface of the earth. We can solve this simple second-orter differential equation. by integrating twice and using the initial conditions to determine the constants of integration. The following formula

$$
x(t)=-\frac{1}{2} g t^{2}+t_{0} t
$$

describes the position of the object at $t \geq 0$ given the initial conditious

$$
r(0)=0 . \quad \frac{d x(0)}{d t}=v_{u},
$$

and assuming that the object is propelled "pward at time $t=0$. From Equation 2.3 , we can predict the maximum height $\left(v v_{c}^{2}: 2 g\right)$ reached by the object and the time it takes the object to fall back to the surface of tue earth $\left(2 v_{0} / g\right)$. Equation 2.3 together with tocis of the differential calculns provide us with a simple model of an object falling through a gravitational field.

We know that Equation 2.3 is only approximate in that it 1 zgiects several important influences on objects falliug through a relatively dense atmosphere under the influence of gravity. For instance, Equation 2.3 treats gravity as a cunstant acceleration whereas we know that Newton's inverse square law provides a more accurate estimate of tiee force due to gravity acting on an ol) ject. If the earth is assumed to be a sphere of radius $R$. and $r$ denotes the distance trom the center of mass of the object to the center of the sphere. then

$$
M \frac{d^{2} x}{d t^{2}}=-\frac{M g R^{2}}{r^{2}}
$$

can provide a more accurate estimate of the position of the object than that provicied by Equation 2.1. especially in the case of an object that travels a significant fraction of the distance $R$.

We can alsu account for the dariping force exerted on the object by the atmosphere as the object moves along its trajectory. If the damping force is pruportinnal to the object s velocity, and $C$ is the damping constant. then

$$
M \frac{d^{2} x}{d t^{2}}=-\frac{M g R^{2}}{r^{2}}-C \frac{d x}{d t}
$$

will, at least potentially. provide a better estimate than eqilations that ne glect friction. Potentially. lecause. having identified that some property of the environment influences a particular process, you still have to determine the form and the magnitude of that influence. There are situations in which 


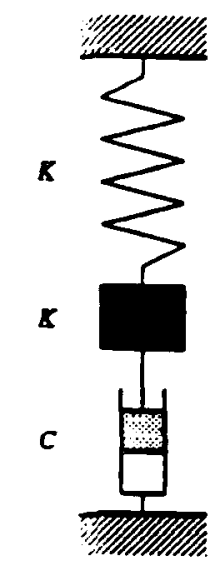

Figure 2.1: A spring-mass-dashpot svstem

the damping force is more nearly proportinnal to the square or the cube of the velocity. In addition. the damping "constant" may not be constant at. all. dependent as it is on the shape of the object and the density of the air through which the object is moving. If you are not careful. yon can actually reduce the predictive accuracy of a model by trying to account for additional properties.

As another example of physical modeling. Figure 2.1 shows a block of mass $M$ suspended from the ceiling by a spring and connected by a rigid rod at its base to a damping device called a dashpot. The spring counteracts the force of gravity and the dashrot lends to inhibit vertical motion in either direction. Suppose that the force exerted by the spring is equal to the product of the distance that the spring is stretched or compressed and $\boldsymbol{K}$. the spring constant. Let $d$ be the distance past the spring s resting length surh that the force of the spring completely offsets the force of gravity, and the block will remain at rest (i.e.. $M g=K d$ ). The equation of motion for the block. neglecting the dash pot. is

$$
M \frac{d^{2} x}{d t^{2}}=M / g-K(x+d)=K x .
$$

To account for the dashpot. We assume that the damping action of the dashpot is proportional to the velocity of the block and introduce another term into Equation 2.5. The result is

$$
M \frac{d^{2} x}{d t^{2}}+C \frac{d x}{d t}+K x=0
$$



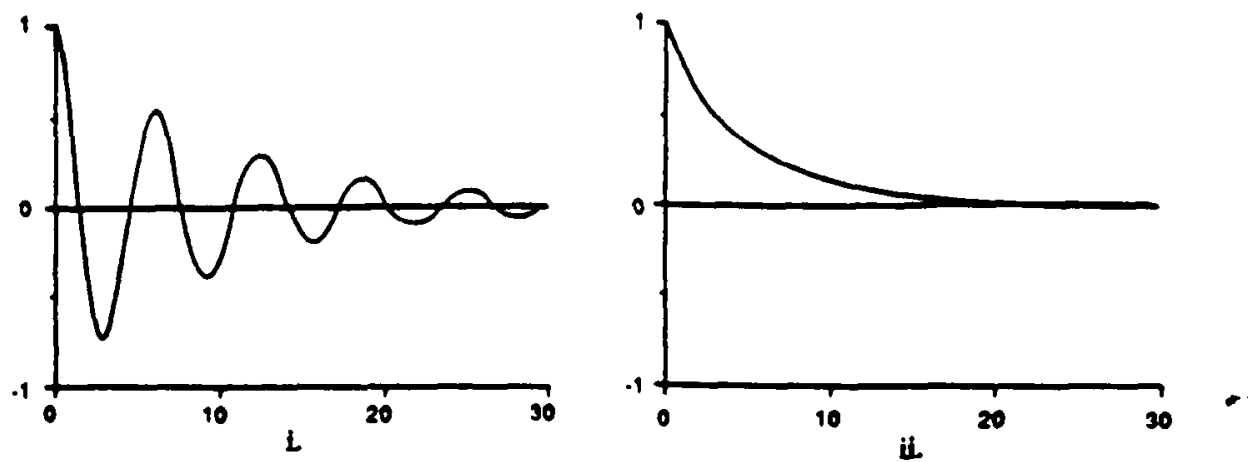

Figure 2.2: Response of the spring-mass-dashpot system in the (i) underdamped and (ii) overdamped cases.

where $C$ is the damping constant.

There are three different solutions to Equation 2.6 depending on whether the quantity $C^{2}$ is less than. greater than. or equal to the quantity $4 M K$. These solutions correspond to the underdamped. overdamped. or critically damped cases. If $C^{2}<+H M$. then the specific solution to Equation 2.6 that satisfies the initial conditions.

$$
x(0)=x_{0}, \quad \frac{d x(0)}{d t}=0,
$$

is given by

$$
x(t)=x_{0} e^{\alpha t}\left(\cos \omega t+\frac{\alpha}{\omega} \sin \omega t\right) .
$$

where

$$
\alpha=\frac{C}{2 M}, \quad \omega=\frac{1}{2 M}\left(4 M K-C^{2}\right)^{1 / 2} .
$$

In this (the underclamped) case. the mass oscillates about the equilibrium point. its amplitude derreasing exponentially with tiuse as shown in Fig"Ire 2.2.j. If $C^{2}>4 K M$, then the specific solution to Equation 2.6 satisfying the same initial conditions is given by

$$
x(t)=\frac{x_{0}}{3-\alpha}\left(\beta \epsilon^{-\alpha t}-\alpha \varepsilon^{-\beta t}\right) .
$$

where

$$
n=-\frac{1}{2 M}\left[-C^{\prime}+\left(C^{\prime 2}-4 M K\right)^{1 / 2}\right] . \quad, \quad 3=-\frac{1}{2 M}\left[-C-\left(C^{2}-4 M M\right)^{1 / 2}\right] .
$$




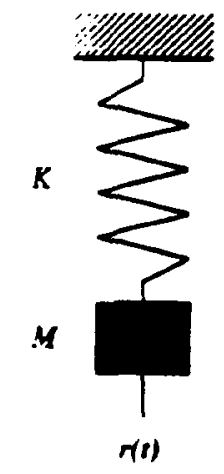

Figure 2.3: An external force acting on a spring-mass system

Figure 2.2.ii illustrates the hehavinr of the resulting overdamped system. The important thing to note here is that. assuming $M$ is fixed. we can vary $I I$ and $C$ to achieve different behaviors.

Control theorists are often iulerested in how a physical syatem responils to a particular input signal. The step input. corresponding to a fixed-size instantaneous change in the reference or a disturbance, provide a convenient basis for comparing performance. In the case of the spring-mass-dashpot, a step input might correspond to the block being displaced froin its equilibrium point or given some initial velocity. Equation 2.6 might serve as a simple model for an automobile shock absorber. The input signal would correspond to a force acting on the mass (e.g.. the automobile hitting a bump in the road). The engineer (lesigning such a system is interested in llie rharacteristics of the output signal corresponding to the changes in the position of the inass. th particular. the engineer wants to know whether or not the control system he or she designs is stable. A system is said to be stahle if its response to a bounded input is itself bounded. In the case of our spring-mass-dashpot system. if wie displace the inass a small amount from it.s equilibrium point, it will eventually return to that point. Similarly; if we give the mass some small initial velocity, it will also eventually return to its equilibrium point.

Unotable systems can manifest undesirable and sonetimes violent behavior (e.g., thernal runaway in a uuclear power plant). Suppose that we eliminate the dashpot from our spring-mass-dashpot system and introduce an additional, external force acting on the mass as pictured in Figure 2.3. 


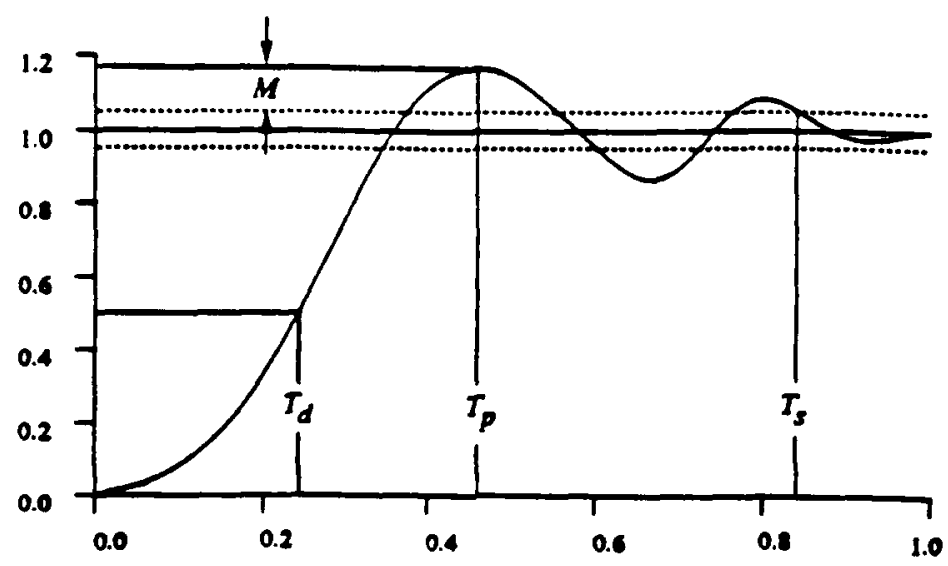

Figure 2.t: Transient response to a step input indicating $T_{d}$ (delay time) the time required for the controlled variable to reach $50 \%$ of the target. $T$, (settling time) the tine required for the controlled variable to achieve and maintain a value $\pm 5 \%$ of the target. $T_{p}$ (peak time) the time at which the coutrolled variable achieves the largest value above the target, and $M$ (peak overshcisi) the largest value of the controlled variable above the target.

Suppose that the external force is periodic of the form

$$
r(t)=R \sin \omega t
$$

where $R$ is a positive constant. The equation of motion is

$$
M \frac{d^{2} x}{d t^{2}}+K x=R \sin \omega t
$$

If $\omega=(K / M)^{1 / 2}$, then the amplitude of the oscillations will increase due to the phenomenon of resunauce [10]. The model predicts that the oscillations will increase indefiuitely, but. of course, there will come a point past which the mathenatical model is no louger appropriate and other physical properties will come into plav (e.y.. the spring breaks or the device generating $r(t)$ reaches saturation ).

Stability is just one aspect of a system's transient response to a step input (i.e. the behavior of the system in transition from one stable state to another as a result of a step input). An engineer usually is also interested in the system's settling time (i.e.. the amount of time it takes the system to 
achieve a state in which .le value of the controlled variable is within some amall percentage of the target value). the system s strady-state error (i.c.. the percent error of the sustem in the limit.). and the sustem sovershomt (i.f.. the maximum past the target that the system achieves in responding 10 step input). Figure 2.4 illustrates some of the important characteristics of a system s transient response to step imput [(6. 12].

Peal overshoot is a particularly important transient response characteristic in a number of applications. In some cases, the sort of underdamped behavior shown in Figure 2.2.i is unacceptable. In attempting to restore equilibrium. the system overshoots the target or equilibrium point. In the case of a robot arm positioning a part. overshoot inight correspond to the part striking a surfare. In the rase of the liquid-level system of C'hapter 1. niershoor might mean that the level of fluid in the tank goes above the top of the tank, spilling Hnid on the floor.

A good deal of control thenry is rnncerned with analyzing the performance of control systems with regard to criteria surh as st.ability, settling time. steady-state error, and overshoot. One way to analyze a control system is to build a mathematical model as a system of differential eqnations. solve the equations. and then examine the behavior of the system in the time domain. This is essentially what was done in our analysis of the springmass-dashpot systein above. This method of analysis can be complicated by the fact that the equations for any reasonably complex control system are likely to be difficult to solve. and. in order to find parameters for the rontrol system that provide good performance, it may be necessary to to look at a large number of special cases. While there exist effective methods for anaivzing control systems in the time domain. one of the great successen of what is called classical control theory has been the development of mathematical techniqueo that enable an engineer to recast a control problem as a problem in the frequency domain. Most of these techniques rely of the use of the Laplace transform.

The Laplace transform enables the control theoriat to avoid working with differemtial equations by replacing these generally difficult-to-solve equationa with simpler algebraic eqfualions. Since the Laplace transform exist.s for many linear differential equalions enconntered in control systems design, methods baced upon the use of the Laplace trausform are widely euployed in the analysis of control systems. The Laplace trausform of a function of 
lime. $f(t)$. is defiued as

$$
F(s)=\int_{0}^{\infty} f(t) \varepsilon^{-s t} d t=\dot{L}(f(t)) .
$$

The Laplace transform of the deritative of a function can be obtained from Equation 2.7 using integration by parts

$$
\dot{L}\left(\frac{d f(t)}{d t}\right)=s \dot{L}(f(t))-f(0) .
$$

However. it is usually not necessary to derive the Laplace transform of a function every time that the cugincer is faced with a new problem. Tables of functions and their Laplace transforns have been compiled for most functions commonly encountered in engineering applications.

The Laplace transform of a sun of two functions is just the sum of the Laplace transform of the first function with that of the second. Using lhis fact and the tables of Laplace transforms, the control engineer can rather easily obtain the Laplace transform for many differential equations used in modeling physical systems. The advantage is that the resulting algebraic equation usually can be exsily solved for the variables of interest. The tmasfer function of a control system is defined to be the ratio of the Laplace transform of the input variable to the Laplace transform of the output rariable. By analyzing a control system in terms of the relation of the Laplace transform of the inputs to the Laplace transform of the outputs. it is possible to gain a good understanding of the system s performance properties. $^{2}$

To make the analysis of control systems even easier, there are tables that provide the transfer functions for many of the differential equation relations encounted in control systems. An engineer can design a control system using various control components connected to one another by the way in which they pass signals. Froin these separate components, the engineer can derive the transfer function for the complete control aystem algebrairally. The familiar block diagranis displayed in the control theory literature provide a convenient graphical representation of the underlying process nodel. The

\footnotetext{
${ }^{2}$ Preyency-domain methods involving trander fnnctions are so named becanse they allow the enginect to analyse the hehavior of a eystem in terma of ite remponme to inputa of varying frequenciea and amplitudes. By evaluating the tranfer function. $T(s)$. al s=ju for any $\omega \in \mathbf{R}^{+}$. we obtain a complex anmber. $T(j \omega)=\alpha(\omega)+j \partial(\omega)$, whone magnitude. $\sqrt{\mathrm{cr}^{2}(\omega)+3^{2}(\omega)}$, represeuts the response of the aystem in stewdy stale to a sinusoidal input of frequency, $\omega$. in terme of the ratio of the ontput to the input amplitude.
} 

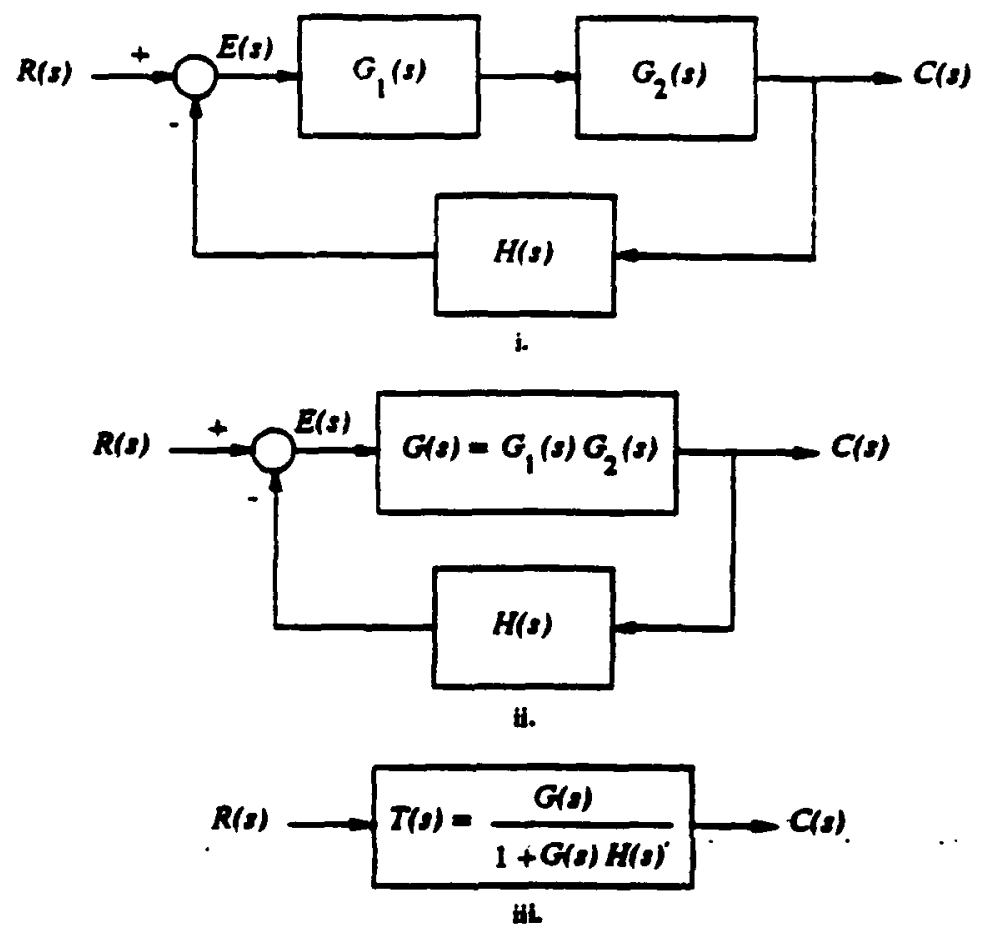

Figure 2.5: Block diagraın of a control system utilizing feedback 
boxes in such diagrams are usually labeled with the transfer function tor the corresponding system component and the arcs indicate the signals passing between components. Figure 2.j.i depicts the block diagram for a control system in which the output of the plant is fed back through some sort of a filter or amplifier and combined with the input to provide an error signal used by a compensator in controlling the plant. The control system pictured in Figure 2.5.i illustrates a simple instance of error-driven feedback. in which the system reference sigual is continuously compared with the system's output in order to adjust various system parameters. ${ }^{3}$

Block diagrams can be simplified by algebrajcally combining the transfer functions of connected components according to a few simple rules [6]. For instance. the two blocks labeled $C_{1}(s)$ and $G_{2}(s)$ in Figure 2.5.i can be combined to form.

$$
G(s)=\frac{C(s)}{E(s)}=G_{1}(s) G_{2}(s) \text {. }
$$

noting that $C(s)=E(s) G_{1}(s) C_{2}(s)$. The simplified block diagram is shown in Figure 2.5.ii. The simplest block diagram is just a single box labeled with the transfer function for the complete coutrol systen. For instance, we can reduce the block diagram for the system showu in Figure 2.5.ii tn a single component with input $R(s)$, output $C(s)$, and transfer function.

$$
T(s)=\frac{C(s)}{R(s)}=\frac{G(s)}{1+G(s) H(s)}
$$

noting that $E(s)=R(s)-H(s) C^{\prime}(s)$ and $C^{\prime}(s)=E(s) G(s)$. This simplest block diagram is shown in Figure 2.5.iii. The function. $T(s)$, known as the rlosed-loop tmnsfer function. is the basis of many existing control systems.

Much of the control theory found in lextbooks deals with what are called linear systens. A system is said to be linear in terms of inputs and outputs if and only if it satisfies the properties of superposition and homogeneity [6]. A system satisfies the property of homogeneity if for any constant $h^{\circ}$ and input $x$ for which the output of the system is $y$, if the systen is input $I^{\circ} x$. the system outputs $\mathbb{R} y$. A system satisfies the superposition property if for any two inpets $x_{1}$ and $x_{2}$ with corresponding outputs $y_{1}$ and $y_{2}$, if the system is input $x_{1}+x_{2}$, the system outputs $y_{1}+y_{2}$. At first blush. the restriction to linear systems would seem to relegate much of control theory to a purely academic pursuit given that most uatural systems are nonlinear at least

\footnotetext{
"In some texts, errnotriven feethack is nynonymons with unty feedbnek. corrempmiling to the case in which $\boldsymbol{H}(\mathrm{s})$, in Figure 2.5.i. is the identity function.
} 
in some range of their variables. Fortunately, we c.n derelop reasonably accurate linear approximations $h$ identifying almost-linear regions in the operating range of uonlinear systems. If ilie natural operating conditions of a system vary over a wide range. it may lue necessary to develup several linear approximations and switch between them when necessary. This nethod of switching between coutrollers is the basis for a technique used in adaptive control called gain scheduling.

Other approximations are often inade to simplify anaiysis and impleinentation. For instauce. it is often possible to eliminate some of the higherorder terms in a model involving differcuitial equations. By eliminating the higher-order terms. the subsequent analysis may ignore effects due to highfrequency inputs. Hopefully, these effects will not pose a problem in practice. but no model should be relied upon without careful experimentation comparing the performance of the modeled system with that of the real one.

While we have emphasized modeling continuous processes. control theory provides tools for modeling discrete processes as well. The discrete analog of a differential equation is called a difference equation and is used extensively not only to model discrete systems. but also to approximate continuous systems using digital hardware. Analog computers still play an important role in eugineering, but, with the iutroduction of inexpensive digital computing hardware. a great deal of attention las been given to discrete nodeling tecliniques.

Digital computers are limited in that they can only sample system variables at discrete points in time. Usually. the delay between samples is fixed of duration $r$. By introducing a new complex variable

$$
z=\epsilon^{o r} \text {. }
$$

we can define a discrete version of the Laplace transform called the :transform for a discrete function $f(k)$ as

$$
\mathcal{Z}(f(k))=F(z)=\sum_{k=0}^{\infty} f(k) z^{-k} .
$$

There exist techuiques, analugons to thuse based on the Laplace 1.ransform, for using line z-trausform to aualyze the response characteristics of control systems [3]. Aualysis using the $=$-trausform is complicated somewhat by the fact that information is irretrievably lost in a sampled system. It is generally necessary to identify the various frequency components of the 
input signal in the Fourier domain, and adjust the sampling rate accordingly to avoid cffects due to signai aliasing (i.c.. mistakenly associating high frequency components of the signat with lower frequency components). According to a theorem of Claude Shannon aliasing can be avoided entirely by onsuring that the sampling frepuency $(1 / \tau$ samples per unit time) is at least twice the frequency of the highest frequency component of the input signal $[+]$. Of course. it may not he possible for the digital hardware to sample that quickly or perform the necessary computations required to generate an appropriate response. The probirm of implementing complex control strategies that keep pace with a rapidy changing environment will be addressed frequently in this monograph.

There exist processes for which we know the form of an appropriate model (c.g.. we know that the process can be modeled using a $k$ th-order linear differential equation with constant coefficients), but we do not know the parameters of the model. For instance. the system we are trying to model might be a black box that re know to be a single-input single-ontput linear system. but the model parameters do not correspond to any known physical parameters such as the spring constant or the damping constant in the model for the spring-mass-dashpot system. In this case, it may he possible to find values for the parameters of the model by sampling the input and output of the system. and "fitting' the parameters of the model to the data. This is a special case of what is called system identification. and constitutes an injportant. part of the branch of control theory known as adaptive contml $[1,11]$. System identification ran be done off line during the design of the control system as prologue to the sort of analysis described above. In adaptive control. system identification is done on line by the control system. and the results of system identification are used to adjust the parameters of a controller. This approach to control is particularly useful if the physical system that you are atteinpting to model changes over time (c.g.. a plant with nechanical parts that are subject to wear).

One particularly convenient feature of the mathematical models used in control theory is that, at least as far as the analysis is concerned. what one learns abont design in one area is immediately applicable in another area for which there exists appropriate analogical apparatus mapping the variables hetween the two systeens [ 6 ]. For instance, the engineer familiar with the analyas and denign of electrical control aystems can often apply what. he or she knows to the analysis and design of merhanical or fluid control systems. The basic models and their corresponding equations appear again and again. and hence much of what is learned can be compiled into tables. tools. and 
ronkbook-style methods for dealing with conimonly occurring spec ic cases $[4]$.

In this section. we considered some of the basic techniques involved in modeling physical systems. We briefly tourhed upon some of the methods and terminology of control theory. specifically what is referred to as classical control theory. As was mentioned. classical control is most closely associated with analysis in the frequency domain. In the next section. we introduce a particular class of physical systems important from the standpoint of control. and consider modeling technicues drawn from modern control theory.

\subsection{Modeling Dynamical Systems}

The techniques described in the previous section are primarily useful for physical systens that can be modeled with a single input and a single output variable. In this sectiou. we consider systems modeled with any finite number of input and output variables. We restrict our attention to a limited class of physical systems called dynamical systems. A dynamical system is defined by the following mathematical objects and axioms governing them. ${ }^{4}$

- A set of time points $T \subset \mathbf{R}$

- A set of states.

- A set of inputs $t$

- A set of outputs $Y$

- A set of input lunctions
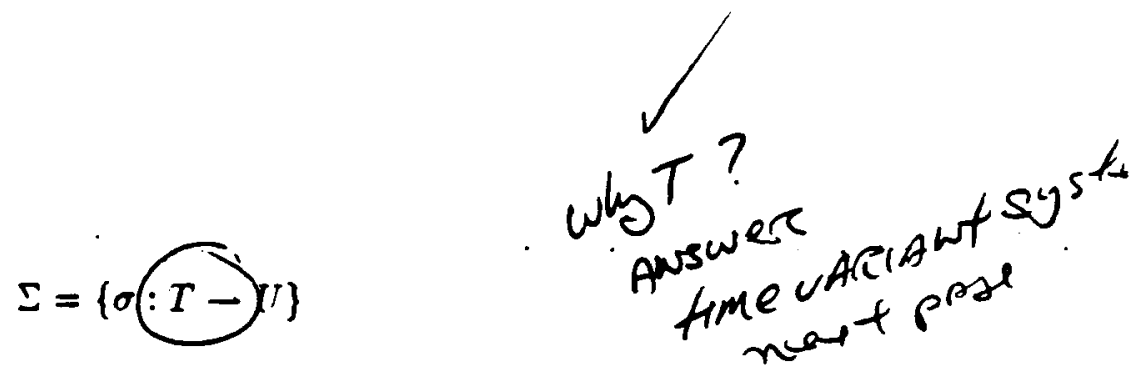

- A state transition function

$$
f: T \times T \times \mathbb{I} \times U-X
$$

whose value is the state $x(t)=f(t ; \tau, x, \sigma) \in X$ resulting at time $t \in T$ starting from all initial state $x(\tau)$ at time $r \in T$ influenced by the action of the input $\sigma$.

\footnotetext{
'The definitions provided liere roughly follow thooe of Kialman [9] though we have sacrificed rigour in some places to avoid lengthy technical commentary. Oor objective liere is to set the atage for a discusaion of practical methods. and not. as in the case of lialinan's work, the precise description of inathematical abatractiona.
} 
- An output function
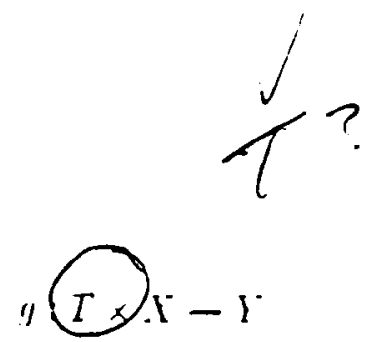

We impose some additional restrictions. In particular. for any $t_{1}<t_{2}<t_{3}$ and $\sigma \in \Sigma$ we have

$$
f\left(t_{3} ; t_{1}, x, \sigma\right)=f\left(t_{3} ; t_{2}, f\left(t_{2} ; t_{1}, x, \sigma\right), \sigma\right) .
$$

and for any two input functions $\sigma$ and $\sigma^{\prime}$ that agree on the interval $(t . \tau)$ we have

$$
f(t ; \tau, x, \sigma)=f\left(1: \tau, x \cdot \sigma^{\prime}\right) .
$$

The first of these restrictions provides a reasonable property that allows us to compose inputs. The sec ond is often referred to as the principle of causality $[2] .{ }^{5}$ Civen an input function $\sigma \in \Sigma$ and an interval of time $\left(t_{1}, t_{2}\right]$, an input segment $\sigma_{\left(t_{1}, t_{2}\right)}$ is just $\sigma$ restricted to $\left(t_{1}, t_{2}\right)$. We require that. if $\sigma, \sigma^{\prime} \in \Sigma$ and $t_{1}<t_{2}<t_{3}$. then there exists $\sigma^{\prime \prime} \in \Sigma$ such that $\sigma_{\left(t_{1}, t_{2}\right]}^{\prime \prime}=\sigma_{\left(t_{1}, t_{2}\right]}$ and $\sigma_{\left(t_{2}, t_{3}\right]}^{\prime \prime}=\sigma_{\left(t_{2}, t_{3}\right)}$. This last property is called conratenation of inputs [9]. aud provides us with a useful closure property for the set of input functions.

We also assume that the response of a dynamical system is independent of the particular time at $v$ hich it is exercised. We say that a dynamical system is time invariant if the following properties hold.

- $T$ is closed under addition.

- $\Sigma$ is closed under the shift opemtor. $z^{s}: \sigma-\sigma^{\prime}$. defined by

$$
\sigma^{\prime}(t)=\sigma(t+s)
$$

for all s.t $\in T$.

- For any s.t. $\tau \in T$. we have

$$
f(t ; \tau, x, \sigma)=f\left(t+s ; \tau+s . x . z^{s} \sigma\right)
$$

- The output function $g(t .$.$) is independent of t$.

\footnotetext{
'Thare is a cendency in mathematical control theory to refer 10 certain acumptions or rentriction a principles. This is particularly the case where the mathematics would be dificult or imponsible without imposing some restrictions. In some cases. auch as the principle of cansality described here. the reatrictions seem innocuous enough. but in otbers Hiey appear to motivated by nothing more than mathematical convenience or neceanity. IVilnew the fact that anperposition. which underlies linearity, is often introduced an the "priaciple of superposition" [9].
} 
We will be concerned with continuous time dynamical systems (i.t. $T$ is the real numbers) and discrete tiine (ynamical systems (i.e.. $T$ is the integers). For mathematical purposes. we mav introduce additional restrictions such as smoothuess and linearity. but it should be pointed out that many physical systems cannot be modeled exactly under such restrictions.

We represent a continuous time-invariant dynamical system as

$$
\begin{aligned}
& \dot{x}(t)=f(x(t), u(t)) \\
& y(t)=g(x(t), u(t))
\end{aligned}
$$

where the first equation is called the state equation and the second the output equation. The state and output equations typically consist of differential equations such that for any initial state $x\left(t_{0}\right)$ and input $u$ both equations have unique solutions. The discrete counterpart of the continuous system is represented as

$$
\begin{aligned}
x(k+1) & =f(x(k), u(k)) \\
y(k) & =g(x(k), u(k))
\end{aligned}
$$

where the state equation in this case is a difference equation.

So far. we have treated states, inputs. and outputs as simple unstructured sets. Generally, the states, inputs. and outputs have considerable structure; it is often reasonable to :epresent each in terms of a multidimensional vector space (e.g., $\mathbf{R}^{n}$ ). Each dimension of the space corresponds to a component variable of the corresponding rector space. For instance. in designing a dynamical system to model the fluid flow in and ont of a holding tank. Whe inight employ three state variables. the height of the fluid in the tank. the angle of the input valve, and the angle of the output valve. The resulting state space would be a subset of $\mathbf{R}^{3}$. In decigning a system to model a robot. we might use the position in $x, y$, and $z$, and orientation in $\theta_{x, y}, \theta_{y, z}$, and $\theta_{r, z}$ for a six-dimensional state space, $\mathbf{R}^{6}$. In general, the state, input. or output variables may be boolean. real, integer, or discrete valued, and cau correspond to any representable quantity or its derivatives, as long as the resulting space satisfies the requirements for being a finite-div.sisional rector epace [5]. By characterizing the states, inputs. and outputs in terms of linear vector spaces, we can bring to bear the considerable power of linear algebra and linear syorems theory.

Much of linear control is concerned with linear time-invariant systems of the form

$$
\dot{x}(t)=A x(t)+B \mathbf{u}(t)
$$

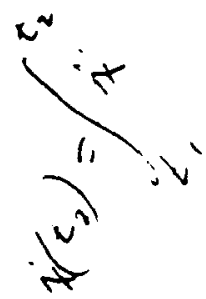




$$
y(t)=C \cdot x(t)
$$

where $\mathbf{x}$ is the $n$-dimensional state vector. $\mathbf{u}$ is the $p$-dimensional input vector. $y$ is the $q$-dimensional out put vector. and $A$. $D$. and $C$ are. respectively. $n \times n . n \times p$, and $q \times n$ real constaut matrices.

As a simple example illustrating how to ronstruct a linear dynanical svstem, consider a single-degree-of-freedom robot of mass. M. acted upon by a force. $\mathcal{F}$. Let $z$ be the position of the robot in some arbitrary frame of reference. We assume that the plate of notion is horizontal and that there are no frictional forces acting on the robot. The relationship between position. $=$. and the force. $\mathcal{F}$. is completely cletermined by Newton 's second law of motion.

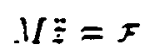

The dyuamic behavior of the rohol can be described in terms of the position and velocity of the robot. and. hence. We define the state vector to be.

$$
\mathbf{x}(t)=\left[\begin{array}{c}
z(t) \\
\dot{z}(t)
\end{array}\right] .
$$

Eqnating the system ontput and the sustem state. we can write down the state and output equations as follows.

$$
\begin{aligned}
& \dot{x}(t)=\left[\begin{array}{ll}
0 & 1 \\
0 & 0
\end{array}\right] x(t)>\left[\begin{array}{c}
0 \\
1 / M
\end{array}\right] \\
& y(t)=x(t)
\end{aligned}
$$

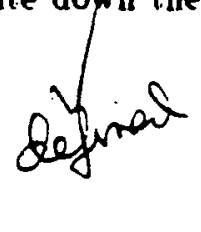

Cienerally. the system output contains incomplete information from which it is necessary to reconstruct the systein state. In subsequent chapters. We consider some of the issues involved in attenpting to infer the system state from incomplete information.

The restriction of inearity is a critical one that causes some researchers to dismise much of nathenatical control theory as a purely academic pursuit with no practical consequences. Mlost physical systems are nonlinear. and. hence. we can ouly approximate these systems using linear models. In many cases. such approximatious are valid over only a limited range of the systems operating conditions. While these problems make it difficult to apply results from linear systems theory. the methods of linear systems theory are so powerful that the effort is often well spent. It seems unlikely that a general method for analyzing nonlinear systems will emerge [i], and that instead 


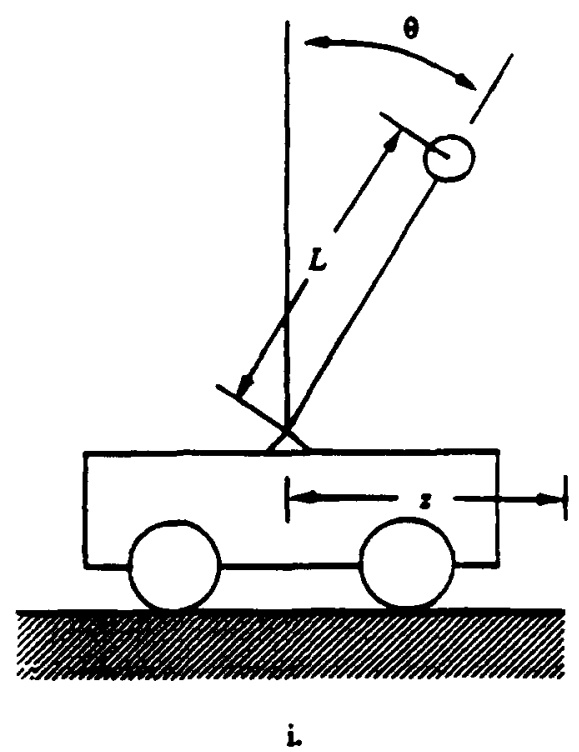

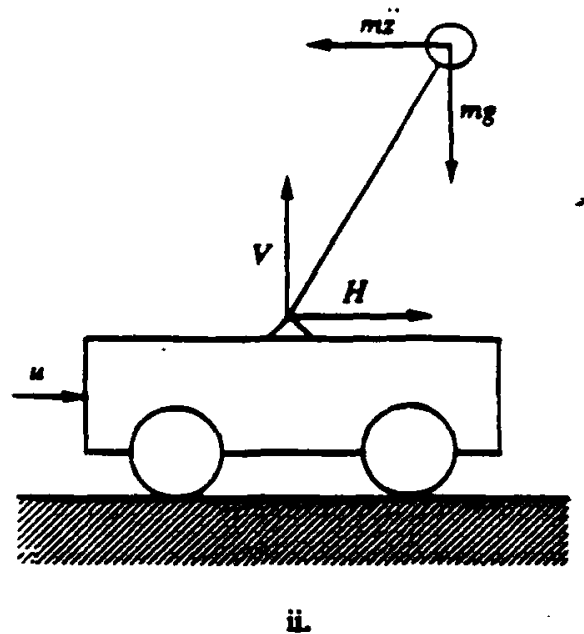

ii.

Figure 2.6: Inverted pendulum mounted on a cart

researchers will divide the class of noulinear systems into a set of more manageable subclasses for which there exist special methods of analysis, much of which will be based on ideas drawu from linear systems theory.

To illustrate how to approximate a nonlinear system by a linear one. we consider a classic example in control that involves unodeling an inverted pendulum mounted on a cart that can move back and forth along a horizontal track. This problem is often cited as an analogue of the problem of controlling a missile balanced atop its booster rockets $[6,8]$. The presentation here follows that of Gopal [8]. We assume that the controller can exert a force on the cart to propel it to the right or left along the horizontal track. Let $z$ be the horizontal position of the cart's center of gravity, and $z+L \sin \theta$ the horizontal position of the center of gravity of the pendulum. where $L$ is the diatance from the pivot to the center of gravity of the pendulum. Similarly, $L \cos \theta$ is the vertical position of the center of gravity of the pendulum. Figure 2.6.i shows the basic configuration of cart and pendulum.

The state of the system is completely described by the position and velocity of the cart and the angular position and angular velocity of the 
pentulum. Thits we trave the state vector:

$$
x(t)=\left[\begin{array}{c}
z(t) \\
\vdots(t) \\
\theta(t) \\
\dot{\theta}(t)
\end{array}\right] .
$$

In order to set up the dynamical equations. we have to establish some additional parameters. Let $m$ he the mass of the pendulum. I/ the mass of the carriage, and $J$ the moment of inertia of the pendulum with respect to its center of gravity.

The forces acting on the pendulum are the force of gravity, mg, acting on its center of gravily, a horizonlal reaction force. $H$. and a vertical reaction force. 1 . Figure 2.6.ii depicts the forces acting on the pendulum and the cart. Taking moments about the center of gravity of the pendulum, we have

$$
J \ddot{\theta}(t)=V L \sin \theta(t)-H L \cos \theta(t) .
$$

Summing all of the forces acting on the pendulum in the horizontal and vertical directions. we have

$$
\begin{aligned}
V-m \cdot g & =m \frac{d^{2}}{d t^{2}}(L \cos \theta(t)) \\
I I & =m \frac{d^{2}}{d t^{2}}(z(t)+L \sin \theta(t)) .
\end{aligned}
$$

Summing all of the forces acting on the cart, we have

$$
u(t)-I I=M(t) .
$$

where $u(t)$ is the (control) input.

Since the task is to keep the pendulum upright. We will assume that $\theta$ aud $\dot{\theta}$ will remain cluse to 0 . On the basis of this assumption. we make the standard approximatious. $\sin \theta \approx \theta$ and $\operatorname{gos} \theta \approx 1$. obtaining

$$
\begin{aligned}
m \dot{\dot{\theta}}(t)+(m+\dot{i x \dot{x}(t)}=u(t) & =0 \\
\left(J-m L^{2}\right) \ddot{\theta}(t)+m L \ddot{z}(t)-m g L \theta(t) & =0
\end{aligned}
$$

We introduce values for the remaining paraneters.

$$
. \stackrel{\mathrm{kg},}{=}, m=0.15 \mathrm{~kg}, L=1 \mathrm{~m}
$$


Using any mechanics or physics textbook. we get

$$
\begin{aligned}
& g=9.81 \mathrm{~m} / \mathrm{sec}^{2} \\
& J=\frac{4}{3} m L^{2}=0.2 \mathrm{~kg}-\mathrm{m}^{2}
\end{aligned}
$$

Using these equations and paraneter values. ye obtain

$$
\begin{aligned}
0.15 \ddot{\theta}(t)+115 \ddot{z}(t) & =u(t) \\
0.35 \ddot{\theta}(t)+0.15 \ddot{z}(t)-0.15 \times 9.81 \theta(t) & =0
\end{aligned}
$$

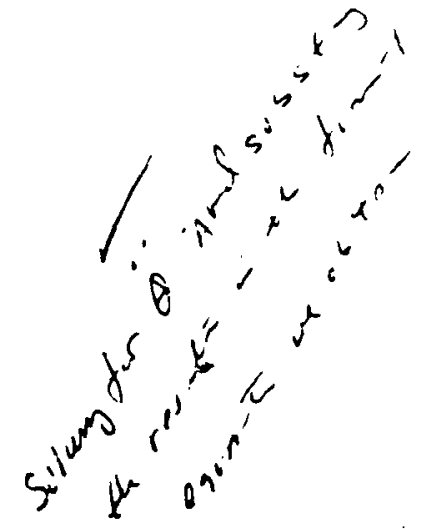

to arrive at the following state and output equations for the dynamical model:

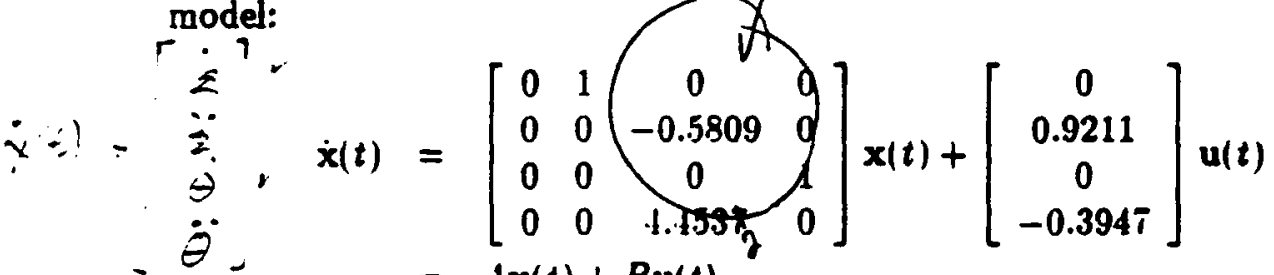

$$
\begin{aligned}
& =A \mathbf{x}(t)+B \mathbf{u}(t) \\
& y(t)=\left[\begin{array}{llll}
0 & 0 & 1 & 0
\end{array}\right] x(t) \\
& =C x(t)
\end{aligned}
$$

where we assume realistically that the only component of the output that is directly observable is the angle, $\theta$, corresponding to the tilt of the missile in the case of the booster rocket.

In Chapter 4, we highlight results from linear systems theory that allow us to establish important properties (e.g., stability and controllability) of dynamical systems, using simple tests on the matrices that define the state and output equations. The inverted pendulum is particularly interesting as it represents a dynamical system that is not stable, but is controllable.

Before leaving this chapler, we introduce some additional concepts and terms. We will develop sinuilar concepts in the next chapter. in some cases using the same terms and in other cases introducing new terminology. Where the terminology differs, we will point out the conceptual similarities. An event is simply a pair cousisting of a time point and a state (e.g., $\langle t, x\rangle$ where $t \in T$ and $x \in X$ ). The event (or phase) space is the space of all possible events, $T \times \mathrm{H}^{\circ}$ A state-space trajectory is simply a mapping from

\footnotetext{
'IVe follow Kalman [9] in our une of the term phace apace. Yon may also see the term used to refer to the apace of pomible ponitiona and velocities. A state variable obtained from a system variable and its derivative is referred to as a phase variable [8].
}

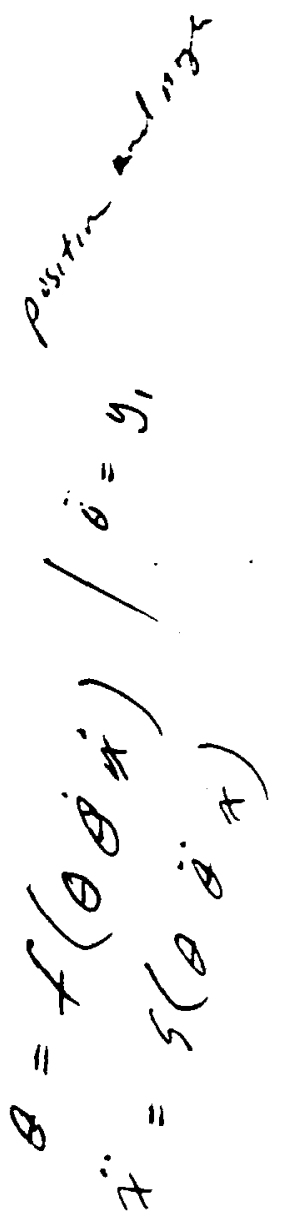


Ihe real interval to the stale space. $h:[0.1]-. j$. defined by a particular Iransition function. $f$. input. " and intial conditions. $x(0)=x_{0}$. In the following chapter. Ne turn our attention to the use of logic in modeling physical systems.

\subsection{Further Reading}

For a genesal introduction to modeling from the perspective of control. see the texts by Dorf [6] or Bollinger [3]. For an eniphasis on modern control. time-domain analysis. and. in particular. linear sustem theory. see ('hen [5] or Copal [8]. Our treat.ment of dynamical systems follows that of halman: Kialınan's chaptcr in [?] provides a very general formulation of dynamical systems and an introduction to the necessary mathenatical a.hstractions. 


\section{Bibliography}

[1] Aström. Käl J. and Wittenmark. Björn, Adaptive Control. (McGrawHill. New York, 1989).

[2] Bellman. Richard. Adaptive Control Processes. (Princeton University Press, Princeton, New Jersey, 1961).

[3] Bollinger, John G. and Duffie. Neil A., Computer Control of Machines and Processes, (Addisou-Wesley, Reading, Massachusetts, 1988).

[4] Borrie, John A., Modern Contml Systems: A Manual of Design Methods, (Prentice-IIall, Englewood Cliffs, New Jersey, 1986).

[5] Chen. C.T.. Introduction to Linear System Theory, (Holt, Rinehart, and Winston. New York, 1970).

[6] Dorf. Richard C., Modern Control Systems, (Addison-Wesley, Reading, Massachusetts, 1989).

[i] Gibeon, John E., Nonlinear Automatic Controh (MeGram-Hill, New York. 1963).

[8] Gopal. M.. Modern Control System Theory, (Halated Press. New York. 1985).

[9] Kilman, R. E., Falb, P. L., aud Arbib, M. A., Topics in Mathematical Sycem Theory, (McGraw-Hill, New York, 1969).

[10] Rabeastein, Albert L.. Elementary Differential Equations with Linear Algebra. (Academic Press, New York, 1975).

[11] Sastry, Shankar and Bodson. Marc. Adaptive Control: Stability. C'onvergence, and Robustness, (Prentice-Ilall, Englewood Cliffs. New Jersey, 1989). 
[12] Wolovich. William A.. Rolvolics: Basic thalysis and Design. IHolt. Rinehart and IIIIston. $\mid y \subseteq i)$. 


\section{Chapter 3}

\section{Temporal Reasoning}

Section 3.1 contiders the use of temporal logic in ressoning about procesces with an emphasis on the issues that arise in dealing with incomplete information. The temporal logic makes use of the differential calculus to reason about continuously changing parameters while at the ame time providing precise semantica for reasoning about discontinuous change and incomplete information. In Section 3.2, we develop a computational language implementing many features of the temporal logic, and inrestigate some inves that arise in building prectical aystems for modeling procesces.

\subsection{Modeling Change in Temporal Logic}

In this section, we consider methods for modeling phyrical syotems based upon the first-order predicate caleulu. We begin by identifying the corts of entitien that we need to reason about. Whereas the methods of the privious chapter focus on the behavior of reah-valued variables over time, in this section the representations are designed primarily to facilitate reasoning about the truth value of propositions at various points in time. The propoaitions that we consider may correspond to atatements about the value of real. valued variables, but we are not restricted to statements of that form.

There is a long history of calculi for resconing about time in philosophy, computer science, and artificial intelligence. Rather than debate the advantagen and diandvantages of the many exinting techriques, we take the expedient of adopting a particular temporal logic that suits our basic needs for modeling phyaical systems. We then augment that logic to handle the

\footnotetext{
'Q1990 Thomes Dean and Miched Wellman. All rights reserved.
} 
s-ecific requirements of the applications considered in this monograph. In Section 3.3, we briefly consider some competing approaches to reasoning about time and provide references to papers dealing with complications not adequately addressed by our treatment.

To model phyoical processes, we need to reason about the truth of propositions over intervals of time. The propositions correspond to properties of the worid that are subject to change over time. For instance, we might want to say something about whether or not a particnlar furnace is turned on at a particular time; to do so, we introduce a relation, on, and a constant, furnace17, denoting the furnace that we heve in mind. Since the furnace is on at some times and off at others, the proponition, on (furace17), must be interpreted differently with respect to different times. The temporal logic ihat we employ here is escentially a calculus for reaconing about the associaicion between time intervils and proponitions.

In the following, we choone to treat time points a primitive and reason about intervals in terms of points. Time points are denoted $t$ or $t i, i \in Z$ (e.g., $t 1, t 2$ ). Variables ranging over time points are denoted $t$ or $t_{i}, i \in \mathbf{Z}$ $\left(e . g ., t_{1}, t_{2}\right)$. Later when we incorporate our temporal notation into PROLOG, we wit adopt standard PROLOG ayntax and notate time variables as $T$ or $T i, i \in Z$ (e.g., $T 1, T 2$ ). We introduce a binary relation, $\preceq$, on time points indieating temporal precedence. If $t 1$ and $t 2$ are time points, then $(t 1, t 2\rangle$ is an interval. The formule $\langle(t 1, t 2\rangle, p)$, where $p$ is a propositional symbol, allowe us to refer to the association between $\langle t 1, t 2\rangle$ and $p$. Following common practice in artificial intelligence, we oubstitnte bolds $(t 1, t 2, p)$ for $((t 1, t 2), p)$. The full specification of the syntax for the logic is described below. ${ }^{1}$

- TC: a set of time point iymbols

- C: a set of constant symbole dijjoint from TC

- TV: a cet of temporal varisbles

- V: a set of variables diajoint from TV

- IIr. a cet of fixed-arity temporal function aymbols

- F: a set of fixed-arity function symbols diajoint from $T F$

\footnotetext{
The grater for the frutorder eace and the cemantio for the propocitional can are borrowed directly from Shoban [5e].
} 
- $R$ : a set of fixed-arity selation symbols

- $\preceq$ : a binary relation symbol

The set of temporal terms (TT) is defined inductively as follows:

1. $(T C \cup T V) \subset T T$

2. If trm $_{1} \in T T, \ldots, t r m_{n} \in T T$, and $\varepsilon \in T F$ is an $n$-ary function symbol, then $f\left(t r m_{1}, \ldots, t r m_{n}\right) \in T T$.

The set of nontemporal terms (NT) is defined similarly with TC replaced by $C, T V$ replaced by $V$, and $T F$ repiaced by $F$.

The set of well-formed formulae (wfts) is defined inductively as follows:

1. If trma $\in T T$ and $t r m_{a} \in T T$, then $t r m_{a}=t r m_{b}$ and $t r m_{a} \preceq$ trmb are wfis.

2. If $t r m_{a} \in T T$ and $t r m_{b} \in T T, t r m_{1} \in N T, \ldots, t r m_{n} \in N T$, and $x \in R$ is an $n$-ary relation symbol, then

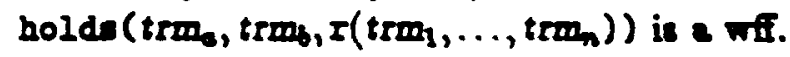

3. If $\varphi_{1}$ and $\varphi_{2}$ are wffs, then so are $\varphi_{1} \wedge \varphi_{2}$ and $\neg \varphi_{1}$.

4. If $\varphi$ is a wf and $x \in(T V \cup V)$, then $\forall x \varphi$ is a wfr.

We assume the standard definitions of $\vee, \supset, \equiv$, and $\exists$, and we make use of the following shorthand: ${ }^{2}$

$\operatorname{holda}\left(t_{1}, t_{2}, \varphi_{1} \wedge \varphi_{2}\right) \Rightarrow \operatorname{bolda}\left(t_{1}, t_{2}, \varphi_{1}\right) \wedge \operatorname{bolds}\left(t_{1}, t_{2}, \varphi_{2}\right)$

holds $\left(t_{1}, t_{2}, \neg \varphi\right) \Rightarrow \neg$ bolds $\left(t_{1}, t_{2}, \varphi\right)$

and $20 \mathrm{on}$. Finally, since the structure of time is generally isomorphic to the integers or the reals, we asume that the addition and subtraction of tempord terms is well defined. For instance,

$\forall t_{1}, t_{2} Y\left(\left(t_{2}-t_{1}\right)>5_{\min }\right) \supset$ holds $\left(t_{1}, t_{2}, \varphi\right)$

is meant to indicate that $\varphi$ holds in any interval longer than five minutes.

By introducing appropriate relation and function aymbols, we can develop notations for representing a variety of phenomens using the above syntex. For instance,

\footnotetext{
'Note that the lea-hand aides are not well formed; benee, we une $\Rightarrow$ indicating a rewrite sule rather that $\mathbf{z}$ indicating logical equivalence.
} 
$\operatorname{holdo}\left(t 1, t 2, t \in \operatorname{mp}(r 00 \mathrm{~m} 32)>72^{\circ}\right)$

is meant to represent the fact that the temperature in a particular room is greater than $72^{\circ}$ throughout the interval $(t 1, t 2\rangle$. The following three formulae illustrate the use of quantification.

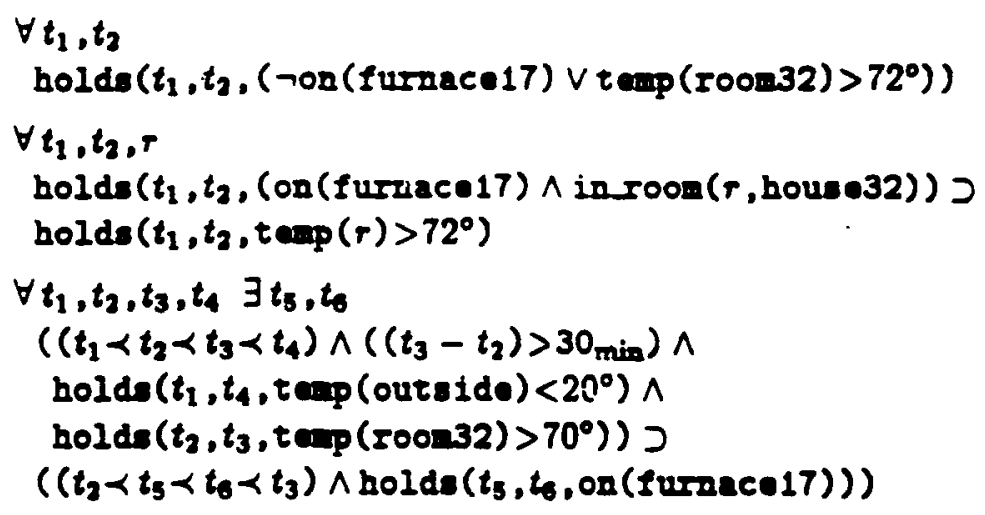

The firat formula is meant to represent the fact that it is always the case that either the temperature in a particular room is greater than $72^{\circ}$ or the furnace is not on. The second formuls is meant to represent the fact that, whenever the furnace is on, all of the rooms in the house are above $72^{\circ}$. The third formula is meant to represent the fact that, if the temperature in a particular room is greater than $70^{\circ}$ throughout an interval of greater than 30 minutes in length during which the outside temperature is leas than $20^{\circ}$, then the furnace was on for some subinterval of duration 5 minutes or longer. There are also thinge that can not be repreneated in this logic. For inetance, the logic is not powerfil enough to reprenent the fact that the furnoce wes on for at leat 5 mincites during a given 10 minute intervl, where that 5 minutes could be spread out over an indeterminate number of subintervels.

We introduce some additional notations and conventions to simplify ous notation. To simplify making statements about an assation being true at a time point, we introduce the following abbreviation:

$\forall t \operatorname{holde}(t, t, \varphi) \equiv \operatorname{holds}(t, \varphi)$

It will froquently be uceful to state that certain properties are timeleasly true; for convenience, we define the "always" operator, $\square$, a

$\forall t_{1}, t_{2} \operatorname{holds}\left(t_{1}, t_{2}, \varphi\right) \equiv \square \varphi$ 
'inally, we dispense with universal quantifiers that range over a textually isolated formula and assume that all free variables are universally quantified of scope the entire formula in which they are contained. For instance, in the following formula

holds $\left(t_{1}, t_{2},\left(\neg 0\right.\right.$ (furnace17) $\vee \operatorname{tamp}\left(\right.$ I00m32) $\left.\left.>72^{\circ}\right)\right)$

we assume that the two temporal variables are universally quantified.

The two things that logicians are most concerned about in a logic is its proof theory and its semantics. Since we will not be concerned with proving theorems in the traditional sense, we will not bother with a proof theory for our logic. We are, however, concerned that our notations have precise meaning. Later, when we consider an algorithm for deriving statements from a set of other statements, we want to be assured that our conclusions are valid; for this, we require a semantic theory for our temporal logic.

Intuitively, the formula holds(t1,t2,on(furnace17)) should be true just in case the furnace is on at every time point between $t 1$ and $t 2$. In a modal logic, we can make that intuition concrete by thinking of time points as possible worlds. A possible world roughly corresponds to a model in traditional Tarskian semantica (i.e., an astignment of true or false to each proposition). The different possible worlds are related to one another by the ordering relationship $\preceq$. In the firat-order temporal logic presented here, we take a different approach to characteriaing the meaning of formulae; we think of each proposition (e.g., on (furnace17)) as denoting a set of time intervals. In this case, holds $(t 1, t 2,02($ furnace17)) should be troe just in case $(t 1, t 2) \in$ on (1urnace17). To make this more precise, we provide the semantice for the propositional form of our temporal logic. ${ }^{3}$

The proponitional case of our temporal lopic is similar to the first-order case described above with the exception that there are no nontemporal variables, constants, or function symbols, and, instead of complex terms and relations, we have $P$ a set of propositional symbols. In order to communicate the caential semantic properties of the logic, it should suffice to provide the comantice for the propositional case.

As interpretation is a triple $(T W, \leq, M)$ consisting of a nonempty univere of time pointe, $T W ;$ a binary relation, $\leq$, on $T W$; and a two-part meaning function, $M=\left(M_{1}, M_{2}\right)$, where $M_{1}: T C \rightarrow T W$ and $M_{2}: P \rightarrow$ $2^{T W} \times \mathrm{TW}$.

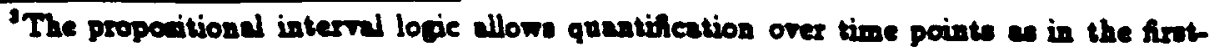
order cace, bet is restricted 0 thet $\varphi$ in holde $\left(t_{1}, t_{2}, \varphi\right)$ in a proporitiond formule.
} 
A variable assignment is function $V A: T V \rightarrow T W$. If $u \in(T C \cup T V)$, we define $\operatorname{VAL}(u)$ to be $M_{1}(u)$ if $u \in T C$, and $V A(u)$ if $u \in T V$. An interpretation $S=\left\langle T W, \leq,\left\langle M_{1}, M_{2}\right\rangle\right\rangle$ is said to satisfy a wf $\varphi$ under the variable assignment $V A$ (written $S \vDash \varphi[V A]$ ) under the following conditions:

1. $S \vDash\left(u_{1}=u_{2}\right)[V A]$ iff $\operatorname{VAL}\left(u_{1}\right)=\operatorname{VAL}\left(u_{2}\right)$

2. $S \vDash\left(u_{1} \mathfrak{u}_{u_{2}}\right)[V A]$ iff $V A L\left(u_{1}\right) \leq \operatorname{VAL}\left(u_{2}\right)$

3. $S \vDash \operatorname{boldg}\left(u_{1}, u_{2}, \varphi\right)[V A]$ iff $\left\langle\operatorname{VAL}\left(u_{1}\right), \operatorname{VAL}\left(u_{2}\right)\right\rangle \in M_{2}(\varphi)$

4. $S \vDash\left(\varphi_{1} \wedge \varphi_{2}\right)[V A]$ iff $S \vDash \varphi_{1}[V A]$ and $S \vDash \varphi_{2}[V A]$

5. $S \vDash \neg \varphi[V A]$ iff $S \not \varphi[V A]$

6. $S \vDash(\forall v \varphi)[V A]$ iff $S \vDash \varphi\left[V A^{\prime}\right]$ for all $V A^{\prime}$ that agrees with VA everywhere except pousibly on $v$.

An interpretation $S$ is said to be a model for a wf $\varphi$ (written $S \vDash \varphi$ ) if $S \vDash \varphi[V A]$ for all variable assignments VA. A wf is said to be satiofiable if it has a model, and a wf is axid to be valid (mitten $F \varphi$ ) if its negation is not satisfiable. We will have to augment the above semantica as we extend the logic to handle more complicated forms of inference, but the basic cemantica relating temporal intervals and proponitions will be setained.

In order to reason about processes, it is often natural to speak in terms of events that precipitate change in the world. For instance, the toggling of a switch corresponds to an event that has as a consequence changes in an electrical circuit. The occurrence of an event corresponds to a particular type of proponition holding over an interval. Shohem [58] provides a clarsification of proponition types that ensbles as to dintinguiah between thoie corresponding to the occurrence of events and thove corresponding to other sorts of phenomens.

Most of the propositions that we have seen so far (e.g., on (furnace), tep (roos32) $\left.>70^{\circ}\right)$ are said to be liquid in Shoham's classification. A propocition type is liquid if, whenever it is is true over an interval, it is true over every anbinterval (except posably the endpoints), and, additionally, wheover it holds for all proper subintervals of some nonpoint interval (except ponaibly the endpoints), it holds over the noupoint interval. Events are generally thought of a corresponding to propositions that are not liquid; they are said to be gestalt in Shoham's clestification scheme. A proponition type is geatalt if, whenever it holds over an intervel, it does not hold over any proper subinterval. To emphasize the role of events in reasoning about 
change, we : se occura $\left(t_{1}, t_{2}, \varphi\right)$ instead of holds $\left(t_{1}, t_{2}, \varphi\right)$ where $\varphi$ is a gestalt proposition type corresponding to the occurrence of an event.

Suppose that the set of time points is isomorphic to the integers. For any given time point $t$, there exists a unique next time point $t+1$. We can specify a simple law of change as follows:

R1: (holde(t, ᄀon(furnace17)) $\wedge$

occurs $(t$, toggle(suitch42) $)) \supset$ holda $(t+1$, on(furnace17)

Of course, this rule is not quite right; the furnace does not always come on when you toggle its switch. Use "ariom" instead of "rule." Indicate that what we really want is a weaker approximation of R1, but that we cannot provide such on approximation within the classical logic. The fuse on the circuit feeding power to the furnace has to be intact, the funnace has to be mechanically and electrically sound, and any number of additional conditions must hold in order for the furnace to come on a a consec uence of toggling its switch. Unfortunately, it generally will not be possible to exumerate all of the necessary conditions, and, even if you could enumerate them, the rule would be useless given that you could never know enough to establish whether or not all of the conditions are met in a given situstion. The conditions specified in the antecedent of a rule such a $\mathrm{BI}$ are meant to correspond to conditions that are readily known and urnally sufficient to warrant the conclusion. The idea is that, if you frequently come to the right conclusion and only occanionally come to the wrong conclusion, then the small reduction in reliability will be offet by potentially enormous computational savings.

However, even if you are willing to accept the reduction in reliability that reavlts from uniag B1, you may not be willing to accept another, more serious consequence of uning rules of this form. The mose serious consequence has to do with handling situations in which it is known that some secessary, but uraceounted for condition is not eatiofied. For instance, you may boow that the fwe on the circuit providing power to the furnace is open, rendering the switch aclees. Unfortunately, the consequent of RI still follow from the antecodat and you are left with a conclusion that you know to be false. What you would like to say is that the furnace will be on if you togele its ewitch unless you have some information to the contrary. Formalising this cort of inference is actually quite complex. The problem of reasoning about the conditions required for an event to have a given consequence is referred to as the qualification problem and is of considerable interest to researchers working in the area of defeult resoning and nonmonotonic 
logic. We introduce some additional synt: $x$ that attempts to address the qualification problem as follows:

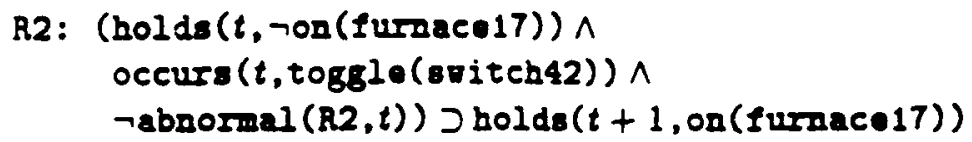

where abrormal $(R 2, t)$ is meant to indicate that $R 2$ is ineppropriate to apply with respect to $t_{;}$in this case, $R 2$ is said to be disabled. The status of the abnormal antecedent in R2 is different from that of the other two antecedents in the rale. The intent that the conclunion should follow as long as there is no evidence that the rule is abnorma. We can now add rules that will serve to disable $R 2$ in appropriate circumstances. For instance,

\section{Q1: (holds $(t$, open(fuse43)) ^ ocevra $(t$, togsle(stitch42) ) ) abnorand $(R 2, t)$}

indicates that the conclusion of $\mathrm{R} 2$ is not warranted whenever a certain fuse is open.

The intent behind $R 2$ is that holds $(t+1,0$ (furnace17)) should follow from the axioms (i.e., be a theorem) just in case holds ( $t$, ᄀon (furnace17)) and occura ( $t$, togglo(avitch 2$)$ ) follow, and $\neg$ abooraral $(R 2, t)$ is consis. tent with the axioms. Unfortunately, if you use such a criterion to construct the set of theorems, you may get different answers depending upon the order in which you consider candidate formulce for membership in the set of theorems. In some cases, we can avoid ambigaity regarding the set of theorems by requiring that only a minimal number of abnormalities are allowed to occur. We can make our intended meaning precies by angmenting our semantic theory.

Firat, we introduce the ides of a partial ordering or prefenence, $<$, on models for a given set of scioms. Let $\mathrm{I}$ be the ret of axioms describing bow events precipitate change in the world. $\Gamma$ would include rules such a $\mathrm{R2}$, qualifications such as $Q 1$, and additional axioms indicating initial conditions, observations, or proposed actions. We denote the set of all models of $\Gamma$ (i.e., $\{\boldsymbol{M}: \boldsymbol{M}=\Gamma\})$ by $\operatorname{Mod}(\Gamma)$. Asouming that there are no infinite (descending) sequences of models $M_{1}, M_{2}, M_{3} \ldots$ sech that $M_{2}<M_{1}, M_{3}<M_{2}, \ldots$, the notion of the set of all minimal (with respect to <) models is well defined; we denote this set as $\operatorname{Min}(<, \operatorname{Mod}(\Gamma))$. We define a particular $<$ such that $M_{1} \ll M_{2}$ just in case:

1. $M_{1}$ and $M_{2}$ agree on the interpretation of all function and relation symbols other than abnoral. 
2. For all $x$ and $t$, if $M_{2} \vDash$ abnormal $(x, t)$, then $M_{2} \vDash \operatorname{abnormal}(x, t)$.

3. There exists some $x$ and $t$. for which $M_{2} \vDash \operatorname{abnomal}(x, t)$, but $M_{1} \not$ abnormal $(x, t)$.

We say that $\Gamma$ preferentially entails $\varphi$ with respect to $\ll$ (written $\Gamma \vDash \ll \varphi$ ) just in case

$$
\forall M \in \operatorname{Min}(\ll, \operatorname{Mod}(\Gamma)), M \vDash \varphi .
$$

To illustrate, consider the following two observations:

01: occurs(1,toggle(stitch42))

02: holds $(1, \neg$ on (furase 17))

indicating that the furnace was not on at time point 1 , and that the switch was togeled at that time. Suppose that the set of axioms is

$$
\Gamma=\{01,02, R 2,01\} .
$$

In this case, holds $(2$, on (furace 17$))$ is true in all models minimal with respect to $<$, and, hence, we have

$$
\Gamma \vDash<\operatorname{bolda}(2 \text {, on(furnace17)). }
$$

Unfortunately, there are situations in which our augmented semantics runs counter to our expectations. For instance, suppose that we complicate our furnace scenario, and add a new rule indicatiog that, whenever a power surge occurs and we have no reason to believe that there are other complicationa, the fure on the circuit providing power to the furnace overheats, leaving the circuit open.

R3: occurs $(t$, surgo $) \wedge$ ᄀabrorsal $(R 3, t) \supset$ bolds $(t+1$, open (fusc43))

In addition, suppose that we have observed a power surge at time 0 .

03: occurs $(0$, surge $)$

Givere the set of exioms

$$
\Gamma=\{01,02,03, R 2, R 3,01\},
$$

one might expect to conciude:

C1: holds $(1$, open(fuse43)) $\wedge$-holds $(2$, on (furnace (17)) 
However, while there are models minimal with respect $t r \ll$ that satisfy $\mathrm{C}_{1}$, there are also minimal models satisfying:

C2: ᄀholds(1, open(fuse43)) $\wedge$ holds $(2$, on (furnace (17))

It seems more plausible that evidence for an abnormality come from the past rather than from the future; hence, we should prefer models that allow us to conclude C1 over those that allow us to conciude C2. In general, we prefer models in which the fewest abnormalities occur, and those that occur do 80 as late as possible. The minimal models with respect to this preference are said to be chronologically minimal. We mate this more precise by defining a new preference, $K_{t}$, such that $M_{1} \ll_{1} M_{2}$ just in case there exints a time $t$ such that:

1. $M_{1}$ and $M_{2}$ agree 0 . the interpretation of all fonction and relation symbols other than abnoranel.

2. For all $x$ and $t^{\prime} \prec t$, if $M_{2} \vDash$ abnormal $\left(x, t^{\prime}\right), M_{1} \vDash \operatorname{abnormal}\left(x, t^{\prime}\right)$.

3. For all $x$ and $t^{\prime} \preceq t$, if $M_{1} \vDash$ abnornal $\left(x, t^{\prime}\right), M_{2} \vDash$ absormen $\left(x, t^{\prime}\right)$.

4. There exists some $x$, for which $M_{2} f=$ abrornal $(x, t)$, but $M_{1} \neq$ abnormal $(x, t)$.

Given the set of axioms $\{01,02,03, \mathrm{R2}, \mathrm{R3}, 01\}, \mathrm{C1}$ is true in all models minimal with respect to $<_{t}$.

The above discussion outlines some techniques for reasoning about what thing change a a consequence of events occurring, bat we haven't said anything about what thinge do not change. If you togie the switch to the furace, what happens to the color of the car in the garage? Preanably the color of the car remains the same a it was before you toggled the switch, but the arioms do not support this inference. We could provide an axiom like

Rs: (holda $(t, \operatorname{color}(\operatorname{car} 45)) \wedge$ occura (togsle(sitch42))) つ bolde $(t+1, \operatorname{color}(\operatorname{car} 45))$

but we would have to write a lot of axioms: one for each event/proposition pair," and mure if we are to acconnt for combinations of events happening

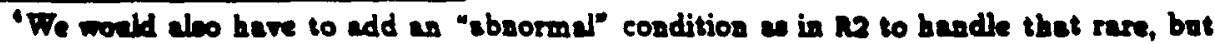
ponible rituation in which togcling the owitch to your furnses comehow does change the color of your cas.
} 
at the same time. R4 is called a frame siom, and the problem of reasoning about what things do not change as a consequence of an event occurring is called the frame problem. ${ }^{5}$ In considering how to deal with the frame problem, we begin by considering the case in which time is modeled after the integers.

In the following, we attempt to augment our temporal logic so that propositions, once they become true, tend to persist in lieu of any informs: tion to the contrary. This augmentation is often referred to as the default rule of persistence [43], or the common-sense law of inertia [37]. The justification for adding this default rule is not based on any natural law. In fact, it does not appear to be appropriate for reasoning about propositions in general. We claim, however, that it is appropriate for reasoning about propositions describing many of the processes that we humans cope with on a day-to-day basis. This claim is based on an assesument of our perceptual and cognitive capabilities; we simply cannot cope with procenses whose im. portant properties are not discernible by our senses or that change so rapidly or seemingly randomly that we cannot keep track of them.

We begin by introducing a special case of abnormality. Since propositions tend to persist, times at which they change should be rare or abnormal. We refer to the abnormality in which a proposition $\varphi$ changes its truth value at time $t$ as a clipping, and notate it as clips $(t, \varphi)$. Note that there are problems with our treatment of clipping. In particular, the predicate clips ranges over other predicates. We took care to indicate that bolds $\left(t_{1}, t_{2}, \varphi\right)$ was just syntactic sugar for $\left\langle\left(t_{1}, t_{2}\right\rangle, \varphi\right\rangle$, but here we will probably jitut let it slide rather than get bogged down in complicated details.

The following axiom schems allows us to infer clippings in appropriate circumstances:

\section{AS1: (holda $(t, \varphi) \wedge \operatorname{bolda}(t+1, \neg \varphi)) \supset$ elips $(t, \varphi)$}

The common-sense law of inertia is captured in the following formula, which is logically equivalent to AS1:

AS2: (holds $(t, \varphi) \wedge \neg$ clips $(t, \varphi)) \supset$ holds $(t+1, \varphi)$

Since theorems of the form $\neg$ clips $(t, \varphi)$ generally do not follow from the arioms, for any $t$ and $\varphi$, there will be modele in which ᄀelips $(t, \varphi)$

\footnotetext{
The name derives from the intuition that aince resy bitle changes from oae frame to the aext in a movie fllm, if you are told what does change, it should be simple to infer what doe not [42].
} 
is true and those in which it is false. We can use the same basi technique of minimizing temporally ordered abnormalities (i.e., clippings in this case) that we used to deal with the qualification problem to ignore models with unwanted or unmotivated elippings. However, we have to be careful that clippings and other sorts of abnormalities do not interact in a counterintuitive manner. One way to control unwanted interactions between the two different sorts of abnormalities is to prioritize them using the following. modification of $<_{t}$ :

2'. For all $x$ and $t^{\prime} \prec t$, if $M_{2} \vDash$ abnormal $\left(x, t^{\prime}\right)$, then $M_{1} \vDash \operatorname{abnormal}\left(x, t^{\prime}\right)$, and, if $M_{2} \vDash \operatorname{clip}\left(x, t^{\prime}\right)$, then $M_{1} \vDash \operatorname{clipo}\left(x, t^{\prime}\right)$.

3'. For all $x$ and $t^{\prime} \leq t$, if $M_{1} \vDash$ abnorand $\left(x, t^{\prime}\right)$, then $M_{2}=\operatorname{abnormal}\left(x, t^{\prime}\right)$, and, if $M_{1} \vDash \operatorname{clipa}(x, t)$, then $M_{2} \vDash \operatorname{clips}\left(x, t^{\prime}\right)$.

4'. Either there exists some $x$, for which $M_{2} \vDash$ clipa $(z, t)$, but $M_{1} \neq$ clips $(x, t)$, or for all $x$, if $M_{2} \neq$ clips $(x, t)$, then $M_{1}=$ elipo $(x, t)$, and there exints some $x$, for which $M_{2} \neq$ abrormal $(x, t)$, but $M_{1} \neq$ absormal $(z, t)$.

Chronological minimization does not alwaye perform sccording to our intuitions. To explain why not, we distinguish between two different sorts of temporal reasoning, referred to as projection and explanation. Projection is the problem of reasoning forward in time from some initial state of affairs to determine the future course of events. Explanation is the problem of reasoning backward in time from some find state of afrain to determine the past course of events. Chronologict minimiation sativfies most of our intuitions regarding projection; unfortunately, it provides some rather counterintuitive results regarding explanation. For instance, suppose that the furnace is observed to be on at 9:00 in the evening and off at 8:00 the next morning. Chronological ignorance would have us conclude that the furnece was on all night and was turned off at the last posoible moment before it was obcerved to be of at 8:00 AM. This inference strikes moat a completely arbitrary, and in thanfore an undenirable consequence of chronological minimiration.

There has been a significant amount of work on designing a temporal logic that satiofies our intuitions regarding both projection and explanation,

\footnotetext{
'Bere we ename the determinintic renvioss of thes problom in which a opeciled initial [Ared] atate of aftein aniquely determine the ancending [preceding] course of events. Note that determinion in one direction does sot necenarily imply the ocher.
} 
and we will review this work briefly at the end of dis section. Most of the deterministic problems that we consider in this book can be posed as projection problems of one sort or another. There is a real advantage to be had in casting a problem in terms of just projection or just explanation. In particular, the decision procedure used to automatically derive conciusions from a given axiomatic theory can exploit the (often linear) structure of time to expedite inference resulting in substantial computational savings. We return to deal with computational issues in Section 3.2 .

Thus far, we have focused on modeling techniques that are suitable for reasoning about processes in which both time and change are discrete. While discrete modeling techniques provide suitable appraximations for many continuous processes, we will find it convenient to extend our temporal logic to reason about continuous time and change. From now on, we asoume that time is isomorphic to the reals. We have to reformulate the axiom schemats for dealing with the frame problem to handle continuous time.

$$
\begin{aligned}
\text { AS1': } & \left(\left(t_{1} \prec t \leq t_{2}\right) \wedge\right. \\
& \text { bolds } \left.\left(t_{1}, t, \varphi\right) \wedge \text { bolds }\left(t, t_{2}, \neg \varphi\right)\right) \supset \text { clips }(t, \varphi) \\
\text { AS2': }( & \left(t_{1} \prec t \preceq t_{2}\right) \wedge \text { bolds }\left(t_{1}, t, \varphi\right) \wedge \\
& \left.\neg \exists t^{\prime}\left(\left(t \leq t^{\prime} \preceq t_{2}\right) \wedge \operatorname{clips}\left(t^{\prime}, \varphi\right)\right)\right) \supset \operatorname{bolds}\left(t, t_{2}, \varphi\right)
\end{aligned}
$$

In addition, our rules of ehange will look a bit different. For instance, we might change $\mathrm{R2}$ to look like:

$$
\begin{aligned}
& \text { R2': }\left(\left(t_{1} \prec t\right) \wedge \text { bolda }\left(t_{1}, t, \neg 0 \text { (furace17) }\right) \wedge\right. \\
& \text { occurs } \left.(t, \text { toggl } 1 \bullet(\text { sitch } 2)) \wedge \neg \text { abnormal }\left(R 2^{\prime}, t\right)\right) \supset \\
& \left.\exists t_{2}\left((t+\epsilon) \prec t_{2}\right) \wedge \text { boldo }\left(t+\epsilon, t_{2}, \text { on (Ituraces } 17\right)\right)
\end{aligned}
$$

where $\epsilon$ corresponds to a small delay betwein the time that the switch is togeled and the time that the furnace actually is on. This delay is meant to capture the intuition that causes precede effects. The delay is particularly appropriate here in that, were we to allow simultaneores cause and effect in this particular case, we would have an instant of time in which the furnace was both on and off. ${ }^{7}$ This has to be corrected. We still have the problem that the furnace is both on and off at some time.

\footnotetext{
This aced sot be true. We have aot been careful to stete whether or not on intervib $\left\langle t_{1}, t_{2}\right\rangle$ ase closed, helf open, or what. Frow our trentment of degenerate intervals (c.g., $(t, t)$ ), however, ope might conclude that of leat come intervib are clowed. The additional notetion and mechinery secenasy to resolve all of the insues concerning the status of time intervels is sot deemed worthwhile for this discuncioa. We will continue to aroid such iserea wherever posible, admitting that they would have to be resolved in a more complete treatment.
} 
Say something more about the preference criterion for continuous time.

We will also find it useful to reason about quantities that change continuonsiy as functions of time. Rather than invent new machinery within the interval temporal logic, we will try to import into the logic as much of the differential calculus as is needed for our anticipated control applications. Our treatment here roughly follows that of Sandewall [54].

Firat, we introduce a set, $U$, of real-valued parameters closed under the differential operator, $\partial$. If $u \in U$, then $\partial^{n} u \in U$, where $\partial^{n} u$ is the $n$th derivative of $u$ with reapect to time. We can trivially extend the syntax to represent statements about the values of parameters at various time points. For instance,

$\operatorname{holda}(t 1, t 2, y=3.1472)$

is meant to indicate that the parameter $y$ has the valne of 3.1472 throughout the interval $(t 1, t 2\rangle$. By restricting $y$ to remain constant throughont the interval $\langle t 1, t 2\rangle$, we also restrict $\partial y$ to remain 0 throughout the same interval.

To garantee this inteaded meaning, we have to angment the camantics somenhat. In addition to set of parameters $U$, we asoume that each interpretation includee a function $Q:(\mathbf{R} \times U) \rightarrow \mathbf{R}$, where we employ the set of real numbers, $\mathbf{R}$, for the set of time points as well a for the set of all parameter values.

Since we will find it convenient on occauion to model abrupt changes in the value of parameters as they change over time, we introduce the notion of a breakpoint. We asume that a phyrical procens is modeled nsing a set of differential equations that deweribe contingous change in the parameters over intervals of time, ind a cet of arioms that determine what equations are appropriate over what intervals. Breakpoints are times at which the arioms signal a change in the differential equations ueed to model a given quantity or ext of quantities. Generally, at a breakpoint, there is a discontinuity in some time-varying parameter.

Wh have to angment the cemantica to account for the behavior of param. etere with reopect to breakpoints. Each interpretation must include a eat of brealpoints $S \subset \mathbf{R}$, so that for all $u \in U, Q(t, u)$ is continuous over every interval not containing an element of $S$, and for all $t \in S$, , t $=Q(t, \partial \psi)$. Strange things can happen at breakpoints, but not so strange that we will allow a parameter to take on two different values. To svoid such anomalies, we will have to introduce some additional machinery. 
At time $t_{0}$, we have a set of differential equations and a set of initial values ${ }^{\mathrm{g}}$ for all of the parameters; these equations and initial values are known to boid until some indeterminate time $t_{1}$, when a breakpoint occurs and the axioms determine a new set of differential equations and a new set of "initial" values. In order to establish breakpoints and the values for parametera immediately following breakpoints, we need to refer to the values of parameters "just before" and "just after" breakpoints. To do so, we defire the left and right limits of a parameter $x$ at time $t$ as:

$$
\begin{aligned}
& Q\left(t, x^{l} \text { det } \lim _{r \rightarrow t^{-}} Q(\tau, x)\right. \\
& Q\left(t, x^{r}\right) \stackrel{\text { def }}{=} \lim _{r \rightarrow t^{+}} Q(\tau, x)
\end{aligned}
$$

A discontinuity occurs at $t$ with regard to a parameter $x$ whenever the left and right limits are not identical:

$$
Q\left(t, x^{l}\right) \neq Q\left(t, x^{r}\right)
$$

As long as there are no discontinuities, the differential equations tell wo exactly how the parameters vary with time. The axioms determine when breakpoints occur and what differential equations and initial conditions should be used to model processes between breakpoints. Discontinuities play a role in reasoning about real-valued quantities analogous to the role played by clippings in ressoning about the persistence of propositions. Just as the arioms do not rule ont spurious modele resulting from unexplained clippings, neither do they rule out models resulting from unexplained discontinuities.

Consider the following example. Suppone that we have two objects moving toward one another along a horisontal line. To keep the example simple, we anonme that the surface is frictionless, the objects are represented as identical point mases, and there are no external forces acting on the objects. Lat $x_{1}$ and $x_{2}$ represent the parameters corresponding to the position of the fint and recond objects, respectively, a meavured from some reference on the horisontal line. At time 0 , the firat object is located at position 0 , and the second object is located 10 meters to the right. A positive velocity indicate movement to the right. We make use of the standard notational conventions for position $(x)$, velocity $(\partial x=\dot{x})$, and acceleration $\left(\partial^{2} x=\bar{x}\right)$. Hese are the axioms indicating the initial conditions:

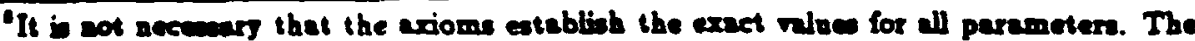
lopie decribed here in well-suited to reseoning sbout inequatities and parnmeter rangen.
} 


$$
\begin{array}{ll}
\operatorname{holds}\left(0, x_{1}=0\right) & \operatorname{holds}\left(0, x_{2}=10\right) \\
\operatorname{holds}\left(0, \dot{x}_{1}=2\right) & \operatorname{holds}\left(0, \dot{x}_{2}=-3\right) \\
\operatorname{holds}\left(0, \ddot{x}_{1}=0\right) & \operatorname{holds}\left(0, \ddot{x}_{2}=0\right)
\end{array}
$$

where velocity is in units of meters per second. The next axiom determines the new velocities immediately following a collision breakpoint.

$\square\left(\left(x_{1}=x_{2}\right) \wedge\left(\left(\dot{x}_{1}-\dot{x}_{2}\right)>0\right)\right) \supset\left(\left(\dot{x}_{1}^{l}=\dot{x}_{2}^{r}\right) \wedge\left(\dot{x}_{2}^{l}=\dot{x}_{1}^{r}\right)\right)$

For the most part, the propositions corresponding to equations involving the parameters in $U$ are constantly changing. In order for us to make useful predictions, however, certain equations have to pertist over intervals of time. Suppose you are told that at time $t_{0}, x=0, \dot{x}=2$, and $\ddot{x}=0$. If $x=0$ pernists, then there will be discontinuities in $\dot{z}$ and $\dot{z}$. If $\bar{z}=0$ penists, then $\dot{x}=2$ has to peraist or be discontinuous in order to aroid a discontinuity in $\dot{x}$, and $x$ is completely determined by $\dot{x}=2$. However, if none of $x=0$, $\dot{x}=2$, or $\ddot{z}=0$ pertist, there need not be a discontinuity in any one of $x, \dot{x}$, or $\dot{z}$, but neither is there any way of predicting the changen in $z$ over time. In this example. we force an interpretation by stating that the accelerations for the two objects art always 0 .

$\square\left(\left(\bar{x}_{1}=0\right) \wedge\left(\tilde{x}_{2}=0\right)\right)$

Using a preference analogous to $k_{t}$ that minimizes discontinuities, there is a single discontinuity in the acceleration of the objects two ceconds after time 0 , after which the objects, having exchanged velocities, head in opponite directions forever. We asume that the vines of parametess are entablinhed in intervin not containing brealpoints by differential equations.

Note that, by our definition of clipping (i.e., axiom scheme 1S1'), a discontinuity is a clipping only in the case that the discontinuity immediately followe positive length interval in which the parameter is constant. We dintinguinh propositions correoponding to real-valued parameters taking on opecile values (e.g., $\ddot{z}=2$ ) from proponitions corresponding to treth-valued paramotion (e.g., on (furacen17)).

In the provious example, $\square\left(\left(\bar{x}_{1}=0\right) \wedge\left(\tilde{z}_{2}=0\right)\right)$ cerves as the model for $x_{1}$ and $x_{2}$. In other caee, it may be convenient to infer a change in a model that persists over some indeterminate interval of time, just as we are able to infer changes in proponitions that persiat over intervals of time. To handle thie cort of inference, we introduce a particular type of propocition $\operatorname{paod}(x, m)$ where $x$ is a real-valued parameter and $m$ is $a$ model for $x$. If 
$m$ is an nth-order differential equation, then it is assumed that the nthorder equation determines all higher-order derivatives, and all lower-order derivatives are known as part of the initial conditions. By stipulating $\square(\bar{x}=$ $0)$, we implicitly indicated bolds $(0, \operatorname{pmod}(x, \ddot{x}=0))$ and that $x=0$ and $\dot{x}=2$ were the initial conditions at 0 . Propositions of the form $\operatorname{pmod}(x, m)$ persist according to chronological minimization. To illustrate how models might change over time, consider the following example.

Suppose that we want to reason about the temperature in a room heated by a furnace, and suppose that the furnace is controlled by a thermostat set to $70^{\circ}$. To make the example more intereating, suppose further that the thermostat has a $4^{\circ}$ differential (i.e., the furnace starts heating iot.en the temperature drope to $68^{\circ}$ and stops Fhen the temperature climbs to $72^{\circ}$ ). To represent parameters "dropping to" or "elimbing to" certain values, we define trans $([|| \uparrow \mid, u, v)$ where $u \in U$ and $v \in \mathbf{R}$ as follows:

$$
\begin{aligned}
& \operatorname{holds}(t, \operatorname{trane}([\downarrow \mid \uparrow], u, v)) \equiv \\
& \quad(Q(t, u)=v) \wedge\left(\exists t^{\prime}<t, \forall t^{\prime} \prec t^{\prime \prime} \prec t, Q\left(t^{\prime \prime}, u\right)|>|<\mid Q(t, u)\right)
\end{aligned}
$$

Propositions of the form trans $(\lfloor\downarrow \mid \uparrow], u, v)$ are used to represent point events of the sort that trigger changes.

To model changes in the room's temperature when the furnace is off, we use Newton's law of cooling

$$
\frac{d r}{d t}=-\kappa_{1}(r-a)
$$

where $r$ is the temperature of the room, $a$ is the temperature outside the room, and $\kappa_{1}$ depends on the imralation surrounding the room. To model changes in the room's temperature when the furnace is running, we nse

$$
\frac{d r}{d t}=\kappa_{2}(f-r)-\kappa_{1}(r-a)
$$

whare $f$ is the temperature of the furnace when it is running, and $\kappa_{2}$ depends on the heat fow characteristics of the furnace. The following arioms describe the tomperature in the room over time.

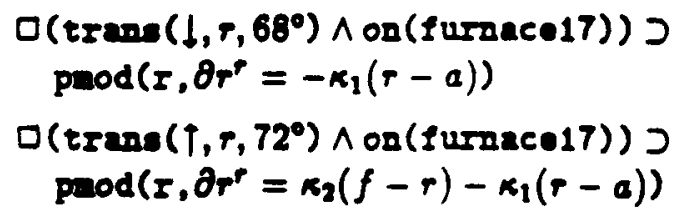



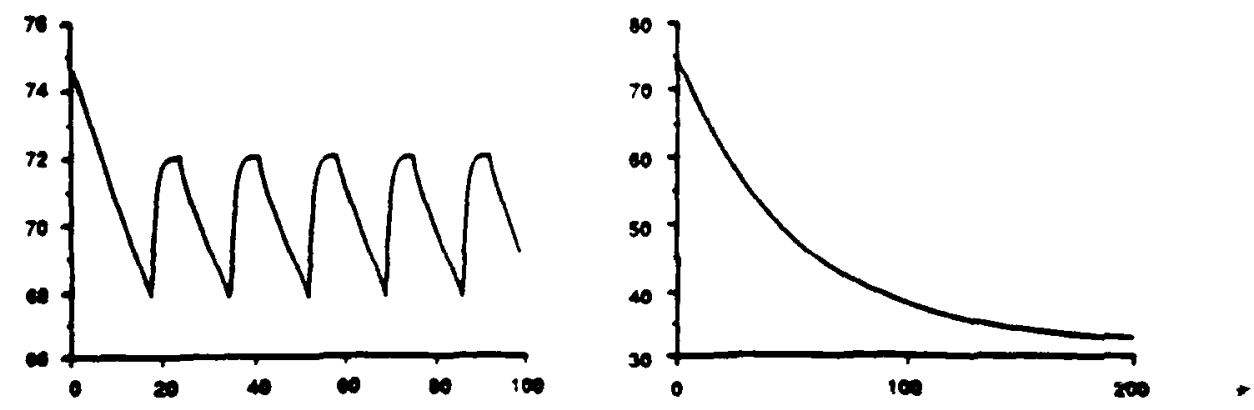

Figare 3.1: Different behaviors for a thermostatically controlled furnace

Suppose that we are interested in the temperature in the room over the interv from time 0 to time 10 . We are told that the temperature outaide is $32^{\circ}$ thronghout this interval, and that at time 0 the room is $75^{\circ}$ with the furnsce on but cursently not heating. We represent these facts as follows:

$\operatorname{bolds}\left(0, r=75^{\circ}\right)$

$\operatorname{bold} s\left(0,10, a=32^{\circ}\right)$

holds $\left(0, \partial r=-\kappa_{2}(r-a)\right)$

$\exists t(0 \prec t) \wedge$ boldo $(0, t$, on $($ furnace 17$))$

We might expect the above axioms to onpport the following inferences. The temperature drope off exponentially' from $75^{\circ}$ to $68^{\circ}$ at which point the furnace starts heating and continues until the temperature reaches $72^{\circ}$, after which the furnace oscillates on and of forever with the temperature al waye betwen $68^{\circ}$ and $72^{\circ}$. This expected behevior is shown on the left in Figure 3.1. Unfortanately, chronologieal minimisation of discontinuities does not support thiv inference. There are chronologically minimal models in which this is the case, but these are aleo chronologically minimal models in which on (fursace17) is clipped just at the time the temperature first drops to $68^{\circ}$, and inatead of cycling forever between $68^{\circ}$ and $72^{\circ}$ the temperature in the room approseches $32^{\circ}$ asymptotically as shown on the right in Figure 3.1.

We can eliminate the unintended model by not allowing simultancous caun and effect. You can think of trane $(|\downarrow| \uparrow \mid, u, v)$ events as a particular cort of canal trigger, and the propositions constraining parameters (e.g.,

\footnotetext{
The bahorior of the syotem can be deceribed is terms of a piecewive costias. ow function in which the specific wolotions for exch piece ure given, alternutely, by $r(t)=32^{\circ}+\left(r_{0}-32^{\circ}\right) e^{-n_{t}}$ and $r(t)=C+\left(r_{0}-C\right) e^{-\left(n_{n}+n_{1}\right) t}$ where $C=\frac{m_{2}\left(00^{\circ}+n_{1} 22^{d}\right.}{n_{1}+n_{2}}$, ro is the initiv temperatare of the room for that purticular piece and the the time elapaed from the beginning of thet piece.
} 
$\partial r=\kappa(r-a))$ as a particular sort of effect. The general form of a causal rule is

holds $(t,[$ entecedent conditions $]) \wedge$

occurs ( $t$, [trigger event type] $) \wedge$

aabnormal $(t,[$ rule identifier] $)]$

$\exists t^{\prime}\left((t+\Delta) \prec t^{\prime}\right) \wedge$ holds $\left(t+\Delta, t^{\prime}\right.$, [conseguent effects] $)$

If $\Delta=0$, then the antecedent conditions, the trigger event, and the consequent effects all compete with one another in the process of chronological minimization. Models in which the antecedent conditions are mysteriously clipped are equi-preferable to models in which the consequent effects occur as expected and result in clippings or discontinuities of their own.

Much of the work in temporal reasoning in artificial intelligence has focused on making precise the intuitions behind cause-and-effect reasoning. By requiring that causes precede effects, we not only avoid certain problems with unintended models, but we aiso subscribe to some of the basic intuitions about causal reasoning.

Our physical model for the thermostatically controlled furnace is not by any means complete. For instance, if we were to add the axiom

\section{$\exists t(8<t) \wedge$ bolds $(8, t$, on (furnace 17$))$}

we would arrive at the inappropriate conclusion that, if the furnace was heating at time 8, then it would continue to do 80 indefinitely. To avoid this unwanted inference, we might add rules saying that whenever anything resuits in the furace "becoming" off, then the temperature in the room is governed by some defapult set of equations. To express thin as an event triggered cansal rule, we might define an analog of trans $([\downarrow \mid \uparrow], u, v)$ for trethvalued parameters. Suppose that beconos $(\varphi)$ corresponds to the event of $\varphi$ becoming true. Adding the following axiom

holda(t, becoses $(\neg$ on $($ furnacel 17$))) \supset$

$\exists t^{\prime}\left(\left((t+\epsilon)<t^{\prime}\right) \wedge \operatorname{holds}\left(t+\epsilon, t^{\prime}, \operatorname{pmod}\left(r, \partial r^{r}=-k_{2}(r-a)\right)\right)\right)$

enourw that we will infer something reasonable in the event that the power to the furnace is cut off.

Note that we can always substitute a set of model that persist over different intervals of time for a single model that is true for all time but with additional parameters that make the model behave differently over different intervals of time. In the furnace example, we might state that 
$\square\left(\partial r=\kappa_{2}(f-a)-\kappa_{1}(r-a)\right)$

and then have rules that govern the value of $f$ over different intervals of time. Whether we vary the model or employ a single model and vary the parameters of the model, we have to provide some means for certain propositions corresponding to equations involving parameters to persist over time.

There remain many open issues in modeling physical systems using temporal logic that are not considered in this section. We will, however, return many times to consider both computational and representational issnes in ressoning about time and change. In particular, the next section is concerned with automating temporal reasoning, Chapter 5 discusses how the temporal logic of this chapter can be used for planning, and Chapter 7 is concerned with temporal reasoning about stochantic processes.

Introduce the concepts of histories, time lines, chronicles and relate them to the notion of state-space trajectories introduced in the previous section.

\subsection{Temporal Logic Programming}

This section is concerned with the design of prectical temporal reasoning systems. We describe a syotem that combines festures from ceveral exinting systems to provide the support that we require for applications in planning and control. The resulting system is presented as an extension of the logic programming language prolog $[9,39]$ angm ated with features, such a forward chaining, normally found in deductive retrieval syotems [31].

In the last section, we preaented a logic without regard to the complexity of determining whether or not a given formuls was velid. Given that boolean sativiability is NP-complete [21], we cannot expect to implement a decicion procedure that is guaranteed to provide correct and timely anewers to all possible queries. To ensure reasonable reapones time for our temporal reesoning system, we reatrict the syntax for both queries and data. In addition, for some types of query, we provide only partial decision procedures (i.e., procedure that occasionally report "don't know" in repponse to a query). This enction represents a catalog of concensions to complexity. Completeneas, expreasiveness, and response time have to be carefully considered in the daiph of any program intended to serve a part of a control eystem. In Chaptes 8, we consider tradeoffe in the dexiga of decicion procedures in some detail; in this section, we are primarily concerned with preanting the basic functions required for practical temporal reasoning, and pointing out potential sources of complexity. 
For the most part, we adopt the syntax of PROLOG. Conditional rules (i.e., PROLOG Horn clauses) are notated $A \leftarrow B$ where $A$ is an atom (i.e., a predicate of zero or more arguments) and $B$ is a conjunction of zero or more atoms. We make use of the negation-as-failure operator, not, to implement various forms of nonmonotonic irference. (The query not $(\varphi)$ succeeds just in case $\varphi$ fails.) We assume the standard semantics for logic programs [3] augmented where needed with informal procedural semsntics.

To speak about the structure of time itselr, we refer to points (or instants) of time, and intervals (or periods) of time. We distinguish between a general type of event or proposition (e.g., "I ate lunch in the cafeteria") and a specific instance of a general type (e.g., "I ate lunch in the cafeteria this afternoon"). The latter are referred to as time tokens or simply tokens. A token asociates a general ty pe of event or proposition with a specific interval of time over which the event is said to occur or the proposition hold.

Our calculus for reasoning about time will be concerned with manipnlating time tokens. Given some set of initial tokens correnponding to events and propositions, we will want to generate additional tokens corresponding to the consequences of the events. Firat, we have to be able to enter new tokens into the PrOLOG database. We notate general types of events and propositions using PROLOG predicates and their negations. For instance, the proposition "the loading dock is unoccupied" might be represented as apty (loading-dock), and its negation a 7 apty (loading-dock). Similarly, the event type "truck \#45 arrives at the loading dock" might appear as arrive(truck45, loading-dock). To enter a new token, we assert an expression of the form, tokon (type, symbol), where type corresponds to a general type of event or proposition, and aymbol is a term that will be asociated with an interval of time. Accerting

token (arrive(truek46, loadingdock), arrival14).

adds a new token of type arriro(truck45,10adingdock) and interval arrival14 to the database.

It is often convenient to refer to the points corresponding to the beginning and end of intervals. If arrival 14 denotes an interval, then bogin (arrival14) denoten its begin point and end (arrival14) denotes its end point. Initially, the interval of time aseociated with a token is completely unconstrained (i.e., it could correapond to any interval). Intervals can be constrained uning ord:nal (e.g., $\prec$ or $\preceq$ ) and metric constraints on their beginning and end points. If arrival14 and departure23 are both intervals, then aserting ond (arrival14) ঝ bogin(departare23) . 
constrains the first interval to end before the second begins. For any interval, int, it is necessarily the case that

begin(int) $\preceq \operatorname{ond}($ int $)$.

Metric constraints allow us to bound the amount of time separiting points. The notation distanee $\left(t_{1}, t_{2}\right) \in[$ low, high $]$ is used to specify that the distance in time separating $t_{1}$ and $t_{2}$ is bounded from above by high and bounded from below by low, where bounds are specified in the form,hours:minutes. For instance, if noon is a reference point corresponding to 12:00 PM today, asserting

distance (noon,begin(arrival14)) $\in[2: 55,3: 05]$.

- natrains the interval ascociated with the arrival of tracks, to occur at 3:00 $\mathrm{rM}$, give or take 5 minutes. If the upper and lower bonnds are the same, we use $=$ instead of $\in$ and one number instead of a pair of numbers.

Given the hours:minutes notation for specifying metric constraints, we have committed to a set of time points isomorphic to $Z$. We could have made it hours:minutes.seconds, but some conceasion vltimately has to be made to the finite precision of arithmetic on the target machine.

To indicate that a bound is unconstrained, we introduce the special symbol $\infty$, so that

- $\infty>n, \forall n \in Z$

- $\infty+\infty=\infty+n=\infty, \forall n \in Z$

- $\infty-\infty=0$

Allowing both metric and ordinal constraints introduces some opecial problems in propagating (i.e., combining) constraints to determine the best bounds on a pair of points (i.e., the grestest lower and least upper bounds on the time separating the two time points). Propagation is simplified by adopting a single representation that captures both types of constraint. We do 20 by introducing yet another symbol $e$ with the following properties:

- $\epsilon$ >

- $n * c<r, \forall n \in \mathbf{Z}, \forall r \in \mathbf{R}^{+}$

$\bullet+\epsilon=2 * \epsilon>\epsilon$ 
Using the above, we define the following ${ }^{10}$

- $t_{1} \prec t_{2} \Rightarrow$ distance $\left(t_{1}, t_{2}\right) \in[\epsilon, \infty]$.

- $t_{1} \preceq t_{2} \Rightarrow$ distance $\left(t_{1}, t_{2}\right) \in[0, \infty]$.

- $t_{1}=t_{2} \Rightarrow \operatorname{distance}\left(t_{1}, t_{2}\right) \in[0,0]$.

We treat events and propositions somewhat differently in our calculus. We assume that the durations of events are specified preciseiy. For instance, we might state that the evert corresponding to the arrival of truck 45 took one minute.

distance (bagin(arrival14), and(arrival14)) $=0: 01$.

For tokens corresponding to propositions, we would like to predict how long the propositions persist once they become true. For instance, suppose that were interested in reasoning about a robot forklift truck that moves appliances around in a warehouse, and suppose we make the following as. sertions to the database:

token(location (forklift, loadingarea), location1).

token(location (forklift, stagingarea), locationz).

distance (noon, brgin(location 1)) $=1: 15$.

distanco (noon, begin(location2)) $=2: 30$.

Assuming the forklift can only be in one of staging_area or loading_area, we conclude that the interval location1 should not persist pant 2:30 PM. In general, we require that the interval corresponding to a token persist no further than the first subsequent interval correaponding to a token of a contredictory type. For any proporition type $\varphi, \varphi$ and $\neg \varphi$ are said to be contradictory. Additional contradictory types have to be explicitly asserted. For instance, the assertion

contradicta(location $(X, L 1)$, location $(X, L 2)) \leftarrow L 1 \neq L 2$.

indicates that any two tokens of type location(ary 1, ang2) are contradictory if their first arguments are the same, and their second arguments are

\footnotetext{
${ }^{10}$ The constraints on time points are repreaented internally as pairs of complex numbers of the form $(\alpha, \rho)$ for $\alpha+p_{e}$, where $\alpha, \beta \in Z$. For inatance the bound, $[e, 1]$ wonld be represeated a $[\langle 0,1\rangle,\langle 1,0)]$. The renalting calculum-firt introduced by Leibaits [32] for studyins the foundations of red analywa-provides convenient basis for propegating and meaipalating ete of equetions including botb ordinal and metric constrainte.
} 




Figure 3.2: Tokens in the TEMPIOG database

different. The process of modifying the bounds on token intervals corresponding to propositions to ensure that tokens of contradictory types do not overiap is refersed to as persistence clipping. One token is clipped by a second in accord with the following rule.

$$
\begin{aligned}
& \text { clips }(K, \operatorname{begin}(J))- \\
& \text { token }(P, K), \\
& \text { toten }(Q, J), \\
& \text { Contradicts }(P, Q), \\
& \text { begin }(X) \prec \text { begin }(J) .
\end{aligned}
$$

The syntax for our temporal logic programming langage severely restricts what can serve as a proposition type and what can be said about two different proposition types being contradictory. The consequences of these restrictions will become clearer as we explore the details query processing.

In the course of our discussions, we will be adding various capabilities to PROLOG to support applications in planning and control. We call this extended logic programming language 'TEM PLOG in recognition of the central -ole of time. For the time being, we asume that TEMPLOC automatically p....roms persistence clipping for all tokens stored in the databave. Later we will have to relax this requirement to deal with the computational complexity of reasoning about partially ordered eveats.

It will help in this and subsequent chapters if we can display the contents of a TEXPLOO database graphically. To that end, we introduce the following graphical conventions. Time tokens are represented with a vertical bar indicating when the corresponding interval begins and either a second vertical ber providing some indication of when the interval ends or an arrow $\rightarrow$, indieating that the end of the interval is far enough in the future that it can't be drawn in the diagram. The delimiters for tokens are connected by a horisontal bar (e.g., $\rightarrow$ ). Each token is labeled with a aymbol corresponding to its associated interval and a formula denoting its type. The tokens are laid out on the page $s 0$ as to indicate their relative offet from some global reference point. Figare 3.2 depicts the information stored in the rEMPLOC database as a consequence of the four ancertions linted in the pre- 
vious paragraph. In Figure 3.2, the token interval location 1 is constrained to end before the beginning of the token interval location 2 by the process of persistence clipping.

Given a database of time tokens, one is generally interested in answering queries concerning what propositions are true over what intervals of time. We begin by defining two primitive queries involving tokeas and the bounds on the distance separating pairs of points. All of our other temporal queries * can be defined in terms of these primitives.

- token (type, int) succeeds once for each token in the database unifying with type and int.

- distanco $\left(t_{1}, t_{2}\right) \in[l, h]$ succeeds just in case $G L B \leq l \leq h \leq L U B$, where $G L B$ and $L O B$ correspond to the least upper and greateat lower bounds on the distance in time separating $t_{1}$ and $t_{2}$ given the closure of the set of constraints. If either $t_{1}$ or $t_{2}$ are not bound, the query will fail. If one or both of $l$ and $h$ are not bound, then, anuming that the query would succeed otherwise, it does so with the variables bound to their respective least restrictive bounds.

A temporal query of the form holde $\left(t_{1}, t_{2}, \varphi\right)$, where $\varphi$ is an atom, should succeed just in case there is a token in the database of type $\varphi$ constrained to begin after or coincident with $t_{1}$ and not constrained to end before $t_{2}$. We can stite this in terms of token and distance as follows.

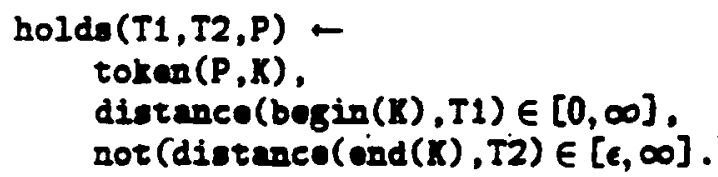

and add an additional prococ rule to handle degenerate intervals holde $(T, P) \leftarrow \operatorname{holdu}(T, T, P)$.

Complex temporal queries involving conjunctions and dinjunctions can be defined in terms of atomic queries uning the standard ProLOG notational conventions (i.e., $(P, Q)$ and $(P ; Q)$ are, renpectively, the conjunction and disjunction of $P$ and $Q$ ). Conjunctive temporal queriea are defined by

holda $(T 1, T 2,(P, Q))-\operatorname{holda}(T 1, T 2, P)$, holda $(T 1, T 2, Q)$.

One way of defining disjunctive queries is

holde $(T 1, T 2,(P ; \lambda) \rightarrow \operatorname{hold}(T 1, T 2, P)$.

holds $(T 1, T 2,(-; Q))-\operatorname{holds}(T 1, T 2, Q)$. 
While this definition is simple to implement, it fails in some cases where we might expect it to succeed. For example, according to the definition above, if all we know is holds $(t 1, t 2, p)$ and bolds $(t 2, t 3, q)$, holds $(t 1, t 3,(p ; q))$ fails. As an alternative definition, we might have bolds $\left(t_{1}, t_{2},\left(\varphi_{1} ; \varphi_{2}\right)\right)$ just in case for all $t_{1} \preceq t \preceq t_{2}$ either bolds $\left(t, \varphi_{1}\right)$ or holds $\left(t, \varphi_{2}\right)$. The alterna. tive definition does not, however, conform to the semantics of our logic of time intervals as given in the previous section; hence, we adopt the original. definition from here on.

Oring negation as failure, we can achieve some, but not all, of the functionality of true negation. For instance, we might define

$\operatorname{bolds}(T 1, T 2, \operatorname{not}(P)) \leftarrow \operatorname{not}(\operatorname{bolds}(T 1, T 2, P))$.

where not $\left(\right.$ bolds $\left.\left(t_{1}, t_{2}, \varphi\right)\right)$ succeeds just in case bolds $\left(t_{1}, t_{2}, \varphi\right)$ fails. ${ }^{11}$

(Queries involving the negation-ab-failure operator can be confusing to the uninitiated. As an example, the behavior of temporal queries in TexPLOC involving unbound variables and the negation-aefailure operator is dependent upon the order of conjuncts just at it is for atemporal queries in proloc. For instance, assuming that holda $(t 1, t 2, p(a))$ and holds $(t 1, t 2, q(b))$, holds $(t 1, t 2,(p(x), \operatorname{not}(q(x))))$ will succeed whereas holds $(t 1, t 2,(\operatorname{not}(q(x)), p(x)))$ will fail.)

While there is no direct mapping from negation in ous logic to negation as failure in PrOLOG, there are certain properties of the negation-as-failure operator that we might want to preserve in our temporal extensions of PROLOG queries. For instance, in PROLOG, not $(\operatorname{not}(\varphi))$ succeeds if and only if $\varphi$ succeeds. Note that holda $\left(t_{1}, t_{2}, \operatorname{not}(\operatorname{not}(\varphi))\right)$ is (procedurally) equiralent to holda $\left(t_{1}, t_{2}, \varphi\right)$ using the first definition but not asing the second. We adopt the firat definition in the following. .

We asume that TEMPLOG procenses both atomic and complex temporal queries efficiently. To illustrate TEXPLOG query procesaing, suppose that the following five queries are initiated in the database depicted in Figure 3.3.

bolds (bogin(sorticel), end(servicel), locat1on(truck45, loadingdock)).

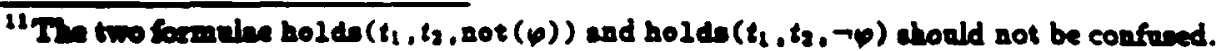
It in best thint of $\neg p$ a a particular atring defined to atand in come relationship to the otrins $p$, when that relationahip in defined by the operstion of dipping. Alterantively, we might dalne holde $\left(t_{1}, t_{2}\right.$, not $\left.(\varphi)\right)$ to soceed just in case there is some point $t$, soch that $t_{1} \preceq t \leq t_{2}$ and holds $(t, \varphi)$ fails.
} 


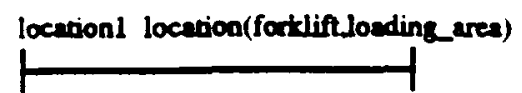

locenoon locesion(fortliftragine_rea)

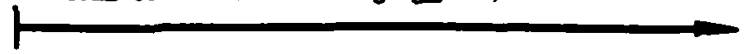

locerion3 location(troct45 Josding_dock)

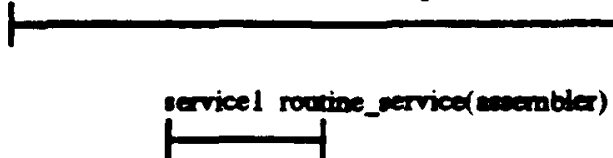

servicel rodine_serviced (ascenbler)

savice2 rouine_envicedfodtin)

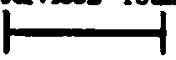

Figare 3.3: TEMPLOG database for illustrating query processing

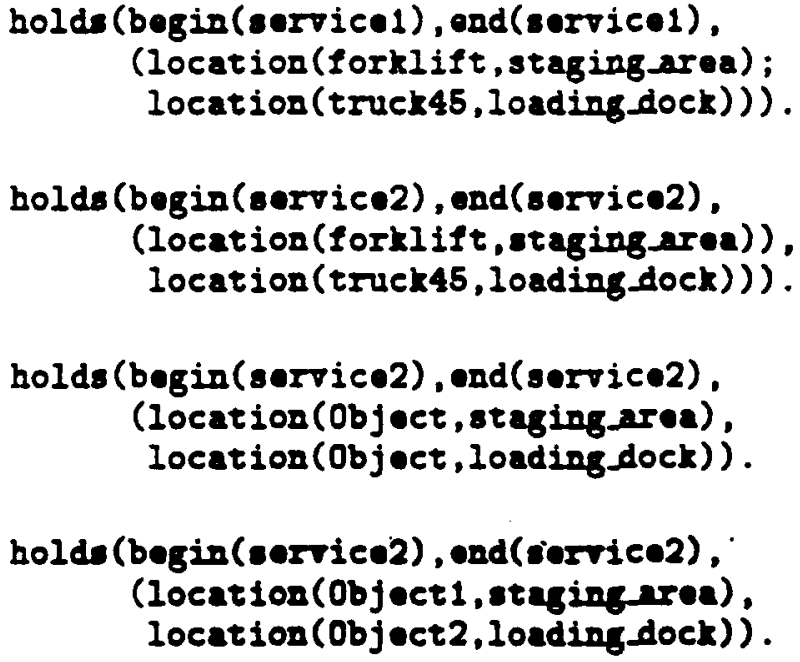

The first three queries succeed; the fourth fails, and the fifth succeeds with Object 1 bound to forklfft and Object 2 bound to trucks5.

There are also abductive vervions of holds that are aseful for building planning iystems. The query bolds $\left(t_{1}, t_{2}, \varphi\right)$ fails if either of $t_{1}$ or $t_{2}$ are unbound. However, the abductive version of this query, Obolda $\left(t_{1}, t_{2}, \varphi\right)$, succeeds under a superset of the conditions that holds $\left(t_{1}, t_{2}, \varphi\right)$ does. In particular, if either $t_{1}$ or $t_{2}$ are not bound, then new (i.e., totally unconstrained) points are created and bound to the variables. Once bound, the query succeeds if the set of constraints can be augmented so that the non. abductive query succeeds. The set of constraints necesuary for the abductive 
query to succeed are referred to as abductive constraints. Abductive constraints are accumulated during backward chaining and withdrawn during backtracining similar to the way in which variable bindings are handled in PROLOG. Consider the database resulting from the following assertions.

token $(p, j)$. $\operatorname{diatanca}(\operatorname{bog} i n(j), \operatorname{and}(j))=5$.

$\operatorname{token}(q, k)$. $\operatorname{distanco}(\operatorname{bog} i n(j), \operatorname{bog} i n(k))=3$.

distance $(t 1, t 2)=3$. distance $(b e g i n(k), t 1) \in[-5,5]$.

Of the following six queries, those on the left fail while those on the right succeed.

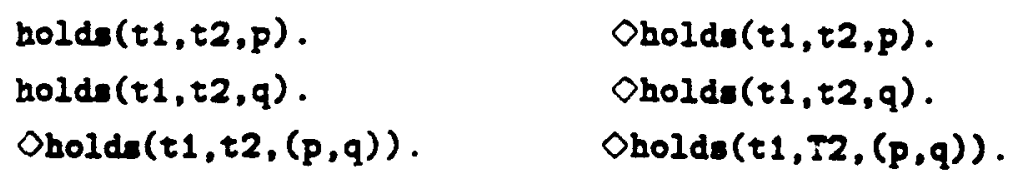

We will say more about abductive query processing in Chapter 5.

Persistence clipping is one type of routine inference important in ressoning about time and change. There is a second type of routine inference, called projection, that we would like Teuploc to perform for us. Projection is concerned with inferring the consequences of events based on a model specified in terms of the cause-and-effect relationships that exist between various event types. To notate such relationships, we use the following form

project (antecedent_conditions, trigger_event, deloy, consequenteffects)

to indicate that, if an event of type trigger event occurs, and the antecedent conditions hold at the outset of the interval associated with trigger event, then the consequent effects are true after an interval of time determined by delay. The trigger event is apecified a a type, the antecedent conditions and consequent effects are specified es types or conjunctions of types, and the delay is apecified a a pair conninting of a lower and an upper bound on the time between the end of the trigger event and the manifeatation of the effects. If the upper and lower bounds are the same, a single bound can be substituted for the pair. We ansme a convenient notationd filter so that the delay argament can be left out of acertions and queries; in the former case, a defanit delay of $[\epsilon, \epsilon]$ is provided. The rule $R 2$ from the previous sective can be encoded as follows.

project ( 70 (furnece17), togslo(oriteh42), op(furance17))

To specify that, whenever the forklift moves from one location to another, it will appear in the nen location after a delay determined by the 
(spatial) distance to be traveled and the minimum and maximum rate of travel allowed by the forklift, we would assert the following

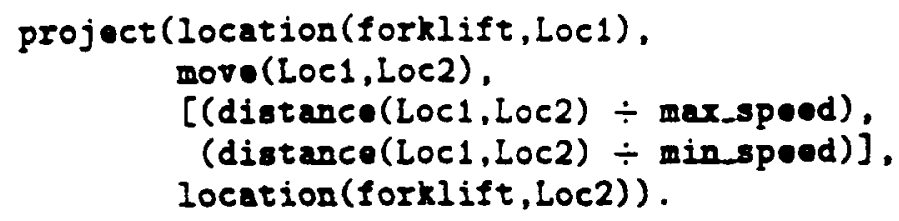

As another example, suppose that the robot forklift is also responsible for installing options in appliances (e.g., installing an ice maker in a stock refrigerator). The following projection stipulates that whenever the robot turas on a particular assembly unit when an appliance and an appropriate option are on the input conveyor, then 30 minutes later, give or take five minutes, the appliance will appear in the output conveyor with the option properiy installed.

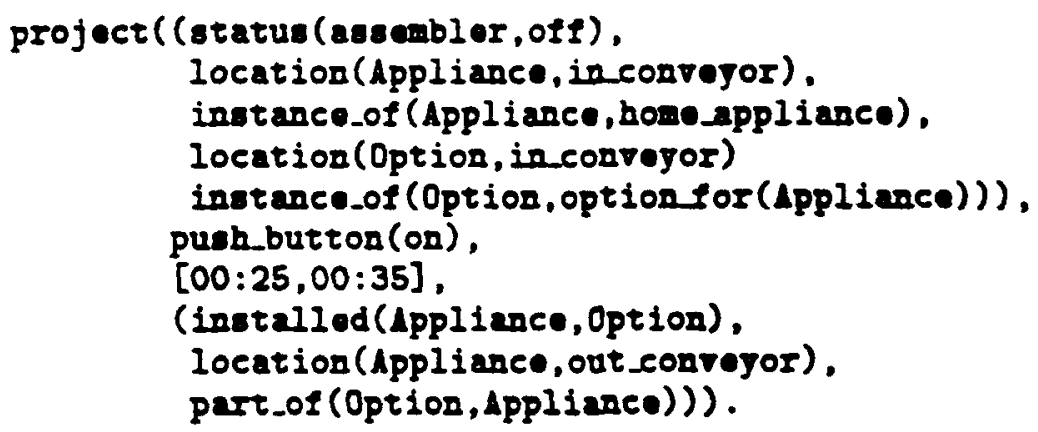

In order to determine whether or not an event has an effect at a particular time, we define the following

causes $(B, R, T)-\operatorname{project}(P, E, R), \operatorname{occure}(E ; T), \operatorname{bolda}(T, P)$.

The projection rules presented above allow for a very restricted form of cansal reaconing. In particular, they do not provide for any means of dealing with the qualification problem described in the previous ection. By modiffing ous causes rule slightly, we can resson about qualifications in a masace similar to that described in the previous section.

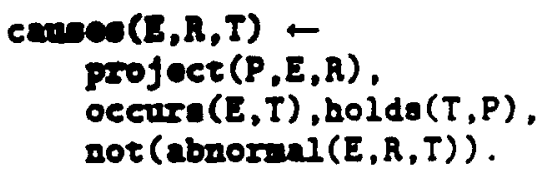

The rule $Q 1$ from the previous section can be encoded as follows.

abnornal (togsle (avitch42), on(furacee17), T) bolde (T,open(fuse43)) 
We inciude the typ: of the trigger event and the type of the consequent effect because the qualuication is likely to depend on them. Note that neither is sufficient alone, since the event of toggling the switch may have other effects (e.g., the switch may make a noise whether or not it makes or breaks a connection), and other events may have the effect of turning the furnace on (e.g., attaching it directly to a backup diesel generator that bypasses the fused circuit). For more complicated applications, it may be useful to allow disabling rules that serve to disable other diabling rules. We do not do $s 0$ here, but it would be straightforward to extend the above to handie a hierarchy of disabling rules (i.e., a set of disabling rules arranged hierarchically with a projection rule at the root so that each disabling rule in the tree is allowed to disable its immediate ancestor in the tree).

Qualifications in projection roles allow as to introduce a very restricted form of quantification. $A:$ an example, consider the following rule.

project ((Clear (X), Clear $(Y)$, on $(X, د), \operatorname{puton}(X, Y), 02(X, Y))$.

For an event of type puton(block1,block2) to have the consequent effect on(block1,block2), there have to be tokens in the database of type clear (block1) and clear(block2). Alteraatively, we can wee the following projection rule

project $(00(X,-), \operatorname{puton}(X, Y)$, on $(X, Y))$.

coupled with the following qualification

abrorand (puton $(X, Y)$, on $(X, Y), T) \leftarrow \operatorname{boldn}(T,(\operatorname{On}(-, X) ;$ on $(-, Y)))$.

to ensure that puton(block1,block2) has the effect on(block1,block2) just in case there are no token in the database with appropriately constrained intervals corresponding to romething being on either blockl or blocke.

Projection is the process of generating new tokens from some set of initial tokeno-roughly corresponding to the boundary conditions in a phyaics problem-uning a sat of projection rules. The bavic alforithm for handling both projection and persintence clipping is rather simple to implement. To aimplify its description, we assume that all trigger events are point events. Wheanere tokens or constraints are added to or deleted from the database, the sytam carrice out the following oteps.

1. Delete all tokens and constraints added the last time the algorithm was ran. 
2. Place all tokens in the database whose types correspond to events on the open list.

3. Let token be the earliest occurring token in the open list.

4. Find all rules whose trigger event type unifies with the type of token.

5. For each rule found in Step 4 whose antecedent conditions are satisfied, add to the database tokens corresponding to the types specified in the consequent effects, and constrain them according to the specified delay.

6. For each new token added in Step 5 whose type corresponds to an event, place it on the open list.

7. For each new token added in Step 5 whose type does not correspond to a finent, find all tokens of a contradictory type that begin before the newly added token and constrain them to end before the beginning of the new token.

8. Remove token from the open list.

9. If there are no tokens remaining on the open lint, then quit, else go to Step 3.

We will assume that TEMPLOG uses an algorithm similar to the above to ensure that the database contains all and only those tokens warranted by the set of initial tokens, and the projection rules stored in the database. Updaten can be performed in time polynomial in the size of the initial conditions and the set of projection rules. Query procenaing is performed by rearching through the set of tokens generated by the projection algorithm, using the types of the tokens and the constraints on token intervals to guide the rearch. The above projection algorithm supports basic reseoning about the truth or falsity of propositional formulae; in the following, we consider extencions to handle real-valued parameters.

Le $\boldsymbol{U}$ be set of real-valued parameters, and $P$ be a set of booleanvalued propositional variables. ${ }^{12}$ In addition, we introduce two mappinga $Q: \mathbf{R} \times \boldsymbol{\sigma} \rightarrow 2^{\mathbf{R}}$ and $V: \mathbf{R} \times \boldsymbol{P} \rightarrow 2^{\{(r m, f d m\}}$. The task of projection is to determine $Q$ and $V$ for some cloned interval of $\mathbf{R}$. We begin by considerin: the completely determined case in which both $Q$ and $V$ map to aingleton sets (i.e., $Q: \mathbf{R} \times U \rightarrow \mathbf{R}$ and $V: \mathbf{R} \times \boldsymbol{P} \rightarrow$ \{true, false $\}$ ).

\footnotetext{
${ }^{13}$ Due to the presence of variables and complex terms, templos rules are sehemata for proporitiond aroms. The underlying lope remain purely proponitionel.
} 
At the initial time point, assume that the values of all parameters and propositional variables are known. In addition, we are given a set of events specified to occur at various times over the time interval of interest. We assume a set of projection rules as before. In addition, we sssume a set of modeling rules for parameters in $U$. A modeling rule is just a special sort of projection rule; the basic form is the same as that introduced earlier in this section, the only difference being that the delay is always assumed to be $\epsilon$, and the consequent effects consist of parameter assignments in the form of ordinary differential equations ${ }^{13}$ with constant coefficients (e.g., $\partial u=2$ or $\left.\partial^{2} u=3 \partial u+5 u+4\right)$.

The projection rule from the last section for ressoning about the temperature of the room in the case that the furnace is off is encoded as follows.

project (on $\left(\right.$ furace17), trans $\left.\left(T, r, 68^{\circ}\right), \operatorname{prod}\left(r, \partial r^{r}=-\kappa_{1}(r-a)\right)\right)$.

To make sure that persistence clipping is handled correctly, we state that a given parameter can have only one zoodel at a time.

contradicts $(\operatorname{pmod}(X, M 1), \operatorname{Pmod}(X, M 2)) \leftarrow M 1 \neq M 2$.

Now we can state the basic algorithm for perforraing projection given some set of initial conditions and a projection interval $\left[t_{s}, t_{f}\right]$. To simplify the description of the algorithm, we asume that all events are point events (i.e., if $e$ is a type corresponding to the occurrence of an event, token $(e, k) \supset($ begin $(k)=\operatorname{ond}(k)))$, and all events described in the initial conditions begin after $t_{\text {. }}$. Let $\mathcal{A}$ be the set of all currently active process models (i.e., all $m$ ouch that holda $\left(t_{c}, \operatorname{prod}(x, m)\right.$ ) for some $\left.x\right)$. Let $\varepsilon$ be the set of pending events (i.e., the set of all events, tokeal $(e, k)$, generated so far such that $t_{c} \prec$ bogin( $(k)$ ). Let $C$ be the set of current conditions (i.e., all $u^{p}=v$ such that there exists $m \in \mathcal{A}$ such that holda $\left(t_{e}, \operatorname{prod}(x, m)\right)$, $u=\partial^{n} x$ for some $n$, and holds $\left(t_{c}, u^{r}=v\right)$.

In the cases that we are intereated in, we can recast a set of ordinary differential equations and their initial conditions as a oystem of first-order difierential equations. We can then colve these equations uning aumerical mathods based on the Taylor expantion (e.g., the Runge-Kutta methodo (60]) and varions forms of lineas and nonlinear extrapolation (e.g., the Adams-Banforth and Adams-Moulton methods $[56,46])$. The particulas

\footnotetext{
"To expodite the neceasury compotation, we ameme that all cquationo are sth order or lew, and that they can be rewritten 00 that hiphestorder ters in algebraically ivolnted os the lefthand ade of the equation.
} 
numerical method chosen is not important for our discussion. In the following, we simply assume the ability to generate solutions to ordinary differential equations efficientiy, and refer to the procedure for generating such solutions as the extrapolation procedure. Given a set of initial conditions and a projection interval $\left[t_{s}, t_{f}\right]$, projection is carried out by the following algorithm.

1. Set $t_{e}$ to be $t_{s}$.

2. Set $\mathcal{E}$ to be the set of events specified in the initial conditions.

3. Osing $\mathcal{A}, \mathcal{C}$, and the extrapolation procedure, find $t_{n}$ correnponding to the earliest point in time following $t_{c}$ such that the trigger for some projection rale is satiofied or $t_{f}$ whichever comen first. If $t_{n} \neq t_{f}$, then $t_{n}$ could be the time of occurrence of ihe earlieat event in $\mathcal{E}$, or it conid be earlier, corresponding to the solution of a set of equations (e.g., $\left.\left(\left(x_{1}=x_{2}\right) \wedge\left(\left(\dot{x}_{1}-\dot{x}_{2}\right)>0\right)\right)\right)$.

4. If $t_{n}=t_{f}$, then quit, else set $t_{c}$ to be $t_{n}$.

5. Find all projection rules with the trigger found in Step 3.

6. For each rule found in Step 5 whose antecedent conditions are satisfied, add to the database tokens corresponding to the types of the concequent effects except in the case of consequent effects corresponding to parameter assignments (e.g., $x_{1}^{r}=x_{j}^{r}$ ). Constrain the new tokens according the delay specified in the corresponding rale.

7. For each token added in Step 6 whose type correpond to an event, add it to $\mathcal{E}$.

8. For each token added in Step 6 whose type does not correspond to an event, find all tokens of a contradictory type that begin before the newly added token and constrain them to end before the beginning of the new token.

9. If the trigger found in Step 3 correaponds to the type of an event token in $\mathcal{E}$ whose time of occurrence is $t_{e}$, remove it from $\mathcal{E}$.

10. Ose the consequent effects corresponding to parameter asuignments found in Step 6 and the reaulta of extrapolation to determine $\mathcal{C}$. The parameter assignments corresponding to the consequent effects of projection rules take precedence over the extrapolation results. 


\section{Go to Step 3.}

There are lots of other rules that we would have to specify in order to model the operation of the assembler in enough detail to support useful prediction. We would have to state that pushing the on button when the assembler is off causes it to become on,



and that a machine can not be on and off at the same time,

contradicts (status $(X, S 1)$, status $(X, S 2)$ ) $\leftarrow$ S $1 \neq S 2$.

In fact, there are potentially an infinite number of rales that would be required to correctly model the behavior of the assembler nader every set of circumstances. Note that the assembler requires power, and the appliance and the options to be installed must be in some reasonable state of repair, and there can't be anything blocking the output conveyor; all of these conditions and more would have to be made explicit in the rules if re required a model guaranteed to produce correct predictions in every conceivable situation. This proliferation of antecedent conditions was addressed in the context of the qualification problem discuseed in Section 3.1. There is also a problem with consequent effects; If the robot pleces a part in a box, then the part is in the box. If the robot then places the box in a truck, then the part is still in the box, but it is also in the truck. If the robot then drives the truck to a new location, then, by virtue of being in the box which is in the truck, the part is in the new location aloo. Keeping track of all of the consequences of an action has been termed the ramification problem [18], and constitutes a aignificant problem in building practical temporal reaconing systems.

The TEMPLOG rules that comprise a physical model are intended as an approximation. Greater accuracy can often be obtained by adding more rulee, but there is a price to be paid in terms of computational overhead, and the increased accuracy may not result in a signifiesat increase in performance. The ides behind causal modeling is that an appropriate model will eficienth semerate those common-sense predictions that are likely to have the gratent impact on the performance of the robot. It is up to the programmer to determine what rules are necessary to generate these common-sense predictions.

This is where the material on reasoning about partial onders and uncertainty should go (separate section?). What if the initial conditions are not 
sact, but, rather, are specified in terms of intervals or distributions. Talt about the use (and abuse) of Monte-Carlo methods for reasoning about underspecified initial conditions. Introduce the notion of possible time lines, and connect this with model theory developed in Section 3.1. Finally, motivate the uncertainty issues developed in Chapter 7.

\subsection{Further Reading}

Perhaps the beat known approach to reasoning about change in artificial intelligence is the situation calculus. $[40,42,34]$. MeCarthy is generally given credit for the basic idea, but many researchers have contributed to the development of what today is referred to as the situation calcuins. A situction corresponds to the state of the world at a particular instant in time. Change results a a consequence of actionn occurring in situations, where an action can be thought of as a funetion from situations to situations that maps the situation in which the action occurs into the next situation. While some attempts have been made to incorporate reasoning about continuous processes within the situation calculus [30], many researchers have considered other approaches for reasoning about real-world processes.

In the late 1970's, Hayes issued a challenge to the researeh community to formalize a large corpus of knowledge about phyuical processes [28]. Hayea got thinga started by proposing an axiomatic theory of how liquids behave [29]. Hayes's theory describen change over time using four-dimensional piecen of space-time called histories. Other researchers, interested in rensoning about phyaical phenomens whoce opatiel properties are leas central, adopt a variety of temporal logies in which change is modelad in terms of some form of canal relation $[2,43]$. The frame problem appeared in all of these logies in one form or another and come revenrehers believed that the frame problem could be solved by employing some form of noamonotonic reasoning $[41,52,44]$.

This belief that nonmonotonic reasoning would solve the frame problem we dealt a blow by the work of Hanks and McDermott, which showed that a itraightforward epplication of existing nonmonotonic logies was not sufficient to colve the probler: $[24,25]$. The rewe: reh community immediately countered with ceveral proposals for solving the particular temporal reasoning problem posed by Hanks and MeDermott $[36,33,57]$, all based on some variation on the idea of chronological minimiration. Subsequent work has focused on formalising causation to solve the frame problem $[37,27]$, and 
coping with problems that involve reasoning both forwarc (projection) and backward (explanation) in tirse $445,38,4,55]$. Say something about the possible worlds approach to recisoning about action: [22, 63].

The idea of preferring certain models over others in order to define a notion of semantic entailment for nonmonotonic logics is due to Bossu and Siegel [6] and (independently) Shoham [58]. Shoiam's formulation is the more general of the two. The idea of selecting models that are minimal with respect to some property and some ordering relation is developed in Iifschitt [36], Kautz [33], and Shoham [57]. The term "chronologicai minimization" idue to Shoham [57]. See also Doyle and Wellman [16] an some fundamental limitations of nonmonotonic lagics based on preference orders.

Much of the work in the philosophical litersture hes focused on the use of modai logies to model time $[49,53,59]$. This has also been the case for theortical computer science in derigning logica to reason about computational processes $[2648,19,47]$. In the case of computer science, one important reason for the emphasis on modal logic is that such logics are somewhat easier to analyze in terms of the complexity of their respective decision problems. As far a expreani-e power is concerned, given that it is posaible to translate any modal logic with standard Kripke semantics into classical logic, it would seem that the interval logic presented here is at least as expressive as any modal logic of time $[58,59]$.

The syntax and semantics for the proponitional case of the temporal logic that we adict were introduced to the artificial intelligence community by McDermott [43]. Shoham [58] provided the semantics for the firtt-order case, and it is a antactic variast of bis formulation that we use hese.

There hes bees a significant amount of work in arti? ial intelligence on modeling pbysicel processes without employing the eort of quantitative andyris prevalent in engineering. This work, involving qualitative reasoning about physical vitemi eicerally make nee of discrete vilue spaces and a special type of differential equation to draw conciuaions about the behavior of continnous processes [5'. Given that the applications that we consider in this monograph typically require some sort of quantitative andysis, it seems reacomable to incorporate into our logic those parts of the differentiel calculus that sem made fns the job [51]. The sementic treatment presented here is based an the wort of Sandewall [54j, but the basic approsech to reasoning about proceases wa influenced significantly by the work of Forbus [20] and de Kleer [11].

The practi:al problems in building useful temporal reasoning systems are manifold, and have given rive to a rich technical literature. Moch $c i$ the 
early work rrakes use of the situation calculus. Green describes a method for applying automated theorem proving to reasoning about time in the situation calculus [23]. Later work sought to avoid the need for frame axioms by introducing some form of nonmonotonic inference into the operation of the temporal ressoning algorithm. Fikes et al.\} implicitly make use of the common-sense law of inertia in their implementation of STRIPS [17]. The temporal reasoning system described in this section is based on the work" of Dean $[15,12]$, but was influenced significantly by other event-based approaches to reasoning about time and causality (e.g., $[1,35,60,61])$.

Davis discusses the computational issues involved in propagating metric constraints for reasoning about time [10], and Dean considers the issues involved in onganizing large amounts of temporal information so as to expedite the sort of causal reasoning described in this section [19]. Willins provides a wealth of prectical advice for systems designers building the temporal reasoning component of a planning system; in particular, his discussion regarding the limited use of quantifiers in causal rules is worth reading [62]. It should be mentioned that the simple projection algorithm described above is not guaranteed to work property if the tokens corresponding to the initial conditions are partially ondered. The general problem of predicting the consequences of a set of partially ordered events is potentially intractable [8]. To deal with this potential source of complexity, partial decioion procedures have been developed to avoid expending too much effort in performing projection [14]. 


\section{Bibliography}

(1) James Allen. Maintaining knowledge about temponal intervals. Communications of the ACM, 26:832-843, 1989.

[2] James Allen. Towards a general theory of action and time. Artificial Intelligence, 29:129-154, 1984.

[3] Krzysztof Apt and M.B. van Emden. Contributions to the theory of logic programming. Journal of the ACM, 29:841-862, 1982.

[4] Andrew B. Baker and Matthew L. Ginsberg. Temporal projection and exlanation. In Proceedings IJCAI 11. IJCAI, 1989.

[5] Deniel G. Bobrow, editor. Qualitative Reaconing and Phyaical Syetems. MIT Press, Cambridge, Massachusetts, 1985.

[6] G. Bossu and $P$ Siegel. Saturation nonmonotonic reasoning, and the closed-world assumption. Artificial Intelligence, 25:179-189, 1985.

[7] Ronald J. Brochman, Bector J. Levesque, and Roymond Reiter, editors. Proceedings of the First International Conference on Principles of Knowledge Representation and Resoning. Mongan-Kaufmann, Los Altos, California, 1989.

(8) Desid Chapman. Planning for conjunctive goals. Artificial Intelligence, s2:sss-s77, 1987.

[9] W.I. Clocksin and C.S. Mellish. Programming in Prolog. Springer Verleg, New York, 1984.

(10) Emest Davis. Constraint propagation with interval labels. Artificial Intelligence, 32:281-331, 1987. 
[11] Johan de Kleer. Mult: ${ }^{2}$ le representations of knowledge in a mechanics problem solver. In Proceedings IJCAI 5, pages 299-304. IJCAI, 1977.

[12] Thomas Dean. An approach to reasoning about the effects of actions for automated planning systems. Annals of Operations Research, 12:147167, 1988.

[13] Thomas Dean. Using temporal hierarchies to efficiently maintain large temporal databases. Journai of the ACM, 36(1):687-718, 1989.

[14] Thomas Dean and Mark Boddy. Rewoning about partially ordered events. Artificial Intelligence, 36(9):375-399, 1988.

[15] Thomas Deen and Drew V. MCDermott. Temporal data base management. Artificial Intelligence, 32(1):1-55, 1987.

[16] Jon Doyle and Michoel P. Wellman. Impediments to universal preference-based defoult theories. pages 94-102, San Mateo, CA, 1989. Morgan-Koufmann.

[17] Richand Fikes and Nits J. Nibson. Strips: A new approach to the application of theorem proving to problem solving. Artificial Intelligence, 2:189-208, 1971.

[18] Joseph Jeffrey Finger. Exploiting constraints in derign symthesis. Ph.D. Thesis, Stanford University, 1987.

[19] Michael Fischer and Richand Ladner. Propositional modal lagic of programe. In Proceedinge of the Ninth Annual ACM Symposinm on The ory of Computing, pages 286-294, 1977.

[20] Kenneth D. Forbus. Qualitative process theory. In Bobrov [5], pages 85-168.

[21] Michoel R. Garey and David S. Johrson. Computing and Intractibility: A Guide to the Theory of NP-Completeneas. W. B. Freemen and Company, New York, 1979.

[22] Matthew L. Ginsbery and David E. Smith. Reasoning about ection i: a possible worlds approach. Artificial Intelligence, 35:165-195, 1988.

[2s] Conill C. Green. Application of theorem proving to problem solving. In Proceedinge IJCA" 1, pages 219-239. IJCAI, 1969. 
[24] Steve Hanks and Drew V. AfCDermott. Default reasoning, nonmonotonic logics, and the frame problem. In Proceedings AAAI-86, pages 328-333. AAAI, 1986.

[25] Steve Banks and Drew V. McDermott. Nonmonotonic logic and temporal projection. Artificial Intelligence, 33:379-412, 1987.

[26] David Harel. First-Order Dynamic Logic. Springer-Verlag, New York, 1979.

[27] Brian Baugh. Simple causal minimizations for temporal persistence and projection. In Proceedings AAAl-87, pages 218-223. AAAI, 1987.

[28] Patrick Hayes. The naive physics manifesto. In Donald Michie, editor, Expert Systems in the Microelectronic Age, pages 242-270. Edinburgh University Press, 1979.

[29] Patrick J. Hayes. Naive physics i: Ontology for liquids. In Jerry E. Bobbs and Robert C. Moore, editors, Formal Theories of the Common Sense World, pages 71-107. Ablex, Norwood, New Jersey, 1985.

[30] Gary Bendrix. Modeling simultaneous actions and continuous processes. Artificial Intelligence, 4:145-180, 1979.

[31] Carl Bewitt. Planner: $A$ language for proving theorems in robots. In Proceedings IJCAI 1, pages 295-301. IJCAI, 1969.

[32] Albert E. Burd and Peter A. Loeb. An Introduction to Nonstandard Real Analysis. Barcourt Brece Jovenovich, New York, 1985.

[33] Henry Kautz. The logic of persistence. In Proceedings AAAI-86; pages 401-405. AAAI, 1986.

[34] Robert Kowalski. A Logic for Problem Solving. North-Bolland, New York, 1979.

[35] Robert Kowalski and M. J. Sergot. A logic-based calculus of events. Now Generation Computing, 4:67-95, 1986.

(30) Vadimir Lifschitz. Pointwise cincumscription: preliminary report. In Proceedings AAAI-86, pages 406-110. AAAI, 1986.

[37] Vladimir Lifschitz. Formal theories of action. In Proceedings IJCAI 10, pages 966-972. IJCAI, 1987. 
(38) Vadimir Lifschitz and Arkady Rab nov. Miracles in formal theories of action. Artificial Intelligence, 38:225-237, 1989.

(39) David Maier and David Warren. Computing with Logic: Logic Programming with Prolog. Addison-Wesley, Reading, Massachusetts, 1988.

[10] John McCarthy. Programs with common sense. In Marvin Minsiby, editor, Semantic Information Processing, pages 403-118. MIT Press, Cambridge, Massachusetts, 1968.

(41) John McCarthy. Circumscription - a form of nonmonotonic reasoning. Artificial Intelligence, 13:295-323, 1980.

(42) John MeCarthy and Patrick J. Hayes. Some philosophical problems from the standpoint of artificial intelligence. Mechine Intelligence, 4:463-502, 1969.

(43) Drew V. McDermott. A temporal logic for reasoning about processes and plans. Cognitive Science, 6:101-155, 1982.

[41] Drew V. MeDermott and Jon Doyle. Non-monotonic logic i. Artificial Intelligence, 13:41-72, 1980.

[45] L. Morgenstern and Stein L.A. Why things go urong: A formal theory of cousal reasoning. In Proceeding AAAI-88, pages 518-52s. AAAI, 1988.

(16) R. H. Pennington, editor. Introductory Computer Methode and Nvmerieal Aralyis:" Mecmilleon, 1971.

[17] Amir Pnueli. The temporal logic of programs. In Proceedinge of the 18th Annual Symposium on Foundations of Computer Science, pages 46-67, 1977.

[48] V. R. Pratt. Process logic. In Proceedings of the 6th POPL, pages 92-100. ACM, 1969.

[49] A.N. Prior. Pant, Present, and Future. Clarendon Press, 1967.

[50) A. Raloton and P. Rabinowitz. A Firat Course in Numerical Analyais. McGrew-Bill, New York, 1978. 
[51] Manny Rayner. Did newton solve the "extended prediction problem?". In Brachman et al. [7], pages 381-385.

[52] Raymond Reiter. A logic for default reasoning. Artificial Intelligence, 13:81-132, 1980.

[53) Nicholas Rescher and Alasdair Urquhart. Temporal Logic. SpringerVerlag, New York, 1971.

[54] Erik Sandewall. Combining logic and differential equations for describing real-world systems. In Brachman et al. [7], pages \$12-\$20.

[55] Erik Sandewall. Filter preferential entailment for the logic of action in aimost continuous worlds. In Proceedings IJCAI 11. IJCAI, 1989.

(56) L. F. Shampine and M. K. Gordon. Computer Solution of Ordinary Differential Equations. W. B. Freeman and Company, 1975.

[57) Youv Shoham. Chronological ignorance: Time, nonmonotonicity, necessity, and causal theories. In Proceedings AAAI-86, pages 989-99s. A.AAI, 1986.

[58] Yoav Shoham. Reasoning About Change: Time and Cansation from the Standpoint of Artificial Intelligence. MIT Press, Cambridge, Massachusetts, 1988.

[59] J. Van Benthem. The Logic of Time. Kluwer Academic Publishers, Boston, Massachusetts, 1989.

[60] Steven Vere. Planning in time: Windows and durations for activities and goals. IEEE Transactions on Pattern Analyuis and Machine Intelligence, 5:246-267, 1989.

[61] Devid E. Wilkins. Domain independent plenning: Representation and plen genenation. Artificial Intelligence, 22:269-302, 1984.

(68) David E. Wilkins. Practical Planning: Extending the Classical AI Plamning Paradigm. Morgan-Kaufmann, Los Altos, California, 1988.

(69) Marianne Winslett. Reasoning about action using a possible worlds approach. In Proceedings AAAI-88, pages 89-99. AAAI, 1988. 


\section{Chapter 4}

\section{Controlling Processes}

This book is concerned with the beliavior of proresses. The world we live in can be described in terms of a set of interacting processes. In the previous chapter. we discussed how to model the behavior of processes. In this chapter, we begin to consider how to influence that behavior.

Some processes are easier to control than others. For instance, someone typing at a word processor generally has a fair amount of control over what characters appear on the screen. Other processes are influenced by a large number of factors only a few of which we are able to directly observe or influence. In sending an electronir mail message, for example, the speed with which the message arrives at its destination is determined in part by the path provided and in part hy the traffic on the networks specified in that path. Electronir mail users can directly control the former bnt have little control over the latter. If you could somehow predict the traffic on the network. then you'night be better prepared to specify a path that would speed your message to its destination. Unfortunately, predicting network traffic flow is itself a complicated and time consuming task.

In studying the control of processes, it is often convenjent to describe the world in terms of two processes: one of which we have absolnte control over, and a second process that we wish to control. The first is called the rontrolling process and the second the rontmlled proress. The hehavior of the controlling proress is deterinined in part hy the control-system designer. Civen some desired beliavior for the controlled process, the task is to denign a device that realizes the controlling process and forces the deaired hehavior in the controlled process.

\footnotetext{
${ }^{\circ}$ (C) 1990 Thomas Dean. All rights reserved.
} 


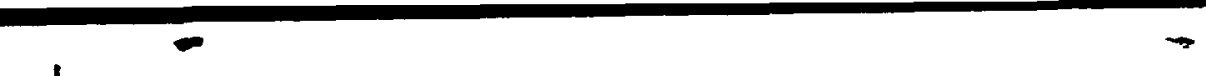

The interaction between controlling and controlled processes can be quite complex. We generally think of the controlling process as calling all the shots. but the control exerted by the controlling process over the controlled process is seldom complete. Factors that intluence the controlled process but are not under the control of the controlling process have to be accounted for. The coutrolled process can. and in many cases unst. influence the controlling process in order to bring about the desired behavior. This influence is wediated through the use of special devices used by the controlling process to observe the behavior of the controlled process.

Information about the observed behavior of the controlled process is often used by the coutrolling process in determining what action to take next. This basic idea that the responses of the controlling process are computed from the observed behavior of the controlled process is generally referred to as feedback control. In some cases. the need for observation can be reduced or even eliminated by usiug models to predict the behavior of the controlled process.

In this chapter, we consider techniques drawn primarily from control theory and control systems engineering. We focus paim ily on the role of feedback in the design of control systeins with an emphasis on representations and techniques that stress computational issues. We introduce criteria for controllability, observability. stability, and optimality, and consider a variety of problems to illustrate these concepts. We then consider some basic feedback controllers and how they might be embedded in a conputational framework. In the context of discussing feedback control. we introduce programming approaches that are well suited to building control systems that have to be particulariy responsive to change. We end this chapter by considering a problem in robotics that.lies at the boundary between those problems traditionally conoidered within the purview of control theory and problems associated with artificial intelligence. The objective here is not to provide a comprehensive survey of control techniques, but rather to draw on the control disciplines for insights and general techniques that apply to the full range of planning and control problems. Before launching into the more technical discussions drawing on results from control theory, we consider a particular problem to illustrate some basic issues.

\subsection{Robot Navigation as a Control Problem}




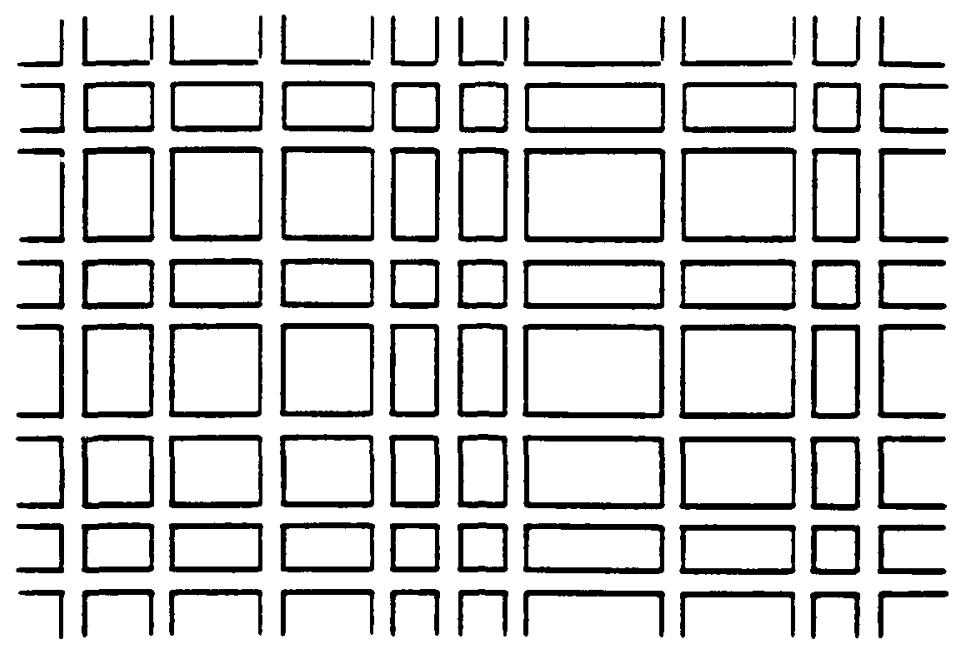

Figure 4.1: A city street layout

Consider the following control problem. Suppose that you want to control a robot to move from one location to another in a city. The robot has to travel using city streets that are arranged as au irregularly-spaced grid of two-way streets (see figure 4.1). You have to devise a control algorithm to direct the robot to move from its present location to a destination location defined in terms of global coordinates. Or course. the problem is not yet well enough specified that you can run of and start writiug down an algorithw. There are a number of other factors that we have to consider.

First. what sort of control can we exert over the robot? Most likely there will be some means of controlling the robot's speed and direction of travel. but it's not likely that the robot will move exactly where we tell it nor will it move at precisely the speed that we specify. If we indicate that the robot is to move due South at 12 kilometers per hour and there is a brick wall in the way, then we might expect some difference between the specified and the actual speed and heading. Usually, however, the differences between actual and specified control variables are more sublle. Errors accumulate and combine in executing a sequence of control actious. Sooner or later it becomes necessary to compare the actual effect against the intended effect. and this is where sensors enter into the picture.

Sensors are used to nonitor the progress of the robot and to determine the state of the euvirounent. Seusors can determiue and correct for movement error. For instance, the robot might be equipped with shaft encoders 
for determining how many revolutions the drive wheels have turned or what direction the wheels are pointing. From this information. We can compute an estimate of where the robot is relative to where it started out. Sensors and the estimates derived from sensor data are also subject to errors. Somehow or another we have to take such errors into account. For instance. it may be that the errors are known to satisfy a particular statistical distribution from which we can calculate a measure of how certain we are in the inferences derived from sensor data. If our confidence in our inferences is low. then that could niean that we lack sufficient information to formulate a good answer to the control problen we are faced with. In some cases. being left with insufficient information is unavoidable and we nust proceed to schedule critical control actions with whatever information we have at hand. In other cases. we can use sensors to gather additional information so as to make inferences that ve are inore confident in.

Sensors tell us about morc than just the state of the robot: they tell us about the state of the larget world in which the robot is embedded. In the simplest robot navigation tasks. the only thiug that changes is the robot itself and its position in the world. The environment is said to be static. If we know something about the fixed state of the environment, then we can take advantage of this in designing a control algorithm. Knowledge of the environment might take the form of a map labeled with street names. whether or not traffic moves in one direction or both, and whether there are stop signs or other impedimeuts to trafic flow.

In more realistic problems. the environment changes; there are other vehicles on the road, traffic lights cliange, roads are blocked by construction. and pedestrians occasionally dart out into trafic. The static map may still be useful. but often we can supplement our knowledge of the environment to account for dynamic phenomena. For instance, we might have access to a construction schedule indicating where and when certain streets will be closed to traffic. In some cases, we might be able to model certain disturbances as predictable processes. A construction crew might be laying new gas pipe under a particular street at the rate of one block per night so that at most one block-long section of the atreet is impassable on any given night. If you notice the crew laying pipe on any two nights, you can predict what block will be closed off for auy subsequent night.

While some processes are predictable. others are either difficult to predict (e.g.. jay-walking pedestrians) or not worth the trouble (e.g., traffic lights). In order to deal with such processes, the control algorithm has to be alert to changes in the environment that indicate the existence of processes whose 
behavior might have an impact on the performance of the robot. The robot lias to be continually alert for evidence of certain processes ( $f$.g.. pedestrians straying into the street in fromt of the robot). Other processes need only be monitored in certain circumstances. For instance. the robot has to check for the state of the traffic light at the next intersection only as it approaches that intersection. The design of the control algorithm must take into account the sensors available and the tasks they are to be put to. Sensors often constitute a scarce resource in need of careful management.

There is another aspect of the coutrol of our nobile robot that we have carefully avoided up until now. and that concerns how the algorithm that we devise is to be implemented. In order to implement a control algorithm. We nee it to specify the algorithn in terms of a language, and we have to provide a. cunpiler for that language, and a larget marhine for the code generated by the compiler. In fact. it getlerally is difficult to specify a control algorilhm without some specific implementation in mind.

llow long a series of program statements takes to execute on a particular marhine may be critical in deternining the consequences of a control action. For instance. suppose that you want to compute how to respond in the case in which a pedestrian runs out into the street in front of the robot. Certainly it would be a good iclea to apply the brakes as soou as possible if indeed that is an appropriate thing to do. llow long the algorithm takes to compute whether or not to apply the brakes will have a profound inupact on the heallh of the pedestrian in question. If the robot is to swerve in an altempt 10 avoid hitting the pedestrian. then the direction in which the wheels are turned will depend upon the time that they are turned, and this will depend upon the lime it takes to compats the direction.

In some cases. we can just assume that the time required to compute responses is shorter than the time available for computation. For instance. suppose that at time $t$ the robot interprets its sensor data as indicating a pedeatrian standing in the street 5 meters directly in front of it. The robot. atlempts to compute what artion to take at time $1+\Delta$. The coutrol algorithm is implemented so that the time required to compute such a response is leas than $\Delta$. Having compuied an appropriate answer. the control algorithm might simply wait out the remaining time, or hand the action and the time it is to be executed.$u$ a sequencer responsible for executing actions at specified times. Of course. if the robot is traveling at a meter a second and $J$ is longer than a couple of seconds. then the response will likely be 100 late to be of any use.

Some of the decisions concerning how long to spend computing an appro- 


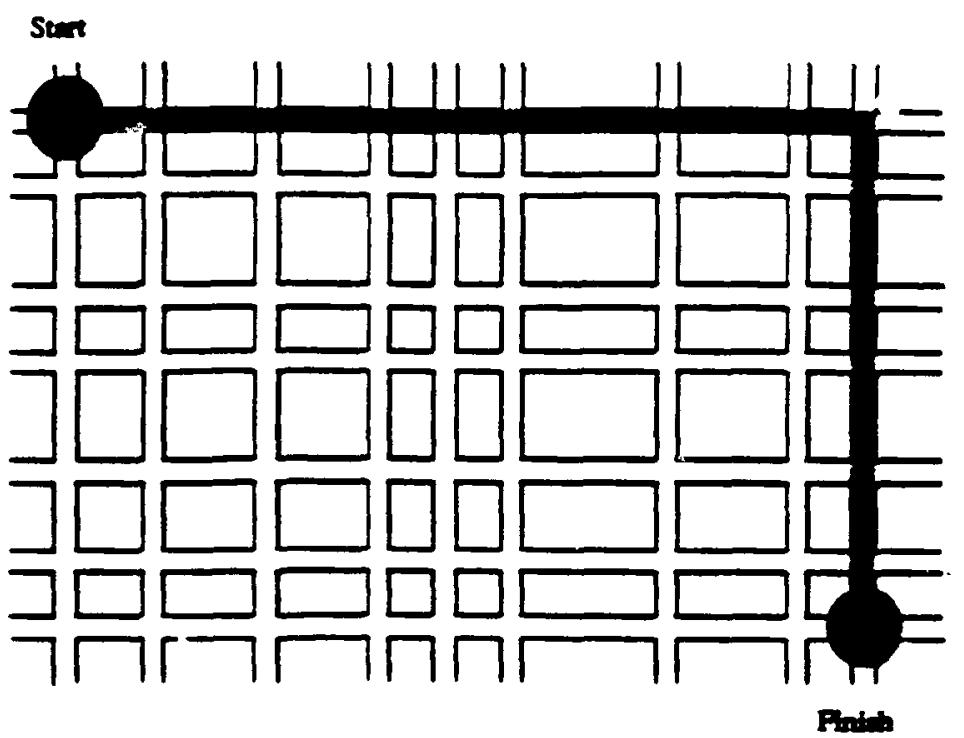

Figure 4.2: A path generated by dead reckoning

priate response in a giveu set of circumstances can be carried ont at design time. Other decisions concerning how to long to compute are better left until run time when the allocation of computational resources cau be based on more data about the situation at hand. If the lead time for respouring to a certain sort of phenomena varies. then having a rigid scheme for compnting a response may lead to poor performauce on average. Jamming on the brakes is ouly appropriate as a last resort. In situations where more time is available to arrive at a decision. a nore careful analysis is often called for. In this chapter. we ignore many of the issues that relate to the run-time allocation of processor time to optimize decision making. ('hapter 8 directly addresses these issues. In this chapter, we take a conservative approach to ensure that the algorithms that we develup perform reasunably for even the worat-case situatious auticipated.

So far we have considered several factors that are iuportant in specifying control problems. Now, we consider some specific control problems. In an ideal world. when the robot is told to turn left $15^{\circ}$ and move forward at 2 ineters per second for 5 seconds. the robot ends up exactly 10 ineters from its original position facing $15^{\circ}$ counter clock $u$ ise from its original heading. Consider the problem involving a static environment in which all of the 
streets allow two-way traffir and are ninstacle free and the rohot is standing in the center of an intersection and is instructed to move to the center of a second intersection specified in $x$ and $y$ coordinates in the frame of reference of the rohot's initial position. In this case. au appropriate control algorithm would direct the robot to complete the traversal in two steps following the paths indicated by the $x$ and $y$ offset: ( see Figure 4.2).

In the above ideal world. the rchut is said to direct itself by "dead reckoning." Aside from a clock to measure the passage of time. and thereby gauge the distance traveled. the robot requires no sensors to direct its motion. Suppose that we relax the requirement that the robot be able to control its velocity precisely. In this case. it is possille that the robot s estimates of distance traveled are subject to error. Ilow is the problem changed as a consequence? If the errors ase suall relative to the length of a city block. a simple variation on the dead-reckoning approach will work just fine. If the errors are large, then the problem may be inpossible to solve since the robot will have no way to determine if it reaches its destination. Even if the robot has some other means of detecting that it has arrived at its sought-after destination, significant movement errors may force the control algorithm to randounly choose paths.

Suppose that the robot can determine its position at any time in some global coordinate system. Now movement errors can be corrected by wha! is generally referred to as feclluck. The control algorithm attempts to move 5 meters to the left: it cliecks to see how far it actually moved: it attempts to correct for the error olsserved. As long as the errors are some fraction of the distance attempted, this techuique will converge quickly on the desired distance. If determining global position is fast enough. then this technique reduces to the previous dead-reckoning method.

Now suppose that all streets are not passable; some streets are one way and others are blocked by construction equipment. The dead-reckoning approach will obviously not work, but a simple path-following strategy will sulfice to find a path if oue exists. Figure 4.3 shows the streets traversed by the robot wader the control of a simple path-following algorithm that tries 10 shorten the Euclidean distance to the destination whenever possible, backing up only when its way becomes blocked. The problem is that directing the robot using the simple path-finding strategy causes the robot to traverse streets that it might not have if it possessed a more global perspective of the city.

Suppose that the robot has all arcurate map of the city indicating oneway streets and construction road blucks. Katlier than actually traversing 


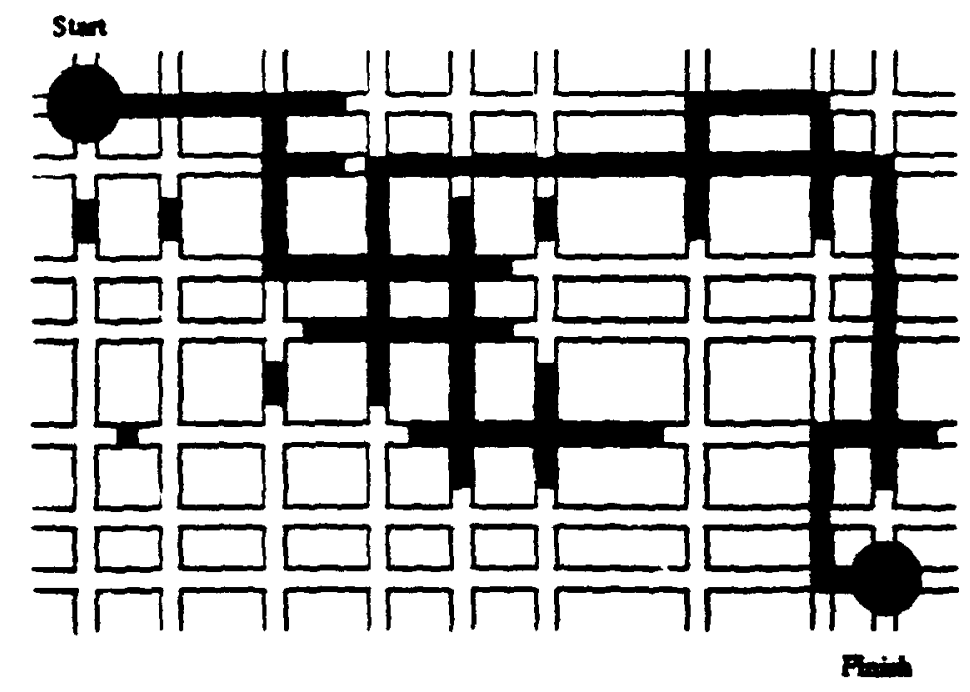

Figare 4.3: Navigation without the aid of a map



Figure 4.4: Navigatiou using path planning aud a global nap 
the streets. the rnutrol algorithm conld use the map to simulate traversing the streets and therehy find a short path. Computing the shortest parh between any two locations can be dune in $O\left(n^{2} \log n\right)$ time using Jjkstras algorithm [1]. assmming a square grid of strcets with n streets alomg each axis of the grid. ligure 4.4 shows the streets 1 raversed by the robot under the control an algorithm with access to a map. This method of sinulating, the behavior of the robot in order to eliminate unnecessary work or avoid an undesirable effect represents an instance of feedforund. The control algorithu generates and analyzes possible actions and their consequences so that it can choose anong the arailable options.

The use of feedlack and feedforward are common in the desigu of control svstems. Feedlback compensales for a system inability to accurately predict the effects of a control action on the behavior of a controlled process. leedback relies on being able 10 accurately monitor the behavior of a process. Feedforward enables a system to anticipate both desirable and undesirable consequences and take steps to, respectively, take advantage of or avoid them. Feedforward relies on a system having an accurate model for the process being controlled.

Feedforward and feedback complement one another. In situations in which the controlled process cannot be accurately predicled but can be closely mouitored, light leedluack loops enable a control algorithm to generate control actions on the basis of inumediately past performance. Such a sclieme is likely to work assuming that the facturs influencing the process at one puint in time are similar in type and magnitude to the factors intluencing the process a short time previously. In situations in which the controlled process cannot be accurately monitored but can be accurately predicted. control actions are generated in response to predictions concerning the processes behavior. If the process can't be monitored at all, then control proceeds blindly relving on the accuracy of the predictive inodel.

Traditional methods in planning stress the use of feedforward methods whereas traditional melliurls in contrul st.ress the use of feedluack. The reason for their different empliases is easy to explain. First of all, planning is by defuition concerned with predicling the future in order to guide behavior. Much of the early work in planning was concerued with processes that interact with one another in a complex manner, and, hence. influencing the behavior of these processes required anticipating these interactions. This early work generally assumed that the coutrolled process. while complex. was understood well enough to be accuralely modeled. More recent work has begun to relax this assumption by either using feedback to supplement

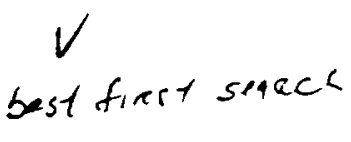


predi-tions or using stochastic models that take uncertainty into account.

lin contrast with the work in planning. much of the early work in control assuned that the controlled process was subjert 1.0 a inultitude of factors that either were not well understood or required run-time data that simply was not available. Precise adjustments to the control paraneters were needed to achieve the desired behavior requiring that the controlling process be able to generate the necessary control actious at a high rate. A more complex algorithn for determining the uext control action lowers the rate at which control actions can be generated. whereas, the more inaccurate the models are in predicting the effect of control actions, the more freyuently the controlling process has to be mouitured and the control paramelers adjusted to compensate for the inaccuracies of the model. In the past. many industrial control applications have favored trading model complexity for increased teliance on feedback and higher parameter-adjustment rates. As computers become faster and our modeling techniques wore reliable. there has been a teudeucy to incorporate more and more complex modeling techniques into industrial controllers. If this trend continues. industrial controllers will begin to look wore like planuers.

As the control community begins to realize the advantages of increased computatioual power for supporting complex modeling, so the planuing community is beginuing to realize the problems in relying solely on the predictions of a conplex model. ('orrectiug these problems is not simply a matter of building an interpreter that executes a sequence of actions generated by a traditional planner and occasionally senses the environnent to see if the activus have had their desired effect. The problem with this approach is that the controlled and the controlling processes are often out of synch with une another.

A control action generated one moment may be deemed inappropriate at the next as new information becumes available. To simply generate a sequence of actions and expect that the sequence can be carried out without modification is for many problems absurd. In asking directions in Boston. a local may tell you to turn left on Commonwealth Avenue and follow it for three blocks until you get to Massachusetts, but if you find four fire trucks tying up traffic on Comnonwealth Avenue. then you would be well advined to disregard their directions and find an alternative route. There was nothing wrong with the directions provided given what was known at the time they were solicited. but knowledge changes over time and such rhanges should be taken into account when deciding how to act.

Of course, the preceding paragraple shouldu't be taken as an argunent 
against planning: we ve already secn that path planning can lead to improved performance in certain circumstances. What we have to beware of is blindly cxecuting plans in the face of information that warns against their use. The traditional noljon of a plan as a sequence of actions las to be rethought. Plans should be interpreted as suggestions about how to behave. Some suggestions require a long time 10 generate. but the processes that they are desigued to help control may proceed at a similarly slow pace. In real-world problems. there are ally number of processes that require some amount of control. Some processes proceed slowly and require attention only at widely-spaced intervals ( $\epsilon . g .$. the pipe-laying process discussed earlier). Otlicr processes are faster pared and refuire almost coustant attention (. .g. pedestrian traffic). The trick is to deal effectively with the fast-paced proresses (f.g.. st.eer clear of periest rians a.nd st.op) al. appropriate t.raffic signals) while at the same time directing behavior so as to take into account suggestions regarding the slower processes (f.g.. avoid routes that are believed to be obstructed by construction I and suggestions generated off-line as it were regarding faster-paced processes (e.g. if you see a ball rolling out into the street. (brake hard as a child may be following closely behind).

In the following, it will be useful to separate ont two kinds of control algorithm. One that generates suggestions concerning certain low-level behaviors and that is likely to perform out of synch with the processes whose behavior it is meant to influence. and a second that is closely tied to the processes that it is meant to influence. The distinction is artificial: it serves primarily to identify two distinct mind sets that have to be merged in order to develop a coherent theory of control. To provide a label for the two kinds of control and identify the source for the corresponding nind sets, we call the first high-level planning and the second low-level control. din example of a high-level planning algorithm would be a path planniug algorithm designed to influence the novement of the robot. An example of a low-level control algorithm would be the algorithin that directs the speed and heading of the robot as it traverses the city streets avoiding obstacles and maneuvering around corners.

One possible arrhitecture for a system integrating high-level planning and low-level coutrol might consist of two components: a reactive component that determines what to do at the next instant, and a strategic component that attempts to mediate the behavior of the reactive component by imposing constraints on the behavior of the iuti-level systems. It is up to the low-level system to interpret these constraints so as to adjust its behavior while at the same time maintaining real-time performance. 
In this chapter. we are primarily interested in what we have called lowlevel control. Luwarci the end of this chnpter. however. We begin to address high-level control issues as proingue to the next chapter which will deal alnıost exclusively with high-level strategic planning. Now. we draw upon the disciplines of control theory and control systems engineering to develop some terminology and explore techirgues that will be used in subsequent chapters.

\subsection{Controllability}

Consider the following tine-invariant discrete-time dynamical system.

$$
\begin{aligned}
x(k+1) & =f(x(k) \cdot u(k)) \\
y(k) & =g(x(k))
\end{aligned}
$$

The state transition function. $f$. completely deternines the state of the system at time $k+1$ given the state and the input at time $k$. Initially; we assume that the state of the system is directly observable. and so the output function, $g$, is defiued

$$
g(x(k))=x(k) .
$$

In solving a particular control problem. we are interested in generating appropriate inputs so as to constrain the behavior of the dynanical systen. In Chapter 1. we introduced a general formulatiou of the control problem. representing the behavior of a dynamical system in terms of the set of possible state-space trajectories.

$$
H_{X} \triangleq\{\dot{h} x: T-X\}
$$

In this formulation of the problem. the desired behavior of the system is specified in terms of a goal set.

$$
\text { (ic } H_{X} \text {. }
$$

There are several special cases of this formulation that we consider in the following sectious.

In the servo problf $m$. we are given a reference trijectory, and expected to repeat or truck that trajectory as closely as possible. In the set-point regulation problem, the objective is for the system to achieve aud maintain a particular state or set of states starting from any initial state. In the 
terminology of Chapter 2. We wish to lind some input function $c \in\{c: T-$ $\left.l^{*}\right\}$ so that for any initial time $\tau \subseteq I$ and initial state $x(\tau) \in I^{*}$ there exists $t>r$ such that for all $t^{\prime}>t$ we have

$$
f\left(x\left(t^{\prime}\right) \cdot\left(\cdot\left(t^{\prime}\right)\right) \in C\right.
$$

where $C^{\prime} C I^{-}$is the set of target states.

We can generalize on our formulation of the set-point regulation problem to restrict not only the final states of the system. but the intermediate states as well. thereby restricting the motions (state space trajectories) of the system. For instance. We might require that the system avoid a certain set of states. hy stipulating that for all $t>\tau$ we have

$$
f(x(t) \cdot \cdot(t)) \notin Q
$$

where $Q \subset \mathrm{X}$ is the set of slates 10 avoid and $C \cap Q=0$.

Amung the ylualitalive properties ol dynamical systenus and their controllers, the following nolion of controll hubility is particularly relevant to the set-point regulation problem. An event $\langle\tau, x\rangle$ in the phase space defined by $T \times X$ is said to be controllable urith respect to a set of target states, $C^{\prime} \subset X$, if and only if there is some time $t$ and some input $v$ which moves $\langle\tau, x\rangle$ into the set $\{t: t \geq \tau\} \times C$. A dynamical system is rompletely controllable with respect to $C$ if and only if every event in $T \times X$ is controllable with respect to $C^{\prime}$. This notion of complete controllability with respect to a set of target stales provides necessary and sullicient conditions for there being a solution to the set-point regulation problem.

As was mentioned in C'hapter 2. One of the best developed areas of modern control theory concerns the aualysis of dynamical systems that can be modeled as linear multivariable systems. In this chapter, we illustrate the power of linear systems theory by defiming three important qualitative properties of dyuanical systens. and stating simple mathematical criteria for these properties to be satisfied.

We begin with the uotion of controllability. Criteria for controllability are generally specific to a particular method of modeling dynamical systems. In general. we are interested in whether or not it is possible to transfer auy state $x\left(t_{0}\right) \in X$ to any other stale in $X$ in a fiuite amount of time $t_{1}-t_{0}$ where $l_{0}<l_{1}$ by approprialely chousing $u(1)$ for $l_{0} \leq l \leq l_{1}$. If such arbit.rary transfers are possible, we say that the system is complelely controlluble (no restriction to a particular set of target states). 
Consider the following lincar time-invariant stystem represented by

$$
\begin{aligned}
& \dot{x}(t)=-t \mathbf{x}(t)+B \mathbf{u}(t) \\
& \mathbf{y}(t)=C \mathbf{x}(t)
\end{aligned}
$$

where $x$ is the $n$-dimensional state vector. $u$ is the $p$-dimensional input vector. $y$ is the $q$-dimensional output vector. and $A . B$. and $C$ are. respectively. $n \times n . n \times p$, and $q \times n$ real constant matrices. There are a unniber of retatively simple mathematical couditions for such a system being completely controllable. One of the simplest is providled by the following theorem which is stated here without prool (see (hen [9] or Ciopal [1H] for proufs and related theorems).

Theorem 1 The system is complctcly controllable if and only if the rank of the $n \times n p$ controllability matrix. $\left[B|A B| \cdots \mid A^{n-1} B\right]$. is $n$.

As a simple example, the divnamical system for the siugle-degree-offreedom robot introduced in Chapter 2 with state equation.

$$
\dot{x}(t)=\left[\begin{array}{ll}
0 & 1 \\
0 & 0
\end{array}\right] x(i)+\left[\begin{array}{c}
0 \\
1 / M
\end{array}\right] u(t)
$$

is completely controllahle since the rank of its controllability matrix,

$$
[B \mid A B]=\left[\begin{array}{cc}
0 & 1 / M \\
1 / M & 0
\end{array}\right]
$$

is 2. However, the system described by

$$
\dot{x}(t)=\left[\begin{array}{cc}
0 & C_{1} \\
C_{2}^{\prime} & 0
\end{array}\right] x(t)+\left[\begin{array}{l}
1 \\
1
\end{array}\right] u(t),
$$

has a coutrollability matrix.

$$
[B \mid A B]=\left[\begin{array}{ll}
1 & C_{1} \\
1 & C_{2}
\end{array}\right] .
$$

\footnotetext{
'The ronk of an $n \times m$ rectangulas matrix. $A$, is defined as the maximum number of linearly independent colunin vectors. or, eppivalently. the order of the largent muare array whose determinant in non-zero, where the square array is obtained by removing rown and columm from $A$.
} 
indicating that the system is controllable only if $C_{1} \neq C_{2}$.

There are onher similarly concise and equitalent conditions stated in the literature. Both ('hen [9] and (iopal [1t] provide similar results for linear time-varving sustems. as well as constructive proofs that identify the appropriate input functions. It is testimony to the power of linear systems theory that such precise conditions ran be stated for such a general class of dynainical sustems. ${ }^{2}$

It should be noted that the above stated notion of controllability places no constraint on the input (controller) or on the trajectory followed by the system. A system may he determined as uncontrollable hy the above criterion, while being controllable in most practical respects. For instance. the sustem may move to any given state from all initial states that will arise in practice. As another example. we nay not care about certain components of the state vertor: it may be that we are only roncerned with controlling the output of the systein.

To investigate further the nution of controllability, we consider some examples of dynamical sustems that can be represented in terms of finite state automala. These dynamical systems are referred to as discrete event systems in the literature [25]. We represent a discrete event system as an automaton. $G=\left(U, X . f, x_{0}\right)$, where. in keeping with our previous notation. $l$ is the set of inputs (think of $l$ as a set of primitive events), $X$ is the set of states. $f: U \times r-X$ is the state transition function. and $x_{U}$ is the initial state.

We partition $l l$ into lwo sets: $l_{c}$. l lie set. of controllable evettis. and $l_{u}$, the set of uncontmillable events. An admissible control for such a dynanical svistem cousists of a subset $\gamma \subseteq l$ such that $U_{u} \subseteq \gamma$. Let $\Gamma \subseteq 2^{U}$ represent the set of all admisaible controls. If $\gamma \in \Gamma$ and $\dot{u} \in \gamma$, we say that is is enabled by $\gamma$. otherwise we say that it is disabled. A controller for a given dvnamical system is specified as a map

$$
\eta: \mathbf{I}-\Gamma
$$

The idee is that disabled events are prevented from occurring and enabled events are allowed to occur if permitted by the underlying dynamics. The

\footnotetext{
${ }^{2}$ As was noted in Chapter 2. it is standard practice in engincering control syatems to model reat-work nontinear systems using linear approximations. Since snall perturbationo of the elemente of the matrices $A$ and $B$ inay signal the difference hetween controllability and its lack. it should be noted that statements of systen controllability tuan be carefully iveighed in the procese of design.
} 


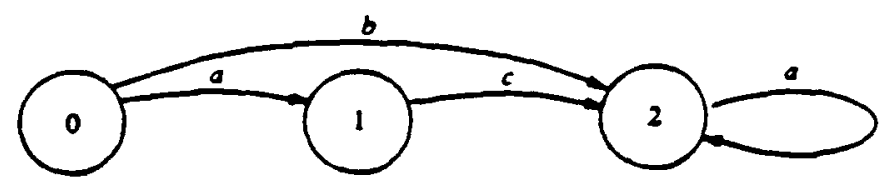

Figure 4.5: A dynamical system represented as a finite state automaton

stipulation that $U_{u} \subseteq \gamma$ for all $\gamma \in \Gamma$ captures the intuition that the controller cannot prevent the uncontrolled eveuts from occurring if the dinamics dictates otherwise. An issue arises regarding what happens if all of the events for a given state are disabled. We resolve the issue by simply requiring that the controller ensure that for any state there is at least one enabled event for which the transition function is defined: the system can remain in the same state ondy if that is permitted by the dynanics.

Consider the dynamical systeen depicted in Figure 4.5 in which $U=$ $\{a . b . c\} . X=\{0.1 .2\} . x_{0}=0$. and $f$ is defined so that

$$
(0 . a) \mapsto 1,(0, b) \sqcap 2 .(1, c)-2 \text {, and }(2 . a)-2 \text {. }
$$

Let $U_{c}=\{a, b\}$ and suppose that we wish to design a controller that achieves $\{2\}$ while avoiding $\{1\}$. The controller defined by

$$
0 \mapsto\{b\} \text { and } 2 \mapsto\{a\}
$$

will suffice to do exactly what we want. The sane coutroller will work if $t_{c}=\{a\}$. However. if we have $t_{c}=\{b\}$, then there is no controller satisfying the requirements given.

There is an alteruative approach to characterizing the behsvior of diecrete event systens modeled as finite state automata. In formal language theory. a finite state automaton can be viewed as a generator for a language. Let $C^{*}$ denote the set of all finite strings of elements of the set $U$. A subset $L \subseteq U^{*}$ is called a language over $U$. The automaton described above is a generator for the language

$$
L=b \boldsymbol{c}^{*}+\mathrm{aca} \boldsymbol{a}^{*}
$$

indicating the union of the set of strings consisting of $b$ followed by a finite number of $a$ 's, and the set of strings consisting of $a$ followed by $c$ followed by a finite number of $a \mathrm{~s}$. Instead of asking if we can design a controller that achieves $\{2\}$ while avoiding $\{1\}$, we ask if we can design a controller for the automaton so that it generates the language $L^{\prime}=b a^{\circ} \subseteq L$. 


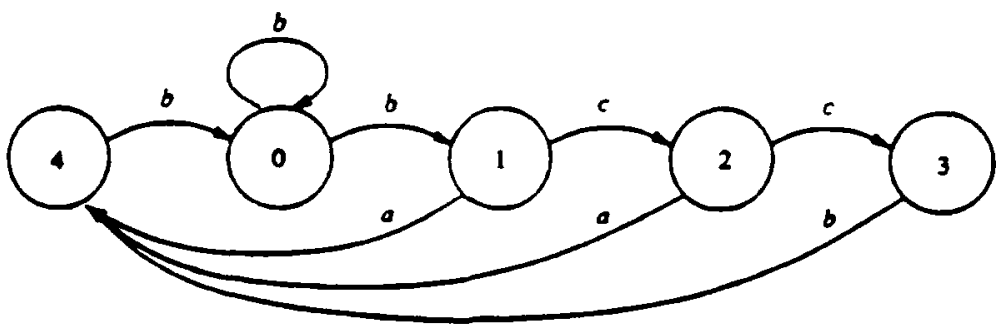

Figure 4.6: One component of a product system

Ramadge and Wonham [25] define a supervisor for a discrete event systenl as a map

$$
\eta: L-\Gamma \text {. }
$$

where $L$ is the language (or behatior) generated by the discrete event system. The prefix closure of $L \subseteq l^{*}$ is that subset $L \subseteq l^{*}$ defined by

$$
\bar{L}=\left\{u: u v \in L \text { for some } v \in U^{*}\right\} \text {. }
$$

A language $K \subseteq L$ is said to be contmllable with respect to a given discrete event system if

$$
\bar{K} U_{u} \cap L \subseteq \overline{\bar{K}},
$$

where $\bar{S} t_{u}$ represents the set of all strings consisting of a string from the prefix closure of $\hbar^{\circ}$ concatenated with an event from $l_{u}$. In [25], Kamadge and Wouham prove the following, thus providing neressary and sufficient conditions for the existence of supervisors for discrete event systems.

Theorem 2 For any discrete event system A with closed behavior $L$ and any subset $K \subseteq L$. there exists a supervisor that serves to restrict A to exactly $K^{\circ}$ if and only if $\overline{K^{-}}=\Pi^{\circ}$ and $\Pi^{\circ}$ is controllable.

In sone cases, it is convenient to represent a dyuanical system as a collection of fiuite state ... toiuata loosely coupled through the state space resulting from taking the cross product of the state spaces for the individual antomata. As an example. suppose that we wish to model a collection of $n$ identical chenical processes. Each individual process is modeled by all antomatou $G_{i}=\left(l_{i}, X_{i}, f_{i}, r_{0}\right)$ where the $i h_{1}$ antomaton is defined by $l_{i}=\left\{a_{i}, b_{i}, c_{i}\right\}, x_{i}=\left\{0_{i}, 1_{1}, 2_{i}, 3_{i}, t_{i}\right\}, x_{0}=0$, and $f_{i}$ is as indicated in Figure 4.6. Let $t_{c_{1}}=\left\{a_{i}, c_{1}\right\}$. Suppose that all $n$ urocesses run independently 
of one another with one important exception: state 4 iuvcives the use of a piece of equipment with limited capacity such that only one process can be in state 4 al a lime. We wish to design a controller that will guarantee this. Note that once a process enters state 1. we can exercise some control over when it enters State 4 . but we can only delav this event. we cannot prevent it Irom Lappening.

To represent the combined hehavior of the collection of processes. we

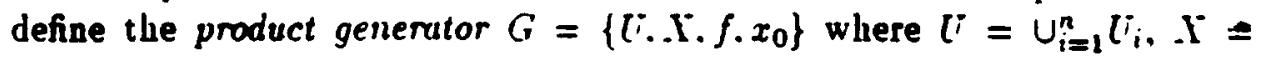
$\Pi_{i=1}^{n}, \dot{x}_{i}, U_{i}=\cup_{i=1}^{n}\left[i_{i}, x_{0}=\left(x_{0_{1}}, x_{0_{2}}, \ldots x_{a_{n}}\right)\right.$ and for each $u \in C_{i}^{i}$ we have

$$
\left.f\left(u \cdot\left(x_{1}, x_{2}, \ldots, x_{i}, \ldots, x_{n}\right)\right)=\left(x_{1}, x_{2}, \ldots f_{i}\left(u, x_{i}\right), \ldots, x_{n}\right)\right) .
$$

The ohjective is to huild a controller for $G$ such that at most one of the chemical processes is in the state requiring the piece of equipment at. any given point in time.

In the worst case. all of the processes will simultaneonsly arrive at state 1 in their respective state spaces. At this point, exactly one process can transition to state 4 , while the $n-1$ remaining processes are forced to enter state 2. The same simple analysis applied to state 1 can be applied to state 2 with the conclusion that $n-2$ processes are forced to enter state 3. The controller has no control over the processes in state 3 , and hence we conclude that there exists a controller for the product system if and only if $n \leq 3$.

Discrete event systems can be used to nodel inanufacturing systems, comninnication networks, vehicular traffic prohlems, and a variety of other dynamical systems requiring coordination and control. In addition to answering matheinatical questions concerning the existence of supervisors. the rurrent theory provides const.rictive methods for realizing certain clasien of supervinors. In the hest circnmatancen, these methods require time and storage polynomial it the size of the state space. For practical problems. one generally has to be clever in searrhing the space of posaible controllers for one that satisfies the domain constraints.

\subsection{Observability}

So far, we have had little to say about the role of the system output function. In fact, we initially assuned that $y(t)=y(x(t))=x(1)$, so that the state of the system was directly observable as output. In general. the entire system state will not be directly observable. If the controller requires 
either the entir system state vertor or specific components of this rector. then an additional module has to be added to the control system in order to recover the state by observing the system output. Such modules are gencrally referred as obscrucrs. If the function $g$ is known and invertible. then the construction of an observer is trivial. Cienerally, $g$ is not invertible and the state has to be recovered hy observing the output of the system over some interval of tinne. In the following, we consider a notion of olsservability which. at least in the case of linear multivariable systems. turns out to he closely related to controllability.

A system is said to be completely observable if it is possible to identify any state $x\left(t_{0}\right) \in X$ by observing the output $y(t)$ for $t_{0} \leq t \leq t_{1}$ where $t_{0}<t_{1}$. observation problem. The problem stated is traditionally called the observation problem. but it is actually just one of several so-called state. determination problems. The observation problem involves determining the state from future outputs. There is a related problem called the roronstrur: tion problem that involves identifying the state from past outputs: identify the state $x\left(t_{1}\right) \in X$ hy observing the outpnt $y(t)$ for $t_{0} \leq t \leq t_{1}$ where $t_{0}<t_{1}$. As in the case of controllability. there are simple mathematical criteria for observability in linear innltivariable systems ( see ('hen [9] or Ciopal [14] for proofs and equivalent conditions).

Theorem 3 The system is completely observable if and only if the rank of the $n q \times n$ observability matrix.

$$
\left[\begin{array}{c}
C \\
C A \\
\vdots \\
C A^{n}-1
\end{array}\right] \text { is } n \text {. }
$$

Given the similarity of the statement of Theorems 1 and 3 one might sunpect that there is a rather deep relalionship between controllability aud ohservability for linear multivariable systems. It would be particularly convenieut if one conld prove that a system is observable if and only if it is controllable. This happens in be true in a somewhat convoluterl mathemat. ical sense as we see in the following theorem.

Theorem 4 (The Principle of Duality) The system represented by

$$
\begin{aligned}
& \dot{\mathbf{x}}(t)=A \mathbf{x}(t)+B \mathbf{u}(t) \\
& \mathbf{y}(t)=C \mathbf{x}(t)
\end{aligned}
$$


is rnintrollable (observable) at time io if and only if the dual system repr:sented by

$$
\begin{aligned}
\dot{z}(t) & =-t^{\prime} z(t)+C^{\prime \prime} v(t) \\
w(t) & =B^{\prime} z(t)
\end{aligned}
$$

is obsermable (contmllable) at to. where the prine (e.g.. $B^{\prime}$ ) indirates matrix tmnsposition, and the sfrond system (ralled the adjoint) is mathematically. closely related to the first.

One practical consequence of Theorem + is that once you have constructed a controller (ouserver). you have done all the necessary work required to construct the associated observer (controller): the algorithms required for one task are almost identical to the algorithins required for the other task. It is also interesting to note that observability and controllability in linear systems can be considered independently. The two problems of buildiug a controller and building an observer can be pursued independently of one another. 'The two problems are sajd to be seprirable. This separation property does not liold in genteral.

Hesults similar to that of 'Theorem 4 hold for linear systems corrupted with Gaussian noise. In Clapter 6 . we consider the prohlen of building a determimistic regulator (controller) and a stochastic estimator (observer) for dyuamical systems modeled as linear systems corrupted with Gaussian noise. It turus out that these two problems are also separable: by coupling the optimal deterninistic regulator to the optinal stochastic estimator one has constructed an optinual control system.

It should be emphasized that the notion of observability introduced in this sectiou is quite strong. Lu general, a controller need not reconstruct the eutire system state in order to provide satisfactory performance for a given coutrol problein. In many cases. the task of reconstructing the entire system state would impose a significant computational burden. Practically speaking, we are interested in demand-driven observation strotegies that allocate resources to measurenent and interpretation in keeping with the immediate denuands on the system. The task-based planning nethods presented in ('bapter 5 employ this sort of denaud-driven observation strategies.

\subsection{Stability}

When we first introduced the notion of controllability in Section 4.2. we were iuterested in the ability to first achieve a given state or set of states in 
a finite amount of lime. and hen maintain the system in that state of set of slates for all time hence. When we subsennently considered controllability criteria for linear systents. We dropped the latter requirement. In many applications. however. it is not enough for a controller to simply move the system to a particular state. Neither is it reasonable to expect that the controller maintain a given state in the lace of arbitrary disturbances or perturbations of the dynamical system. Stability is a property of dynamical systems which implies that small clianges in input or initial conditions cio not result in large changes in system behavior. Stability is not a prerequizite for being able to control a system. bit it makes the task of desigwng a cont: col system somewhat easier. The system describing the inverted pendulum presented in Chapter 2 is not stable by the criteri-s that we will present shortly. but it is controllable. The concept of stability introduced in the following is attributed to the Russian malher atician A. M. Iyapunov.

We will be coucerned with the same linear multivariable system introduced earlier.

$$
\begin{aligned}
& \dot{\mathbf{x}}(t)=A \mathbf{x}(t)+B \mathbf{u}(i) \\
& \mathbf{y}(t)=C^{\prime} \mathbf{x}(t)
\end{aligned}
$$

a

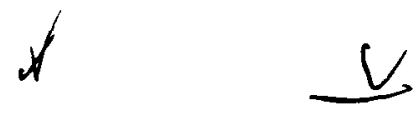
Let $u(t)=u_{c}$ be any constan. input. If there existspoint $x_{e} \in \mathbf{R}^{n}$ such
that

$$
A \mathbf{x}_{e}+B \mathbf{u}_{c}=\mathbf{0}
$$

then $\lambda_{e}$ is said to be an fquilibrium print of the system corresponding to the input $\mathbf{u}_{c}$. We assume that the systeut has only one equilibriun point, and. without loss of generality, take the origin of the state space to be that equilibrium point. Finally. we cotisider only the case in which $y=0$ so that

$$
\dot{\mathbf{x}}(t)=A \mathbf{x}(t) \text {. }
$$<smiles>C1C[As]C1</smiles>

This syatem is stable in the sense of Lynpunon at the origin if. for exrry $\epsilon>0$. there exists $\delta>0$ such that $\left\|x\left(t_{0}\right)\right\| \leq \delta$ implies $\|x(t)\| \leq \epsilon$ for all $t \geq t_{0}$. where $\|x\|$ denotes the Euclidcan norm for a vector $x$ of $n$ components $x_{1}, x_{2} \ldots \ldots x_{n}$ defined hy

$$
\|\mathbf{x}\|=\left(x_{1}^{2} \cdot 2_{2}^{2}, \ldots, x_{n}^{2}\right)^{1 / 2} .
$$

The hyper-spherical region defined by the set of all points such that $\|\mathbf{x}\| \leq \epsilon$ serves to eusure a bound on the system respouse.

We say that the above system is asymptolically stable at the origin if 
1. it is stable in the sense of Lyapunov. and

2. there exists a real number $r>0$ such that

$$
\left\|\mathbf{x}\left(t_{0}\right)\right\| \leq r \text { inplies } \mathbf{x}(t)-0 \text { as } t-\infty .
$$

The stability of a linear multivariable system can be determined using a relatively simple mathematical test provided in the following theorem (see. [14] for proof).

Theorem 5 The system described by the state equation,

$$
\dot{\mathbf{x}}=d \mathbf{x}(t)+B \mathbf{u}(t) \text {. }
$$

is asymptotically strable if and only if all of the eigenvelues of the matrix A huve negative real parts.

Recall that the eigenvalues of a matrix $A$ correspond to those values of $\lambda$ such that $\operatorname{Det}(\lambda I-A)=0$. where $I$ is the identity matrix and $\operatorname{Det}(M)$ indicates the determinant of the matrix $M$. One particularly convenient advantage of the stability test introduced in Theorem 5 is that it does not require one to solve the systen state equations. In the case of the singledegree-of-freedom robot. the eigenvalues correspond to solutions of

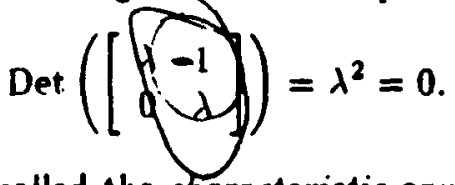

The equation $\lambda^{2}=0$ is called the chameteristic equation, and, in this case. the characteristic equation has no solutions indicating that the the dynamical svstem for the single-degree-of-freedom robot is stable.

In the case of the inverted pendulum example of C'hapter 2.

$$
\dot{x}(t)=\left[\begin{array}{cccc}
0 & 1 & 0 & 0 \\
0 & 0 & -0.5809 & 0 \\
0 & 0 & 0 & 1 \\
0 & 0 & 4.453 i & 0
\end{array}\right] x(t)+\left[\begin{array}{c}
0 \\
0.9211 \\
0 \\
-0.394 i
\end{array}\right] u(t) .
$$

the charecteristic equation is

$$
\operatorname{Det}\left(\left[\begin{array}{cccc}
\lambda & 1 & 0 & 0 \\
0 & \lambda & -0.5809 & 0 \\
0 & 0 & \lambda & 1 \\
0 & 0 & 4.453 i & \lambda
\end{array}\right]\right)=\lambda\left(\lambda\left(\lambda^{2}-4.453 i\right)\right)=0 \text {. }
$$




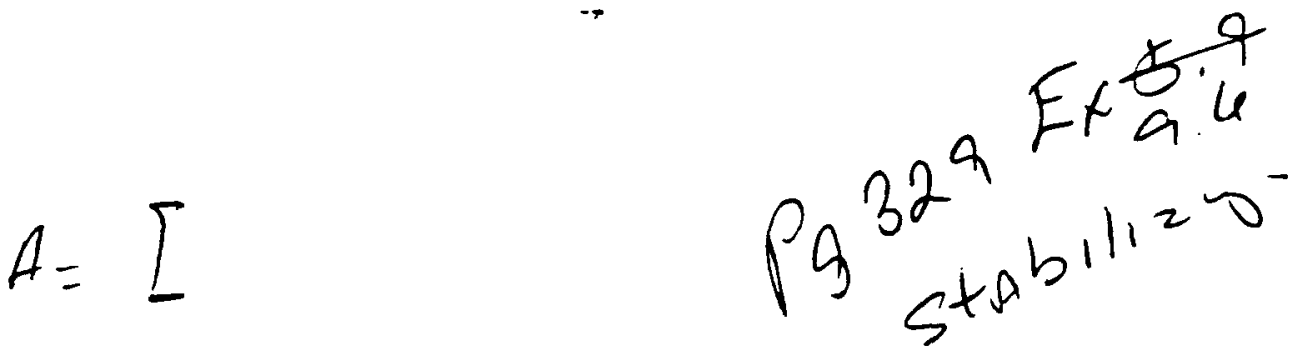

According to criterinn established in Thec eII 5. the dynamical system for the inverted pendulum is not stable since one of the solutions of the characteristic equluation is $\lambda=+v \overline{4.4 .537}$.

Before we leave the subject of stability. it is worth mentioning one particularly useful techuigue referred to as the mot-locus method developed by W.R. Evans for investigating the stability of linear systens. The root-locus method is most closely associated with what is called classical control theory which. as was mentioned in (hapter 2 . is based prinarily upon the use of the Laplace transform and analysis in the frequency clonain.

Many control systens have a siugle input variable and a single output variable. The input is referred to as a ifference signal indicating the desired value for the output or controlled rariable. The transfer function of such a control system is defined to be the ratio of the Laplace transform of the input variable to the Laplace transform of the output variable. Consider the spring-mass-dashpo' system described in Chapter 2. and suppose that we allow an external force to act on the block. The equation of motion of the block is

$$
M \frac{d^{2} x}{d t^{2}}+C \frac{d x}{d t}+K x=u(t)
$$

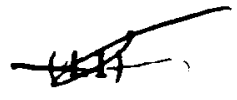

where the output of the systen is defined to be $x$ and the input is $u$. The Laplace transform of Equation 4.1 is

$$
M s^{2} X(s)+C \cdot s(s)+K I(s)=l^{\prime}(s)
$$

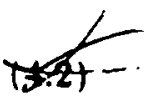

assuming the initial conditious

$$
x(0)=x_{0}, \quad \frac{d x(0)}{d t}=0 .
$$

The transfer function for the system corresponding to Equation 4.2 is

$$
T(s)=\frac{I(s)}{[(s)}=\frac{1}{I s^{2}+C s+K^{*}}
$$

By analyzing the sustem's poles (the roots of the denominator or chamcteristic equation of the trausfer function) and zeros (the roots of the numerator of the transfer function). oue cau tell a great deal about the transient respouse characteristics of the control systen. For instance. it is well bnown [10] that. for a system to be stable. it is necessary and sufficient that all of

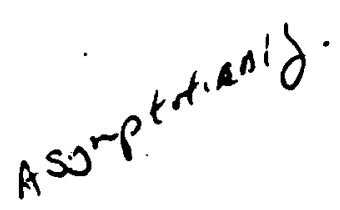
the poles of the system transfer function have negative real parts. ${ }^{3}$

\footnotetext{
${ }^{3}$ The Laplace variable is a complex variable and lieuce the roots of the characteristic equation are generally complex as well.
} 

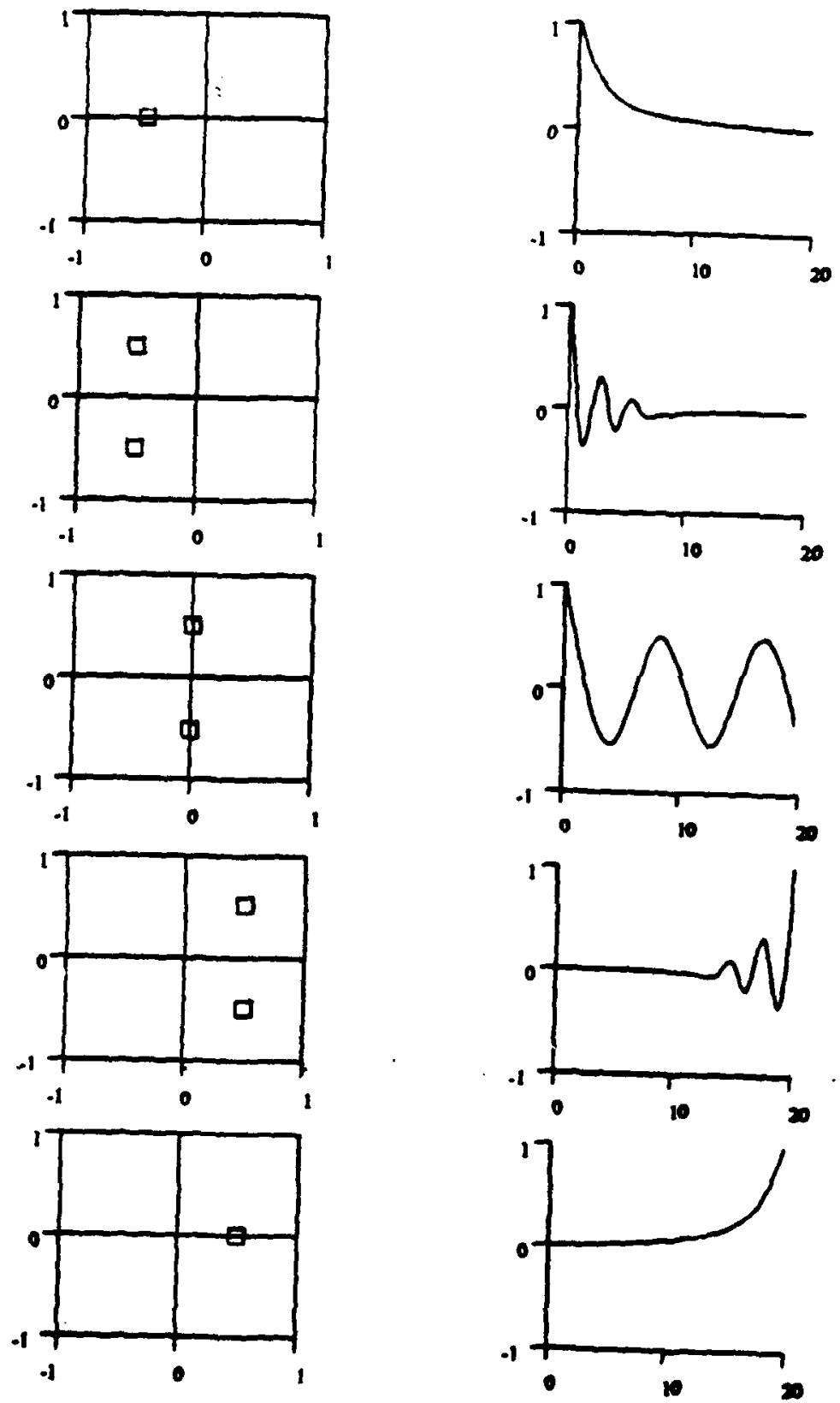
Figure t.i: The connection hetreen pole placement in the complex s-plane
and performance in the tinie domain. 
Figure $4 . i$ shows the relation between the poles of the transfer function for a second order system and the system's corresponding behavior in the time domain. Ln Figure t.i. each plot on the left hand side indicates one particular placemcut of the poles in the complex s-plaue. and the corresponding plot on the right indicates the resulting performance in the time domain. This tuethod of analyzing control systems by deterwining the placement of poles is known as the root locus method.

Not surprisingly. there is close connection between the frequency-and time-domain methods for determining stability. In the case of multipleinput. multiple-output systems. We have to generalize on the notion of a trausfer function. which is defined ouly for single-input. single-output systeins. The transfer matrix of a linear multivariable dynamical system as introduced in the beginning of this section is uniquely defined by

$$
T(s)=C^{\prime}(s I-A)^{-1} B \text {. }
$$

where $I$ is the identity matrix [29]. It should be noted that there is information lost in this conversion. In particular. the state and input equations specify the internal state as well as the input/output behavior of the dynamical system, whereas the transfer iuatrix only specifies the latter. It 1.urns out that the poles of the system represented by the transfer matrix are exactly the eigenvalues of the malrix $A$ [29].

One convenient property of trausfer functions and transfer uatrices is that. in certain cases, such representations call be obtained experimentally ly subjecting the dyuanical system to sillusoidal inputs and measuring the steady-state response. The close conuection between frequency-and timedomain methods allows the engineer to shift back and forth between these two perspectives as the problem dictates.

Stability can simplify the design of control systems; it is not, however. a presequisite for control. The linear system for the inverted pendulum is not stable. lout it is controllable. If we a re clesigniug a device. it is generally worthwhile to design it in such a way that its corresponding dynamical system is stable. In cases in which the plant (environment) is given. we have little choice and must proceed whether or not the associated system is stable. 


\subsection{Optimality}

In previous sections. We have stressed primarily the qualitative properties of dynamical systens (e.g.. controllability. observability. and stability). With the exception of criteria concerning whether or not a given controller can achieve a particular state from some arbit rary initial state. we have had very little in say about the performaure of a control system. In this section. we consider control problens in which some quantitative measure (or index) of performance is provided. It is natural within this context to consider problems of optimal contml that involve maximizing or minimizing such a performance iudex.

In describing optinal control problems. we generally restrict our attention to some restricted interval of tine, either continuous. $\left[t_{0}, t_{1}\right]$. or discrete. [1.n]. The behavior of the dynamical system is described by either a set of dilferential equations

$$
\dot{x}(t)=\int(x(t), u(t)), \text { restricted to } t_{0} \leq t \leq t_{1}
$$

in the continuous case, or a set of difference equations

$$
x(k \div 1)=f(x(k), u(k)) \text {, restricted to } 1 \leq k \leq n
$$

in the discrete case. In addition to the model for the dynamical syistem. it is ofteu convenient to place restrictions ou both the inputs (e.g.. you might want to place a bound on control torques to keep the cost of servo motors within budget coustraints) and the outputs (e.g.. you may want to restrict the trajectories of a robot arn to a confined work space). The input restrictions define a set of admissible contmis (see the discussion in Section 4.2 on adnissible controls for discrete events svstems). Finally, it will be necessary lo formulate a jerformance index in terms of a scalar value function, $V$.

The choice of performance index is largely subjective. but geuerally a particular application will suggest something reasouable. In some cases, it may make seuse simply to minimize time:

$$
V=\int_{t_{1}}^{t_{2}} 1 d t=t_{1}-t_{0}
$$

In other cases, there may be an obvious cost function. $c(x, u)$. such as the amount of fuel or other resource spent:

$$
V=\int_{t_{1}}^{t_{2}} c(x(t) \cdot u(t)) d t
$$


For the ser-point regulation and servo problems a good measure of $p$,rformalle is the squared error:

$$
V=\int_{t_{1}}^{t_{2}}\left(x(t)-r^{*}(t)\right)^{2} d t
$$

where $x^{-}(t)$ is the desired state at time $t$. The squared error index is an example of a quadratic performance inclex. ${ }^{+}$.lore generally. the performance index is defined as

$$
V=h\left(x\left(t_{1}\right)\right)+\int_{t_{1}}^{t_{2}} g(x(t), u(t)) d t
$$

where $h$ and $g$ are scalar functions ucant to capture the value of the terninal state and the state/input trajectory respectivcly. The problem of designing optimal coutrols consists of finding an admissible control that nuinimizes (maximizes) the performance index. $V$.

There are two classes of uptimal coutrol problems involving linear multivariable systeins for which general results have been obtained. The first class involves the use of a quadratic performance index as in the example of the uninimum squared error index. and includes optimal versions of the linear set-point regulation and servo problems. In the second class of problems. the objective is to ininimize the time required to drive the system to a desired state. In hoth of these two classes of problenis. optimal controllers can wake use of feedback. which. as covered in the next section. provides for more robust contrcl in the presence of external disturbances and errors in inodeling. The optimal linear mininum-time controller is of a particularly simple form: it can be viewed as a function that simply switches between the extreme values dictated by the class of admissable controls. A controller that operates at a constant level either in one mode or another (e.g., $\forall t . u(t) \in\{-1.0 .1\})$ is called a bnng-bang controller.

Moat of the work on optimal control builds upon basic technique in the calculus of rariations [12]. The nethod of Lagrange uultipliers ${ }^{5}$ for findins extrems of functious subject to constraints is one techniques from

\footnotetext{
'Tw fonction $V=\int f(t) d t$ is a quadratic pefformance index if $f(t)=x(t)^{\prime} A x(t)$ where $A$ i an $n \times n$ matrix with $a_{i}, \in \mathbf{R}$ and $x \in \mathbf{R}^{n}$.

${ }^{3}$ Leonard Euler (170i-1783) devetoped the bavic approach to colving constrained exiremum probtems. Joseph Larange (1736-1813) studied Eulet approach and worked out the details for some inportant special cases. The basic method is generally referred 10 as the tnethod of Lagrange multipliers. but in some texts the equations are referred 10 as the Euler-Lagronge equations recognizing Euler's contribations.
} 
the calculus of variations that students Iypically encounter in college calculus rourses.

As a simple example illustrating the use of the method of Lagrange multipliers. let $\boldsymbol{\varphi}(x, y)$ and $s(x, y)$ be functions of two variables. The object is to find values of $x$ and $y$ that maximize (or mimimize) the objective function $\hat{r}(x, y)$ while at the same time satisfying the constraint equation. $\varsigma(x, y)=0$. We replace $-(x, y)$ with an auxiliary function of three rariables called the Hamiltonian function. $\Phi(x, y, \lambda)$. defined as

$$
\Phi(x, y, \lambda)=\boldsymbol{f}(x, y)+\lambda s(x, y) .
$$

The new variable. $\lambda$. is called a Lagmnge multiplier. The Euler-Lagrange multiplier theorem (12) inplies that. if we locate all points $(x, y, \lambda)$ where the partial derivatives of $\Phi(x, y, \lambda)$ are all 0 . then among the corresponding $(x, y)$ we will find all of the points at which the function $\forall(x, y)$ will have a constrained extremum.

In the method of Lagrange multipliers. we solve for $x, y$, and $\lambda$ in the equations formed by setting the partial derivatives to 0 :

$$
\frac{\partial \Phi}{\partial x}=0, \frac{\partial \varphi}{\partial y}=0, \text { and } \frac{\partial \Phi}{\partial \lambda}=0 .
$$

Since $\partial \Phi / \partial \lambda=\varsigma(x, y)$, if we find a solution $(x, y, \lambda)$ to the above three equations. the constraiut equation $\varsigma(x, y)=0$ will automatically be satisfied.

To illustrate how to apply the method of Lagrange multipliers to problems in optimal control. consider the discrete-time system

$$
x_{k+1}=f\left(x_{k}, u_{k}\right),
$$

and the performance index defined by

$$
V=\sum_{k=1}^{n} g\left(x_{k}, u_{k}\right),
$$

where we have changed our notation somewhat. $x(k)=x_{k}$ and $u(k)=u_{k}$. to simpify subsequent equations. The only constraint that we impose is that the optimal solution obey the state difference equations. We enforce this constraint by augmenting the performance index a follows

$$
V^{\prime}=\sum_{k=1}^{n}\left[g\left(x_{k}, u_{k}\right)+\lambda_{k+1}\left(f\left(x_{k}, u_{k}\right)-x_{k+1}\right)\right]
$$


We define the Hanultonian somewhat differcnty from above as

$$
\Phi_{k}=g\left(x_{k} \cdot u_{k}\right)+\lambda_{k+1} \int\left(x_{k} \cdot u_{k}\right) .
$$

so that we can rewrite the angmented performance index as

$$
V^{\prime \prime}=\sum_{k=1}^{n}\left[\Phi_{k}-\lambda_{k+1} \alpha_{k+1}\right]
$$

By the Euler-Lagrange multiplier thenrem. the change in the total derivative. $d V^{\prime}$. defined as

$$
d V^{\prime}=\sum_{k=1}^{n}\left[\left(\frac{\partial \Phi_{k}}{\partial x \delta k+1 \delta}-\lambda_{k}\right) d \underline{x_{k}}+\left(\frac{\partial \Phi_{k}}{\partial \lambda_{k+1}}-x_{k}\right) d \lambda_{k}+\frac{\partial \Phi_{k}}{\partial u(k)} d \underline{u_{k}}\right] .
$$

suould be zero at a constrained minimum. As a consequence. the necessary conditions for a constrained minimum are defined by

$$
x_{k+1}=\frac{\partial \Phi_{k}}{\partial \lambda_{k+1}}=f\left(x_{k}, u_{k}\right), 1 \leq k \leq n .
$$

referred to as the stute equations.

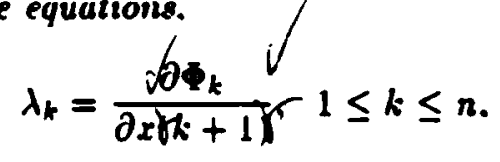

referred to as the costate equations.

$$
0=\frac{\partial \phi_{k}}{\partial u / k)}(1 \leq k \leq n
$$

referred to as the stationary conditions, and, finally, we require that the $x_{1}$ be the initial state. The state and costate equations are coupled difference equations. and together they define a two-point boundary value problem. In the special case of linear systems with quadratic performance indices. numerked solutions cau be obtained rat her easily:

In goncral, it can be quite difficult to solve the two-point boundary value probleme resulting from Lagrange multiplier formulations. However, in some

\footnotetext{
'Specifically. it is posoble to derive open-toop (the syotem atate in not emplored in computing the next input) controllers for the case in which the final state is sperified (fixed) in adrance. and closed-loop (the system state is employed in computing the next inpnt) controllen for the case in which the find otate is not specified (free) in adrance [21].
} 
cases. finding global maxima or minima can still be achieved by searching the space defined $y$ the variational tariables (f.g.. $x$ and $y$ in the case of mininizing $\boldsymbol{f}(x, y)$ ) One approach is to use mumeriral mel hods to solve the urigiual equations relaling to lise performance index and cunst rajnts. and then search the resulting surface looking for global extrema. The gradient. clefined as

$$
\Gamma_{\hat{r}}=\left[\begin{array}{l}
\partial_{\varphi} / \partial x \\
\partial_{\varphi} / \partial y
\end{array}\right]
$$

in the case of $\varphi(x, y)$, is used to guide search in a method that proceeds by taking many small steps. each one in the direction indicated by the (negated) gradient. This search method is called gmdient descent. If the surface has a single (global) inininum. then gradient descent search is guaranteet to find it. If. however. there are many local minima. as is often the case. then one has to be a lot more rlever in directing the search. It is this aspect of optimal control involving search in a space of possible controls that primarily iuterests us in this section.

In soine rases, we call resort to exhallstive search. For instance. if $x$ and $y$ are bounded, we might try to discretize the domain of $q$, allowing each of $x$ and $y$ to take on $r \in Z$ possible values. In this rase. there are only $r^{2}$ points at which to evaluate $r$; however. in the case of $m$ tariational variables each having $r$ possible values, there will he $r^{\prime n}$ points to evaluate. As we will see. the dimensionality, $n$. of a control problem is a critical factor in the design of optimal control systems.

Bellman [3] and Pontryagin [24] were largely responsible for formulat.ing the necessary prohlems and developing many of the basic approaches to solving optimal control problems. The requisite mathematics is complicated enough that the backgronnd required to even state the basic theorems doen not seem warranted for our treatment here. Suffice it to say that the results for linear systems are exteusive, and that, additionally; there are powerful nnmerical methords that have proved successful for a range of nonlinear systems. For a good overview of the field the reader is enconraged to consult the text by Athans and Falb [2]. In the remainder of this section. we focus on a particular class of optinal coutrol problezns called multistnge decision pmeraves. and a particular approarh to solving such problems optimally dynamir programming due to Richard Hellman.

Consider a deterministic discrete-time "1-st.age proress consisting of all initial state $\tau_{1}$, a serquence of inpnts $\left\|_{1},\right\|_{2}, \ldots, \|_{n}$, and a sequence of reanlt. 
iug states $x_{2}, x_{3} \ldots \ldots t_{n}$ such that

$$
r_{k+1}=f\left(x_{k}, u_{k}\right) \text {. }
$$

Following standard practice. the $\left\{u_{k}\right\}$ and $\left\{r_{k}\right\}$ are treared as variables ranging over $i i$ and $X$ respectively. We introduce a performance index.

$$
V\left(u_{1} \ldots \ldots u_{n} ; x_{1} \ldots \ldots x_{n}\right) .
$$

We wish to find input sequences that maximize $V$.

As we indicated earlier. in general. this problen of maximizing a function of $n$ variables is computationally quite hard. In the worst case, it will be necessary to search through the set of $\left|U^{\top}\right|^{n}$ possible sequences of length $n$ in order to choose the sequence with the highest value. In some cases. however. we call do much better. In the following, we consider some easier problems that result from introducinf restrictions on $V$. In particular. we consider the case in which at ? age in the process. say the $k$ th stage. the effect of the remaining $n-k$ ages on the total value depends only on the state of the system following the $k$ th decision and the subsequent $n-k$ decisions [4]. Let $\mathbf{R}:(I \times I-\mathbf{R}$ represent a reuard function, where $\mathbf{R}(u . x)$ corresponds to the (iminediate) benefit derived from perforning action $u$ in state $x$. We write $R(u, x)$ if both the input and the state matter in determining the amount of reward and $R(x)$ if only the state matters. As an example of the sort of performance functions we are interested in, we might have

$$
V\left(u_{1}, \ldots u_{n} ; x_{1}, \ldots, x_{n}\right)=\sum_{k=1}^{n} R\left(u_{k}, x_{k}\right)
$$

in which we are interested in the sum of rewards (referred to in the sequel as sepamble control), or

$$
\mathrm{V}\left(u_{1}, \ldots, u_{n} ; x_{1}, \ldots, x_{n}\right)=\mathrm{R}\left(x_{n}\right)
$$

in which we are interested only in the reward associated with the final state (referred to an terminal mintrol).

We proced by generating a sequence of functions, $\left\{V_{n}\right\}$, so that

$$
V_{n}\left(x_{1}\right)=\max _{u_{k}} \sum_{k=1}^{n} R\left(u_{k}, x_{k}\right)
$$


Expanding. we have

$$
\begin{aligned}
V_{n}\left(x_{1}\right) & =\max _{u_{k}} \sum_{k=1}^{n} \mathrm{R}\left(u_{k}, x_{k}\right) \\
& =\max _{u_{k}}\left[\mathrm{R}\left(u_{1}, x_{1}\right)+\mathrm{R}\left(u_{2}, x_{2}\right)+\cdots+\mathrm{R}\left(u_{n}, x_{n}\right)\right] \\
& =\max _{u_{1}} \max _{u_{2}} \ldots \max _{u_{n}}\left[\mathrm{R}\left(u_{1} \cdot x_{1}\right)+\mathrm{R}\left(u_{2}, x_{2}\right)+\cdots+\mathrm{R}\left(u_{n}, \alpha_{n}\right)\right] .
\end{aligned}
$$

Rearranging, we obtain

$$
\begin{aligned}
V_{n}\left(x_{1}\right)= & \max _{u_{1}}\left[R\left(u_{1}, x_{1}\right)+\right. \\
& \left.\max _{u_{2}} \max _{u_{3}} \ldots \max _{u_{n}}\left[R\left(u_{2}, x_{2}\right)+R\left(u_{3}, x_{3}\right)+\cdots+R\left(u_{n}, x_{n}\right)\right]\right] .
\end{aligned}
$$

Note that

$$
V_{n-1}=\max _{u_{2}} \max _{u_{3}} \ldots \max _{u_{n}}\left[R\left(u_{2}, x_{2}\right)+R\left(u_{3}, x_{3}\right)+\cdots+R\left(u_{n}, x_{n}\right)\right] .
$$

Substituting, we have in the case of separable control,

$$
V_{n}\left(x_{1}\right)=\max _{u_{1}}\left[R\left(u_{1}, x_{1}\right)+V_{n-1}\left(x_{2}\right)\right]
$$

or just

$$
V_{n}(x)=\max _{u}\left[R(u, x)+V_{n-1}(f(x, u))\right]
$$

for $n \geq 2$. and

$$
V_{1}(x)=\max _{u} R(u, x) .
$$

for $n=1$. For the case of terminal control, we have

$$
V_{n}(x)=\max _{u}\left[V_{n-1}(f(x, u))\right], \text { for } n=2,3, \ldots
$$

and

$$
V_{1}(x)=R(x) \text {. }
$$

The time to compnte $V_{i}(x)$ for all $x \in X$ given that invoking $V_{i-1}$ has unit cont is $O\left(\left|X^{-} \| U^{\top}\right|\right)$. From this observation, it follows that the time required to compute $V_{n}(x)$ for all $x \in X$ given that invoking $V_{1}$ has unit cost is $O(n|X||U|)$.

This general method of computing the performance index recursively is called dynamic pragmmning. The basic constrained minimization variational problem essentially involves choosing a point in an $n$-dimensional 



\begin{tabular}{|c|c|c|c|c|c|c|c|c|c|c|c|c|c|c|c|}
\hline$x$ & $x$ & $\pi$ & $x$ & $x$ & $x$ & $x$ & $x$ & $x$ & $\mathrm{X}$ & I & .1 & $x$ & $x$ & $\mathrm{X}$ & $x$ \\
\hline$x$ & $\therefore$ & -4 & .5 & fi & .7 & -8 & .9 & -111 & -11 & -12 & .131 & .14 & -15 & $-11 i$ & $\pi$ \\
\hline $\bar{x}$ & -4 &. .3 & $-t$ & $\therefore i$ & $-1 i$ & $\therefore$ & -.8 & -11 & -10 & .11 & -12 & -13 & .14 & -15 & $x$ \\
\hline$i$ & .3 & -2 & -.3 & $x$ & $-\bar{T}$ & -8 & $-!$ & .10 & -11 & -12 & -1.3 & -14 & -1.5 & -16 & $x$ \\
\hline$x$ & -2 & -1 & .2 & $x$ & -8 & -9 & .10 & -11 & .12 & .13 & -14 & .15 & .16 & -17 & X \\
\hline $\mathrm{X}$ & -1 & $G$ & -1 & $\mathrm{X}$ & -9 & -10 & -11 & -12 & -13 & -14 & -15 & .16 & -17 & -18 & $x$ \\
\hline$x$ & -2 & -1 & -2 & $x$ & -8 & -9 & -10 & -11 & -12 & -13 & -14 & -15 & -16 & -17 & $x$ \\
\hline$x$ & .3 & -2 & -3 & $x$ & -7 & -8 & -9 & $x$ & $x$ & $x$ & $x$ & -18 & -17 & -18 & $x$ \\
\hline$x$ & -4 & -3 & -4 & -5 & -6 & $-\pi$ & -8 & $x$ & -20 & -19 & -18 & .17 & -18 & -19 & $x$ \\
\hline $\bar{x}$ & .5 & -4 & .5 & $-i$ & $\pi$ & -8 & $-y$ & $x$ & -21 & .20 & -19 & .18 & .19 & -20 & $x$ \\
\hline$x$ & -6 & .5 & -6 &.$i$ & -8 & -9 & .10 & $x$ & .22 & .21 & -20 & -19 & -20 & -21 & $\bar{x}$ \\
\hline $\bar{x}$ & $-i$ & .6 & $-i$ & -8 & -9 & .10 & -11 & $\therefore$ & $x$ & $x$ & $x$ & -18 & -19 & -20 & $x$ \\
\hline$x$ & -8 & $-i$ & -8 & .9 & -10 & -11 & .12 & -13 & -14 & -15 & -16 & .17 & -18 & -19 & $x$ \\
\hline$x$ & -9 & .8 & -9 & .10 & -11 & -12 & .13 & -14 & -15 & -16 & -17 & -18 & -19 & -20 & $x$ \\
\hline$\dot{x}$ & -10 & -9 & -10 & -11 & -12 & -13 & -14 & -15 & -16 & .17 & -18 & -19 & -20 & -21 & $\bar{x}$ \\
\hline $\bar{x}$ & $x$ & $x$ & $x$ & $x$ & $x$ & $x$ & $x$ & $x$ & $x$ & 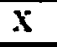 & $x$ & $\mathrm{x}$ & $x$ & $x$ & $x$ \\
\hline
\end{tabular}

Figure 4.9: $\mathrm{V}(\langle x, y\rangle)$ for the Cirid world

We compute $V_{1}, V_{2}$, up to $V_{k}$ such that $V_{i}=V_{i-1}$ and set $V=V_{i}$. Figure 4.9 shows $V(\langle x . y\rangle)$ for each state (location $\langle x, y\rangle)$ in the grid world of Figare 4.8 .

If you look carefully at the numbers shown in Figure 4.9. ynu will notice that by always moving to the neighboring location with the highest value you will eventually end up at the goal location no matter what location you start out in. This property can illustrated graphically by considering the elevation map showu in Figure 4.10 defined using $V(\langle x, y\rangle)$ as the elevation at coordinates $(x . y)$ in the grid with interior obstacles represented as small negative ralues. Notice that the goal location is a global naximum in the elevation map. This will always be the case no matter what the arrangement of obstackes. It turns out that the strategy of always moriug to the location with the highest value is optimal in the following sense.

We drine a control law or policy as a mapping from states to acions:

$$
\eta: x-t l
$$

We are interested in policies that are optimal according to the following principle of Bellnan. Aprinciple of optimality. An optimal policy has the $\forall \underbrace{21}$<smiles>[Mg][TeH]</smiles> 


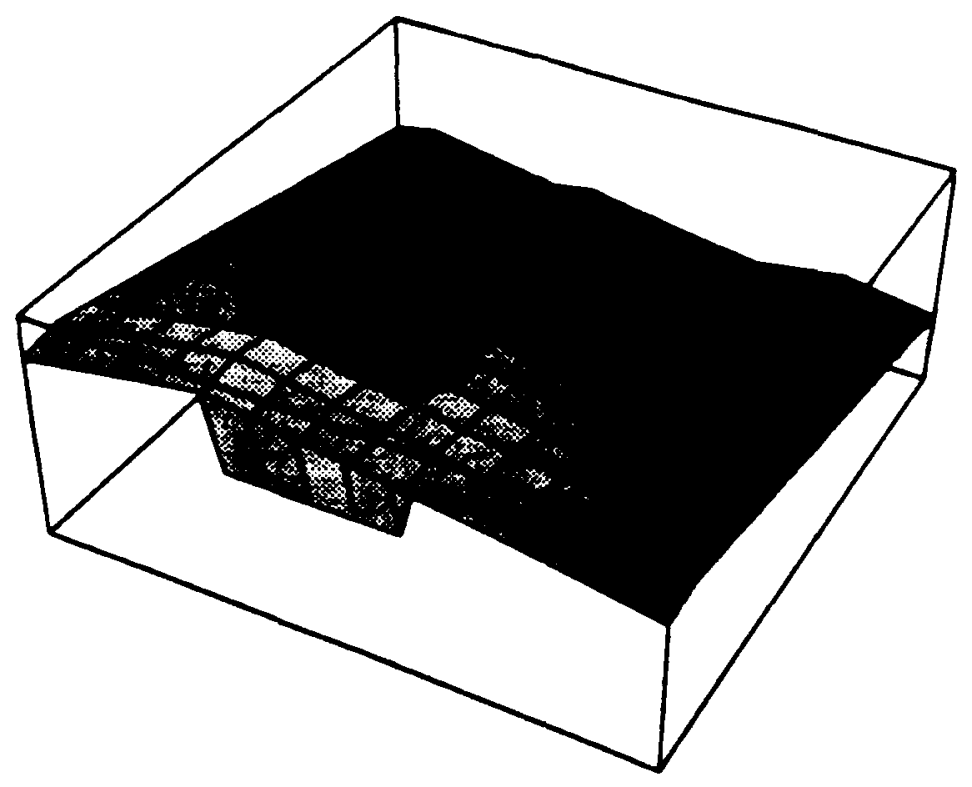

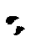

Figure 4.10: Representation-o[ $V(\langle x, y\rangle)$ as an elevation map

property that whatever the initial state and the initial decision are, the remaining decisions must constitute an optimal policy with regard to the state resulting from the first decision." ([4] Ps. 5i) Given Bellwan's principle of optimality, the following policy

$$
\eta(x)=\arg \max _{u} V(f(x, u))
$$

is optimal.

Figure 4.11 shows the optimal policy for the grid world shown in Figure 4.8. where.,- 1 , and $\perp$ indicate the direction of novement for the indicated state as specified by the optinal policy.

Becames the trausitious in state space are so localized in the grid world. we can we a much more efficient dyuamic programming algorithm for computing the optinal policy than the one described above. In particular, we compute $V_{2}$ only for grid cells correspouding to one of the four neighbors of the goal adjacent atung the grid axes, and. in su duing, treat $V_{1}$ as undefined for all cells other than the goal. In general, we compute $V_{i}$ only for previously unconsidered grid cells correspouding to one of the four neighbors of cells considered in $i$ - 1 th iteration, and treat $V_{i-1}$ as undefined for all 


\begin{tabular}{|c|c|c|c|c|c|c|c|c|c|c|c|c|c|c|c|}
\hline $\mathrm{x}$ & $i$ & $x$ & $\because$ & $x$ & $x$ & $x$ & 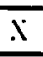 & $x$ & $x$ & $x$ & $x$ & $\therefore$ & 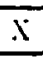 & $x$ & $\therefore$ \\
\hline $\mathbf{X}$ & 1 & 1 & - & - & - & - & - & - & - & - & - & - & - & - & $\lambda$ \\
\hline $\bar{x}$ & 1 & 1 & - & - & - & - & $\overline{-}$ & - & - & - & - & - & - & - & $x$ \\
\hline $\bar{\lambda}$ & 1 & 1 & - & $x$ & 1 & - & - & - & - & - & $=$ & - & - & - & $x$ \\
\hline $\bar{x}$ & $T$ & 1 & - & $\bar{x}$ & 1 & - & - & - & - & - & - & - & - & - & $x$ \\
\hline$x$ & - & $\mathbf{G}$ & - & $\bar{x}$ & 1 & - & $=$ & - & - & - & - & - & - & - & $\bar{\lambda}$ \\
\hline $\bar{x}$ & 1 & 1 & - & $\mathrm{x}$ & 1 & - & - & - & - & - & - & - & - & - & $\dot{\lambda}$ \\
\hline $\bar{x}$ & 1 & 1 & - & $\mathrm{x}$ & 1 & - & - & $x$ & $\bar{x}$ & $x$ & $x$ & 1 & - & $=$ & $x$ \\
\hline$x$ & 1 & 1 & - & - & - & $=$ & - & $x$ & $=$ & $=$ & $=$ & 1 & - & $=$ & 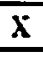 \\
\hline $\bar{x}$ & 1 & 1 & - & - & - & - & - & $x$ & 1 & 1 & 1 & 1 & - & - & $x$ \\
\hline $\bar{x}$ & 1 & 1 & - & - & - & - & - & $x$ & 1 & 1 & 1 & 1 & - & - & $x$ \\
\hline$x$ & 1 & 1 & - & - & - & - & - & $x$ & $x$ & $x$ & $x$ & 1 & - & - & $\lambda$ \\
\hline $\bar{x}$ & 1 & 1 & - & - & - & - & - & $=$ & $=$ & - & $=$ & $=$ & - & $=$ & $x$ \\
\hline $\bar{x}$ & 1 & 1 & - & - & - & - & - & - & - & - & - & - & - & - & $\lambda$ \\
\hline $\bar{x}$ & 1 & 1 & - & - & - & - & - & - & - & $=$ & - & - & - & - & $\bar{x}$ \\
\hline $\bar{x}$ & $x$ & $\lambda$ & $x$ & $\bar{x}$ & $x$ & $x$ & $x$ & $x$ & $\bar{\lambda}$ & $x$ & $x$ & $x$ & $x$ & $\bar{\lambda}$ & $x$ \\
\hline
\end{tabular}

Figure 4.11: An optimal policy

cells not considered in the $i-1$ or earlicr iterations. If $k$ is the last iteration in which there are uncousidered cells. then $V_{k}$ is defined for all cells in the grid. and we set $V=V_{k}$. This specialized dunamic programming algorithn runs in $O(|X|)$.

The example application of dynamic programming given above involves a discrete deterministic dynamical system. Dynamic programming can be applied to continuous dynanical syintenis to achieve solutions of arbitrary accuracy using a variety of namerical techniques. Dynamic programming can be seen as a method of efficiently solving variational problenıs involving multiple local minima by cleverly guiding the search. Dynamic programming can aloo be applied to stochastic processes. and we will return to this subject in Chaperer 6.

Hew as elwewhere the dimensionality of the problem severely restricts the applieation of this and most other methods to generating solutions effiriently. Dyaamic programning is often referred to as an "appronch" rather than a "method." where the distinction generally made is that an approach provides a way of looking at problems that still requires considerable creativity to actually apply, whereas a methor is more a matter of tuming a 
crank. Dynamic programming suggests that we try to view optinization problems as multistage decision problens in which the performance index is some simple ( $\epsilon . g$.. additive) function of the state and input at each stage. If it is possible to view a problem thus. we can effectively reduce the dimensionality of the problen thereby, availing ourselves of substantial computational savings. Uufortunately, there are many aspects of a problem that serve to determine its dimensionality. For example, at best, the solution methods that we considered above involved computations linear in the size of the state space. and the dimeusionality of the state space is determined by the number of state variables that comprise the state vector. In practical problems. nethods that require quantifying over the entire state space can be computationally prohibitive. In subsequent chapters. we consider methods that sifl allow us to decompose certain problems into independent subproblems each of which requires quantifving over ouly a small portion of the state space.

\subsection{Feedback Control Systems}

In Section 4.2 on controllability, we considered a controller as a function from states to inputs (control actions). While there are many different types of controllers mentioned in the literatnre, this particular formulation is perhaps the inost common. It is so common. in fact, that traditionally a control lau is defined to be a function $\eta: T \times . I \rightarrow U$,

$$
u(t)=\eta(x(t), t) .
$$

However, in the problems we will be considering, $\eta$ wilt not depend on the current time.

This basic jdes that the inputs to a dynamical system should be computed from the state is quite important. Kalman describes it as "the fundamental idea of control theory, ${ }^{-}$and "a scieutific explanation of the great invention known as 'feedback, which is the foundation of control engineering- ([19) pr. 46).

It is worth anking why, if we have an accurate model of the process that ive are trying to control. must we resort to sampling the state of this process on a continual basis. The answer is that uncertainty can and. generally. does arise from several sources besides the dynamical model. For instance. we have to sample the state of the system at some point in order to supply the initial conditions to the model. If there is any error in our measurement 


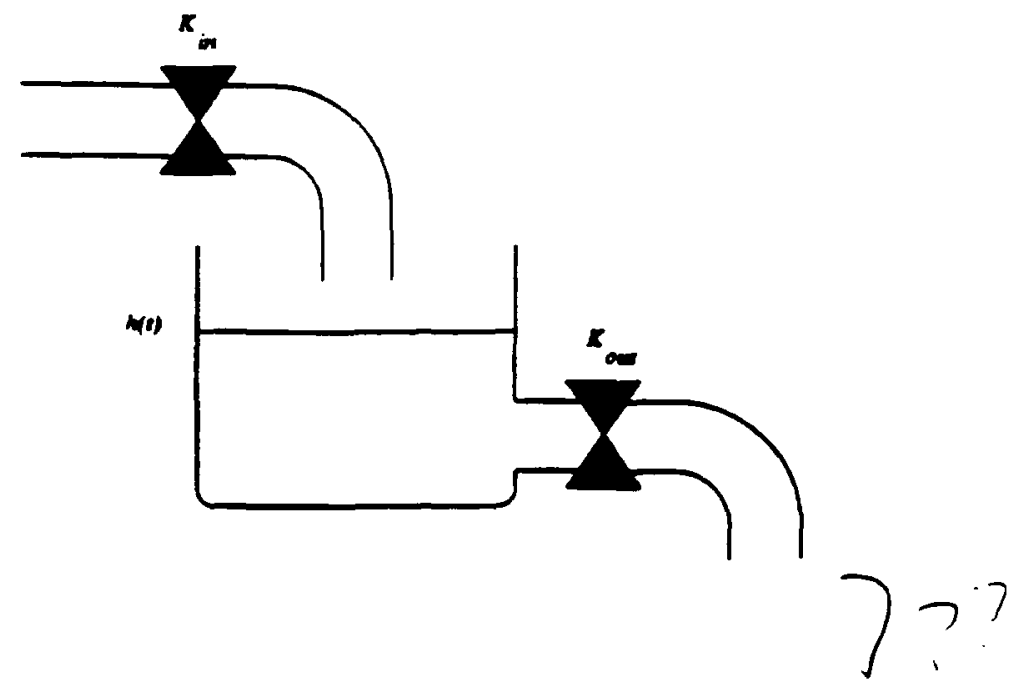

Figure 4.12: Controlling the level of fluid in a tank

of the state variables, then that error will likely be exacerbated with the $i$ :assage of time and as a consequence of inappropriate inputs generated on the hasis of incorrect state information. Even if we are able to observe the state precisely, there will inevitably be some delay hetween our observation of the state and our initiation of a control action. This delay may be due to time spent in computing inpuls. the response time of the actuators used to realize an input. or lags int.rudinced by the sensors. We return to these issues in ( /aapler $\mathbf{6}$ when we consider the problems that arise in dealing wit// uncertainty in control.

In the following, we consider the applitation of feedback control to some of the problems introduced in Chapter We begin by considering the problem of regulating the level of fluid in a tank using a closed-loop feedback controller. Figure $\$ .12$ depicts the tank and its associated input and output pipes.

We model the controlled process as a first-order differential equation:

$$
K_{\text {in }} \theta(t)-K_{\text {out }} h(t)=d \frac{d h(t)}{d t}
$$

where $K_{\text {in }}$ is the flow constant in cubir meters per degree minute for the valie governing flow through the input pipe, $K_{\text {out }}$ is the flow constant in square meters per minute for the output pipe. $A$ is the surface ares of the 


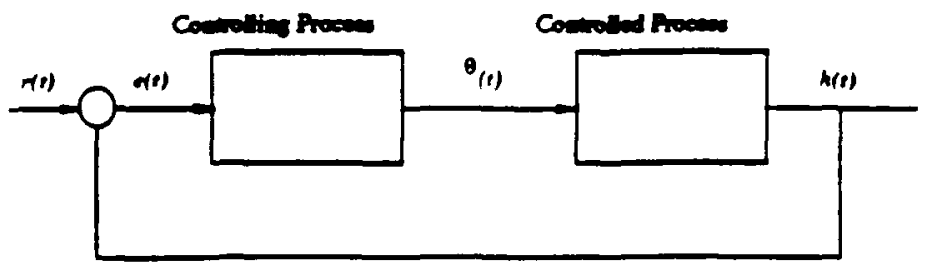

rigure 4.13: Block diagram for a closed-loop process controller

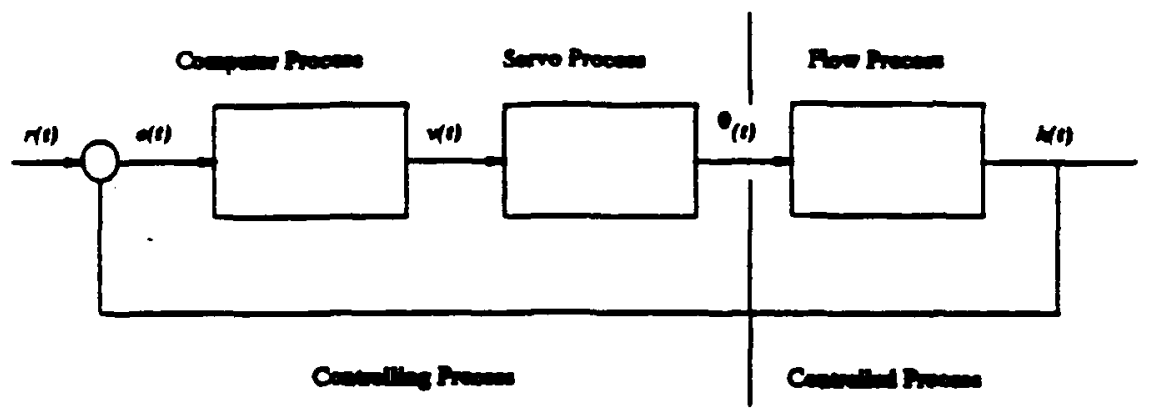

Figure 4.14: Decomposing the coutrolling process into subprocesses

tank. $\theta(t)$ is the position of the valve governing flow through the input pipe at tine $t$, and $h(t)$ is the height of the fluid in the tank at time $t$.

Now we have to specify a controlling process that changes $\theta$ in order to cause changes in $h$. In the simplest model. the controlling process directly determines $\theta$ by looking at the difference between the reference (or target) level and last measured value of $h$; this difference is referned to as the error. The block diagram shown in Figure 4.13 depicts this model with $r(t)$ indicating the reference and $e(t)$ indicating the error.

In Chapter 1, we defined a control algorithm that could cause instantaneous changes in $\theta$. Needless to say, the typica! interface between the controlling and controlled processes is more complex. In a somewhat nore realintic model, the control computer might determine a voltage that is input to a servo system consisting of an amplifier and a DC: motor attached to the input valve. The servo system in just auother process, and we night model it usiug the equation:

$$
\frac{d \theta(t)}{d t}=K_{g} v(t)
$$



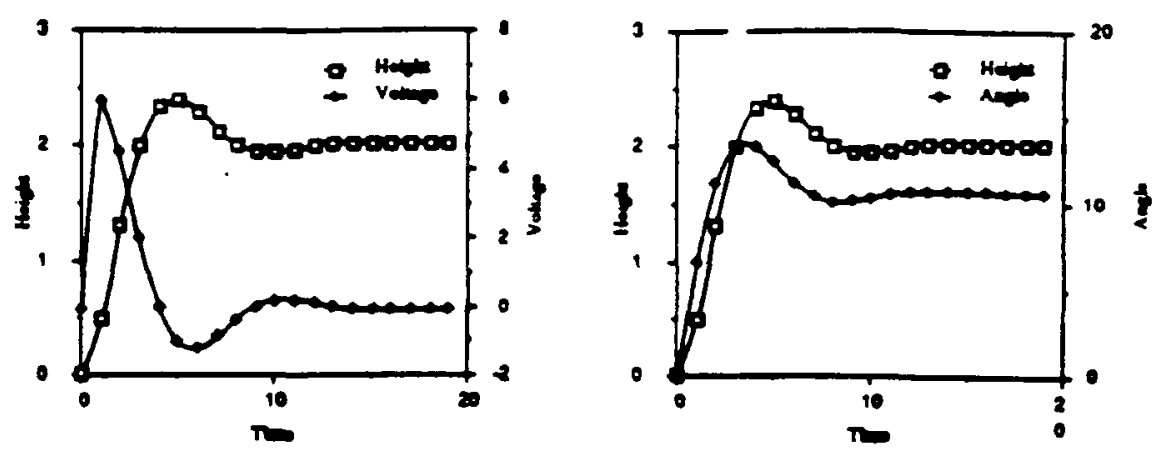

Figure 4.15: The behavior of the discrete proportional controller

where $r(t)$ is the input voltage and $K_{j}$ is a constant that depends on the characteristics of the servo. Figure $t .1 t$ provides a block diagram of this more complex model.

To define a process that determines the voltage input to the servo. we employ a standard technique from control theory. In many control schemes. the output of the controller is a simple function of the error. For controlling certain processes. an effective controller can be designed in which the output of the controller, $v(t)$ in this case. is directly proportional to the error:

$$
v(t)=K_{p} \epsilon(t)
$$

where $K_{p}$ represents the controller proportionality constant. Not surprisingly. this sort of control is called proportional control.

For a control algorithm running on a digital conputer. we have to specify a discrete controller that samples the output of the controlled process and outputs a control action at discrete intervals. The discrete proportional controller is just a computer program running on a specific machine that saunples the output of the controlled process every so many clock cycles and outputs a value proportional to the computed error.

To maintain the level of fluid in the tank depicted in Figure 4.12 at two meters. we might use the following loop:

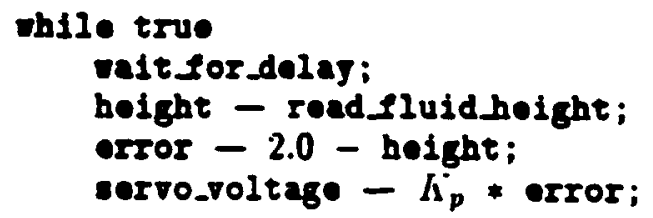

where roadfluid_hoight rearls the height sensor. vait_for_delay canses the coutroller to pause for the specified sample period, and sorvo_roltage 
is a machine register that directiv dctermines the voltage fed to the serro. Figure 4.15 shows two graphs descrithing the bchavior of the above control algorithm with a sample period of 1 mimute and a proportionality constant of 3.0. One graph compares clinnges in $h$ with changes in $r$. and a second compares clianges in $h$ with changes in $\theta$. The particular proportionality. constant 3.0 was chosen after a small amount of experimentation.

Proportional controllers are suitable for controlling only a limited class of processes. Two other popular forms of control are integral control and derivative control. The output $u(t)$ of an integral controller is proportional to the accumulated error:

$$
u(t)=R_{i} \int_{0}^{t} r(t) d t
$$

whereas the output of a derivative controller is proportional to the change in the error:

$$
u(t)=K_{d} \frac{d \epsilon(t)}{d t}
$$

The proportional-plus-integral-plus-derivative (or PID) controller generalizes the above three types of controllers:

$$
u(t)=K_{p} \epsilon(t)+K_{i} \int_{0}^{t} f(t) d t+K_{d} \frac{d \epsilon(t)}{d t}
$$

For the simple tank-filling process, proportional control is quite aclequate. Other, less stable processes. such as the inverted pendulum introduced in Chapter 1. may require an integrator and a differeutiator to damp oscillations and counpensate for ahrupt disturbances.

It should be noted that the constants used in a discrete PW controller are lependent apon the sample period. Of course, once you have the coefficients for the continuous PID controller youl can derive the coefficients for a discrete controller of any sample period.

The matheinatical discipline of control theory is largely concerned with the formal analysis of coutrol systems. As was unentioned in Section 4.j, in some canco. optimal contrul processes cau be derived auadytically providiug that accurate models of the controlled processes are available. Siuce the characteristics of the controlled processes rarely are known precisely. control theoriats are interested in systems that are insensitive to minor deviations in the models used in the design process. In cases withere significant deviations are likely, or the nodlets are kuown to be inconplete. adaptive sysiems are 
designed to conıensate hy adjusting the "pdel as information hecomes
arailable.
Adaptive control techniques attenupt to cope with uncertainty about the process being controlled by automating certain aspects of controller design. The basic idea is quite simple. The clesigner generally has some sort of model of the process or plant that he is trying to build a controller for. This model. while it is known to provide only a rongh idea of the hehavior of the plant. is sulficient to determine the form of the baxic controller (te.g. a parameterized PID controller). The designer lien builis a prograin that refines the basic controller as it observes this controller attempting to control the plant. In the case of a PID cuntroller. refinement consists of adjusting the control coefficients. Adaptive control is one approach to niaking controllers more responsive to a complex and often unpredictable environnent. Adaptive control also provides a means for coping with complexity in the design process by allowing a control system to monitor its own behavior and adjust accordingly. ('hapter 9 deals with some aspects of aclaptive control in the coutext of a discussion of learning techniques. Now we turu our attention lo soine more practical issues in building cuntrol systems.

Control systems are complex devices that involve the interaction of mechanical and compulatioual processes. In considering the computational aspects of control, it is important to keep in mind that someone has to write the programs or design the circuits that perform the necessary computations. For problens like controlling a power plant or an automated assembly line. these programs and circuits can become quile complex. Despite our best elforts. large programs develop organically as a proceso only partly under the control of any one individual. Continual redeaign is impractical. and sooner or later the deaigner has to commit to a apecific implementation of a module, interface, or subroutine. Once in a while, a deaigner has the luxury of rewriting an interface. oplinuizing an algorithm, or cousolidating several functious in a single module, but oflen enough the or she has to make do with whatever is available. Il would be convenient if contrul knowledge could be eacapoulated in small general-purpose functional units that could be applind in a wide variety of circumstances. This has long been a dream of researcters in artificial intelligeuce. and. in the following, we consider some poacible approsches to realizing that dream. Two critical issues that have to be addressed in the context of coutrulling processes are:

1. Cin general-pnrpose control knowledge he used to snpport real-time control of interesting procesnes? 
2. (all disparate behaviors be made 10 cooperate so as to achieve coordinated behavior across a range of situations?

In attempting to address these issues. we consider a class of programning techniques called ractive system.s that were specifically designed to address shortcomings in classical approaches to planning relying primarily on offline computation and perfect information. Reactive systeins are meant to be responsive to the processes heing cont rolled. They tend not to employ any complicaterl predictive merhanisms in order to avoid the compntational orerhead generally associated with such mechanisns. A reactive system has to he prepared to respond quickly to changes perceived in the controlled process. If the system is engaged in a complex and time-consnming computation. it will likely miss opportunities to generate appropriate responsen. In the applications for which reartive sustents are best suited. it shonld he possible to arhieve the dexired helavior using simple modeis that can he quickly computed.

Mnch of the work on reartive systems done in artificial intelligence has been concerned with building systems that are capable of representing and nanipulating precompiled procedural knowledge about how to control things. Different behaviors can be separately realized in terms of distinct procedures each naaking nse of the available sensors and effectors as needed. The differences between such systems usually revolve aronnd the complexity of the primitive operations allowed hy a given procedure and the means whereby procedures are selected. coordinaled, and allowed to communicate with one another. In the following, we ronsider two approaches to building reactive systems. For the most part. the two approarhes look like programming languages. and our analysis concerns what features of the different languages make them more or less suitable for writing and thinking about control systems.

Every programming langmage is designed to support a particular level of abatraction. High-level languages can introduce barriers to abstraction by forcing the programmer to aulopt a particular way of thinking. For instance, a language that provides only sequential control constructs can make it diffienlt to deal with parallel or axyuchronous processes. Low-level languagen can also introdnce barriers to abstraction simply by failing to provide the progranmer with adequate means to deal with the complexity of programlming large systems. Of course. One can simulate any computational process given any Turing-equivalent machiue/language coubination. In looking at approsches desigued to facilitate controlling processes. we should be alert to 
notice features that allow us in naturally map our understanding of con col problems onto computational processes.

Almost every programning language provides support for procedures of one sort or another. Procedures encapsulate procedural knowitedge: how to go about achieving certain tasks. In speaking about the control of processes. procedures are usually associated with specific behaviors. The first approach to implementing reactive systens that we look at is called a procedural reasoning aystem [13]. A procedural reasoning system consists of a set of procedures and a schrdulcr for selecting what procedures to run and when. Each procedure has associated with it a specific task-achieving behavior that it inplements, and an invocation condition or goal specifying what the procedure is meant to arhieve.

Procedures are represented as labeled tmnsition gmphe. A laheled transition graph is a directed graph whose arcs are labeled with statements in some logic or programming language. In the following. we use Prolog statements to label arcs. The statements are exanined by the scheduler to determine transitious froun one node in the graph to some adjacent node in the graph. Each node in a labeled transition graph has one or more arcs leading out of it. Some statements correspond to predicates or queries and others have an imperative content. The statements labeling arcs are generally seen as giving rise to the goals of the system.

The scheduler is charged with keeping trark of what goals the system has and invoking whatever procedures are appropriate to achieving those goals. At any given moment, the scheduler has some number of active procedures that it is employing to pursue its present goals. For each of those procedures, the scheduler maintains a pointer to some node in the associsted labeled transition graph. The scheduler chooses a particular procedure to work on and attempts to transit to a new node by exaniuning the statements on the arcs leading out of the node currently associaled with the chosen procedure. An example should help clarify.

Figure 4.16 shows a labeled transition graph implementing the discrete proportional controller discussed earlier. The procedure shown also implements an overfiow test to issue an alarm if the fluid runs over the top of the tank. Statements labeling ares such as lluidhoight (Tank, Hoight). and $V$ is $R$ (Targot - Hoight) correspond to queries: "what is the current height of the fluid in the tank?" and "what voltage is $X$ times the difference between the current height and reference value?" Statements such as ant servo_rol tage (Tank, $V$ ) and sot al arn (Tank, 1) correnpond to imperatives to adjust parameters used by the procedures associated with the 

servo attached to the input valse and the alarm device.

Doll queries and inperatives. can be seen as giving rise $t 0$ additional goals. For some of these goals. the scheduler invokes additional procedures. l'or other goals. special-purpose systems may kick in to try to satisfy the goal. For a given goal there may be many different procedures running. I procedure can be revoked if its associated goal becomes satisfied or if some competing goal becomes satisfied. Most labeled transition graphs have terminal nodes indicating exil conditions for the associated procedure. The scheduler is responsible for starting new procedures and terminating old ones. Procedures communicate with one another by posting goals to a global database in a manner similar to that used in blackboard systems $\{15]$. A possible scheduling algorithw for a procedural reasouing system is described as follows. The scheduler maintains two queues ACTIVE and PENDING to keep track of procedures that are in various stages of processing.

1. Choose a procedure $p$ from ACTIVE.

2. Post goals corresponding to each statement labeling an arc emanating from the current node of the procedure $p$.

3. Move $p$ from aCtive to PENDING.

4. Add to ACTIVE each procedure whose invocation condition matches a goal posted in Step 2.

5. For each procedure $q$ in PENUING such that any of the posted goals corresponding to the statements labeling arcs emanating from the current node of $q$ are satiafied:

(a) Choose one satisfied goal $g$.

(b) Retract the other posted goals and remove any associated procedures from ACTIVE and PENDING.

(c) Set the current node of $q$ to be the node terminating the arc labeled with the statement corresponding to $\mathrm{g}$.

(d) Remove $q$ from PEN DING.

(e) If the cntrent node of $q$ is not a terminal node. move $q$ to Active.

6. Gio to Step 1. 
It is important to note that the schedule: never waits aromin to compute anything: the scheduler simply posts new goals. invokes procedures where required. and notires when postcd goals are satisfed. Suppose that the procedure shown !n Figure 4.16 is the only active procedure and its current node is N2. The scheduler posts the goal fluid_hoight (Tank, Height) with Tant bound and Height unbound. and the procedure is moved to the list of pending procedures. The substrstem responsible for monitoring the level of fluid in the tank notices the posted goal. reads the sensor for fluid level. and narks the goal Iluidheight (Tank, Høight) as satisfied with Heighe bound to xhatever the sensor read. The next time the scheduler looks at the pending procedures it notices the satisfied goal. updates the procedure's current node to N3. and places the procedure hack on the list of active procedures.

The procedural reasoning system supports subroutine calls in that a transition in one procedure may require involing a second procedure. Several procedures can run in parallel and communicate asyachronously by posting goals to the global database. As an example of how two procedures might work together in parallel. we consider a type of feedforward control that can be implemented easily in a pr.sedural reasoning system.

The reference or target value specified in a control problem can be thought of as a command for the controller to achieve a particular condition (e.g.. a fluid level of the specified height). In many problems. the reference changes-sometimes continuously-over an interval. The controller has to track these changes so as to minimize errors. If the reference changes can be predicted or are simply provided in advance. the controller can take advantage of this to help eliminate certain errors by nsing feedforward control. For example. if the controller for a robot arm knows the exact trajectory it is to nove the end effertor along, it can often precompnte a sequence of control actions. and then execute an error-free path without any feedback control whatsoever. In most cases. however, feetforward and feedback are used in conjunction, with feedforward taking advantage of know'n changee in the target value, and feedbark compensating for the inevitable errors that nopmp in dealing with real-world processes.

In the cave of our tank-filling process. a feedforward controller could be added to the feedback controller of Figure 4.14. The feedforward controller anticipates the next reference value and mediates the output of the feedhack controller if a change is detected. This sort of controller is referred to as a command feedforward controller and its block diagram is shown in Figure $4.1 \bar{T}$.

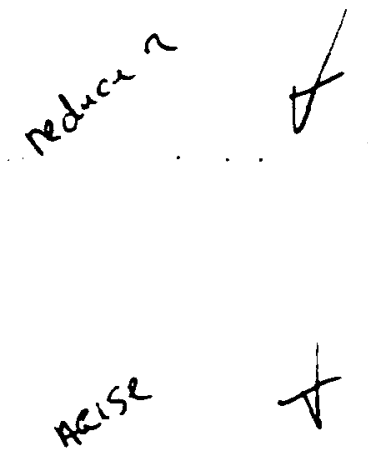




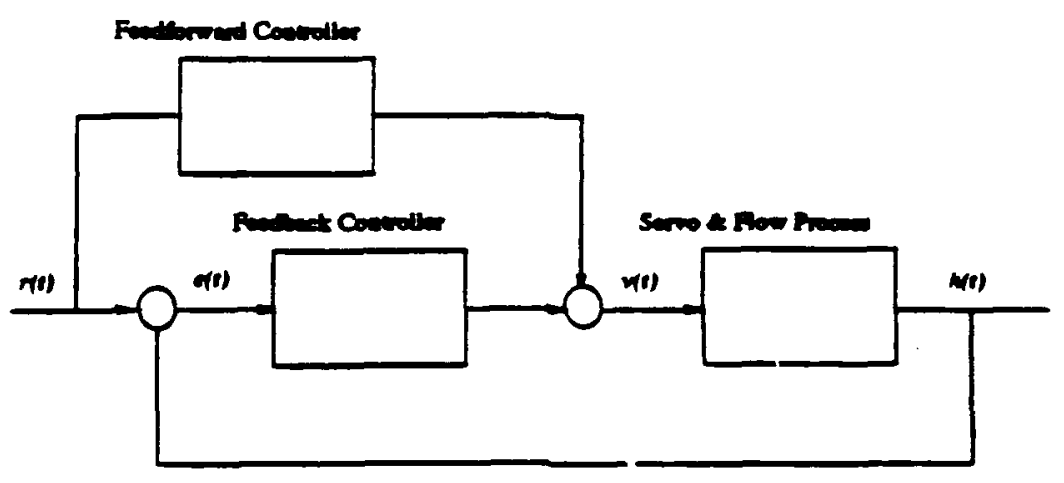

Figure 4.17: Block diagram for a coutroller with command feedforward

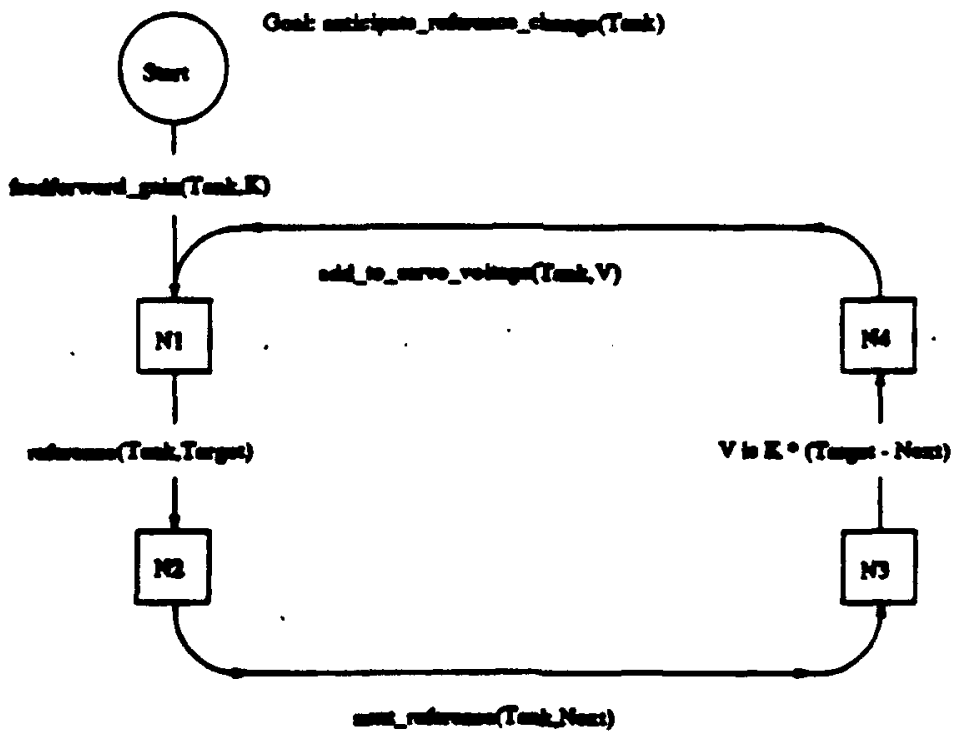

Figure 4.18: Labeled transition graph for a command feedforward controller 

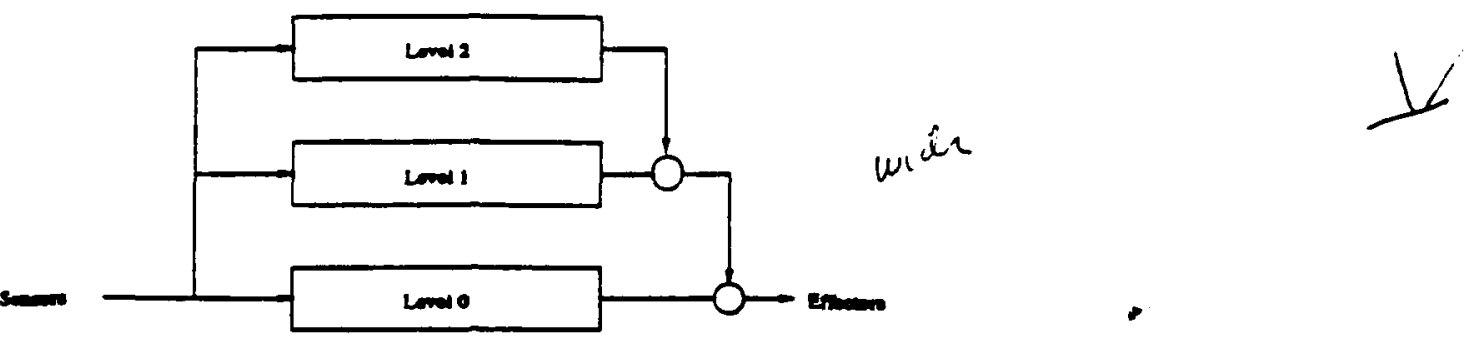

Figure 4.19: A hierarchical control systen

To inplement command feedforward control in a procedural reasoning svstem. we define a uew procedure to monitor changes in the reference value. This procedure specifies a value proportional to the change in reference to be added to that specified by the leedback controller. The labeled transition graph for the command fedforward procedure is shown in Figure 4.18. The two procedures shown in Figure 4.16 and Figure 4.18 run at the same 'ine. The servo process operates on a voltage which is the sum of that specified by each of the two procedures. This control scheme works particularly well for tracking a continuously changing reference: for instance. if you wanted the level in the tank to decrease to 0 at a fixed rate.

In describing the coinmand feedforward control system above. we started with an existing feedback control system and then added a feedforward controller without changing the basic architecture of the feedback control system. Afierarchical control systemsgeneralizet on this basic idea. A Lierarchical coutrul system is coustructed of several lavers so that each laver serves as a coutroller for the layer immediately below and is controlled bv the laver immediately above. There are different types of hierarchical control systems. They differ in how the various lavers are controlled by and impose control on the lavers imnediately above and below. As our second approach to building reactive systems. we consider a hierarchical control system in which one layer is allowed to impose control on a lower layer by modifying control aigals used for communicating between componeuts of the lower layer [1].

Figure 4.19 depicts the general forin of the sort of hierarchical coutrol system we are considering. Each level is composed of a set of conponents each of which is responsible for a simple prinutive behavior. The conponeuts conununicate with one another by passing signals. For the most part. the signals consist of bit or byte streams. The components can be implemented 


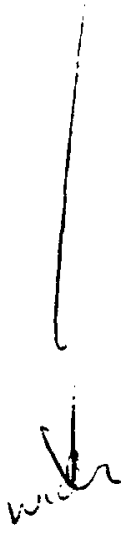

$\forall$

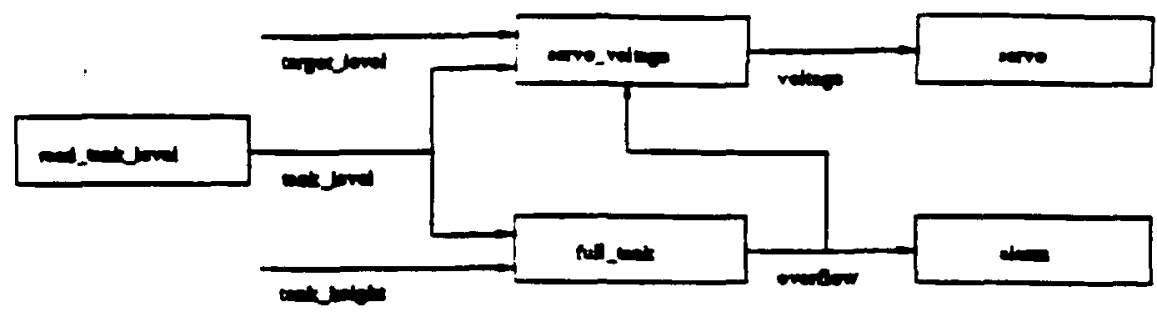

Figure 4.20: A single-level control system

any way that you want. but it is a good discipline to think of them as very simple computing devices. For instance, the components might be inplemented as regular finite state machines augmented with a small amo.!nr. of local state. a combinatorial circuit. and a local clock. The combinatoria' circuit and local state are used to keep track of signals originating from other components. The clock is used to provide simple timing capabilities. There is no global state and the different components communicate asynchronously by writing values into the local memory of other components.

Figure 4.20 shows a single-level control system for thaintaining the fluid level in a holding tank. The component labeled roadtank_level continuously samples the sensor indicating the level of fluid in the holding tank and outputs the value read on the wire labeled tank_lerel which subsequently appears in registers in the components labeled sorvo_roltage and full_tant. The sorvo_roltage component implements the same procedure as the labeled transition graph of Figure 4.16. The full_tank component detects when the level in the tank is equal to the height of the tank and pasces this information on to the the serro_roltage component and to the alare component which is responsible for sounding an alarm.

To illustrate how one level in a hierarchical control system might influence a lower level in the saune system, we consider a second form of feedforward control referred to as disturbnnce feedfortund control. A disturbance is a proceses that affects the controlled process but is not taken into account by the controlled process model. In the fluid-level process we have been consindhs we might model a process restricting the flow through the pipe leading ont of the tank shown in Figure 4.12 as a disturbance. Suppose that the output pipe is being used to fill containers that are moved into position under the pipe using a conveyor system. When a containet is filled, the flow through the output pipe is temporarily restricted so that a new container can be positioned under the pipe. Figure 4.21 shows how a simple propor- 

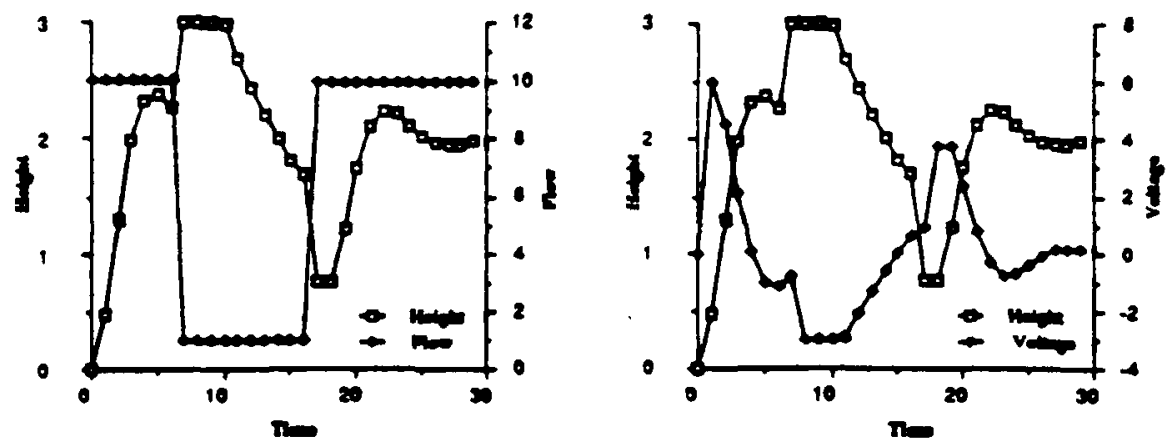

Figure 4.21: Overflow due in a disturbance restricting ontflow

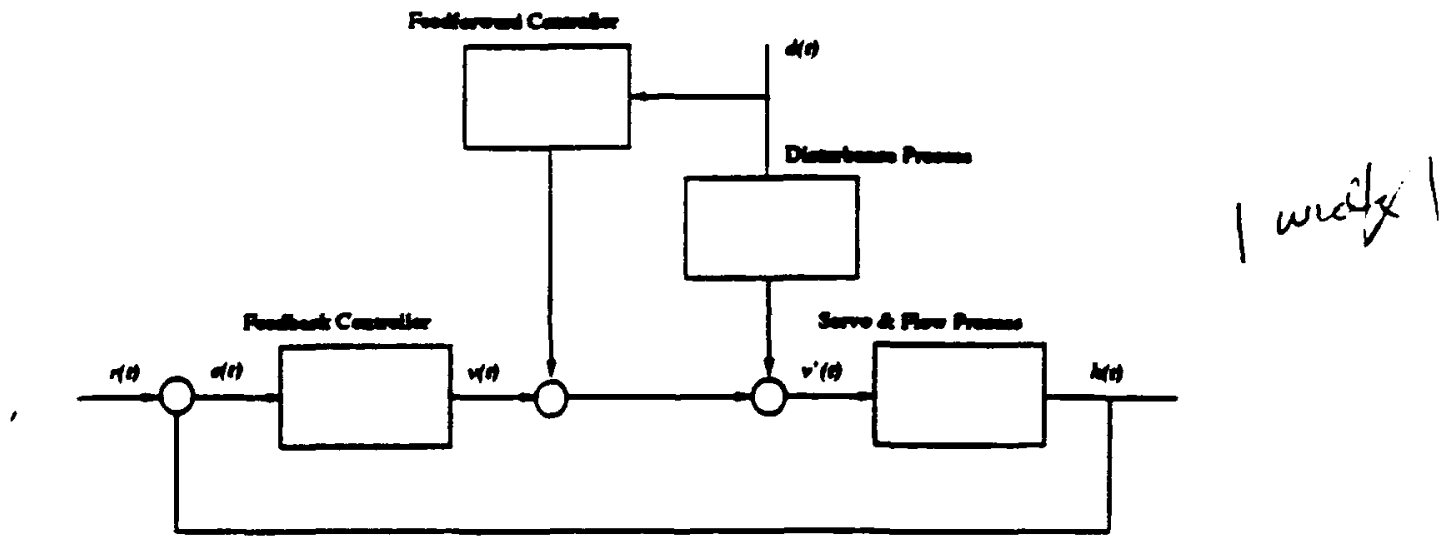

Figure 4.22: Block diagram for a controller with disturbance feedforward

tioual coutroller reacts to a brief restriction in the output flow: the reduced flow effectively reduces the gain of the proportional controller and fluid spills over the top of the tank before the coulroller caul react and appropriately compensate.

Let us suppose that it is possible to anticipate a restriction in the out put flow as would be the case for the coutainer-filling example described above. Figure 4.22 shows a bluck diagrau for a disturbance feedforward coutroller for the fuid-level problem. We assume that it is possible to sense restrictions in the output flow and use this information to increase the voltage fed to the servo motor thereby temporarily increasing the gain of the feedback coutroller.

Given the single-level proportional controller shown in Figure 4.20 . we 


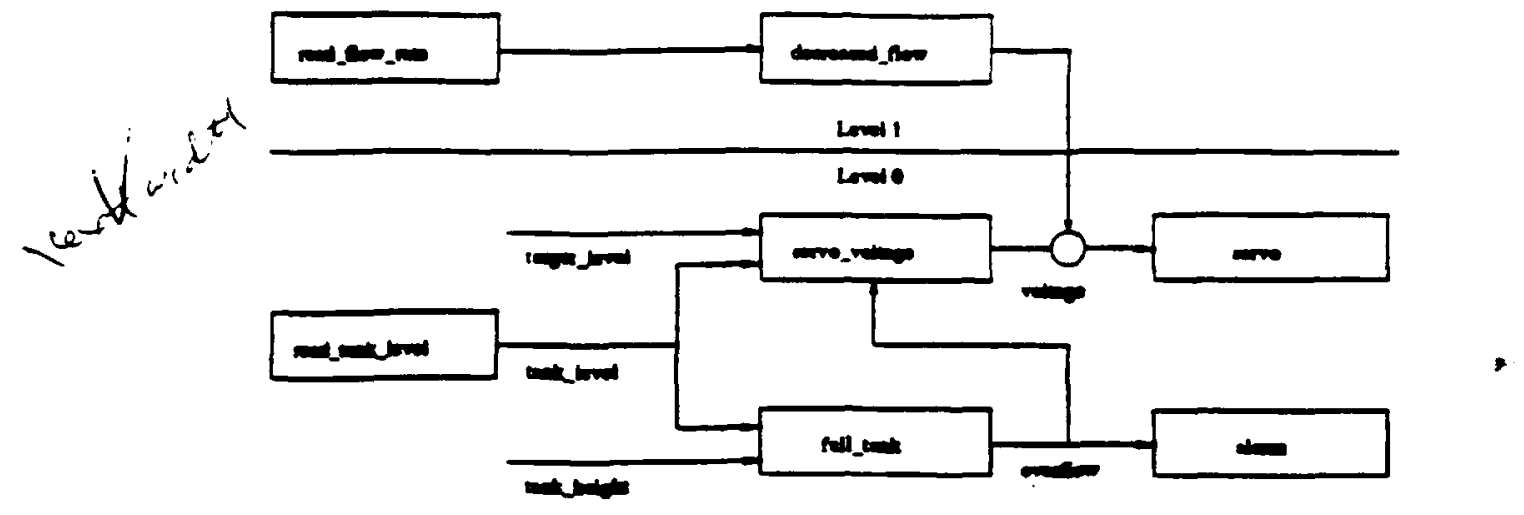

Figvre 4.23: A two-level system with disturbance feedforward control
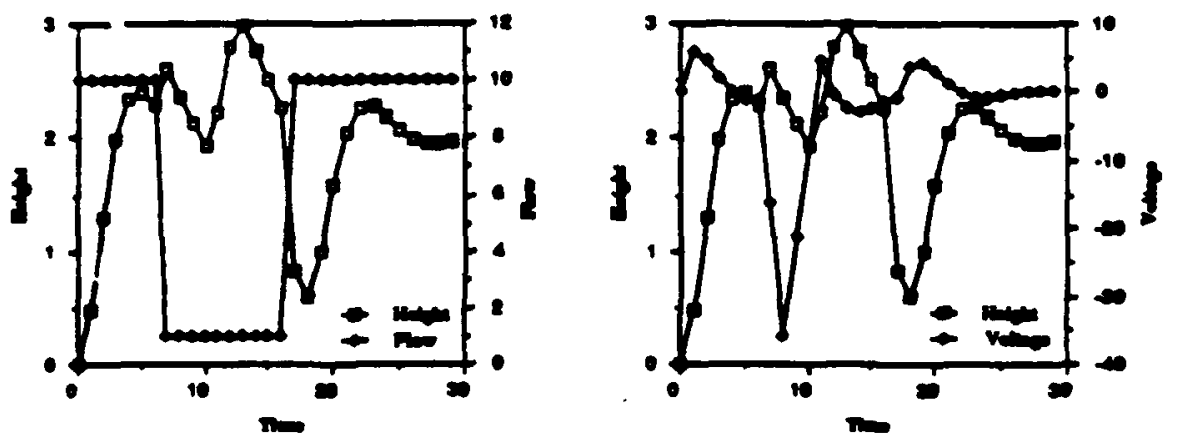

Figure 4.24: Disturbance feedforward controller preventing overflow

can add a second coutrol level in order to reduce or eliminate the amount of spillage resulting from momentary reatrictious. The resulting two-level system is shown in Figure 4.23.

The perfornance of the two-level system is somewhat less than optinal: as indicated in Figure 4.24. the two-level system does avoid spilling any fluid. but the fluid height is somewhat erratic around the time of the restriction. We unight be able to further tune the feedforward compouent to eliminate or 4 reduce this erratic behavior. However, it is often the case that, in buildiug $\{$ on top of an existing coutrol system, we simply have to accept the limitatious of what we started out with, or do it over. The hierarchical system described above makes it rather easy to build on an existing coutrol system. Given the discipline described earlier for building modular stand-alone computatioual components. adding new functionality or enhancing old often cousists of 
cimply adding some new components and wiring them together with the old ones. To the extent that this call be realized in practice. it makes building and experimenting with control systems remarkably easy.

The procedural reasoning system and the hierarchical control system described above are sinilar in many resperts. Both support multiple processes runniug in parallel. Both support procedural abstractiou aud asynchronous control. There are some differences. however. The procedural reasoning system encourages the explicit representation of intentions. behaviors. and goals. The hierarchical control system encourages one to think in terms of evolving control systems and distributed computation. We say "encourage" as both systems are no more thau general-purpose programming languages. ITnless you specify a compiler aud a target machine. the two systems are essentially equivaleut.

There are other approaches to building reactive systems some of which will be discussed in subsequent chapters. In some cases. the reactive system looks more like the sort of planning systenns that we will investigate in Chapter 5 in that it manipulates a representation of its pending tasks imposing ordering constraints and dealing witl certain classes of interactions between 1.asks [11]. In others cases, the system is realized as a boolean circuit [\$. 26] or as a network of processes that communicate using a specjalized message passing protocol [23]. The process of compiling reactive systems from a behavioral specifications is of particular interest. and we will return to this issue in C'hapter 5.

$$
8^{9} \text { io? }
$$

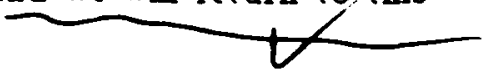

\subsection{Navigation and Control}

Traditionally, the problem of navigation. involving spatial and geometrical modeling, and the problen of control. involving kinematics and dynamical modeliug have beell considered separately. The former is believed to be in the reaim of planning; the latter in the realm of control. In the first problen. we are given a geonetrical model describing a robot, the objects surrounding it. thet current relative positions and orientatious. and some goal state deacribing a final position of the robot. and we are asked to generate a trajectory or path through the associatcd space of possible configurations of the robot and the surrounding objects. In the second problem. we are given a dynamical model of the robot. and asked to geverate a feedback control law that issues torques to manipulator joints and drive wheels in order to track a supplied reference trajectory. In this section. we consider a unified 
approach that addresses both of these problems.

To represent the state of the rubol with respect to its environnent. We introduce the idea of configuntion simice laken from Mechanics and adapted fur use in robotics [22]. Fullowing Latombe [20], we represent the robot. $A$. and the objects-we will referichem as obstacles-in its environment. $\mathcal{B}_{1}, \mathcal{B}_{2}, \ldots B_{m}$, as closed subsets of the urork space. $\mathcal{W}=\mathbf{R}^{n}$, where $n=2$ or 3. Both the robot and the obstacles in the workspace are assuned to be rigid. Let $\mathcal{F}_{A}$ and $\mathcal{F}_{W}$ be Cartesian frames of reference embedded in $\mathcal{A}$ and $W$ respectively. $\mathcal{F}_{A}$ is a moving frame while $\mathcal{F}_{W}$ is fixed.

A configuration. $q$. of au object is a specification of the position and orientation of $\mathcal{F}_{\mathcal{A}}$ with respect to $\mathcal{F}_{W}$. The configuration space. $\mathcal{C}$. is the set of all configurations of $\mathcal{A}$. We employ the Euclidean metric and the following distance function to induce a topology ou $C$. The distance between two coufigurations. $q, \varphi^{\prime} \in C^{\prime}$. is defiucd as

$$
\text { distanco }\left(q \cdot q^{\prime}\right)=\max _{a \in \mathcal{A}}\left\|a(q)-a\left(q^{\prime}\right)\right\| \text {. }
$$

where $\left\|x-x^{\prime}\right\|$ denotes the Euclidean distance between auy two points, $x . x^{\prime} \in \mathbf{R}^{n}$, and $a(q)$ is the point in $\mathcal{W}$ occupied by $a \in \mathcal{A}$ when $\mathcal{A}$ is in configuration $q$. We define the free space, $c_{\text {free, }}$ to be

$$
C_{\text {free }}=\left\{q \mid q \in \mathcal{C} \wedge \mathcal{A}(q) \cap\left(\bigcup_{i=1}^{m} B_{i}\right)=0\right\} \text {. }
$$

where $\mathcal{A}(q)$ is that subset of $\mathcal{W}$ occupied by $\mathcal{A}$ in configuration $q$. A free path (or just a path) of $\mathcal{A}$ (rom some initial configuration. 4 , to the goul configuration, $q^{*}$, is a coutinuous map

$$
\pi:[0,1] \rightarrow \mathcal{C}_{\text {freses }}
$$

subject to the constraints that $\pi(0)=q$ and $\pi(1)=q^{*}$.

The literature is fnll of approarhes to solving the problem of finding obstacifye paths in configuration space. In the following, we consider the artificial potential field approach first introduced to the robotics community. by $\mathbf{K}$ atib [1i] which unifies navigation (or path planning) and control. Onr treatment here borrows the notatiou of Latombe [20], as well as some of the insights of Koditschek [19] on the connections between planning and control. To simplify the subsequent discussion. we assume that the robot is a point object and the workspace is $\mathbf{R}^{\mathbf{2}}$. In this case. it is meaningless to talk abont 
the rubot s orientation. and. hence. the configuration space is identical to the work space.

Ile wish to design an artificial potential find so that the robot will he attracted torard the goal configuration in $C^{\prime}$ and repulsed by obstacles. This field of forces is modeled as a function. $F$. defined by

$$
F(q)=-\Gamma(i) \text {, }
$$

where $I^{T}: C_{\text {free }}-\mathbf{R}$ is a differentiable potential function. and the gradient. $\Gamma$. is defued in the case of $C^{*}=R^{2}$ as

$$
\Gamma t i=\left[\begin{array}{l}
\partial \tau / \partial x \\
\partial U / \partial y
\end{array}\right] .
$$

We represent the potential function as a sum of attractive and repulsive romponent polential functions:

$$
l^{\prime}(q)=l_{\text {aut }}(q)+l_{\text {rep }}(q) .
$$

Generally. the attractive force is represented either as a conic potential well using the Euclidean distance. as in

$$
U_{\text {aet }}(q)=\xi\left\|q-q^{-}\right\|
$$

where $\xi$ is a positive scaling factor, or as a parabolic potential well using the Euclidean distance squared. as in

$$
U_{a u c}(q)=\frac{1}{2} \xi\left\|q-q^{*}\right\|^{2},
$$

where the constant $1 / 2$ is just to make $\Gamma$ come out a little neater. In the former case. we have

$$
\nabla l_{\text {set }}(\varphi)=\xi \frac{\left(q-q^{*}\right)}{\left\|q-q^{*}\right\|}
$$

and in the latter

$$
\Gamma^{i} \text { att }(q)=\xi\left(q-q^{*}\right) .
$$

There are advantages and disadvantages to both approaches to representing the attractive potential. In some cases. it is useful to define a hribrid potential using a parabolic potential within some fixed radius of the goal (facilitating gradient descent search in the proximity of the goal) and a conic 
potential outside that radius (keeping the potential value smaller at points far from the goal) [20].

We decompose the repulsive component of the potential function into $\mathrm{m}$. additive components. one for each obstacle. In desiguing a repulsive field for a particular obstacle. We waut to make it impossible for the robot to come in contact with the surface of the obstacle while allowing movement to proceed uninpeded when the robot is sufficiently distant from the obstacle. For a convex object, $\mathcal{B}_{i}$, the following polential function performs well

$$
U_{B_{1}}(q)=\left\{\begin{array}{cl}
\frac{1}{2} \eta\left(\frac{1}{\rho_{1}(\eta)}-\frac{1}{6}\right)^{2} & \text { if } \rho_{i}(q) \leq \zeta \\
0 & \text { if } \rho_{i}(q)>\zeta
\end{array} .\right.
$$

where $\zeta$ is a positive scalar called the distance of influence. and $\mu$, is defined as

$$
\rho_{i}(q)=\operatorname{nuin}_{\eta^{\prime} \in B_{1}}\left\|q-q^{\prime}\right\|,
$$

where we do not bother to distingmish between the configuration space and the work space, since in the rases considered here they are the same.

The gradient of $U_{B_{i}}$ is defined by

$$
\nabla U_{B_{i}}(q)=\left\{\begin{array}{cc}
\eta\left(\frac{1}{\rho_{1}(q)}-\frac{1}{\zeta}\right) \frac{1}{\rho_{i}^{2}(q)} \nabla \rho_{i}(q) & \text { if } \rho_{i}(q) \leq \zeta \\
0 & \text { if } \rho_{i}(q)>\zeta
\end{array},\right.
$$

Where $\Gamma \rho_{i}(q)$ is defined as follows. Let $q_{c}$ be the unique configuration in $B_{i}$ such that $\left\|q-q_{c}\right\|=\rho_{i}(q)$. $\nabla \rho_{i}(q)$ is the unit vector pointing away from $B_{i}$ in the direction determined by the line passing through $q$ and $q_{c}$.

We combine the repulaive fields for the set of obotacles, $\left\{B_{1}, B_{2}, \ldots B_{m}\right\}$, by taking a simple sum.

$$
U_{r e p}(q)=\sum_{i=1}^{m} r_{B_{i}}(q) .
$$

The grodient of the sum is simply the sum of the gradients.

$$
\nabla U_{\text {rep }}=-\sum_{i=1}^{m} \nabla U_{Q_{1}}(q) \text {. }
$$

Combining the attractive and repulsive force fields, we have

$$
F(q)=\Gamma l_{\text {eut }}+\Gamma l_{\text {rep }}
$$




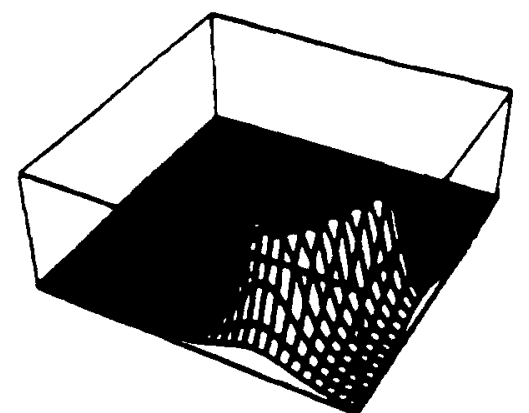

iii.

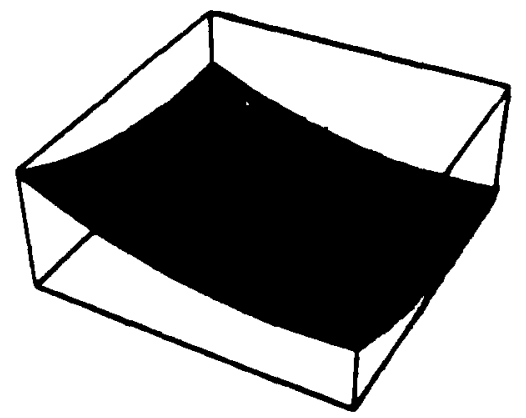

ii.

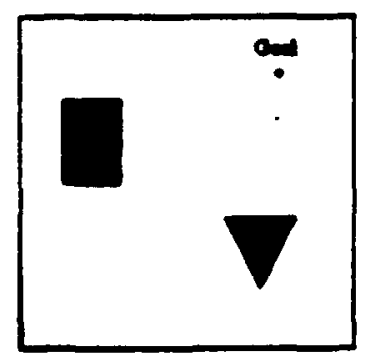

i.

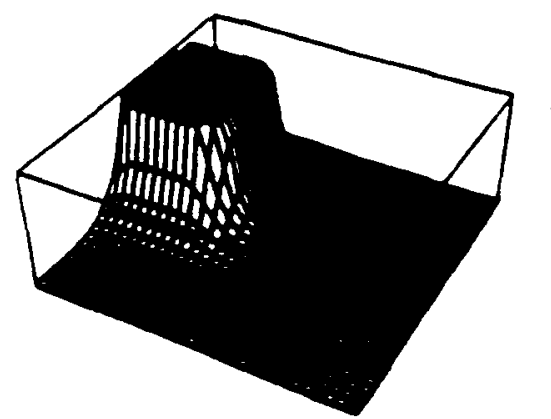

iv.

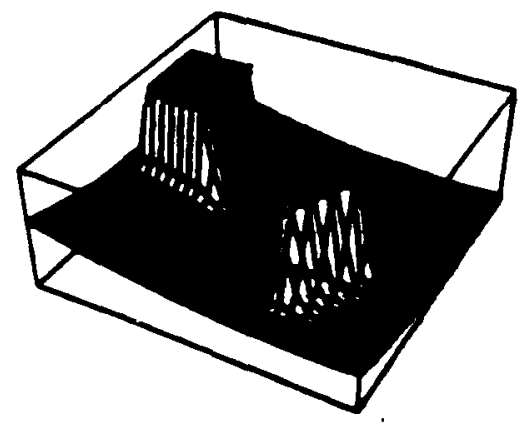

v.

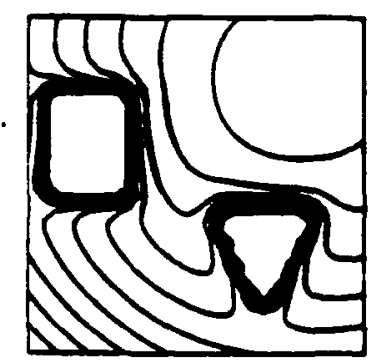

vi.

Figure 4.25: A 2.D configuration spare (i) containing two obatacles. The attractive potential field (ii) along with the repulsive potential fields (iii) and (iv) for each of the two obstacles, the sum (v) of the attractive aud repalaive potential fields, and a 2-D plot (vi) showing several equipotential colltours. 
Fig" se 4.25 sliows a 2 -D configuration space. the resulting potential fields. and several equipotential contours indicating that the potential field lras a single mininum. The attractive potential is modeled as a parabolic potential well.

The potential field approach was originally conceived of as a method fur real-line ubstacle avoidance. The basic idea was to regard the robot in coufiguration space as a particle moving under the influeuce of the field. $F=-\Gamma U$. The acceleration is determiued by $F(q)$ for every $q \in \mathcal{C}$. Given the dyuauics of $\mathcal{A}$ and assuming perfect seusing and motors that deliver exact and unlimited torque. we can compute the torques that should be issued to each of the actuators so that the robot behaves exactly as the varticle metaphor predicts.

Consider a very simple robot with a single degree of freedom (€.g., a p.ismatic (sliding) joint). We assume that its position (configuration). \& $\in$ $\boldsymbol{c}=\mathbf{R}$. and velocity. $\dot{q}$. can be ineasured precisely by a perfect sensor and controlled by a servo that delivers exact and unlimited force, $r$. We model the dynamical system using Newton's second law of motion.

$$
\boldsymbol{M} \ddot{q}=\mathcal{F},
$$

where $M$ is the mass of the robot. The object is to move the robot from its present configuration to some fiul configuration $q^{\circ}$.

In the potential field approach described above. we address the geometrical side of the problem in terms of optimizing a cost function disguised as a potential function. This approach is quite similar to the dynamic programning example that we investigated in Section 4.5. The cost function that we are trying to minimize in this case is just the attractive potential function introduced earlier

$$
\varphi=\frac{1}{2} K_{p}\left\|q-q^{*}\right\|^{2} .
$$

where $K_{p}$ is any ponitive scalar. To simplify the prenent discussion. we ignore the problem of avoiding obstacles. From this equation. e obtain

$$
\dot{q}=-\nabla \varphi=-\Gamma_{p}\left(q-q^{*}\right),
$$

and note that, since in this case $q^{*}$ is the only minimum of $\varphi$. this linear differential equation generates a solution to the geometric problem of finding a path from any initial starting configuration to $q^{*}$. Now we set out to derive a control law that will serve to track the path (or reference trajectory) so defined. 
Having interpreted $;$ in terms of potential energy: we define the kinetic sllerg.,$k$. as

$$
i=\frac{1}{2} \cdot M i^{2} \text {. }
$$

and obtain the total energy. $\lambda$. as the difference of the kinetic and potential energies

$$
\lambda=\kappa-\hat{r} \text {. }
$$

A dynamical inodel can be obtained using the Lagrangian formulation of Newton's equations defined by.

$$
\frac{d}{d t}\left(\frac{\partial \lambda}{\partial \dot{q}}\right)-\frac{\partial \lambda}{\partial q}=F_{e r t \cdot}
$$

where $\mathcal{F}_{\text {ext }}$ represents all of the external (non-conservative) forces acting on the robot. The resulting Newtonian law of motion is

$$
M \ddot{q}-\operatorname{Sp}\left(q-q^{*}\right)=F_{\text {ere }} .
$$

Let us assume that $\mathcal{F}_{\text {ext }}$ represents a dissipative force (we can add this if necessary) proportional to the relocity.

$$
F_{\text {ext }}=-\boldsymbol{K}_{D} \dot{q}
$$

where $I_{D}$ is a positive scalar. The resulting system is asymptotically stable, and converges to the goal $q^{*}$ from all initial configuratious $q \in \mathcal{C}$.

Finally, we have

$$
: 1 \dot{\ddot{q}}+K_{D \dot{q}}-\mathscr{K}_{P}\left(q-q^{\bullet}\right)=0
$$

Returning to our original dynamical model

$$
M \ddot{q}=F,
$$

we can obtain the following cont rol law

$$
F=-\Pi_{D} \dot{q}+K_{p} p\left(\varphi-\varphi^{*}\right),
$$

an instance of proportional derivative feedback control. The proportional component captures the essence of a simple one-dimensional planning sistem that deterunines an appropriate reference trajectory in configuration space. The derivative component enables the controller to respond appropriatcly 
to the behavior of the two-dimensional (one spatial and one temporal diueusiont physical system.

lihatib's motivation for emploving artificial potential fields was to provide real-time obstacle avoidauce capability for multi-link mauipulators [1i]. In his original formulation. it was assumed that there would exist a higher level of control that would compute a global strategy in terms of internediate goals. The low-level ststem would produce the necessary forces to achieve these goals, accounting for the detailed geometry. kinematics, and dynamics in real time. In the following. We say a bit more about the highlevel problem of computing a global strategy corresponding 10 a path from the curreut configuration to the goal configuration.

The approach to building poteutial fields described earlier has a number of problems: some of which can be easily remedied and others of which are more difficult to overcome. Ile address some of these problems now. beginning with the easiest ones. Working our way up to the more difficult.

The repudsive field for obstacles in the workspace was defined only for convex objects. We can exlend the method to haudle more general objects by decomposing each obstacle into some number of (possibly overlapping) convex objects. assuciating a repulsive polential with each compouent. aud summing the result. There are somte subthes with this approach (see [20]). but this basic method of decompusition works well in practice.

The next problem concerus the assumptions regarding the dimensions of the workspace and the degrees of freedon of the robot. For the idealized point robot operatiug in two dimensions. the two-dimeusional configuration space was equivalent to the Euclidean plane. In general. the number of parameters required to deacribe the coufiguration of the robot will determine the dimension of the configuration space. For a rigid robot operating in three dimensious. it takes six parameters to describe the coufiguration of the robot. For manipulators consisting of rigid links serially connected by singledegree-of-freedom joints (e.g.. revolute (rotating) and prismatic (sliding) joints). the number of parameters required is equal to the number of joints. For exiating mobile robots and manipulators, it is possible to construct the requime configuration spaces and extend the techniques described above to handle the resulting motion planning problems. However. assuming $P \neq$ NP. the complexity of plauning free paths is exponential in the dimension of the configuration space.

In general. computing free paths for multi-link manipulators and mobile robots in cluttered euvironments cau be quite expeusive [2i]. From the perspective of computatioual complexity. this high-level geometric planning 


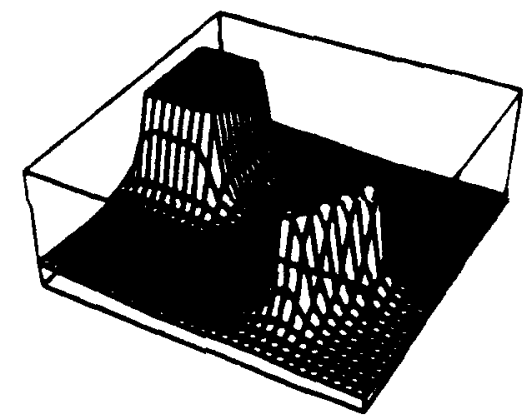

i.

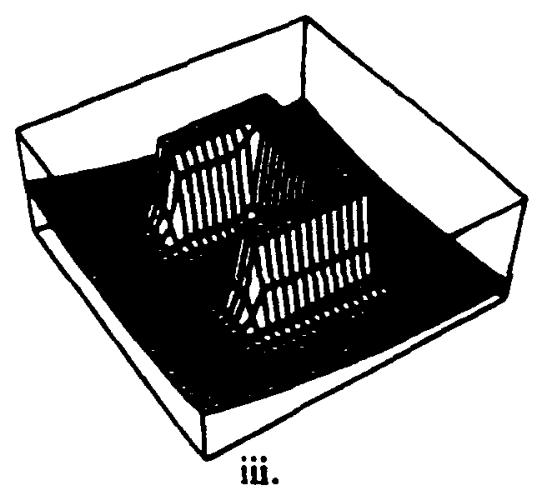

iii.

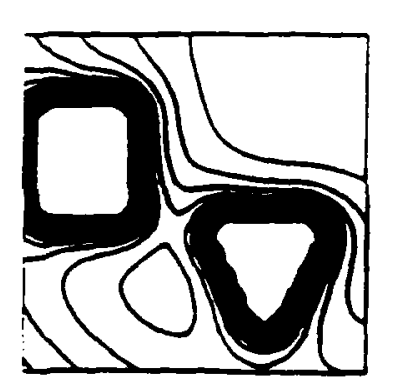

ii.

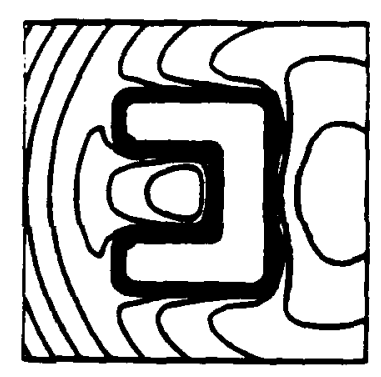

iv.

Figure 4.26: Two potential fieids with multiple extrema: one (i) resuiting from two closely situated convex obatacles. and a second (iii) resulting from a single concave obstacle. A set of corresponding equipotential contours is shown (ii) and (iv) for each of the two potential fields.

problem is typical of the sort of probleus that we will encounter in the next chaples. Solutious to problems iuvolving a significaut number of coustraints (e.g., an caviroument cluttered with obst acles) and many alternative control action (e.g.e robots with several degrees of freedom) tend to be computalioually prohibitive. For real-time applicatious involving such problems. it is generally necesaary to make simplifyiug assumptions therebr. decreasing the complexity of the resulting decision problem while at the same time sacrificiug generality and possibly risking soundness or completeness.

Another problem with the artificial potential function approach outlined 
earlier concerns with the problen c' multiple extrema in potential fields. In general. a potential field for a cluttered work space may include several exirema. Vinder such conditions. using the gradient to guide search may result in paths that terminate at extrena other than the one corresponding to the goal configuration. Concave objects are one potential source of misleading local extrema (see ligure 4.26 .iii). but such extrema can also result in the rase of rlusely situated convex nhst.acles if the distance of influence. $\zeta_{\text {, is }}$ greater than twice the distauce between the obstacles (see Figure 4.26.i).

In order to avoid falling into local minima. it is necessary to employ more sophisticated search methods than simple gradient descent. In the following, we consider one such method for finding collision-free paths in a two-dinensional configuration space. ${ }^{i}$

We begin by tessellating the configuration space to form a grid of equally sized cells. In the case of a point robot on a planar surface. the discretized configuration space. $C_{\mathbf{Z}}$, is a subset of the integer plane. $\mathbf{Z} \times \mathbf{Z}$ :

$$
c_{\mathrm{Z}}=\{(i, j) \mid 0 \leq i, j \leq r\},
$$

where $r$ is a intrger parameter used to hound the size of the configuration space. The potential at the coordinates, $(i, j)$, in the integer plane is $U(i l, j l)$ where $l$ is the length of the side of a cell. We assume that both the initial and the goal configurations are configurations in $\mathrm{C}_{\mathbf{Z}}$, and that, if two configurations are neighbors in $\mathcal{C}_{Z}$ and bolls of them belong to $\mathcal{C}_{\text {frees }}$ then the st.raight line segment connecting them also lies in $C_{\text {free }}$

In the following, $T$ is a tree whose nodes are configurations in $c_{\mathbf{Z}}$. We define a best-first path planning algorithm as follows.

1. Initialize $T$ to be the tree conninting of the single (root) node corresponding to the current configuration.

2. Choose a leas node. $q$. of $T$ with unexplored neighbors in $C_{Z}$ whose potential value is equal to or less than the potential value of all the other leave in $T$ with unexplored neighbors.

3. Add to $T$ as children of $q$ all configurations not already in $T$ whose petcatial value is less than some (large) threshold. (This threshold is ent to avoid paths that get too close to obstacles. Recall that at the surfaces of obstacles the potential is infinite.)

'The method for searching tro-dimensional configuration opace deacribed here can be extended to higher-dimensional conffgration apeces with little modification, bat is only practical for dinimaion $\leq 4$ [30]. 
4. If $q^{*}$ is a leaf node in $T$. then go to Step 6 .

5. If there are no leaf nodes in $T$ with unexplored neighbors. then return failure. clse go to Step 2 .

6. Return the path from the rout of $T$ to $q^{*}$.

The algorithun described above is guaranteerl to find a free path if one exists or report failure otherwise. The algorithm deals with multiple extrema by following a discrete approxination to gradient descent until reaching a local minimum. Once in a local minimum. it proceeds to "fill in" the well of this unininum by exploring the surrounding cells until a saddle point is reached and the local ininimun is avoidecl. By adding simple optinizations to facilitate finding the next node to explore. it is possible to achieve a runuing time of $O\left(m r^{m} \log r\right)$ for a configuration space of dimension $m$. The algorithm works for configuration spaces of arbitracy dimension. but fog dimension much greater thau four the runuing time is prohibitive.

IIt should be noted that the best-first planuing algorithm will find a path if oue exists. but not necessarily the shortest path or the optimal path by auy given usetric. The discretized configuratiou space can be used as part of a dynamic programming approach to linding optimal paths. Indeed, using a dynamic programming approach, we can design an algorithm that will coustruct a polential field with a single tuinima at $\varphi^{*}$ in $O\left(\mathrm{mr}^{\mathrm{m}}\right)$. Using this potential field, oue can generate the shortest path from any initial location to $q^{*}$ using a discrete approximation to gradient desceut in time linear in the leugth of the path.

Koditschek [18] provides a method of generating potential functions which he calls novigation functions that have a single global minimum. The advantage is that sinple local methods (e.g., gradient descent) suffice for uavigation and control. However. as with other approaches to motion planuing, the cost of generaling navigation functions can be quite high in the case of cluttered enviromments and robols with many degrees of treedom.

This section was meant as a bridge between the ceutral issues of this chaptes and thowe of the next. In this chapter, we considered basic properties of dynamical systems such as coutrollability, observability, and stability that are critical in the design of control systems. We investigated the fundanental idea of leedback control and considered the use of performance measures in optimal coutrol. Finally. in this section. we considered the idea of providing higher-level directiou for control in the context of navigation problens. In particular. we considered methods for eucoding navigation tasks in terms 
of potential functions that provide a convenjent hasis for the control of manipulators and mobile robots. The next chapter considers the issues involved in encorling ligh-level tasks in much more detail. Like the problems involved in motion plauning. the problems we the lookimg at in the next chapter are computationally complex.

\subsection{Further Reading}

The literature on control systems theory and practice is vast. In the following, we point out sone books and articles that have been particularly useful in understanding the basic control issues and their attendant mathenatical formulations. For a good overview of classical and inodern approaches to control. the introductory lext by Dorf [10] is excellent. Most control texts assume a relatively high level of nathematical sophistication. In particular. some familiarity with linear sistems analysis is generally assumed. The text by ('hen [9] provides a good introduction to linear systems theory. Ciopal's book [14] on the control of linear uultivariable systems is an excellent introduction to that subject. For nore of an eugineering perspective on control. the interested reader is advised to consult Bollinger [5] or Borrie [6].

The survey article by Ramadge and Wonhan [25] provides a good introduction to work in the area of discrete events systems. Optimal control texts generally rely on a good background in the differential and integral calculus. and, in particular, the calculus of variations [12]. Athans and Falb [2] provide an introduction to optimal control. There have been many books written on dynanic programming. The original text by Bellman [3] is still generally available and provides a good introduction to the subject with plenty of illustrative exanuples.

For a careful treatment of the configuration space representation and a variety of approaches to finding free paths in configuration space. the rearler is enconraged to read Latombe's hook on robot motion planning [20]. Koditschek [19] provides a technical and historical survey of navigation techniques uning potential functions inclucling a cliscussion of stability issues. For a survey of complexity results pertaining to motion plauning, see Schwartz. Sharis, and llopcroft [28]. 


\section{Bibliography}

(1) Aho, Alfred V.. Hopcroft. John E.. and Ullunau. Jeffrey D.. Data Structures and Algorithms. (Addison-Weslev. Reading, Massachusetts. 1983).

[2] Athans, Michnel and Falh. Peter L.. Optimal Contod: An Intmdurtion to the Theory and Its Applications. (McCiraw-Ilill. New York, 1966).

[3] Bellman. Richard. Dynamic Programming. (Princeton University Press. 195i).

[4] Bellman, Richard, Adaptive Control Processes, (Princeton University Press, Princeton, New Jersev, 1961).

[5] Bollinger, John Ci. and Duffie, Neil A., Computer Control of Machines and Processcs, (Addison-Wesley, Reading, Massachusetts, 1988).

[6] Borrie, John A.. Modern Control Systerns: A Manual of Design Methode. (Prentice-Hall. Englewood Cliffs, New Jersey, 1986).

[i] Brooke, Rodney A.. A Robuat Layered Control System for a Mobile Robot, IEEE Journal of Robolics and Autoniation, 2 (1986) 14-23.

[R] Chapman, David and Agre. Philip F.., Pengi: An Implementation of a Theory of Activity, Proreedings AA/I-87. Sicnttle, Washington. AAAI, $1997,268-272$.

[9] Cren C. T., Introduction to Linear System Theory, (Holt, Rinehart. and Whaton, New lork. 1970).

[10] Dorf. Richard C.. Modern C'onirol Systems. (Addison-Weslev. Reading, Massachusetts, 1989). 
[11] Fir.y. R. James. An Investigation in Reartive Plamning in ('omplex Do-

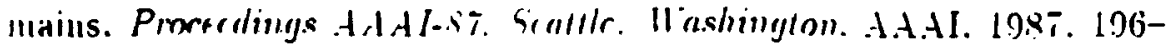
201.

[12] Cielfand. I. M. and Foniu. S. V.. C'alrulus of Iariations. (Prentice-Hall. Euglewood Cliffs. New Jersev. 196;3).

[13] Ceorgeff. Michael P. aud Lansky. Auy L.. Reactive Reasoning and Planuing. Procetdings .1.AI-s7. Scillle. I'ashinglon. AAAI. 198T. 6Ti-68:2.

[14] Ciopal, M.. Ifolem C'ontrol System Theory, (Halsted Press. New York. $1985)$.

[15] Hayes-Roth. Barbara. A Blackboard Architecture for Control. Artificial Intelligence. 26 (1985) 251-.321.

[10] Kalman. R. E.. Falb. P. L.. and Arbib. M. A.. Topics in Mathenuatical System Theory. (McGraw-Hill. New York, 1969).

[1i] Khatib, On.sama, Real-time olsstacle avoidance for manipulators and mobile robuts. International Journal of Robvtics Research. 5 (1986) 90-99.

[1.8] Koditschek. D., Exact Rohot Navigation hy Means of Potential Functions: Some Topological Considerations. IFFF, International Conferrnce on Robotics and Automation. Ralcigh, NC', 1987. 16.

[19] Koditschek. D.. Robot Planning and Coutrol Via Potential Functions. Kihatib. Oussana. C'rajg. John H., and Lozano-Pérez. Tomás. (Eds.). Robotics Review 1. (MIT Press. Cambridge, Massachusetts. 1989). 34936 i.

[20] Latoube. Jean-Claude. Robot Motinn Planning, (Kluwer Academic Publishers. Bostou, Massachusetts, 1990).

[21] Lewia. Frank I.. Optimal Control. (John Wiley and Sons. New York. (8m).

[22] Lozano-Pérez. Touás. Spatial Planniug: $\Lambda$ C'oufiguration Space. $\mathrm{p}$ proach. IEEE Transactions on C'omputers. 32 (1983) 108-120. 
[23] Nilsson. Xils ... Action Yelworks. Tenenberg. Jush. Weleer. Jay. and Allen. James. (Eds.). Prowerlings from the Rerhester Plamning Ilorkshop: From Formal Systems to Practical Systems. 1989. 36-68.

[24] Pontryagin. L. S.. Bullyanskii. I. (i.. Ciankrelidze. R. I.. and Msichenko. E. F.. The Vathematirul Theory of Optimal Processes. (John Wiley and Sons. New lork. 1962).

[25] Ramadge. Peter and Wonham. MInrray. The C'ontrol of Discrete Event. Systeus. Proreedings of the IEEE. 77(1) (1989) 81-98.

[26] Rosenschein. Stan and Kaelbling. Leslie Pack. The Synthesis of Digital Machines with Provable Epistemic Properties. Halpern. Joseph Y.. (Ed.). Theortical Aspects of Rensoning Abut Kinouledge. Proceedings of the 1986 Conference. Los ditos. C'alifornia. Morgan-Kaufmann. 198T. 8.3-98.

[2i] Schwartz. J. T. and Sharir. M.. On the Pianos Movers Problem: I. Communications on Pure and Applied Mathematics. 36 (198.3) 34.5398.

[28] Schwartz. J. T.. Sharir. M.. and Hopcrolt. J.. Planning. Geometry. and Complexity of Robot Motion. (Ablex. Norwood. New Jerses. 1987).

[20] Wolovich. William ... Linear .Vultivariable Systems. (Springer-Verlag. New York. 1!id). 


\section{Chapter 5}

\section{Knowledge-Based Planning}

Control theory provides a framework for constructing strategiea to control procenses modeled as dynamic systems. Sometimes, however, it is more convenient to represent the controlled procens in terms of causal event models of the sort investigated in Chapter $3 .^{1}$ The problem of constructing courses of action based on properties of causal event models is called planning, and the specification for intended actions of the robot over time is called a plan. By planning, the robot in effect programs itself to act in a particular way in the future. AI researchers have developed a variety of planning techniques, applicable for a wide aseortment of plan and event representations.

In the general planning setup, the robot is given a causal event model, with a distinguished subset of events, called actions, deemed under the robot's control. In other words, the robot can direetly eatablish the truth of actions, but cas infinence other events only indirectly through their caneal relations to actions. The robot aleo has some objectives describing deairable propertits of the controlled process in terms of patterns of events: Planning is the process of asembling bavic actions into a componite plan object designed to further these objectives.

A large fraction of planning effort is typically devoted to reasoning about the effecte, or potential consequences, of actions. One important reasoning tack is to determine whether a particular property should be expected to hold it come point after or during the plen's execution. Planners perform this ack by applying their truth criterion to the causal event model. The

\footnotetext{
'It would th nice to provide some reggestions ebout what features of the process indicate the best choice of ropresentetion. Potential adventeges of event-based (lingristic) ontoloyy include fecilitice for ropresenting incomplete information and the intuitive appad of caued events. Perhops a comparative discussion belongs at the start or end of Chepter $s$.
} 
computational expense of determiring which propositions hold at various points in time depends strongly ou the representation for the effects of actions and the accuracy of the algorithm implementing the truth criterion. For the planning techniques described below, we use deducibility with respect to TEMPLOG causal models as the truth criterion.

Usually it is not possible to predict perfectly the effects of actions on the controlled process. These limitations are manifest by indeterminacy or even incorrectness of the truth criterion. To plan effectively under these circumstances, the robot may need to gather information directly from the controlled process, augmenting the predictions drawn from its causal model. This approach is directly analogous to the use of feedback in control systems. In robot planning, the process of sensing the state to influence subsequent action is called execution monitoring.

Planning is deliberative, in that it generally calls for a broad consideration of the available courses of action and their potential consequences. However, in mont aituations the robot does not have the luxury of unbounded deliberation, because the process of interest progresses in time as the robot computes its plan. To produce effective action under the stress of real time, the planner muat have some capability to react to its perceived oituation without necessarily invo'cing its full deliberative powers. For any planning problem there is a spectrum of computational strategien, expected to produce better plans as more time is devoted to deliberation. Managing this tradeoff is a significant issue in the design of comprehensive planning architectures.

The final issue we consider in this chapter concerns the specification and interpretations of the robot's fundamental objectives in control. In the corimon approachen to planning (including the one we present here), objectives are represented as a set of predieatea, called goalo, on states of the controlled process. The planning tark then amounts to finding a course of action guaranteed to achieve thece goals. As we have seen in several ex_mples, however, absolute goal conditions cannot express gradations of preference needed to capture the realiatic objectives of a control problem. The bacic difficulty is that predicates coaruely partition the outcomes into twc. axts, failing to distinguish among states where the goals are achieved, and providing no guidance whatever for problems where it is impossible to guaratan goal achievement. When objectives can be achieved to varying degrees or with some probability, the more general preference representation is required to properly account for the tradeoffs inhereat in choosing alternative cources of action. On the other hand, the goal representation meshes well with the event ontology for causal modeling, and with plan evaluation 
procedures based on the truth criterion. Moreover, goals have significant heuristic value in focusing the search for good plans, and therefore constitute a useful approximation for more expressive preference structures. At the end of this chapter, we analyze the preferential interpretation of goals as a first step toward a reconciliation of common planning practice with the general theories of decision and control.

\subsection{A Task Reduction Approach}

The approach to planning we describe here is organized around the concept of a task, which is an abstract operation that the robot is committed to performing. Task are abstract in the sense that they dictate the general nature of what the operation is to accomplish without necessarily specifying its precise implementation. Before an abstract task can be carried out, the planner must supply sufficient detail so that it can be executed direetly by the robot hardware.

One way of increasing detail is to replace an abstract task with a more specific task or collection of more specific tasks. This process of refining the level of abstraction is called task reduction. Upon reducing an abstract task, the robot commits to carrying out the more specific tasks. The reduction process continues until all the tasks are specified in sufficient detail or all avenues of reduction are exhausted.

A task detailed enough to be executed by robot hardware is called primitive. Of course, primitiveness is a relative property, defined with respect to the capabilities of a particular execution module. For complex planning problems, it is often useful to conutruct a hierarchy of abstraction levels, each corresponding to a virtual robot with its own set of actions that are considered primitive. In this scheme, the planner at each level generates tasks at the next lowest level of detail, but is viewed as an execution module by the level immediately sbove.

One important type of nonprimitive task comprises those committing the robot to make a given proposition hold. These achievement tasks are denoted achlove $(P)$, where $P$ is the particular proposition to be achieved. Such tack may be reduced by finding a primitive task that necessarily achiens $P$, or by finding some other tanks achieving propositions that collectively entail $P$.

\footnotetext{
'Othar types include maintenence and prevention. Mention these, but do not introduce them into the logic. Perhops give interpretation for them in terms of achieve.
} 
Reduction is complicated by the fact that $a^{+}$any instant the robot is likely to have many tasks, and several methods fo. reducing any given one. In other words, finding a method to achieve a proposition is a search problem. It is quite possible that a choice for reducing one task may preclude potential reductions for some of the other tasks, requiring backtracking. Sometimes these conflicts can be detected and avoided, by coordinating the reduction of separate task vis constraints. In the remainder of this section, we present a seheme for task reduction, developing a set of data structures and associated techniques for organizing and managing the search process.

As far a our temporal model is concerned, a task is just a special sort of time token. An instance of a task is created by asserting an expression of the form

token (task (type), symbol).

The assertion declares that the robot has a task of type type throughout the interval from bogin (symbol) to and (symbol). For instance, the following expressions assert that the robot has two particular taks, one primitive and the other an echievement.

task (push_button (button42)).

task(achiore(locat 1on(robot, valre1))).

Primitive tasks are specified by their type. If the query primitivo(Q) succeeds, then $Q$ is the type of a task that can be directly executed on robot hardware. In the warehouse domain, we assume that pusbbutton $(B)$ is primitive, ${ }^{3}$ where $B$ is the label of a known push-button control switch. Being primitive does not imply that executing an action will necessarily achieve the proposition of the achievement tank it was reduced fr'um. The intended reaults are typically. guaranteed only under certain çonditions, which may or may not be entirely under the robot's control.

Tasks come and go as the robot discovers information about its environment. If the robot enters the loading area and noticen a truck that was not there the last time it visited, then it will formulate a new task to load that truck. Conversely, if the robot currently has the task to load trucks5, and it sotices that truek45 is no longer waiting, the robot will give up on this tank. To institute the general policy of servicing trucks waiting in the loading area, we ascert a task covering that policy, and add a projection rule to the database relating this task to its more specific inatances.

\footnotetext{
${ }^{3}$ Comment from Jean-Claude Latombe that this can actually be complas oporation from the robot control paropective.
} 


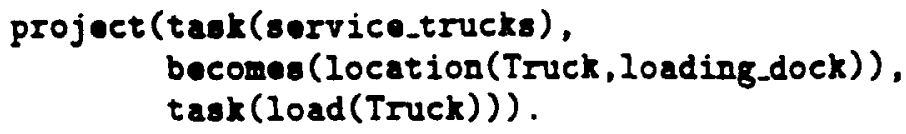

along with a corresponding policy to give up on load tasks when they are no longer feasible. ${ }^{4}$

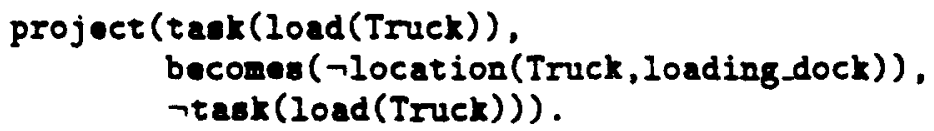

Of course, for the above polices to work as intended, the robot has to be continually aware of new arrivals and unexpected departures, and, hence, it might be reasonable to have policies that call for the robot to occasionally scan the loading area looking for changes. This points out a problem with our representation of time and action; we do not distinguish between what is true of the world and what the robot knows to be true of the world. We returs to this issue in Section 5.2.

Some policies should be ignored in certain situations. For instance, whenever the robot is in an area where an assembly operation is in progress, it should check to see if the anembler's melfunction light is on, and, if so, generate a task to push the reset button. However, if the robot is in a hurry or has only recently checked the malfunction light, it might not generate the task to check. The decision whether or not to check will depend upon what other tasks the robot currently has pending.

Some types of policies are more difficult to administer than others. For instance, a policy to clean up concrete spills might generate a specific task in response to each detected spill, but what about a policy to prevent or minimize concrete spills? In the latter case, the robot's response to a predicted spill might simply be to change its current plan by, say, opening an input valve a little lese or an output valve a little more, brit the robot might instead decide that the valve settings are perfect and choose to prevent spillage by raising the walls of the mixing tank. Whether this latter approach is acceptable will depend upon the cost of raising the walls. In Section 5.4, we conaider how more precise specifications of objectives, in the form of value functions, may provide the information necessary for such decisions.

In the tank reduction approsch, planning knowledge is encoded in expreasions of the form

todo (what, when, how).

\footnotetext{
"What if the lood task is alrody reduced! Presents complicated problem of hov to maintain stater of tasks in reduction search.
} 
where what is a task type, when is an interval, and how is either $\lrcorner$ nother task type or a compound task description specifying how to reduce the what task type. If how is a simple task, the result of interpreting the todo expression is to introduce a new task

token(task (how), when).

and mark the original what task as "reduced," to note that we need not search for another method.

One common how task type is the no_op, or do-nothing action. In general, when you have a task to accomplish something that is already true, the obvious action to perform is none at all. We can represent this simple strategy as:

todo (achiore(P),K,no_op) - holds $(\operatorname{ond}(X), P)$.

where, in order to absolve the robut of its commitment to achieve $P$, all that is important is that $\boldsymbol{P}$ is true at the end of the interval $\mathrm{x}$.

Note that providing methods for achievement tasks in todo expressions significantly simplifies the search process. Without these methods, the plan. ner would have to examine the causal model directly to find controllable events that would result in the proposition to be achieved. By relying on them, however, the robot will not in general conoider every possible way of accomplishing its task. The task reduction approach implicitly assumes that the computational benefits of using todo directives exceeds the cost of supplying them and the loss of opportunities potentially derived from a direct analysis of the causal model.

It is often useful to group together a collection of tasks coordinated for a common purpose. We call the deacription of such componite action a plan. Actually, these plan objects only partially ipecify the full course of ection, and we sometimes empharize this by calling them abstract or partial plans. In contrast, a complete plan is comprised entirely of primitive actions with a precise specification of the time that each is to be executed.

In our tank reduction scheme, a plan consists of a set of steps with ascociated constraints that determine their order and duration. For instance, a plan to fil a tank might include the following tasks a steps:

Step1: achiero(location(truck42, loading_tock))

Stop2: achlore(location(robot, ralre1))

Step3: achiore(position (valvo1) $=35^{\circ}$ )

Stop4: achiore( $\{$ loor(robot, floor 1 ))

Step5: achiore(location(robot,valve2)) 
along with constraints on those steps as follows:

end (Step1) $\preceq$ begin(Step2)

distanco (bogin(Stop2), ond (Step2)) $\in[00: 00,00: 01]$

The steps in a plan are transformed into a set of tokens in the course of formulating a specific instance of that plan. For example, the above steps might be instantiated as

token (task (achiove(location(truck42, loading-dock))), step 141).

token(task (achiove(location(robot, valve1))), stop 142).

tokon (task (achiove(position(valve1) $=35^{\circ}$ )), step 143).

token(tack (achioro(floor(robot, floor1))), stop 144).

tokon (task (achiove(location(robot, valve2))), stop 145).

and then constrained temporally by instantiating the specified constraints:

ond (step 141) \bogin(stop 142).

distance(bogis(step 142)), and (stop 142)) $\in[0,00: 01]$.

where stop141 through step 145 are newly minted symbols identifying the intervals associated with the task instances.

Plans are represented in our scheme by expressions of the form

plan (steps, time-constraints, protections)

where the steps indicate the new task involved in the reduction, the timeconstraints restrict the order of those tasks, and the protections specify special properties that must be maintained during the plan's execution. The new tasks are referred to as subtals of the task they were reduced from, inversely designated the supertask of the new tasks. All subtaks are implicitly constrained to occur during the interval of the supertask, an specified in the todo expresuion. Protections are important in detecting problems that arise when one tak interferes with another. ${ }^{5}$ Consider the following general method for making two propositions true at the same time:

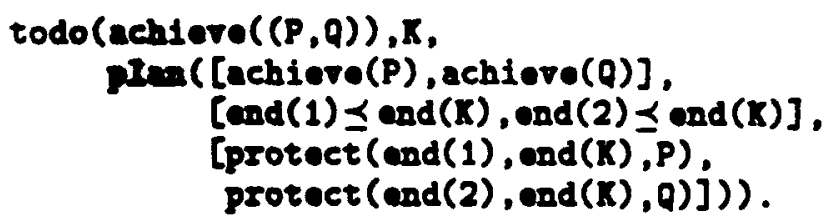

\footnotetext{
Latomb: what about simplifications possible by merying identical subtasks for different supertashe?
} 
The steps are numbered by their position in the list of steps. ${ }^{6}$ The con. straints refer to these numbers and are used to constrain the corresponding tokens created in the process of instantiating a particular plan. The two protections stipulate that to achieve the conjunction of $P$ and $Q$, achieve each of $P$ and $Q$ individually, and ensure that once each proposition is made true it remains so at least until the end of time interval $\mathrm{X}$. A protection is said to be violated when the robot becomes committed to an action with an effect whose type contradicts the type of the protection. ${ }^{7}$ Certain combinations of tasks can make it impossible to avoid violating protections. ${ }^{8}$ In some cases, conflicts among propositions to achieve are easy to detect, for instance: ${ }^{2}$

achieve((status (asecobler,on), status (assenbler,off)))

In general, however, the interactions between tasks can be arbitrarily complex, requiring considerable effort to detect and resolve.

Most of the plans for a given application encode domain-specific strategies for reducing abstract tasks to more concrete ones. The set of all such strategies constitutes a plan library. In the following, we provide examples of plans that might appear in the plan library for a robot operating in the warehouse domain. We take the liberty of simplifying the plans somewhat (e.g., by leaving out certain steps and conotraints) in order to make the text more readable. Here is a plan for installing an option in an appliance:

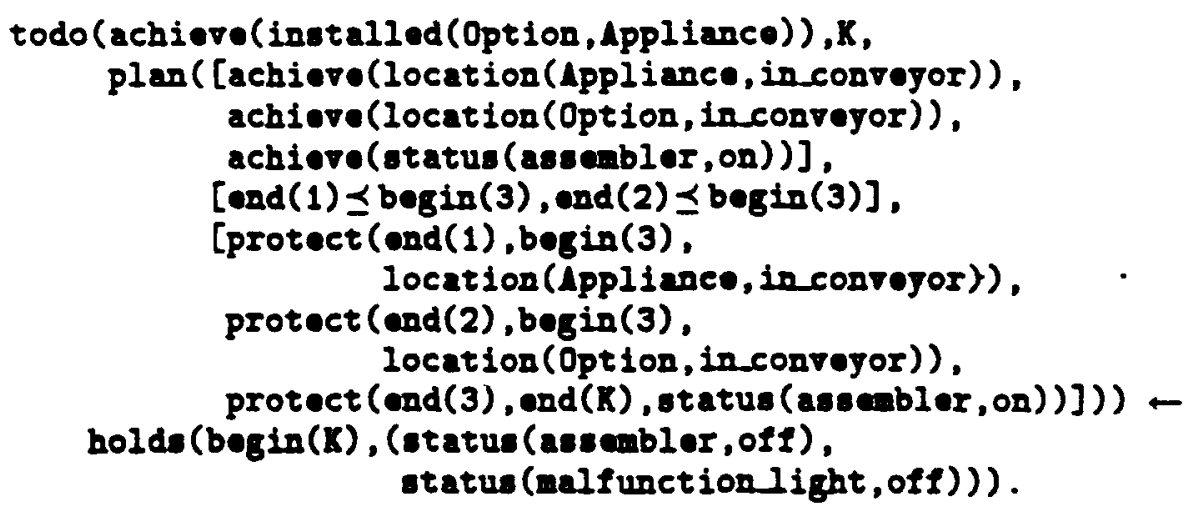

\footnotetext{
'Drimin how this might be implemented in prolos using a pre-processor, and how the suntes mingt be further sugared to wee step identifiers.

'I ctomb: What about temporery violations! Is there any vey to allow them! Answer: never rally weful; corsider modal truth eriterion.

-e.e., the Swomen enomaly.

- Mate cleer that conficts are not errors but can represent legitimate compatition among goals and oubgoals.
} 
Take note of the role protections play in this plan. The first two protections ensure that, once placed on the assembler's input conveyor, the appliance and the option to be installed will remain there until the robot starts the assembly. The third protection prevents the robot from inadvertently scheduling some other activity that would result in turning the assembler off during its execution of the installation task.

The robot will also need plans for changing the location of objects. The following general rule specifies how to change the location of something other than the robot:

todo(achiore(location (Object, Loc1)), K, plan([achievo(location(robot, Loc2)), pickup (Object), achiove (location(robot, [oc1)), sot dotn(Object)], [and(1) $\preceq$ bogin(2), ond (2) $\preceq$ bogin(3), $\operatorname{and}(3) \preceq$ bogin(4)], [protect(ond(1), bogin(2), location(robot, Loc2)), protect (ond(2), bogin(3), bolding (robot, object)), protect (ond(3), bogin(4), location(robot, Loc1))])) holda(begin(X), (location(Object, Loc2), Object $\neq$ robot , Loc1 $\neq$ Loc2)).

The above plan assumes a somewhat implausible model of robotic movement. In order to move an appliance onto the input conveyor, the robot would have to move itself onto the conveyor while holding the appliance, then set the appliance down so that it rests on the conveyor. Although we continue to make use of such simplifications as required to keep the discussion focused, we return to consider continuously changing parameters in general and spatial inference in particular later in this chapter. ${ }^{10}$ To plan for moving the robot about, we use the following rule, and assume that the task type more (source, destination) is primitive:

todo(achiere(location(robot, Loc1)), K, more(Loc2, Loc1)) holda (begin(K), location (robot, Loc2)).

Finally, the robot needs a plan for turning the assembler on or off: todo(achiore (atatus (assembler, Stat 1)), K, plen ([achiere(location(robot, assembly area)), puab_button(Stat1)], $[$ and (1) $\preceq$ bogin(2)], [protest (and (1), bogin(2), location(robot, asaonbly_araa)) ])) bolds $(\operatorname{ard}(K),($ status (asseabler, Stat 2$)$, Stat $1 \neq$ Stat 2$))$.

\footnotetext{
${ }^{10}$ Will we? I don't think 10.
} 
Now we are ready to consider how to go about reducing a set of abstract tasks to primitive tasks. In general, the reduction process can be quite complex. We start by sketching an algorithm for performing the reduction, give an example illustrating the algorithm in operation, and then comment on complications not explicitly handled by the algorithm. The task reduction procedure is specified as follows:

1. Find some task, token ( $\operatorname{task}$ (what), when), which is neither primitive : nor marked as already reduced. If no such task exists, wait until a new task is added to the database.

2. Using the query, todo (what, when, how), try to find some method how for carrying out the task found in Step 1.

3. If the query a - - 1 in Step 2 fails, try adding constraints to reatrict the ordering of the existing tasks. This may trigger rules permitting the todo query to succeed on the next attempt.

4. If the query specified in Step 2 fails even after trying various additional constraints, try removing one or more of the existing tasks along with all associated protections and other constrainta. Be careful to reinstate the original supertask.

5. If Step 2 through Step 4 fail to produce an applicable method, return to Step 1 and try another task.

6. If the query succeeded, mark the orig : takk as reduced and add the new how task or plan to the databave, along with any specified constraints and protections.

7. Upon effecting the reduction, TEMPLOG will have updated the databare using the projection and persistence clipping algorithm, and the projection rules that describe the sffects of the actions. Check to see if any protections are violated by the addition of the new tasks.

8. If any protections are violated, resolve the violation by either reordering or removing one or more of the existing tasks.

9. Go to Step 1.

A concrete example should help illustrate the basic operation of the reduction algorithm. Figure 5.1 shows a TEMPLOG database containing one 


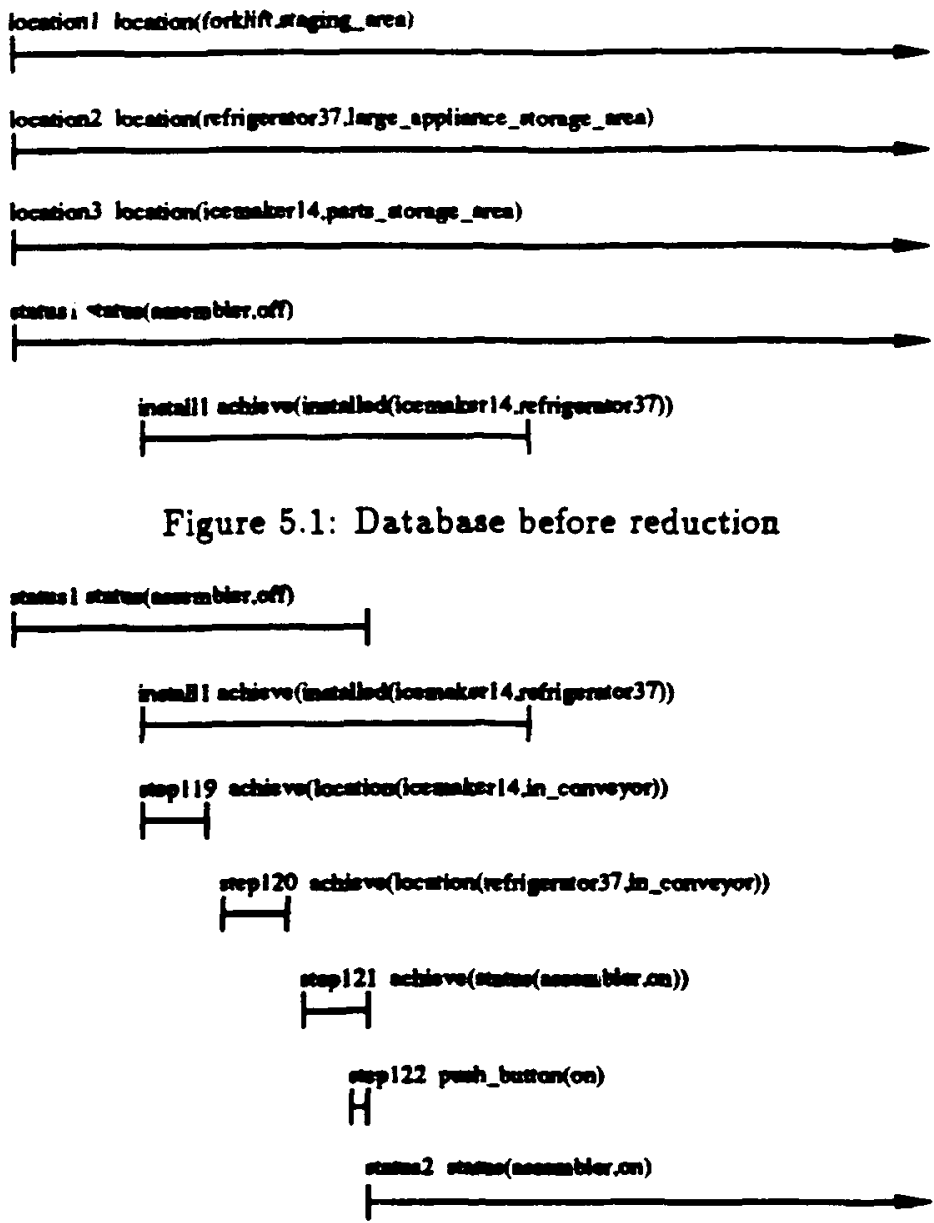

Figure 5.2: D̀atabase after reduction

nonprimitive unreduced task to install an ice maker in a refrigerator. Figure 5.2 shows the TEMPLOG database resulting from applying the reduction aigorithm, using the planning knowledge specified in this section and the knowlodge of cause-and-effect relationships described in Chapter 2. (Only selected stepe are depicted in Figure 5.2 to keep the display readable.) The reduction illustrated in Figure 5.2 is a particularly simple one; we consider next some problems that may arise in more complicated situations.

Returning to the previous listing of the reduction algorithm, note that there are a number of steps where choices are made. In Step 1, the robot 
will generally have to choose from a number of unreduced nonprimitive tasks. In Step 2, there are likely to be severas methods for reducing the chosen task. If the todo query does not immediately succeed, the robot may have to consider several alternative orderings in Step 3, or several reduced sets of tasks in Step 4, before it is able to find a reduction strategy that works. In fact, the iteration of Steps 1 through 5 can cause the algorithm to loop indefinitely, continually removing tasks and adding new ones. In general, the algorithm is not guaranteed to eventually terminate with a complete reduction. The problem of resolving protection violations in Step 9 can be particularly troublesome; sometimes involving numerous attempts at reordering or modifying the set of tasks. If the robot makes the wrong choice early in the planning process, it may expend a great deal of effort before it "backs up" and tries an alternative option. All of these problems and more have to be routinely solved by a robot control aystem that generates plans by task reduction. Researchers have developed an array of techniques for dealing with these problems, although none offer s complete solution.

For an example of how the procedure detects and resolves negative interactions among task, suppose that the TEMPLOG database depicted in Figure 5.1 also contains a task committing the robot to perform routine service on the sseembler. Suppose further that this routine service task is currently scheduled to overlap with the task to install the ice maker in the refrigerator. The plan for isutine-service tasks is specified below:

todo (routine sorvice (aseambler), $K$,

plan ([achiove(status (asesembler, off)), lubricate (aseanbler), roplonish_coolant (ascambler), puahbutton(reset)],

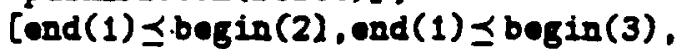
$\operatorname{end}(2) \preceq \operatorname{bogin}(4), \operatorname{ead}(3) \preceq \operatorname{bogin}(4)]$, [protect (ond(1), bogin(4), otatus (ascambler, off))]).

Note that the routine service plan requires that the ascembler be turned off before the lubrication and coolant-replacement taks are initiated. The task to tere the asembler off conflicts with the installation plan, which requires that the secembler be on.

Figure 5.3 depiets the databace resulting from reducing both the installation and routine service tasks. Note that the database predicts that the assembler will not remain on throughout the required portion of the installation interval. In the course of reducing the two task, the robot should have generated two protections, the first asociated with the installation task: 


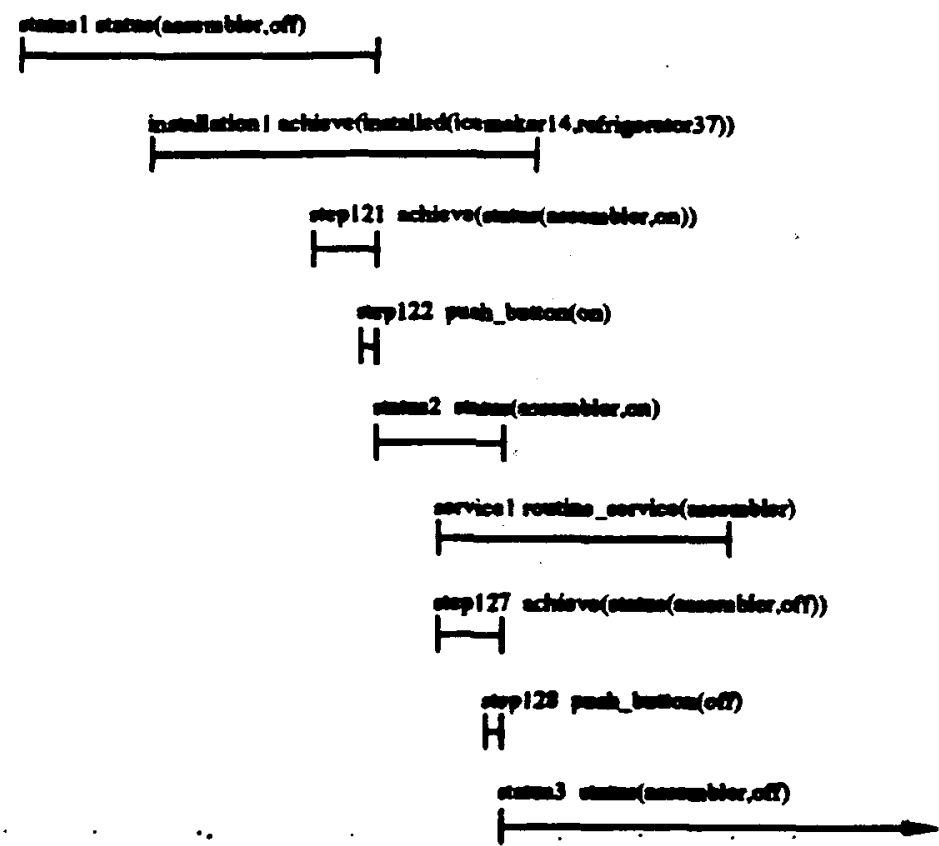

Figure 5.3: Database with a protection violation 
protect (ond (step 121), end(installation1), status (assembler, on))

and the second associated with the routine service task:

protect (ond(stop 127), ond(service1), status (assembler, off))

These two protections conflict with one another (i.e., they require the persistence of tokens of contradictory types over a common subinterval). The easiest way to resolve this particular conflict between the installation task and the routine-service task is to reorder the two tasks: either constrain the interval sorvicel to end before the beginning of stop 127, or constrain servicol to begin after installationl. For other conflicts, reordering may not suffice, necessitating more drastic measures.

There are other problems that can arise besides protection violations. Many of the rules specifying reduction methods have conditions that must hold if the reduction method is to apply. We refer to these conditions as reduction assumptions. For instance, consider the general rule for avoiding unnecessary work:

todo $(\operatorname{achiore}(P), K$, no_op $) \leftarrow \operatorname{holda}(\operatorname{and}(K), P)$.

If the robot has a task of type achieve (atatus (assembler, off)) during token intorral81, when the assembler is already expected to be off, then it will reduce the task to a no_op. The reduction assumption is that status (assembler, off) holds at ond(interval81). The robot will check at reduction time that the reduction assumption holds, but the assumption may become false during subsequent planning as additional tasks are added to the database. Reduction asumptions have to be carefully monitored in much the same way that protections are, and steps taken when the assump. tions are found to be violated. ${ }^{11}$

The general problem of reducing s set of taiks to primitive tauks 80 as to avoid violating any protections or falaifying any reduction asumptions is believed to be computationally intractable (i.e., it has been shown to be in the class of NP-hand problems). Deadlines and reasoning about resources are obvious sources of complexity, but, even if we were to ignore deadlines and rwourcas, mont interesting planning problems remain in the company of them dincult problems. For certain versions of the problem, there is no

\footnotetext{
"If the ruluction trigger neads to hold at tank time, why enen't bhese always protected! Or allemetely, why not allow protections with simple task ruductions! Clarify the utility of defining the concept of nduction asmimptions distinct from protections. Confusing factor: protections easm to guard againot inter-task conficicts as a ride effect of preventing intre. task conficts, porforming some of the function of reduction asoumptions.
} 
effective method for generating plans (i.e., the problem is undecidable). For the problems that are decidable, it is fairly simple to write an algorithm that finds a solution if one exists, and signals that no solution exists otherwise. Unfortunately, such an algorithm may take an unacceptably long time to return its answer. While these observations are somewhat discouraging, we at least know that good approximate solutions are possible (e.g., bumans perform reasonably well driving forklifts in warehouses). In artificial intelligence, planning problems are typically recast as search problems, and a standard methods have been applied to develop heuristic algorithms that perform well in practice. In this chapter, we have not explored the various search techniques, concentrating instead on the basic problem of how a robot might use symbolic representations to guide its behavior.

In each iteration of the reduction algorithm, a partially completed plan is analyzed and modified. For some planning problems, such incremental analyois is problematic. The projection rule describing the process of moving from one location to another (specified in Chapter 2) indicates that the distance in time between when the move is initiated and when the robot is in the final location is a function of the distance in space between the robot's initial and final position. This rule brings up an important issue that we have avoided so far. The order in which tasks are executed determines to a large extent how long they take to execute. If the robot is trying to minimize the time spent in execution or avoid violating deadlines, then it has to consider not only the order in which to perform each task, but the location that it has to be in to perform each taek and how to travel between those locations. Task scheduling with deadlines and travel time inevitably involves nasty combinatorics and NP-hard problems.

There are all sorts of deadlines that a robot might have to contend with in practice. In addition to absolute deadlines (e.gi, fiaish before noon), there are graded deadlines (e.g., the longer you take, the more it will cost you), and relative deadlines (e.g., finish before the tub overflows). The last are particularly interesting from the perspective of control. How do you coordinate the behavior of a robot with that of other processes over which the robot an only partial or intermittent control? We have already mentioned how wight accomplish ouch coordination for the tank-filling problem using falbeck. In the following, we consider how we might accomplish the necesary coordination using planning, for a somewhat more complex problem.

Recall the problem presented in Chapter 1 involving a robot in a concrete plant scurrying about from one valve to another trying to fill trucks with 


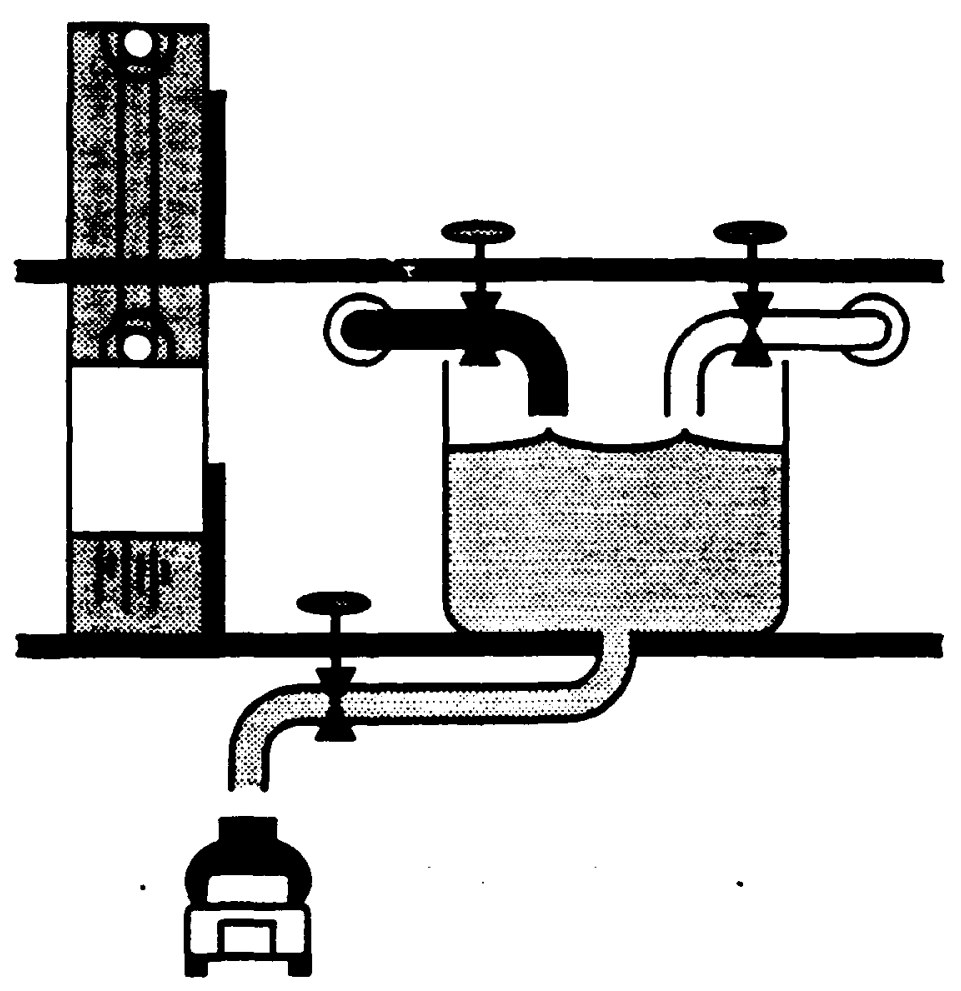

Figure 5.4: The concrete factory domain 


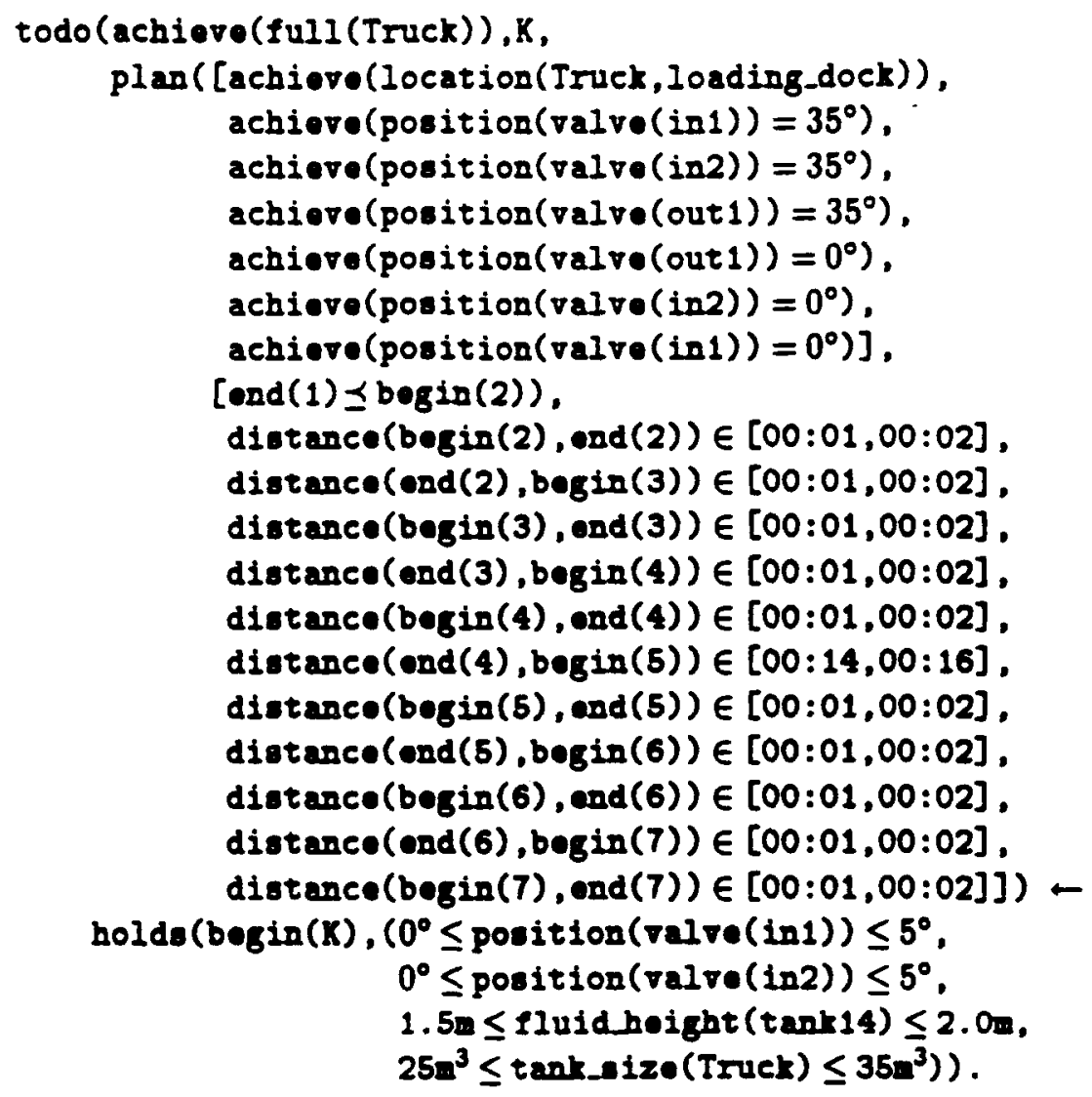

Figure 5.5: A plan for filling a single truck

properly mixed concrete. Figure 5.4 depicts the basic layout of the concrete factory.

The simplent approach is to provide a amall number of canned solutions, each covering a subset of the situations that the robot might find itself in. For intance, Figure 5.5 shows a plen for filling a single truck. If the tasks are carsind ont within the specified time constraints, then this plan guarantees that 20 concrete is upilled, the two ingredients, cement and agregate, are mixed in the proper proportion (i.e., 50/50 give or take $5 \%$ ), and that the tank is filled to at least $90 \%$ of its capacity. To achieve the required degree of coordination, the task are tightly constrained with respect to one 
another. Figuring out how the individual tasks are achieved will require further reduction. If the robot is to carry out all of the tasks itself, it will have to move between the various valve locations (or stations) and perform the indicated valve adjustments in the times allotted. The plan for changing the position of a valve is simply:

todo (achiere(position (Valva) $=$ Theta),$K$, plan([achioro(location(robot, station(Va]re))), turn (Valve, Theta)], [ond(1) $\preceq$ bogin(2)] , [protect (ond (1), ond (2), location(robot, station(Valva))) ])).

The process of turning a valve is modeled by the following projection rule, which bounds the time it takes for the turning to complete.

project (position(Valve) = Thotal, tura (Valre,Theta2), [(|Theta1-Theta2 $\mid \div$ max.turning-speed $)$, (|Thota1-Thota2 $\mid \div$ min-turningspoed)], position (Valve) $=$ Thota2).

Moving from one location to another is complicated by the fact that the stations for the input and output valves are located on different foors. We assume that there are two ways of going from one floor to another: by elevator or stairs. When it is in service, uning the elevator is always preferred to taking the stairs.

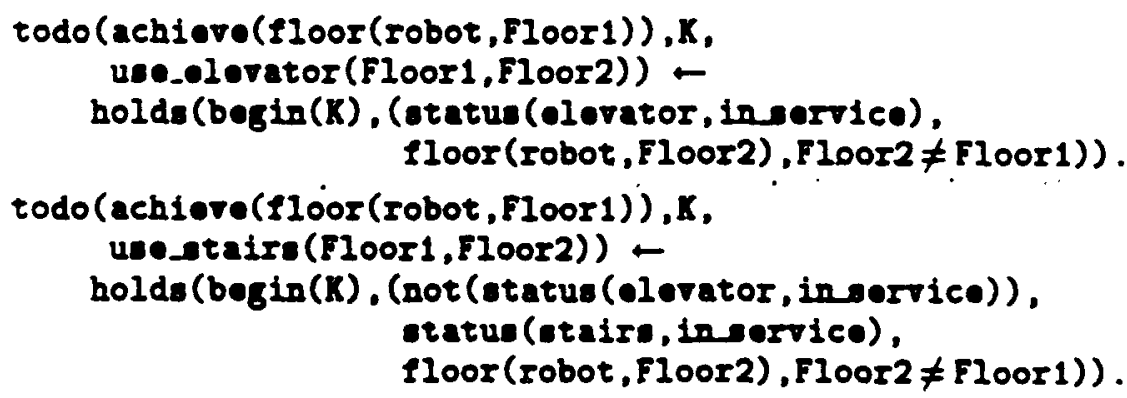

The phan for using the elevator and atairs are straightforward. 


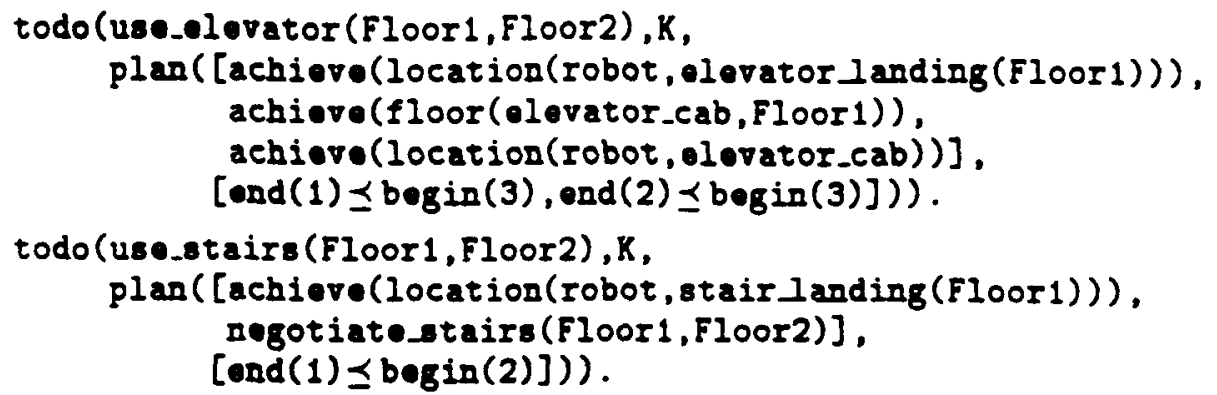

where we assume that negotiating the stairs is primitive:

project (location(robot,Floor1), nogotiate_tairs (Floor 1, Floor2), [00:03,00:05], locat1on(robot, Floor2)).

and the elevator begins to operate as $800 \mathrm{n}$ as the robot enters the cab:

project(floor(elevator_cab,Floor), becomes(location(robot, elevator_cab)), [00:01,00:02], location(robot, other(Floor)) ).

Now, suppose that the robot is given the task to fill a particular truck, truck42. The robot's task is indicated by the following token.

token (task (achiere(full (truck42))), fill45).

State the initial conditions, valve flow factors, tank area and height, truck capacity, and status of stairs and elevator. To avoid introducing plans for summoning the elevator, assume that the elevator, if it is in service, is always on the same floor as the robot. Get material from Dean and Siegle, AAAI-90.

We can reduce 121146 using the plan shown in Figure 5.5 and either the elevetor plan or the stairs plan. The reduction uning the elevetor plan is preferable becanse it managea to fill the truck three minutes earlier than the reduction uning the stairs plan. Although we have provided no mecha. nism to express this general preference, the relative time requirements are taken into account in reasoning about interactions between competing tasks. For anple, suppose that the robot bes another task constrained to oceur during 111145, which involves running a systerr ' diagnontic program requirins to rumin idle for ten minutes. In this case, there is only one solution conaintent with the constraints: the reduction uning the elevator plan.

There are a number of potential problems with the type of plan shown in Figure 5.5. One ariee in trying to apply such plans to coordinate two simultaneous fillings or to orchentrate series of fllings. It would be necensary 
in general to provide special plans for each particular filling combination. Another difficulty is that if the flow rate for one of the valves or the volume of the mixing tank changes, then the plan no longer guarantees avoidance of spillage and suitable mixture proportions. For instance, if the flow rate of valve(in1) is increased by a factor of $10 \%$, then the reduction using the elevator plan will result in a task duration of 24 minutes, but there will be $2 \mathrm{~m}^{3}$ of concrete spilled on the floor and an unacceptable 2:3 ratio of cement to aggregate in truck 42.

As an alternative to excessively specific plans, we could provide general plans that do not specify exact valve positions and task durations, and hence give up the guarantees regarding results like spillage and mixture. A search aigorithm would then heuristically choose positions and durations to use in generating candidate plans, and the candidate satisfying the mixture constraints that provides the least spillage would be chosen for execution. The advantage of such a scheme is its improved prospect for finding a solution over a broad range of task situations. The disadvantage is that the set of all combinations of valve positions and task durations is quite large, only a small subset of which are likely to yield good solutions. ${ }^{12}$

A compromise is to have a small number of highly specific plans that are likely to produce solutions close to satisfying the achievement task and then heuristically adjust the plan parameters to improve performance. For example, heuristice might include "if the truck is not filled to $90 \%$ of its capacity, then start closing the output valve later" or "if the mixing tank spills over, then open the output valve more and close it earlier." Research in planning tends to focus on general-purpose domain-independent methods. It is important to remember, however, that the performance of a particular planning system can be dramatically enhanced by bodies of special-purpose knowledge encoded in the form of domain-dependent rules.

One important issue that we avoided in the previous examples involves the representation of plans in which an action is repeated some number of times. For instance, how do you represent a plan to unload a truck containing erven appliances? Using the list manipulation routines in PROLOG, this turne oat to be relatively easy. A more difficult problem involves planning to unbaed s truck with some unknown number of appliances. We would like to be able to predict the type of the subtaks involved and how long the anloading is likely to take. We might opecify a recursive plan such as:

\footnotetext{
12 What about parameterizad plens, where the precise settings an spocified as a function of the ocher variables! This is a form of conditional plan, to be discussed in nest section.
} 


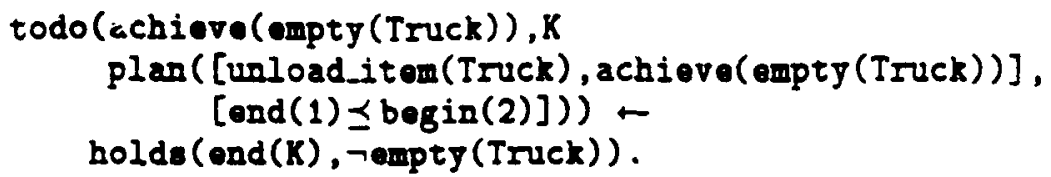

This gives us $=n$ idea of the types of subtasks involved, but we cannot determine their number because it does not make sense to reduce the recursive (second) step until after some item is unloaded. Thus, we are still left with the problem of estimating how long the unloading task will take. We could estimate how many items are likely to be on a given truck, and expand a plan with this number of subtasks. This remains short of a complete reduction, as we cannot determine where the robot will have to travel until we know the exact contents of the truck.

A more general problem with the sort of approach specified above is that it relies on execution-time replanning. Because the effects of the plan are not completely predictable, the subsequent course of action cannot be specified until after the results are known, at which time the task reduction process is resumed. The drawback of this strategy is that task reduction involves deliberate search, and thus may entail a considerable panse in the robot's constructive activity. This pattern of alternation between planning and execution can waste through idleness a considerable fraction of the robot's resources. Worse, the continuing evolution of the controlled process during deliberation may erode or eliminate the robot's opportunity to effectively promote its objectives.

One way to addreas this problem is to provide, at plan time, for alternate courses of action depending on conditions bolding at execution time. In the following, we consider methode for constructing and reaconing about plans that explicitly refer to such contingencies. These plans include knowledge aequisition steps to collect information, associated with alternative subplans to be performed or not, conditional on the information gained during plan execution.

\subsection{Conditional Plans}

Facad with the tank to unload a particular truck with unknown cargo, there are (a bant) two approaches. The robot might construct a plan to find out what eppliances are on the truck, and postpone planning their removal until the contente are known. Alternatively, it might create a plan that includes a step to determine the appliances in need of unloading, plus some additional steps conditional upon the outcome of the initial information- 
gathering operation. This second approach preduces a conditional plan, and has a number of advantages over postponing planning entirely. For instance, while the robot may not know exactly what appliances are on the truck, it does know that in order to move them it will need a screwdriver to remove the restraining straps that protect them from damage in transit. The plan to unload the truck will require a step to remove the restraining straps no matter what appliances are on the truck. If the robot is currently near a tool box, it can asve itself a trip by appending a task to fetch a screwdriver a to the beginning of the plan to unload the truck.

More importantly, the conditional plan provides the robot with the means to commit to an answer conditional upon information gathered at execution time. Given a conditional plan, the robot can avoid reinvoling the planner upon determining the contents, and can proceed immediately with the uninading plan specified for the situation actually encountered. However, this readiness is achieved only at the price of computing contingency plans for unloading all potential types of cargo. As all but one of these plans goes unused, there is a considerable computational overhead in generating the contingency plans. This is the fundamental tradeoff in generating conditional plans, an issue we discuss further in Section 5.3. In this section, we present some simple mechanisms for expreasing and reasoning about conditional action.

To specify conditional actions in plans, we introduce a new task type:

Cp (condition, conditionalaction, alternate_action)

If the condition holds at task execution time (i.e., the interval specified in its task token), then the robot is to perform the conditional action; otherwice it is to perform the alternate action. To illnatrate the nse of $T$, consider the following method for moving to a particular floor. The plan is to use the elevator if it is in service, otherwice to take the stairs.

todo (achiere( 1 loor (robot, Floor1)), X, cp (statue (olerator, inservice), use_elevator(Floor1, Floor2), usedetairs(Floor1, Floor2)) ) -

bolde(bogta(X), (location(robot, Floor2), Floc: $1 \neq F$ Foor2)).

Note the although it includes no temporal argument, the conditional expreacion implicitly refers to the status of the elevator during $X$, the interval in which the taks are operative. Recall that in reducing taks using todo, the new tank (in this case, conditional) inherits the interval of the original task. 
The appropriate spplication of a ip inethod relies on two assumptions. First, it makes sen-e to introduce a conditional task only if the value of the $r$ indition is not already known at the time of introduction. In the example above, this means that the robot cannot determine at planning time whether the elevator will be in service during $K$. We can verify this assumption by augmenting the rule's antecedent:

$$
\begin{gathered}
\text { holde(begin(K), (location(robot,Floor2), Floor1 } \neq \text { Floor2, } \\
\text { not (status (elovator, inservice))) }) .
\end{gathered}
$$

We rely here on negation as failure to satisfy the query in cases where the elevator status at bogin $(K)$ cannot be determined. Having modified the rule, we should also add to the plan library an unconditional todo method for the case where the elevator is known to be in ser"ice during $\mathbf{K}$.

The second assumption underlying conditionalization is that the value of the condition will be known at the time of task execution. This prerequisite is much more difficult to ensure. Suppose we implement the conditionalization using a pair of projection rules:

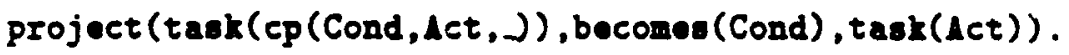

project (task (cp (Cond, , , $\Delta c t)$ ), becones ( $\neg$ Cond), task ( $\Delta c t)$ ).

The problem with this approach is that we have no assurance that either Cond or $\neg$ Cond will become true during the interval of interest. Moreover, it confuses what is true in the model with what the robot knows to be true. We can alter the syntax all too easily.

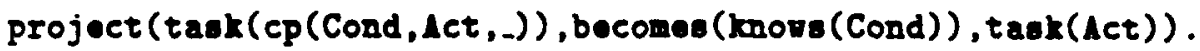

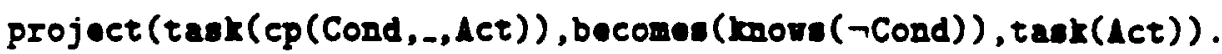

Unfortunately, it is not at all straightforward to define expressione of the form knove $(\varphi)$ in a manner consistent with both our intuitions sbout the meaning of knowledge and the behavior of our temporal logic. Instead, we present a simpler approach based on explicit declarations of the observability of events. Although this scheme does not provide for complicated inferences about the knowledge state of the robot, it covers many useful situations with minimal additional machinery. In Section 5.5, we evaluate the limitations of our obcervability approsech with respect to more general theories of knowloly.

Our first step toward managing the genuration of conditional pians is to reatrict the clase of propositions that are eligible for conditioning. The basic constraint is that the robot can execute a conditional action only if the condition is part of its available information. To impose this constraint, 
we define a special clar; of propositions, called observables, that comprise the exclusive domain of conditional expressions.

A proposition $\varphi$ is declared observable during the interval $\left\langle t_{1}, t_{2}\right\rangle$ by an assertion of the form observable $\left(t_{1}, t_{2}, \varphi\right) .^{13}$ Given this declaration, the planner is permitted to specify cp tasks for proposition $\varphi$ during subintervals of $\left\langle t_{1}, t_{2}\right\rangle$.

It is important to distinguish the temporal extent of the observable proposition from the time the robot observes it. For example, the robot might find out at $t_{1}$ (when it reads the maintenance schedule) whether the elevator will be in service at some subsequent time $t_{2}$. We would express such a situation by asserting:

holds $\left(t_{1}\right.$, observable $\left(t_{2}\right.$, status (elevator,,$\left.)\right)$ ).

Observability at a given time has implications for observability at other times. For instance, it is reasonable to postulate that observability is persistent; that is, the robot does not forget:

bolds $(T 1$, observable $(t, \varphi)) \leftarrow \operatorname{holde}(T 2$, obeervable $(t, \varphi)), T 2 \preceq T 1$.

But of course we cannot assume that, just because the robot can observe whether $\varphi$ holds at $t$, it can also observe whether $\varphi$ holds at $t+\epsilon$. Ir other words, a similar persintence relation does not apply for the temporal extent of the observable proposition.

In the common conditional planning situation, the time of observation and the temporal extent of the observable proposition coincide. Given a conditional task of the form $c p(\varphi, \ldots, \ldots)$ during interval $K$, we are most concerned with whether:

holds (bogin $(K)$, ond $(K)$, obserrablo(bogin $(K)$, ond $(K), \varphi)$ ).

It is precisely this fact that determines whether the ep task is executable by the robot. If the robot is committed to a conditional plan, therefore, it follows that it should be committed to making the condition observable. We might encode this antomatic commitment as a projection rule.

project (troe, beconoe(taek $(C P(P, \ldots,-))$ ), tank (achlete(obsertablo(P)))).

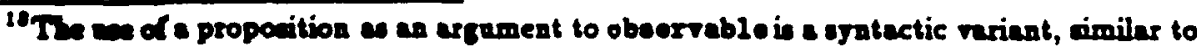
conotrect ins eldpa, holds, and others introduced in Chapter 3. As for those predicates, we edopt the ereal syatectic conrentions in opecifying its temporal arguments as either pointe or intervel. Moren.er, we cometimes omit the temporal arcument when its value is implicit in the context (e.g., within a talk asertion).
} 
The problem of ensuring the txecutability of conditional plans thus reduces to achieving the necessary observability prerequisites. While this is a difficult problem in general, there are typically a wide range of propositions that are rendered directly observable by primitive actions. Let us call such propositions testable, and assume that the query tostablo(P) succeeds if and only if there exists a primitive action, indexed by test $(P)$, that tests for the proposition $P$. We therefore have:

todo (achiere(observablo(P)), K, test $(P)) \leftarrow$ testablo(P).

We could enforce observability syntactically by requiring that all propositions appearing in conditional expressions be potentially testable. This approach is not as restrictive as it might sound, since we can always push off the complexity to reasoning about the relation of directly testable propositions to properties more central to the robot's planning decisions. Nevertheless, such indirection may be unnatural, and it is often possible-albeit more complicated-to achieve observability of useful conditions by means of explicit planning. In allowing more complex information-gathering behavior, we gain flexibility at the expense of sacrificing the guarantee that all conditional tanks will be executable. For completeness, we note that the meaning of a task that conditions on an unobservable proposition is simply that of the no_op action.

To illustrate some of the potential dificulties involved in ressoning about information-gathering, consider the following plan to determine the level of fluid in a truck sitting in the loading dock at a particular point in time.

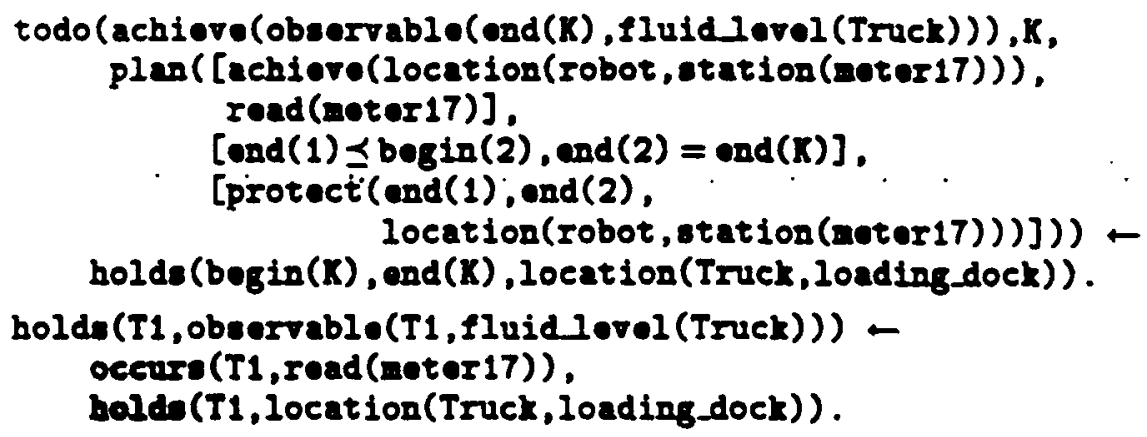

If the robot has the task to obcerve the level of the fluid in the truck currently located in the loading dock, then it can do so by positioning itself in the appropriate place to read the fluid-level meter, and invoking the subroutines necensary to read the meter and procese the resulting data. Other knowledge acquisition tasks may require significantly more complicated synchronization. 


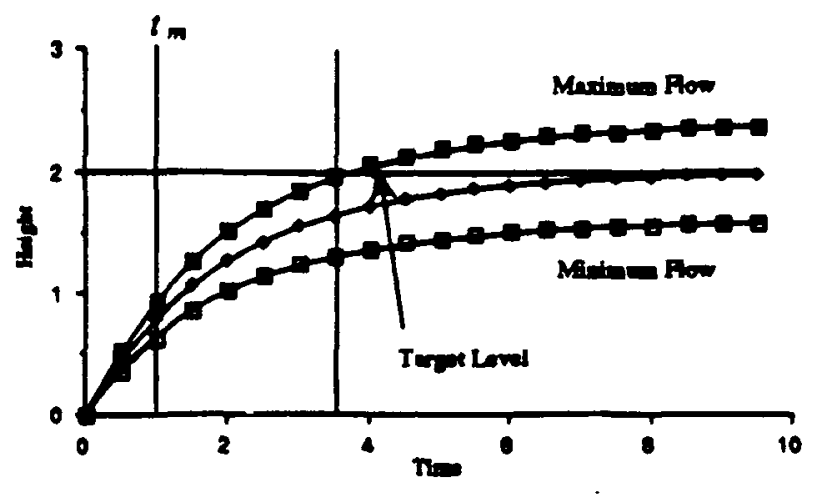

Figure 5.6: Planning with an approximate model

Suppose that the robot wats to close a valve when the fluid level of the truck being filled reaches a particular height. In order to do so, the robot will need to know when the level achieves this height. If the robot lacks a predictive model of the tank-filling process, then it must stand in the appropriate location and monitor the fluid-level meter continuously. If the robot knows the initial conditions and has a precise model of the tank-filling process, then, it can predict exactly when the fluid will reach the target level without consulting the meter at all. If the robot does not know the initial conditions but has a precise model, then it is sufficient that the robot observe the values of the parameters at some point in time in order to predict the height of the tank for all subsequent times. The most likely situstion is that the robot will have some eatimates for the parameters (perhaps based on measurements at different points in time) and an approximate model whose predictions decrease in accuracy as they extrapolate into. the future. Using this information, the robot can generate expectations or worst.case scenarios about when the tank will reach the target level. ${ }^{14}$ For instance, suppose that the robot knows the initial conditions for its model at time 0 , but its tankfilling model is subject to bounded errors (see Figure 5.6). In planning when to read the meter, the robot must take into account the earlient that the fluid level ningt reach the target level, as well as the amount of time required to move tron the meter to the valve and close it. One possible approsch would be for the robot to find its way to the meter at or before the time marked $t_{m}$ in Figure 5.6, and then replan on the basis of the observed height of the thid. It could avoid execution-time replanning by indentifying, in advance,

\footnotetext{
${ }^{10}$ This is a case of resoning about the noed for feedbeck, a difficull general problem.
} 
a threshold on the fluid level upon which it would proceed to the valve. To exhibit maximal robustness, however, the rolut must be flexible enough to apply more complex dynamic replanning strategies. For example, if it seems on monitoring for some time that the level is not rioing fast enough, the robot might contider opening the valve a bit and rescheduling its subsequent meter readings based on the revised predictions of its flow model.

It should be clear that we could make the dynamic decision problem facing the robot arbitrarily complex. Use this example to motivate fuller exploration of reactivity in next section. Also point ahead to Chapters 6 and 7, which focus on sensing and reasoning under uncertainty.

\subsection{Planning and Reaction}

Discuss in this section, among other things.13

- Conditions as the first step toward reactivity. Continuum between unconditional plan languages and univernal plans. Conditional plan language defines middle ground.

- Relation of observability approsech to control framework.

- Making plans more robust by convidering perturbations. Provides for the role of monitoring in plan er-cution.

Talk about expectations and expectation monitoring during plan execution. What happens when your expectations fail? For ezample, you try to turn a valve and it doesn't appear to turn or the water level goes up when you close the valve. Talk about replanning and recovering from execution er rors. What does it mean to lose, regain, or maintain control? What do you do when things go wrong and you're in the middle of doing something? For instance, the tub is running over and you're on the phone or trying to rescue your dinner from the oven. Develop the analogy between difference-reducing plannere and error-driven control strategies.

The ide of reactivity and its contrast to deliberate planning. Architectures for integrating planning methods of the sort discussed in Section 5.1 with rasetive systems. Task interpretation systems (see old material). Firby's RAPs. Ties to sections on reactive control in Chapter 4. The "obvious" solution: different levels of competence with varying degrees of reactivity, asynchronous control, run-time artitration, and off-line compilation for real-time

\footnotetext{
16 Fis trenaition from preceding rection.
} 
*Draft* of December 10, 1990

responsiveness. Prelude to architecture for decisi-n-theoretic control of inference, presented in later chapter.

\subsection{Goals and Utilities}

Limitations of task reduction approach (and classical planning framework) in treatment of goals as predicates. Present more general view of preferences, utility functions, tie to goals, point to decision-theoretic analysis of Chapter 7. How will this be coordinated with the introduction of value functions in Chapter 4?

Paragraph moved from task reduction section. It should also be noted that the reduction planning method described above is not able to handle planning problems in which the criteria for a good plan involve minimizing execution time or maximizing income. While finding a solution that minimizes or maximizes some quan:ity is generally computationally complex, it is still useful to be able to compare candidate solutions. The standard technique for comparing candidate solutions is to use a value function to define a metric on the outcomes associated with candidate solutions. The basic idea behind using a value function is simple. Given two candidate solutions (plans), determine the changes over time (referred to as time lines) that are predicted to occur as a consequence of executing each plan. The value function is then applied to the resulting time lines and the plan with the lowest cost (highest value) is determined to be the better of the two. Given a set of candidate solutions, one can then seluct the best. Planning consists of (heuristically) generating a set of casdidate solutions, evaluating each candidate, and selecting the beat. We discuss this sort of planning in the context of reasoning about desdlines and control.-

Generally, chooning an appropriate action requires considering several possible actions and anticipating the consequences of each action. In the case of PID control, the designer does all the neceasary considering and anticipating at deaign time and cimply encodea his findings in the coeficients of the PD controller. This sort of deaign-time compilation is difficult to do in ganer. For instance, finding the shortest tour visiting a set of loc3tions in a factory is a type of problem that might occur frequently for a mobile robot. Computing the colution to even one instance of this type is known to be a hard problem. It would be quite dificult to enumerate and then compute, in advance, the solution to all possible instances of this problem, and, even if you could, it would be difficult if not imponsible to store the 
results of such a prodigious effort on any practical machine.

For any interesting problem, it is impossible or impractic al to write down $\Phi$. In the decision sciences, they never even attempt to; rather, they specify belief functions, preferences, and a utility (or value) function. The notion of task is implicit in whatever maximizes expected utility. ${ }^{16}$ The introduction of beliefs and expectations is crucial here; what constitutes a task depends critically on a given agent's knowledge, which in turn depends upon what the agent has observed, not just at the last clock tick, but over time, and the agent's ability to reason about those observations. The notion of task in AI is similar despite the fact that the use of value functions is not universally accepted.

Normative vs computational theories of decision-making. The decision sciences provide a "normative" theory of decision making, in that any rational decision maker posseased with the same information and unlimited time to reflect on it would come to the same conclusion. AI, starting with Herb Simon's Nobel-prise-winning model of administrative man, has taken the idea of a resource-bounded agent as a starting point [17].

Motivate need for utility in terms of complications involving $\Phi$. Start with preference onder on $\Omega$, then intraduce onder-preserving, reab-valued utility function. Perhaps notation Util is best, by parallel to Val and given that $u$ and $U$ are already taken in the presentation of control. State the obvious problem with reasoning about elements of $\Omega$ and introduce machinery to get around the problem. Introduce a set of time points $T$, and define time lines in terms of functions from $\tau$ to $\Omega$. Redefine $\Phi$ accondingly. Introduce the notion of error-driven control laws in terms of a variant on means/ends analysis. If we allow the reference signal to comespond to an arbitrary world state and the controlled variables to include any condition, then the solution to almost any control problem can be charectericed in terme of a suitable error-driven control law.

Explain how goals fit in with this expressive framework. A goal predicate specifies that a state achieving the goal is preferred to one that does not, all eloe equal. Combining all the expressed goals yields a partial onder on states, with mofinence between competing gouls or alternate ways of achieving the same sad not defined. This suggests that goals do not provide sufficient guidance for rational choice of action. Must augment with more precise specification, either by providing strength of preference or finer-grained descriptions of goal predicates and combinations.

\footnotetext{
10 Just as in clessical plenning it is implicit in what echieves the top-level goal.
} 


\subsection{Further Reading}

The material presented on planning is a distillation of a great deal of research. The need for protections was first identified by Sussman [18], and indeed the simplest example of a problem requiring nonlinear plan construction is known as the "Sussman anomaly." The basic idea of reduction interleaved with resolving interactions originated with Sacerdoti's influential NOAB system [14]. Our development of the task reduction approach follows Charniak and MeDermott [3], who provide a more comprehensive treatment of protections and search algorithms. The reduction algorithm itself is based loosely on Tate's NONLIM [19] (see Vere [20] for extensions to handle metric time constraints). The notion of policy projection is borrowed from McDermott [10].

Pointers to other work on planning, not necessarily taking task reduction approech. Truth criterion: implicit in mech work, made explicit by Chap-

man. Problems for temporal reasoning about nonlinear plans explored by Dean and Boddy. For a discussion of issues in representing and reasoning about resources, see [22, 5]. General discussions of partial plans (Wellman, Hsu?).

Reasoning about knowledge, action, and perception [4, 9, 11, 12] (especially Morgenstern, Moore). Discussion of observable events and test actions follows Wellman. Evaluate with respect to the more general theories (essentially, the latter allow reasoning about how observability of some facts implies observability of others). General capability for reasoning about knowledge in planning an area of active investigation, with many open questions (see Halperm overview in TARK-86, or survey in Annual Review).

The ides of debugging almost right plans is characteristic of many approaches to planning in artificial intelligence $[8,16,18]$.

Reactive planning: AI interest spurred by work of Agre and Chapman [2], Brooks [1], Rocenschein [13], Schoppers [15]. Early example: triangle tables in sTrips.

Goals and utilities: see our discussion [6], also Haddawy and Hanks [7], Loui, now paper. 


\section{Bibliography}

[1] Rodney A. Brooks. A robust layered control system for a mobile robot. IEEE Journal of Robotics and Automation, 2:14-23, 1986.

[2] David Chapman and Philip E. Agre. Pengi: An implementation of a theory of activity. In Proceedings AAA1-87, page 268-272. AAAI, 1987.

[3] Eugene Charniak and Drew V. McDermott. Introduction to Artificial Intelligence. Addison-Wealey, Reading, Maseschusetts, 1985.

[4] Erneat Davis. Inferring ignorance from the locality of visual perception. In Proceedings AAAI-88, pages 786-790. AAAI, 1988.

[5] Thomas Dean and Keiji Kanasewr. A model for reasoning about persistence and causation. Computational Intelligence, 5(3):142-150, 1989.

[6] Thomas Dean and Michael Wellman. On the value of gouls. In Jooh Tenenberg, Jay Weber, and James Allen, editors, Proceedinge from the Rochester Planning Workshop: From Formal Systems to Practical Syotems, pagee 129-140, 1989.

[7] Peter Haddawy and Steve Hanks. Issues in deciaion-theoretic planning: Symbolic goale and numeric utilities. In Proceedings of the DARPA Workshop on Innovative Approaches to Planning, Scheduling, and Contol DARPA, 1990.

[8] It Bammond. Chef: A model of case-based planning. In Proceedings AAAI-86, pagee 267-271. AAAI, 1986.

[9] Kurt Konolige. A Deduction Model of Belief. Pitmen Publishing, London, 1986. 
[10] Drew V. McDermott. Flexibility and efficiency in a computer program for designing circuits. Technical Report 402, MIT AI Laboratory, 1977.

[11] Robert C. Moore. Reasoning about knowledge and action. Technical Report Technical Note 191, SRI International, 1980.

[12] Leora Morgenstern. A first order theory of planning, knowledge, and action. In Joseph Y. Halpern, editor, Theoretical Aspects of Reasoning About Knowledge, Proceedings of the 1986 Conference, pages 83-98, Los Altos, California, 1987. Morgan-Kaufmann.

[13] Stan Rosenschein. Synthesizing information-tracking automata from environment descriptions. In Ronald J. Brachman, Hector J. Levesque, and Zaymond Rejter, editors, Proceedings of the First International Conference on Principles of Knowledge Representation and Reasoning, pagea 386-393. Morgan-Kaufmann, Los Altos, California, 1989.

[14] Earl Sacerdoti. A Structure for Plans and Behavior. American Elsevier, New York, 1977.

[15] Marcel J. Schoppers. Universal plans for reactive robots in unpredictable environments. In Proceedings IJCAI 10, pages 1039-1046. IJ. CAI, 1987.

[16] Reid Simmons and Randall Davis. Generate, test and debug: Combining associational rules and causal models. In Proceedings IJCAI 10, pages 1071-1078. IJCAI, 1987.

[17] Herbert A. Simon. The Sciences of the Artificial MIT Press, Cambridge, Massachusetts, 1981.

[18] Gerald J. Sussman. A Computer Model of Skill Acquisition. American Eleevier, New York, 1975.

[19] Austin Tate. Generating project networks. In Proceedings IJCAI 5, pares 888-893. IJCAI, 1977.

[20] Steven Vere. Planning lu time: Windows and durations for activities and goals. IEEE Thansactions on Pattern Analysis and Machine Intelligence, 5:246-267, 1983.

[21] Michael P. Wellman. Formulation of Tradeoffs in Planning Under Uncertainty. Pitman and Morgan Kaufmann, 1990. 
[22] David E. Wilkins. Domain independent planning: Representation and plan generation. Artificial Intelligence, 22:269-302, 1984. 


\section{Chapter 6}

\section{Uncertainty in Control}

In predicting and controlling the behavior of processes, it is nearly impossible to avoid some degree of uncertainty. Even in cases where an engineer carefully designs a piece of equipment to behave in a particular manner, sourc $:$ of uncertainty are introduced in manufacturing, in the wear on parts during subsequent use, and through unanticipated interaction with the environment. In this chapter and the next, we ronsider various approaches to dealing with uncertainty in planning and control. This chapter focuses on uncertainty issues in the context of control systems engineering.

Here, as elsewhere in this book, we make no attempt to provide a comprehensive survey of techniques. Our objective in this chapter is to make several observations about the nature of control as a problem involving uncertainty, and to introduce two techniques that illustrate key issues.

The first technique involves an approach to recovering the state of a dynamical system from observations of its autput. The general problem was introduced in Chapter 3 in the discussion of system observability. The solution that we consider here, the Kalman filter, is somewhat specialized, but of broad practical import. In the introduction to a collection of papers on the theory and applications $c_{\text {f }}$ the Kalman filter, Sorenson [16] writes that, "It is probably not an overstatement $\bullet 0$ assert that the Kalman filter represents the mont widely applied and demonstrably useful result to emerge from the stata variable approach of 'modern control theory.' " Our introduction to Kalman filtering emphasizes a basic cycle of activity that is central in the application of the Kalman filtering equations, and is applicable to a wide variety of state estimation problems that do not satisfy the assumptions

\footnotetext{
${ }^{\circ}$ C1990 Thoma Dean. All rights reserved.
} 
required for the Kalman filter.

The second technique involves an extension of the dynamic programming approach considered in Chapter $\$$. The extension is concerned with multistage decision problems in which the dynamical system can be modeled as a stochastic process. We introduce the basic theory in this chapter as it is generally considered $s s$ a part of the repertoire of techniques of coutrol. In Chapters $q$ and $\&$ we return to consider the connection between stochastic dynamic programming and various techniques in planning (Chapter 1 ) ara vearning (Chapter 8 ). We begin this chapter by considering just . 0 w deeply the issues involving uncertainty enter into the proble: 'of controlling dynamical systems. Our treatment here follows that of Koditschek [12].

\subsection{Uncertainty and Delay in Dynamical Systems}

In both Chapters 2 and $\$$, we considered a single-degree-of-freedom robot as an example of a simple dynamical system. We continue to resort to such simplified models in this chapter to illustrate our basic points. Let $M$ be the mass of the robot, $z$ its position in some arbitrary frame of reference, and $F$ the force acting upon the robot. As in Chapter we assume that the plane of motion is horizontal and that there are no frictional forces acting on the robot. The relationship between position, $z$, and the force, $\mathcal{F}$, is completely determined by Newton's second law of motion.

$$
M \ddot{z}=\boldsymbol{F}
$$

The state vector for the dynamical system is defined to be

$$
x(i)=\left[\begin{array}{c}
z(t) \\
\dot{z}(t)
\end{array}\right],
$$

and system state equation is

$$
\dot{x}(t)=\left[\begin{array}{ll}
0 & 1 \\
0 & 0
\end{array}\right] x(t)+\left[\begin{array}{c}
0 \\
1 / M
\end{array}\right] u(t)
$$

In the set-point regulation problem, the task is to transfer the robot som its initial location, $z\left(t_{0}\right)$, to some final (goal) location, $z^{*}$, and then keep it there. We begin by giving the controller every advantage in an attempt to avoid the problems introduced by uncertainty. In particular, we assume that the control actuator can exert an arbitrary amount of force, $\mathcal{F}(t)$, at an 
instant in time, $t$. We model this using the Dirac delta (impulse) function defined by

$$
\int_{-\infty}^{\infty} \delta_{\tau}(t) d t=1
$$

where

$$
\delta_{r}(t)=0 \quad \forall t \neq \tau,
$$

so that our actuator is able to deliver a pulse of infinite magnitude over an infinitesimally short interval of time possessed of unit area and involving a finite amount of energy.

The controller begins by getting the robot headed in the right direction, namely towards the goal, $z^{*}$. We measure the current position and velocity,

$$
\mathbf{x}\left(t_{0}\right)=\left[\begin{array}{c}
z\left(t_{0}\right) \\
\dot{z}\left(t_{0}\right)
\end{array}\right]
$$

and at the same instant apply an impulse defined by

$$
u_{\text {start }}(t)=M\left(1-\dot{z}\left(t_{0}\right)\right) \delta_{t_{0}}(t) .
$$

The impulse has the effect resetting the initial conditions so that

$$
x(t)=\left[\begin{array}{c}
t+z\left(t_{0}\right) \\
1
\end{array}\right] \text { for } t>t_{0}
$$

and the goal position is achieved at time $t^{*}=z^{*}-z\left(t_{0}\right)$. At $t^{*}$, we apply a force to exactly cancel the velocity achieved by the first impulse. The second impulse is defined by

$$
u_{\text {itop }}(t)=-M \dot{\delta}_{t} \cdot(t)
$$

The control strategy defined by

$$
u(t)=u_{\text {atart }}(t)+u_{\text {atop }}(t)
$$

provilm a colution to our idealized set-point regulation problem. In addition to the aremptions made regarding the Dirac impulse function, this solution relie on the following assumptions.

- We krow the exact mass, $M$, of the robot.

- We can instantaneously and exactly measure the robot's position, z, and velocity, $\dot{z}$. 
- We can instantaneously perform all calculations required for control.

- We can exactly measure the elapsed time in order to sequence the velocity canceling impulse.

If any one of the above assumptions fails to hold, then some error will be introduced and this error will become magnified with the passage of time. For instance, suppose that there is some error in the estimate, $\dot{M}$, used for : the mass, $M$. If we apply the same control strategy as before, we obtain

$$
\mathbf{x}(t)=\left[\begin{array}{c}
z\left(t_{0}\right)+\left[\frac{\dot{M}}{M}+\left(1-\frac{\dot{M}}{M}\right) \dot{z}\left(t_{0}\right)\right] t^{*}+\left(1-\frac{\dot{M}}{M}\right) \dot{z}\left(t_{0}\right) t \\
\left(1-\frac{\dot{M}}{M}\right) \dot{z}\left(t_{0}\right)
\end{array}\right] \forall t>t^{*},
$$

where we have substituted $\hat{M}$ for $M$ in the specification of $u_{\text {utar }}$ and $u_{\text {stop. }}$ From this description of the system state, it should be apparent that small inaccuracies in estimating $M$ will result in finite and increasing error in the position of $z$ relative to the goal $z^{*}$. Similar errors would occur due to imprecision in measuring the position or velocity at $t_{0}$.

This simple example is meant to illustrate hnw deeply the issue of uncertainty is rooted in the problems of control. Koditschek [12] writes in the same article from which we adapted the above analysis, "The origins of control theory, then, rest in the following observations. Dynamic systems give rise to delay that must be taken into account by any control strategy regardless of available actuator power or sensor accuracy. Moreover, information regarding the real world is inevitably uncertain and may have an adverse effect on performance no matter how small the uncertainty or powerful and accurate the apparatus."

As was pointed out in Chapter \&, feedback control strategies achieve their robust performance because they continuously account for the error between the measured state of the system and the goal state. Such feedback control systems tend to compensate for measurement and modeling errors. If the measurement and modeling errors systematically mislead the controller, then parformance will most certainly be poor; however, feedback controllers ofter perform well in the presence of certain benign forms of random errors. In the following section, we consider a class of problems for which it is possible to design a module to estimate the system state. This module can be coupled to a deterministic feedback regulator to obtain a controller that is optimal by most accepted criteria. 


\subsection{State Estimation}

Suppose that you are designing a system to control the movements of a mobile robot that has to navigate in an office or industrial environment. If you could obtain the exact geometric description for the surfaces of the objects in the surrounding environment, then you could use the planning and control algorithms described in the last section of Chapter 3 , $\mathrm{br}$ any of a host of other deterministic control strategies to guide the robot on its appointed rounds. Using path planning methods and an exact geometric model for navigation requires that the robot not err in its movement or that the robot correct for errors in movement by reestablishing its position and orientation with respect to the geometric model. This process of reestablishing position and orientation with respect to a geometric model is called registration or localization in the literature. To help generate a geometric model or maintain registration with an existing model, suppose that the robot has been equipped with a variety of sensors: ultrasonics, infrared, inertial guidance, compass, odometry, laser ranging, tactile sensing. Unfortunately, all of these sensors are prone to errors. In this section, we consider how to design an algorithm ${ }^{3}$ that combine (fuseq) the data from all of the sensors, accounting for their tendency to err, to provide as accurate a picture of the geometry of the robot's environment as is possible from the data supplied.

Consider the following problem in fusing data from different sensors. Suppose we are interested in the distance from the robot to the nearest obstacle surface in the direction the robot is traveling. Sensor 1 reports that the distance is 2 meters, but Sensor 2 reports 5 meters, and Sensor 3 pretty much agrees with Sensor 2, reporting 5.15 meters. The close agreement of two of the sensors would suggest relying on a value close to 5 meters, but it may be that Sensors 2 and 3 are wrong quite often, even systematically

$i$ wepg, while Sensor 1 is hardly ever wrong. Without additional information about the sensors, it is difficult to know what to do with conflicting evidence. Howner, if we have prior knowledge about the errors that can be expected from different sensors, then we may be able to combine the data in a dinclinad, perhaps even optimal manner.

1 tis following, we adopt a Bayesian perspective, and represent our knowlodoe about sensor errors in terms of conditional probabilities. In particular, if $x \in R^{n}$ represents the system state vector, and $z \in \mathbf{R}^{\mathbf{m}}$ represents the measurement vector providing information about $x$, then we represent our knowledge about the performance of the sensors that produced $z$ as a conditional probability density function, $p(\mathbf{x} \mid \mathbf{z})$, indicating the probabil-

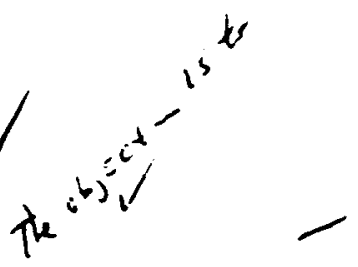




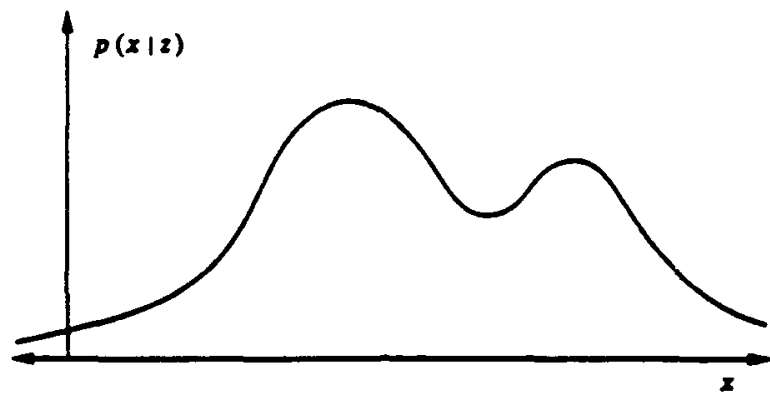

Figure 6.1: The conditional probability density for $I$ given $z$

ity that $X$ is the true state of nature given that we have observed 2 . For a scalar $x$, the density function might take the form shown in Figure 6.1. More generally, given a discrete dynamical system

$$
\begin{aligned}
\mathbf{x}(k+1) & =f(\mathbf{x}(k), \mathbf{u}(k)) \\
\mathbf{z}(k) & =h(\mathbf{x}(k)),
\end{aligned}
$$

where $h$ is a measurement function, we will want to calculate a density function of the form

$$
p(\mathbf{x}(k) \mid z(1), z(2), \ldots, z(k)),
$$

where $z(t)$ indicates the measurements made at time $t$.

Given a conditional probability density function, we wish to determine an estimate of the system state, denoted $\dot{x}$, to be used for control purposes. Possible candidates for such an estimate are the average or mean of the probability distribution corresponding to the density, the peak or mode of the distribution, and the median of the distribution. ${ }^{1}$

In the following, we assume a linear dynamical system corrupted by "white Gavecian" noise. The assumption that the noise be white requires that the noise value not be correlated in time (i.e., knowledge of the value of the aim at one point in time tells you nothing about the value of the noise at later timen). ${ }^{2}$ The assumption that the noise be Ganssian requires that

\footnotetext{
'For a ccalar quantity, the median is that value of a such that half of the probability man lies to the left of it and half to the right.

${ }^{2}$ Whitenea aloo requires that the noive have equal power at all frequenciea; a requirement that in imposable to achieve in practice given that all real phyacal aytems respond
} 
the probability density for the amplitude of the noise at any particular point in time take on the familiar bell-shaped curve of a Gaussian distribution. ${ }^{3}$

The assumption of Gaussian noise is often justified by observing that, if :the noise is generated by a large number of separate processes, then the sum of their effect can be approximated by a Gaussian distribution. However, the most compelling reason for accepting the assumption of white Gaussian noise is the same as that for accepting the assumption of linearity, namely, it makes the mathematics tractable. As an example of how the Gaussian assumption simplifies things, a Gaussian distribution is completely determined by its first- and second-order statistics, its mean and variance. The Gaussian assumption will also simplify our choice for an estimate of the state given the density; under the assumption of Gaussian noise, the mean, mode, and median all coincide. What is surprising is that, despite the fact that the assumptions seldom if ever are met in dealing with real physical systems, the basic methods that we describe in the sequel have met with extraordinary success in practice [16].

To make our assumptions explicit in the model, we represent the state of the system at time $k+1$ by

$$
\mathbf{x}(k+1)=f(\mathbf{x}(k), \mathbf{u}(k))+\mathbf{v}(k),
$$

where $f$ models the response of the dynamical system to a given input, and $\mathbf{v}(k)$ is a vector of zero-mean, white, Gaussian noise processes, modeling the input disturbance or process noise. Let $z(k)$ represent the (observable) output of the system at time $k$, so that

$$
z(k)=h(x(k))+\mathbf{w}(k),
$$

where $h$ models the physics of the measurement process and $w(k)$ is a vector of zero-mean, white, Gaussian noise processes, modeling the measurement

only within a narrow range of frequenciea called the system bandpans. For practical purposea, however, the noive will often behave as if white within the bandpan of the aystem. In cartain cans in which the noise is not constant over the system bandpas or is correlated a the, apecial "ahaping filter" can be added to the aystem to achieve a model of a dy

In Con in or nomal dintribution, $N\left(\mu, \sigma^{2}\right)$, for a (ecalar) random rariable, $I$, with ane and rariance $\sigma^{2}$ ( $\sigma$ denoten the standard deviation) in characterized by the normal probability dencity:

$$
p(x)=\frac{1}{\sqrt{2 \pi \sigma}} \exp \left[-\frac{(x-\mu)^{2}}{2 \sigma^{2}}\right] .
$$




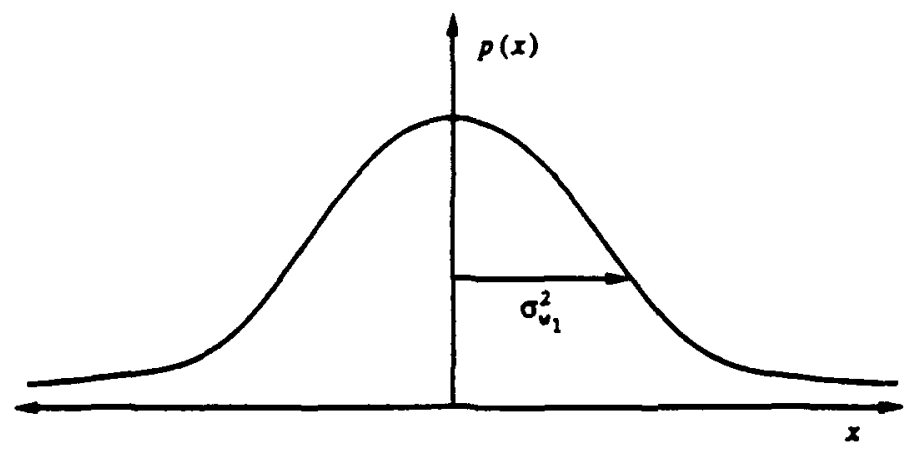

i.

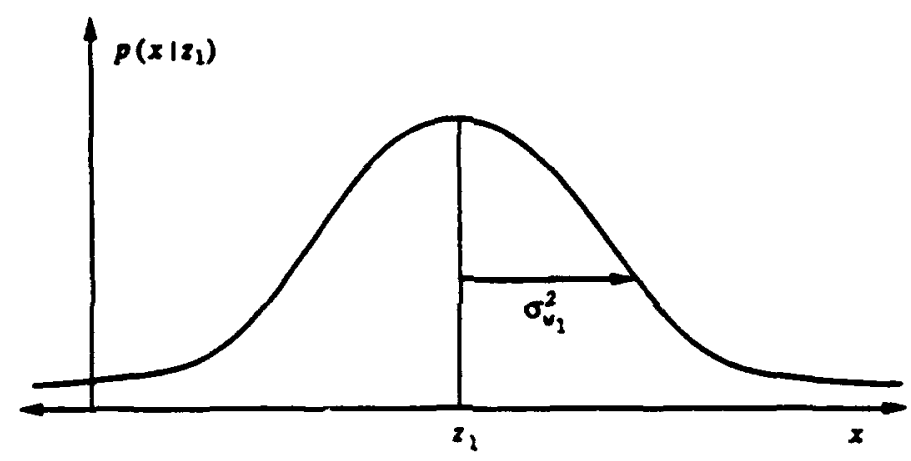

ii.

Figure 6.2: The densities for (i) the zero-mean Gaussian distribution $N\left(0, \sigma_{w_{1}}^{2}\right)$ modeling the measurement noise for the first sensor, and (ii) the Gaussian distribution $N\left(z(1), \sigma_{w_{1}}^{2}\right)$ modeling the measurement itself.

erros. Before we write down the equations for the Kalman filter, we consider some aimple examples adapted from Maybeck [14] to illustrate the basic issuce.

We return to our single-degree-of-freedom robot, moving back and forth on a horizontal track. Here we use the scalar $x$ to represent the state of the system corresponding to the position of the robot on the track. Suppose that there are two sensors that allow the robot to obtain measurements its position. Each of the two sensors returns an estimate of the robot's 
location corrupted by Gaussian noise: $N\left(0, \sigma_{w_{1}}^{2}\right)$ in the case of the first sensor and $N\left(0, \sigma_{u_{2}}^{2}\right)$ in the case of the second. At time 1 , the first sensor is deployed to obtain a measurement $z(1)$ of the robot's position. We model the measurement as a sum of the robot's actual position and the zero-mean Gaussian noise process shown in Figure 6.2.i. The conditional probability density for the actual position, $x$, given the measurement, $z(1)$, is shown in Figure 6.2.ii. The mean of the distribution is just $z(1)$ in this case, and the variance, $\sigma_{w_{1}}^{2}$, is rather large, indicating a sensor with significant potential for error.

Based on the density shown in Figure 6.2.ii, the best estimate of the robot's position is

$$
\hat{x}(1)=z(1),
$$

and the variance of the error in the estimate is

$$
\sigma_{x}^{2}(1)=\sigma_{w_{1}}^{2} .
$$

At time 2, following the first measurement and assuming that the robot has not moved, you obtain a second measurement, $z(2)$, from the second, and generally more reliable of the two sensors. The fact that this second sensor is generally more reliable is indicated by the density for the second measurement being more peaked (having a smaller variance) than the density for the first measurement as shown in Figure 6.3.i. In this case, the mean of the distribution is $z(2)$, and the variance is $\sigma_{w_{2}}^{2}$.

We can combine the two measurements to obtain a conditional density for the position of the robot given both measurements. The result is a Gaussian density, $N\left(\mu, \sigma^{2}\right)$, with mean, $\mu$, given by

$$
\mu=\left[\frac{\sigma_{u_{2}}^{2}}{\sigma_{w_{1}}^{2}+\sigma_{w_{2}}^{2}}\right] z(1)+\left[\frac{\sigma_{w_{1}}^{2}}{\sigma_{w_{1}}^{2}+\sigma_{w_{2}}^{2}}\right] z(2)
$$

and variance, $\sigma^{2}$, given by

$$
\sigma^{2}=\frac{\sigma_{w_{1}}^{2} \sigma_{w_{2}}^{2}}{\sigma_{w_{1}}^{2}+\sigma_{w_{2}}^{2}} .
$$

Figan a.3.ii depicts the resulting density superimposed over the densities for each of the individual measurements. Notice that $N\left(\mu, \sigma^{2}\right)$ is more peaked than either of the densities for the measurements taken separately. Given $N\left(\mu, \sigma^{2}\right)$, the best estimate for the robot's position at time 2 is

$$
\hat{\boldsymbol{x}}(2)=\mu,
$$




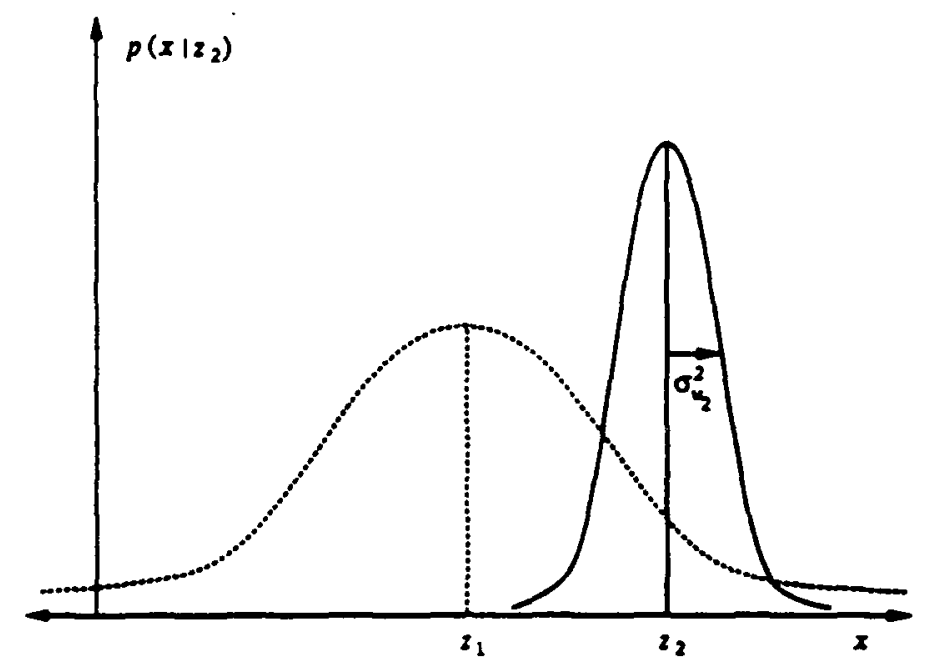

i.

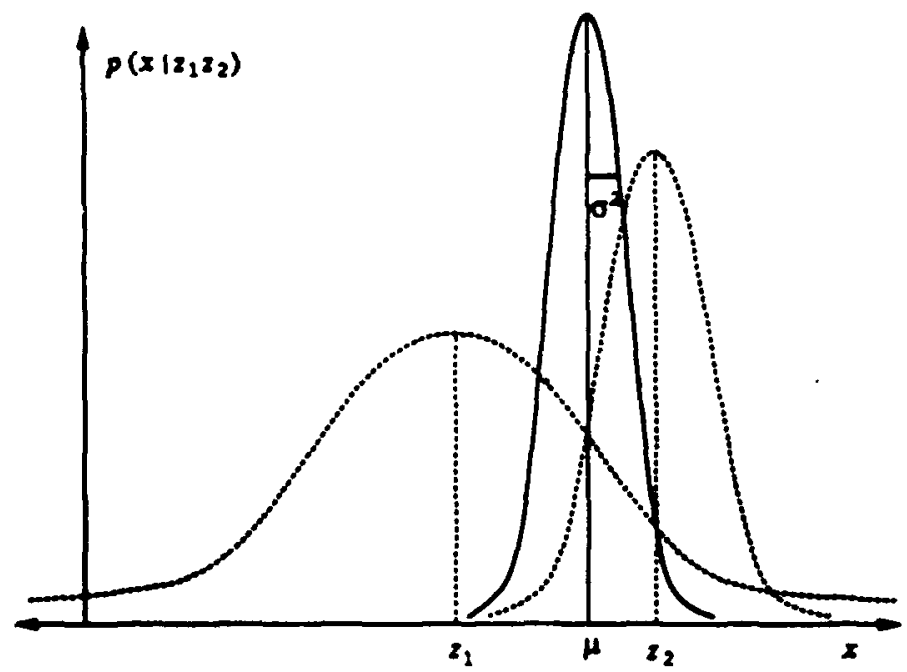

ii.

Figure 6.3: The densities for (i) the second measurement superimposed over the first, and (ii) the combined measurements superimposed over the first and second. 
with -a associated error variance

$$
\sigma_{x}^{2}=\sigma^{2}
$$

We will not provide a proof that this is the best estimate. We will, however, provide some intuitions as to why it is a plausible estimate.

The variances provide information to assist in establishing the relative weight to attach to the evidence from the previous measurement(s) and that from the latest measurement. If the two variances are equal, then the two measurements are equally reliable and we simply take their average. If, on the other hand, the variance for the previous measurement(s) is large and the variance for the latest measurement small, then we give more weight to the latest measurement. The variance will always decrease in the case of two or more measurements taken at the same time, reflecting the fact that additional (consistent) information should serve to sharpen the estimate. Casting the problem of state estimation in terms of optimizaiion, the recursive update algorithm described in this section is optimal in the sense that it minimizes the variance. ${ }^{4}$

To adopt the form generally used in describing the Kalman filter, we rewrite the equation for $\hat{x}(2)$,

$$
\begin{aligned}
\hat{x}(2) & =\left[\frac{\sigma_{u_{2}}^{2}}{\sigma_{w_{1}}^{2}+\sigma_{w_{2}}^{2}}\right] z(1)+\left[\frac{\sigma_{w_{1}}^{2}}{\sigma_{w_{1}}^{2}+\sigma_{u_{2}}^{2}}\right] z(2) \\
& =z(1)+\left[\frac{\sigma_{u_{2}}^{2}}{\sigma_{w_{1}}^{2}+\sigma_{w_{2}}^{2}}\right](z(2)-z(1))
\end{aligned}
$$

and, substituting $\hat{x}(1)$ for $z(1)$, we obtain

$$
\hat{x}(2)=\hat{x}(1)+K(2)(z(2)-\hat{x}(1)),
$$

\footnotetext{
'The variance is just the expectation of error. In the case of no prior expectations, we want to find the eatimate, $s$, minimizing the mean of the aquared error,

$$
\frac{1}{n} \sum_{i=1}^{n}\left(i-x_{i}\right)^{2}
$$

whes of si are the meanrements. We obtain this eatimate by cetting the derivative to zero,

$$
\frac{h_{1} \sum_{i=1}^{n}\left(z-x_{i}\right)^{2}}{d z}=2 \sum_{i=1}^{n}\left(z-x_{i}\right)=0 \text {, }
$$

and colving for $\dot{x}$. The eatimate provided by the method deacribed bere is juat the mean of the meaurements, $\frac{1}{n} \sum_{i=1}^{n} x_{i}$, which is a colution to the above equation.
} 


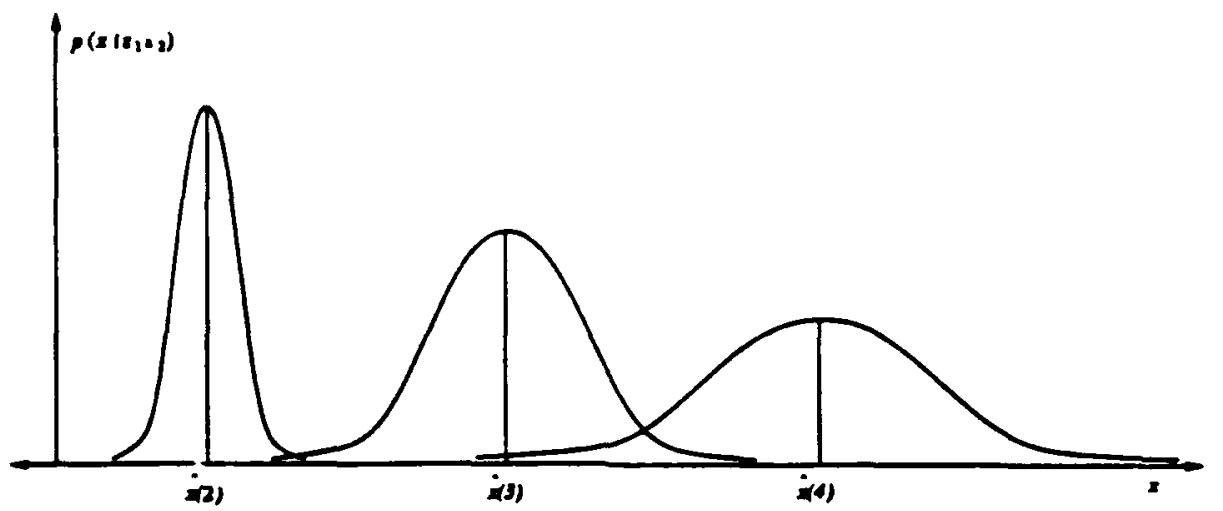

Figure 6.4: Evolving state estimates without additional measurements

where $K(2)$ is defined as

$$
K(2)=\frac{\sigma_{u_{2}}^{2}}{\sigma_{w_{1}}^{2}+\sigma_{u_{2}}^{2}}
$$

Our objective is to provide an algorithm that computes an estimate of the evolving state of a dynamical system. We have not as yet made any real use of the equations describing the dynamical system. The method of combining measurements in the static case is generally referred to as minimum mean-square estimation, and is attributed to Carl Friedrich Gauss (17771855). The primary contribution of Kalman and the other researchers who developed and refined the Kalman filter is the recursive solution of minimum mean-square state estimation problems involving dynamical systems.

Given an estimate of the system state at time $t$, we wish to compute an estimate of system state at time $t+1$, which accounts for the most recent measurements and also for the system dynamics. Continuing with our example, we assume the following simple dynamics

$$
x(k+1)=x(k)+u(k)+v(k),
$$

where $x(t)$ is the distance moved, and $v(t)$ is a zero-mean, white, Gaussian noise process with variance, $\sigma_{v}^{2}$.

We denote the estimate of the system state at time 3 given only the measurements taken at time 2 or earlier as $\dot{x}(3 \mid 2)$ defined by

$$
\hat{x}(3 \mid 2)=\hat{x}(2)+u(2),
$$


with corresponding variance

$$
\sigma_{x}^{2}(3 \mid 2)=\sigma_{z}^{2}(2)+\sigma_{v}^{2} .
$$

If we made no additional measurements, the estimate of the system state would degrade over time, as shown in Figure 6.4. In general, however, we will make at least one measurement at every time step. To incorporate measurements taken at time 3 , we employ the same basic equations used for combining $z(1)$ and $z(2)$.

Generalizing the previous examples, we present the Kalman filtering equations for the following one-dimensional dynamical system,

$$
\begin{aligned}
x(k+1) & =f(x(k), u(k))+v(k) \\
z(k) & =h(x(k))+w(k),
\end{aligned}
$$

where $x, u$ and $z$ are scalar quantities, $f$ and $h$ are linear functions, and $v$ and $w$ are zero-mean, Gaussian noise processes with associated variance, $\sigma_{v}^{2}$ and $\sigma_{w}^{2}$ respectively. Since $f$ and $g$ are linear we can rewrite the above equations as

$$
\begin{aligned}
x(k+1) & =C_{1} x(k)+C_{2} u(k)+v(k) \\
z(k) & =C_{3} x(k)+w(k),
\end{aligned}
$$

where $C_{1}, C_{2}$, and $C_{3}$ are constants. We assume exactly one measurement taken at each time step.

Recall that the objective is to maintain an estimate of the state of the system at all times. The estimate of the system state at time $k$ given all of the measurements up until time $j$ is denoted $\hat{x}(k \mid j)$. Similarly, we denote the variance in the estimate at time $k$ given all of the measurements up until time $j$ as $\sigma_{x}^{2}(k \mid j)$. We write $\bar{x}(k \mid k)$ and $\sigma_{x}^{2}(k \mid k)$ simply as $\bar{x}(k)$ and $\sigma_{x}^{2}(k)$. At each time $k$, all of the past measurements are summarized by the estimate, $\hat{x}(k)$, and its associated variance, $\sigma_{x}^{2}(k)$.

There are three basic steps performed in updating the estimate of the syste atate to reflect the measurement made at $k+1$. These steps are refarred to as the prediction, observation, and estimation steps. We consider each of theat in thifn.

In the prediction step, we compute what we expect to observe at $k+1$. This involves first computing an estimate of the state at $k+1$ given all the measurements at time $k$ or earier, dofined by $b$

$$
\hat{x}(k+1 \mid k)=C_{1} \hat{x}(k)+C_{2} u(k) .
$$


The variance associate with this estimate is

$$
\sigma_{x}^{2}(k+1 \mid k)=\sigma_{2}^{2}(k)+\sigma_{v}^{2}
$$

Notice that the control is not considered in computing the variance. The predicted measurement is then

$$
\hat{z}(k+1 \mid k)=\omega_{\varnothing} \hat{x}(k+1 \mid k)
$$

and the variance associated with the predicted measurement is

$$
\sigma_{z}^{2}(k+1 \mid k)=\psi_{4}^{2} \sigma_{x}^{2}(k+1 \mid k)+\sigma_{w}^{2}
$$

In the observation step, we make the observation and then compare the resulting measurement with what we expected. The difference between the actual and predicted measurement, $V$

$$
\nu(k+1)=z(k+1)-\hat{z}(k+1 \mid k),
$$

is called the innovation.

In the third and final step, called the estimation step, we compute $\hat{x}(k+1)$ as

and the associated variance as

$$
\dot{x}(k+1)=\hat{x}(k+1 \mid k)+K(k+1) \nu(k+1),
$$

$$
\sigma_{x}^{2}(k+1)=\sigma_{x}^{2}(k+1 \mid k)-\gamma(K(k+1))\left(\sigma_{x}^{2}(k+1 \mid k),\right.
$$

where $K(k+1)$ is called the filter gain and defined by

$$
K(k+1)=\frac{\operatorname{gog}_{x}^{2}(k+1 \mid k)}{\sigma_{z}^{2}(k+1 \mid k) \cdot t \sigma_{x}^{2}(k+1 \mid k)}
$$

It should be noted that we have to invert the measurement function in order to compute the filter gain. In general, this inversion can be difficult if not imponibl. However, for linear systems, inversion simply involves taking a recipsued in the scalar case or inverting a matrix in the vector case.

A good way of convincing yourself that these equations make sense is to consider limiting cases. For instance, consider cases in which the there is no error in movement or measurement (i.e., $\sigma_{v}^{2}$ and $\sigma_{w}^{2}$ are 0 ) or cases in which $C_{1}, C_{2}$, and $C_{3}$ are 1 . 
In the above, we made use of nodels for predicting not only the current and future states of the system, but also the current and future measirements made in observing the system. These models account for uncertainty in the underlying process by incorporating probabilistic noise models for disturbances in the dynamical system and errors in measurement. At each point in time, we compare what we expect to observe with what we actually observe in order to determine how much weight to attribute to each, based on the sort of errors we expect from the corresponding noise models.

Extending the above equations to handle finite vector spaces and multiple measurements is reasonably straightforward though notationally tedious, and we will not attempt it here. Instead of the mean and variance of the distribution of a single random variable, it is necessary to generalize to the mean and covariance of a multidimensional distribution of a vector of random variables. 3 Once you understand the equations for the singledimensional case, it is relatively easy to understand the multidimensional case. It is quite another matter, however, to apply the equations to real problems which invariably deviate from the assumptions stated above. In the following, we consider some of the issues that arise in the application of the Kalman filter to robotics problems.

In many problems in robotics, linearity is hard to come by and one has to appeal to an extension of the Kalman filter designed to handle nonlinear state equations. For instance, in the case of even the simplest holonomic (turn-in-place) mobile robot, the state vector might consist of the robot's position along the $x$ axis, its position along the $y$ axis, and its orientation, $\theta$, all specified with respect to some coordinate frame of reference in the

\footnotetext{
${ }^{5}$ For a vector, $x$, of $n$ random variables the n-dimensional normal (Gencaina) density is defined by

$$
p(x)=\frac{1}{(2 \pi)^{\frac{2}{3}}|P|^{\frac{1}{3}}} \exp \left[-\frac{1}{2}(x-\mu)^{\prime} P^{-1}(x-\mu)\right],
$$

where $\mu$ and $P=E[(x-\mu)(x-\mu)]$ are the mean and covariance of the vector $x$, and the prime $\left(a\right.$ in $\left.(x-\mu)^{\prime}\right)$ indicates vector (or matrix) transponition. The covariance of two randon verables, $x$ and $y$, indicates the degree to which $x$ is related to $y$, and is defined by

$$
E[(x-E(x))(y-E(y))]=E[x y]-E[x] E[y] \text {. }
$$
}

The cuminace (matrix) of the $n$ dimensional vector, $x$, in the symmetric matrix whose ijth eatry is the conriance of the ith and $j$ th componente of $x$. 
workspace:

$$
\mathbf{x}(k)=\left[\begin{array}{c}
x(k) \\
y(k) \\
\theta(k)
\end{array}\right],
$$

where we notate the state vector, $x$, using a bold font to distinguish it from the state variable corresponding to position along the $x$ axis. The input vector in this case is just

$$
\mathbf{u}=\left[\begin{array}{c}
D(k) \\
\Delta \theta(k)
\end{array}\right]
$$

where $D(k)$ is the distance traveled in a single time step, and $\Delta \theta(k)$ is the rotation turned through in a single time step. We can write the state equation as

$$
\begin{aligned}
\mathbf{x}(k+1) & =f(\mathbf{x}(k), \mathbf{u}(k))+\mathbf{v}(k) \\
& =\left[\begin{array}{c}
x(k)+D(k) \cos \theta(k) \\
y(k)+D(k) \sin \theta(k) \\
\theta(k)+\Delta \theta(k)
\end{array}\right]+\mathbf{v}(k),
\end{aligned}
$$

which is clearly nonlinear.

The standard approach to dealing with such nonlinearities is to linearize the state equation by expanding the nonlinear function in Taylor series around the current estimate, $\dot{\mathbf{x}}$, with terms up to first or second order to obtain, respectively, the first- or second-order extended Kalman filter. In the case of the first-order extended Kalman filter for the nonlinear state equation above, we would have

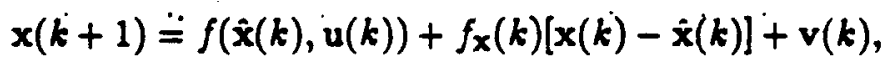

where $f_{\mathbf{X}}(k)$ is the Jacobian matrix ${ }^{6}$ of $f$ defined by

$$
f_{X}(k)=\left[\begin{array}{ccc}
1 & 0 & -D(k) \sin \theta(k) \\
0 & 1 & D(k) \cos \theta(k) \\
0 & 0 & 1
\end{array}\right] .
$$

TI Jecobian in to vector-valued functions what the gradient is to calar-valued function. If $f$ is a rector-rileed fuaction,

$$
f(x)=\left[\begin{array}{c}
f_{1}\left(x_{1}, x_{2}, \ldots, x_{n}\right) \\
f_{2}\left(x_{1}, x_{2}, \ldots, x_{n}\right) \\
\vdots \\
f_{m}\left(x_{1}, x_{2}, \ldots, x_{n}\right)
\end{array}\right],
$$


Generally, the measurement functions are also i.onlinear and require similar linearization. Having obtained the necessary linearizations, we then proceed as in the linear case, and hope that the resulting approximations will provide acceptable state estimates.

Modeling sensors so as to satisfy the Gaussian noise requirement is another problem frequently encountered in robotics applications. Most sensors cannot be modeled as simple functions of one or more of the state variables corrupted with Gaussian noise. Consider, for example, some of the problems that arise in modeling ultrasonic (sonar) sensors of the sort typically found on mobile robots.

A sonar sensor consists of an ultrasonic transducer, a receiver, and some signal-processing hardware. Information about the distance from the senscr to nearby surfaces is obtained by measuring the round-trip time of flight of an ultrasonic pulse that is emitted by the transducer, bounces off an object surface, and returns to the receiver.

If the transducer is pointed along a line perpendicular to a nearby planar surface, then the sensor can be modeled as ti.e actual distance vo the surface corrupted by zero-mean Gaussian noise However, if the transducer is not pointed perpendicular to the nearest object urface, then there is some chance that not enough of the energ: from the ultrasonic pulse will be returned to the receiver to de...mine the true time of flight to the nearest surface. Instead, the pulse may be reflected, bouncing off possibly several objects before a signal with enough energy is cetected by the receiver. In this case, the information returned by the sensor may deviate significantly from the distance to the nearest " jject. Figure 6.5 (from [13]) shows the range data sbtained from a single sensor rotated $360^{\circ}$; the range data is superimposed over a line drawing of the room in which the sensor is located.

If you know tiat your sensor is pointing perpendicular to a planar surface, then you can use the Kalman filtering equations to obtain a good estimate of the distance separating the robot from the surface. The problem, of course, is that it is generally very difficult to know that you are

then its Jecobian matris is defined by

$$
\frac{\partial f(x)}{\partial x}=\left[\begin{array}{cccc}
\partial f_{1} / \partial x_{1} & \partial f_{1} / \partial x_{2} & \cdots & \partial f_{1} / \partial x_{n} \\
\partial f_{2} / \partial x_{1} & \partial f_{2} / \partial x_{2} & \cdots & \partial f_{1} / \partial x_{n} \\
\vdots & \vdots & & \vdots \\
\partial f_{m} / \partial x_{1} & \partial f_{m} / \partial x_{2} & \cdots & \partial f_{m} / \partial x_{n}
\end{array}\right] .
$$




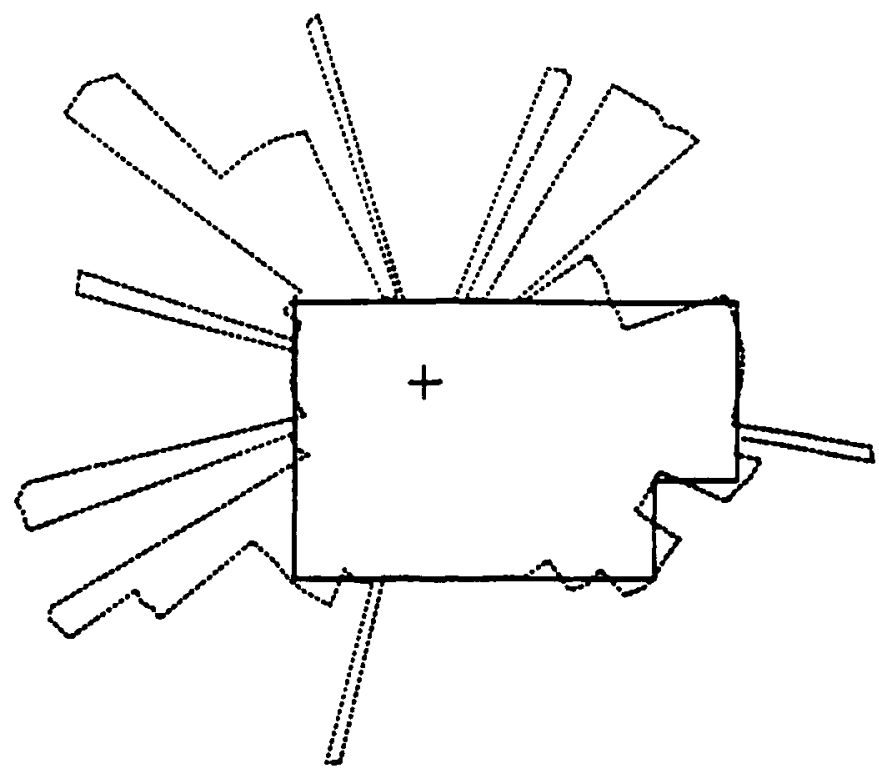

Figure 6.5: A $360^{\circ}$ sonar scan of an indoor environment

pointing perpendicular to a planar surface.

If you have some a priori knowledge about the surfaces of the objects in the form of a map, then you can often make good guesses abont what surfaces are out there and align your sensors so as to obtain reliable range data. In the following, we outline some basic steps in sonar guided navigation using an existing map and the Kalman filter.

1. Consult the map and extract some number of beacons corresponding to geometric features found in the map. This process of extracting beacons involves using the current estimate of the robot's state (position and orientation with respect to the frame of reference of the map). Uecful geometric features are those whose sonar signature is distinctive. Flat walls (planar surfaces), round columns (cylindrical surfaces), and corners (intersection of planar surfaces) are examples of geometric features with distinctive sonar signatures. Having obtained a set of candidate beacons, we attempt to ascertain if they really stand in the expected relationship to the robot (and ultimately to one another).

2. For each candidate beacon, construct a model for the measurements that would be obtained from the sensor if the beacon was in the rel- 
ative position and orientation predicted by the map. Note that the model may require that the sensor be aligned with the bezcon in some particular configuration to avoid errors due to multiple reflections. We assume that there is a library of parameterized models, one for each type of geometric feature deemed useful. The model for a particular candidate beacon is obtained by instantiating one of the parameterized models using relative position and orientation information from the map. There would be a separate model for each beacon of the form

$$
\mathbf{z}_{i}(k)=h_{i}(\mathbf{x}(k))+\mathbf{w}_{i}(k),
$$

where $h_{i}$ is the nonlinear measurement function for the ith candidate beacon, and $w_{i}$ models the measurement noise. Using the estimated state $\dot{\mathbf{x}}(k+1 \mid k)$, we obtain a prediction for each observation

$$
\dot{\mathbf{z}}_{i}(k+1 \mid k)=h_{i}(\dot{\mathbf{x}}(k+1 \mid k)) \text {. }
$$

3. We now make the next observations, using heuristic strategies where appropriate in an attempt to align the sensors according to the requirements of the corresponding model. ${ }^{7}$ Given the actual and predicted observations, we compute the innovation

$$
\nu_{i}(k+1)=z_{i}(k+1)-\dot{z}_{i}(k+1 \mid k),
$$

and the corresponding prediction variance which is obtained by linearizing the $h_{i}$. Up until this point, we have essentially followed the basic steps of the Kalman filter. However, in the next step, we deviate somewhat.

4. We have only hypothesized the existence of the candidate beacons, and we could easily turn out to be mistaken. Because of the possibility of making mistakes in identifying beacons, we cannot immediately use the innovations and their associated variances to obtain $\dot{\boldsymbol{x}}(k+1)$. It will not hurt if we are off a bit in our estimation of the geometric feature's relative location and orientation; the Kalman filtering

\footnotetext{
'Idedy the robot would be equipped with several independent rotating cencor arrays. Each array would consint of a pair of ultraconic sensors mounted at come amall dintance apart on a rigid platform wo that the two sensors are always pointing in the same direction. Each candidate beacon would be axigned an arras and the beacos conld then be aligned with the bescon onfiace(s) using a feedback controller that exploits the diference between values returned by the two eensore.
} 


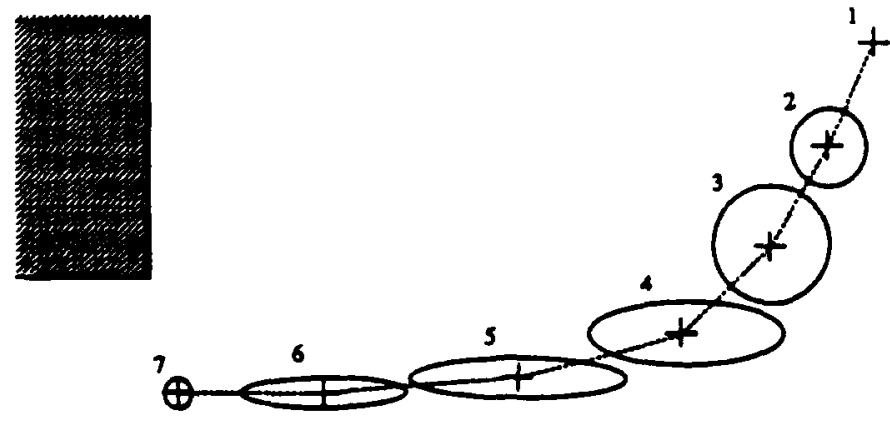

Figure 6.6: Localization using the extended Kalman filter

equations will weight the new measurements appropriately and, over time, the estimate should converge to the actual state. However, if the measurements are due not to the hypothesized beacon but rather to some other geometric feature, then incorporating those measurements into the state estimate using the Kalman filtering equations will lead to significant estimation errors. To avoid such errors, we subject the observations to the following test. We determine a range of possible values for each beacon such that, if the beacon is actually present, then the measurement will fall within that range with some reasonably high probability. We select only those measurements that fall within the range determined by the specified threshold probability.

5. Finally, we compute the latest estimate as

$$
\hat{\mathbf{x}}(k+1)=\hat{\mathbf{x}}(k+1 \mid k)+\sum_{i=1}^{m} K_{i}(k+1) \nu_{i}(k+1),
$$

where $K_{i}$ is the filter gain (matrix) for the ith measurement out of the $m$ menourements obtained in the previous step.

Th approach sketched above is conceptually quite simple but somewhat tricky to implement for a real robot. Determining an appropriate threshold probability requires a certain amount of experimentation. Achieving proper alignment is difficult in the case of highly specular (glossy) metal or painted surfaces. Unexpected objects, either moving or fixed but not accounted for in the map, can cause problems. If, however, there are plenty of potential 
beacons and there are enough sensors to track several of them at any o..e time, then quite robust performance can be achieved.

Figure 6.6 illustrates how the method described above would perform in a particular environment. The robot's location in the plane is represented at 7 discrete points in time. Initially, the robot knows its exact location with respect to the frame of reference of the global map. In the next two time steps, its estimated position becomes increasingly uncertain due to . movement errors. This uncertainty is represented in Figure 6.6 in terms of ellipses corresponding to contours of constant probability of the error distribution. We assume that at time points 2 and 3 the robot is not tracking any beacons. At time point 4 , the robot acquires a beacon corresponding to the wall shown at the bottom of Figure 6.6. This beacon allows the robot to decrease its uncertainty with respect to the $y$ axis. The robot continues to track the wall beacon thereby obtaining an increasingly more accurate estimate for its position with respect to the $y$ axis. At time point 6 , the robot acquires the beacon corresponding to the corner at the left of Figure 6.6, obtaining more accurate estimates for its position with respect to the $x$ axis.

This example illustrates a special case of a more general approach employing the Kalman filter as a basic subroutine. In the general approach, we assume that the world is in one of several states; it is our task to determine which is the actual state. For each of the possible states, we provide a dynamical model in terms of a linear system corrupted by Gaussian noise. For each model, we interpret the data as though produced by the model. We then choose the model whose predictions conform most closely to the data.

We had several motivations in presenting the material on state estima. tion and the Kalman filter. Mathematically, the Kalman filter is simple and elegant. Practically, the Kalman filter provides a powerful tool that can yield extremely precise and robust control systems. Approaches based on the Kalman filter are well suited for implementation on digital computers. They provide a disciplined approach to combining the data from any number of sources. Finally, the recursive update equations for the Kalman filter illuntuate a cycle of activity involving prediction, observation, and estimation, that aloeld play a part in any approach to dealing with uncertainty.

The atate regulation problem for a linear dynamical system, quadratic performance index, linear control law, and Gaussian disturbance and measurement noise can be cast in terms of two separate problems. The problem of deterministic optimal control and the problem of stochastic optimal estimation. It has been shown that the two problems can be solved separately to yield an optimal solution to the combined control problem. While this 


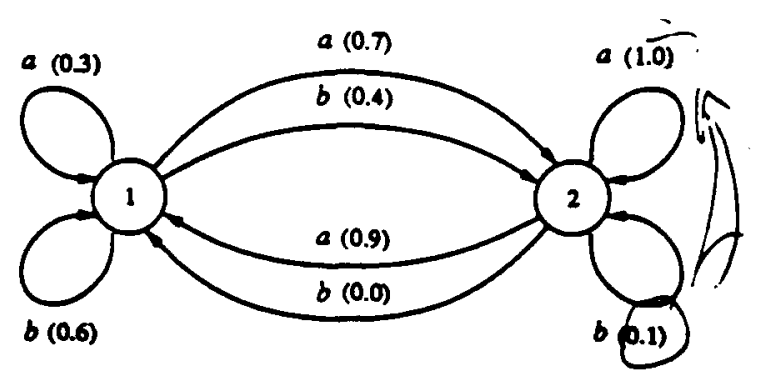

Figure 6.7: A stochastic process with two states

separation property does not hold for nonlinear systems, in many cases, engineers proceed as if it did, designing controllers and state estimators separately and then connecting them to obtain a complete control system. In estimation as elsexhere in control, the linear case serves as the basis for design. In Chapter (1) we consider problems in which observation and control interact strongly, requiring that the robot consider both state regulation and state reconstruction when choosing control actions.

\subsection{Stochastic Dynamic Programming}

$$
\text { A }
$$

In Chapter $\mathbf{3}$, we considered the problem of determining an optimal policy for multistage decision processes. In this section, we reconsider this problem in the context of stochastic processes. The material in this section is ifportant in its own right, but it will also figure prominently in Chapters a ahd 8

For our purposes, a finite-state, time-invariant, discrete-time stochastic process is a four tuple $(T, X, U, P)$ consisting of the following.

- A set of time points $T=Z$

- A finite set of states $X=\left\{x_{1}, x_{2}, \ldots, x_{|X|}\right\}$

- A finite set of inputs $U=\left\{u_{1}, u_{2}, \ldots, u_{|U|}\right\}$

- A cet, $P=\left\{\rho_{i j}(u)\right\}$, of state-transition conditional probability distributions, one for each state/input pair, $\left\langle x_{i}, u\right)$ where $x_{i} \in X$ and $u \in U$, such that for each $x_{j} \in X$ we have the distribution,

$$
\rho_{i j}(u)=\operatorname{Pr}\left(x(t+1)=x_{j} \mid x(t)=x_{i}, u(t)=u\right),
$$


probability distributions,

$$
0 \leq \rho_{i j}(u) \leq 1, \forall x_{i}, x_{j} \in X, u \in U,
$$

and

$$
\sum_{x, \in X} \rho_{i j}(u)=1, \quad \forall x_{i} \in X, u \in U
$$

We notate the state-transition distributions as $\rho_{i j}(u)$ so that in the sequel we can drop the explicit input argument by assuming an implicit control law or policy of the form,

$$
\eta: X \rightarrow U
$$

so that

$$
\rho_{i j}=\rho_{i j}\left(\eta\left(x_{i}\right)\right) \text {. }
$$

Figure 6.7 shows a simple stochastic process with two possible states, $X=$ $\{1,2\}$, and two possible inputs, $U=\{a, b\}$.

The stochastic processes we are considering here are guaranteed to transition to every state infinitely often no matter what initial state the process is started in. Such processes are said to be completely ergodic.

In addition to the requirements stated above, the stochastic processes that we will be concerned with have the following Markov property,

$$
\operatorname{Pr}(x(t+1) \mid x(t), u(t))=\operatorname{Pr}(x(t+1) \mid x(t), u(t), x(t-1), u(t-1), \ldots),
$$

indicating that the transition probabilities depends only on the last state and not on any prior history of the system.

Finally, we introduce a reward function,

$$
\mathrm{R}: U \times X \rightarrow \mathbf{R},
$$

such that $R(u, x)$ corresponds to the (immediate) benefit derived from performing action $u$ in state $x$. In Chapter 34 we were concerned with n-stage decision problems and maximizing performance indices such as

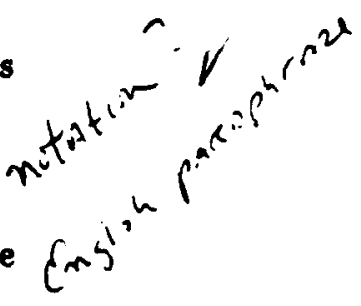

$$
V(u(1), \ldots, u(n) ; x(1), \ldots, x(n))=\sum_{i=1}^{n} R(u(i), x(i)) .
$$

We were able to solve such problems using the following recurrence,

$$
\begin{aligned}
& V_{n}(x)=\max _{u}\left[R(u, x)+V_{n-1}(f(x, u))\right], n \geq 2 \\
& V_{1}(x)=\max _{u} R(u, x),
\end{aligned}
$$


where $f$ is the deterministic state-transition function.

In the case of stochastic processes, there is generally some uncertainty in the outcome resulting from performing a given action in a particular state, and so we maximize expected value to account for this uncertainty. We can extend the recurrence for the deterministic case to handle stochastic processes by summing over the possible next states weighted by their probability of occurring. The extended recurrence is defined by

$$
\begin{aligned}
& V_{n}\left(x_{i}\right)=\max _{u} \sum_{x_{j} \in X} \rho_{i j}(u)\left[R\left(u, q_{i}\right)+V_{n-1}\left(x_{j}\right)\right], n \geq 2 \\
& V_{1}\left(x_{i}\right)=\max _{u} \sum_{x, \in X} \rho_{i j}(u)\left[R\left(u, x_{j}\right)\right] .
\end{aligned}
$$

The above recurrence represents the application of Bellman's principle of optimality, as discussed in Chapter 34 to Markov decision processes. The method of solving Markov decision processes by solving this recurrence is referred to as value iteration since the value functions are determined iteratively [9].

There are other variations on this basic recurrence relation. For instance, we could specify boundary conditions (e.g., initial amount of fuel or other resource) by redefining $V_{1}$ to include some initial value. We could also define a set of admissible controls thereby restricting which actions are allowed under what circumstances. The primary limitation of value iteration concerns its ability to handle processes of indefinite duration. Under some circumstances the above recurrence can be shown to converge asymptotically, so that, in the limit as $n \rightarrow \infty$, an agent using the policy defined by

$$
\eta\left(x_{i}\right)=\arg \max _{u} \sum_{x_{j} \in X} \rho_{i j}(u)\left[R\left(u, \mathcal{I}_{i}\right)+V_{n-1}\left(x_{j}\right)\right],
$$

will act 80 as to maximize its average expected return [3]. However, in certain cases, we can do much better, and, in the following, we consider a method due to Howard [9] for solving processes of indefinite duration.

If a completely ergodic stochastic process is allowed to transition indefinitely, the cumblative reward will increase without bound given a strictly positiv reward function. A more appropriate performance index for processes of indefinite duration is the average reward per transition. We define the average reward per transition or system gain with respect to a given policy. In the following, we always assume a current policy of the form,

$$
\eta: X \rightarrow U
$$


allowing us to make the following abbreviations,

$$
\begin{aligned}
\rho_{i j} & =\rho_{i j}\left(\eta\left(x_{i}\right)\right) \\
\mathrm{R}(x) & =\mathrm{R}(\eta(x), x)
\end{aligned}
$$

Using these abbreviations, we can rewrite the basic recurrence used in value iteration as follows.

$$
\begin{aligned}
V_{n}\left(x_{i}\right) & =\sum_{x, \in X} \rho_{i j}\left[R\left(x_{i}\right)+V_{n-1}\left(x_{j}\right)\right] \\
& =\sum_{x, \in X} \rho_{i j}\left[R\left(x_{i}\right)\right]+\sum_{x, \in X} \rho_{i j}\left[V_{n-1}\left(x_{j}\right)\right]
\end{aligned}
$$

We introduce new notation for the expected immediate (quick) returns corresponding to the first summation term in the above equation,

$$
\mathrm{Q}\left(x_{i}\right)=\sum_{x, \in X} \rho_{i j}\left[R\left(x_{i}\right)\right],
$$

allowing us to simplify the recurrence once more as

$$
V_{n}\left(x_{i}\right)=Q\left(x_{i}\right)+\sum_{x, \in X} \rho_{i j}\left[V_{n-1}\left(x_{j}\right)\right] .
$$

Note that the quick returns can be computed directly from the reward function and the state-transition probabilities. To evaluate the quick return for an input other than that specified by the current policy, we simply add a control argument,

$$
\mathrm{Q}\left(x_{i}, u\right)=\sum_{x, \in X} \rho_{i j}(u)\left[\mathrm{R}\left(u, x_{i}\right)\right] .
$$

In considering processes with indefinite duration, we are interested in how often a given process will end up in a particular state. Let $\pi_{j}(n)$ indicate the probability that the system will be in state $x_{i}$ after $n$ transitions given that the initial state is known. Let $\pi_{i}$ be the limit of $\pi_{i}(n)$ as $n \rightarrow \infty$. Clearly $\sum$ aex $\pi_{i}=1$. For completely ergodic processes, the $\pi_{i}$ are completely inderace of the starting state and provide us with the frequency that the system will enter a given state given that it is allowed to run indefinitely. Using these limiting state transition probabilities, we can define the system gain (average reward per transition) with respect to a given policy as

$$
G=\sum_{x_{i} \in X} \pi_{i}\left[R\left(x_{i}\right)\right]
$$


As $n$ gets large, the quantity, $V_{n}(x)$, increases without bound, but the difference, $V_{n}(x)-V_{n-1}(x)$, is bounded. As a consequence, we can determine the equation of a line,

$$
y(n)=g n+v_{0},
$$

bounding the values of $V_{n}(x)$, where $g n$ represents the steady-state component of the behavior as $n \rightarrow \infty$, and $v_{0}$ represents the transient component, depending only on the starting state. This bounding line is referred to as the asymptote of $\mathrm{V}_{n}(x)$. The slope, $g$, of the asymptote is just the system gain, $G$, and the $y$-intercept, $v_{0}$, we denote $V(x)$ (no subscript) for starting state, $x$. For completely ergodic processes, the slope is independent of the starting state. As $n$ gets large, we have the following approximation,

$$
\mathrm{V}_{n}(x)=n \mathrm{G}+\mathrm{V}(x) \text {. }
$$

Substituting in our recurrence, we obtain

$$
\begin{aligned}
& n G+V\left(x_{i}\right)=Q\left(x_{i}\right)+\sum_{x, \in X} \rho_{i j}\left[(n-1) G+V\left(x_{j}\right)\right] \\
& n G+V\left(x_{i}\right)=Q\left(x_{i}\right)+(n-1) G \sum_{x, \in X} f_{i j}+\sum_{x_{j} \in X} \rho_{i j}\left[V\left(x_{j}\right)\right] .
\end{aligned}
$$

Noting that $\sum_{x_{j} \in X} \rho_{i j}=1$, we finally obtain a set of equations of the form,

$$
\mathrm{G}+\mathrm{V}\left(x_{i}\right)=\mathrm{Q}\left(x_{i}\right)+\sum_{x_{j} \in X} \rho_{i j}\left[\mathrm{~V}\left(x_{j}\right)\right],
$$

one for each $x_{i} \in X$. This constitutes a set of $|X|$ linear similtaneous equations in $|X|+1$ unknowns: the values of $G$ and the $|X| V\left(x_{i}\right)$. In order to solve this system of equations, we can eliminate one unknown by setting one of the $V\left(x_{i}\right)$ equal to zero. The values for the $V\left(x_{i}\right)$ obtained from the solution to the set of simultaneous equation with, say, $V\left(x_{\mid} X \mid\right)=0$ will differ from those defined in

$$
V_{n}\left(x_{i}\right)=n G+V\left(x_{i}\right)
$$

by a constant amount, but this difference is not significant for processes with a large number of transitions, and the values obtained for the $V\left(x_{i}\right)$ will suffice for determining the relative merit of two policies, hence they are referred to as relative values. 
We now have a method, referred to as value determination, for establishing the expected value of a given policy for a stochastic decision process of indefinite duration. We now need a method of choosing an optimal policy. In the following, we consider a method due to Howard [9] called policy iteration which allows us to generate an optimal policy by successive approximation. Policy iteration starts with an arbitrary policy, generates an improved (higher gain) policy on every iteration, and is guaranteed to terminate in a finite number of iterations with the optimal (bighest possible attainable gain) policy. The policy iteration algorithm cycles between the value-determination procedure outlined above and a policy-improvement procedure that involves selecting an improved policy on the basis of the relative values for the current policy. As Howard [9] puts it, "the valuedetermination operation yields values as a function of policy, whereas the policy-improvement routine yields policy as a function of the values."

The policy iteration algorithm is defined as follows.

1. Let $k \leftarrow 0$.

2. Choose an arbitrary ${ }^{8}$ policy, $\eta_{0}$, compute the corresponding values for the $Q\left(x_{i}\right)$, and then use the value determination method described above to compute the values for the $V\left(x_{i}\right)$.

3. For each state, $x_{i}$, find $u_{i}$ maximizing

$$
\mathrm{Q}\left(x_{i}, u_{i}\right)+\sum_{x_{j} \in X} \rho_{i j}\left(u_{i}\right)\left[\mathrm{V}\left(x_{j}\right)\right],
$$

using the current value function. For each $x_{i}$, if $u_{i}$ yields a better return based on the current value function, that is we have

$$
\left(\mathrm{Q}\left(x_{i}, u_{i}\right)+\sum_{x_{j} \in X} \rho_{i j}\left(u_{i}\right)\left[\mathrm{V}\left(x_{j}\right)\right]\right)>\left(\mathrm{Q}\left(x_{i}\right)+\sum_{x_{j} \in X} \rho_{i j}\left[\mathrm{~V}\left(x_{j}\right)\right]\right),
$$

then $u_{i}^{\prime} \leftarrow u_{i}$, otherwise $u_{i}^{\prime}-\eta_{k}\left(x_{i}\right)$.

"Wh the chaice of initial policy does not affect whether or not the algorithm converges on the opind policy, a good initial choice can often result in finter convergence. If there is no o priori rescon for chocaing any particular policy, Howard recommends chooxing $\eta_{0}$ so that

$$
\eta_{0}\left(x_{i}\right)=\max Q\left(x_{i}, x\right) \text {. }
$$

This is effectively the same as setting $V\left(x_{i}\right)=0$ for all $x_{i} \in X$, and then running the policy improvement step in the algorithm. 
4. Define a new policy such that

$$
\eta_{k+1}\left(x_{i}\right)=u_{i}^{\prime}
$$

5. If $\eta_{k}=\eta_{k+1}$, then exit returning $\eta_{k}$.

6. Using $\eta_{k+1}$, compute the values for the $Q\left(x_{i}\right)$, and then use these to corspute $V\left(x_{i}\right)$ using value determination.

7. Let $k \leftarrow k+1$.

8. Go to Step 3.

Step 6 and Step 2 both of which involve value determination are the most expensive steps computationally. However, the solution of the set of simultaneous equations required for value determination can be easily handled by means of existing efficient linear programming algorithms. The limiting factor is the size of the state and input spaces.

To illustrate how policy iteration works, we consider a variation on a classic problem found in $[9,3]$. The classic formulation involves a taxicab driver searching for fares; we have changed the problem slightly to reflect our interest in mobile robots. Our treatment here follows that of [9].

Consider the problem faced by a robot courier assigned the task of delivering files, office supplies, and other assorted small items in a three-story office building. The robot is rewarded for making its deliveries and the rewards differ depending on where the robot is and how far it is required to travel.

For the most part, the robot just waits around-for the next delivery job, but it has a few options that can influence how quickly the next job arrives and how much of a reward it is likely to obtain in carrying out this job. Each floor of the building is dedicated to a different department of a company, and each floor has its own separate reception area and copy room. The offices on the first and third floors are equipped with computer worktations linked by local area networks, and the robot can plug into the networt on a given floor using a receptacle located near the elevator. Using their personal workstations, office workers can issue requests to the robot through the network.

Let $X=\{1,2,3\}$, corresponding to the first, second, and third floors of the office building, and $U=\{c, r, n\}$, corresponding to the three options open to the robot, wait in the copyroom, wait in the reception area, and 


\begin{tabular}{|c|c|ccc|cccc|c|}
\hline$i$ & $u$ & \multicolumn{3}{|c|}{$\rho_{i j}(u)$} & \multicolumn{3}{|c|}{$\mathrm{R}_{i j}(u)$} & $Q_{i}(u)$ \\
\hline & & $j=$ & 1 & 2 & 3 & $j=$ & 2 & 3 & \\
\hline 1 & $c$ & $1 / 2$ & $1 / 4$ & $1 / 4$ & 10 & 4 & 8 & 8.00 \\
& $r$ & $1 / 16$ & $3 / 4$ & $3 / 16$ & 8 & 2 & 4 & 2.75 \\
& $n$ & $1 / 4$ & $1 / 8$ & $5 / 8$ & 4 & 6 & 4 & 4.25 \\
\hline 2 & $c$ & $1 / 2$ & 0 & $1 / 2$ & 14 & 0 & 18 & 16.00 \\
& $r$ & $1 / 16$ & $7 / 8$ & $1 / 16$ & 8 & 16 & 8 & 15.00 \\
\hline 3 & $c$ & $1 / 4$ & $1 / 4$ & $1 / 2$ & 10 & 2 & 8 & 7.00 \\
& $r$ & $1 / 8$ & $3 / 4$ & $1 / 8$ & 6 & 4 & 2 & 4.00 \\
& $n$ & $3 / 4$ & $1 / 16$ & $3 / 16$ & 4 & 0 & 8 & 4.50 \\
\hline
\end{tabular}

Table 6.1: Specification for the robotcourier problem

plug into the local area network, where the last option is only available in States 1 and 3. Since the reward depends not only on the action taken and the initial state, but also upon the final state, we modify the reward function to take a third argument, $\mathrm{R}: U \times X \times X \rightarrow \mathbf{R}$, so that $\mathbf{R}_{i j}(u)$ corresponds to the (immediate) benefit derived from performing action $u$ in state $x_{i}$ and ending up in state $x_{j}$. We also modify the definition of the immediate (quick) reward function to reflect the dependence on the final state,

$$
Q_{i}(u)=\sum_{x, \in X} \rho_{i j}(u)\left[R_{i j}(u)\right] .
$$

The complete specification for the robot courier problem is shown in Table 6.1 where the transition probabilities and rewards are shown in matrix form.

We begin by assuming that the expected values for all states are zero,

$$
\mathrm{V}(1)=\mathrm{V}(2)=\mathrm{V}(3)=0 \text {, }
$$

so that the initial policy will depend only upon immediate rewards. Looking at the last column in Table 6.1, it should be clear that the robot should wait in the copyroom no matter what floor it finds itself on, and so we define the initial policy, no, as

$$
\eta_{0}(1)=\eta_{0}(2)=\eta_{0}(3)=c
$$


The state transition probabilities and reward values for this policy are given by the following matrices,

$$
\left[\rho_{i j}\right]=\left[\begin{array}{ccc}
1 / 2 & 1 / 4 & 1 / 4 \\
1 / 2 & 0 & 1 / 2 \\
1 / 4 & 1 / 4 & 1 / 2
\end{array}\right] \quad\left[\mathrm{R}_{i j}\right]=\left[\begin{array}{c}
8 \\
16 \\
7
\end{array}\right]
$$

From the general equations used in value determination,

$$
\mathrm{G}+\mathrm{V}\left(x_{i}\right)=Q_{i}+\sum_{x_{j} \in X} \rho_{i j}\left[\mathrm{~V}\left(x_{j}\right)\right]
$$

we construct the particular equations for the current policy,

$$
\begin{aligned}
& G+V(1)=8+\frac{1}{2} V(1)+\frac{1}{4} V(2)+\frac{1}{4} V(3) \\
& G+V(2)=8+\frac{1}{2} V(1)+0 V(2)+\frac{1}{2} V(3) \\
& G+V(3)=8+\frac{1}{4} V(1)+\frac{1}{4} V(2)+\frac{1}{2} V(3)
\end{aligned}
$$

Setting $V(3)$ equal to zero and solving, we obtain

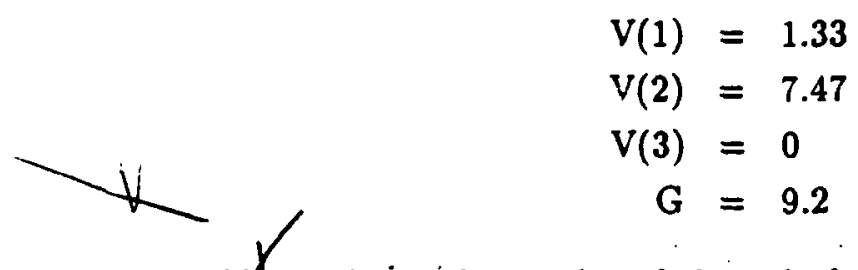

Tabif6.2 shows the results of the calculations made in the process of improving ufon the initial policy, $\eta_{0}$. For each state, $x_{i}$, we choose the option, $u$, that maximizes the quantity,

$$
Q_{i}(u)+\sum_{x_{j} \in X} \rho_{i j}(u)\left[V\left(x_{j}\right)\right]
$$

and abet the imprnved policy, $\eta_{1}$, defined by

$$
\eta_{1}(1)=c, \eta_{1}(2)=-, \eta_{1}(3)=r,
$$

indicating that the robot should wait in the reception area un the second and third floor, but wait in the copyroom on the first. 


\begin{tabular}{|c|c|c|}
\hline$i$ & $u$ & $Q_{i}(u)+\sum_{x_{,} \in X} \rho_{i j}(u)\left[\mathrm{V}\left(x_{j}\right)\right]$ \\
\hline 1 & $c$ & 10.53 \\
& $r$ & 8.43 \\
& $n$ & 5.52 \\
\hline 2 & $c$ & 16.67 \\
& $r$ & 21.62 \\
\hline 3 & $c$ & 9.20 \\
& $r$ & 9.77 \\
& $n$ & 5.97 \\
\hline
\end{tabular}

Table 6.2: First round of policy improvement for the robot courier

If we perform another cycle of value determination and policy improvement, we arrive at the policy, $\eta_{2}$ defined by

$$
\eta_{2}(1)=r, \quad \eta_{2}(2)=r, \eta_{2}(3)=r,
$$

indicating the robot should wait in the reception area no matter what floor it is located on. If we perform yet another cycle we obtain, $\eta_{3}$, defined by

$$
\eta_{3}(1)=r, \quad \eta_{3}(2)=r, \quad \eta_{3}(3)=r .
$$

Noticing that $\eta_{2}=\eta_{5}$, we now have an optimal policy,

$$
\eta(1)=r, \quad \eta(2)=r, \quad \eta(3)=r,
$$

for the robot courier problem, reinforcing the belief held by'many office workers that the reception area is one of the busiest areas in an office and one to be avoided if you wish to avoid work.

As might be expected, policy iteration is sensitive to a variety of changes in the initial conditions. For instance, if you reverse the transition probabilition, $\rho_{2,2}(r)$ and $\rho_{2,3}(r)$, you obtain a different optimal policy,

$$
\eta(1)=c, \quad \eta(2)=c, \quad \eta(3)=r .
$$

In addition, the number of iterations (most importantly, the number of times we have to perform value determination) depends critically on the choice of an initial policy. If, for example, we start with the initial policy,

$$
\eta_{0}(1)=n \eta_{0}(2)=r \eta_{0}(3)=n,
$$


policy iteration takes only two iterations instead of the three required for $\eta_{0}(1)=\eta_{0}(2)=\eta_{0}(3)=c$. In many cases, the choice of an initial policy that is close to optimal can improve the performance of policy iteration dramatically.

In some cases, it is unrealistic to count consequences in the distant future on an equal basis with more immediate consequences. For instance, we may mistrust our model for making accurate long term predictions, or future rewards may actually lose value due to some inflationary process. Most biological organisms tend to discount longer term rewards and focus on more immediate rewards. We can model this outlook on rewards iy adding a discounting factor to our value function.

$$
\mathrm{V}_{n}\left(x_{i}\right)=\mathrm{Q}\left(x_{i}\right)+\lambda \sum_{x_{j} \in X} \rho_{i j}\left[\mathrm{~V}_{n-1}\left(x_{j}\right)\right],
$$

where $0 \leq \lambda<1$ is the discount rate. In the case of discounting, the notion of gain (average reward per transition) no longer makes sense, as the optimal policy is simply the one that maximizes expected value in all possible states.

Value determination is actually simpler for stochastic processes with discounting, as we no longer have to account for the system gain. Eliminating the system gain and appealing once more to the asymptotic limit of $V_{n}$, namely $V$, we obtain a set of equations of the form,

$$
\mathrm{V}\left(x_{i}\right)=\mathrm{Q}\left(x_{i}\right)+\lambda \sum_{x, \in X} \rho_{i j}\left[\mathrm{~V}\left(x_{j}\right)\right]
$$

one for each $x_{i} \in X$. This constitutes a set of $|X|$ linear simultaneous equations in $|X|$ unknowns (the $V\left(x_{i}\right)$ ) that can be easily solved for the unknowns. Policy iteration works in the case of discounting exactly as before with the substitution of the simplified value determination procedure.

If we add discounting to the robot-gourier problem, we get a different policy depending upon the value of $\lambda$. Por $0 \leq \lambda<0.13$, we get the policy,

$$
\eta(1)=c, \quad \eta(2)=c, \quad \eta(3)=c,
$$

for $0.13 \leq \lambda<0.53$, we get

$$
\eta(1)=c, \quad \eta(2)=r, \quad \eta(3)=c,
$$

for $0.53 \leq \lambda<0.77$, we get

$$
\eta(1)=c, \quad \eta(2)=r, \quad \eta(3)=r
$$


and, finally, for $0.77 \leq \lambda<1.0$, we get

$$
\eta(1)=r, \quad \eta(2)=r, \quad \eta(3)=r,
$$

As one might guess, the closer $\lambda$ is to $!$, the more iterations of value determination and policy improvement will be required to obtain the optimal policy.

a

In Chapter 8, we consider a form of learning that is closely related to the approach used here to compute an optimal policy for stochastic decision processes with discounting. We will employ the same basic form of successive policy improvement. The main departure from the techniques of this section is that value determination will be done without the aid of frodal. Value determination will occur over time as the agent interacts with its environment obtaining rewards and punishments intermittently and occasionally inappropriately. This sort of reinforcement learning provides a good model of learning in biological organisms and also appears to be a good model for many automated planning and control applications.

\subsection{Fuzzy Set Theory and Fuzzy Control}

Uncertainty arises in many different forms. Probability theory provides a basis for reasoning about uncertainty due to randomness, but there are other forms of uncertainty that cannot be easily captured using the tools of probability theory. In this section, we consider some alternative tools provided by fuzzy set theory and fuzzy control.

Fuzzy set theory provides a mathematical basis for capturing knowledge in a form close to that used in everyday zommunication. Using fuzzy set theory, we can assign meaning to terms associated with sets for which there are no clearly defined boundaries separating elements from non-elements, terms like large, small, close, far, hot, cold, short, and tall.

The standard interpretations of probabilities in terms of frequencies or likcliboods make it difficult to model linguistic phenomena characterized by rord like "heavy" or "tall." The word "tall" denotes a fuzzy set not becases there is randomness in the process of measurement, but because there is general dispute and uncertainty about whether a borderline case belongs to the set or not.

Our interest here stems from the considerable success that fuzzy set theory and its counterpart, fuzzy control, have had in practical applications. Fuzzy control systems have been used in video cameras, automobiles, and 
high-speed public transportation systems, just to name a few of the more successful applications. Fuzzy control and fuzzy decision-support systems provide a focus on knowledge acquisition and representation similar to that found in the work on so-called expert rule-based systems. We mention fuzzy methods in this chapter because they have shown themselves to provide a viable alternative to other more traditional approaches to dealing with uncertainty in control, and because they share with other rule-based approaches to reasoning an emphasis on symbolic representations.

We begin with a brief introduction to fuzzy set theory [17]. Let $X$ denote the universe set of elements, and $x$ an instance of this set. A fuzzy set $A$ in $X$ is characterized by a membership or characteristic function from $X$ to the real interval $[0,1]$,

$$
I_{A}: X \rightarrow[0,1]
$$

The value of $I_{A}$ at $x$ indicates the "degree" to which $x$ is considered to be a member of $A$. In standard set theory, $I_{A}$ is either 0 or 1 . In the sort of sets that fuzzy set theory is primarily concerned with, such binary distinctions are often difficult to make. For instance, let $X$ be the set of all people, and $A$ be the set of "tall" people. Suppose yuu consider people over seven feet to be tall, under six feet not to be tall, and between six and seven feet to be to some degree (between zero and one) tall. In this case, you might characterize the set of tall people using the following function,

$$
I_{A}(x)= \begin{cases}1 & \text { if } 7 \geq h(x) \\ h(x)-6 & \text { if } 6 \geq h(x)<7 \\ 0 & \text { otherwise }\end{cases}
$$

where $h(x)$ denotes the height of $x$.

We now provide fuzzy versions of some common set-theoretic notions. The fuzzy complement, $\bar{A}$, of the set, $A$, is defined by the function,

$$
I_{\lambda}(x)=1-I_{A}(x)
$$

The fussy union, $A \cup B$, of two fuzzy sets, $A$ and $B$, is defined

$$
\mathcal{I}_{A \cup B}(x)=\max \left(\mathcal{I}_{A}(x), \mathcal{I}_{B}(x)\right)
$$

and the fuzzy intersection, $A \cap B$, is defined

$$
I_{A \cap B}(x)=\min \left(I_{A}(x), I_{B}(x)\right) \text {. }
$$


Note that, in the case of boolean-valuer characteristic functions, these definitions coincide with the standard set-theoretic definitions of complement, union, and intersection.

For building rule-based control systems, we are not so much interested in a generalization of set theory as we are in a generalization of predicate logic. The standard (Tarskian) semantics for predicate logic is based on standard set theory; predicates denote sets, the (truth-functional) interpretation of atomic sentences is defined in terms of membership, and the meaning of the connectives, $\neg, \vee$ and $\wedge$, defined, respectively, in terms of complementation, union, and intersection. In a similar manner, one can provide semantics for fuzzy logic using fuzzy set theory. Since our objectives in this section are modest, we only introduce those concepts that are necessary for our discussion, and refer the reader to a more detailed treatment in [10].

The syntax for the propositional case is as follows. Let $\mathcal{A}$ be a set of fuzzy propositional variables. We define the set of well-formed formulae (wffs) inductively as consisting of any propositional variable, the negation of any wff (written $\neg \varphi$ where $\varphi$ is a wff), the conjunction of any two wffs (written $\left(\varphi_{1} \wedge \varphi_{2}\right)$ where $\varphi_{1}$ and $\varphi_{2}$ are wffs), or the disjunction of any two wffs (written $\left(\varphi_{1} \vee \varphi_{2}\right)$ where $\varphi_{1}$ and $\varphi_{2}$ are wffs).

Next, we provide the semantics for the propositional case. An interpretation, $M$, is a function from propositional variables to the real interval $[0,1]$. An interpretation, $M$, is said to be an $\alpha$-model for a wff, $\varphi$, (written $M \vDash \propto \varphi$ ) under the following conditions.

- $M \models_{a} A$ iff $M(A)=\alpha$, where $A \in \mathcal{A}$

- $M \vDash_{\alpha} \neg \varphi$ iff $M \vDash_{1-\alpha} \varphi$

- $M \models_{\alpha} \varphi_{1} \wedge \varphi_{2}$ iff $\alpha=\min \left(\alpha_{1}, \alpha_{2}\right)$, where $M \vDash_{\alpha_{1}} \varphi_{1}$, and $M \models_{\alpha_{2}} \varphi_{2}$,

In analogy to two-valued propositional logic, a wff, $\varphi$, is said to be $\alpha$ satisfiable if it has an $\alpha$-model, and is said to be $\alpha$-valid (written $F_{a} \varphi$ ) if all modul are a-models. We can also define an analog of semantic entailment. A $\sim \boldsymbol{I}_{1} \varphi_{1}$, is said to $\alpha$-entail another wf, $\varphi_{2}$, (written $\varphi_{1} \vDash a, \varphi_{2}$ ) if for any $\bmod , \mathcal{M}, M F_{\alpha_{1}} \varphi_{1}$ implies that $\alpha_{2} / \alpha_{1} \geq \alpha$, where $M F_{\alpha_{2}} \varphi_{2}$.

For a particular control problem, we would construct a set of fuzzy propositional variables as follows. Let $F$ be the set of fuzzy sets, and $X$ the universe set (generally the state space of a dynamical system) for the problem at hand. For each $A \in F$ and $x \in X$, we define a propositional variable of the form, $A(x)$, as shorthand for $x \in A$. 
Assigning $A(x)$ a real number is like assigning a proposition a truth value; such assignments restrict the interpretations we are willing to consider and therefore restrict what formulae are valid. In a two-valued propositional logic, if you are told that $P$ must be true in all interpretations, then, subject to that restriction, $Q$ is true in all models for $\neg P \vee Q$. Similarly, in fuzzy logic, if you are told that $A(x)$ must be assigned 0.7 in all interpretations, then $B(x)$ must be assigned 0.4 in all models for which $\neg A(x) \vee B(x)$ is assigned 0.4 . Note that, in the case of boolean-valued characteristic functions, the above fuzzy semantics reduces to standard truth-functional semantics.

For the cases we consider in the sequel, we are interested in the unique model, $M$, such that, for all $A \in F$ and $x \in X, M(A(x))=I_{A}(x)$. Ilustrating the connection to the fuzzy set-theoretic concepts introduced earlier, note that $M$ satisfies the following conditions 9

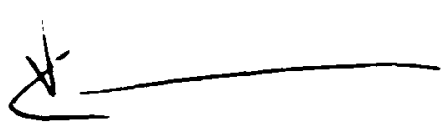

$$
\begin{aligned}
& M \vDash I_{I_{A}(x)} \neg A(x) \\
& M \vDash I_{A \cap B}(x) A(x) \wedge B(x) \text {, } \\
& M \vDash_{I_{A \cup B}(x)} A(x) \vee B(x)
\end{aligned}
$$

for all $A, B \in F$ and $x \in X$.

The primitive notions presented above provide us with all the logical machinery we require for building simple fuzzy control systems. We could use fuzzy logic directly to obtain assignments to fuzzy propositional variables in an analog of the way in which boolean logic is used in some control systems. Instead, we consider how fuzzy logic formulae are used to construct fuzzy algorithms [18]. For our purposes, a fuzzy control system consists of a set of statements (or rules) of the form,

$$
\text { - . If } A_{1} \wedge A_{2} \wedge \cdots \wedge A_{n} \text {, then } C \text {. }
$$

where the $A_{i}$ are the antecedent conditions and $C$ is the consequent action. Generally, the antecedents correspond to fuzzy propositions involving the system state variables, and the consequent corresponds to a fuzzy assignment statement involving the system input variables.

Fo instance, suppose you are trying to control a robot to move parallel to the planar surface of a wall in the direction right facing the wall and maimaining a distance of about one meter from the wall. You would need fuzby cets characterizing the distance separating the robot from the wall, and the angle of the robot with respect to the surface of the wall. The distance to the wall might be captured using six fuzzy sets, corresponding to being next to the wall, VERY NEAR, some distance but cloce, NEAR, somewhat further but still relatively close, SOMEWHAT_NEAR, even further, 


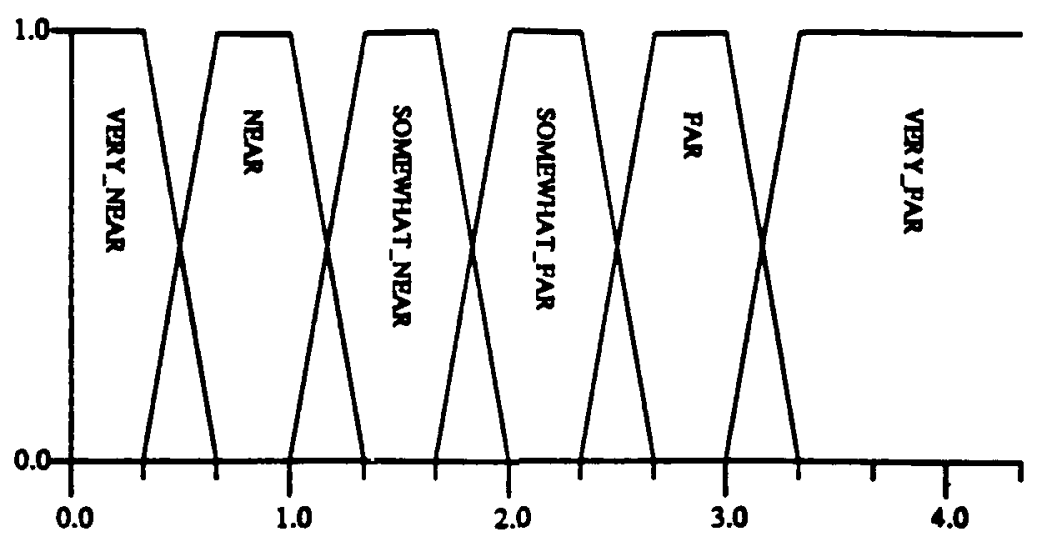

Figure 6.8: Fuzzy membership functions for the wall-following problem

SOMEWHAT FAR, further still, FAR, and very far VERY_FAR. Possible characteristic functions for these six fuzzy sets are shown in Figure 6.8.

To control the robot, you might specify that, if the robot is within a meter or so of the wall and moving nearly perpendicular but slightly toward the wall's surface, then steer a little further to the right. Such a specification would be represented by the rule,

\section{R1: If NEAR(x)^SOMEWHAT_TOWARD(x) then $u \leftarrow u+$ SOMEWHAT_RIGHT,}

where NEAR, SOMEWHAT_TOWARD, and SOMEWHAT_RIGHT correspond to fuzzy sets, $x$ is the system state indicating the position and orientation of the robot with respect to the wall, and $i$ is the system input indicating the steering angle.

Furzy logic indicates how to interpret the antecedent of R1. For instance, given that

$$
\begin{aligned}
\operatorname{NEAR}(x) & =1.0 \\
\text { SOMEWHAT_TOWARD }(x) & =0.9
\end{aligned}
$$

we have

$$
\operatorname{NEAR}(x) \wedge \text { SOMEWHAT }-T O W A R D(x)=0.9 \text {. }
$$

However, the statements in a fuzzy algorithm are not formulae in a fuzzy logic. What we require is a procedural interpretation. In particular, we have to determine the result of execnting Rl? 
If SOMEWHAT RIGHT were a constant, say $5^{\circ}$, then the result of executing R1, might be that the value of $u$ is increased by 5 over what it was formerly, where the general rule might be, if the value of the antecedent is greater than 0.75 , then treat the consequent as a statement in a conventional programming language and execute it accordingly.

In the case of SOMEWHAT RIGHT being a fuzzy set, we will want to consider a different evaluation strategy. Suppose that we define the fuzzy set, SOMEWHAT_RIGHT, as follows,

$$
\text { ISOMEWHAT_RIGHT } T^{(x)}= \begin{cases}0.2 & \text { if } x=1^{\circ} \\ 0.4 & \text { if } x=2^{\circ} \\ 0.6 & \text { if } x=3^{\circ} \\ 0.8 & \text { if } x=4^{\circ} \\ 1.0 & \text { if } x=5^{\circ} \\ 0.8 & \text { if } x=6^{\circ} \\ 0.6 & \text { if } x=7^{\circ} \\ 0.4 & \text { if } x=8^{\circ} \\ 0.2 & \text { if } x=9^{\circ} \\ 0.0 & \text { otherwise }\end{cases}
$$

Then we might define the result of executing $R 1$ as another fuzzy set, RESULT_R1, defined by weighting the fuzzy set, SOMEWHAT_RIGHT, using the value assigned to the antecedent condition.

$$
I_{\text {RESULT_RI }}(x)= \begin{cases}0.2 * 0.9 & \text { if } x=u+1^{\circ} \\ 0.4 * 0.9 & \text { if } x=u+2^{\circ} \\ 0.6 * 0.9 & \text { if } x=u+3^{\circ} \\ 0.8 * 0.9 & \text { if } x=u+4^{\circ} \\ 1.0 * 0.9 & \text { if } x=u+5^{\circ} \\ 0.8 * 0.9 & \text { if } x=u+6^{\circ} \\ 0.6 * 0.9 & \text { if } x=u+7^{\circ} \\ 0.4 * 0.9 & \text { if } x=u+8^{\circ} \\ 0.2 * 0.9 & \text { if } x=u+9^{\circ} \\ 0.0 & \text { otherwise }\end{cases}
$$

We still need a unique result, and one obvious possibility is to choose the result with the highest rating, breaking ties randomly if necessary.

The method of using thresholds to determine whether or not to execute the consequent of fuzzy roles is inadequate in the case in which there are several rules all attempting to perform conflicting action, say setting a 
control variable to different vajues, and all having antecedent conditions that pass the threshold. For instance, in addition to Rl, we might have the following rule,

$$
\begin{aligned}
& \text { R2: If } N E A R(x) \wedge \text { SOMEWHAT_AWAY }(x) \\
& \text { then } u \leftarrow u+S O M E W H A T \_E F T .
\end{aligned}
$$

As an alternative to thresholds, we could define a corresponding fuzzy result, RESULT_R2, for R1, and set $u$ according to the following,

$$
u \leftarrow \arg \max _{x}\left(I_{R E S U L T \_R 1}(x), I_{R E S U L T \_R 2}(x)\right) .
$$

We can generalize on the above method for any number of rules. In practice, the set of rules is represented using an $n$-dimensional table, with one dimension for each state variable and some number of fuzzy sets to cover the domain of each such variable. At each point in time, all of the rules are evaluated to determine their corresponding fuzzy results, and the maximal

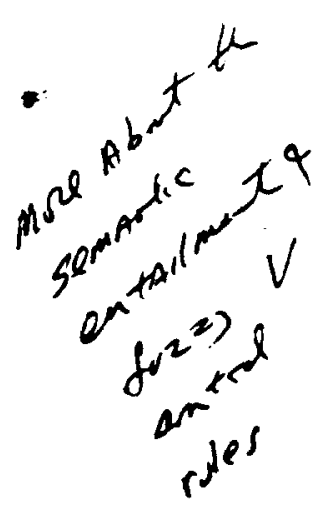
control action taken.

There are many different schemes for executing fuzzy algorithms. There are methods that combine the results from several rules, using a variety of weighting schemes. There are fuzzy algorithmic versions of integer programming, dynamic programming, database query processing, as well as a host of specialized techniques for financial decision making, natural-language processing, circuit layout, and speech recognition, just to name a few. Our purpose here is not to survey fuzzy methods, but simply to make the reader aware of a large and active area of control, and provide a comewhat different perspective on uncertainty than that offered by the probabilists.

\subsection{Further Reading}

For a more thorough treatment of state estimation techniques in general and the Kalman filter in particular, the reader is encouraged to read Bar-Shalom and Rutrann [1], Brammer and Siffling [4], Gelb [6], or Maybeck [14]. It is also worth returning to some of the original papers on the theory and appiration of the Kalman filter. A number of the original papers appear in a collection by Sorenson [16] which is particularly interesting for the broad range of applications considered.

For approaches to geometrical reasoning under uncertainty involving static estimation and using minimum mean-square parameter estimation 
techniques, see the work of Durrant-Whyte [5] and Smith and Cheeseman [15]. Hager [7] presents a game-theoretic analysis of the errors that arise in applying minimum mean-square estimation methods and develops alternative techniques for stochastic geometrical reasoning that allow more flexibility in modeling uncertainty.

Leonard and Durrant-Whyte [13] describe techniques to obtain estimates of the distance separating a mobile robot from nearby walls, corners, and other environmental features that exhibit well-behaved sonar signatures. These estimates are then used to update the robot's position with respect to a global map. The discussion in Section 6.2 is based on their work.

While there are any number of more recent books on dynamic programming and stochastic decisi. nn processes, the texts by Bellman [2] and Bellman and Dreyfus [3] are well worth reading. The method of policy iteration discussed in this chapter is due to Howard [9], and his book is an excellent source of examples as well as proofs of correctness for the basic method and a number of interesting variations. Among the variations, Howard discusses nonergodic (multichain) and continuous-time processes. For an introduction to finite Markov processes. the texts by Kemeny and Snell [11] and Hoel, Port, and Stone [8] are recommended.

The original paper by Zadeh [17] is still an excellent introduction to fuzzy set theory. In a later paper, Zadeh [18] considers the use of fuzzy set theory for reasoning about complex systems and decision processes. In this same paper, Zadeh elaborates on the notion of a fuzzy algorithm, providing a number of interesting examples. The text by Kaufman [10] covers some of the mathematics of fuzzy logic and fuzzy set theory. 


\section{Bibliography}

[1] Bar-Shalom, Yaakov and Fortmann, Thomas E., Tracking and Data Association, (Academic Press, New York, 1988).

[2] Bellman, Richard, Dynamic Programming, (Princeton University Press, 1957).

[3] Bellman, Richard and Dreyfus, Stuart, Applied Dynamic Programming, (Princeton University Press, Princeton, New Jersey, 1962).

[4] Brammer, Karl and Siffing, Gerhard, Kalman-Bucy Filters, (Artech House, Norwood, Massachnsetts, 1989).

[5] Durrant-Whyte, Hugh F., Integration, Coordination and Control of Multi-Sensor Robot Systems, (Kluwer Academic Publishers, Boston, Massachusetts, 1988).

[6] Gelb, A., (Ed.), Applied Optimal Estimation, (MIT Preas, Cambridge, Massachusetts, 1974).

[7] Hager, Gregory D., Task-Directed Sensor Fusion and Planning: A Computational Approach, (Kluwer Academic Publishers, Boston, Massachusetts, 1990).

[8] Hoed, Panl G., Port, Sidney C., and Stone, Charles J., Introduction to Sechastic Processes, (Houghton Mifflin, Boston, Massachusetts, 1971).

[9] Foward, Ronald A., Dynamic Pragramming and Markov Processes, (MT Preas, Cambridge, Massachusetts, 1960).

[10] Kanfmann, Arnold, Introduction to the Theory of Furzy Subsets, (Academic Press, New York, 1975). 
[.1] Kemeny, J. G. and Snell, J. L., Finite Markov Chains, (D. Van Nostrand, New York, 1960).

[12] Koditschek, D., Robot Control Systems, Shapiro, Stuart, (Ed.), Encyclopedia of Artificial Intelligence, (John Wiley and Sons, New York, 1987), 902-923.

[13] Leonard, John J. and Durrant-Whyte, Hugh F., Active Sensor Control 8 for Mobile Robotics, Technical Report OUEL-1756/89, Oxford University Robotics Research Group, 1989.

[14] Maybeck, Peter S., The Kalman Filter-An Introduction to Potential Users, Technical Report TM-72-3, Air Force Flight Dynamics Laboratory, Wright Patterson AFB, Ohio, 1972.

[15] Smith, Randall and Cheeseman, Peter, On the Representation and Estimation of Spatial Uncertainty, The International Journal of Robotics Research, 5 (1986) 56-68.

[16] Soronson, Harold W., (Ed.), Kalman Filtering: Theory and Application, (IEEE Press, New York, 1985).

[17] Zadeh, Lofti A., Fuzzy Sets, Information and Control, 8 (1965) 338353.

[18] Zadeh, Lofti A., Outline of a New Approach to the Analysis of Complex Systems and Decision Processes, IEEE Transactions on Systems, Man and Cybernetics, 3 (1973) 28-44. 


\section{Chapter 7}

\section{Planning Under Uncertainty}

This rhapter is still liery murh in flur. It rurnently consists of early dmfts of a couplc of intmductory sections along with somc cxample sorstions dmun rerbntim from conferrner and journal pmpers. Th further apologirs will be madc for its state of disarmy.

The approaches to planning that we considered in earlier chapters involve generating possible states of affairs from some initial information and a model. In this and the next two chapters. we focns on problems in which the present and future states of affairs are not completely determined by the morlel and the information at hain. We have already seen some problems of this sort. In the case in whiri a robot is uncertain of the outcome of an action. but the ontcome will he apparent once the action is completed, we suggested that the rohot construrt a conditional plan indicating what subsequent course of action to take for each possible outcome. In this chapter. we consider cases in which the agent has somewhat more information about the possible outcomes before the action is.completed, and somewhat less information about the actual outcome after the action is completed.

\subsection{Decision Theory}

Let $\Omega$ be a set of possible states. Suppose that we have some means of assigning numerical values to possible states:

$$
V: \Omega \rightarrow \mathbf{R} \text {. }
$$

\footnotetext{
${ }^{\circ}$ (c) 1990 'Thomes Denn. All rights reserved.
} 
This function is ;enerally referred to as a ralue or utriity inclion. In some cases. depending on our measure of value. it may be more convenient to think of value in terms of its inverse. cost. In the case of value or utility. we generally seek to increase it: in the case of cost. we generally seek to decrease it.

If you could choose some $\omega \in \Omega$. yo" would want to choose 4 such that $V(\omega)$ is maximum:

$$
\arg \max _{\omega \in S} V(\omega)
$$

('nfortunately, we cannot simply select at will irom $\Omega$. We assume. however. that we can select our actions from a set of actions. $\mathcal{A}$. Let $[\mathrm{cr} \mid \boldsymbol{\omega}]$ denote the state resulting from executing action $\alpha$ in state $\omega^{2}$. If the state is unimportant or clear froin context, we simply write $[\alpha]$.

Suppose that each action $\alpha \in \mathcal{A}$ has a unique outcone $[\alpha] \in \Omega$. Then we could simply choose the action whose outcone is riost desirable:

$$
\arg \max _{v \in A} V([\alpha]) \text {. }
$$

Of course. an action seldom, if ever. completely determines a uniquie state. 'lo represent an agent's uncertajuty about the consequeuces of its actions. We assume that the state resulting from a given action is governed by a random process. In this case, we let [o] dcuole a randon variable witl probability space $\Omega$, and assume that we have conditiona: probability distributions of the form:

$$
\operatorname{Pr}([\alpha] \mid \mathcal{E}),
$$

for all $n \in \mathcal{A}$. where $\mathcal{E}$ represents the agent's background knowledge. Now. $V([n])$ is a real-valued function of a random variable, and its expertation is rlefined to he:

$$
\left.E(V([n]) \mid \varepsilon)=\sum_{\omega \in \Omega} V(ن) \operatorname{Pr}(\mid n]=\omega \mid \mathcal{E}\right)
$$

The agent will want to choose the action with the highest expected value:

$$
\arg \max _{a \in \lambda} E(V([n]) \mid \mathcal{E}) .
$$

One asoumption underlying the decision strategy captured in Equatinns 7.1 and 7.2 is that the agent is often going to find itself in the situation of having 10 choose what action to take. Ilence. the agent wants to clioose actions so that its long-term payoff, as predicted by the value function and 


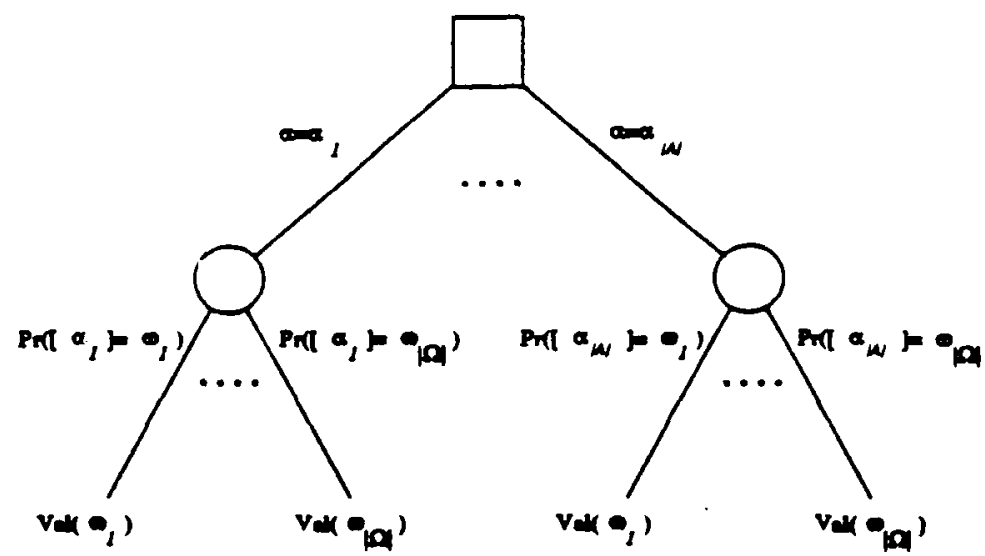

Figure i.1: Simple decision tree

its expectations concerning outcomes is maximized. A decision that maximizes expected value is called an optimal decision.

A significant portion of the next two chapters will involve variations on this basic idea of choosing actions on the basis of expectations about their outcomes. so it is important that you understand it. You can picture the decision process embodied in Equations 7.1 and 7.2 as a decision tree. in which the root node corresponds to a choice by the agent of what action to take. and the children of the root node correspond to a choice by nature of what state should result from the agent's action. Figure $i .1$ shows a simple decision tree in which the agent's choices are represented by boxes called decision nodes. and nature's choices are represented by circles called chance norles. The terminal nodes in the decision tree are labeled with the values assigned to the outcomes. The edges leading out of chance-nodes are lobeled with the probabilities of the outcomes. The edges leading out of decision nodes are labeled with the agent's choices.

In general, decision trees can be of any depth. not just depth 2 as in the decinion tree shown in Figure $\mathrm{i} .1$. Often decision trees are arranged with thm alternating between decision and chance nodes. but this is not reques There is uo requirement that decision trees be symmetrical though they civen appear 80 in textbooks. Indeed. we will often sacrifice symnietry to reduce the size of the decision tree and the computational effort required to evaluate the optimal decision.

An example should help to make the approach to decision making de- 


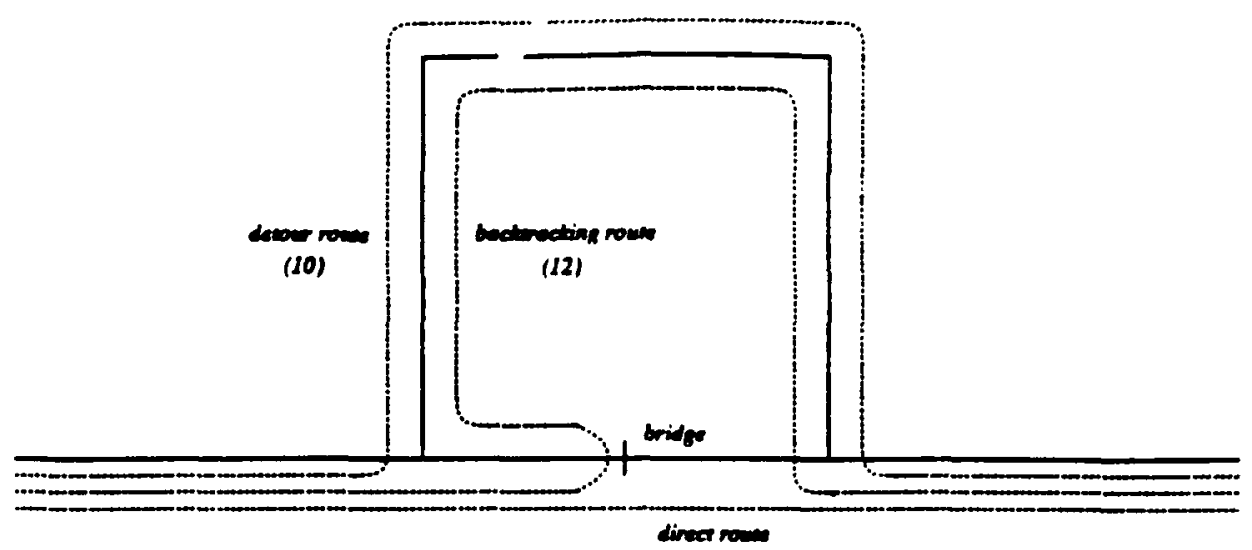

(6)

Fignre 7.2: Alternative rontes to the beach

scribed here inore concrete. Suppose that you live in the city and are taking your summer vacation at a beach some distance from the city. Suppose further that there are two routes to the beach: a direct route that takes six hours and roundabout route that takes ten hours. We will call these the direct and detour routes. The direct route requires that you cross a bridge which, as luck would have it, is undergoing najor repairs this summer. There is a $50 \%$ chance that the bridge will be closed at the time you wish to cross it. If you attempt the direct route and find the bridge closed. you will have to backtrack to the detour route. and your total transit time will be twelve hours.

lour decision involves choosing whether to try the direct or detour route first. Figure $i .2$ shows the three possible outcones of your decisiou. If you choose the delour route, the trip will take ten hours. If you choose the direct route, the trip will take either six hours or twelve hours depending on whether or not the bridge is closed. We need to assign a value or cost to each of the possible outcomes, and, in this case, a natural measure of cost is time erent in transit.

F 7.3 provides a graphical representation of the decision problen for chasing which route to take. Note that the terminals of the subtree emanating from the end of the detour brauch have the same cost. The probabilities on the edges of this subtree govern whether or not the bridge is closed. but this factor has no impact on the outcome if we take the detour. Such uninteresting subtrees are generally eliminated and replaced with the 


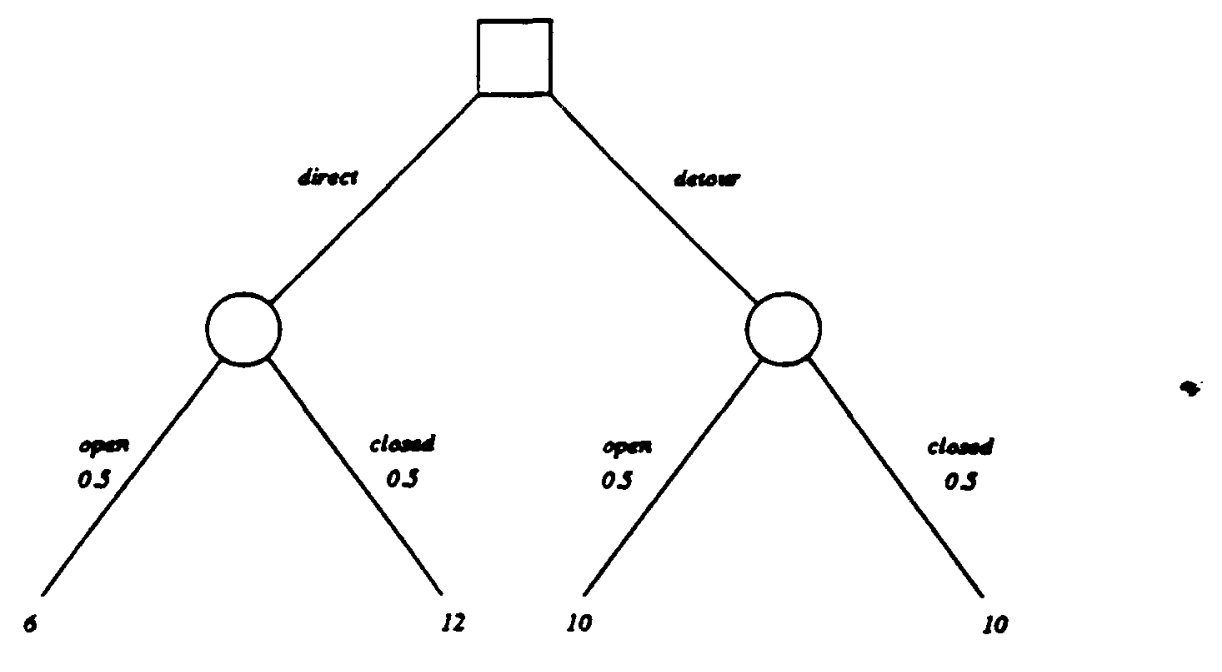

Figure 7.3 : Decision tree for the vacation trip problem

value of the appropriate outcome.

Given a decision tree such as that depicted in Figure 7.3. we can calculate the optinal decision and its expected cost using the following simple procedure. Initially, all of the nodes in the tree except terminal nodes have null labels. Terminal nodes are labeled with the cost of outcomes.

1. For each chance node with a null label all of whose children have non null labels. label it with the expected cost for the node calculated as the sum over all children of the product of the probability of the child (as indicated on the edge from the chance node to the child) and the child's label.

2. For each decision node with a mull label all of whose children have non null labels, label it with the minimum cost of the labels of its children. and st rike from consideration all edges except that one leading to the child with minimum cost.

3. If there are any nodes with null labels. go to Step 1, otherwise find a pal from the root to a terminal node consisting of action edges that have not been stricken from consideration. The sequence of actions along this path indicates the optimal decision and its expected cost is the label of the root. 


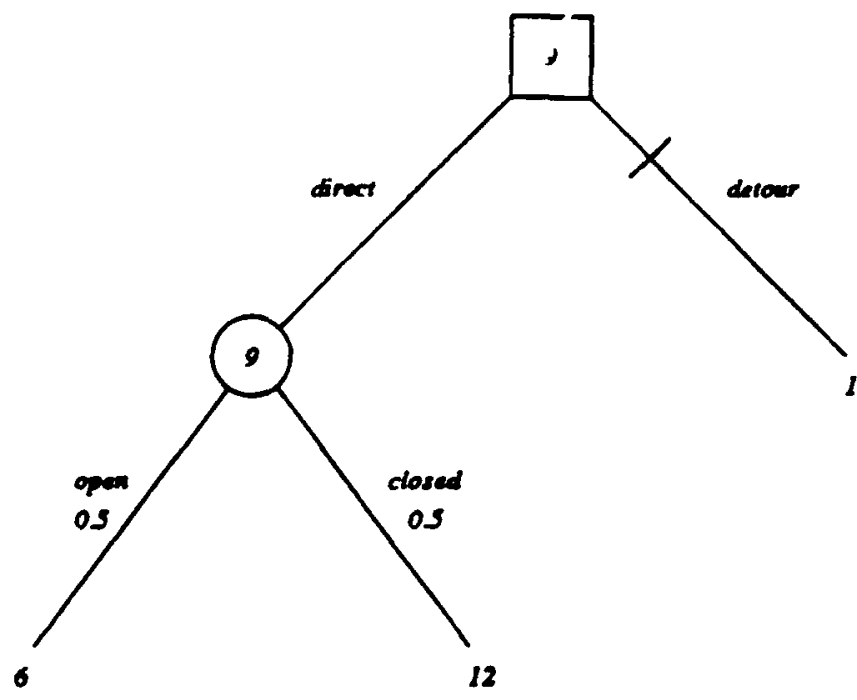

Figure i.t: Evaluated decision tree for the vacation trip problem

If we are concerned with value instead of cost. substitute cost everywhere for value. and naximum and maximize everywhere for nunimum and mininize.

Figure $i . t$ shows the labeled and marked decision tree for the vacation trip problem obtained using the above procedure. The optimal decision is to try the direct route first. and the expected transit time in this case is 9 hours.

We can extend the above analysis to handle sequences of actions of length $n$. Let $\bar{\alpha}=\alpha_{1}, \alpha_{2}, \ldots, \alpha_{n}$, where $\bar{\alpha} \in \mathcal{A} \times \mathcal{A} \times \ldots \mathcal{A}$. The result of executing the sequence of actions $\alpha_{1}, \alpha_{2}, \ldots, \alpha_{n}$ in $\omega$ is

$$
\left.\left[\alpha_{n} \| \alpha_{n-1} \mid\left[\ldots\left[\alpha_{1} \mid \omega\right] \ldots\right]\right]\right] \text {, }
$$

abbreviated $\left[\alpha_{1}, \alpha_{2}, \ldots, \alpha_{n} \mid \omega\right]$. We dente the $k$ th action in the sequence $\bar{r}=$ $\alpha_{1}, \alpha_{2}, \ldots, \alpha_{k}, \ldots, \alpha_{n}$ as $\bar{\alpha}_{k}$. The corresponding decision tree is shown in Figure 7.5. There are two things to note about the tree shown in Figure i.5. Fint, the tree is likely to be quile large, $O\left(|\mathcal{A}|^{n}|\Omega 2|\right)$ nodes. Second, the tree is not interesting in terms of capturing the structure of the clecision problum. We unight as well just use the simple two-level decision tree shown in Figure 7.1, and let the choice of what action to take range over the complex actious in $\mathcal{A} \times \mathcal{A} \times \ldots \mathcal{A}$.

There are cases. however. in which actions can alter an agent's decision making capability by providing additional information. For instance, if 


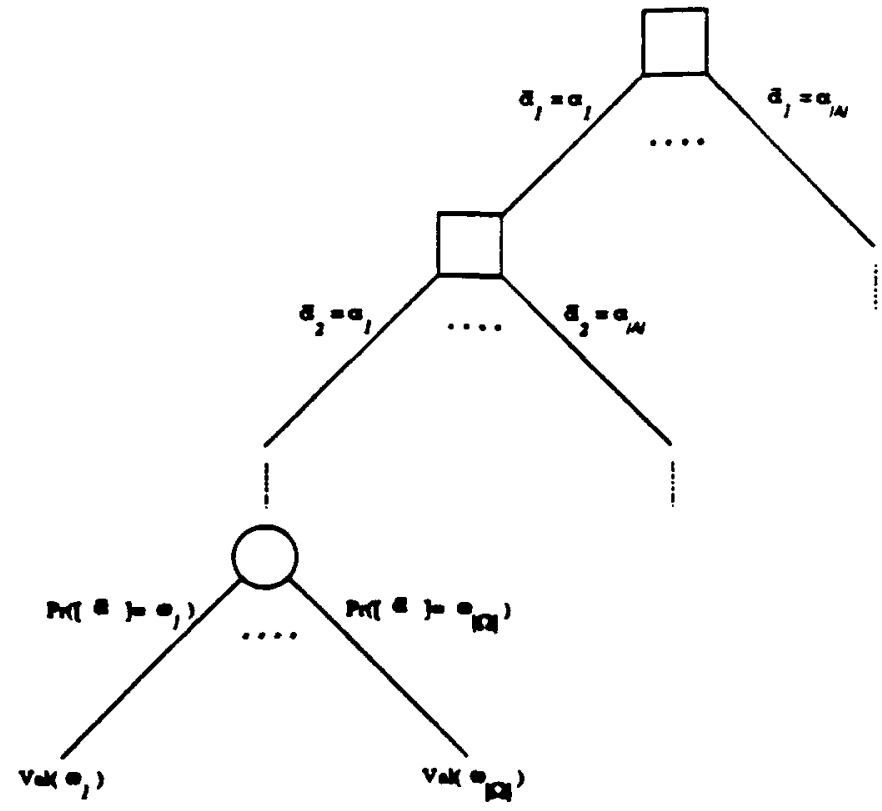

Figure i.5: Sequential Decision Tree

yon are interested in buying a used car. hiring a mechanic to check the car's cundition before making a purchase will probably reluce the possibility that you end up buving a car with high repair costs. By representing the consequences of such information-gathering actious explicilly in our graphical representations for decision problems, we can gain sone additional iusight into the structure of such problems.

In our vacation trip example, suppose there is a state police station located near the lighway prior to the point at which we have to decide between the direct and detour routes. We will assume that the state police call provide us with information about the current status of the bridge. Suppose that stopping at the police station requires getting off the highway and traving to a nearby lown, and that the total time spent in acquiring the inforation about the bridge is estimated to be 30 minutes.

Now wave an additional decision to make besides simply whether to take the direct or detour route. You can think of the trip to the police station as particular type of test with two possible findings: the state police believe that the bridge is open or they believe that it is closed. The findings may not provide conclusive evidence with regard to the primary question 
we are interested in. namely whether or not the bridge is slosed. but let us suppose in this case that the beliefs of the state police are veridical.

We represent possible findings of our test as a chance node in the clecision tree. The probabilities correspond to our priors regarding the status of the bridge. since at this time we have no beller information. Inder each of the two possible findings, we attach the tree shown in Figure $\mathbf{i . 3}$ with one change: the probabilities for the chance nodes corresponding to whether or not the bridge is closed are now conditioned on the findings. Civen our assumption that the police know the true status of the bridge, the probahility the bridge is closed given the police say it is closed is 1 , the probability the bridge is rlosed given the police say it is open is 0 , and so on.

Figure $\mathbf{7 . 6}$ shows the decision tree for the vacation trip problem with the decision node corresponding to driving to the police station or not. The two options are labeled check and not cherk. We also label test options with their associated costs. Information costs. Every tine that you get operator assistance in dialing a long-distance number or consult an accountant abour your incone tax you are paying for information. In the vacation trip problem, the cost of the information regarding the status of the hridge is in terms of increased driving time: 1/2 hour for the check option and un increase in tine for the not rheck option. In computing the optimal derision for a decision problem with decision nodes correnponding to test.s. We ralcnlate the maxinum values for the labels of such nodes accounting for these costs.

In the case of decision problems with actions to acquire information. the optimal decision is a conditional plan sperifying what to do at each point in time given the information available at the time. This conditional plan is called the optimal policy in the decision sciences: The optimal policy for the decision tree sliown in Figure $i .6$ is to rheck with the state police, and then take the direct ronte if the police say the bridge is open and the detour otherwise. For this policy, the expected transit time is 8 and $1 / 2$ hours.

It is often usefnl to be able to assess the value of information so as to make reaconable decisions regarding whether or not to pay for it. We call quantity the value of information in decision-theoretic terms.

In the vacation trip example. we were able to compnte the expected value of making the trip by selecting actions that minimize expected travel time hased on the information at hand. Let

$$
\mathrm{E}(T \mid \varepsilon)
$$

be the expected travel time, $T$. for the optimal course of action based on 


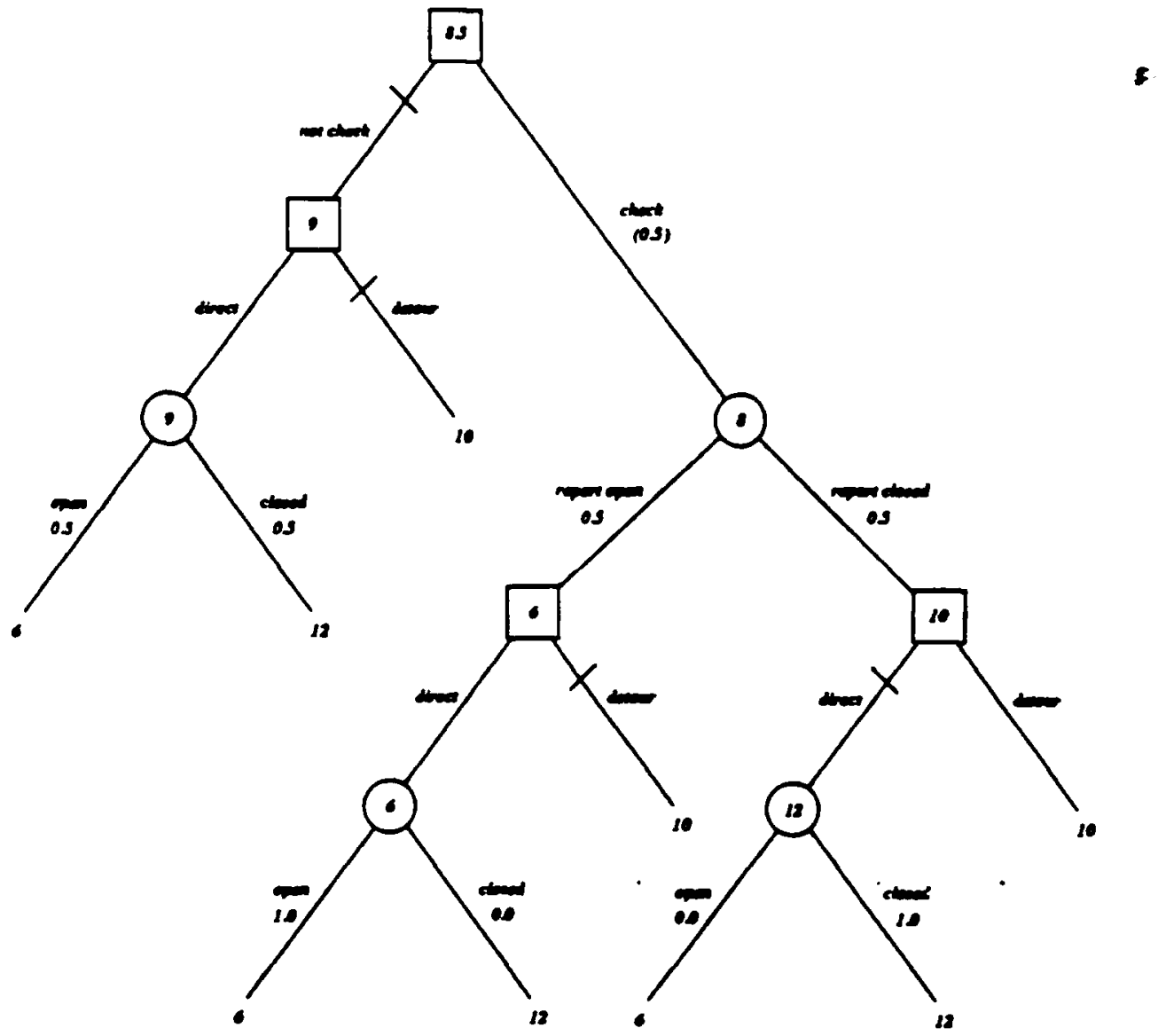

Figure i.6: Reasoning about information gathering actions 
the barkground information. s. In reasoning ahout whether or not to top at the state pulice station. we computed the expected travel lime given the additional information obtained from the police:

$$
\mathrm{E}\left(T \mid I_{S}, \varepsilon\right)
$$

where $I_{S}$ represents the event of obtaining information from the police regardiug the status, $S$. of the bridge. either open or clused. The expected value of the information obtained from stopping at the police station is

$$
\mathrm{E}\left(\mathrm{V}\left(I_{S}\right) \mid \mathcal{E}\right)=E\left(T \mid I_{S}, \mathcal{E}\right)-\mathrm{E}(T \mid \mathcal{E})
$$

where

$$
\begin{aligned}
& \mathrm{E}\left(T \mid I_{S}, \mathcal{E}\right)= \\
& \mathrm{E}\left(T \mid S^{\prime}=\text { closed. } \mathcal{E}\right) \operatorname{Pr}(S=\text { closed } \mid \mathcal{E})+\mathrm{E}(T \mid S=\text { open. } \mathcal{E}) \operatorname{Pr}(S=\text { open } \mid \mathcal{E}) .
\end{aligned}
$$

In the example, $E\left(V\left(I_{S}\right) \mid \mathcal{E}\right)=1.0$, implying that we should be willing to spend up to one hour to obtain the information regarding the status of the bridge.

More generally, let $E\left(V\left([]_{1}\right) \mid \vec{E}\right)$ be the expected value of carrying out your present policy. Suppose that, prior to carrying out your present policy, someone offers to sell you information pertaining to some variable. $x$, used in calculating $E(V)[] \mid \xi)$. To be more specific, suppose that the informant is clairvoyant and knows the actual value of $\boldsymbol{X}$. Let $I_{X}$ correspond to the event of obtaining the information regarding $x$.

The expected value of obtaining this information is given by

$$
\mathrm{E}\left(\mathrm{V}\left(I_{\boldsymbol{X}}\right) \mid \mathcal{E}\right)=\mathrm{E}\left(\mathrm{V}([)) \mid I_{\boldsymbol{X}}, \mathcal{E}\right)-\mathrm{E}(\mathrm{V}(\boldsymbol{D}) \mid \mathcal{E})
$$

To compute $E\left(\operatorname{Val}\left([) \mid I_{X} \cdot \mathcal{E}\right)\right.$, we evaluate the expectation given knowledge about $I$ for each possible value of $X$ provided by the informant. summing over these expectations weighted by our prior on. $\mathrm{I}$

$$
E\left(V(D) \mid I_{X}, \mathcal{E}\right)=\sum_{x \in \Omega_{X}} \mathrm{E}(\mathrm{V}(\mathbb{D}) \mid X=x, \mathcal{S}) \operatorname{Pr}(X=x \mid \varepsilon)
$$

It is important to note. as did Howard in the 1960 paper [19] in which he int roduced Equations $\mathbf{i} .3$ and $\mathbf{i} .4$. lhat we use the prior distribution $\operatorname{Pr}(\boldsymbol{X} \mid \boldsymbol{E}$. $)$ for $\boldsymbol{H}$ becanse, until the informant provides the information about $\boldsymbol{X}$, our knowledge of $\boldsymbol{X}$ is based entirely on our background knowledge $\mathcal{E}$. 
A good deal of the discussion in this and the next chapter will concern 1. asoning about the value of information and using the results of this reasoning to direct action. Before we can progress much further. we need to provide some additional machinery for probabilistic reasoning. In the next section. We consider a particular framework for modeling the world in the presence of uncertainty. We show how this framework can be extended to handle decision making, and then we demonstrate the power of the extended framework using applications involving sensing and mobile robolics.

IIace to establish a generic name for uhat huve been called Bayes nets, Bnyesian neturorks, belief networks. probnbilistic networks. influence diayrums and uho knous u'kat else.

\subsection{Probabilistic Networks}

I probabilistic network is a clirected acyclic graph $\mathcal{G}=(\nabla . E)$, where $V$ is a finite set of vertices. and $E$. the set of edges. is a subset of $V \times r$. the set of ordered pairs of distinct vertices. Before we discuss how to use these probabilistic networks to build decision models, we introduce and define some standard graph theoretic terms.

If $\left(v_{1}, v_{2}\right) \in \mathcal{G}$. then $v_{1}$ is said to be a parent of $v_{2}$, and $v_{2}$ a child of $v_{1}$. The set of all parents of $v$ is denoted $\mathrm{Pa}(v)$ and the set of all children $\mathrm{C} h(v)$.

A path of length $n$ from $v_{0}$ to $v_{n}$ is a sequence $r_{0} . r_{1} \ldots . . n_{n}$ such that $\left(r_{i-1}, r_{i}\right) \in E$ for $i=1 \ldots \ldots n$. If there is a path from $r_{1}$ to $r_{2}$. then $r_{1}$ is said to be an ancrstor of $v_{22}$. The set of all ancestors of $r$ is denoted $A n(v)$. $A$ subset $S \subseteq V$ is said to sfmmte $V^{\prime} \subseteq V$ from $V^{\prime \prime} \subseteq V$ if every path from a vertex in $V^{\prime \prime}$ to a vertex in $V^{\prime \prime}$ intersects $S$.

We can obtain an undirected graph from $\mathcal{G}$ by ignoring the ordering on the pairs of vertices in $E$. The graph 80 obtained is called the undirected graph corresponding to $\mathcal{G}$. If $V^{\prime \prime} \subseteq V$, then $l^{\prime \prime}$ induces a subgraph $\mathcal{G}_{\nu^{\prime \prime}}=$ $\left(I^{\prime \prime} . E_{\left.l^{\prime \prime}\right)}\right.$ where $E_{l}$ is that subset of $E$ restricted to $V^{\prime \prime} \times I^{\prime \prime}$. A graph ( $\left.V^{*} E\right)$ is complctc if for all $v_{1}, v_{2} \in V$ either $\left(v_{1}, v_{2}\right) \in E$ or $\left(v_{2}, v_{1}\right) \in E$. If $V^{-1} \subseteq V$ induces a complete subgraph, then $V^{\prime \prime}$ is sajd to be complete. A complete subem that is maximal with respect to set inclusion is called a cliquc.

For th directed acyclic graph $(V, E)$, we define its moml gmph as the undireted graph with the same vertex set in which $n_{1}$ is adjacent to $n_{2}$ juat in case either $\left(v_{1}, v_{2}\right) \in E$. $\left(v_{2}, v_{1}\right) \in E$. or there exists $r_{3} \in I^{-}$such that both $\left(r_{1}, v_{3}\right) \in E$ and $\left(v_{2}, v_{3}\right) \in E$.

The vertices in $V$ correspond to randon variables and are called chance 
nodes as in decision trees. The edges in $E$ define the causal and informational dependencies between the random variables. In the models described here. chance nodes are discrete-valued variables that encode states of knowledge about the world. We use upper-case italic letters (e.g.. I) to represent raudom variables. and lower-case italic letlers $(e . g . . x)$ to represent their possible values. Let $\Omega_{X}$ denole the set of possible values (state sprice) of the chance node $X$. In order to quantify a probabilistic network. we have to specify a probability distribution for each node. If the chance node has no parents, then this is its unconditional (manginal) probability distribution. $\operatorname{Pr}(X)$; otherwise, it is a conditioual probability distribution dependent on the states of the parents. $\operatorname{Pr}(X \mid \mathrm{Pa}(X))$.

If $V=\left\{I_{1}, X_{2}, \ldots, X_{n}\right\}$, we can write down the joint distribution using the chain rule as follows:

$$
\begin{aligned}
& \operatorname{Pr}\left(x_{1}, x_{2}, \ldots, x_{n}\right)= \\
& \quad \operatorname{Pr}\left(x_{n} \mid x_{n-1}, \ldots x_{1}\right) \operatorname{Pr}\left(x_{n-1} \mid x_{n-2}, \ldots x_{1}\right) \ldots \operatorname{Pr}\left(x_{2} \mid x_{1}\right) \operatorname{Pr}\left(x_{1}\right) .
\end{aligned}
$$

There are certain independence assumptions implicit in the structure of probabilistic networks that enable us to simplify this expression somewhat. A complete characterization of the conditional independencies embodied in the structure of a given probabilistic network can be given in graph theoretic lerms. For a given $\mathcal{G}=\left(V^{\circ} E\right)$ and subsets $V^{\prime \prime} . V^{\prime \prime} . S \subseteq V . V^{\prime \prime}$ is conditionally independent of $V^{\prime \prime}$ given $S$ if $S$ separates $V^{\prime \prime}$ from $V^{\prime \prime}$ in the moral graph for $\dot{G}$. From this characterization. it follows that a chance node is conditionally independent of its ancestors given its parents:

$$
\operatorname{Pr}(x \mid A n(x))=\operatorname{Pr}(x \mid \operatorname{Pa}(X)) \text {. }
$$

If the indices. $1, \ldots n$, of the variables. $x_{1}, x_{2}, \ldots, x_{n}$, are consistent with the partial ordering in $\mathcal{G}$ (i.e. $\left.\left(X_{i}, X_{i+k}\right) \in E \supset k>0\right)$, then we can use this conditional independence property to simplify our expression for the joint distribution:

$$
\operatorname{Pr}\left(x_{1}, x_{2}, \ldots, x_{n}\right)=\prod_{i=1}^{n} \operatorname{Pr}\left(x_{i} \mid \operatorname{Pa}\left(x_{i}\right)\right)
$$

The aice thing abont Equation 7.5 is that the product terms the righthand side are exactly the marginal and conditional probabilities required to quantify the network.

In using probabilistic networks for planning and control. we generally wish to compute the posterior distribution for sone ranclom variable givell 
some evidence o some proposed artion or conten plated observation. Intuitively. we are 'uterested in uprating our beliefs given the evidence obtained so far, and reasoning hypothetically about possible future courses of action. In the vacation trip example. before we slart out on the trip. we hyporhesize abont taking various rontes and making information gathering side trips. After stopping at the state police station, we upclate our beliefs regarding the status of the bridge by incorporating the evidence obtained from the police.

To capture this process of updating beliefs and reasoning hypothetically, we introduce the notion of a belief function defined on each of the random variables in $\mathcal{G}$ as

$$
\operatorname{Bel}(\boldsymbol{X})=\operatorname{Pr}(\boldsymbol{X} \mid \mathcal{E}),
$$

where $\mathfrak{f}$ represents all of the evidence obtained so far. Whenever we obtain new evidence, we extend $\mathcal{S}$ and update $\operatorname{Bel}(X)$ for all $X$ of interest. Itypothetical reasoning is handled by including additional conditioning infurmation. as in

$$
\operatorname{Bel}\left(X^{*}\right)=\operatorname{Pr}(X \mid Y, \varepsilon) .
$$

We can compute Bel( $X$ ) directly using the joint distribution defined in Equation 7.5. For instance, suppose that $V=\{A . B . C\}$. and we have obtained as evidence the actual value of $B$. To compute the belief function on $A$ given the eviclence regarding $B$. we need $\operatorname{Pr}(\lambda \mid B)$. By the definition of conditional probability. we have

$$
\operatorname{Pr}(A \mid B)=\frac{\operatorname{Pr}(A, B)}{\operatorname{Pr}(A)}
$$

We can obtain $\operatorname{Pr}(A)$ by summing the joint distribution over all variables except $A$ as in

$$
\operatorname{Pr}(\dot{A})=\sum_{c \in \Omega_{C}} \sum_{b \in \Omega_{B}} \operatorname{Pr}(A, B=b, C=c) .
$$

This is referred to as maminalizing the joint probability distribution to $\mathrm{A}$. We obtain $\operatorname{Pr}(A, B)$ in a similar manner as

$$
\operatorname{Pr}(A, B)=\sum_{c \in n_{c}} \operatorname{Pr}(A, B, C=c) .
$$

This particular method of compnting belief functions can involve a number of arithmetic operations linear in the size of the joint probability space:

$$
\prod_{x \in 1^{*}}\left|\Omega_{x}\right|
$$




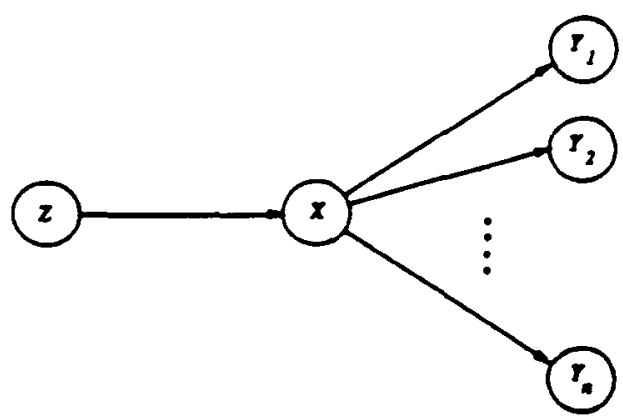

Figure $7.7:$ Simple tree-structured probabilistic network

In many cases, we can do significantly better from a computational standpoint by exploiting the struct.ure of the graph. In particular, if $\dot{G}$ is a t.ree (i.e., for all $v \in I^{\circ} . \operatorname{la}(v) \leq 1$ ), then we can compute the helief function for all variables in $r$ in time proportional to

$$
\sum_{x \in V^{\prime}}\left(\prod_{V \in \mathrm{P}_{\mathrm{a}(X)}} \mid \Omega_{Y} !\right) .
$$

For trees. the only information required to compute the belief function at a given node can be obtained from adjacent nodes in the graph. The conplexity arises from the local structure of the graph.

In the following, we describe how to compute the belief function for trees. While trees occur infrequently in practice, the exercise provides some additional insight iuto probabilistic networks. Following the description of the method for haudling trees, we describe a method of transforming arbitrary probabilistic networks into hyper graphs with tree-like structure that can be handled by methods similar to those used for trees.

Consider how we might compute $\operatorname{Pr}\left(X \mid Z, Y_{1}, \ldots, Y_{n}\right)$ giveu the treestructured probabilistic network shown in Figure i.i. Applying Bayes rule. we have

$$
\operatorname{Pr}\left(X \mid Z, Y_{1}, \ldots, Y_{n}\right)=\frac{\operatorname{Pr}\left(Z . Y_{1}, \ldots Y_{n} \mid X\right) \operatorname{Pr}(X)}{\operatorname{Pr}\left(Z, Y_{1}, \ldots Y_{n}\right)}
$$

Marginalizing in the denoninator. we have

$$
\operatorname{Pr}\left(Z, Y_{1}, \ldots, Y_{n}\right)=\sum_{r \in \Omega_{x}} \operatorname{Pr}\left(Z, Y_{1}, \ldots Y_{n}|.| \mid \operatorname{Pr} . Y\right)
$$


I'sing conditional independence and applying Bayes rule again. we have

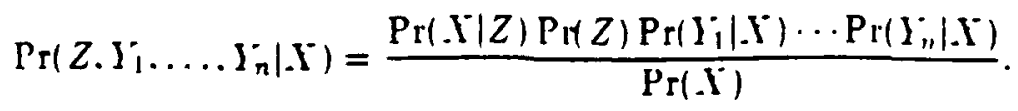

Substiluting, we have

$$
\begin{aligned}
& \operatorname{Pr}\left(X \mid Z . Y_{1} \ldots \ldots Y_{n}\right)= \\
& \frac{\operatorname{Pr}(X \mid Z) \operatorname{Pr}(Z) \operatorname{Pr}\left(I_{1} \mid X\right) \ldots \operatorname{Pr}\left(Y_{n} \mid X\right)}{\sum_{x \in \Omega_{X}} \operatorname{Pr}(X=x \mid Z) \operatorname{Pr}(Z) \operatorname{Pr}\left(Y_{1} \mid X=x\right) \cdots \operatorname{Pr}\left(I_{n} \mid X=x\right)} .
\end{aligned}
$$

which requires only the marginal and conditional probabilities necessary to quantify the probabilistic network shown in Figure $7 . \overline{7}$.

For the problems we will be considering. evidence corresponds to the instantiation of variables at the boundary of the network (i.e.. variables with no parents or no children). The impact of evidence on variables not on the boundary has to be assessed by propagating the effects of evidence through intervening variables. In Figure $i . i$, the set $\left\{Z . Y_{1} \ldots \ldots I_{n}\right\}$ corresponds to the boundary. Some or all of the variables tit the boundary may be instantiated in response to observations nade by the agent. After each observation. the belief function will require updating. For instance. having determined $\operatorname{Pr}\left(X \mid Z . Y_{1} \ldots \ldots Y_{n}\right)$, we can compute $\operatorname{Bel}(X)$ for $\varepsilon=Z=z . Y_{1}=y_{1} \ldots \ldots Y_{n}=y_{n}$.

Let e represent all of the evidence obtained thus far. Removing $X$ separates $\mathcal{G}$ into $n+1$ subtrees associated with the single parent of $\mathcal{X}$ and its $n$ children. We partition $e$ into $n+1$ components corresponding to these $n+1$ subtrees. Let $e^{+}$be the evidence associated with the parent of $X$. nnd $e_{i}^{-}$be the evidence associated with the $i$ th child of $x$. Figure $\bar{i} .8$ illustrates this partition graphically. Suppose that $X$ can obtain $\operatorname{Pr}\left(Z / e^{+}\right)$from $Z$, and $\operatorname{Pr}\left(e_{i}^{-} \mid Y_{i}\right)$ froin $Y_{i}$. Given this information. we can compute $\operatorname{Pr}\left(I^{-} \mid e\right)$ in a manner similar to that used in computing $\operatorname{Pr}\left(X \mid Z . Y_{1} \ldots \ldots Y_{n}\right)$ above:

$$
\begin{aligned}
& \operatorname{Pr}(X \mid e)=\operatorname{Pr}\left(X \mid e^{+}, e_{1}^{-}, \ldots, e_{n}^{-}\right)= \\
& \frac{\operatorname{Pr}\left(X \mid e^{+}\right) \operatorname{Pr}\left(e_{1}^{-} \mid X\right) \cdots \operatorname{Pr}\left(e_{n}^{-} \mid X^{-}\right)}{\sum_{x \in n_{X}} \operatorname{Pr}\left(X=x \mid e^{+}\right) \operatorname{Pr}\left(e_{1}^{-} \mid X=x\right) \cdots \operatorname{Pr}\left(e_{n}^{-} \mid X^{-}=x\right)} .
\end{aligned}
$$

where $\operatorname{Pr}\left(X \mid e^{+}\right)$is obtained from $\operatorname{Pr}(X \mid Z)$ and $\operatorname{Pr}\left(Z \mid e^{+}\right)$as follows:

$$
\operatorname{Pr}\left(X \mid e^{+}\right)=\sum_{z \in \Omega_{Z}} \operatorname{Pr}(X \mid Z=z) \operatorname{Pr}\left(Z=z \mid e^{+}\right) \text {. }
$$




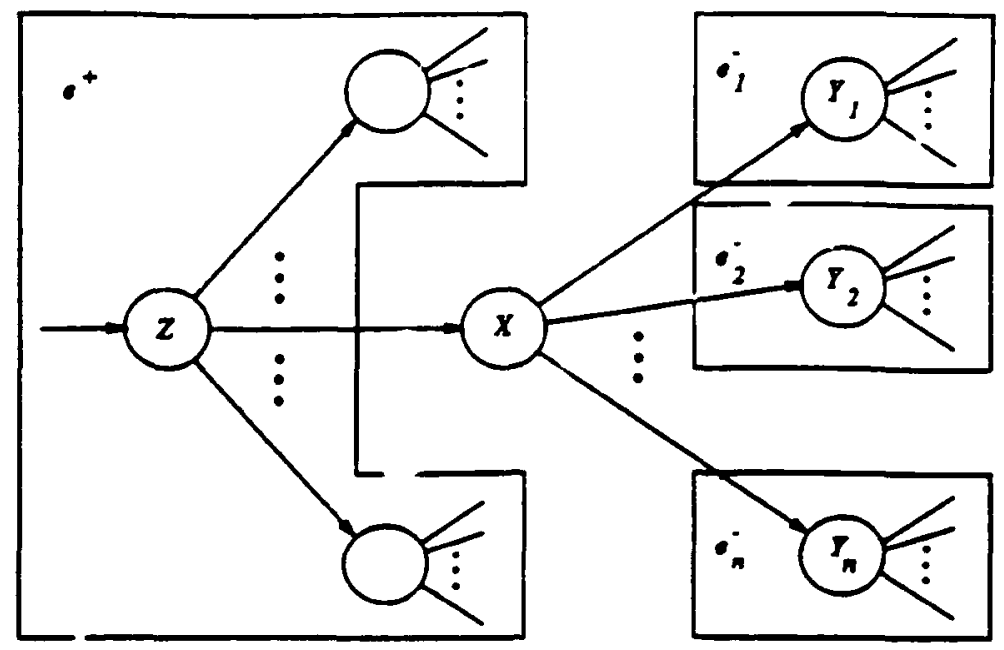

Figure 7.8 : Partitioniug the evidence bearing on $X$ into subtrees

Evidence propagation occurs by local message passing. Each node kceps track of $n+1$ messages corresponding to the last nessages received from its single parent and each of its $n$ children. The node corresponding to $X$ recomputes $\operatorname{Pr}(X \mid e)$ only in the event that it receives a message from a parent or child that differs from the last message received from that same parent or child. Nodes corresponding to evidence ignore incoming messages. If $\mathrm{I}$ recomputes $\operatorname{Pr}(\mathrm{X} \mid \mathrm{e})$, it also recomputes appropriate messages to send to its parent and children. and then sends these niessages. The message $I$ sends to its parent $Z$ is computed as

$$
\operatorname{Pr}\left(e_{\bar{X}} \mid Z\right)=\sum_{r \in \Omega_{X}}\left(\operatorname{Pr}(X=r \mid Z) \prod_{i=1}^{n} \operatorname{Pr}\left(e_{i}^{-} \mid x\right)\right),
$$

where $e_{\bar{x}}$ indicatcs the cvidence in the subtree rooted at $X$. The message $r$ sends to its $k$ th child is computed as

$$
\operatorname{Pr}\left(X \mid e_{X / I_{k}}^{+}\right)=\operatorname{Pr}\left(X \mid e^{+}, e_{1}^{-}, \ldots, e_{k-1}^{-}, e_{k+1}^{-}, \ldots, e_{n}^{-}\right) .
$$

where $e_{x / Y_{h}}^{+}$indicates all of the evidence in the tree rooted at $X_{\text {except that }}$ found in the subtree rooted at $\zeta_{k}$. Note that the right-hand side of this expression can be computed in a manner similar to that used in computing $\operatorname{Pr}(X \mid e)$ by simply eliminating the $\operatorname{Pr}\left(\mathbf{e}_{k}^{-} \mid X\right)$ factor. 


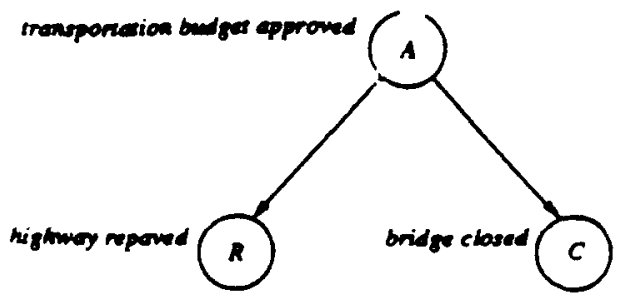

Figure i.9: Propagating eviclence in trees

All of these messages require only information available from the either originating node or fronı messages sent by parents and children. If we assune unit cost for updating the local information at. stored at a node in response to a new message. then the cost of updating $\operatorname{Bel}(X)$ for all $X \in I^{\circ}$ in response to new evidence originating at a single node is proportional to $V^{\circ}$ in the worst case.

Consider the following example illustrating how evidence propagates through a tree-structured network. The example extends the earlier example concerning the status of a critical bridge in planning a vacation trip. Suppose that we know some additional information regarding the status of this bridge. In particular, suppose we know that the repairs to the bridge that would result in its closing are contingent. upon an increase in the state transportation budget. This budget. increase was to be voted on in the stat.e legislature earlier in the year. Unfortunatcly, we did not hear the ontcome of the vote. but the same increase was to be used to repave a portion of the highway that will have to be traversed near the beginning of the trip.

We introduce three boolean-valued random variables: $A$ representing the proposition that the budget increase was approved. $C$ representing the proposition that the bridge is rlnscd, and $R$ representing the proposition that the highway portion in question was repaved. Suppose that we have a prior distribution on the budget approval,

\begin{tabular}{|c|c|}
\hline & $\overline{\operatorname{Pr}}(A)$ \\
\hline$A=$ true & 0.1 \\
\hline$A=$ false & 0.9 \\
\hline
\end{tabular}

a conditional probability distribution for the bridge being closed given that the budget is approved. 


\begin{tabular}{|l|c|c|}
\hline \multirow{2}{*}{$C=$ true } & \multicolumn{2}{|c|}{$\operatorname{Pr}(C \mid . A)$} \\
\cline { 2 - 3 } & $A=$ rrue & $A=$ false \\
\hline$C=$ false & 0.1 & 0.2 \\
\hline
\end{tabular}

and a conditional probability distribution for the highway being repaved given that the budget is approved.

\begin{tabular}{|l|c|c|}
\hline \multirow{2}{*}{} & \multicolumn{2}{|c|}{$\operatorname{Pr}(R \mid A)$} \\
\cline { 2 - 3 } & $A=$ true & $A=$ false \\
\hline$R=$ true & 0.6 & 0.1 \\
\hline$R=$ talse & 0.4 & 0.9 \\
\hline
\end{tabular}

The resulting network is shown in Figure 7.9. Now. suppose that during the early part of our trip we discover that the portion of the highway iu question has indeed been repaved. We want to update the network to reflect the evidence: $R=$ irue. For the purposes of the trip example. we are interested in

$$
\operatorname{Bel}(C=\text { true })=\operatorname{Pr}\left(C^{\prime}=\operatorname{true} \mid R=\text { true }\right)
$$

in order to determine whet her to take the direct or detour routes. To npilate $(\therefore$ we will also update $A$ in the process of propagating the ismpact of the evidenre.

For the simple network shown in Figure 7.9, we can easily compute the belief function using the joint distribution,

$$
\operatorname{Pr}\left(A, C^{\prime}, R\right)=\operatorname{Pr}\left(C^{\prime} \mid A\right) \operatorname{Pr}(R \mid A) \operatorname{Pr}(A),
$$

As described earlier, by defiuition we have

$$
\operatorname{Pr}(C=\text { true } \mid R=\text { true })=\frac{\operatorname{Pr}(C=\text { true. } R=\text { true })}{\operatorname{Pr}(R=\text { true })} .
$$

Marginalizing, we compute the numerator by summing over $\Omega_{A}$.

$$
\begin{aligned}
& \operatorname{Pr}(C=\text { true. } R=\text { true })= \\
& \operatorname{Pr}(C=\text { true } A=\text { true }) \operatorname{Pr}(R=\text { true } A=\text { true }) \operatorname{Pr}(A=\text { true })+ \\
& \operatorname{Pr}(C=\text { true } A=\text { talse }) \operatorname{Pr}(R=\text { true } A=\text { talse }) \operatorname{Pr}(A=\text { talse }),
\end{aligned}
$$

and the denominator by sumning over $\Omega_{4} \times \Omega_{C}$.

$$
\operatorname{Pr}(R=\text { true })=
$$




$$
\begin{aligned}
& \operatorname{Pr}\left(C^{\prime}=\text { true } d=\text { true }\right) \operatorname{Pr}(R=\text { true } d=\text { true }) \operatorname{Pr}(d=\text { true })+ \\
& \operatorname{Pr}\left(C^{\prime}=\text { true } d=\text { talse }\right) \operatorname{Pr}(R=\text { true } d=\text { talse }) \operatorname{l}^{\prime}(A=\text { false })+ \\
& \operatorname{Pr}\left(C^{\prime}=\text { false } d=\text { true }\right) \operatorname{Pr}(R=\text { true } d=\text { true } \operatorname{Pr}(A=\text { true })+ \\
& \operatorname{Pr}(C=\text { false } A=\text { false }) \operatorname{Pr}(R=\text { true } d=\text { false }) \operatorname{Pr}(A=\text { false }) .
\end{aligned}
$$

to oht ain the value 0.4 for $\operatorname{Pr}(C=$ true $R=$ trne $)$. Now. consider how we might obtain the same value by local message passing.

Prior to obtaining any evirlence $A$. just sends $\operatorname{Pr}(A)$ to $C$ and $R$. and $C$ : and $R$ send the function that maps all of $\Omega_{C}$. to 1.0. After $C^{\prime}$ updates itself. we have $\operatorname{Pr}(C=$ true $)=0.25$. After obtaining the evidence $R=$ true, $R$ computes

$$
\begin{aligned}
\operatorname{Pr}(R=\operatorname{true} A)= & \operatorname{Pr}(K=(\text { rue } \mid R=\text { (rue) } \operatorname{Pr}(R=\operatorname{true} \mid A)+ \\
& \operatorname{Pr}(K=\text { (rue } R=\text { lalse }) \operatorname{Pr}(R=\text { talse } A),
\end{aligned}
$$

and sends this message to $A$.

In response to this message. $A$ updates its belief using the new message from $R$ and the old one from $C$ :

$\operatorname{Pr}(A \mid R=(r u e)=$

$\operatorname{Pr}(A) \operatorname{Pr}(R=\operatorname{trme} \mid A)$

$\overline{\operatorname{Pr}(A=\text { true }) \operatorname{Pr}(R=\text { rue } \mid A=\text { true })+\operatorname{Pr}(A=\text { false }) \operatorname{Pr}(R=\text { true } A=\text { false })}$

The message sent to $C$ from $A$ is just $\operatorname{Pr}(A \mid R=$ true $)$, and $C$ updates its belief as

$$
\begin{aligned}
\operatorname{Pr}(C \mid R=\text { true })= & \operatorname{Pr}(C \mid A=\text { true }) \operatorname{Pr}(A=\text { true } \mid R=\text { true })+ \\
& \operatorname{Pr}(C \mid A=\text { talse }) \operatorname{Pr}(A=\text { talse } \mid R=\text { true }),
\end{aligned}
$$

from which we compute $\operatorname{Pr}(C=$ true $\mid R=$ true $)=0.4$, the same value obtained using the joint distribution.

It is fairly straightforward to extend the above method for evaluating probeuniatic models to liandle networks in which a given node has more than owe parent, but there is at most one path between any two nodes in the corresponding undirected graph. Such networks are called singlyconnected. The exteusion involves keeping track of the evidence originating from subgraphs associated with the nodes of parents. Since there is a one-toone correspondence between the parents and children of a given node and the set of subgraphs resulting from renoving that node, keeping track of 


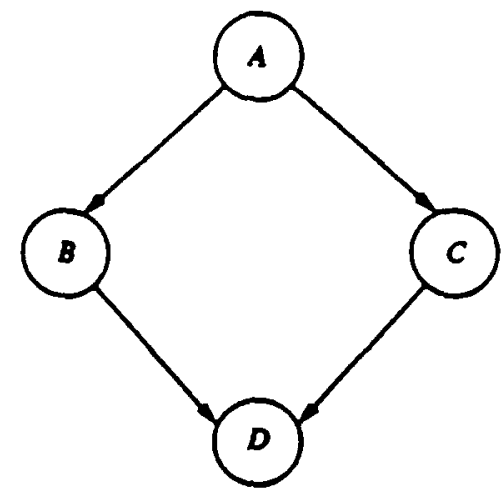

Figure i.10: $A$ multiply-connected network

evidence is relalively simple in singly-connected networks. The same cannot be said for multiply-connected nelu'orks. networks in which there are cycles in the corresponding undirected graph. Figure 7.10 shows a simple multiplyconnected network. Problems arise in trying to distribute the impact of the evidence on $A$ to $B$ and $C$. In the worst case, correctly routing evidence about in a multiply-connected network requires a global perspective. ('ouper has shown that exact evaluation of general probabilistic networks is NP-hard [9].

While computing the belief function for variables in probabilistic networks is intractable in the general case, we can often exploit the structure inherent in particular networks to reluce the cost of computation. One approach involves finding a set of variables, $\left\{x_{1}, \ldots x_{n}\right\}$, which, if removed from the network. would render it singly connected $\{$ e.g. the set $\{B\}$ in Figure $\mathbf{i} .10$ ). The belief function for a given node is taken as the weighted sum of the belief functions computed for all possible instantiations of the variables in $\left\{x_{1}, \ldots, x_{n}\right\}$. Calculating the weights is a rather complex, but the real trick involves linding a small set of variables to render the network singly connected. This is crucial since you have to calculate the betief function for $\prod_{i=1}^{n}\left|\Omega_{X_{1}}\right|$ variable instantialiuns.

A econd approach to evaluating general probabilistic networks also involve converting multiply-connected networks into singly-connected unes. This approach involves constructing a hyper graph whose vertices correspoud to the cliques of the chordal graph formed by triaugulating the moral graph for the given network. [ISay a little more about triangulation and churdal graphs.]] From this hyper graph, we extract a maximal spanumg 


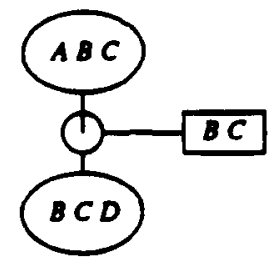

Figure i.11: Join tree for a multiply-connected network

tree which is referred to as a join trce. [ISay a little more about maxinal spanning trees.]] Figure 7.11 shows a join tree for the network of Figure i.10.

$$
\left.\operatorname{Pr}\left(A . B, C^{\prime}, D\right)=\operatorname{Pr}\left(D \mid B, C^{\prime}\right) \operatorname{Pr}(B \mid A)\right]^{\prime} r\left(C^{\prime} \mid A\right) \operatorname{Pr}(A)
$$

[[Say something about the messages passed in evaluating join trees. Provide some insight into Jenseu's variation on Lauritzen and Spiegelhaller by updating the graph shown in Figure $i .11$ (e.g., the role of the running intersection property).]]

The cost of evaluating a probabilistic network using the join-tree approach is largely determined by the sizes of the stale spaces formed by taking the cross product of the state spaces of the nudes in each vertex (clique) of the join tree. We can ubtain an accurate estimate of the cost of evaluating a probabilistic nel work. $\dot{\mathcal{G}}=(\mathbb{V}, E)$ as follows. Let $C=\left\{C_{i}\right\}$ be the set of cliques in the chordal graph described earlier. where each clique represents a subset of $V$. We define the function, Card : $C-\{1 \ldots \ldots|C|-1\}$, so that Card $\left(C_{i}\right)$ is the rank of the lighest ranked node in $C_{i}$. where rank is determined by the maximal cardinality ordering of $v$. [Say a little more about maximum cardinality ordering.]] We define the function. Adj : $C \rightarrow 2^{C}$, by:

$$
\left.\operatorname{Adj}\left(C_{i}\right)=\left\{C_{j} \| C_{j} \neq C_{i}\right) \wedge\left(C_{i} \cap C_{j} \neq 0\right)\right\}
$$

The join tree for $C^{\prime}$ is constructed as follows. Each clique $C_{i} \in C$ is connected to the clique $C_{j}$ in $\operatorname{Adj}\left(C_{i}\right)$ that has lower rank by $C^{\prime}$ ard $($.$) and has$ the riment number of nodes in common with $C_{i}$ (ties are broken arbitrarily). Where we conuect two cliques $C_{i}$ and $C_{j}$, we create the scpamtion set $S_{i j}=C_{i} \cap C_{j}$. The set of separation sets $S$ is all the $S_{i j}$ s. We define the function, Sep : $C \rightarrow 2^{S}$. by:

$$
\operatorname{Sep}\left(C_{i}^{\prime}\right)=\left\{S_{i k} \mid S_{j k} \in S,(j=i) \vee(k=i)\right\} .
$$


Finalli: we define the join-liet cost as

$$
\sum_{C_{i} \in C^{-}}\left(\left|\operatorname{Sep}\left(C_{t}\right)\right| \prod_{n \in C_{i}}\left|\Omega_{n}\right|\right) .
$$

where $\Omega_{n}$ is the state space of node $n$.

Say something about the mulliply-connected rasp. Given that the subsequent sections will refer to Jensen's uariation on Lauritien and Spiegelhalter. that algorithm should bo described as some level deeper than alrcarly attempted. An extremely detailed description is probnbly not warranted given that the material is readily available in a number of recent textbsoks (e.g., [32. 31]).

Introduce influence dingmms and relate them to the decision trees described earlier in the intioductory sections.

The first two examples of applying Bayesian netuorks to plunning and continl problems come from [11]. The first example considers the relatively simple problem of recormizing locally distinctive places. The second example considers the problem of choosing betu'een paths through knoun and unknoun teiritory. The latter frample can be used to illustmte some of the tmiteoff involved in working with multiply connected networks.

\subsection{Robot Navigation}

I siguificant problem in designing mobile robot control systems involves coping with the uncertainty that arises in moving about in an unknown or partially unkuown euviroument and relying on noisy or ambiguous sensor data to acquire knowledge about that euvironment. In this section. we consider a control system that chooses what activity to engage in next on the basis of expectations about how the information returned as a result of a given activity will improve its knowledge about the spatial lavout of its envirounent. Certain of the higher-level components of the control system are specified in terms of probabilistic decision models whose output is used to madiate the behavior of lower-level control components responsible for movement and sensing. The objective it to design control systems capable of dinectiag the behavior of a mobile robot in the exploration and napping of its environment. while attending to the real-time requirements of navigation and obstacle avoidance.

We are interested in building systems that construct and maintain representations of their enviromment for tasks involving navigation. Such systems 
should expend effort on the construction and maintenance of these representatious commensurate with expectations about their value for immediate and anticipated tasks. Such sisteuts should employ expectations about the information returned from sensors to assist in choosing activities that are most likely to improve the accuracy of its representations. Finally: in addition to reasoning about the future conseguences of acting. such systems nust attend to the immediate consequences of acting in a changing environnent: consequences that gencrally cannot be anticipated and hence require some amount of continuous attention and commitment in terms of computational resources.

We start with the premise that laving a map of your environment is generally a good thing if you need to move between specific places whose locations are clearly indicated on that map. The more frequent your need 10 move between locations. the nore useful you will probably find a good map. If you are not supplied with a map and you find yourself spending an inordinate annount of time blundering about. it might occur to you to build one. but the annount of time you spend in building a map will probably depend upon how much you anticipate using it. Once you have decider to build a map. you will have to decide when and exactly how to go about building it. Suppose that you are on an errand to deliver a package and you know of two possible routes. one of which is guaranteed to take you to your destination and a second which is not. By trying the second route. you inay learn something new about your environment that may turn out to be useful later. but you may also delay the completion of your errand.

The mobile robot that we cousider in the examples in the rest of this cliapter is a simple holonomic (turn-in-place) robot equipped with a number of sensors. The most inportaut sensor for our immediate purposes is the ultrasonic sonar sensor considered in the previous chapter. The robot's ultrasonic sensors provide it with information about the distance to nearby objects. With a little care, the robot can detect the presence of a variety of geometric features using these sensors. In gathering information about the office enviroument, the robot will drive up to a surface to be investigated. aligen one of the sensors to the right or to the left of its direction of travel alon the surface, and then move parallel to that surface looking for abrupt chan in the information returned by the aligned sensor that would indicate some geometric feature such as a $90^{\circ}$ corner. In doing this, it is possible to keep track of the accumulated error in its movement and the variation in its sensor data to assign a probability to whether or not a feature is present.

We assume that the robot has strategies for checking out many simple 
georr etric features found in typical office cuviromnents: we refer to these stri.cegies as fenture detertors. Each feature detector is realized as a control process that directs the robot's movement and sensing. On the basis of the data gathered during the execution of a given feature detector. a probability distribution is determined for the random variable corresponding to the proposition that the feature is present at a specific location.

The robot that we consider here is designed to explore its environment in order to build up a representation of that environment suitable for route planniug. In the course of exploration. the robot induces a graph that capt ures certain qualitative features of its environnent. In addition to detecting geometric leatures like corners and duor jaubs. the robot is able to classify locations. In particular. it is able lo distinguish between corridors and places where corridors meet or are punclualed by durs leading to olfices. labs, and storerooms. A corridur is defined as a piece of rectangular space bounded on two sides by uminterrupted parallel surfaces 1.5 to 2 meters apart and bounded on the other two sides by ports indicated by abrupt changes in one of the two parallel surfaces. The ports signal lorally distinctive places (LUP's) (after [23]) which generally correspoud to hallway junctious. Uninterrupted corridors are represented as arcs in the induced graph while junctious are represented as vertices. Junction; are further partitioned iuto classes of junctions (e.g.. L-sliaped junctions where t.wo corridors meet at right angles, or $T$-shaped junctions where one corridor is interrupted by a second perpendicular corridor). We will assume that the robot is given a set of junction classes that it uses to classify and the label the locations encountered during exploration.

In the following sectious. we consider two of the main decision processes that comprise the robot's control system. but first we consider briefly the overall architecture in which these decision processes are embedded.

In the following, we assume a nulti-level control system composed of a set of clecision processes rumning concurrently uncler a multi-lasking priorilized operating system. There is no shared stale information; all communication is handled by inter-process message passing. Run-lime process arbitration is handlat by dyuamically altering the process priorities. Courdination amoug procenem in achieved through a set of message-passing protocols.

The different processes that make up the controller are partitioued into levels (see Figure i.12). For each level, there is a corresponding arbitrator desigued to cuordinate the different processes located at that level. At Level 0 , we find the processes responsible for coutrol of the different seusor/effector systems on board the mobile base. Each Level 0 process is 


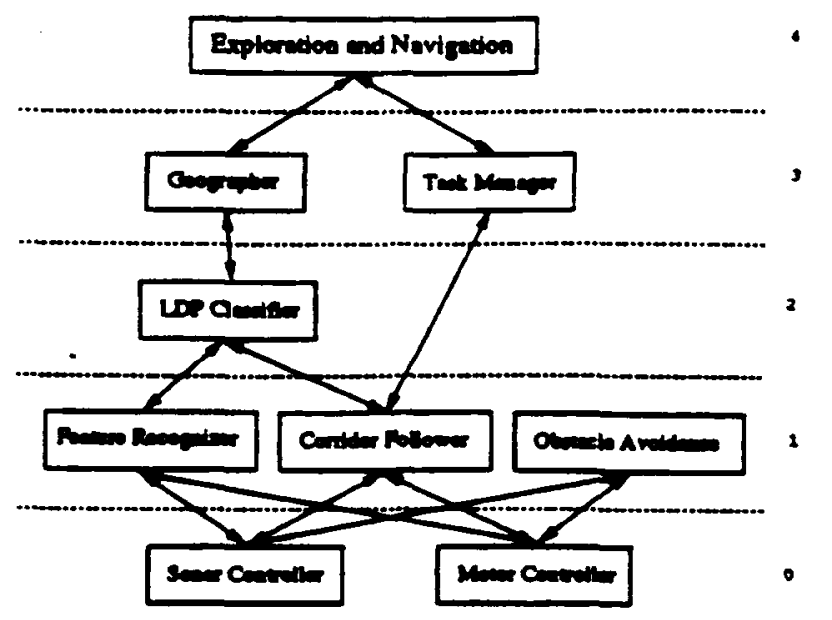

Figure 7.12 : Mobile robot control architecture

completely independent of the other processes, so no arbitration is needed. At Level 1 , we find the processes responsible for the low-level control of the robot. Level 1 processes are coordinated using a simple priority scheme: the obstacle avoidance process always takes priority over the other Level 1 processes. The activities of the feature recognition and corridor following processes are coordinated by higher-Invel proresses.

In the design shown in Figure $i .12$. there is only one Level 2 process. the LDP classificr, but. in a more complicated architecture. one could easily imagine several processes on this level. At Level 3, we find the two processes responsible for the robot 's higher-level behaviors: the task manager in charge of running user-specified errands. and the geographer in charge of exploration and map building. Both the geograplier and the task manager are special-purpose route planners: the geographer tends to construct paths through unknown territory and the task manager through known territory. The activities of these two processes are coordinated by a Level 4 decision process that takes into account the possible costs and benefits to be derived from drifereat strategies for mixing exploration and errand running. In the followises we consider the decision processes at Levels 2 and $t$.

\subsubsection{Classifying Locally Distinctive Places}

'pon exiting a corridor through a port. the robot will want to determine what sort of LDP it has entered. If the robot is in a reell-explored portion 
of its environment. this determmation should match its expectations as inelicated in it.s $\mathrm{m} \mathrm{p}$. If. On the other liand. the robot is in some unknown or only partially-explored area. this dotcrmination will be used to extend the map. possibly aldling new vertices or identifying the current. LDP with existing vertices. In this section. we clescribe how the robot might classify LDPs encountered during exploration.

Let $L$ be the set of all locally distinctive places in the robot 's environment. $C^{\prime}=\left\{C_{1}^{\prime}, C_{2}^{\prime}, \ldots, C_{n}^{\prime}\right\}$ be a set of equivalence classes that partitions $L$. and $F$ be a set of primitive geomet ric feat.ures (e.g. convex and conrave corners. flat walls). Each class in $C$ can be characterized as a set of features in $F$ that stand in some spatial relationship to one anuther. As the robot exits a port. a local coordinate sustelu is set up with its origin on the imaginary line defined by the exit port and centered in the corridor. The space about the origiv enclosing the LDP is divided into a set of equi-angular wedges IV. For each feature/wedge pair $\left(f, u^{\prime}\right)$ in $F \times W$, we define a specialized feature detector $d_{f, u}$ that is used to determine if the current LDP' satisties the feature $f$ at location $w$ in the coordinate system established upon entering the LDP. Let $U$ be the set of all such feature detectors plus no_op, a psendn-detector that results in no new information and takes no time or effort to : xernte.

The IDP-classification modnle maintains a a probabilistic assessment. of the hypotheses concerning the class of the current LDP given the evidence arquired thus far. At any given time, the robot will have tried some number of feature detectors. Let $P_{t}$ be the pool of detectors available for use at time $t: P_{\imath}$ is just $D$ less the set of detectors executed up until $t$ in classifying the current LDP. The LDP-classification module is responsible for choosing the next feature detector to invoke front the set $P_{t}$. It does so using a decision model cast in terms of an influence diagram.

The I.DP-rlassification morlule s influence diagran inclucles a set. of chance nodes corresponding to random rarialbles, a decision node corresponding to actions that the robot might take, and a value node representing the expected utility of invoking the different feature detectors in various circumstances. The chance nodes include a liypothesis variable, $I I$, that can take on vales from $C$, and a set of boolean variables of the form. $x_{f, w}$, used to reprement whether or not the feature $f$ is present at location ${ }^{\prime}$. Each $\boldsymbol{X}_{f, u}$ is conditioned on the bypothesis $H$ according to the distribution $\operatorname{Pr}\left(\boldsymbol{X}_{f, \ldots 1} \mid C_{i}\right)$ deternined by whether or not the class requires the feature at the specified lucalion. The decision node, $P_{t}$, indicates the feature detectors available for use at time $t$, and the value node. $v$, represents the utility of invoking each 


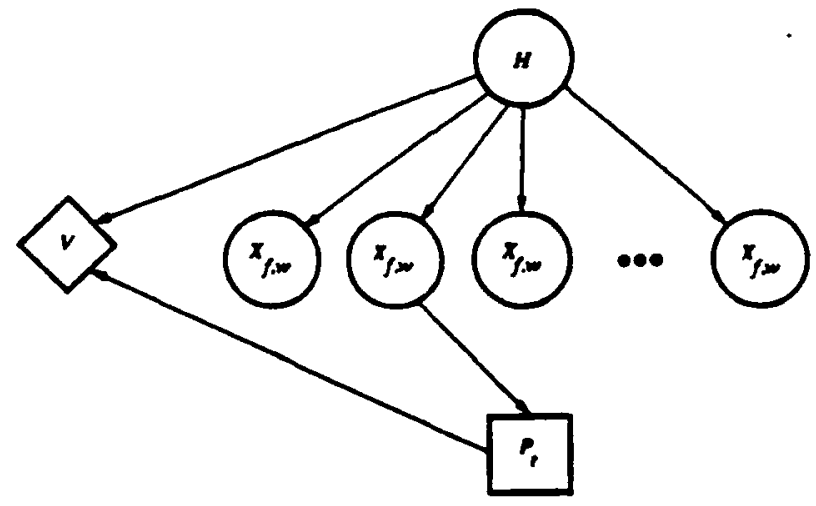

Figure i.13: LDP-classification module's influence diagram

feature detector. $V$ is dependent only upon the hypothesis and decision norles. The preileressors of $P_{t}$ are just the feature detectors invoked so far, thereby indicating temporal precedence and informational dependence. A graphical representation of the influence diagram is shown in Figure i.13.

The utility of invoking each detertor is based on (i) the ability of the cletector to discriminate among the hypotheses, (ii) the cost of deploying the detector. (iii) the probability that the current best hypothesis is correct. and (iv) the cost of nisidentifying the LDP. The first two are used to selert from ainong $D-\{n o-o p\}$ and the last two are nsed to choose between the best detertor from $D-\{$ no_op $\}$ and no.op. The LDP-classification inodule selects from $D-\{$ uo_op $\}$, using the function, $\mu: P_{t} \times H-t$. defined by $\mu\left(d_{f, w}, h\right)=$

$$
\kappa_{1} \operatorname{Discrim}\left(d_{f, w^{\prime}}\right)-\kappa_{2} \operatorname{Cost}\left(d_{f, w}, h\right),
$$

where $k_{1}$ and $k_{2}$ are constants used for scaling, $\operatorname{Cost}\left(d_{f, w^{*}}, h\right)$ is a function of the expected time spent in executing $d_{f, w}$ for an LDP of a given class. and Discrim $\left(d_{f, w}\right)$ is a variation on a standard discrimination function used in pattern recognition, and defined by

$$
\sum_{i=1}^{n} \operatorname{Pr}\left(C_{i}\right) \sum_{v \in\{0.1\}}\left|\operatorname{Pr}\left(d_{f, u}=n \mid C_{i}\right)-\operatorname{Pr}\left(d_{f, w}=n\right)\right|
$$

where $d_{f . u}=v$ is meant to represent the proposition that the detector $d_{f . n}$ returus the value $r$. The terms in the above formula are easily obtained. $\operatorname{Pr}\left(d_{f, w}=v \mid C_{i}\right)$ is the distribution associaled with the corresponding $I_{f, n}$ 
node. and $\operatorname{Pr}\left(A_{f . w}=r\right)$ can be calculated using

$$
\operatorname{Pr}\left(d_{f, u^{\prime}}=v\right)=\sum_{i=1}^{n} \operatorname{Pr}\left(d_{f . u}=n \mid C_{i}\right) \operatorname{Pr}\left(C_{1}^{\prime}\right)
$$

The L.DP-classification module evaluates the influence diagran using one of the methods described in Section 7.2 to obtain a decision policy and an expected value function for choosing from anoug $D-\left\{n 0_{-}\right.$op $\}$. The LDP-classification module can also choose to do nothing by selecting no_op, thereby committing to the class $C_{i}$ with the highest posterior probability given the information returned by the feature detectors invoked thus far. In a more realistic decision model. we might employ an additional set of chance nodes corresponding to micro fealures and a more extensive the set of feature than indicaled here. We would also want to allow for a feature detector to be invoked mulliple times.

\subsubsection{Expected Value of Exploration}

One could imagine several decision models for reasoning about the expected value of exploration. In the simple model presented in this sortion, we assume that the system of junctions and corridors that make up the rohot's environment can be registered on a grid so that every corridor is aligned with a gridl line and every junction is coincident with the intersertion of two grid lines. In the following, the set. of junction types. $J$, corresponds to all possilule conligurations of rorridors inrident. on the intersection of two grid lines. Intersections with at. least one incident corridor correspond to LDl's. Since we also assume that the rohol knows the diniensions of the grid (i.f., the number of $x$ and $y$ grid lines), we can enumerate the set of possible maps $M=\left\{M_{1}, M_{2}, \ldots, M_{m}\right\}$, where a nap corresponds to an assignment of a junction type to each intersection of grid lines. For most purposes, we can think of a map as a labeled graph.

We can rentrict $M$ hy inaking a number of assumptions ahout office building of the sort that the robot will finl itself in (c.g.. all LDPs are connerted). To further restrict. $M$, the rohot engages in an initial phase of taok-driwm exploration. Each task specifies a destination location in $x, y$ grid coordinates. The robot computes the shortest path assunning that all intersections have as many coincident corriclors as is consistent with what. is known about the intersection and its arljacent intersections. The robot then follows this path. acquiring additional information as it moves through 


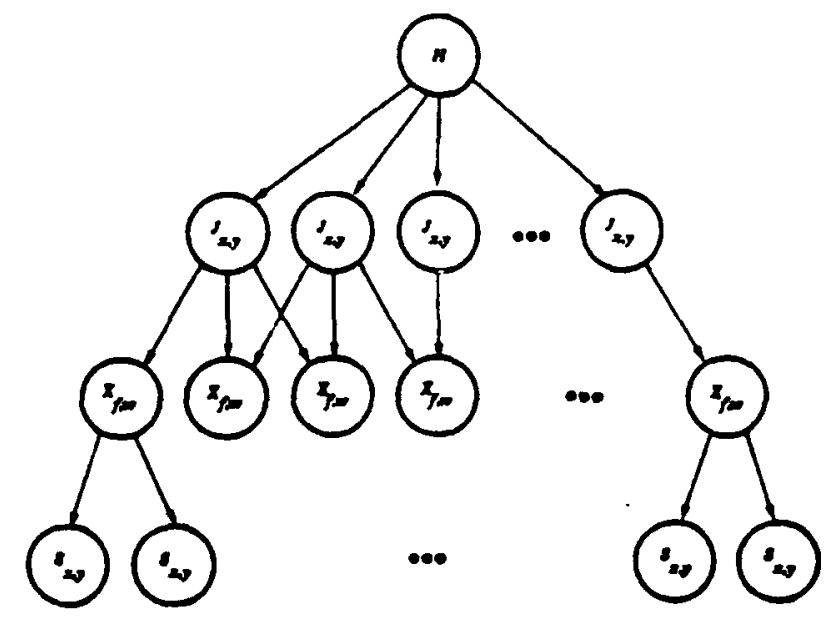

Figure 7.14 : The probabilistic model for map building

unknown intersections until it either finds its path blocked, in which case it recomputes the shortest path to the goal taking into account its new knowledge, or it reaches the goal.

The robot continues in this task-driven exploration phase until it is likely-based on the spatial distribution of known locations-that all locations have been visited at least once. From this point on, given a task to move to specific location, it is likely that it will be able to compute a path through known territory. The robot now faces the decision whether to take the known path or to try an alternative path through unknown territory. In the model considered here, the robot has to choose between taking the shortest path through known territory, and trying the shortest path consigtent with what is known. In the latter case. it will learn something new, but it nay end up taking longer to complete its task.

Let $\boldsymbol{H}$ be a random variable corresponding to the actual configuration of the environment; $\boldsymbol{H}$ takes on values from $M$. Let $J_{x, y}$ be a random variable corresponding to the junction type of the intersection at the coordinates, $\langle x, y\rangle$, in the grid; $J_{x, y}$ can take ou values from the set $C$ defined previously. Let $\boldsymbol{I}_{\mathrm{f}, \mathrm{e}}$ be as previously defined, a boolean variable corresponding the presence of a feature at a particular position. Let $S_{x, y}$ be a random variable corresponding to a possible sensing action taken at the coordinates. $\langle x, y\rangle$, in the grid. Let $\mathcal{E}$ correspond to the set of seusing actiois taken thus far. The complete probabilistic model is shown in Figure $\mathbf{i . 1 4}$. 
In our simple model. the rohot lias in deride between the two alternatives. $P_{K}$ and $P_{L}$, corresponding to paths throngh known and unknown territory. To compute $\operatorname{Pr}(I / \mid \mathcal{E}) . \operatorname{Pr}(I /)$ is assumed to be uniform. $\operatorname{Pr}\left(J_{x, y} \mid / I\right)$ and $\operatorname{Pr}\left(X_{f . u} \mid J_{x, y}\right)$ are cletermined by the geometry, and $\operatorname{Pr}\left(S_{x, y} \mid X_{f . u}\right)$ is determined experimentally. Let $T=\left\{T_{1} . T_{2}, \ldots, T_{r}\right\}$ denote the set of all tasks corresponding to point-lo-point traversals. and $E\left(\left|T_{i}\right|\right)$ denote the expected number of tasks of type $T_{i}$. Let $\operatorname{Cost}\left(T_{i}, M_{j}, M_{k}\right)$ be the time required for the task $T_{i}$ using the map $M_{j}$, given that the actual configuration of the environment is $M_{k}$; if $M_{j}$ is a subgraph of $M_{k}$, then $\operatorname{Cost}\left(T_{i}, M_{j}, M_{k}\right)$ is just the length of the shortest path in $M_{j}$. Let $T^{*}$ denote the robot's current lask. For evaluation purposes. We assume that the robol will take al inust one additional exploratory step.

To complete the derision model. we need a means of computing the expected value of $P_{K}$ and $P_{T}$. In general, the value of a given action is the sum of the immediale costs related to $T^{*}$ and the costs for expected future tasks. Let

$$
\text { Futures }\left(M_{i} . I\right)=\sum_{j=1}^{r} \mathrm{E}\left(\left|T_{j}\right|\right) \operatorname{Cost}\left(T_{j}, M_{I}^{*}, M I_{i}\right) .
$$

where $M_{i}^{*}=M_{\text {arg max }} \operatorname{Pr}(M ! . j)$.

If classification is perfect. the robol correctly classifies any location it passes through, and $M_{\varepsilon}^{*}$ is the minimal assigument cousistent with what it has classified so far. In this case. the expected value of $P_{K}$ is

$$
\operatorname{Cost}\left(T^{*} . . Y^{*} . .\right)+\text { Futures }(., \mathcal{E}) \text {. }
$$

If classification is imperfect. the expected value of $P_{K}$ is

$$
\sum_{j=1}^{m} \operatorname{Pr}\left(M_{j} \mid \varepsilon\right)\left[\operatorname{Cost}\left(T^{*}, M I^{*}, M_{j}\right)+\dot{\operatorname{Futures}}\left(M_{j}, \varepsilon\right)\right]
$$

Handling $P U$ is just a bit more complicated. Suppose that the robot is contemplating exactly one sensiug action that will result in one of several posaibh obeervations $O_{1}, \ldots, O_{n}$, then the expected value of $P_{t}$ is

$$
\begin{aligned}
& \sum_{j=1}^{m} \operatorname{Pr}\left(M_{j} \mid \varepsilon\right) \operatorname{Cost}\left(I^{*^{\prime}}, M^{*}, M I_{j}\right)+ \\
& \sum_{i=1}^{n} \operatorname{Pr}\left(O_{i}\right) \sum_{j=1}^{m} \operatorname{Pr}\left(M, \mid O_{i}, \varepsilon\right) \text { Futures }\left(M_{j},\left[O_{i}, \varepsilon\right]\right)
\end{aligned}
$$


where $T^{*^{\prime}}$ is a modification of $T^{*}$ that accounts for the proposed exploratory sensing action.

We use Jensen's [21] variation on Lauritzen and Spiegelhalter's [25] algorithm to evaluate the network shown in Figure $\mathbf{i . 1 4}$. The time required for evaluation is determined by the size of the sample spaces for the individual random rariables and the connectivity of the network used to specify the decision model. In the case of a singly-connected network. the cost of computation is polynomial in the number of nodes and the size of the largest sample space-generally the space of possible maps. The network shown in Figure $i .14$ would be singly-comected if each feature. $x_{f, w}$, had at most one parent correspouding to a junction. $J_{x, y}$; a network of this form with 100 possible maps can be evaluated in about 10 seconds. assuming an $8 \times 8$ grid.

In the case of a multiply-connected network. the cost of computation is a function of the product of the sizes of the sample spaces for the nodes in the largest clique of the graph formed by triangulating the DAC, corresponding to the original network. By making use of the information gathered in the initial exploratory phase. the robot is able to reduce the connectivity of the network used to encode the decision inodel. Multiply-connected networks accounting for approximately 50 possible maps require on the order of a lew minutes to evaluate.

The space of possible maps chosen may not include the map corresponding to the actual configuration of the environment. To handle such possible omissions. we add a special value, 1 . to the sample space for $H$. and make all of the $\operatorname{Pr}\left(J_{x . y} \mid \perp\right)$ entries in the conditional probability tahles $1 / s$ where .$s$ is the number of junction types. If the robot ever detects tliat $M_{\varepsilon}^{*}=1$, then it assumes that it has excluded the real map, and dynamically adjusts its decision model by computing a new sample space-for $H$ guided by the results of the exploratory actions takeu thus far.

\subsubsection{Designing Robot Control Systems}

One approach to desiguiug control systems employing a decision-theoretic perspective is described as follows. We begin by considering the overall decirio problem, deternining an optimal decision procedure according to a precisely stated decision-lheoretic criteria. neglecting computational costs. We use an influence diagram to represent the underlying decision model and define the optimal procedure in terms of evaluating this model.

In the case described above, the robol's overall decision problem in- 
volves several component problems associated with specific classes of events occurring in the environment. These component decision problems inclucle what action to take when appronchcil by an unexpected object in a corridor. what sensor action to take ncxt when classifying a junction. and what path to take in combining exploration and task execution. Each of these problems is recurrent.

Problems involving what sensor action to take in classification or what. path to take in navigation are predictably recurrent. For instance. during classification each sensor action takes about thirty seconds to a ninute. so the robot has that ainount of time to decide what the next action should be if it wishes to avoid standing idle lost in computation. The frequency with which choices concerning what path to take occur is depentent on how long the robot takes to traverse the rorridor on ronte to the next I,DP. With the current mobile platform opernting in the halls of the computer science department. moving between two consecutive LDPs takes about four minutes. The problem of deciding what to do when approached by an unexperted object occurs unpredictably, and the time between when the approaching object is detected and when the robot must react to avoid a collision is on the order of a few seconds.

By unaking various (in)dependence assumptions and eliminating noncritical variables from the overall complex decision problem. we are able to decompose the glohally optimal decisi in problem into sets of simpler compn nent decision problems. Fach of the sets of romponent prohlenis are solverl by a separate module. The computations carried ont hy these morlules are optimized using a variety of techuiques to take advantage of the expecter time av. for decision making. The different decision procedures rommunicate by passing probability distributions back and forth. For instance. the module responsible for making decisions regarding exploration aind the module responsible for classifying LDPs pass back and forth distributions regarding the junction types of LDPs.

The control system descrihed above combines high-level decision making with low-level control and sensor interpretation to provide for navigation, rad-time obstacle avoidance, and exploralion in an unfainiliar environment. The basic controller handles multiple asynchronous processes communicating via simple meskage-passing protocols. The architecture support.s a variety of arbitration schemes from fixed-priority processor scheduling to decision-theoretic control. This section has emphasized two decision processes: one responsible for reasoning about the uncertainty inherent in dealing with noisy and ambiguous sensor data. and a second responsible for 
assessing the expected value of varions explorato y actions. Our basic approach to designing robot control systems invoives constructing a decision model for the overall problem and then decomposing it into component nodels guided by the time criticality of the associated decision problems.

The third example involves sequential decisior: naking. and for this we have to introluce some additional machinery. In particular, the probatilistic projection approach described in [13. 15] and particularly [14]. Relate this to Tatman and Schacter's uork on connecting influence diagmms and dynamic progrnmming methads for sequential decision making involving Markov processes.

\subsection{Change Over Time}

Reasoning about change requires predicting how long a proposition, having become true. will continue to be so. Lacking perfect knowledge. an ageut may be constrained to believe that a proposition persists indefinitely simply because there is no way for the agent to infer a contravening proposition with certainty. In this section, we describe a model of causal reasoning that accounts for knowledge concerning cause-and-effect relationships and knowledge concerning the tendency for propositions to persist or not as a function of time passing. The model has a natural encoding in the form of a network representation for probabilistic models. We will also consider how our probabilistic model addresses certain classical problems in temporal reasoning (e.g., the frame and qualification problems).

The common-sense law $0 ;$ inertia [27] states that a proposition once made true remains so until something makes it false. Given perfect knowledge of initial conditions and a complete predictive model. the law of inertia is sufficient for accurately inferring the persistence of propositions. In nost circumstances, however, our predictive models and our knowledge of initial conditious are less than perfect. The law of inertia requires that. in order to infer that a proposition ceases to be true, we nust predict an event with a contravening effect. Such predictions are often difficult to make. Consider the fonwing examples:

- eca is sleeping on the couch in your living room

- you leave your umbrella on the $8: 15$ commuter train

- a client on the telephove is asked to hold 


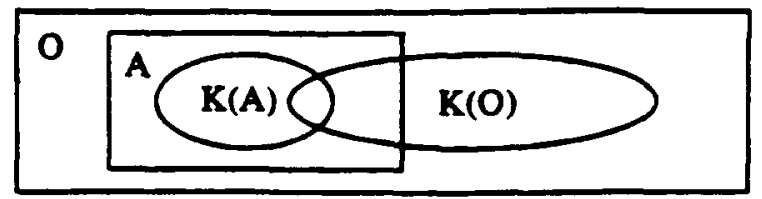

Figure i.1.5: Events precipitate change in the world

In each case. there is some proposition initially observed to he true. and the task is to deterunine if it will be true at some later time. The cat may sleep undisturbed for an hour or more. hut it is extremely unlikely to remain in the same spot for more than six hours. Your umbrella will probably not be sitting on the seat when yon catch the train the next morning. The client will probably hold for a few nuinutes. but on!y : he most determined of clients will be on the line after 15 minutes. Sometimes we can make more accurate predictions ( $\epsilon . g$.. a large barking dog runs int.o the living room). butt. lacking specific evidence. we would like past experience to provide all estimale of how long certain propositions are likely to persist.

Events precipitate change in the world. and it is our knowledge of events that enables us to make useful predictions atout the future. For any proposition $P$ that can hold in a situation. there are some number of general sorts of events (referred to as enent types) that can affect $P$ (i.e.. make $P$ true or false). For any particular situation. there are some number of specific events (referred to as cuent instanres) that occur. Let $O$ correspond to the set of events that occur at time t. A correspond to that subset of $O$ that affect $P . H(O)$ that subset of $O$ kllown to occur at time $t$, and $H(A)$ that subset of $A$ whose type is known to affect $P$. Figure 7.15 illustrates how these sets might relate to one another in a specific situation. In many cases, $\Lambda(O) \cap h(A)$ will be empty while $A$ is not, and it may still be possible to provide a reasonable assessnent of whether or not $P$ is true at $t$. In this section, we provide an account of how such assessments can be made prohabiliatically.

\subsubsection{Prediction and Persistence}

In the following, we distinguish between two kinds of propositions: propositions, traditionally referred to as fuents [28]. which. if they become trne. tend to persist without additional effort. and propositions. corresponding to the occurrence of events. which. if true at a point. tend to precipitate or trigger change in the world. Let $(P . t)$ indicate that the fluent $P$ is true at 
time $t$. alld $\langle E . t\rangle$ indicate that an event of trpe $E$ occurs at tiscte $t$. We use the notation $E_{P}$ to indicate an erent corresponding to the fluelit $P$ becoming true.

Given our characterization of fluents as propositions that tend to persist. whether or not $P$ is true at some time 1 may depend upon whether or not it was true at $t-\Delta$. where $د>0$. We can represent this dependency as follows: ${ }^{1}$

$$
\begin{aligned}
\operatorname{Pr}(\langle P . t\rangle)= & \operatorname{Pr}(\langle P . t\rangle \mid\langle P . t-\Delta\rangle) \operatorname{Pr}(\langle P . t-\Delta\rangle)+ \\
& \operatorname{Pr}(\langle P . t\rangle \mid \neg\langle P . t-\Delta\rangle) \operatorname{Pr}(\neg\langle P . t-\Delta\rangle)
\end{aligned}
$$

where $\neg\langle P . t\rangle \equiv\langle\neg P . t\rangle$.

The conditional probabilities $\operatorname{Pr}(\langle P . t\rangle \mid(P . t-\Delta))$ and $\operatorname{Pr}(\langle P . t\rangle \mid \neg\langle F . t-\Delta\rangle)$ are related to the survivor function in classical queuing theory [35]. Survivor functions encode the changing cxpectation of a fluent remaining true over the course of time. We employ survivor functions to capture the tendency of propositions to becoine false as a consequence of events with contravesing effects. With survivor functions. one need not be aware of a sperific instance of an event with a contravening effect in order to predict that $P$ will cease being true. As an example of a survivor function.

$$
\left.\operatorname{Pr}(\langle P . t\rangle)=r^{-1 \Delta} P(P . t-\Delta)\right)
$$

indicates that the probability that $P$ persists drops off as a function of the time since $P$ was last observed to be true at an exponential rate determinerl by $\lambda$ (Figure $i .16$ ). The exponential decay survivor function is equivalent to the case where

$$
\operatorname{Pr}(\langle P . t\rangle)(P, t-\Delta\rangle)=e^{-. \lambda \Delta}
$$

and

$$
\operatorname{Pr}(\langle P . t\rangle|\neg\langle P . t-\Delta)|=0 .
$$

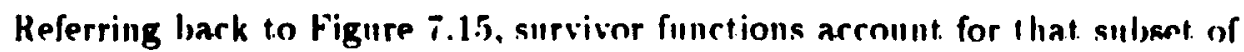
$A$ correaponding to events that make $P$ false, assuming that $I(A)=\{\}$.

\footnotetext{
'The apality in Formula $\$ .6$ follows from the generalized addition lar: if $t_{1} \ldots \ldots t_{n}$ are erachers and exhautive and $B$ is any event, then

$$
\operatorname{Pr}(B)=\sum_{i=1}^{n} \operatorname{Pr}\left(B \mid . f_{1}\right) \operatorname{Pr}\left(f_{1}\right)
$$
}




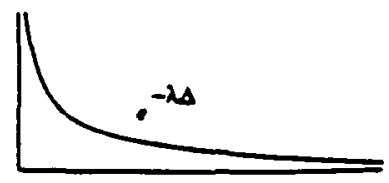

Figure i.16: A survivor function with exponential decay

If we have evidence concerning specific events known to affect $P$ (i.e., $K(A) \cap K(O) \neq\{\})$, Formula $\bar{T} .6$ is intarlequate. As an interesting special case of how to deal with events know in to affect $P$. suppose that we know about all events that make $\Gamma$ irue (i.e., we know $\operatorname{Pr}\left(\left\langle E_{P}, t\right\rangle\right)$ for any value of $t)$. and none of the events that nake $P$ false. In particular, suppose that $P$ corresponds to John being at the airport. and $E_{P}$ corresponds to the arrival of John's flight. We are interested in whether or not John will still be waiting at the airport when we arrive 1.0 pick hin up. Let $\sigma(t)=\epsilon^{-\lambda t}$ represent Juln's tendency to hang around airports, where $\lambda$ is a measure of his impatience. If $f(t)=\operatorname{Pr}\left(\left\langle E_{P}, t\right\rangle\right)$, then we can compute the probability of $P$ being true at $t$ by convolving $f$ with the survivor function $\sigma$ as in

$$
\operatorname{Pr}(\langle P, t))=\int_{-\infty}^{t} \operatorname{Pr}\left(\left(E_{P}, z\right)\right) \sigma(t-z) d z
$$

A shortcoming of Formula $T . T$ is that it fails to account for evidence conceruing specific events known to nıake $P$ false. Suppose. for instance. that $E_{\neg P}$ corresponds to Fred meeting John at the airport and giving him a ride to his hotel. In certain cases.

$$
\operatorname{Pr}((P, t))=\int_{-\infty}^{t} p\left(\left\langle E_{P}, z\right\rangle\right) \sigma(t-z)\left[1-\int_{z}^{t} \operatorname{Pr}\left(\left(E_{-P}, x\right)\right) d x\right] d z
$$

provides a good approximation. Figure $i .1 i$ illustrates the sort of inference licensed by Formula 7.8 .

'There are some potential prohlems with Formula 7.8 . The survivor function $\sigma$ wa meant to account for all events that nake $P$ false. but Formula $T$.s connts on such event, John leaving the airport with Fred. twice: once in the function and ouce in $\operatorname{Pr}\left(\left(E_{-P}, 1\right)\right)$. In certain cases, this can lead to aignificant errors (e.g., fred always picks up John at the airport). To combine the availahle evidence corrertly, it will help if we dintinguish the different sorts of knowledge that might be bronght to bear on eatimating whether or not $P$ is true. We will also reinterpret the event type $E_{P}$ to 


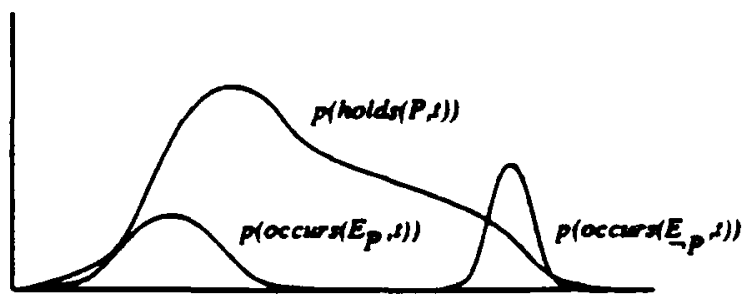

Figure i.1i: Probabilistic predictions

mean an event knoun to nake $P$ true. The following formula makes the necessary distinctions and indicates how the evidence should be combined:2

$$
\begin{aligned}
& \operatorname{Pr}((P, t))= \\
& \operatorname{Pr}\left(\langle P, t) \mid\langle P, t-\Delta\rangle \wedge \neg\left(\left\langle E_{P}, t\right\rangle \vee\left\langle E_{-P}, t\right\rangle\right)\right) \\
& \text { * } \operatorname{Pr}\left(\langle P . t-\Delta\rangle \wedge \neg\left(\left\langle E_{P}, l\right\rangle \vee\left\langle E_{\neg P, 1}\right)\right)\right) \\
& +\operatorname{Pr}\left(\langle P, t\rangle \mid\langle P, t-\Delta\rangle \wedge\left\langle E_{P,} l\right\rangle\right) \\
& * \operatorname{Pr}\left(\langle P, t-\Delta) \wedge\left\langle E_{P}, t\right\rangle\right) \\
& +\operatorname{Pr}\left(\langle P, t) \mid\langle P, t-\Delta\rangle \wedge\left\langle E_{\neg P, t}\right\rangle\right) \\
& * \operatorname{Pr}\left(\langle P, t-\Delta\rangle \wedge\left\langle E_{\rightarrow P}, t\right\rangle\right) \\
& +\operatorname{Pr}\left(\langle P, t) \mid \neg(P, t-\Delta) \wedge \neg\left(\left\langle E_{P}, t\right\rangle \vee\left(E_{-P}, t\right\rangle\right)\right) \\
& \text { * } \operatorname{Pr}\left(\neg\langle P, t-\Delta) \wedge \neg\left(\left\langle E_{P}, t\right\rangle \vee\left(E_{\neg P, t}\right\rangle\right)\right) \\
& +\operatorname{Pr}\left(\langle P, t) \mid \neg\langle P, t-\Delta) \wedge\left\langle E_{P}, t\right\rangle\right) \\
& \text { * } \operatorname{Pr}\left(\neg\langle P, t-\Delta) \wedge\left\langle E_{P,}(\rangle\right)\right. \\
& +\operatorname{Pr}\left(\langle P, t) \mid \neg\langle P, t-\Delta) \wedge\left\langle E_{\neg}, t\right\rangle\right) \\
& * \operatorname{Pr}\left(\neg(P, t-\Delta) \wedge\left\langle E_{-P,} t\right\rangle\right)
\end{aligned}
$$

C'onsider the contribntion of the individual terms corresponding to the conditional probabilities labeled N1 through N6 in Formula i.9. N1 accounts for natuml attrition: the tendency fur proprositions to become false given no direct evidence of events known to affect $P$. N2 and N.5 account for camal accretion: accumulating evidence for $P$ due to events known to make $P$ true. N2 and N5 are generally 1 . N3 and N6, on the other hand, are gencercil 0 , since evidence of $\neg P$ becoming true does little to couvince us that $D \mathrm{~b}$ true. Finally, $\mathrm{N} 4$ accounts for spontaneous causation: the tendency for propadition to suddenly becone true with uo direct evideuce of events known to afied $P$.

\footnotetext{
${ }^{2}$ In order 10 jutify our use of the generalized addition law in Formula $i .9$. We anme that $\operatorname{Pr}\left(\left\langle E_{p}, t\right\rangle \wedge\left\langle E_{-}, t\right)\right\rangle=0$ for all $t$.
} 
By using a discrete approximation of time and fixing $د$. it is possible both 10 acquire the necessary values for the terms $\mathrm{NI}$ through $\mathrm{Nt}$ and to use them in making useful predictions. If time is represented as the integers. and $\Delta=1$. We nole that the law of inertia applies in those situations in which the terms N1. N2. and N.5 are always $\perp$ and the other terms are always 0 . In the rest of this section. we assume that time is cliscrete and linear and that the time scparating any two ronsecutive time points is some constant $\delta$. Only evidence concerning events known to make $P$ true is hrought to bear on $\operatorname{Pr}\left(\left\langle E_{P}, t\right\rangle\right)$. If $\operatorname{Pr}\left(\left\langle E_{P}, t\right\rangle\right)$ were used to sunmarize all evidence concerning events that make $P$ true, then $N 1$ would be 1 .

\subsubsection{Reasoning About Causation}

Before we consider the issues involved in making predictions using knowledge concerning N1 through N6. we ueed to add to our theory some neans of preuicting additional events. We consider the case of one event causing another event. Deterministic theories of causation often use implication to model cause-and-effect relationships. For instance. to indicate that the occurrence of an event of type $E_{1}$ at time $t$ causes the occurrence of an event of type $E_{2}$ following $t$ by some $\delta>0$ just in case the conjunction $P_{1} \wedge P_{2} \ldots \wedge P_{n}$ holds at $t$, we uight write

$$
\left(\left\langle P_{1} \wedge P_{2} \ldots \wedge P_{n}, t\right\rangle \wedge\left\langle E_{1}, t\right\rangle\right) \supset\left\langle E_{2}, t+\delta\right\rangle .
$$

If the caused event is of a type $E_{P}$. this is often refetred to as persistence rausation [29]. In our model, the conditional probability

$$
\operatorname{Pr}\left(\left\langle E_{2}, t+\delta\right\rangle\left|\left\langle P_{1} \wedge P_{2} \ldots \wedge P_{n}, t\right\rangle \wedge\left\langle E_{1}, t\right)\right\rangle=\pi\right.
$$

is used to indicate that. given an event of type $E_{1}$ occurs at time $t$. and $l_{1}$ through $P_{n}$ are true at $t$. an event of type $F_{2}$ will occur following $t$ by some $\delta>0$ uith prohability $\pi$.

In noving to a probabilistic moclel of cansation, there are some conplication that we have to deal wilh. Consider, for example. the two rules:

$$
\because \quad(\langle P, t\rangle \wedge\langle E, t\rangle) \supset\left\langle E_{R}, t+\delta\right\rangle
$$

and

$$
(\langle P \wedge Q . t\rangle \wedge\langle E \cdot t\rangle) \supset\left(E_{R} \cdot t+\delta\right)
$$

These two rules pose no problems for the deterministic theory of causation, since $P$ and $Q$ are either true or false. and the rules either apply or not. 
In fact, the second rule is redundant Ilowever, in a probabilistic model.

- $P$ and $Q$ usually are not unambigucusly Irue or false. Therefore. in the probabilistic causal theory consisting of

$$
\begin{gathered}
\operatorname{Pr}\left(\left(E_{R} \cdot t+\delta\right) \mid\langle P . t) \wedge\langle E . t\rangle\right)=\pi_{1} \\
\operatorname{Pr}\left(\left\langle E_{R} \cdot t+\delta\right) \mid(P \wedge Q . t\rangle \wedge\langle E . t\rangle\right)=\pi_{2}
\end{gathered}
$$

the second rule can no longer be considered redundant. Since the second rule is more specific than the first. it provides us with valuable additional information. In a complete account of the causes for $E_{R}$, we would also need

$$
\operatorname{Pr}\left(\left\langle E_{R}, t+\delta\right\rangle \mid\langle P \wedge \neg Q, t\rangle \wedge\langle E, t\rangle\right)=\pi_{3}
$$

and other information as nell. Provirling a complete account of the interactions antoug causes and between causes and their elfects is important in modeling change in a probabilistic framework. In the following two sections, we will consider this issue in more detail.

\subsubsection{An Example}

The task in probnbilintic projertion is to assign each proponitional variable of the furm $(\varphi, t)$ a certainty measure consistent with the constraints specified in a prohlem. In this section. we provide examples drawn from a simple factory domain that illustrate the sort of inference required in probabilistic projection. We hegin by introduring some new event types:

$$
\begin{aligned}
& C l=\text { "The mechanic on duty cleans up the shop" } \\
& A s=\text { "Fred tries to assemble Widget } 17 \text { in Room } 101 \text { " }
\end{aligned}
$$

and fluents:

$$
\begin{aligned}
& W_{r}=\text { "The location of Wrench14 is Roon 101" } \\
& \text { Sc }=\text { "The location of Screwdriver31 is Room 101" } \\
& \text { IVi }={ }^{\text {"Widget } 1} i \text { is completely assembled" }
\end{aligned}
$$

Wh acoume that toole are occasionally displaced in a busy shop, and that Wir and Se are both snbject to an exponential persistence decay with a half life of one day; this determines N1 in Formula 7.9 :

$$
\begin{aligned}
& \operatorname{Pr}\left(\left\langle W_{r}, t\right) \mid\left\langle W_{r, t}-\Delta\right\rangle \wedge \neg\left(\left\langle E_{M_{r}, t}\right) \vee\left(E_{-M_{r}, t} t\right)\right)=e^{-1 \Delta}\right. \\
& \operatorname{Pr}\left(\langle S r, t) \mid\left\langle(S c, t-\Delta) \wedge \neg\left(\left\langle E_{S e}, t\right) \vee\left(E_{-S_{e}, t} t\right)\right)=e^{-1 \Delta}\right.\right.
\end{aligned}
$$


where $e^{-.1 \Delta}=0.5$ when $\triangle$ is one day.

The other terms in Forınula i.9. N2, N3. N4. N.5. and NG. we will assume to be. respectively. $1,0,0.1$. and 0 . When the mechanic on duty cleans up the shop. he is supposed to put all of the tools in their appropriate places. In particular. Wirench 14 and Screwdriver 31 are supposed to be returned to Room 101. We assume that the mechanic is very diligent:

$$
\begin{aligned}
& \operatorname{Pr}\left(\left\langleE_{\left.\mid \psi_{r}, t+\epsilon\right) \mid\left\langle\left(C^{\prime}|t\rangle\right)\right.}=1.0\right.\right. \\
& \operatorname{Pr}\left(\left\langle E_{S c}, t+\epsilon\right) \mid\langle C l . t\rangle\right)=1.0
\end{aligned}
$$

Fred's competence in assembling widgets depends "pon his tools being in the right place. In particular. if Screwdriver 31 and Wrenchl 4 are in lloom 101, then it is certain that Fred will successfully assemble Widget $1 i$.

$$
\operatorname{Pr}\left(\left(E_{u_{i}, t} t+\epsilon\right) \mid\left(\| I_{r} . t\right\rangle \wedge\langle(S c . t\rangle \wedge\langle A s, t\rangle)=1.0\right.
$$

Let TO correspond to 12:00 PM 2/29/88. and T1 correspoud to 12:00 PM on the following day. Assume that $\epsilon$ is uegligible given the events we are concerned with (i.e.. we will add or subtract $\epsilon$ in order to sinplify the allalysis).

$$
\begin{aligned}
& \operatorname{Pr}(\langle C l . T O\rangle)=0.7 \\
& \operatorname{Pr}(\langle\text { As. } T 1\rangle)=1.0
\end{aligned}
$$

We are interested in assigning the propositions of the form $\langle\varphi, t\rangle$ a rertainty measure consistent with the axioms of probability theory. We will work through an example showing how one night derive such a measure, noting some of the assumptions required to make the derivations follow from the problem spetification and the axioms of prolsability. In the following. we will denote this measure of belief hy Bel. What can re say about. Bel $((W i, T I+\epsilon))$ ? In this particular example. we begin with

$$
\begin{aligned}
& \operatorname{Bel}(\langle I I i, T 1+\epsilon)) \\
& =\operatorname{Pr}\left(\left\langle E_{W i} \cdot T 1+\epsilon\right)\right) \\
& =\operatorname{Pr}\left(\left\langle E_{W_{i}}, T 1+\epsilon\right) \|(W r . T 1) \wedge\langle S c . T 1\rangle \wedge\langle\text { As. } T 1\rangle\right) \\
& -\operatorname{Pr}\left(\left\langle W_{r}, T 1\right\rangle \wedge\langle\text { Sc. } T 1\rangle \wedge\langle\text { As. } T 1)\right) \\
& =\operatorname{Pr}((W r . T l) \wedge\langle\text { Sc. } T I) \wedge\langle\text { As. } T I\rangle) \\
& =\operatorname{Pr}(\langle\operatorname{Ir} . T 1\rangle \wedge\langle\text { Sc. TI }))
\end{aligned}
$$


The first step follows from our interpretation of " ir $_{r}$. and the fact that there is no additional evidence for or against $I r$ at $\Gamma l+\epsilon$. The second step employs the addition rule aud the assumption that the assembly will fail in have the effect of $\left(E_{w_{r}}, T 1\right)$ if any one of (Wr, T1). (Sr. T1). or (As. Tl) is false. The third step relies on the fact that assembly is always surcessful given that the altenipt is made and Wrench 14 and Screwdriver31 are in Room 101. The last step depends on the assumption that the evidence supporting ( $\left.W^{\prime} \wedge S c . T 1\right\rangle$ and $\langle A s, T 1$ ) are independent. The assunption is warranted in this case given that the particular instance of As occurring at $T 1$ does not affect $\operatorname{Ir} \wedge S c$ at $T 1$. and the evidence for $A s$ at $T 1$ is independent of any eveuts prior to $T$. Note that. if the evidence for $A s$ at $T 1$ involved events prior to $T 1$, then the analysis would be more involved. It is clear that $\operatorname{Pr}(\langle W r . T 1\rangle) \geq 0.35$. and that $\operatorname{Pr}(\langle S c . T 1\rangle) \geq 0.35$; unfortunately, we cannot simply combine this information to obtain an estimate of $\operatorname{Pr}\left(\left\langle W_{r} \wedge S c . T 1\right\rangle\right)$, since the evidence supporting these two claims is dependent. We can, however. determine that

$$
\begin{aligned}
& \operatorname{Pr}(\langle\operatorname{Ir} . T 1\rangle \wedge\langle S c, T 1\rangle) \\
& =\operatorname{Pr}(\langle\operatorname{Wr} . T 1\rangle \wedge(S c, T 1\rangle \mid\langle\text { Wr. T0 }) \wedge\langle S c . T 0\rangle) \operatorname{Pr}(\langle\text { Wr. T0 }) \wedge\langle\text { Sc. T0 }\rangle) \\
& =\operatorname{Pr}\left(\left\langle W_{r} . T 1\right\rangle \mid\langle W r, T 0\rangle \wedge\langle S c . T 0\rangle\right) \\
& \text { * } \operatorname{Pr}(\langle S c, T 1\rangle \mid\langle W r, T 0\rangle \wedge\langle S c, T 0\rangle) \operatorname{Pr}\left(\left\langle H_{r}, T 0\right\rangle \wedge\langle\text { Sc. TO }\rangle\right) \\
& =\operatorname{Pr}(\langle\text { IVr. TO }\rangle \wedge\langle S c . T 0\rangle) * 0.5 * 0.5 \\
& =\operatorname{Pr}\left(\left\langle E_{W_{r}}, T O\right\rangle \wedge\left\langle E_{S e}, T 0\right\rangle\right) * 0.5 * 0.5 \\
& =\operatorname{Pr}\left(\left\langle E_{U_{r},}, T O+\epsilon\right\rangle \wedge\left(E_{S e}, T O+\epsilon\right) \mid\langle C ! I, T O\rangle\right) \operatorname{Pr}(\langle C I . T 0\rangle) * 0.5 * 0.5 \\
& =0.7 * 0.5 * 0.5 \\
& =0.175
\end{aligned}
$$

assuming that there is no evidence concerning events that are known to affect ejther II'r or Sc in the interval from To to T1, that Irr and Sc are independeut, and that $E_{W_{r}}$ and $E_{S c}$ are conditionally independent of one auother given (?).

Throughout our analysis, we were forced to make assumptions of independence. In many cases, such assumptions are unwarranted or introduce inconnintencies. The inference process is further complicated by the fact that probabilistic constraints tend to propagate both forward and backward in time. This bi-directional flow of eridence can render the analysis described above useless. In the next section. we consider a model that simplifies specifying independence assumptions. and that allows us to handle both forward 
and backward propagation of probabilistic constraints.

\subsubsection{A Model for Reasoning About Change}

In this section. we take a slight modification of Formula 7.9 as the basis for a model of persistence. Formula $\bar{i} .9$ predicts $\langle P . t\rangle$ on the basis of $\langle P . t-J\rangle$. $\left\langle E_{P} . t\right\rangle$. and $\left\langle E_{-P} . t\right\rangle$. where $\Delta$ is allowed to vary. In the model presented in this section, we only consider pairs of conserutive time points, $t$ and $t+\delta$, and arrange things so that. the value of a fluent at time $t$ is compleiply determined by the state of the world at $\delta$ in the past. In Formula 7.9 , we interpret. events of type $E_{P}$ orrurring at $f$ as providing evirlence for $P$ heing true at $t$. In our new morlel, we interpret events of type $E_{P}$ occurring at $t$ as providing evidence for $P$ heing true at $t+\delta$. This seinterpretation is not strictly necessary, but we prefer it since the expressiveness of the resulting models can easily be characterized in ternis of the properties of Markov processes. In our new inodel. we prealict $A \equiv\langle P, t+d\rangle$ hy conditioning on

$$
\begin{aligned}
& C_{1} \equiv\langle P, t\rangle \\
& C_{2} \equiv\left\langle E_{P} \cdot t\right\rangle \\
& C_{3} \equiv\left\langle E_{-P} \cdot t\right\rangle
\end{aligned}
$$

and sperify a complete model for the persistence of $P$ as

$$
\operatorname{Pr}(A)=\sum \operatorname{Pr}\left(A \mid C_{1} \wedge C_{2} \wedge C_{3}\right) \operatorname{Pr}\left(C_{1} \wedge C_{2} \wedge C_{3}\right)
$$

where the sum is over the eight possible truth assigunents for the variables $C_{1}, C_{2}$. and $C_{3}$. Note that this model requires that we have probabilities of the form $\operatorname{Pr}\left(\lambda \mid C_{1} \wedge C_{2} \wedge C_{3}^{\prime}\right)$ and $\operatorname{Pr}\left(C_{1} \wedge C_{2} \wedge C_{3}\right)$ for all possible valuations of the $C_{i}$.

In the following, we will make use of a network model that will serve to clearly isuicate the necessary indepedence assumptions. We will use the generic term belief network to refer to a network that satisfies the fullowing basic properties cominon to all three of the above representations. A belief network represents the variables or propositions of a probabilistic theory as node in a graph. The variables in our networks correspond to propositional rariables of the form $(\varphi, t)$. Dependence between two variables is indicated by a directed arc between the two nodes associated with the rariables.

Because dependence is always indicated by an arc. belief networks make it easy to identify the conditional independence inherent in a model simply by inspecting the graph. Two nodes which are linked via a common 


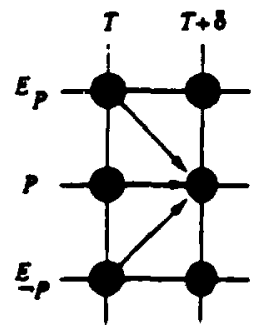

Figure i.18: The evidence for $P$ al time $T+\delta$

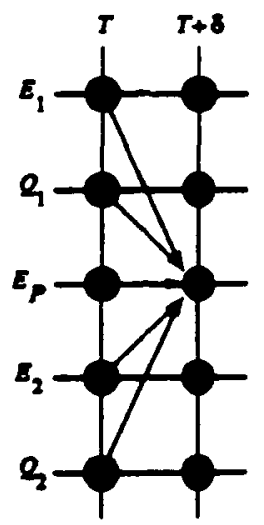

Figure $7.19:$ The evidence for $E_{P}$ at time $T+\delta$

neighbor, but for which there are no other connecting paths are conditionally independent given the common node. For instance, in the models described in this section, $\langle P, t-\delta\rangle$ is independent of $\langle P, t+\delta\rangle$ given $\cdot\langle P, t\rangle$. Belief networks make it easy to construct and verify the correctness and reasonableness of a model directly in terms of the corresponding graphical representation. Our model for persistence can be represented by the network shown in Figure i.18. As soon as wic provide a model for causation. we will show how this simple model for persistence can be embedded in a more complex model for reasoning about change over time.

Concrally, we expect that the callse-and-effect relations involving $E_{P}$ will be specified in terms of constraints of the form:

$$
\begin{aligned}
& \operatorname{Pr}\left(\left\langle E_{P}, t+\delta\right\rangle \mid\left\langle E_{1}, t\right) \wedge\left\langle Q_{1}, t\right\rangle\right)=\pi_{1} \\
& \operatorname{Pr}\left(\left\langle E_{P}, t+\delta\right) \mid\left(E_{2}, t\right) \wedge\left\langle Q_{2}, t\right\rangle\right)=\pi_{2}
\end{aligned}
$$




$$
\operatorname{Pr}\left(\left\langle E_{P}, t+\lambda\right) \mid\left\langle E_{n}, t\right\rangle \wedge \cdot\left\langle Q_{n}, t\right\rangle\right)=\pi_{n}
$$

However. to specify a complete morlct. we will need some more inforniation. To predict $A \equiv\left\langle E_{P} . t+\delta\right\rangle$. we condition on

$$
\begin{aligned}
C_{1} & \equiv\left\langle E_{1}, t\right\rangle \wedge\left\langle Q_{1}, t\right\rangle \\
C_{2} & \equiv\left\langle E_{2}, t\right\rangle \wedge\left\langle Q_{2}, t\right\rangle \\
& \cdots \\
C_{n} & \equiv\left\langle E_{n}, t\right\rangle \wedge\left\langle Q_{n}, t\right\rangle
\end{aligned}
$$

and specify a complete model as:

$$
\operatorname{Pr}(A)=\sum \operatorname{Pr}\left(A \mid C_{1} \wedge C_{2} \wedge \ldots \wedge C_{n}\right) \operatorname{Pr}\left(C_{1} \wedge C_{2} \wedge \ldots \wedge C_{n}\right)
$$

Nule that we need on the order oi $2^{n}$ probabilities corresponding to the $2^{n}$ possible valuations of the propositional variables $C_{1}$ through $C_{n}$ to specify this model. The associated belief network is shown in Figure i.19. Similar networks would be constructed for event $t_{y}$ pes other than those involving propositions becouning true or false.

Now we call construct a complete model fc. reasoning about change over time. figure $i .20$ illustrales the temporal belief network for such a complete mociel. For each prousositional variable of the form $(\varphi .1)$. there is a node in the belier network. "'he arcs are sp.ecilied according to the isolated models for persistence and causation illustrated in Figure 7.18 and Figure 7.18 . following Pearl (i988), we can write down the unique distributicn corresponding to the mocisl show" :ar Figure 7.20 as

$$
\operatorname{Pr}\left(x_{1}, x_{2}, \ldots, x_{n}\right)=\prod_{i=1}^{n} \operatorname{Pr}\left(x_{i} \mid S_{i}\right) \operatorname{Pr}\left(S_{i}\right)
$$

where the $x_{i}$ denote the propositiunal variables in the model. and $S_{i}$ is the conjunction of the propositional varjables associated with those nodes for which there exist arcs to $x_{i}$ in the network.

Ae apecific insiance of a temporal belief netuork. we reconsider the factosy exanuple of Soclion i.4.3. We will need models for the persistence of wrenches and screwdrivers remaining in place. and unodels for seasoning alout the cousequences of cleaning and assembling actions. Figure 7.21 .i shows a portion of a belief network dedicated to modeling the persistence of $11 r$ (i.e. the proposition corresponding to Wrench 14 being in Room 101$)$. 


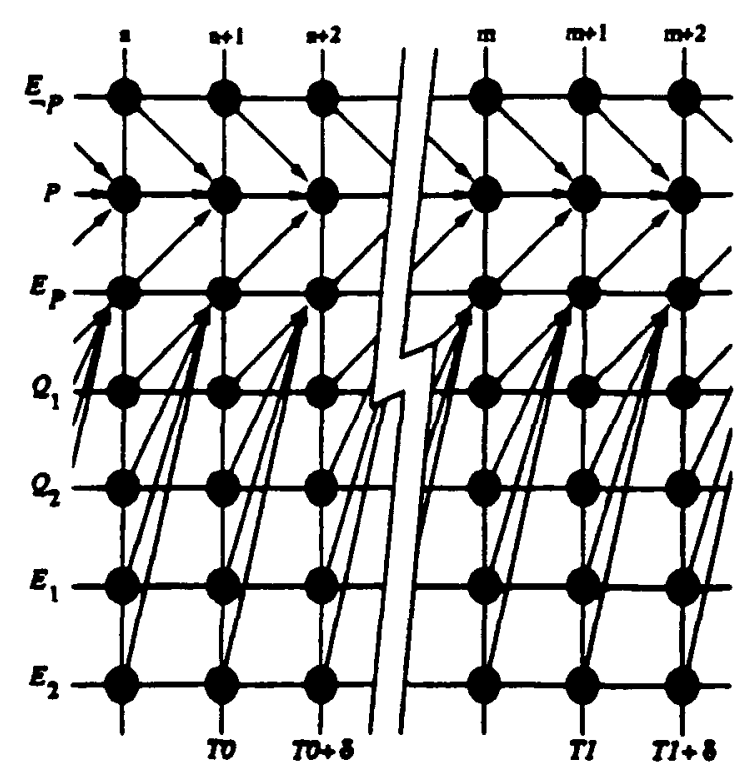

Figure 7.20: A temporal beliel network

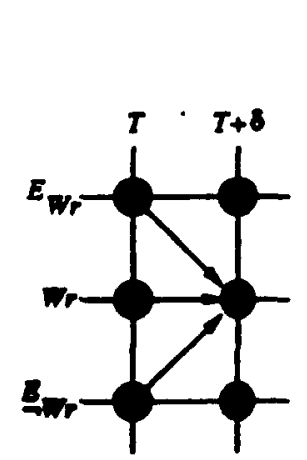

1

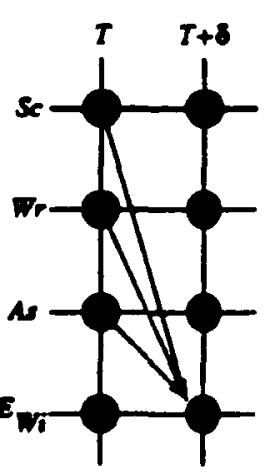

H.

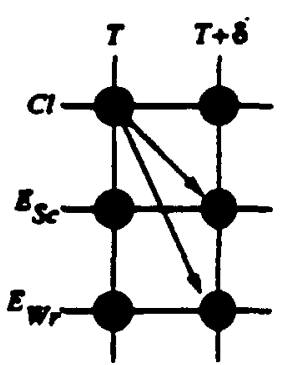

판

Figure 7.21: Models for the factory example 


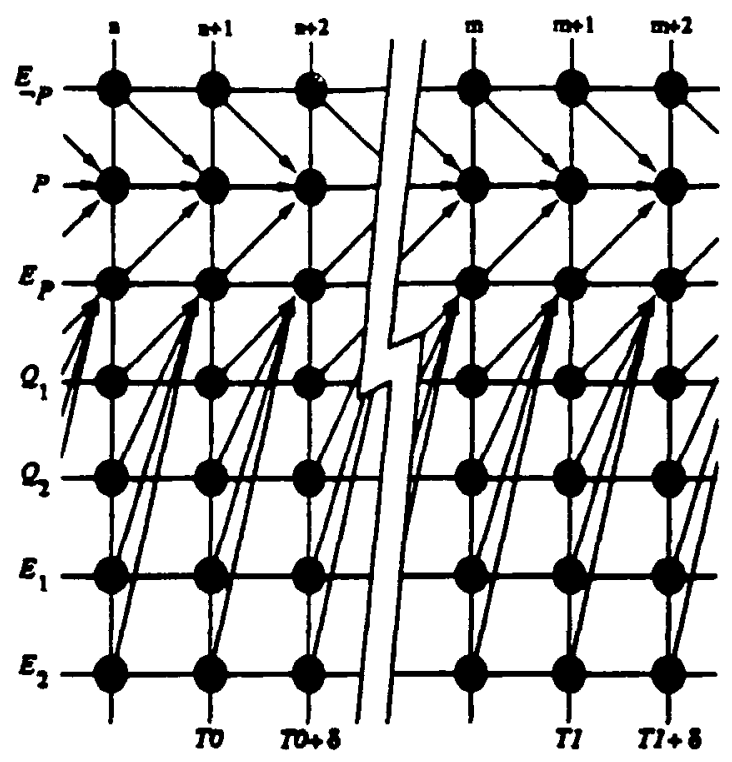

Figure 7.20: A temporal beliel network



4

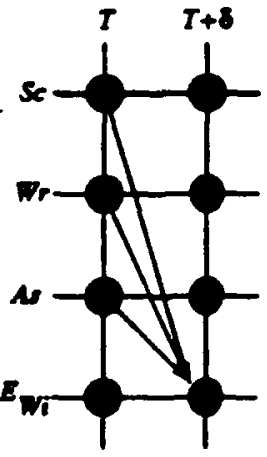

i.

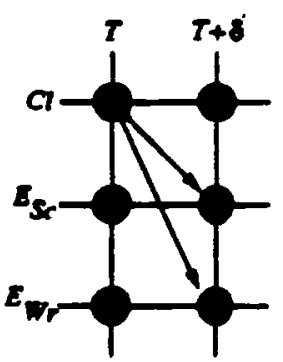

a.

Figure 7.21: Models for the factory example 
In order to completely specify the model for Ir persisting. we need the following information:

\begin{tabular}{|c||c|c|c|}
\hline $\operatorname{Pr}\left(\left\langle W_{r} t\right) \mid \ldots\right)$ & $\left\langle W_{r . t}-\Delta\right\rangle$ & $\left\langle E_{\text {ur.t }}-\Delta\right\rangle$ & $\left\langle E_{-u_{r} . t}-\Delta\right\rangle$ \\
\hline \hline$\epsilon^{-\lambda \Delta}$ & True & False & False \\
\hline$\epsilon^{-\lambda \Delta}$ & True & True & False \\
\hline 0.0 & True & F'alse & True \\
\hline 0.0 & l'alse & False & False \\
\hline$e^{-\lambda \Delta}$ & False & True & False \\
\hline 0.0 & False & False & True \\
\hline- & True & True & True \\
\hline- & False & True & True \\
\hline
\end{tabular}

The first six entries entries in the table correspond to terms N1-6 in Formula i.9. Note that the entries corresponding to N2 and N.5-assumed to be 1 in Section i.4.3-are now the same as $\mathrm{N} 1$ lo account for our revised interpretation of events of type $E_{P}$.

Figure 7.21 .ii shows a portion of a belief net for modeling the effects of the assembly action. The complete model is specified as follows:

\begin{tabular}{|c||c|c|c|}
\hline $\operatorname{Pr}\left(\left(E_{H_{i}, t}\right) \mid \ldots\right)$ & $\langle$ Sc.t $-\epsilon)$ & $\left\langle\|_{r, t}-\epsilon\right\rangle$ & $\langle$ As.t $-\epsilon\rangle$ \\
\hline 0.0 & False & False & False \\
\hline 0.0 & True & False & False \\
\hline 0.0 & False & True & False \\
\hline 0.0 & True & True & False \\
\hline 0.0 & False & False & True \\
\hline 0.0 & True & False & True \\
\hline 0.0 & False & True & True \\
\hline 1.0 & True & True & True \\
\hline
\end{tabular}

Finally, Figure i.21.iii shows a portion of a belief net for morleling the effects of the cleaning action. 'The complete model for the effect of cleaning on the beation of Wrench14 are shown below:

\begin{tabular}{|c||c|}
\hline $\operatorname{Pr}\left(\left\langle E_{\text {ur. }} t\right) \mid \ldots\right)$ & $(C l . t-\epsilon\rangle$ \\
\hline \hline 0.0 & False \\
\hline 1.0 & True \\
\hline
\end{tabular}

and similarly for the effect of cleaning on the location of Screwdriver:31: 


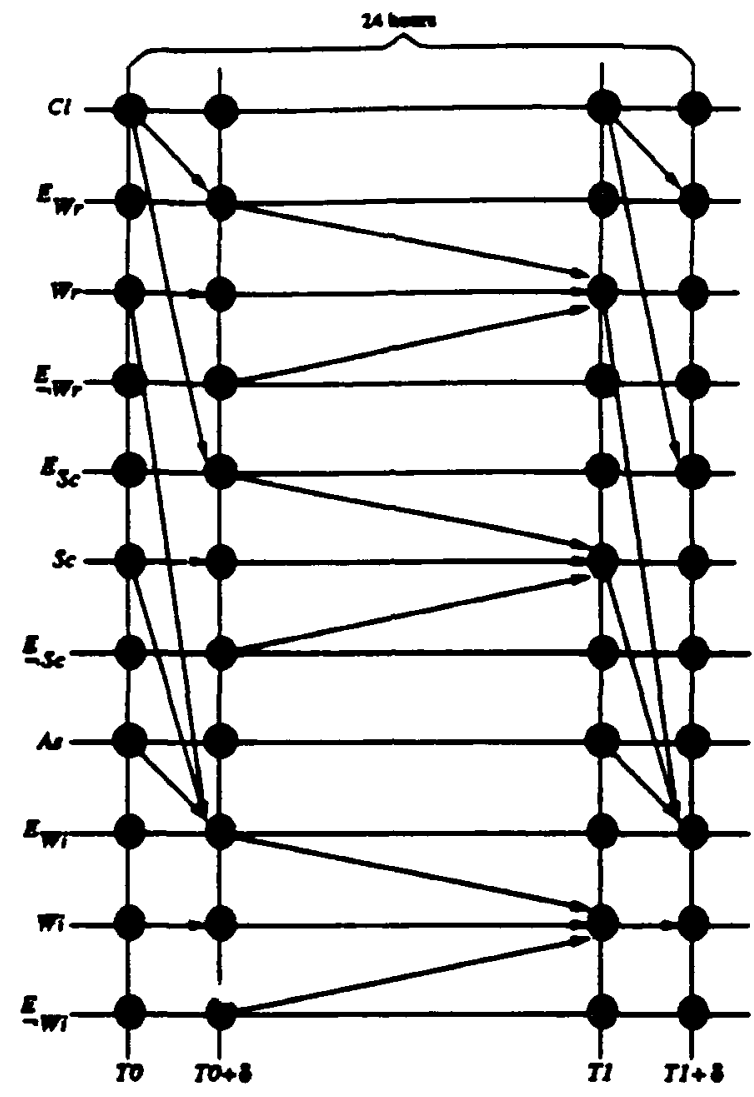

Figure 7.22: A belief network for the factory example

\begin{tabular}{|c||c|}
\hline $\operatorname{Pr}\left(\left(E_{\text {Sc }} ; t\right) \mid \ldots\right)$ & $(C, t-\epsilon)$ \\
\hline \hline 0.0 & False \\
\hline 1.0 & True \\
\hline
\end{tabular}

In the discussion of the general model, the amount of time separating time points was assumed to be the same for all pairs of consecutive time pointe. In reasoning about the factory example. it will be useful to have the time eparating pairs of consecutive time points differ, and to have different models for handling different separations. We will need time points close together for propagating the (almost immediate) consecquences of actions. and time points separated by several hours so as not to incur the coniputational expense of reasoning about intervals of time during which little of interest 
happens. To reduce the complexity of the network for the factory exantple. we assmure that evidence concerning the ocrurrence of actions surh as cleaning and assembling is alwavs with regard to the end points of $2 \pm$ hour intervals. Figure $\mathbf{i} .22$ shows the complete network for the factory example. Note that. since the evidence for actions appears only at 2.4 hour intervals. we encode the morlels for action only at the time points $T 0$ and $T I$ : similarly, since additional evidence for events of type $E_{P}$ is only available at $T 0+\epsilon$ and $T 1+\epsilon$, we use a simpler model for persistence at $T 0$ and $T 1$ in which. for example. $\langle W r . T O+\epsilon\rangle$ is completely determined by $\langle\mathrm{Wr}$. TO $\rangle$. If we assume a prior probability of 0 for all norles without predecessors in Figure $\mathbf{i} .22$ excepting $\langle C l . T O\rangle$ and $\langle A s . T l\rangle$ which are, respectively, 0.7 and 1.0 , then $\operatorname{Pr}\left(\left\langle E_{W_{i}}, T 1+\epsilon\right\rangle\right)$ is 0.175 in the unique posterior distribution deternined by the network. This is the sane as that established by the analysis of Section i.4.3. but. in this case. we have made all of our assumptions of independence explicit in the structure of the temporal belief network.

It is strajghtforward to extend the model described above to arcount for new observations and updating beliefs. Suppose we have the observations $o_{1}, o_{2}, \ldots, o_{n}$, where each observation is of the form $(O, t)$ and $O$ is an event type corresponding to a particular type of observation. We assume some prior distribution specified in terms of constraints of the form:

$$
\operatorname{Pr}(\langle O, t))=0.001
$$

There are also constraints indicating prior helief regarding the occurrence of events other than observations. For instance, we might have

$$
\operatorname{Pr}(\langle E, t\rangle)=0.001 \text {. }
$$

Observations are related to eyents by constraints such as

$$
\operatorname{Pr}(\langle E, t\rangle \mid\langle O . t\rangle)=0 . i 0
$$

and

$$
\operatorname{Pr}(\langle E, t) \mid \neg\langle O, t\rangle)=0.025
$$

To update an agent's beliefs you can either change the priors:

$$
\operatorname{Pr}(\langle O, t\rangle)=1.0
$$

or you can compute the posterior distribution:

$$
\operatorname{Bel}(A)=\operatorname{Pr}\left(A \mid o_{1}, o_{2}, \ldots o_{n}\right)
$$


Most of the standard techniques for representing and reasoning about evidence in belief networks apply directly to our mudel.

Veed material on the expressive limilations of this model. Relation to Markov processes and Markov chains.

Suppose that the instantaneous state of the world can be completely specified in terms of a vector of values assigued to a finite set of boulean variables $\mathcal{F}=\left\{P_{1}, P_{2}, \ldots, P_{n}\right\}$, and suppose further that the environment can be accurately modeled as a Markov process in which time is discrete and the state space $\ \Omega$ corresponds to all possible valuations of the variables in $\mathcal{F}$. Given such a model including a transition matrix defined on $\Omega$. We can generate a lemporal belief network to compute the probability of any proposition in $\mathcal{P}$ being true at any time $I$ based upon evidence concerning the values of variables in $\mathcal{F}$ at various times, and do so in accord with the trausilion probabilities specified in the Markov model. Conversely. given a lemporal belief network such that. for all $t$ and $P \in \mathcal{P}$. all of the predecessors of $\langle P . t\rangle$ are in the set $\left\{\left\langle P_{i}, t-\delta\right\rangle\right\}$, the network is said to satisfy the Markov property for temporal belief networks. and, from this network. one can coustruct an equivaleut Markov chain.

The reason that one might use a lluent-and-event-based tempora: belief network model ratler than an equivalent state-based Markov model is because the belief network representation facilitates reasoung of the sort required for applicatious in planuing and decision support (e.g., computing answers to questions of the form. "What is the probability of $P$ at $t$ given everything else we know alsout the situation?"). These same answers can be computed using the Markor model. but the process is cousiderably less direct.

Satisfying the Markov property for temporal belief networks allows us to establish the connection between temporal belief networks and Markov chains. but it sometimes results in unintuitive network structures. Lntroducing a delay between an action and its consequences may appear reasonable give the intuition that causes precede elfects. However, introducing a delay between $E_{P}$ and $P$ simply to eusure the Markov property may seem a little extras. We can eliminate the delay between $E_{P}$ and $P$ by returning to the modet for persistence in Formula i.9. The resulting networks do not satisfy the Marbor property described in this section, but they are perfectly legitimate temporal belief nets and provide a somewhat more intuitive model for representing change than networks that do satisfy the Markov property. 


\subsubsection{Fundamental Problens in Temporal Reasoning}

Civen that our morlel actdresses many of the same problems that concern logicians working on temporal logic. we will briefly mention how our nodel deals with rertain classic problens in temporal reasoning: the franne. ramification. and qualification problems. We will begin by considering the frame problem stated in probabilistic terms: "Does our model accurately capture our expectations regarding fluents that are considered not likely to change as a consequence of a particular event occurring?" The answier is yes insofar as frame avioms can be said to solve the frame problem in temporal logic: persistence constraints are the probabilistic equivalent of frame axioms.

In considering the ramification problem. we will consider two possible interpretations. First. "Does our model enable us to compute appropriate expectations regarding the value of a particular fluent at a particular point in time without bothering with a myriad of seemingly unimportant consequences?"- The answer to this is a resounding no: our model commits us to predicting every possible consequence of every possible action no unatter how implausible. A second interpretation (or perhaps facet is a better word) of the ranification problem is "Does our model enable ns to handle additional ronsequences that follow from a set of cansal predictions?" For instance, if $A$ is in box $B$ and I move $B$ to a new location. I should be able to predict that $A$ will be in the new location along with $B$. Our model provides no provision at all for this sort of reasoning. The basic idea of Bayesian illference can be extended to handle this sort of reasoning, but we have not investigated this to date.

The last problem we consider concerns reasoning about exceptions involving the rules governing canse-and-effect relationships. Does our model solve the qualification problem? That is to say, "Does our model accurately" capture our expectations regarding the possible exceptions to knowledge about cause-and-effect relationships?" The answer is yes: conditional probabilities would seem to be exactly suited for this sort of reasoning. It should be noted, however, that our model imposes a considerable burden on the person setting up the model. The model described in this section requires specifying all possible causes for each possible effect and the probability of each ext for every possible combination of possible causes. It is not clear. however, that one can get away with less. Given the prohlems inherent in eliciting such information from experts. it would appear that we will have to alltomate the process of setting up our probabilistic models.

The thimd example is dmon frmm [1:] and ooncerns the sfquential dr- 
rision problem for the mobile tanft loralization (M/TL) problem. Be sure to addiess the issue concerning the dumtion of the time interval sepamting points in the trnuporal Bayes network. There are two possible appronches for the MTL problem. Either the intervals are of a fixed dumtion independent of the action performed. or they are dcpendent on the action performed in which case additional arcs have to be ailded between the action norles for the mbot at one point in time and all of the other nodes at the next point in time. In the first appronch. the model is simple and control is tricky; in the serond appronch. the model is complex and rontrol is simple.

\subsection{Sequential Decision Making}

In this section, we consider an approach to building planning and control systems that integrates sensor fusion. preduction. and seyuential decision making. The approach is based on Bayesian decision theory, and involves encoding the underlying planuing and cuntrol problem in terms of probabilistic models We illustrate the approach using a robotics problem that re quires spatial and temporal reasoning under uncertainty and time pressure. We use the estimated computational cost of evaluation to justify representational tradeoffs required for practical application.

In this secton, we view planuing in terms of enumerating a set of possible courses of action. evaluating the consequences of those courses of action. and selecting a course of action whose consequences naximize a particular performance (or value) function. We adopt Bayesian decision theory as the theoretical framework for our discussion, since it provides a convenient basis for dealing with decision making under uncertainty.

One interesting thing about nost planning problems is that the results of actions can increase our knowledge, polentially inproving our ability to make decisions. From a decision theoretic perspective, there is no difference between actious that involve sensing or movenent to facilitate sensing aud any other actions; a decision maker simply tries to choose actions that maximizs expected value. In the approach described in this section. an ageut enged in a particular perceptual lask selects a set of seusor views by phuteally moving about.

Haring committed to a decision theoretic approach. there are specific problem that we have to deal with. The most difficult concern representing the problem and obtaining the necessary statistics to quantify the underlying decision model. In the robotics problems we are working on. the latter is 
relatively straightforwaid, and so we will concern ourselves primarily with the former.

In building a decision mudel for cont rol purposes. it is not enough to write down all of your preferences and expectations; this information might provide the basis for constructing some decision model. but it will likely be inupractical from a computational standpoint. It is frustrating when you know what you want to compute but cannol afford the time to do so. Some researchers respond by saying that eventually computing machinery will be up to the task and ignore the computational difficulties. It is our contention, however. that the combinalorics inherent in sequential decision making will continue to outstrip computing technolugies.

In the following, we describe a concrete problem to ground our discussicn, present the general sequential decision making model and its application to the concrete problem. show how to estinate the computational costs associated with using the model. and. finally, describe how to reduce those costs to manageable levels by making various represeutational tradeoffs.

\subsubsection{Mobile Target Localization}

The application that we have chosen to illustrate our approach involves a molsile rohot navigating and tracking moving targets in a cluttered environment. The robot is provided with sonar and rudimentary vision. The moving target condl be a person or another mobile robot. The mobile base consists of a holonomic (turn-in-place) synchro-drive robot equipped with a C C D camera mounted on a pan-and-tilt head, and 8 fixed Polaroid sonar sensors arranged in pairs directed forward, backward, right. and left.

The robot's task is to detect and track moving objects. reporting their location in the coordiuate system of a global map. The environment consists of one floor of an office building. 'The robot is supplied with a floor plan of the office showing the position of permanent walls and major pieces of furuiture such as deaks and tables. Smaller pieces of furuit ure, potted plants and other assorted clutter constitute obstacles that the robot has to detect and anin

Wence thai there is error in the robot s movement requiring it to contertion este its position with respect to the floor plan so as not to become loat. Position estimation (localization) is performed by having the robot track beacons corresponding to walls and corners and then use these beacons to reduce error in its position estimate.

Localization and tracking are frequently at odds with one another. A 
particular localization strategy may reduce position errors while making tracking difficult. or improve tracking while losing registration with the global map. The trick is to balance the demands of localization against the demands of tracking. The molsile target localization (MITL) problem is particularly appropriate for planning research as it requires considerable complexity in terms of tenporal and spatial representation. and involves tine pressure and uncertainty in sensing and action.

\subsubsection{Model for Time and Action}

In this section. we provide a decision model for the MTL problem. To specify the model, we quantize the space in which the robot and its target are embedded. A natural quantization can be derived from the robot's sellsor y capabilities.

The robot's sonar sensors enable it to recoguize particular patterns of free space corresponding to various configurations of walls and other permanent objects in its environment (e.g.. corridors. $L$ junctions and $T$ junclions). We tessellate the area of the global map into regions such that the same pattern is detectable anywhere within a given region. This tessellation provides a set of incations $\mathcal{L}$ corresjonding to the regions that are used to encode the location of both the robot and its target.

Our decision model includes two variables $S_{T}$ and $S_{R}$, where $S_{T}$ represents the location of the target and ranges over $\mathcal{L}$, and $S_{R}$ represents the location and orientation of the robot and ranges over an extension of $\mathcal{L}$ including orientation information specific to each type of location. For any particular instance of the MTL problem, we assume that a geometric description of the environment is provided in the form of a CAD model. Given this geometric description and a model for the robot's sensors, we generate L. $S_{R}$, and $S_{T}$.

The model described here is based on the approach of Section i.t. Given a set of discrete variables, $x$, and a finite ordered set of tine points, $\mathcal{T}$, we construct a set of chance nodes. $\mathcal{C}=\boldsymbol{X} \times \mathcal{T}$, where each elenent of $\mathcal{C}$ correaponds to the value of some particular $x \in l^{\prime}$ at some $t \in T$. Let $C^{\prime}$; correspond to the subset of $\mathcal{C}$ restricted to $t$. The temporal belief networks discuand in this section are distinguished by the following Markov property:

$$
\operatorname{Pr}\left(C_{t} \mid C_{t-1} \cdot C_{t-2}^{\prime}, \ldots\right)=\operatorname{Pr}\left(C_{t} \mid C_{t-1}^{\prime}\right) \text {. }
$$

Let $S_{R}$ and $S_{T}$ be variables ranging over the possible locations of the robot and the target respectively: Let $A_{R}$ be a variable ranging over the ac- 




Figure 7.23: Probabilistic model for the MTL problem

tions available to the robot. At any given point in time. the robot can make observations regarding its position with respect to nearby walls and corners and the target's position with respect to the robot. Let $O_{R}$ and $O_{T}$ be varialyles ranging these observations with respect to the robot's surroundings and the target's relative location.

Figure $7.2: 3$ shows a temporal belief network for $\mathfrak{l}=\left\{S_{R}, S_{T}, A_{R}, O_{R}, O_{T}\right\}$ and $\mathcal{T}=\left\{T_{1}, T_{2}, T_{3}, T_{4}\right\}$. To quantify the model shown in Figure 7.23 . we have to provirle distributions for each of the variables in $\boldsymbol{l}^{\prime} \times \mathcal{T}$. We assume that the model does not depend on time, and, hence, we need only provide one probability distribution for each $x \in d$. For instance. the conditional probability distribution for $S_{T}$,

$$
\operatorname{Pr}\left(\left\langle S_{T}, t\right\rangle \mid\left\langle S_{T}, t-1\right\rangle,\left\langle O_{T}, t\right\rangle,\left\langle S_{R}, t\right\rangle\right),
$$

is the ame for any $t \in \mathcal{T}$. The numbers for the probability distributions can be obtained by experimentation without regard to any particular global map.

In a practical model consisting of more than just the four time points shown in Figure 7.23. some points will refer to the past and some to the future. One particular point is designated the current time or Now. Representing the past and present will allow us to incorporate evidence into the 


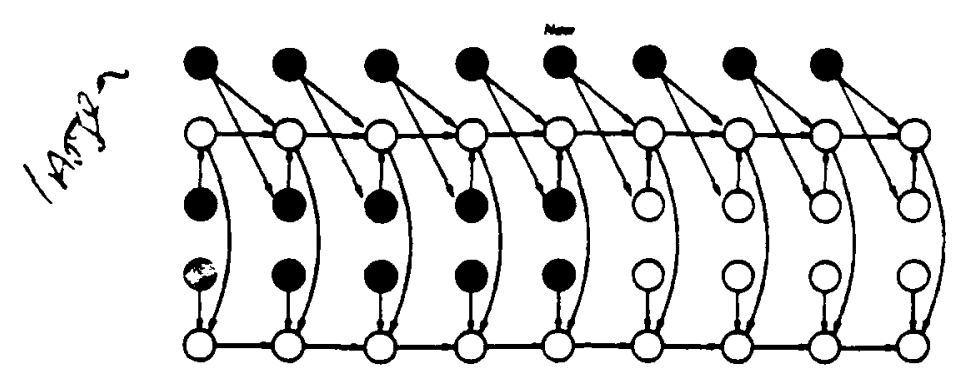

Figure 7.24: Evidence and action sequences

model. By convention, the nodes corresponding to obser vations are nieant to indicate observations completed at the associated tine pnint, and nodes corresponding to actions are meant to indicate actions initiuted at the associated time point. The actions of the robot at past time points and the observations of the robot at past and present time points serve as evidence to provide conditioning events for computing a posterior distribution. For instance. having ohserved $\sigma$ at $T$, denoterl $\left\langle O_{F=0, T}\right.$, and initiated $\alpha$ at $T-1$, denoted $\left(A_{R}=R-1\right\rangle$, we will want lo compute the posterior distribution for $S_{H}$ at $T$ given the evidence:

$$
\operatorname{Pr}\left(\left\langle S_{\mp \omega}, T\right\rangle . \omega \in \Omega_{S_{R}} \mid\left\langle O_{\mp}, T\right\rangle .\left\langle A_{\mp \alpha, T-1\rangle) .}\right.\right.
$$

To update the model as time passes, all of the evidence nodes are shifted into the past. discarding the oldest evidence in the process. Figure $\mathbf{7 . 2 1}$ shows a network with uine time points. The lighter shaded nodes correspond to evidence. As new actions are initialed and observations are mide, the appropriate nodes are instantiated as conditioning nodes. and all of the evidence is shifted to the left by one time point.

The darker shaded nodes shown in Figure $i .21$ indicate nodes that are instantiated in the process of evaluating possible sequeuces of actions. For evaluation purposes, we employ a sinple time-separable value function. By time separable, we mean that the total value is a (perhaps weighted) sum of the value at the dilferent time points. If $V_{t}$ is the value function at lime $t$, then the lotal value, $r$, is defined as

$$
V=\sum_{t \in T} g(t) l_{i}
$$

where $\gamma: T \rightarrow\{x \mid 0 \leq x \leq 1\}$ is a decreasing function of time used to discount the inpact of future consequences. Since our model assumes a 
finite $\mathcal{T}$. we already discount some future consen'sences by ignoring them altogether; 9 just gives us a little more control over the imnediate future. For $l_{i}$. we use the following function

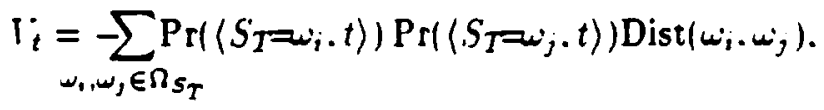

where Dist : $\Omega_{s_{T}} \times \Omega_{S_{T}} \rightarrow \mathfrak{H}$ determines the relative Euclidean distance between pairs of locations. The $i_{i}$ function reflects how much uncertainty there is in the expected location fo: the target. For instance, if the distrihution for $\left\langle S_{T}, t\right\rangle$ is strongly weighted toward one possible location in $\Omega_{S_{T}}$, then $V_{t}$ will be close to zero. The more places the target could be and the further their relative distance, the more negative $V_{i}$.

The actions in $\Omega_{A_{R}}$ consist of tracking and localization rontines (e.g. move aloug the wall on your left until you reach a corner). Each action has its own termination criteria (e.g., reachiug a corner). We assume that the robot has a set of strategies, $\mathcal{S}$, consisting of serfuences of such actions, where the length of seqnences in $\mathcal{S}$ is limited by the number of present and future tine points. For the network sliown in Figure i.24, we have

$$
S \subset \Omega_{A_{R}} \times \Omega_{A_{R}} \times \Omega_{A_{R}} \times \Omega_{A_{R}} .
$$

The size of $S$ is rather important. since we propose to evaluate the network $|S|$ tinues at every decision point. The strategy with the highest expected value is that strategy, $\varphi=\alpha_{0}, \alpha_{1}, \alpha_{2}, \alpha_{3}$, for which $V$ is a maximun. couditioning on $\left(A=\alpha_{0}\right.$, Now $\rangle .\left\langle A=\alpha_{1}\right.$, Nout 1$\rangle .\left\langle A=\alpha_{2}\right.$, Nout 2$\rangle$. and $\left\langle A,-r_{3}\right.$, Vow 3$\rangle$. The best strategy to pursue is reevaluated every time that an action terminates.

Wie use Jensen : [21] variation on Lauritzen and Spiegelhalter's [25] àgorithm to evaluate the decision network. Jensen's algorithm involves constructing a hyper graph (called a cliquc tree) whose vertices correspond to the (maximal) cliques of the chordal graph formed by triangulating the undirected graph obtained by first connecting the parents of each note in the netwon and then eliminating the directions on all of the edges. The cost of evalus a Bayesian network using this algorithm is largely determined by the of the state spaces formed by taking the cross product of the state spaces of the nodes in each vertex (clique) of the clique tree.

Following Kanazawa [22], we can obtain an accurate estimate of the cost of evaluating a Bayesian network, $G=(r, E)$, using Jensen 's algorithm. Let $C=\left\{C_{i}\right\}$ be the set of (maximal) cliques in the chordal graph described 
In the previous paragraph. where each cliguc represents a subset of $1^{\circ}$. We define the function. card : $C^{\prime}-\{1 \ldots .|C|-1\}$. so that $\operatorname{card}\left(C_{\text {, }}\right)$ is the rank of the highest ranked node in $C_{1}$. where rank is determined by the maximal cardinality ordering of $I^{-}$(see [32]). We define the function. adj : $C^{\prime}-2^{C}$. hy:

$$
\operatorname{adj}\left(C_{i}\right)=\left\{C_{3} \mid\left(C_{j} \neq C_{i}\right) \wedge\left(C_{i} \cap C_{j} \neq \theta\right)\right\} .
$$

The clique tree for $G$ is constructed as follows. Each clique $C_{i} \in C^{\prime}$ is connected to the clique $C_{j}$ in $\operatorname{adj}\left(C_{1}\right)$ that has lower rank by card(.) and has the highest number of nodes in common with $C_{i}$ (ties are broken arbitrarily). Whenever we counect two cliques $C_{i}$ and $C_{j}$. we create the separntion set $S_{i j}=C_{i} \cap C_{j}^{\prime}$. The set of separation sets $S^{\prime}$ is all the $S_{i j}$ 's. We define the function, sep : $C-2^{s}$. by:

$$
\operatorname{sep}\left(C_{i}\right)=\left\{S_{j k} \mid S_{j k} \in S_{.}(j=i) \vee(k=i)\right\} .
$$

Finally, we define the ueight of $C_{i}, u_{i}=\prod_{n \in C_{i}}\left|\Omega_{n}\right|$, where $\Omega_{n}$ is the state space of node 11 . The cost of compulation is proportional to $\sum_{C_{i} \in C}$ "i $_{i} \mid \operatorname{sep}\left(C_{i}\right)$. We refer to this cost estimate as the clique-tree cost.

The approach described in this section allows us to integrate prediction. observation. and control in a single model. It also allows us to haudle uncertainty in sensing, movement. aud moleling. Behavioral properties emerge as a consequence of the probabilistic model and the value function provided, not as a cousequence of explicitly programming specific behaviors. The main drawback of the approach is that. while the model is quite compact. the computational costs involved in evaluating the model can easily get out of hand. For instance. in our model for the MTL problem. the clique-tree cost is bounded from below by the product of $|\tau|,\left|\Omega_{S_{T}}\right|^{2}$, and $\left|\Omega_{S_{R}}\right|^{2}$. In the next section, we provide several metliods that, taken together. allow us to reciuce computalional costs to practical levels.

\subsubsection{Coping with Complexity}

To reduce the cost of evaluating the MTL decision model. we nse the following three methods: (i) carefully tailor the spatial representation to the robot's sensory capalsilities, reducing the size of the state space for the spartial variables in the decision nodel. (ii) enalsle the robot to dynamically narrow the range of the spatial variables nsing heuristics to further reduce the size of the state space for the spatial variables, and (iii) consider unly a few candidate action secquences from a fixed library of tracking strategies 


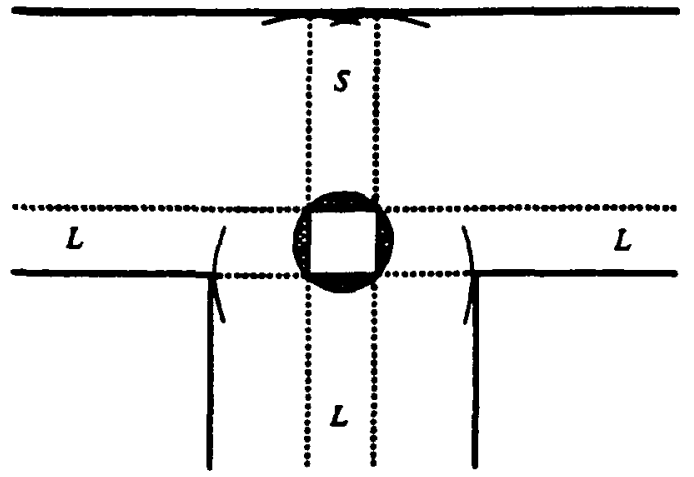

Figure $i .25$ : Sonar data entering a $T$ junction

by taking into account the reduced state space of the spaiiai variables. In the rest of this section. we consider each of these three methods.

The use of a high-resolution representation of space has disadvantages in the wodel proposed here: increasing the resolution of the representation of space results in an increase in the sizes of $\Omega_{S_{R}}$ and $\Omega_{S_{T}}$. and thus raises the cost of evaluating the network. Heeping the sizes of $\Omega_{S_{R}}$ and $\Omega_{S_{T}}$ small makes the task of evaluating the nodel we propose feasible.

A further consideration arises from the real-world sensory and data processing systems available to our robot. Finer-resolution representations of space place larger demands on the robot's on-board system in terms of both run-time processing time and sensor accuracy. To allow our robot to achieve (near) real-tine performance, it seems appropriate to liwit the represeutation to that level of detail that can be obtained economically from the hardware available.

In our current inplementation. we have 8 sonar transducers positioned on a square platform, two to a side, spaced about $25 \mathrm{~cm}$. apart. We take distance readings from each transducer. and threshold the values at about 1 meter. Anything above the threshold is "long." anything below is "short." The readings aloug each side are then combined by voting. with ties going to "loug." In this way, the data from the souar is reduced to 4 bits. Figure $\mathbf{7 . 2 5}$ showe the result of this scheme on eutering a $T$ junction. In addition. we use the shas encoders on our platform to provide very rough metric information for the decision model. Currently: 2 additional bits are used for this purpose. but ouly when the robot is positioned in a hallway, which corresponds to ouly one sonar configuration. So the total number of possible states for $O_{R}$ 


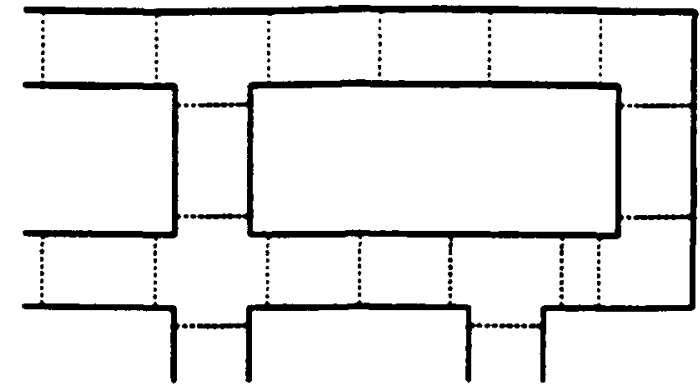

Figure $7.20:$ Tessellation of office lavout

is 19.1 .5 for various kinds of hallway junctions and 4 more for corridors.

This technique results in a tessellation of space like that shown in Figure $\mathbf{i} .26$. Our experiment $z$ have shown that this tessellation is quite robust in the seuse that the readings are consistent anywhere in a given tile. The exception to this occurs when the robot is not well-aligned with the surrounding walls. In these cases, reflections frequently make the data unreliable. One of the tasks of the controllers that uuderlie the actious described in the previous sections is to maintain good alignment, or achieve it if it is lost.

In addition. to reducing the size of the overall spatial representation, we can restrict the range of particular spatial variables on the basis of evidence not explicitly accounted for in the decision model (e.g., odometry and compass information). For instance. if we know that the robot is in one of two locations at time 1 and the robot can move at most a single location during a given time step, then $\left\langle S_{R}, 1\right\rangle$ ranges over the two locations, and, for $i>1 .\left\langle S_{R}, i\right\rangle$ ueed only range over the locatious in or adjacent to those in $\left\langle S_{R}, i-1\right)$. Similar restrictions can be obtained for $S_{T}$. For invdels with limiled lookahead (i.e., sinall $|T|$ ), these restrictions can result in significant computational savings.

Consider a temporal Bayesian network of the forn shown in Figure $\mathbf{7 . 2 3}$ with $n$ steps of lookaliead. Let $(X, i)$ represent an element of $\left\{S_{R}, S_{T}, A_{R}, O_{R}, O_{T}\right\} \times$ $\left\{1 \ldots \ldots\right.$, . The largest cliques in one possible ${ }^{3}$ clique tree for this network consint of eets of variables of the form:

$$
\left\{\left\langle S_{R}, i\right\rangle,\left\langle S_{R}, i+1\right\rangle,\left\langle S_{T}, i\right\rangle,\left\langle S_{T}, i+1\right\rangle\right\}
$$

\footnotetext{
${ }^{3}$ The triangolation algorithm attempts to minimize the size of the largest clique in the resulting chordal graph. 'There may be more than one way to triangulate a graph so as to ninimize the clique size.
} 


\begin{tabular}{|c|c|c|c|}
\hline \multirow[b]{2}{*}{ State space size } & \multicolumn{3}{|c|}{ Number of time points } \\
\hline & 3 & 5 & 8 \\
\hline \multirow[t]{2}{*}{ Coustaut (6) } & $4091-1$ & 78066 & 133794 \\
\hline & $(0.58)$ & (1.11) & $(1.90)$ \\
\hline \multirow{2}{*}{ Constant (16) } & 624944 & 1232176 & $21+3024$ \\
\hline & $(8.87)$ & $(17.49)$ & $(30.42)$ \\
\hline \multirow[t]{2}{*}{ Constant (30) } & 3846330 & 7669530 & 13404330 \\
\hline & $(54.60)$ & $(108.86)$ & $(190.26)$ \\
\hline \multirow[t]{2}{*}{ Linear $(2 t+1)$} & 5844 & 5.5088 & $43: 5759$ \\
\hline & $(0.08)$ & $(0.78)$ & (6.16) \\
\hline \multirow[t]{2}{*}{ Quadralic $\left(l^{2}+1\right)$} & 3691 & 160701 & 37.565 .59 \\
\hline & $(0.0 .5)$ & $(2.28)$ & (53.32) \\
\hline \multirow[t]{2}{*}{ Expouential $\left(2^{\prime}\right)$} & $28 \pi 5$ & 107.515 & 4131611 \\
\hline & $(0.05)$ & (1.53) & (58.64) \\
\hline
\end{tabular}

Table T.1: Clique-tree costs for sample networks

for $i=1$ to $n-1$, and the size of the corresponding cross product space is the product of $\left|\Omega_{\left(S_{R, i}\right)}\right|,\left|\Omega_{\left(S_{R}, i+1\right)}\right|,\left|\Omega_{\left\langle S_{T, i}\right\rangle}\right|$, and $\left|\Omega_{\left(S_{T}, i+1\right)}\right|$. For fixed state spaces. this prodnct is just $\left|\Omega_{S_{R}}\right|^{2}\left|\Omega_{S_{T}}\right|^{2}$. However, if we restrict the state spaces for the spatial variables on the hasis of some initial location estimate and some bounds on how quickly the robot and the target can move about, we can do considerably better.

Table $\mathbf{7 . 1}$ shows the clique-tree costs for three MTL decision model networks of size $n=3,5$, and 8 time points. For each size of model, we consider rases in which $\Omega_{\left(S_{R, i}\right)}$ and $\Omega_{\left(S_{T, i}\right)}$ are constant for all $1 \geq i \geq n$, and cases in which $\left|\Omega_{\left\langle S_{R}, 1\right\rangle}\right|=\left|\Omega_{\left\langle S_{T}, 1\right\rangle}\right|=1$ and the sizes of the state spaces for subsequent spatial variables, $\Omega_{\left(S_{R}, i\right)}$ and $\Omega_{\left(S_{T}, i\right)}$, for $1>i \geq n$ grow by linear, quadratic, and exponential factors bounded by $\left|\Omega_{S_{T}}\right|=\left|\Omega S_{R}\right|=30$. For these evaluations, $\left|\Omega_{A_{R}}\right|=6 .\left|\Omega_{O_{T}}\right|=32$, and $\left|\Omega_{O_{R}}\right|=19$ in keeping with the and and wovement routines of onr cursent robot. The number in bractun underneath the clique tree cost is the time in cpu seconds required for eviliallon.

Our current idea for restricting the present location of the robot and the target involves using a fixed threshold and the most up-lo-date estimates for these locations to eliniuate unlikely possibilities. Occasionally, the actual 
locations will be mistaken': eliminated, and the robot will fail to track the target. There will have tc be a recovery strategy and a criterion for iuvoking it to deal with such failures.

There are certain costs involved with evaluatiug Bavesian networks that we have ignored so far. These costs involve triangulating the graph. constructing the clique tree. and performing the storage allocation for building the necessary data structures. For our approach of dynamically restricting the range of spatial variables. the state spaces for the randonn variables cliange. but the sizes of these state spaces and the topology of the Bayesian network remain constant. As a consequence. these ignored costs are incurred once. and the associated computatioual tasks can be carried out at design time. Dynamically adjusting the state spaces for the spatial variables is straightforward and computationally inexpensive.

The third method for reducing the cost of decision making involves reducing the size of $\mathcal{S}$. the set of sequences of actions corresponding to tracking and localization strategies. For an $n$ step lookahead. the set of useful strategies of length $n$ or less is a very small subset of $\Omega_{A_{R}}{ }^{n}$. Still. given that we have to evaluate the network $|\mathcal{S}|$ times. even a relatively small $\mathcal{S}$ can cause problems. To reduce $S$ to an acceptable size. we only evaluate the network for strategies that are possible given the current restrictions on the spatial variables. For instance. if the robot knows that it is moving down a corridor toward a left-pointing $L$ junction. it can elinunate from consideration any strategy that involves it moving to the end of the corridor and turning right. With appropriate preprocessing, it is computationally simple to dynamically reduce $\mathcal{S}$ to just a few possible strategies in most cases.

\subsection{Further Reading}

Bavesian decision theory [5. 8. 33]. Value of information [19]. It should be noted that Howard's is not the only theory proposed for assessing the value of information sonrces. In particular, information value theory is closely related to the theory of experimental design $[16,30]$. Experimental design is concerned with the problem of maximizing the information gainerl from performing experiments under cost constraints. Information value theory represents one approach to experinental design based on Bayesian decision theory.

Influence diagrams [20]. Dynamic programming [i]. Conditioning [18]. lieiji's join-tree cost [22]. Jensen's [21] variation on Lauritzen and Spiegel- 
lialter's clustering algorithm [25]. Causal polv trees [32]. Evaluating influence diagrams [3:4]. Influence diagrams for control applications [1].

The notion of lucally distinctive place as it is used in Section 7.3 is due to Kuipers [23]. The design of the geographer nodule was based on the work of kuipers [24] and Levitt [20] on learning maps of large-scale space. and the extensions of Basye $\epsilon t$ al $[6]$ to handle uncertainty.

See Dean and hanazawa [13] and Ilanks [1i] for competing approaches. See Cooper ef al [10] for a discussion of a related approach to probabilistic reasoning about change using a discrete model of time.

References to work on active perception $[2,3,4]$. 


\section{Bibliography}

[1] Agogino. A. M. and Ramamurthi. K.. Real-Time Influence Dingrams for Monitoring and Controlling Mcchanical Systems. Techuical report. Dopartment of Mechanical Engineering. University of California, Berkeley. 1988.

[2] Aloimonos. J.. Bandyopadlyyay. A., and Weiss, I., Active Vision, Prvceedings of the First Inlemational Conference on Compuler lision. 198i. 35-5.5.

[3] Rajcsy. R., Active Perception. Prorecdings of the IEEE. 76(8) (1988) 996-1005.

[t] Ballard. Dana HI., Reference Frames for Auimate Visiou. Proceedings IJCAI 11. Detroit. Michigan. IJC.dI. 1989, 1635-1641.

[5] Barnett. V., Comparntive Slatistical Inference. (Juhn Wiley and Suns. New York, 1982).

[0] Basye. Kenneth, Dean, Thomas. and Vitter, Jeffreqv Scott. Coping With I'ncertainty in Map Learning. Proceedings IJCAI 11, Detmit. Michigan. IJCAI. 1989, 663-668.

[i] Bellman. Richard. Dynamic Progmmming, (Princeton University Press. 195i).

[8] Cherwolf, Herwan and Moses, Lincoln E., Elementary Decision Theory, (John Wiley and Suns, New York. 19:59).

[9] C'ooper. Gregory F., Probabilistir Inference l'sing Beliff Neturorks is IP.Hand, Technical Report KSL-8T-2i, Stanford Knowledge Systems Laboratory, 1987. 
[10] Cooper. Giregory F., Horvitz. Eric J., and Heckerman. David E.. A Method for Temporal Probnbilistic Reasoning. Technical Report KSL88-30. Stauford Knowledge Sịstems Laboratory, 1988.

[11] Dean, Thomas, Basve, Kennetlı. ('hekaluk, Robert, Hyun, Seungseok, Lejter. Moises, and Randazza. Margaret. Coping with Uncertainty in a C'ontrol System for Navigation and Exploration. Proceedings AAAI-90. Boston. Massachusetts, AAAI. 1990. 1010-1015.

[12] Dean. Thomas, Basye, Kenneth, and Lejter. Moises, Planning and Active P'erception, Proceedings of the UAMPA Horkshop on Innovative Approaches to Planning, Scheduling, and Control, DAKPA, 1990.

[13] Dean. Thoinas and Kanazawa. Keiji, Probabilistic Temporal Reasoning, Proceedings AAAI-88. St. Paul. Minnesota. AAAI. 1988. 524-528.

[14] Dean. Thomas and Kanazawa. Keiji, A Model for Reasoning About Persistence and Causation, Computational Intelligence, $5(3)$ (1989) 142150 .

[15] Dean, Thomas and Kanazawa, Keiji, Persistence and Probabilistic Inference, IEEE Tmnanctions on Systems, Man, and Cybernetics, 10(3) (198.9) $574-5.85$.

[16] Fedorov, V., Theory of Optimal Experimental Design, (Academic Press, New lork, 1972).

[1i] Ilanks, Steve, Representing and Computing Temporally Scoped Beliels, Proceedings AAA1-88, St. Paul, Minnesota, AAAl, 1988, 501-505.

[18] Horvitz, Eric J.:- Suermondt, H. Jacqnes, and Cooper, Giregory F., Bounded Conditioning: Flexible Inference for Decisions (Inder Scare Rr.sourrs, Technical Report KSL-89-42, Stanford Knowledge Systems Laboratory, 1989.

[19] Howard, Ronald A., Information Value Theory. IEEE Transactions on Syctems Science and Cybernetics. 2(1) (1966) 22-26.

[20] Howard, Ronald A. and Matheson. James E., Intluence Diagrams. lloward. Rouald A. and Matheson. James E., (Eds.). The Principles and Applications of Decision Analysis, (Strategic Decisions (iroup, Meulo Park, (A 94025, 1984). 
[21] Jensen. Finn V.. Lauritzen. Steffen L.. and Olesen. Listian Ci., Baycsian Ipplating in Recursire Cimphiral Voxlels by Loral Compuirations. Techniral Report R 89-15. Institute for Electronic Sistems. Department of Mathenatics and C'omputer Science. University of Aalborg. 1989.

[22] Kauazawa. liejii. Probability. Time. and Action. PhD thesis. Brown Iniversity, Providence. Rl. Furtlicuming.

[23] Kuipers. Benjamin. Modeling Spatial Knowledge. Cognitive Science. 2 (19i8) 129-153.

[24] Kuipers. Benjamin J. and Byun. lung-Tai. A Robust. Qualitative Method for Robot Spatial Reasoning. Proccedings AA AJ-s8. St. Paul. Minnesota. AAAI. 1988, iT\&-iig.

[25] Lauritzen. Stephen L. and Spiegelhalter. David J.. Local computations with probabilities on graphical structures and their application to expert systemis. Journal of the Royal Siatistical Society. 50(2) (1988) 15i194.

[26] Levitt, Tod S., Lawton, Daryl T., C'hellerg, David M.. and Nelson, Philip ('.. Qualitative Landmark-hased Path Planning and Following, Proceedings AAA1-87, Senttle, Ilashington, AAAl. 198i, 689-694.

[27] McC'arthy. John. Applications of Circumscription to Formalizing ('ommonsense kinowledge. Arlificial Inlelligence, 28 (1986) 89-116.

[28] McCarthy. John and Hayes. Palrick J., Some Philosophical Problems from the Staudpoint of Artificial lntelligence. Machine Intelligence. 4 (1969) 463-502.

[29] McDermott. Drew V.. A temporal logic for reasoning about processes and plans. Cognitive Scicnce. 6 (1982) 101-15.5.

[30] Mendenhall, W., Intmduction to Lincar Modcls and the Design and Andynis of Experinents. (Wadsworth, Belmont, California, 1968).

[31] Neapolitan, Riclard E., Pmbabilistir Rersonning in Expert Systemsa: Theory and Algorithms, (John Wiley and Sons, New York, 1990).

[32] Pearl. Judea, Probabilistic Rensonin! in Inlelligent Systems: Ne1urorks of Plausible Inference. (Morgan-Kaufmam. Los Altos. Califurnia. 1988). 
[33] Raifla. Iloward and Schlaifer. R.. Applied Statistical Derision Theory. (llarvard ('niversity Press. 1961).

[34] Shachter. Ross D.. Evaluating Influence Diagrams. Opemtions Researrh. 34(6) (1986) $871-8.82$.

[35] Siski. Hyszard. Random Proresscs.s. (Marcel Dekker. New York. 1979). 


\section{Chapter 8}

\section{Controlling Inference}

This chapter describes approaches for designing systems that are capable of taking their own computational resources into consideration during planning and problem solving. In partirular. we are interested in systems that manage their computational resources by using expectations about the performance of decision making procedures and preferences over outcomes resulting from applying such procedures. Careful management of computational resourres is important for complex problein solving tasks in which the time spent in decision making affects the quality of the responses generated by a system.

Much of the work described in this chapter can be seen as a response to a movement, started in the early 1980's, away from systems that inake use of complex representations and engage in lengthy deliberations, and towards systems rapable of making many very simple decisions quickly. This moveinent brought about the advent of the so-called "reactive systems" described in Chapter 4. Most. reactive systems are essentially programming languages for building systems that nunst be rexponsive to their environment. Such languages generally allow for multiple asyuchronous decision processes. farilitate conmunication among processes, and provide support for interrupts and process arbitration.

Ming of the renearchers hildling reactive strstems were interested in robotibe and decision-snpport applications requiring real-time response. The rempondreness of reactive syktems was in stark contrast with the performance of most planning and problem solving systems in use at that time. Mont existing planning systems rere essentially off-line data processing proredures that accepted as input some initial (and generally complete) descrip-

\footnotetext{
${ }^{\circ}$ O 1990 Thomas Dean. All rights reserved.
} 
tion of the current state of the environment. and. after some indeterminate (and generally lengthy) delay. returned a rigid sequence of actions which. if the environment was particularly cooperative. might result in the successful achievement of some goal.

Reactive systems unight be seen as an extreme response to the shortcomings of the existing planning systems. Reactive systems provided respunsiveness at the cost of shallow and often short-sighted decision making. Since there were no proposals for how to coutrol decision making in time-critical situations. researchers turned away from the traditional approaches to planning and attempted to incorporate more sophisticated decision making into reactive systems. Unwilling to sacrifice response tine. the researchers that were trying to improve the decision-making capabilities of reactive systems were forced to trade space for time. oflen without a great deal of attentiou to the consequences.

Some of the dissatisfaction with complex representations and coniplicated deliberation strategies was due to misinterpreting asymptotic complexity results as evidence of the existence of inpassable computational barriers. Proofs of NP-hardness certainly indicate that re must be prepared to make concessions to complexity in the form of tradeoffs. The lesson to be learned, however, is that we have to control inference, and not that we have to abandon it altogether.

In the 1970's. a great deal of effort was spent studying systems capable of explicitly reasoning about their own decision-making capabilities. This sort of reasoning about reasoning is generally referred to as meta-reasoning. As the research in this area matured, some researchers were concerned with how to learn to control decision making, while others were interested in the basic mechanisms required to guide decision making under time pressure. Many of the mechanisms studied had in common the use of expectations regarding the performance of decision procedures to help in selecting from among a set of such decision procedures.

As researchers began looking in the literature. it hecanne clear that many of the tools required for reasoning about the costs and benefits of applying decisjon-making rontines were already available. Indeed. researchers in the decivion eciences had already considered some of the problems involved in reasoning about the costs and benefits of inference. However. with rare exception. ${ }^{1}$ the decision analysts assumed that the agent was possessed of

'I. J. Good was one of those exceptions. and, in an amaxingly forward lookiag paper [22]. Good talked about what he called tupe $I I$ mtionality which involres an agent reanoning 
unlimited conmputational capabilities for reasoning about its current knowleilge: the issue must. often addressed concerned whether or not an agent should consider adding to its current knowledge. We are interested in the case of all agent currently biased to act in a certain way and consiclering if it should expend further computational resources and risk the consequences of delay in order to deliberate further about its options. It is tiiis basic idea of an agent with limited computational capabitities. embedded in a complex environment with other agents and processes not under its control. and reasoning about the custs and benefits of continued deliberation that is the subject of this chapter.

\subsection{Decision Theory and the Control of Inference}

We begin with the idea of a decision procedure: a procedure used by an agent to select an action which. if executed. changes the world. Sonie actions are purely computational. For our purposes, such computational actions correspond to an agent running a decision procedure. and we refer to such actions as inferential. The results of inferential actions have no immediate effect on the world external to the agent, but they do have an effect on the internal state of the agent. In particular. inferential actious consume computational resources that might be spent otherwise, and generally result in the agent revising its estintations regarding how to act in various circumstances. In addition to the purnly computational actions. there are physical actions that change the state of the world external to the agent. but that may also recpuire some allocation of computational resources.

Real agents have severely limited computational capabilities. and. since inferential actions take time. an inferential action mav end up selecting a physical action that night have been appropriate at some earlier time. but that is no longer so. Inferential actions are useful only insofar as they enable an agent to select physical actions that lead to desirable outcomes. ${ }^{2}$ Decision theory provides us with the language required to talk precisely about what it would mean for an action to lead to a desirable outcome. Betore mecan proceed further. we will need some precise language for talking about possible outcomes and stating preferences among them. The language that

about ils own abilities to rescon.

${ }^{2}$ Inferential actions are also useful for learning purposes, as in learning search st rategies. hit. even here the actions are ultimately in service to selecting plivsical actions that leal lu desiralile uulicuines. 
we adopt is borrowed directly from statistical decision theory.

The fallowing porrigiphes slinuld be subormed by the discussion of dte-

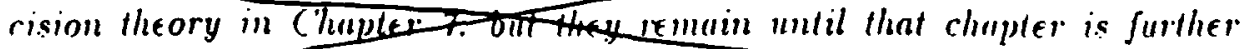
along.

In thie simplest case. we might consider an agent laced with choosing from antomg a set of completely defined and immediately attainable alternatives (6.g.. a student might be faced with choosing between seeing a movie or studying for all vam). The agent might ignore/some of the implications of its actions and focunon inmediate rewards $(6 . g .$. a relaxing respile from work or an increase in knawledge about a givell subject). but. more often than not. the agent will be concerned wiyh the long-term implications or consequences of $\mathrm{its}$ actions (e.g.. Whe possibility of achieving a higi score on au exan which in turn unght rabe the chances of getting into graduate school). In general. we caunol guaraytee these consequences: they are " seldom immediately attaimable gnd they are usually only partiall" defined. If we ignore the long-term implications of our actions, the alternatives can be viewed as rewards, and 2 rational agent wo Id sply choose the reward that it considers best. In the case in which the agent is concerned about the consequences of its actions and those co' seynences are not - ' aly under its coutrol. the picture ts more complirates. In this case, 'he agent might have a probability diotributio. vier the set of rossible consequences and some way of assiguing values to tha iudividual consequences so as to form expectalions regardiug the value of the pe isible outcomes or prospects resulting from performing alternative actions.

Let $\Omega$ correspond to a set of possible states of the world. We assume that the agent has a fur : ion.

$$
\cdot \mathrm{U}: \boldsymbol{\Omega} \rightarrow \mathbf{R}
$$

that assigns a real number to each state of the world. This is referred to as the agent's utility function. ${ }^{3}$ These numbers enable the agent to compare rarious states of the world that might result as a consequence of its actions. It is acoumed that a rational agent will act so as to maximize its uriity. The quantity, $U(\omega)$ where $\omega \in \Omega$. is generally meant to account for both the immediate coats and benefits of being in the state $\omega$ and the delayed costs and benefits derived from future possible states. We assume that there is some process deterministic or storhastic governing the transition between

\footnotetext{
See Chernoff and Moses [9]. Barnett [2]. or Pearl [37] for discunaions regarding the axions of utility theory.
} 
states. and that this process is partially determined or biased by the agent's choice of action. In the case of a stochastic process. the agent cannot know what state will result from a given action and hence the agent must make use of expectations regarding the consequences of its actions. In order to account for these longer-term consequences. it is often useful to think of the agent as having a particular long-term plan or policy. In such cases. the agent will generally assign an mpreterl "tility in a given state based upon the imnediate rewards available in that state and expectations abont the subsequent states. given that the agent continues to select actions based upon its current policy.

In addition to expectations abont the possible future consequences of its physical actions. an agent capable of reasoning about its computational capabilities must also have expectations regarding the potential value of its computations. and estimates of how long those computations are likely to take. In most of the work discussed in this paper. an agent is assumed to engage in some sort of meta-reasoning. For our purposes, meta-reasouing consists of running a decision procedure whose purpose it is to deternine what other decision procedures should run and when. We prefer the term deliberation scheduling (13] to the unore general meta-reasoning and will use the two interchangeably in this raper If the meta-level decision procedure takes a siguificant amount of time to run, it must be argued that this time is well spent. In some cases. the time spent in meta-reasouing is small enough that it can be safely ignored; in other cases, it may be useful to invoke a meta-meta-level decision procedure to reason about the costs and benefits of nieta-reasoning.

Refer back to the material in Chapter 7 on the value of information.

Note that so far we have not accounted for the computational cost of de: libcrating about the value of a particular information source. In information processing systems. information costs in terms of the time and resonrces expended in compnting an answer to a query. Nejther have we closely considered how an agent might compute an expectation such as $E[r \mid \varepsilon]$. We may know to compute such an expectation. but it may be that an agent can not and to compute it. In the following sections, we build on the basic idea behin information value theory to account for systems that have limited compntational capabilities.

The rest of this cliapter is organized as follows. In Section $\$ .2$. we consider a general approach to studying the contiol of reasoning that cast.s the general problem in terms of search. In this same section. we also investigate some of the practical issues that constrain how an agent might reason 
ahout its computational capalilities: these constraints and the measures taken to deal with them apply to all of the work discussed in this chapter. Section 8.3 considers all approach to reasoning about computational capabilities that relies on a particular class of algorithms for implementing decision procedures. Section 8.5 briefly considers some related issues in desigu-time mela-reasoning for compiling run-time sustems for time-critical applications.

\subsection{Control of Problem Solving}

In this section. we consider a general approach to reasoniug about decisiontheoretic control of inference due to Russell and Wefald $[10 .+1]$. As in most decision problens. the basic goal is for the agent to maximize its utility function $U(u)$ on stales of the world $\omega \in \Omega$. We assume that the agent has some set of base-level actions $\mathcal{A}$ that it can execute to affect its environnent. Borrowing Russell and Wefald's notation. we denote the outcome of an action $A$ performed in state $\omega$ as $[A . \omega]$ or just $[A]$ if the action is performed in the current state.

At suy given time the agent has a default action $\alpha \in \mathcal{A}$ which is the action that currently appears to be best. In addition. the agent has a set of computational actions $\left\{S_{i}\right\}$ which might cause the agent to revise its default action. The agent is faced with the decision to choose from annong the available optious: $\alpha, S_{1}, S_{2}, \ldots, S_{k}$. Computational actions only affect the agent's internal state. However. time passes while the agent is deliberating and opportunities are lost, so the net value of computation is defined to be the difference between the utility of the state resulting from the computation minus the utility of the state resulting from executing at

$$
V\left(S_{j}\right)=U\left(\left[S_{j}\right]\right)-U([\alpha])
$$

If the rompulation $S_{j}$ results in a revised assessulent of the best action. $r_{s}$, and a commitment to perform this action, then

$$
\mathrm{U}\left(\left[S_{j}\right]\right)=\mathrm{U}\left(\left[\mathrm{er}, s_{,},\left[S_{j}\right]\right]\right) .
$$

where $\left[a, s_{,} \cdot\left[S_{j}\right]\right]$ indicates the outcome of the action as, in the state follcwing the computation $S_{j}$. Alternatively. if $S_{j}$ is a partial computation (i.c.. a computation that doesn't inmediately result in a revised assessnent of a best action. but that provides intermediate results leading to a revised 
assessme IIt). then

$$
\operatorname{lr}\left(\left[S_{j}\right]\right)=\sum_{T} \operatorname{Pr}(T) \operatorname{L}\left(\left[a_{T} \cdot\left[S_{j}, T\right]\right)\right)
$$

where $T$ ranges over all possible complete computalions following $r_{,}, S, . T$ denotes the computation corresponding to $S_{j}$ inmediately followed by $T$. and $\operatorname{Pr}\left(T^{\circ}\right)$ is the probability that the agent will perform the computation $T$.

Generally. the agent doesn' know the exact utilities or probabilities. and so it must compule an estimate using some amount of its computational resources. Let $\dot{Q}^{S}$ denote the estimate of the quantity $Q$ following a computation $S$. In this case, we have

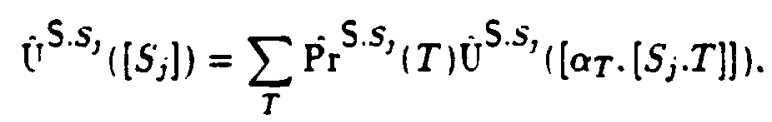

where $S$ is the total computation prior to considering $S_{j}$. and

$$
\hat{U}^{S . S,}\left(\left[\alpha_{T} \cdot\left[S_{j} . T\right]\right]\right)=\max _{i} \dot{U}^{S . S,}\left(\left[A_{i} \cdot\left[S_{j} . T\right]\right]\right) \text {. }
$$

where the $d_{i}$ range over all possible base-level actions in $\mathcal{A}$. By superscripting quantities to indicate the computations required to generate the corresponding estimates. Russell and Wefald are attempting to capture the behavior of real agents with realistically limited computational capabilities. At each point in time. the agent decides how to act based upon whatever estimates it currently has, using a meta-reasoning decision procedure whose time cost is assumed to be negligible. The meta-reasoning decision procedure is responsible for deciding whether further deliberation is warranted. and it does so on the basis of the estimated net value of computation,

$$
\hat{v}^{S . s_{j}}\left(S_{j}\right)=\hat{U}^{S . S_{j}}\left(\left[S_{j}\right]\right)-\hat{U}^{S^{S . S_{j}}}([\alpha]) \text {. }
$$

As Russell aud Wefald point onl. liefore the computation $S_{j}$ is performed $\hat{V}\left(S_{j}\right)$ is just a randon variable, and so the agent, not knowing the exact value. computes an expectation,

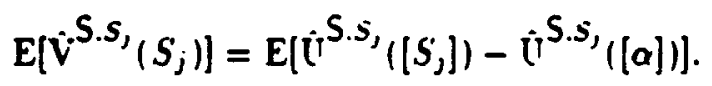

It is worth noting the difference between Equation 8.1 and the following equation introduced in Chapter $i$ in presenting Howard s value of information theorye

$$
\mathrm{E}\left(V\left(I_{X}\right) \mid \mathcal{E}\right)=\mathrm{E}\left(V([]) \mid I_{X} \cdot \mathcal{E}\right)-\mathrm{E}(V([]) \mid \mathcal{E})
$$


The important difference is that both terms in the right-hand-side expectation in Equation 8.1 cliange as a consequnence of further inference. If an agent had unlinited computational capabilities. it would not be computing estimates. and only the first of the rwo terms would require expending computational resources since it would be the case that

$$
\left.\left.i^{S . S,}([a])\right]=\hat{U}^{S}([\alpha])\right] \text {. }
$$

However, we are concerned with agents with limited computational capabilities. and further computation will likely result in a better estimate of the utilities for $[\alpha]$ as well as for $\left[a^{\prime} .\left[S_{j}\right]\right]$ for any action $\alpha^{\prime} \in \mathcal{A}$.

In order to sinplify reasoning about the utility of combined computational and base-level actions, Russell and Wefald separate the intrinsic utility. that is the utility of an action independent of time, from the time cost of computational actions. defuning the utility of a state as the difference between these two:

$$
\hat{U}\left(\left[A_{i},\left[S_{j}\right]\right]\right)=\dot{U}_{l}\left(\left[A_{i}\right]\right)-T C\left(S_{j}\right) .
$$

It shonld be noted, however, that determining an appropriate time cost function can become quite complicated in applying Russell and iVefald's approach. In particular, costs concerning hard and soft deadlines will have to be accounted for by this function. In the game-playing application explored in [40], there are 110 hard deadlines on a per-move basis, instead there is a per-game time limit that is factored into the time cost. In many timecritical problem solving applications, calculating the time cost can be quite complicated (e.g., consider the sort of medical care applications investigated in [28] and [25]). Russell and Wefald assume that the time cost is independent of both the computation itself and its recoinmendations. The former is certainly reasonable, and, since the recommendation is not known at metareasoning time, the latter is also reasonable. However, one could easily inlagine employing an expected time cost based on some a priori knowiedge concerning possible recommenclations. It should be noted that all of the approaches described in this chapter make assumptions about time cost similar to those of Russell and W'efald.

Pertape the nicest part of the Russell and Wefald work is their careful treatment of the criterion for deciding whether or not to expend further resources on deliberation. If the agent is considering at most one additional computational step, then it is only interested in computations that serve to update the expected value of a given base-level action so as to supplant 
the current default ar.jon. The experted gain from a given computation is measured in terms of the difference betwen the current expectation regarding one action and the anticipated revised expectations regarding a second action where one of the two actions is the default action. Int uitivelv, further deliberation is called for whenever the difference between the expected gain in Ittility from a computation and the associated cost of clelay is greater than zero. Hussell and Wefalt irlentify two cases to cousider in deciding to perform a computation aimed at providing a revised assessment of the best action to perform. In the first case. we sulppose that there exists a computation $S_{j}$ which allects the agent's estimation of the utility of the alternative action is so that

$$
E\left[V\left(S_{j}\right)\right]=\int_{\hat{U}_{I}([\sigma])}^{x_{1}} p_{.3 . j}(x)\left(x-\dot{\mathrm{U}}_{I}^{S}([\alpha])\right) d x-T C\left(S_{j}\right)
$$

where $p_{3 . j}$ is the probability density function for $\hat{\mathrm{I}}_{I}^{S . S,}([, 3])$. In the second case. there exists a computation $S_{k}$ which affects the agent's estimation of the utility of the default action or so that

$$
E\left[V\left(S_{k}\right)\right]=\int_{-\infty}^{\dot{\mathrm{U}}_{J}([\beta])} p_{a, k}(x)\left(\dot{\mathrm{U}}_{l}^{S_{l}}([, \beta])-x\right) d x-T C\left(S_{k}\right)
$$

where $p_{0 . k}$ is the probability density function for $\hat{\mathrm{i}}_{I}^{\mathrm{S} . S_{k}}([a])$. If there are $n$ computational actions and $m$ base-level actions, then each neta-level reasoning step will require computing earh of Equations 8.2 and $8.3 \mathrm{~nm}$ times. If the dist ributions governing utility estimales are simple in form (c.g., uormal distributions). computing the integrals in Equations 8.2 and 8.3 can be done quile efficiently.

There are a number of assumptions that Russell and Wefald make in their analysis. First. it is assumed that the agent considers only single computation steps, estinates their ultimate effect, and then chooses the step appearing to have highest benelit. This is referred to as the metagreed monaption. Secoud, it is assumed that the ageut will act as though it wit at mout one more search step. This is referred to as the singlestep amption. Finally. it is assumed that a computational actiou will change the expected utility estimate for exactly oue base-level action. In Russell and Wefald's state-space search paradigm. this is referred to as the subtree-independence assumption.

The assumptions stated in the previous paragraph may seem overly restrictive, but it is quite difficult to avoid these or similar assumptions in 
general. Pearl [3i] identifies two assumplions that most practical metareasoning systems ascribe to: no cumpetition. each information source is evaluated in isolation from all the others, and one-step horizon. we consult at most one additional information source before committing to a base-level action. Assessments of information sources based on the no-competition and one-step-horizon assumptions are referred to as myopic. and most practical svstems employ myopic decision poliries.

1 The next piece of research that we consider in this section is due to Etzion [18], and it borrows from the Russell and Welald work, and builds on the early work of Simon and hadane [.13] on satisficing search. I is particularly interesting for the fact that it attempts to combine the sort of goal-driven behavior prevalent in artificial intelligence with the decision theoretic view of maxinizing expected irility. In Etzioni's model. the agent is given a set of goals $\left\{G_{1}, G_{2}, \ldots, G_{n}\right\}$. a set of methods $M$. and a deadline $B$. The agent attenpts to determine a sequence of methods

$$
\sigma=m_{1,1}, m_{2,1} \ldots \ldots m_{k_{1}, 1}, m_{1,2}, m_{2,2}, \ldots m_{k_{2}, 2}, \ldots m_{1, n}, m_{2, n} \ldots \ldots m_{k_{n}, n}
$$

where $m_{i, j}$ is the $i$ th inethod to be applied to solving the $j$ th goal. The idea is that the agent will apply cach methorl in turn until it cither runs out of methods or achieves the goal, at which point it will turn its attention to the next goal. The expected utitity of $\sigma$ is

$$
\begin{aligned}
E[\hat{\gamma}(\sigma)]= & E\left[\hat{U}\left(m_{1,1}\right)\right]+\cdots+E\left[\hat{U}\left(m_{k_{1}, 1}\right)\right] \prod_{i=1}^{k_{1}-1}\left(1-\operatorname{Pr}\left(m_{i, 1}\right)\right)+ \\
& E\left[\hat{\varphi}\left(m_{1,2}\right)\right]+\cdots+E\left[\hat{U}\left(m_{k_{2}, 2}\right)\right] \prod_{i=1}^{k_{2}-1}\left(1-\operatorname{Pr}\left(m_{i, 2}\right)\right)+ \\
& \vdots \\
& E\left[\hat{U}\left(m_{1, n}\right)\right]+\cdots+E\left[\hat{U}\left(m_{k_{n}, n}\right)\right] \prod_{i=1}^{k_{n}-1}\left(1-\operatorname{Pr}\left(m_{i, n}\right)\right) .
\end{aligned}
$$

In this sinuple model, no provision is made for switching back and forth betwre goals, aud, except for ignoring the remaining methods for a given gual oace that goal has been achieved, no provision is made for modifying the search as new information becomes available. Etzioni defines the expected opportumily cost $\left(\gamma_{B}\right)$ of a unethod $m$ for a deadline $B$ as

$$
E\left[\gamma_{B}(m)\right]=E\left[\hat{\bigcup}\left(\sigma_{B}^{*}\right)\right]-E\left[\hat{\varphi}\left(\sigma_{B}^{*}-T C(m)\right)\right] .
$$


where $\sigma_{B}^{*}$ is the optimal nethod sequence for a deadline $B$. and $T C^{\prime}(m)$ is the expected time to carry our method $m$. In addition. the expected gain $\left(C_{B}\right)$ of a method $n$ for a deadline $B$ is defined to be

$$
E\left[G_{B}(m)\right]=E[\dot{Y}(m)]+E\left[\left(\eta_{B}(m)\right] .\right.
$$

Ile then shows that by repeatedly choosing the niethod whose expected gain is mavimal an agent will construct an optimal method sequence.

From one point of view, Etzioni's work is not about meta-reasouing at all; his work is concerned with ordinary sequential decision problems. For these problems. Etzioni points out that. in certain cases, the cost of determining an optimal method sequence can be quite high. In other words. we can't ignore the cost of meta-level reasouing in the decision-making model. Ilis analysis showing that sorting methods on their marginal utility can often result in optimal or near-optimal method sequences is exactly the sort of analysis required to justify a particular meta-level reasoning.

In the case in which the agent has a single goal and nultiple methods for achieving it. the requisite meta-reasoning is easy. In particular, suppose that there is a constant opportunity cost $\gamma$ per unit of time spent on the goal. and for each method $m \in M$ the agent has an expected time cost $E[T C(m)]$. an expected utility estimate $E[\hat{U}(m)]$, and a probability $\operatorname{Pr}(m)$ of achieving the goal using that method. The expected gain of a method $m$ is just

$$
\mathrm{E}[G(m)]=\mathrm{E}[\hat{\mathrm{U}}(m)]-\gamma \mathrm{E}[T C(m)],
$$

and the task is to find $\sigma$ so as to maximize

$$
\mathrm{E}[\dot{\mathrm{U}}(\sigma)]=\sum_{i . . . \mathrm{E}\left[G\left(m_{1}\right)\right]>0} \mathrm{E}\left[G\left(m_{i}\right)\right] \prod_{k=0}^{i-1}\left(1-\operatorname{Pr}\left(m_{k}\right)\right) .
$$

Etziou claims, and it is easy to verify, that. by sorting the methods in increaning order using $\frac{E[G(m)]}{P(m)}$ as a key, an agent can construct an optimal mel hod ordering.

Is the above case, it is plausible to assume that the cost of metareasconing (i.e.. the time spent calculating $\frac{E[G(m))}{P(m)}$ for each method $m$ and sorting using the results) is negligible in comparison with the cost of applying a method. In the case of an agent faced with multiple guals even where there is only one method for each goal. it is more difficult to make such an assuniption. By reduciug the knnpsack problem [20] to the problem 
of compnting the expected opportunity cost. Etzioni shows that computing the expected opportunity cost of a method is NP-complete.

It is not too surprising that there are some hard problens lurking anong the deliberation scheduling problems that underlie decision-theoretic control of inference. It should be pointed out. however. that all we should really be concerned with is the expected cost of neta-reasoning, and that. in many practical applications. approximations are much preferred to even polynomial-time methods for computing exact solutions.

Etzioni suggests nsing Garey and Johnson's factor-of-two approximation [20]. but it should be noted that the knapsack problen is a number problem for which there exist pseudo-polynomial time algorithms and good branch-and-bound approximations. These branch-and-bound algorithms have exponential-time worst-case behavior. but their expected performance is such that many practitioners consider knapsack tractable. In the next section. we see how approximation algorithms for computationally expensive problems can provide us with even greater flexibility in allocating processor time to decision procedures.

While Etzioni's invocation of asymptotic complexity as a measure of difficulty may not be particularly appropriate in this case, it does force the reader to reconsider the assumptions regarding the tine cost of metareasoning. For instance, if $n$ is sinall and the average time cost of the methods in $M$ is high, then it may even be reasouable to perform a metacomputation whose worst-case behavior is exponential in $n$. It may even be useful to add another level of ineta-reasoning to reason about various alternative scheduling algorithms.

Just what is the structure of the decision making process that we are seeking to control? In the Russell and Wefald model, object-level decision inaking involves fixed-duration computations that attempt to provide a better assessinent of a single hase-level action. In the next section. we consider problems in which the ineta-level reasoner sacrifices some of its control over object-level decision making in order to simplify meta-level decision making. In particular. we consider decision procedures that return estimates that inprove with additional allocations of processor time. The ability to prewent decision procedures at any time during their computation simplifies deliberation scheduling in many cases. 

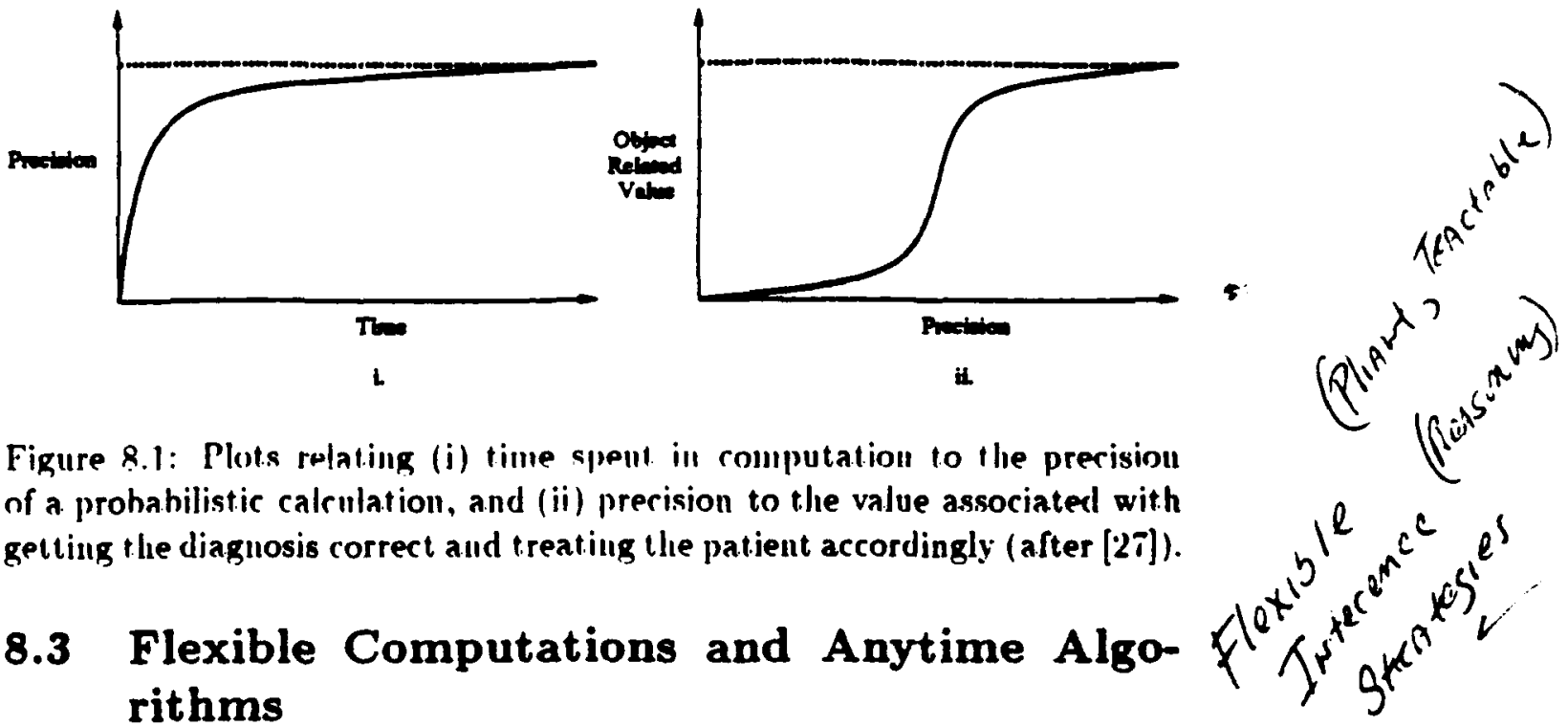

Figure 8.1: Plots ralating (i) time spent in computation to the precision of a prohabilistic calculation, and (ii) precision to the value associated with getting the diagnosis correct and treatiug the patient accordingly (after [2i]).

\subsection{Flexible Computations and Anytime Algo- rithms}

In this section. we consider two indepenciently developed but closely related approaches lo decision-ilieuretic control of problem solving. The two approaches are due to Dean and Boddly [13] and Horvitz [27]. Horvitz refers to his decision procedures as fexible compulations and Dean and Boddy refer to theirs as anytime algurithms, but the basic idea behind the two proposals is the same, and we will use the two lerms interchangeably.

In the ideal flexible computation, the object-related value of the result returned by a decision procedure is a continuous function of the time spent in computation. 'The notion of "object-related value" of a result is to be contrasted with the "comprehensive value" of a system's response to a given stale: the latler refers to the overall utility of the response and the former is some measure of the value of the result apart from its use in a particular set of circumstances. Object-relaled value is exaclly Russell and Wefald's intrinsic itiblity. In come cases. the task of relating a result to the comprehensive value the overall respouse can be quite complex. This is especially so in can in withch there are several results from several different decision procedures. In these cases, it is often couvenient to make the assumption that the value function is separmble so that the compreheusive value of the system's respouse can be computed as the sum of the value of a sequence of outcolnes.

We assume lliat a Mexible computation can be interrupted at any point 

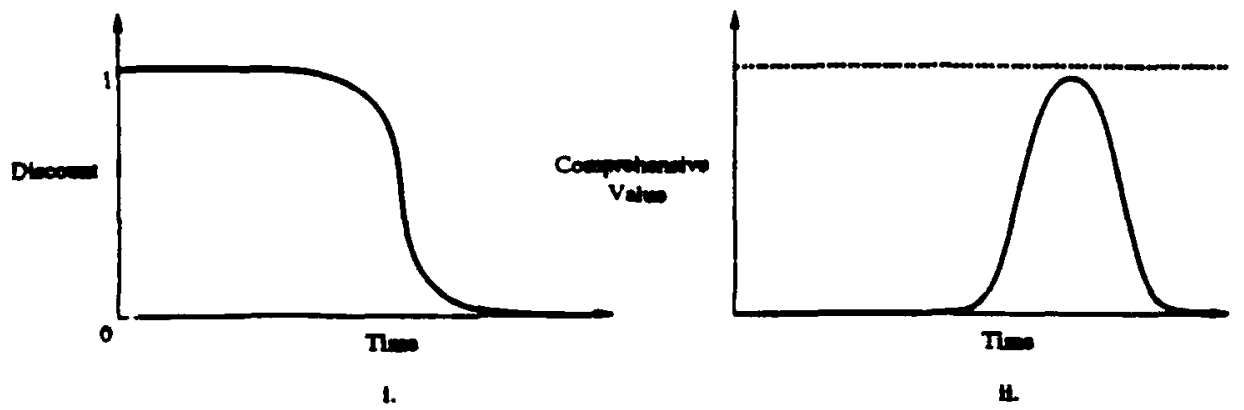

Figure 8.2: P'lots indicating (i) a discounting factor for delayed treatment. and (ii) the conprehensive value of computation as a function of time (after $[2 i])$.

during computation-lience (hilie name "anytime"--to return an answer whose object-related value increases as it is allocated additional time. Horvitz provides a good exs" : e of a flexible computation and an analysis of its object-related value crawn from the health care doniain. Suppose that we have an anytinie - 6 orithm that computes a posterior distribution for a set of possible diaznoses given certain information regarding a particular patient. Figure 8. i.i frou (27) show's a graph that relates the precision of the result returned by this algorithm to the time spent in computation. The objertrelicled value can be determined as a function of precision by consideriug the

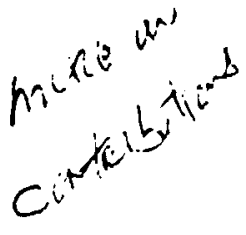
experted utility of administering a treatment based on a diagnosis of a given precision ignoriug when the treatment is administered (see Figure 8.1.ii).

The comprehensive value of computation is meant to account for the costs and benefits related to the time at which the results of decision procedures are made use of to initiate plysical actions. Figure 8.2.i (from [2i]) indicates how a physician unight discount the object-related value of a computation as a function of delay in adninistering treatment. The conprehensive value of computation is shown in Figure 8.2.ii and is obtained by combiung the infornation in Figures 8.1.ii and 8.2.j. This method of combining information assumes both time-cost separability and one-step horinos.

Both Horvitz and Dean and Boddy note that the most useful sort of flexible computations are those whose object-related value increases monotonirally orer some range of computation times. Dean and Boddy [13] employ decision procedures that are monotonic throughout the range of computa- 
tion tines. and exploit this fact to expedite deliberation scheduling for a special class of planuing problems referred to as time-slependent plammmg problems. A planning problem is saicl to be lime-dependent if the time available for responding 10 a given event varies from situation to situation. In their model. a predictive component. whose time cost is not considered. predicts events and their time of occurrence on the basis of observations. and the planning system is givell the task of formulating a response to each event and executing it in the time available before the event occurs.

The model of Dean and Boddy generalizes on the multiple-goals/singlemel hod-for-each model of Etzioni described in the previous section. by alluwing each goal to have a separate dealline. If the responses to the different events are independent, the task of deliberation scheduling can be stated in terms of maximizing the sum

$$
\sum_{\epsilon \in E} v(\text { Response }(\epsilon)),
$$

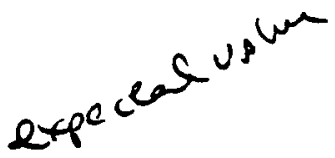

where $E$ is the set of all events predicted thus far. It is assumed that there is exactly one decision procedure for each type of event likely to be encountered, and that there are statistics on the perfornance of these decision procelures. The statistics are summarized in what are called performance profiles which are essentially the same as the graphs used by Horvitz in his analusis (e.g.. see Figure 8.1.ii).

In Section 8.4, we define the class of time-dependent planning problems precisely, and provide polynomial-time algorithms for deliberation scheduling for particular subclasses. These algorithms use a simple greedy strategy working backward from the last predicted event, choosing the decision procedure whose expected gain conputed from the performance profiles is greatest.

It is worth considering why the NP-completeness result reported by Etzioni does not apply in this more general case. In job-shop sclieduling, if it is posaible to suspend, and later resume, a job, lien many ot herwise difficult probmen become trivial [23. 5]. Such (preemptive) scheduling problems are nowhat rare in real job shops given that there is often siguificant overtbad involved is suspending and resuming jobs (e.g., traveling between workstations or changing tools), but they are considerably uore common with regard to purely computational tasks ( $6 . g$.. suspending and resuming ('nix processes). In many scheduling problems. each job has a fixed cost and requires a fixed amount of time to perform: spendiug any less than the full amount yields no advantage. This is the case in the decision procedures

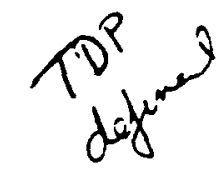

\section{$\cdot$}


considered by Elzioni. If. however. the decision procedures for computing appropriatc actions are preemptible and provide better answers depending upon the time available for deliberation. then the lask of deliberation scheduling is considerably simplified. Anytime decision procedures thus provide nore flexibility in responding to time-critical situations. and simplify the task of allocaling processor time in cases where there is contention among several decision lasks.

fur the multiple-goals/single-methot-for-each problem described in the previous section. Etzioni suggests usiug a factor-of-two approximation to a void potential combinatorirs in deliberation scheduling. Rather than always simply applying the factor-of-two approximation. We can design an anytime approximation algorithm and allocate it some amount of processor time based on expectations regarding its performance. The fully-polynomial approx'nation scheme' of Ibarra and fiim [30] for solving the optinuzation version of the knapsack problem serves uicely as the basis for an asytime approximation algorithm for choosing wethod sequences. The simplest approach would be to classify the base-level problems in terms of, say, the number of goals and the length of time until the deadline. and gather statistics on the utility derived from invoking the approximation scheme with different precision requirements. Whether or not this nore conplicated approach to deliberation scheduling performs better than the factor-of-two approxiination will depend upon the specifics of the application and how efficiently the algorithms are realized. It is easy to imagiue applications. however. for which the expected performance of the system will be improved by using such a scheme.

The use of flexible conputatious can also simplify problems in which one decision procedure produces an intermediate result that is used as input to a second decision procedure: Boddy and Dean $[4]$ investigate one such problem involving a robot courier assigned the task of delivering packages to a set of locations. The robot has to determine both the order in which to visit the lucations. referred to as a tour. and. given a tour. plan paths to traverse in moving between cousecutive locations in the tour. To simplify the analyais. it is assumed that the robot s only concern is time: it seeks in

\footnotetext{
'See Garey and .Johnaon [20] for a diecnasion of fully-polynomial approximation schemes for NP-conplete problems. For our purposes. an approximation ocheme $S$ for a problcm Il take an indance $I n$ and a precision reynirenient $e>0$ and returns a candidate solution that is within e of the optimal solution. Such a scheme in said to be fully protynomial juat in case the lime complexity of $S$ is bounded by a polynomiai fnaction of $\frac{1}{b}$ and the size of In .
} 


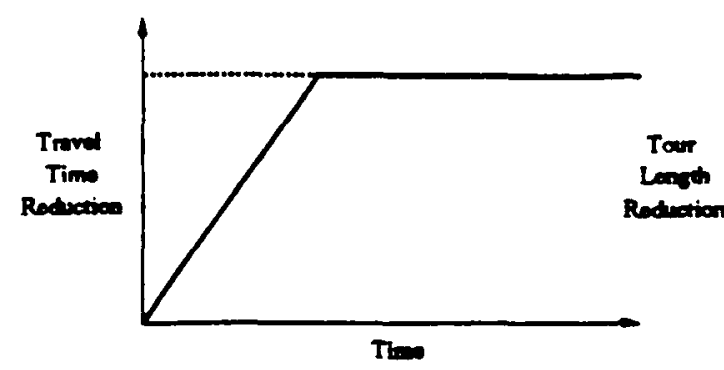

i.

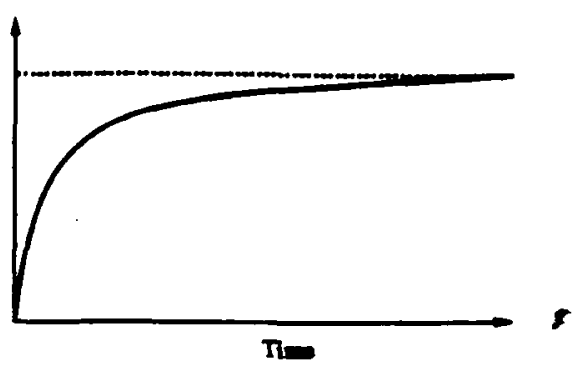

Figure 8.3: Performance profiles relating (i) the expected savings in travel time to time spent in path planning. and (ii) the expected reduction in the length of a returued lour as a function of time speut in tour improvenent.

minimize the total amount of time consumed both in sitting idly deliberating about what to do next, and in actually moving about the environment on its errands. Furthermore, it is assumed that there is no advantage to the robot in starting off in some direction until it knows the first location to be visited on its tour. Finally. while the robot can deliberate about any subsequent paths while traversing a path between consecutive locations in the tour. it must complete the planning for a given path before starting to traverse that path.

The two primary components of the decision making process involve generating the tour and planning the paths between consecutive locations in the tour. The first is referred to as tour improvement and the second as path planning. Boddy and Dean employ iterative refinement approximation routines for solving each of these problems, and gather statistics on their performance to be used at run-tine in guiding deliberation scheduling. The statistics are summarized in what are called performance profiles. Figure 8.3 (from [H]) shows the profiles for path planning and tour improvement. Figure 8.3.j shows how the expected savings in travel time increase as a function of time spent in path planning. Figure 8.3.ii shows how the expected length of the cour decreases as a fraction of the shortest tour for a given amount of time spent in tour improvement. In the analysis described in [t]. this performance estinate is independent of the initially selected tour. We assume that the robot starts out with an initial randomly selected tour. Civen the length of some initial tour. the expected reduction in length as a function of time spent in tour improvenent. and some assumptions about the 
performance of path plann: ug. the rubot can figure out exactly how much time 10 devote to tour improvement in order to minimize the time spent in stationary deliberation and combined deliberation and traversal.

There currently is no general theory about how to combine anytine algorilhus. and neither is there likely 10 be in the near future. For cases in . Which the decision problems are not independent. there is not a great deal

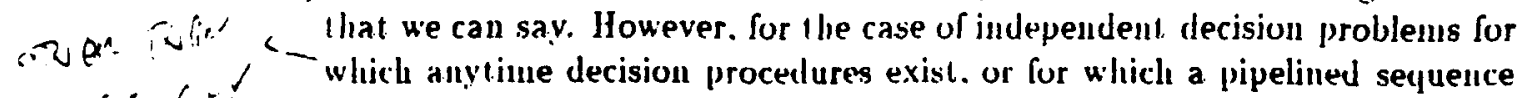
g $\mu$ f t of antine decision procedures exist. as in the robot courier problem. there is a great deal of interesting research to be done: research that can draw heavily on the scheduling and conbinatorial optinization literature.

It is worth pointing out some connectious between the Russell and Wefald work and that of Dean and Boddy and Horvitz. The Russell and Wefald work can be seeu as trying to construct an optimal anvtime algorillum: a single algorithm that operates by calculating a situation-specific estimate of utility using only local infurmation. just as subscribed by information value theory. It should be possible to apply the Russell and Wefald approach to scheduling anvtime algorithms. For some purposes (e.g., the gane-playing and search applicatious described in [41]), the monolithic approach of Russell and Wefald seems perfectly suited. For other applicalions (e.g., the robotic applications described in [1] or the intensive-care applications described in [28]). it is quite convenient to think in terns of scheduling existing approximation algorilhms.

Since this book is concerned with planning and control problems. we now turn our attention to the general class of time-dependent planning problems mentioned earlier. and investigate the deliberation scheduling issues that arise with regard to various subclasses of this general class.

\subsection{Time-Dependent Planning}

We define a class of time-dependent problems in terms of

1. A set of event (or condition) types. $C$

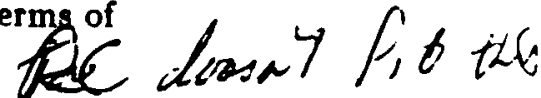

2. A cet of action (or response) types, $A$

3. A set of time points. $T$

4. A set of decision procedures. $\mathcal{D}$

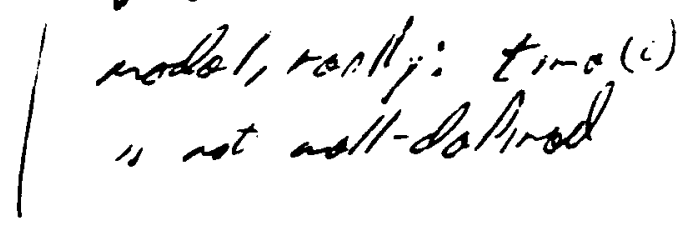




\section{A value functiou. $V$}

We assume that at each point in time the agent knows about some set of pending events that it has to formulate a response to. We are nut concerned with how the agent came to know this information: suffice it to say that the agent has some advance $r$ utice of their type and time of occurrence. To represent its knowledge regarding fut ure events. we say that the agent knows about a sel of tokens drawn from the set $\mathcal{C} \times \mathcal{T}$. When we talk about events or conditions. we will be referring to tokens and not types. Each condition. c. has a type. trpe(c) $\in \mathcal{C}$. and a time of occurrence. time $(c) \in T$. In the following, all conditious are assumed to be instantaneous (i.e. . corresponding to point events).

We evaluate the agent's prfformance entirely on the basis of its responses. Let Response(c) $\in \mathcal{A}$ be the agent's response to the condition $c$. Let $V(a \mid c)$ be the value of responding to the condition $c$ with the action $n \in \mathcal{A}$. To simplify the analysis. we make the strong assumption that the value of the agent s response to one condition is completely independent of the value of the agent's response to any other condition. Given this independence assumption, we can deterinine the total value of the agent 's response to a set of conditions $C$ as the sum,

$$
\sum_{c \in C^{\prime}} V(\operatorname{Response}(c) \mid c) \text {. }
$$

Since we are primarily interested in investigating issues concerning the costs and benefits of conputation. we abstract the problem somerhat more in order to simplify the anadysis. We retuire the agent to formulate a response to every condition it is confronted with. We further require that the agent perform all of its deliberations regarding a given event prior to the time of occurrence of that event. There is no benefit to be had in coming up with a response early.

For each condition type. $c \in \mathcal{C}$. there is a decision procedure in $\mathrm{dp}(c) \in D$. The knows how to select an appropriate decision procedure given the type of an event. The decision proredures in $\mathcal{D}$ have the properties of fledibs computations that we discussed earlier. In addition. the agent has expectations about the performance of these decision procedures in the form of performance profiles. For each condition type. $c \in \mathcal{C}$. there is a corresponding function $\mu_{c}: \mathbf{R}-\mathbf{R}$ that takes an amount of time. $\delta$. and returns the expected value of the response to $c$ generated by $d p(c)$ having 
been run for the specified amount of tim..

$$
\mu_{c}(\delta)=E(V(\text { Response }(c) \mid c . \text { alloc }(\delta . d p(c)))) .
$$

In the following, we consider various restricted classes of decision procedures. We begin by considering, decision procedures whose performance profiles cau be represented or suital)ly approxinated by piecewise linear monolonic increasing functions. We add the further restriction that the slopes of consecutive line segmcuts be decreasing. If the functions representing the performance profiles were everywhere differentiable, this restriction would correspond to the first derivative function being monotonic decreasing. ${ }^{5}$

Let $C^{\prime}=\left\{c_{1} \ldots \ldots c_{n}\right\}$ be the set of conditions that the system has to formulate responses for. and $\hat{t}$ he the present tine. Let $\mu_{i}$ be the function describing the prrformance profile for the decision procednre used to compute responses for the ith condition. We present an algorithm that works backward from the time of occurrence of the last event in $C$. On every iteration through the main loop, the program allocates some interval of time to deliberating about its response to one of the conditions, $c$, whose time of occurrence, time $(c)$. Lies forward of some particular time $t$. The set of all conditions whose tine of occurrence lies forward of some particular time $t$ is denoted as

$$
\Lambda(t)=\{c \mid(c \in C) \wedge(\text { time }(c) \geq t)\} .
$$

The criterion for choosing which decision procedure to allocate processor time to is based on the expected gain in value for those decision procedures associated with the conditions in $\Lambda(t)$. The criterion also has to account for the time already allocated to decision procedures. Let $\gamma_{i}(x)$ be the slope of the linear segment of $\mu_{i}$ at $x$ unless $\mu_{i}$ is discontinuous at $x$ in which case $\gamma_{i}(x)$ is the slope of the linear segment on the positive-side of $x$. We refer to $\gamma_{i}(x)$ as the gain of the $i$ th decision procedure having already been allocated $x$ amount of processor time.

Having allocated all of the time forward of $t$ in previous iterations. figuring out how nuch time to allocate on the next iteration is a bit tricky. It is certainly bounded by $t-\hat{t}$; we cannot nake use of time that is already

'If (1F) we suggested a similar restriction referred to as diminishing returns. and . I defict fown: $\forall c \in C . \exists f \cdot \mu_{e}(t)=f(t)$ anch that $f$ is monotonic increasing. contimnown, and piecewine differentiable. $\vee r . y \in \mathbf{R}^{+}$. such that $f^{\prime}(s)$ and $f^{\prime}(y)$ exist. $(x<y) \supset\left(f^{\prime}(y) \leq f^{\prime}(x)\right)$. For this clas of problems. we provided an approximation algorithm that use time-glicing to come within $e$ of optimal. The algorithm presented liere is exact given the restrictions on the form of performance profiles. 

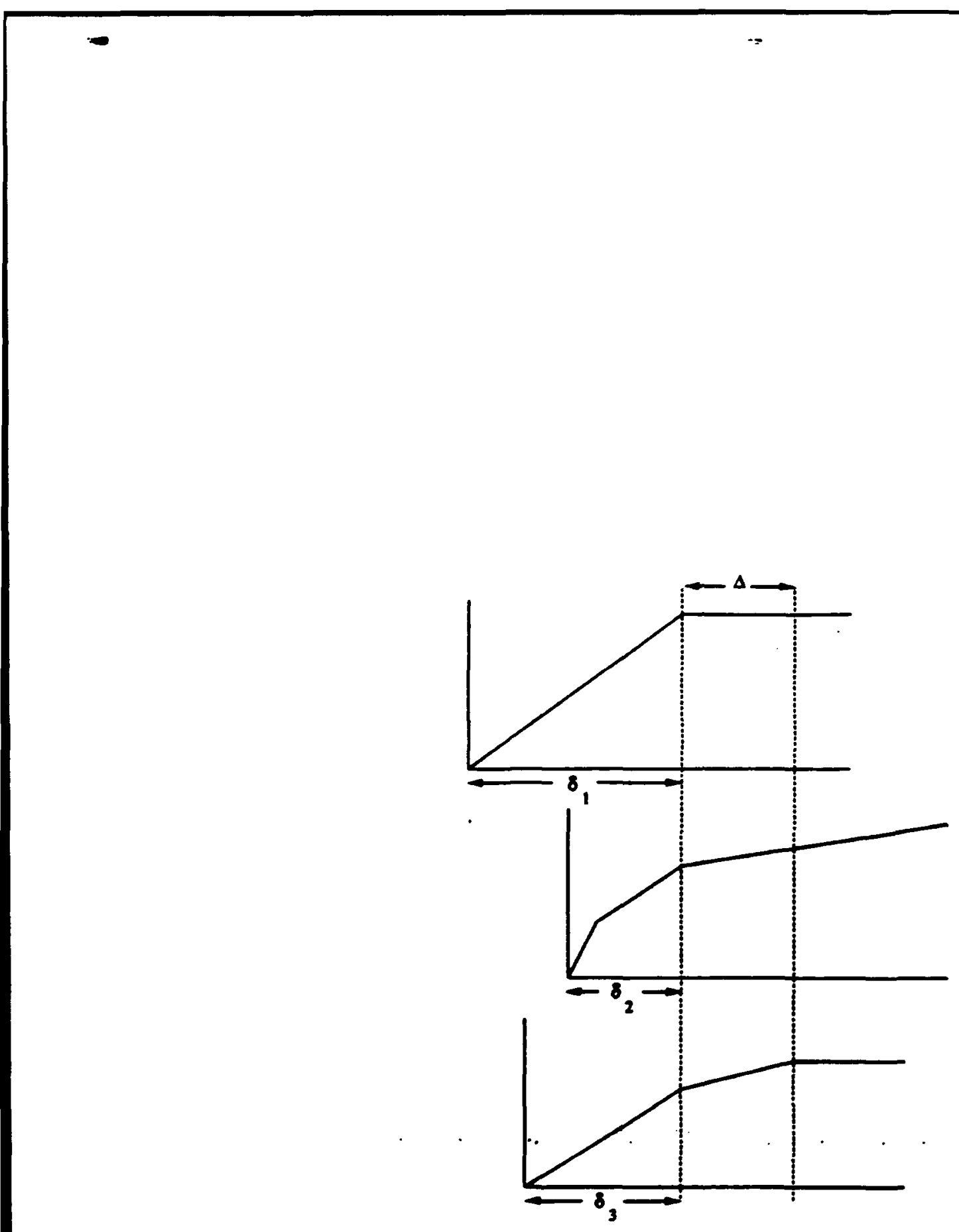

Figure 8.4: Determining min_alloc $\left\{\left\{\delta_{1}, \delta_{2}, \delta_{3}\right\}\right)$ 


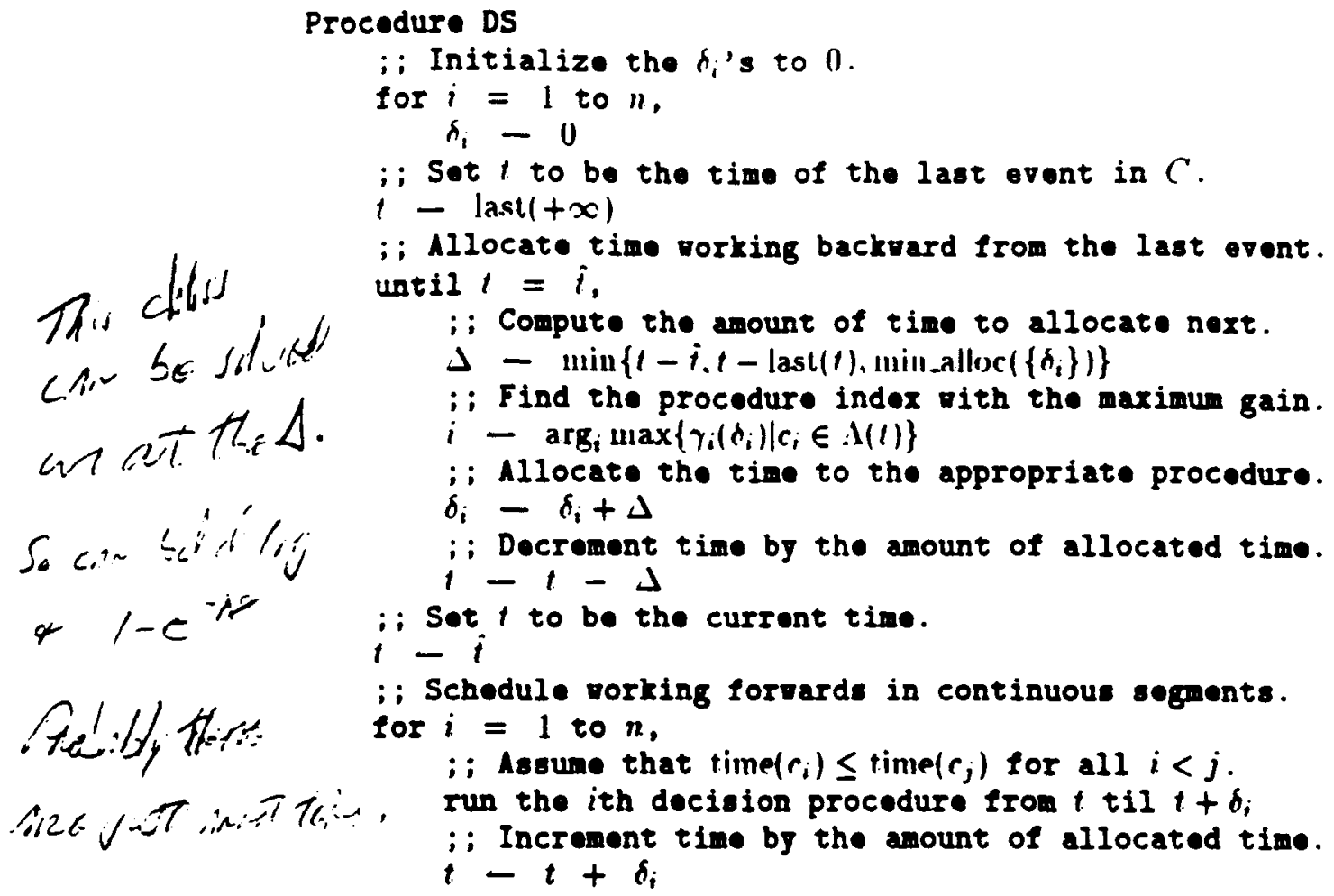

Figure 8.5: Deliberation scheduling procedure

past. In addition, given that we are using the gain of the decision procernres for conditions in $\Lambda(t)$ as part of our allocation criterion, the criterion only applies over intervals in which $\Lambda(t)$ is unchanged. Let last $(t)$ be the first tine prior to $t$ that a condition in $C$ occurs that is not already accounted for in $\Lambda(t)$ :

$$
\text { last }(t)=\max \{\operatorname{time}(c) \mid c \in C-\Lambda(t)\}
$$

Finally, given that the gains determine the slope of particular line segments characturing the performance of the decision procedures. we have to be rarefal to apply our criterion to an interval longer than that over which the current gains are constant. Let nin_alloc $\left(\left\{\delta_{i}\right\}\right)$ be the minimum of the lengths of the intervals of time for the next linear segments for the performance profiles given the time allocated thus far. Figure 8.4 illustrates min_alloc $\left(\left\{\delta_{i}\right\}\right)$ for a particular case. 

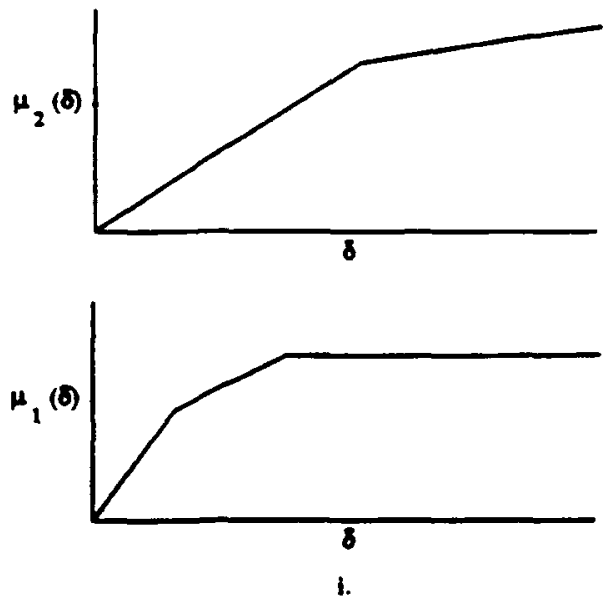
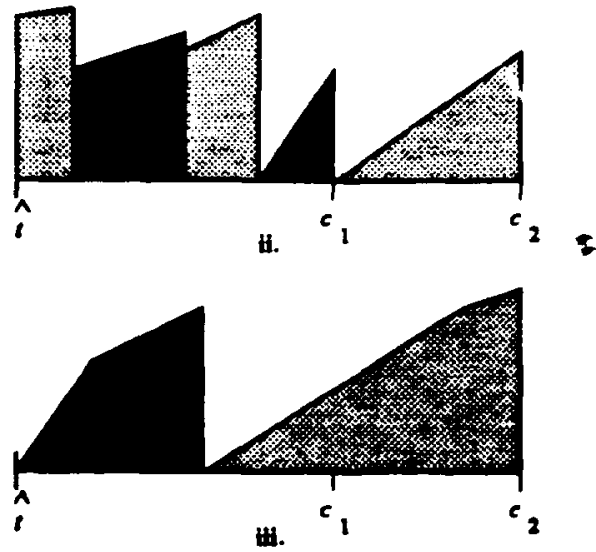

Figure 8.6: A siniple example of deliberation scheduling

Figure 8.5 lists the procedure for deliberation scheduling for the class of problems under consideration. The procedure, DS, consists of three iterative loops. The first initializes the allocation variables, the second determines how much time to allocate to each of the decision procedures. and the third determines when the decision procedures will be run. For convenience. we assunie that the events in $C$ are sorted so that $\operatorname{time}\left(c_{i}\right) \leq \operatorname{time}\left(c_{j}\right)$ for all $i<j$. This assumption is only made use of in determining when decision procedures will be run.

Consider the following simple example to illustrate how DS works. Suppose that we have two events to contend with. $c_{1}$ and $c_{2}$. Figure 8.6.i shows the performance profiles for the decision procedures for $c_{1}$ and $c_{2}$. DS starts by allocating all of the time between $c_{1}$ and $c_{2}$ to the decision procedure for $c_{2}$. The uext interval of time to be allocated $(\Delta)$ is determined by the first linear segment of $\mu_{1}$. and this interval is allocated to $c_{1}$.

At this point, the slope of the second lincar segment of $\mu_{1}$ is less than the slope of the first segmeut of $\mu_{2}$. so the next interval (determined by what is left of the first linear segment of $\mu_{2}$ ) is allocated to $c_{2}$. The next interval corresponds to the second linear segment of $\mu_{1}$. and this eutire interval is allocated to $c_{1}$. Finally. the remainder of $\mu_{1}$ has slope 0 . so the remaining time is allocated to $c_{2}$. Figure 8.6.ii show's the complete history of allocations. and Figure 8.6.iii shoris how the decision procedures are scheduled to run. Now ne prove that DS is optimal. 

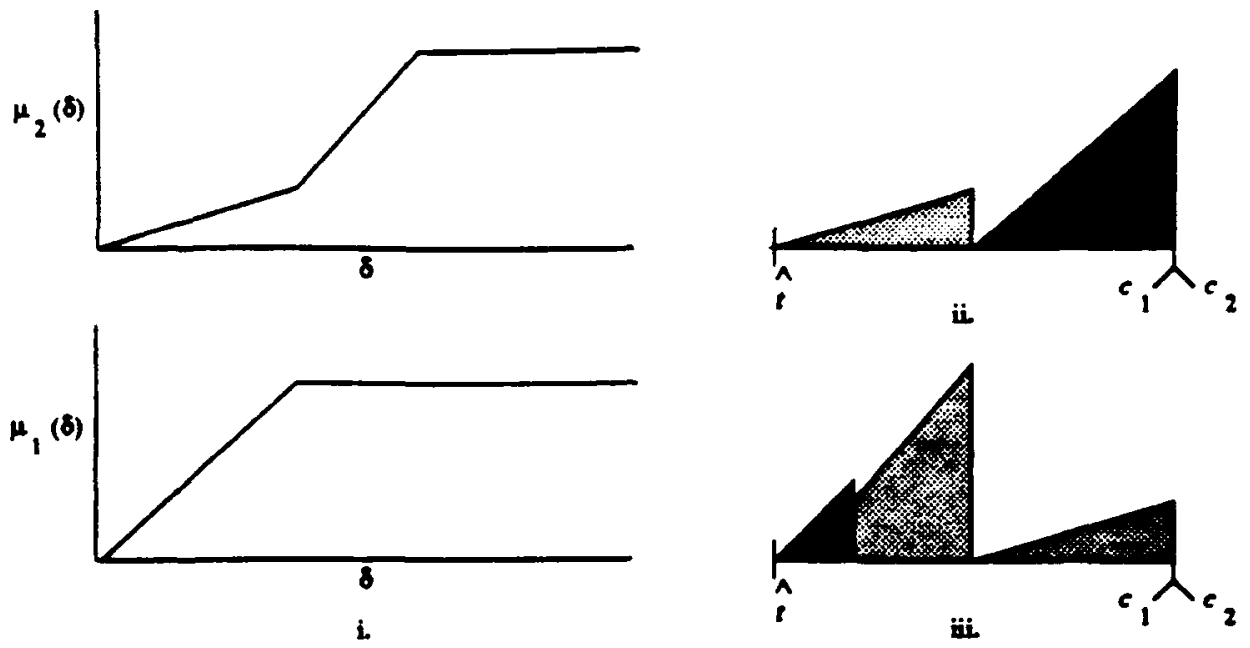

Figure 8.7: Performance profiles that foil DS

Theorem 1 The procedure $D S$ is optimal in the sense that it generates a set of allocations $\left\{\delta_{i}\right\}$ maximizing $\sum_{i}^{n} \mu_{i}\left(\delta_{i}\right)$.

Proof: We proceed by induction on n, the number of conditions in $C$. For the basis step, $n=1$, the algorithm allocates all of the time available to the only event in $C$, and hence is clearly optimal. For the induction step, we assume that DS is optinial for up to and including $n-1$ eveuts. Our strategy is to prove each of the following:

1. Let $\hat{t}^{\prime}$ be the time of the earliest event in $C^{\prime}$. Using $\hat{t}^{\prime}$ as the starting time, DS optimally allocates processor time to the $n-.1$ (or fewer assuning simultaneonsly occurring events) events in $C$ occurring afler $\dot{t}^{\prime}$.

2. DS optimally allocates processor time to all $n$ events in $C$ over the period from $\hat{t} u n t i l$ the time of orrnrrence of the first pvent in $(\therefore$ accownting for the processor time already committed to in the allocations denribed in Step 1.

3. Given Steps 1 and 2. the combined allocations result in optinal allocatious for $C$ starting at $\hat{t}$.

Step 1 follows immediately from the induction premise. To prove Step 2. we have to demonstrate that DS solves the simpler problem of mavinuzing 
$\sum_{1}^{n} \mu_{b}\left(\delta_{1}\right)$ sul) ject to the constraint that $\sum_{1}^{n} \delta_{1}=1$. where 1 is the length of time separating $i$ and the first event in $C^{\prime}$. Lor this demonstration, it is enough to note that. as long as the set of events being considered $(1(t))$ remains unchanged. during each iteration of the main loop. DS chooses an interval with maximal gain. and. by making this choice. DS in no way restricts its future choices givell that all subsequent intervals are bound to have the same or smaller gaills. This last poill is due to the restriction lhat the slopes of conseculive line segments for all performance profiles are decreasing. Note that, if we were tu relax this restriction. the greedy stralegy used by DS would not produce optimal allocations. Figure $8 . i . i$ provides a pair of performance profiles such that DS will produce suboptimal allocations. Figure 8.i.ii shows the allocations made by DS. and Figure 8.i.iii shows the optimal allocations.

Step 3 follows from the olsservation that the allocation of the time from the occurrence of the first event to the occurrence of the last is independent of any consideration of the first event or any time available for deliberation priur to the occurrence of the first event.

Theorem 1 proves that the allocations made by DS are optimal in a welldefined sense. We still have to show that the method for scheduling when to run decision procedures is correct. In particular, we have to show that DS generates a legal schedule, where a legal schedule is one such that for all $c \in C$ the tine allocated to the decision procedure for $c$ is scheduled prior to the time at which $c$ occurs. To see that DS does generate legal schedules. nole that DS eusures that the sum of the time allocated to all conditions that occur prior to $t$ for any $t>t$, is less than $t-t$. DS is guaranteed to generate a legal schedule since it schedules all of the time for any coudition $c$ before any condition occurring later.

In the time-dependent planning problems described above, the exact time of uccurrence of conditions is known by the deliberation scheduler. One can eaily imagine variants in which the scheduler ouly has probabilistic information about the time of occurrence of events.

Fos instance, for each condition. $c_{i}$, the scheduler might possess a probability demaity function.

$$
\rho_{i}(t)=\operatorname{Pr}\left(\text { occura }\left(t . c_{i}\right)\right)
$$

indicating the probability that a particular condition will occur at tine. $t$. For practical reasons. we will assume that for each condition. $c_{i}$. there is a 


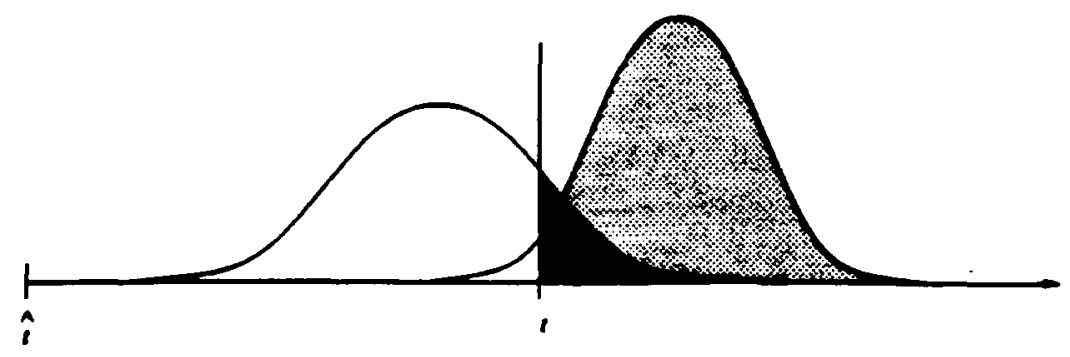

Figure 8.8: Uncertainty abont the occurrence of conditions

latest time. $\sup \left(c_{i}\right)$, and an earliest time. inf $\left(c_{i}\right)$, such that

$$
\rho_{i}\left(\sup \left(c_{i}\right)\right)=\rho_{i}\left(\inf \left(c_{i}\right)\right)=0 .
$$

While the sclieduler does not know exartly when ronditions will orrur. we assume that the excentor will know when to carry out a given action. For instance. conditions might have precursor cvents signaling their inmediate occurrence. The executor would simply take the best response available at the time the prernrsor event. for a given condition is observed.

Our performance criterion for deliberation scheduling is no longer,

$$
\left.\sum_{c \in C^{\circ}} V(\operatorname{Response}(c) \mid c)\right)
$$

but rather,

$$
\sum_{c \in C} \int_{i}^{\infty} \rho(\text { occura }(t, c)) V(\operatorname{Response}(c, t) \mid c) d t
$$

where Response(c, l) indirales the respunse generaleel with respect to condition. $c$, given that $c$ ocrurs at $t$.

In deciding how to allocate an interval of processor time given uncertainty alout the occurrence of conlitions. we have to account for the possibility that the event may have already occurred. Figure 8.8 depicts the probalitity density functions for the time of occurrence of two cunditions. The anes of the shaderl regions indicate the probability that the conditions ocrnt in the future of the time marked $t$.

We extend the $\lambda_{i}$ notation to represent processor schedules. Let each $\lambda_{i}$ be a function.

$$
\phi_{i}: \mathbf{R}-\{0,1\}
$$




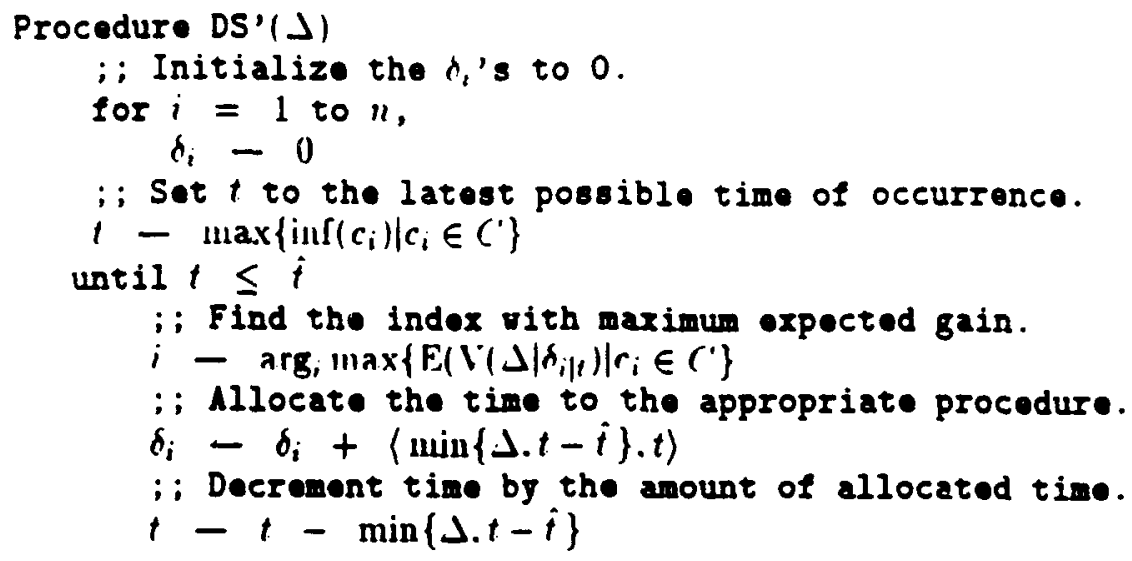

Figure 8.9: Deliberation scheduling with uncertain condition times

where $\delta_{i}(\ell)=1$ if the decision procerlure for $c_{i}$ is allocaled the processor at $t$, and $\delta_{i}(t)=0$ otherwise. The expected value of a given schedule, $\delta_{i}$, beginning at $t$, and allocating processor tine to deliberating about a condition, $c_{i}$. is just the sum over all times, $t^{\prime}$, in the fut ure of $t$, of the probability that $c_{i}$ occurs at $t$ multiplied by the expected value of the respouse generated by the decision procedure for $c_{i}$ given the processor time scheduled between $t$ and $t^{\prime}$. We notate this expected value,

$$
E\left(V\left(\delta_{i \mid t}\right)\right)=\int_{t}^{\infty} \rho_{i}(\tau) \mu_{i}\left(\delta_{i}(t, \tau)\right) d \tau
$$

where $\delta_{i}\left(t, t^{\prime}\right)$ is the total amrount of time allocated to $c_{i}$ by the schedule $\delta_{i}$ letween $t$ and $t^{\prime}$. The expected value of augmenting a given schedule, $\delta_{i}$, starting at $t$, by allocating the time from $t-\Delta$ to $t$ to deliberating about $c_{i}$ is defined by

$$
\operatorname{E}\left(\boldsymbol{V}\left(\Delta \mid \delta_{i k}\right)\right)=\int_{t-\Delta}^{\infty} \rho_{i}(\tau) \mu_{i}\left(\delta_{i}(t . \tau . \Delta)\right) d \tau-\int_{t}^{\infty} \rho_{i}(\tau) \mu_{i}\left(\delta_{i}(t . \tau)\right) d \tau .
$$

where $\delta_{i}(t, \tau, \Delta)$ is the tolad amount of lime between $t$ and $t^{\prime}$ allocated to $c_{i}$ by the $\Delta$-augmented schedule.

Figure 8.9 lists a procedure for deliberation scheduling for the class of problems involving uncertainty in condition times. The procedure listed in Figure 8.9 takes a positive real number. $J \in \mathbf{R}^{+}$. to be used as the length of the interval of time allocated in each iteration of the main loop of the 
proredure. The assignment. $\delta_{i}-0$. results in $\delta_{i}(t)=0$ for all $t \in \mathbf{R}$. The assigument. $\delta_{i}-\delta_{i}+\left\langle t . t^{\prime}\right\rangle$. results in $\delta_{i}(\tau)=1$ for all $\tau$ in the interval $\left\langle t . t^{\prime}\right\rangle$. and for all $\tau$ outside the interval $\left(t . t^{\prime}\right\rangle$ is the same as it was prior to the assignment. In the following. we make several comments regarding DS:

The first comment concerns what exartly it is that DS computes. DS provides an approxination to the optimal deliberation schedule. It is an approximation because we allocate each interval of length on the basis of expectations conputed for a single point at the boundary of that interval: in gencral. this method will result in a suboptimal deliberation schedule. On the positive side. the smaller the allocated intervals are. the better the approximation: the schedules gencrated hy DS' converge to the optimal schedules as $\Delta-0$. Ou the negative side. the smaller $\Delta$ is. the longer it takes to rompute the entire deliberation schedule.

In this chapter. we genernlly ignore the cost of deliberation scheduling. assuming that. if the running time of the scheduling algorithm is linear in the size of the input. then the cost of scheduling is negligible. In this case. however. the cost of deliberation scheduling can be made arbitrarily large hy employing a small enough value for $J$. In practice, it will be necessary to account for the cost of deliberation scheduling. In some cases. it will reasonable to clioose a value for $J$ at compile time by experimenting with various values and expected inputs. In other cases, it might be useful to select a value at run time, using some simple criteria for selection; this constitutes a simple example of meta-meta-reasoning.

The serond coinment regarding DS' concerns the form of the final schedule. IIulike the case in which we know exactly when each condition will occur. tie cannot coalesce all of the time allocated to a given condition into a continuous interval. As a consequence, we have to assume the capability. of switching the processor rapidly between different decision procedures. In most nulti-tasking operating systems, assuming this sort of rapid process switcluing is reasumable.

The final comment regarding DS' concerns the notion of optimality which we employ in rating performance. Clains of optimality are made assunuing that there will be no further opportunities to modify the srherinle. In prartice. Lomever, each time that a condition occurs, it will be useful to coinpute a new deliberation schedule.

In the remainder of this section. we consider one more variant of timedependent planning. In this variant. We assume that there are no external conditions requiring responses of the controller: instead. the controller has some number of tasks it is assigned to carry out. The tasks do not liave 
In be completed by any particular time. but the sooner they are completed the better. As in the previons problems. we assume that there is a decision procedure for each task. Cienerally, the more time the controller deliberates about a given task. the less time it takes to carry ont that task. We assume that the outcome of deliberation concerning one task is independent of the outcome of deliberation concerning any other.

The performance profiles relate the tine spent in deliberation to the time sased in executtion. For instance, suppose that. the task is to navigate from one location to another. and the derision procedure is to plan a pat!? to follow between the two locations: up to a certain limit. the more time spent in path planning. the less time spent in navigation.

In the following. we consider a few special instances of this class of problenus. In the first instance, all of the deliberations are perforned in advance of rarrying ont any task. This model might be appropriate in the case in which a set of instructions are compiled in advauce, and then loaded into a rohot. that carries out the instructions. Deliberation scheduling is simple. For each task, the scheduler allocates time to deliberation as long as the time spent. in cleliberation results in a greater reduction in the time spent in execution. All of the deliberation is then performed in advance of any execrition.

In the second instance. the order in which the tasks are to be carried out is fixed in advance. and all deliberation concerning a given task is performed in advance of carrying out that task, but deliberation concerning one task can be performerl while carrying out another. In this instance, deliberation schedluling is somewhat more complicated. We consider delil)eralion scheduling in terms of three steps, minimal allorntion, dead-time irduction. and free-time optimization. In the minimal allocation step, we proceel as in the previous instance, by determining a minimal allocation for each task. ignoring the possibility of performing additional deliberation during execution.

This minimal alloration for a given task corresponds to that alloration of delberation time minimizing the sum of deliberation and expected execution tine. Figure 8.10.i shows four tasks and the tine they are expected to take, asouming no time spent in clebiberation. Figure 8.10.ii shows the performance profiles for each of the four tasks. The dotted line in each performance profile indicales the minimum slope such that. allocaling deliberation time will result in a net decrease in the sum of deliberation and expected execution time for the minimal allocation. Figure 8.10.iii shows the minimal allocations for each of the four tasks. where the $\delta_{\text {; }}$ indicates the

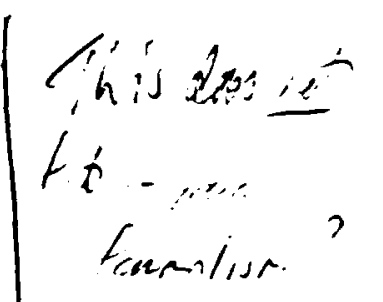

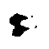




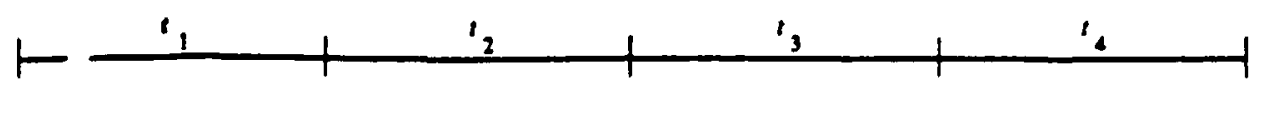

i.
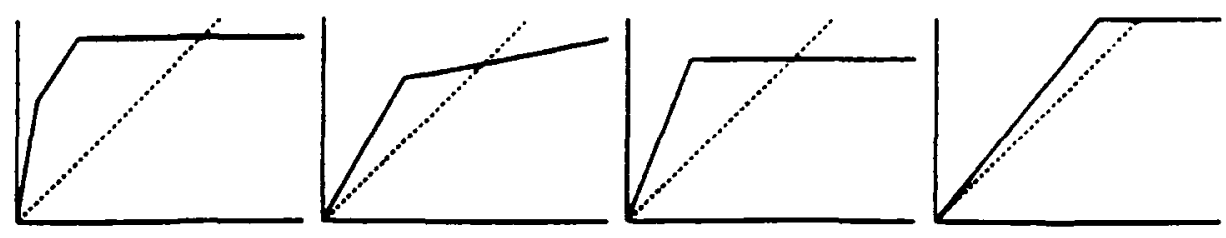

ii.
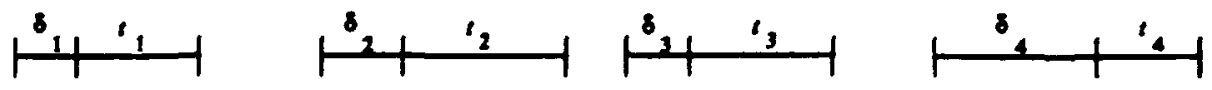

it.

Figure 8.10: Minimal allocations of processor time

time allocated to deliberation for $t_{i}$.

Using the allocations computed in the ininumal allocation step. we construct a schedule in which lasks begin as early as possible subject to the constraint that all of the deliberation for a given task occurs in a continuous block immediately preceding the task and following any deliberation for the previous lask. Figure 8.11.i shows the resulting schedule for the example of Figure 8.10. Note that there are two additional types of intervals labeled in Figure 8.11.i. This first type, notated $f_{i}$, indicates the free time associated with $t_{i}$, corresponding to time when the system is performing a task but not deliberating. The second type. notated $d_{i}$, indicates the deud time associated will $t_{i}$, correspouding to tịme when the system is deliberating but not performing any task.

In the dead-time reduction step, we attempt to rednce the amount of dead time in the minimal-allucations schedule by making use of earlier free time. Where possible, we allocate earlier free time to performing the deliberation gevionsly performed duriug the dead time. starting with latest dead time $f^{2}$ als and working backward froin the end of the schedule and using the bossible intervals of free time. Figure 8.11.ii shows 1.he schedule of Fifin 8.11.i modified to eliminate one of the dead time intervals. It is not always possible to eliminate all dead time intervals. In particular. any deliberation time allocaled for the first task will always correspond to dead linue. 

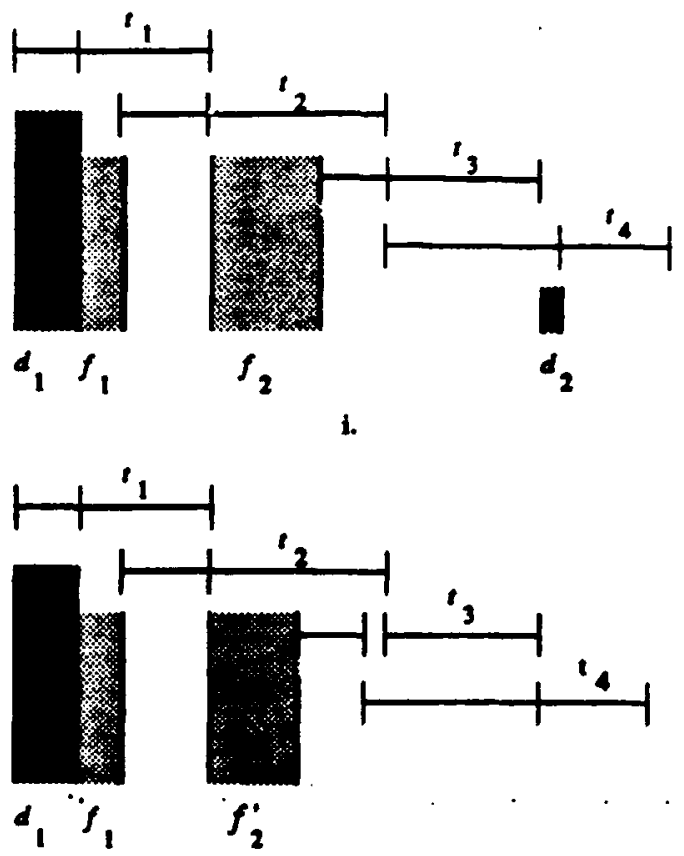

ii.

Fignre 8.11: Reduring dead time nsing available free time

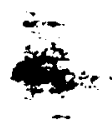


I $19 ! T_{-i}^{\prime}$ 舟, $1: \because$
Following this rocess of dead-time reduction. if there is any free time left. We attemipt to allocate it for deliberating about other tasks. This is just a bit tricky. since by performing additional deliberation we eliminate previonsly available free time. Not only do we eliminate the free time we are filling in by scheduling deliberation. but the deliberation reduces execution time thereby eliminating aclditional free time. There is one special case for which optimally allocating the additional free tine is easy. This is the case in which all of the performance profiles are piecewise linear composed of two linear segments such that slope of the first segment is the same for all profiles and the slope of the second is 0 . This corresponds to the specification of the robot courrier problem described in the previous section, regarding the task of optimally allocating processor time for planning several paths between locations in a tour of such locations to be visited.

The reason that optinally allocating the additional free time in this case is easy is explained as follows. If the slope of the first linear segment for all of the performance profiles is greater than 1 , then all of the time corresponding nonzero slope will be allocated in making the minimal allocations, and any additional allocations will yield no decrease in execution time. If the slope of the first linear segment for all of the performance profiles is less than 1 . then all of the minimal allocations will be 0 , and there will be no free time to allocate.

There are many variations on the problems described above. This section is meant as a sampler of problems and associated deliberation scheduling techniques. Deliberation scheduling should be seen as a means of programming in knowledge about how to improve run-time performance. There are occasions. however, in which the time required to apply that knowledge is not arailable at run time, and it beconies reasonable to make certain choices concerning the allocation of computational resources at design time. In the next section. we cousider design-tine tradeoffs for improving system performance.

\subsection{Compiling Problem Solving Systems}

In the previous sectiuns, we were concerned with the design of systems that. given expectatious alsoul the performance of decision-making routines, were able to make appropriale I radeofts al ruil-time so as to maximize expected ntility. Another approach to building systems capable of good performance in time-critical situations involves making certain inferences al design time 


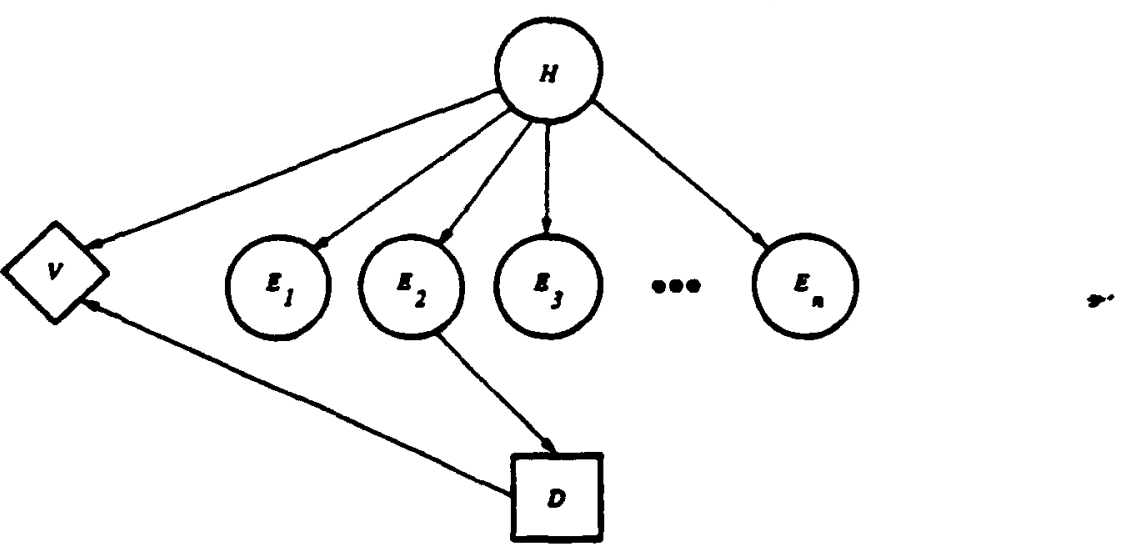

Figure 8.12: Decision model for the diagnosis problem (after [26])

and caching those inferences for use at run time in order to improve the syslem's response time. Other researchers have suggested compiling domain models to guarantee bounded response time $[31,39]$. Generally, the result of compilation is a table or circuit whose space requirements are an important factor in assessing the value of a given compilation method. Usually, the object is to improve response lime withont sacrificing decision quality; when this cannot be done ( $\epsilon . g$., the storage requirements for caching are substantial) it becones necessary to consider tradeoffs. The approaches described in this section are noteworthy for their use of a decision theoretic criterion for trading space for response time.

Heckerman, Breese, and Horvitz [26] investigate a simple form of tradeoff that involves improving response time by compiling decision models. In their model, the utility of a state depends on whether or nol a particular hypothesis $H$ is true and whether or not an action $D$ is taken. We will assume that, if $H$ is true, the action $D$ should be taken, and otherwise the action $\neg D$ is appropriate. We can define a threshold probability of $H$. call it $p^{*}$, that the agent is indifferent about acting one way or the of her:

$$
p^{*} \text { O }(A, D)+\left(1-p^{*}\right)\left((-H . D)=p^{-}\left(1(H, \neg D)+\left(1-p^{*}\right)(1(-H .-D) \text {. }\right.\right.
$$

The agent is not able to observe $H$ directly, and, hence, must infer whether or not $H$ is true on the hasis of the nhserverl evidence. $E_{1}, E_{2}, \ldots, E_{n}$. Thus the agent should perform the action $D$ if and only if

$$
\operatorname{Pr}\left(H \mid E_{1}, E_{2}, \ldots E_{n}\right)>p^{*}
$$


The resulting decision mode' (depicted graphically in Figure 8.12) is represented as an influenre dingrun that captures the causal and informational rependencies between chance variables (indicated as circles) and between rhance variables and decision variables (indicated as boxes). and the value of states of the work corresponding to particular instantiations of the chance and decision variables (indicated as dianonds).

lleckerman. Hreese. and Horvitz reformulate the decision problem in terns of log-likelilood ratios, and. Wy making certain inclependence assump- tinns, they reduce the decision problem to computing

$$
W^{*}=\sum_{i=1}^{n} w_{i}
$$

where the $u_{i}$ are the weights accorded to the $E_{i}$. The agent should perform the action $D$ if and ouly if $W^{\prime}>W^{*}$ where $W^{*}$ is the log-likelihood equivalent of $p^{*}$. We will refer to the strategy of computing the weights at run time as the compule strategy.

As an alteruative to computing the sum of the weights of evidence at run time, the agent might consider all possible combinations of evidence and compile a table indicating whether or not to act for each possible combination. If memory is inexpensive and response time critical. then this might be an attractive alternative. In general. however. it will be prohibitively expensive to compile a table for all possible combinations of the evidence. and. hence. if the agent wants to speed its response time by compiling a table. it will have to limit its atteution to a subset of the evidence. Suppose that the agent chooses $m$ pieces of evidence.

$$
\left\{E_{c_{1}}, E_{c_{2}} \ldots, E_{c_{m}}\right\} \subseteq\left\{E_{1}, E_{2}, \ldots, E_{n}\right\},
$$

to use in compiling a table of responses. For each of the $2^{m}$ possible combinations of the $m$ variables. we compute the sun.

$$
W_{m}=\sum_{i=1}^{m} w_{c_{1}}
$$

al. compile time, and store $I)$ in lile table if $\| I_{m}^{\prime}>I^{\prime *}$ and $-D$ ullierwise. At run tine, the agent simply uses the evidence as an index to lookup the appropriate eutry in the table. We will refer to a strateg! of compiling a table for $m$ pieces of evidence as a compile strategy.

Note that the advantage of the compute strategy is that it takes all of the evidence into account: the disadvantage is that there may be some delay 


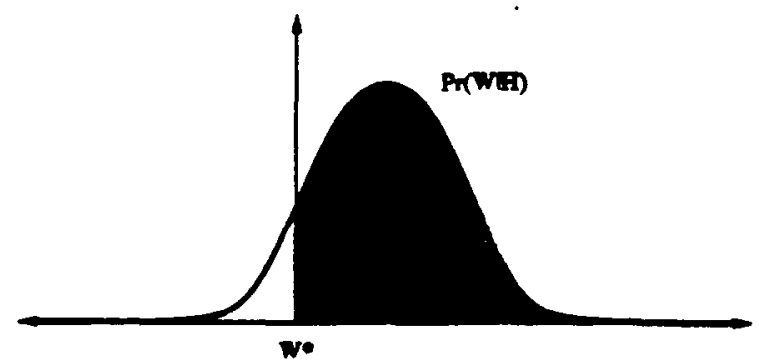

Figure 8.13: The probability that the total evidential weight will exceed the threshold is determined by sumining the area under the curve for the distribution of $W^{r}$ given $H$ and ahove the threshold weight $W_{m}$ (after [26]).

betreen the time that the evidence is observed and the time that the agent responds to the evidence. The coinpile strategy may enable the agent to respond more quickly, but at the cost of ignoring some of the evidence and providing storage for a decision table whose size is exponential in the number of pieces of evidence accounted for in the reduced model.

In the following. let $E V_{\text {compune }}$ indicate the expected value of the agent using the compute strategy for a single instance of the decision problem, $P C_{\text {compuse }}^{H}$ and $P C_{\text {compute }}^{-H}$ indicate the cost dne to computing delays in the case in which $H$ is true and the case in which $H$ is not, and $M C_{\text {compur indicate the }}$ one time cost of memory for the compute strategy. Assume similar quantities for the compile strategy. In order to compare the compute strategy against different compile strategies (i.c.. conipilar in involving different subsets of $\left.\left\{E_{1}, E_{2}, \ldots, E_{n}\right\}\right)$, Heckerman. $c t$ al. introduce formulse for determining the nct inferential valuc of a given strategy.

$$
\begin{aligned}
& N I \mathrm{~V}_{\text {compule }}= \\
& \rho\left[E V_{\text {compree }}-\operatorname{Pr}(H) P C_{\text {tompule }}^{H}-\operatorname{Pr}(\neg H) P C_{\text {compoute }}^{-H}\right]-I / C_{\text {compue. }}
\end{aligned}
$$

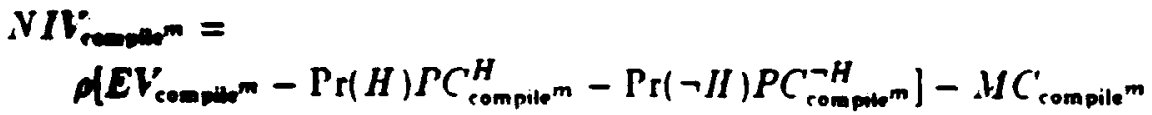

where the $N I V_{\text {enmpilem }}$ depends upon the particular choice of evideuce. and $\rho$ is a factor "that converts the expected value of each policy on a single instance to a summary (present) value for a series of problem instances over the life of the ststem." Civen the above. the agent desiguer should choose

$$
\text { i }
$$


the compute strategy over the compile stratc $y$ if and only if

$$
X I H_{\text {compule }}>N I T_{\text {compilem }}
$$

In the analysis presented in [26]. $P C_{\text {compute }}^{-H} P C_{\text {compute }}^{H}$ and $M / C_{\text {compuse }}$ are linear functions of $n$. the total number of evidence variables in the coinplete model. $P C_{\text {compilen }}^{-H}$ and $P C_{\text {compilem }}^{H}$ are linear functions of $m$. the total number of evidence variables in the restricted compilation model, and $M C_{\text {compilem }}$ is a linear function of $2^{m}$. The fornulae for the expected value of using the compute and compile strategies for a single instance of the decision problem are given as follows:

$$
\begin{aligned}
& E V_{\text {eompere }}= \\
& \left.\quad \operatorname{Pr}\left(W>W^{*} \mid I\right) U(I I, D)+\operatorname{Pr}\left(W \leq W^{*} \mid \Pi\right) U(I I, \neg D)\right] \operatorname{Pr}(I)+ \\
& \left.\left.\quad \operatorname{Pr}\left(W^{\prime}>W^{*} \mid \neg H\right) U(\neg I . D)+\operatorname{Pr}\left(W^{\prime} \leq W^{*} \mid \neg H\right) U(\neg I I . \neg D)\right] \operatorname{Pr} \neg I I\right) \\
& E V_{\text {rompilem }}^{.} \\
& \quad\left[\operatorname{Pr}\left(W_{m}^{\prime}>W^{*} \mid H\right) U(H, D)+\operatorname{Pr}\left(W_{m} \leq W^{*} \mid H\right) U(H . \neg D)\right] \operatorname{Pr}(H)+ \\
& \quad\left[\operatorname{Pr}\left(W_{m}>W^{*} \mid \neg H\right) U(\neg H . D)+\operatorname{Pr}\left(W_{m} \leq W^{* *} \mid \neg H\right) U(\neg H . \neg D)\right] \operatorname{Pr}(\neg H)
\end{aligned}
$$

The only trirk to using the above to decide whether to use the compnte or compile strategy is determining the probabilities involving the weights (e.g., $\operatorname{Pr}\left(W>W^{*} \mid H\right)$ ). Assuming that $n$ is large (as it should be for us to take seriously the cost of computing $W$ ), then we can compute the first two monents for the each of the weights given $I I$ and combine them to approximate the distribution of $W$ given $I I$ using the central linit theorem. Using the resulting approxinations for $\operatorname{Pr}\left(W_{m} \mid H\right), \operatorname{Pr}\left(W_{m} \mid \neg H\right), \operatorname{Pr}(W \mid H)$, and $\operatorname{Pr}(W \mid \neg H)$, we can determine the values for the terms needed to compute $E l_{\text {compuce }}$ and $E V_{\text {compile }}$ ( see Figure 8.1.3).

Heckerman, et al. go on to consider relaxing certain assumptions (specifically, allowing multiple-valued hypothesis. evidence, and decision varial)les and introducing alternatives to caching romplete tables, in the form of carhine situation/action rules in asymmetrical trees), and methorls for considering what subsets of the set of all evidence variables to consider for compilation. What they don't cousider. and what might be worth pursuing, are mixed strategies involving some amount of design-time compilation and some amount of run-time inference.

If the basic methods described by lleckerman. et al. for evaluating the expected performance of decision models used for tine-critical applications 


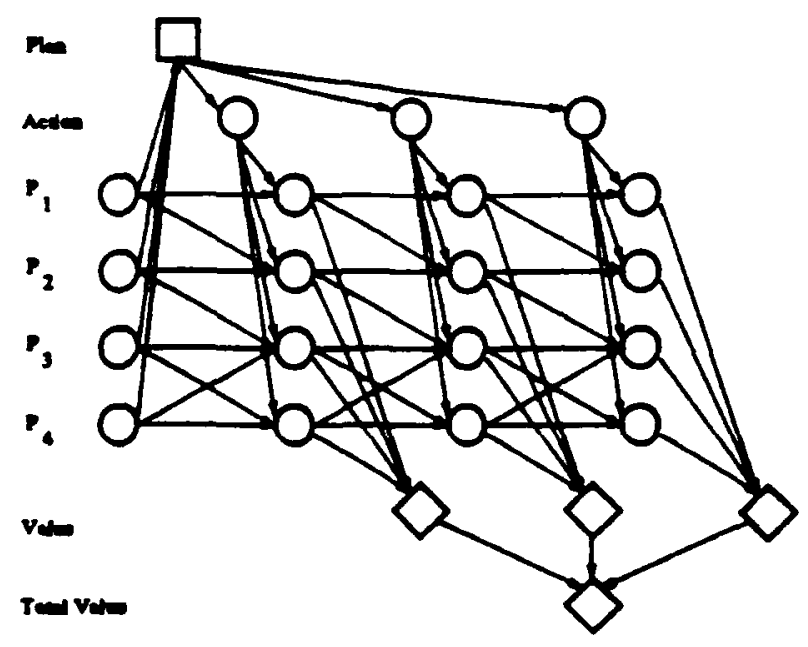

1.

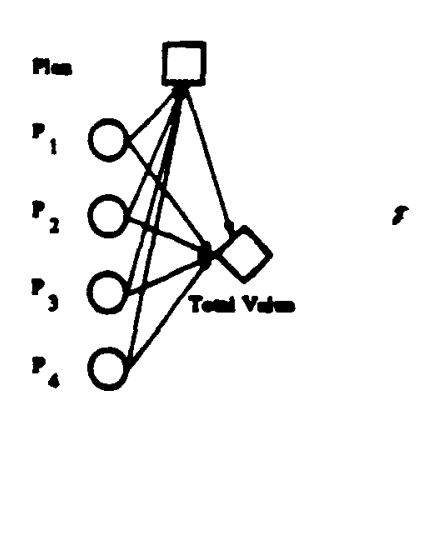

t.

Figure 8.14: Two influence diagrams indicating ( $\mathrm{i}$ ) a complete decision model for reasoning about plans, and (ii) a reduced version of the decision model obtained by absorbing chance nodes in the complete model.

turn out to be practical for realistic decision problems, then we will want to try out more sophistirated models for reasoning about plans and change over time. Kanazawa and Dean [32] describe a model for reasoning ahout. time, causation, and action that can be cast an an influence diagram. Civen a set $\mathcal{F}$ of propositions and a set $\mathcal{T}$ of time points, we can define a set of chance variahles froin $\mathcal{F} \times \mathcal{T}$ representing the truth of various propositions at different points in time. By quantifying the dependencies between these chance inriables, we can specify a model of change over time referred to as a lempoml Bayes not [14].

The model described in [32] generalizes on this basic model of rhange over time to include actions 80 as to provide a decision model for selecting plam. Pigare 8.14.j shows an example of such a model clepicted as an inflnence dingram. Each row. except those corresponding to decision variables or value fenctions, indicates a proposition or quantity that changes over time, and each column indicates a difierent point in time. Kanazawa and Dean consider possible tradeoffs involved in improving the performance of reasoning systems using such a model for decision making. In particnlar, they consicler trading accuracy for time by employing approximation schemes for 
evaluating probabilistir models [ $8,2 x$ ]. They also consider " ading ce for lime by eliminaling cliance variables in the decision modes using a "hod of conditjoning called nulf "lsomplion [.12]. By eliminating chance variables at design time. it is possible to dramatically improve the tine required 10 evaluate the model. Such improvements occur for both exact and approxintate eraluation techuiqnes. Figure 8.14.ii shows a version of the model shown in Figure 8.14.i obtained by repeated use of node absorption.

In general. node absorption can result in an increase in the space required to store the moclel: there will be fewer nodes in the resulting graph. but the space required to store the conditional probabilities quautifying the dependencies mav increase significantly. llowever. given the structure of temporal Bayes nets. the net increase in space is generally acceptable and more than offset by the resulting reduction in evaluation time. It would be interesting to extend the techuiques of Heckerman. $f t$ al. 10 evaluate at design time various alternative approximation schenes and methods of simplifying the clecision mode!. The biggest harriers to making such extensions practical will likely be due to the combinatorics of action selection and the difficulties involved in obtaining an accurate model of the euvironnent in the first place.

\subsection{Directions for Future Research}

This chapter provicles only a sketch of current work on problem solving methods for time-critical applications. There is a great deal of excellent rescarch that we did not cover. simply hecanse it did not. fit into the st.ruct.ure of the presentation. In particular. we did not say anything significant ahout architectures for real-time control [ $1, \overline{7}]$, or relate how the search communit.y is beginning to address real-time issues $[24,33,44]$. Regarding search, Hansson and Masyer's work [2.1] predicts that we will find many of the standard techniqnes in heuristic search as emergent properties of inechanisms that. emplox Bayesian inference and decision-theoretic control of inference. All of this work is serving to shape a new field of research.

The next few years will see a marked increase in the effort directed at. timerritleal problem solving and resource-linited reasoning. We need to extend the current approaches to handle computational models that reflert the complexity of existing problem-solving systrms. For instance. how might. all agent deal with inultiple tasks. perhaps deciding to act with regard to one task while continuing to deliberate ahout others. We need experience 
with real applications so that the research will be driven by real issues and not artifacts of our matheniatical inodels. We need to reconcile the goal-oriented. resource-bounded perspective of artificial intelligence with the idealized. optimizing perspective taken in the decision sciences.

This rhapter nakes use of Howard's information value theory as a basis from which to start in analyzing systems with linnited computatioual resources. All of the approaches descrihed in this chapter call be seen as extensious of the basic idea of assessing the value of infornation sources. The approaches surveved here depart from information value theory when they at.tempt to account for the cost of inference. including the computational cost of assessing the value of information sources. It would seem that the theory of experimental design [19. 35] which is concerned with the problem of maximizing the information gained from performing experiments under cost constraints night provide a source of additional techniques that could be applied in controlling inference for time-critical applications.

All of the approaches described in chapter make rather restrictive assumptions in order to avoid the combinatorics involved in dealing with unlimited decision-making horizons and complicated interactions between information sources. For practical problems, it is unlikely that we will be able to entirely relax the one-step horizon and no-competition assumptions that characterize myopic decision policies. An interesting area for future research involves identifying and dealing with restricted types of interactions and providing a disciplined approach to extending derision-making horizons. It would also be usefnl to explore methorls of extending the anytime algorithm approaches to handle more situation-specific information.

The research on compiling decision models is just beginning, and one area that appears particularly interesting to investigate involves mixed strategies for combining design-time compilation and run-time inference. Another area that was not covered in this survey, but is of considerable interest involves learning control knowledge in the form of statistics to support decisiontheoretir control of inference. Two of the papers covered in this chapter 14l. 1.t. tweribe interesting terhniques that addrens learning isnues.

Now abut learning in general and speedup learning in particular.

The work in time-critical problem solving will liave far reaching implications for the whole research community. Time is, after all. an issue in any problem solving task. Theoretical resnits concerning agents with limited computational resources should shed light on a number of basic representation issues. For instance, the notion of a "plan" as a persistent belief does not make sense until you take computational considerations into account. 
P'lans enalsle a sustem to amortize the cost of doliberation over an inter ial of time. If time were not an issue. there wonld be no justification in committing to a plan. What are the tradeofls involved in generating a partial plan? What are the rosts and henefits of compiling a cletailed plan to use in a siluation in which there will be very litte time for computing appropriate responses. These are just a few of the questions that can be addressed once we begin to account for the time spent in problem solving.

\subsection{Further Reading}

Meta-reasoning [10, 11, 15, 16. 21, 45].

Speedup learning [34. 36].

Early work in the decision sciences on the costs and benefits of inference

[17. 29. 38].

Examples of mỵopic decision makiug [3. 12]. 


\section{Bibliography}

[1] Agogino. A. M... Srinivas. S.. and Schneider. K., Multiple Sensor Expert System for Diagnostic Keasoning, Moniloring, and Control of Mechanical Systems, Merhanical Systems and Signal Processing, (1988).

[2] Barnett. V.. Comparative Statistical Inference. (John Wilev and Sons. New York. 1982).

[3] Ben-Basal, M.. Myopic Policies in Sequential Classification. IEEE Transactions on Computers, 27 (19i8) 170-1i4.

[4! Boddy, Mark and Dean, Thomas, Solving Time-Dependent Planning Problems, Proceedings IJCAI 11, Detroit. Mirhignn, IJCAl. 1989. 9i's984.

[5] Bodin. L. and Golden, B., Classification in Vehicle Routing and Scheduling, Networks. 11 (1981) 9i-108.

[6] Brachman. Ronald J.. Levesque, llector J., and Reiter, Raymond, (Eds.), Proceedings of the First Interinational Conference on Principles of Kinouledge Representation and Reasoning, (Morgan-Kaufmann. Los Altos, C'alifornia, 1989).

[i] Breme, Jolin S. and Fehling, Michael R., Decision-Theoretic Control of Problem Solving: Principles and Architecture, Procefdings of the 1988 Werbabop on Inrertainty in Artifirial Intellige "re. Minnenpolis. MN. 1900, 30-37.

[8] Chavez. R. Martin. Fully Polynominl Randomized ftppmrimation Schcmes for the Bayesian Infererencing Problem. Report KSL-88-i2. Section on Medical Informatics. Stanford I'niversity School of Medicine. 1088 . 
[9] C'hernoff. Herman and Moses. Lincoln E.. Elcmentary Decision Theory. (John Wiley and Sons. New York. 1959).

[10] Davis. Randall. Teiresias: Applicatinus of Meta-Level Kinowledge, Davis. Randall and Lellat. Douglas B3.. (Vids.). Kinouledgf-Basfd Sy.stems in Artifirinl Intelligenre. (MrCiraw-Hill International Book Compauv. $19 \times 2) .22 \pi-490$.

[11] ile Kleer, Johan, Doyle, Jon, Sieele Jr., Ciuy L., and Sussman. Gerald Jay. AMORD: Explicit ('ont rol of Reasoning. Brachuan. Ronald J. aud Levesque. Ilector J.. (Eds.), Reudings in Knouledge Representation. (Morgan-Kaufmann. Los Altos. C.A. 198.5). chapter 19, 346-3.5.5. Origiually published in $197 \%$.

[12] de Kleer. Johan and Wijliams. Brian ('.. Diagnosing Multiple Faults. Artificial Intelligence. 32(1) (1987) $9 \bar{i}-130$.

[13] Dean. Thomas and Boddy. Mark. An Analysis of Time-Dependent Plauning. Procetdings A AA I-88. St. Paul. Minnesota. AAAI. 1988. 4954.

(14) Dean. Thomas and Kanazawa. Keiji, A Model for Reasoning Abont Persistence and Causation. Computational Intelligence. 5(3) (1989) 142150 .

[15] Doyle, Jon. A Moslel for Dflibemution. Action, and Introspection, Techniral Report. Al-TR-581, MI'I Al Laboralory, 1980.

[16] Doyle. Jon. Reasoning, Representation, and Rational Self-Goverument. Ras. Zbigniew W.. (Ed.). Methodologies for Intelligent Systems, 1, New lurk. Nurth-Ilullaind, 1989; 30i-380.

[1i] Edwards. Ward. Dynamic Decision Theory and Probabilistic Information Processiug, Human Factors. 4 (1962) 50-i3.

[18] Etzioni. Oren. Tractable Decision- $\Lambda$ ualytic Control. In Brachman et al. [0. $114-125$.

(19) Fedorov. V.. Throry of Optimal Erprrimental Design. (Academic Press. New lork. 1972).

[20] Ciarev. Michael R. and Johnson. David S.. Computing and Intmrtibilily: A cinide to the Theory of NP.C'nmplete ness. (W. H. Freemau and ('ompany. New lork. 197!)). 
[21] Cieneseretin. Michael R.. An Overvipw of Metalevel Architerlire. Proreedings A.t.A-8.3. IInshinglon. D.C'.. AAAl. 198:3. 119-12:3.

[22] Ciood. I. J.. A Five lear Plan for Automatic Chess. Machinc Intclligence. 4 (1962) 5!)-i.3.

[23] Cirahaın. R. L.. Lawler. E. L.. Lenstra. J. K.. and Rinnooy Kan. A. II. G.. Optinuzation and Approximation in Determninistic Sequencing and Scheduling: A Surver, Prvcedings Discrete Optimization. I'ancouver. 197 i.

[24] Hansson. Othar and Maver. Andrew. The Optimality of Satisfiring Solutions. Proceedings of the 1988 IInrkishop on Incertainty in Artificial Intelligence. Minnenpolia. MN. 1988. 148-15T.

[25] Ifayes-Rotll. Barbara. Washingtou. Richard. Hewett. Rattikoru. Hewett. Michael. and Seiver. Adam. Intelligent Monitoring and Coutrol. Proceedings IJC'Al 11. Detruit. Michigan. IJCAI. 1989. 243-249.

[26] Heckerman, David E., Breese. John S.. and Horvitz, Eric J., The ('ompilation of Derision Models. III'89. IIindsor, Ontario. 1949. 162-173.

[2i] Horvitz. Eric J., Reasoning About Beliefs and Actions Under Computational Resource Constraints. Proceedings of the 1987 Workshop on Incertainly in Artificial Intelligence. Seattle. Washington. 1087.

[28] Horvitz. Eric J., Snermondt. H. Jarques, and Cooper. Ciregory F., Bounded C'onditioning: Flexible Inference for Decisions IInder Scarce Resaurces, Technical Report KSL-89-42, Stanford Knowledge Systems Laboratory, 1989.

[20] Howard. Ronald A.. Information Value Thenry, IEEE Transactions on Syeteme Science and Cybernetics. 2(1) (1966) 22-26.

[30] Anze. O. II. and Kitu. C.. E.. Fast Approximation Algorithms for the Iframack and Sum of the Sulsset Problems, Journal of the $A C, 1,22$ (15) $403-468$.

[31] Kaelbling. Lealie Pack. Cioals as Parallel Program Specifications. Pmreedings AAA I-8.S. St. Pnul. .Vinnr.asta. AAAl. 1989. (i0-65.5.

[32] Kanazawa. Keiji and Dean. Thomas. A Model for Projection and Actiun. Proceetings IJCAI 11. Detroit. .Vichigan. IJCAI. 1989. 98.5-990. 
[33] Korf. Richard. Real-Time Heuristic Snarch: New Results. Prorccdings A.4.1I-88. St. Paul. Vimuesotn. A.A.1. 1989. 139-144.

[34] Laird. J. E.. Newell. A.. and Rosenbloom. P. S., SOAR: An Architecture for Ciencral Intelligence. Artificial Intclligence. 33 (1985) 1-(j4.

[35] Menclenhall. WV.. Intmdurtion to Linear Mostels and the Design and dnalysis of Eaperiments. (Wadsworth. Belmont. ('alifornia. 1968).

[36] Mlinton. S.. Carbonell. J. C... Kinoblock. C'. A.. Kuokka. D. R.. Etzioni. O.. and (iil. Y.. Explanalion-Based Learning: A Problem Solving Perspective. Artificial Intrlligcncc. 40 (1989) 63:118.

[3i] Pearl. Judea. Probubilislic Reuscinia! in Inlelligent Systems: Neturorks of Plausible Inference. (Morgall-liaufmanu. Los Altos, California. 1988).

[38] Raiffa. Iloward and Srhlaifer. R.. Applied Statistical Decision Theory, (llarvard University P'ress. 1961).

[39] Rosnnschein. Stall. Syntlıssizing Infrrmation-Tracking Automata from Environment. Descriptious. In Brachnan el al. [6], 386-393.

[40] Russell, St.uart J. and Wefalı, Eric H., On Optimal Ciame-Tree Search using Rational Mela-Reasouing, Proceedings IJCAI 11. Detrvit. Michigan. IJCAI. 1989. 334-340.

[41] Russell, Stuart J. and Wefald. Eric II., Principles of Metareasoning, In Brachman et al. [6].

[12] Shachiter. Ross D.. Evaluating Influence Diagrams. Opcrations Rescarch. 34(6) (1!)86) 871-\$82.

[13] Simon, Herbert A. and hadane. Joseph B., Optimal Problem-Solving Search: All-or-None Sulutious. Arlificial Inlelligence. 6 (19i5) 23.5-24i.

[44] Smith. Davill E.. A Derision-Thenitic Appronch to the Control of Plannime Search. Report No. LOCiIC-8T-11. Stanford Logic Group. 19x8.

[45] Weyhrauch. R. IV.. Prolegoinena to a Theory of Mechanized Formal Reasoning. Irtificial Intclligcncc. 13 (1980) 133-1i0. 


\section{Chapter 9}

\section{Learning in Planning and Control}

In the problems considered in previous chapters. we are given a model of the physical process we are trying to control and a specific to goal to achieve or performance index to maxinize. The model provided may not be the most accurate model possible, but once given there is no altempt made to inprove upon it. In order to choose appropriate actions to take, the coutroller has to predict the consequences of its actions as those cousequences relate to the goal or performance index provided in the problem specification. In this chapter, we consider problems in which the system can use its experience. the perceived record of its interaction with the environment. to improve upon its performance by improving its ability to predict the consequences of its actions.

The concept of learning, as it is used in everyday speech, is difficult define precisely. Intuitively, learuing has something to do with clianging behavior in response to experience. However, if we were to equate learning with changing behavior in response to experience, we would be obliged to say that uaing sensor data to delermine what action to take next was a form of learific, Rather than debate what is and what is not learning. we simply: coons word for our own purposes and equate it with certain forms of function epproximation.

In the simplest form of function approximation for control. We assume that some aspect of the environnent can be modeled by a particular function. We generally assume that this function does not change over time. or.

\footnotetext{
${ }^{\circ}\left(C_{1} 1990\right.$ Thoina Dean. All rights reserved.
} 
if it does change, it changes very slumly. The control sistem is given examples in the form of inputs to the function and heir corresponding outputs. From these examples. the sristem is supposed to find an approximation to the function of interest that agrees on the exaluples seen so far and generalizes to those that it has not seen as yet. This type of learning is called supertised leaming since the control system is told exactly wh that is expected for each input provided during learning.

We lalk about approximalions instead of exact functions for a numbrr . of reasuns. By specifying in adrance a parameterized family of functions to represent the function of interest. we can often simplify the search involved in funding a candidate function. The parameterized family of functions also allows us to limit the amount of storage used to represent the function of interest. One drawback to the use of a restricted family oi functions is that the function of interest may not belong to the specified fanily and so we must clioose the function that best approximates the function of interest. A second reason for using approximations is that the control system has to continually respond to its environment. and. at any given point in time. it will want to use whatever information it has so far to guide its choice of action.

What constitutes a good approximation will depend ou any number of factors relating to the performance of the controller. For instance. the amount of storage required to represent the function, the amount of time required to evaluate the function for a given input. and how the results of evaluating the function inpact ou the ability of the controller to achieve its goal or maximize its performance index are all factors that have to be taken into account in evaluating a given approxination.

ln previous chapters. we represented control problems and their solutions using a variety of functions. For instance. the evolution of the state of a dynamical system was represented as a function from states and inputs to states. and a performance index was represented as a function fron sequences of states and inputs to the real numbers. A typical control scheme might involve enumerating a set of possible courses of action. predicting their consequences in terms of the state trajectories corresponding to the predicted evolutiou of the system state. and then comparing the various courses of action by applying a value function to the corresponding state trajectories. This is roughly the approach taken in Chapter 6 with respect to storlaastic dynamic programming and in Chapter $i$ on using Bayesian decision theory for planuing.

In this chapter. we cousider problems simular to thuse investigated in 
C'lapters 6 and $\bar{i}$. In particnlar. We mudel the dynamical system as a stochastic process. and we assume a separable value function in which the rotal value of a state trajectory is the (temporally discounted) sum of the value (reward) at each state. The big difference between the problems of this chapter and those of the eartier chapters is that the controller will not be given the state-transition probabilities for the dynanical system nor will it be given the imnediate reward function.

There are two basic approaches to building a controller for problems in which the dynamics and rewards are not initially specified. In the first approach. the controller attempts to learn the dynanucs and rewards. and then constructs an optimal policy for the resulting model as in Chapters 6 and $i$. We call this approach the cxplicit-morld approach. In the sccond approach. the controller at tenipts to Icarn an optimal policy by constructing an evaluation function to use in sclecting the best action to take when in a given state. The controller constructs this evaluation function withont recourse to an explicit model of the system dynamics. and so. while the system cannot predict what the state resulting from a given action will be, it can determine whether that resulting state is better or worse than the state resulting from any other action. We call the second approach the direct approach.

In the explicit-model appronch, the control system has to learn two functions. First. it has to learn the dynamics. a function fron states and actions to distributions over states. Second, the system has to learn a function from states and actions to the real numbers. From these two functions. the systcm constructs a third function, a policy or control law. from states to actions.

Of course, it is not as simple as. learn the dynamics and rewards. and then construct a policy and follow it ever after. The control system has to continue to operate while it is learning the dynamics and rewards, and this introduces some complications reminiscent of the interaction between observation and control in systems for which the separation property does not hold. The problen is that the controller has to visit all of the states and ery out all of its options in every state sufficiently often to construct an accurate statistical model. This means that the controller has to systematically explore its environment and experiment with various policies in order to ensure that it will construct an optimal policy.

In the direct approach. the system also learns two functions. First. it learns a function from states to the real numbers. This function is essentially the value function for a fixed policy introduced in Chapter 6 . but here we attempt to learn this function without the use of an explicit dynamical 
model. Second, the system learns a function from states and artions to the real numbers that is used for selecting what action to take next. Here again the problem of exploration and experimentation comes up. The calculation of the value function assumes a fixed policy, but the cont roller has to deviate from the fixed policy in order to explore its environment in sufficient detail to find the optimal policy.

In both the explicit model and direct approarhes, the ultimate objective is to learn an optimal policy, a function from states to actions that maximizes expected rumulative discounted reward. The system does not, however, learn by being given examples of states and the optimal actions to take in those states. Rather, the system performs actions in states and is given feedback in the form of rewards. This type of learuing is called reinforcement learuing.

Reinforcement learning is complicated by the fact that the reinforcement in the form of rewards is often intermittent and delaved. The controller may perform a long sequence of actions before receiving any reward. This makes it difficult 10 attribute credit or blame to actions when a reward finally is received. In chess or checkers, reinforcenent occurs in the form of lost pieces or lust games, and the reason for losing a piece or a game is seldom completely dne to the last action taken before the luss. The problem of attributing credit or blame in such circumstances is called the creditassigmment problem, and any solution to the problents addressed in this chapter will require a solution to the credit-assignment problen.

The rest of this chapter is orgauized as follows. First. we cousider some basic lechuiques for learuing functions. We then return to the problem of learning an optimal policy, concentrating on the direct approach described above. In looking at the problem of learning an optimal policy. a number of computational issues become rritical in considering problems with large input spaces. We consider approaches that address the problem of coping with large input spaces. We then lake another look at learning optimal policies in terms of learning rules. Finally, we consider some issues cuncerned with the ability of a learming system to perceive the true state of the vorld.

\subsection{Function Approximation}

We characterize a function-learning problem in terms of

- a dumain set $\boldsymbol{x}$, 
- a range set $Y$. and

- a set of candidate functions $F=\left\{f: \mathrm{I}^{-}-\mathrm{I}^{-}\right\}$.

In the cases we are interested in. Lhe domain is often the state or output space of a dynamic system. and the range is often the input space of a dynamic system or the real numbers in the case of learning a value function. In most cases. the set of candidate functions can chasacterized by a finite set of parameters.

For instance. in the case in which $I=Y=R$. the set.

$$
\left\{C_{0}+C_{1} x+C_{2} x^{2}+C_{3} x^{3} \mid C_{0} \cdot C_{1} \cdot C_{2} \cdot C_{3}^{\prime} \in \mathbf{R}\right\} .
$$

represents the set of all polynonuals of degree 3 or less. and is characterized by four real-valued parameters.

The size of the parameter set is often a good indication of the storage reguired for a given function learning problem. In some cases. the storage required for a problem is equal to the size of the domain set. For instance, suppose that the domain set is a finite subset of the integers, $x \subset Z$, and the range is the real numbers. Consider the set of candidate functions,

$$
\left\{\sum_{i \in X} C_{i} \mathcal{I}_{i}(x) \mid C_{i}^{\prime} \in \mathbf{R}\right\},
$$

where $\mathcal{I}_{i}$ is the characteristic or indicator function for the singleton set consisting of just $i$ and defined by

$$
I_{i}(x)=\left\{\begin{array}{ll}
1 & \text { if } x=i \\
0 & \text { if } x \neq i
\end{array},\right.
$$

In this case. we have one real-valued parameter for each element of $X$.

It may be difficult. impossible or even unnecessary to characterize the set of candidate functions using a finite set of parameters. It may be difficult or impentuly if the function varies erratically or randomly over some portion of id main. It may unnecessary if all we require is an approximation of the funetion. For the problenss we are interested in. a good approximation will snfice for acceptable control. For instance. in learning a value function for control. all the controller carcs about is whether performing one action is better than performing another: being able to compute an exact value or even a value to 10 siguificant digits is not likely to improve the performance of the controller. 
Let $X$ be any set. $\{X, \| \leq i \leq n\}$ partition ${ }^{1} X$. and $Y=R$. Consicler the set of candidate functions.

$$
\left\{\sum_{i=1}^{n} C_{i}^{\prime} I_{i}(x) \mid C_{i}^{\prime} \in \mathbf{R}\right\} .
$$

where. in this case. $\mathcal{I}_{i}$ is the indicator function for the set $X_{i}$,

$$
\mathcal{I}_{i}(x)= \begin{cases}1 & \text { if } x \in X_{i} \\ 0 & \text { if } x \notin X_{i}\end{cases}
$$

In this case. we have partitioned the clomain into a finite set of regions and assigued a single real-valued parameter to each region. This allows us to represent exactly a class of piecewise-constant functions with $n$ pieces where the pieces correspond to the regions of the partition. We can approximately represent a much larger class of functions.

You can probably think of several. more general methods of characterizing classes of candidate functions. For instance. the set of regions need not define a partition; the regions might intersect or the set might not cover the entire domain. In addition. the set of regions need not remain static thoughout the learning process; their boundaries night be characterized by additional paraineters.

The regions referred to above are often called receptice fields in the literature on artificial neural networks. In some cases. each receptive field is characterized by two parameters. a point in the domain set. $\mathbf{R}^{n}$. and a dialleter. together describing an $n$-dimensional spherical region of the domain. Each receptive field has associated with it a small annount of storage used to represent soine aspect of the behavior of the function in the. region covered by the field. These fields can be moved about to obtain a better approximation of the function. Large fields can be used to represent the behavior of the function in regions where not much is going on. Several small fields can be used to represent the behavior of the function in regions where a lot is going on.

In addition to allowing the regions to vary, the behavior of the function in a given region can be characterised by any finitely paraneterizable function. The variety of learning prohlems is considerable. and it is not our purpose here to survey those problems in any detail. In the following. we consider

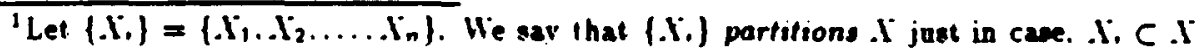
for $1 \leq i \leq n . \bigcup_{i=1}^{n}, X_{1}=X$. and $X, X_{j}=$ for all $i$ and $j$ such that $i \neq j$.
} 
a very restricted sort of function learning in order illustrate some basic principles and provide some machinery lhat will be of use in subsequent sections.

In the following. we assume that the range set is the real numbers. and consider only very simple sets of candidate functions of the form.

$$
F=\left\{\sum_{i=1}^{n} u ; o_{i}(x) \mid u_{i}, o_{i}(x) \in \mathbf{R}\right\},
$$

where $o_{i}: \not-R$ is an arbitrary function, and we use the notation, $u_{i}$, for the parameters to indicate that they are variable ueights.

The set of functions, $\left\{\phi_{i}\right\}$, are oftell called features in the literature. Such features might model measurements taken by differeut sensors that detect. whether or not a specific property holds of the input. $x$. In general, each function. $\phi_{i}$. processes the input in some manner and issues a real number which is weighted by the parameter. $u_{i}$, and combined with the other features. The functions so represented are linear combinations of the features thongh the features themselves ueed not be linear functions.

We can rewrite

$$
\left\{\sum_{i=1}^{n} w_{i} \phi_{i}(x) \mid w_{i}, \phi_{i}(x) \in \mathbf{R}\right\},
$$

in vector notation as

$$
\left\{w \phi(x) \mid w . \phi(x) \in \mathbf{R}^{n}\right\}
$$

where the first terin, called the paraneter vector, is defined by

$$
w=\left\langle w_{1}, w_{2}, \ldots w_{n}\right\rangle \text {. }
$$

the second term. called the ferture vertor, is defined by

$$
\phi(x)=\left\langle\psi_{1}(x), \omega_{2}(x), \ldots \phi_{n}(x)\right\rangle .
$$

and the implied operator separating the two vectors is the inner product.

To indicate a member of $F$. it is enough to specify a vector $w \in \mathbf{R}^{n}$. Learning generally proceeds by incrementally adjustiug the reights to specify an updated parameter vector. It any given point. the learning sustem will have seen a set of input/output pairs.

$$
\left\{\left(x_{i}, y\left(x_{i}\right)\right\rangle \mid 1 \leq i \leq k\right\} \text {. }
$$


where $y(x)$ denotes the output of the function we are trring to learn for the input. $x$. One standard criterion for selecting weights is to determine the parameter vector that mininizes the mean of the squared error. That is. We wish to find $\mathbf{w} \in \mathbf{R}^{n}$ minimizing the silm.

$$
\frac{1}{h} \sum_{i=1}^{k}\left(\left(x_{i}\right)^{2}\right.
$$

where the ermr term. $f(x)$. is defined as

$$
\epsilon(x)=y(x)-\mathbf{w} \phi(x) .
$$

If we are willing to keep around the entire sequence of input/ouput pairs. we could compute the parameter vector minimizing the mean of the squared error directly: The mean of the squared error is a convex function of the weights and hence it has a unique minimum. As a consequence. we can coup pute the parametcr vector nuinimizing the sum of the squared crror by simply setting the gradient.

$$
\nabla\left[\frac{1}{k} \sum_{i=1}^{k} \epsilon\left(x_{i}\right)^{2}\right]=-\frac{2}{k} \sum_{i=1}^{k} c\left(x_{i}\right) \phi\left(x_{i}\right) .
$$

to zero and solving the resulting system of equations for the weights. Alte. liatively. we call use gradient-descent search methods to find the weights. Rer all that gradient-descent search proceeds by making small changes to the parameter vector in the direction indicated by the negative gradient.

It is generally assumed. however. that either the system cannot afford the storage to keep around all of the training data. or that it would be useless to keep around all of the training data given that the function we are trying to learn changes gradually over time. In keeping with this assumption. we are interested in methods that proceed by inaking small changes to the pu ameter vector on the basis of the last example.

Let $w_{t}$ and $x_{t}$ denote. respectively. l he parameter vector and the example at time $t$. In a manner similar to that cniployed in gradient descent. We makc ad.ustments to the parameter vector on the basis of the last example. using the following update rule.

$$
w_{t+1}=w_{t}+b \epsilon_{t}\left(x_{t}\right) \phi\left(x_{t}\right)
$$

where the error term in this case is defined as

$$
\epsilon_{f}(x)=y(x)-w_{t} \phi(x) \text {. }
$$


aud the scalar. 3 . is the learning rate or gain of the update rule. This update rinle is called the Irast monn squar (IASS) rule and is due to Widrou and Iloff [21]. This rule is also closely related to the perreptron learning rule of Rosenblatt developed for pattern classification [15].

If there is storage available. we can improve the estimate of the gradient by taking into account more than just the last example. Ceneralizing on the LISS rule. we have the rule.

$$
w_{t+1}=w_{t}+\beta \frac{1}{k} \sum_{i=t-k-1}^{t} \epsilon_{i}\left(x_{i}\right) \phi\left(x_{i}\right) .
$$

accounting for the last $k$ examples.

In order for the above learning method to converge to a fixed parameter vector closely approximating the function of interest. the serqueuce of training examples has to represent a sufficiently varied subset of the set of all such examples. Exactly what constitutes a sufficiently varied set of exainples will depend upon the class of functions being learned. but. intuitively, you want examples drawn from across the domain with more examples in regions where the behavior of the function is more complex.

Experiment 1 To illustrate the performance of the function-learning approach described above, suppose that the target function is the cubic polynomial.

$$
y(x)=1.20-0.2 x+3.1 x^{2}-0.9 x^{3} .
$$

and the examples are drawn (psendo) randomly from the set.

$$
\{(x, y(x)) \mid-1 \leq x \leq 1\}
$$

Figure 9.1.i shows the performance of the LMS update rule nith $k=\infty$ (i.e. use all of the exainples encountered so far) and $\beta=0.1 .^{2}$ The approximation aftcr 100 examples is

$$
W \phi(x)=1.311+42-0.290810 x+2.900 i 18 x^{2}-0 . i 63296 x^{3}
$$

Figure 9.1.ij $k=1$ shows the performance of the LMS update rule with $k=1$ (i.e.. use only the last example encountered) and $j=0.1$. The approximation after 400 examples is

$$
\mathbf{w} \phi(x)=1.292608-0.238687 x+2.966245 x^{2}-0.81559 x^{3} \text {. }
$$

\footnotetext{
${ }^{2}$ Hideki leozaki supplied the data for the graphs sliown in Figure 9.1.
} 

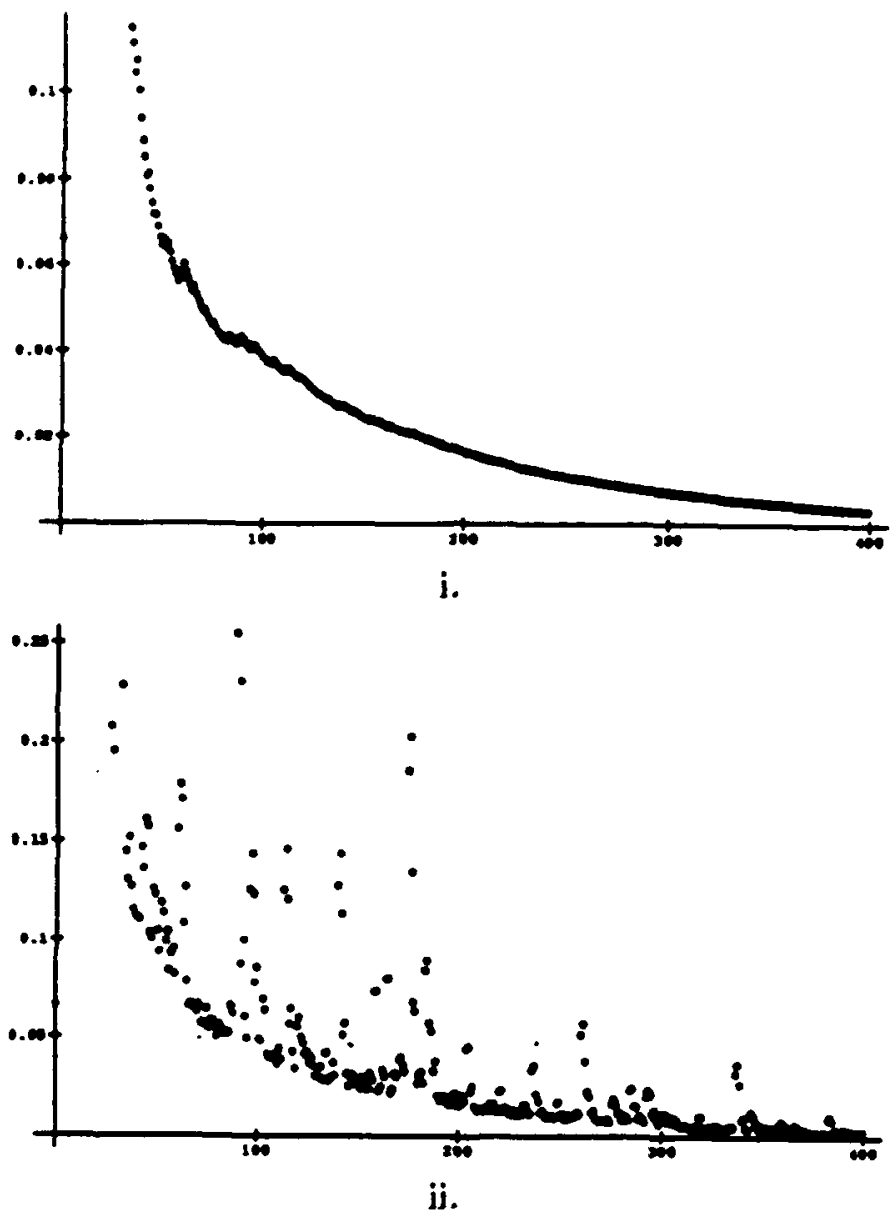

Figure 9.1: Performance of the generalized LMS rule 
Now we have techniques that will allow us to select a good approximation from a set of candidate functions given a set of training examples. We can IItilize any a priori knowledge we have of the function of interest to hias the learning process by selecting appropriate features to constrain the set of candidate functions. In selecting a set of features to represent the problem. one can make the learning problem trivial (c.g.. you select the function of interest as one of the features) or inpossible (c.g., the function of interest cannot be closely approximated by a linear combination of the features).

The performance of a function approximation technique is measured in terms of the a mount of storage required. the time. required for each update. and the expected accurracy of the approxination (E.g.. the nean squared error) as a function of the number of training examples seen so far. There are a host of other function approximation techniques. but their performanre insariably depends "pon starting with a good representation. The linear method utilizing the LMS rule described above is prohally the hest understood method, and, despite its limitations (f.g., it can only be used to represent functions that can be described as a linear combination of the features), it is often the method of choice in building practical learning systems.

The learning methors discussed in this sertion can also be viewed as special-purpose memories. In the case of there being one parameter per member of the domain set, learning corresponds to just filling in the entries in a large table. In soine cases. the set of features allow the learning system to generalize from the set of examples seen so far to those that it has yet. to see. It is this notion of generalization. that people often closely assoriate with learning. Once again, the ability of a system to generalize depends critically npon the representation chosen.

\subsection{Policy and Value Learning}

As indicated in the introduction to this chapter. we intend to narrow the scomif our discussion to focus on learning an optimal policy for a stochastic sequ decision making task. We are interested in any ronte to the goal of kefing an uptimal policy, but the discussion of Chapter 6 suggests unte relatively straightforward approach. The approach is to learn the transitiun probabilities and the reward function and then employ Howard's policy iteration techuique to compute the optimal policy.

Let $X$ be the state space of the dyrumical system. and $C^{*}$ be the input 
space. Assuming "uat it is possible in directly observe the state of the dynamical system. the controller would start by executing a random walk (i.e.. it would select its actions according to a uniform distribution). Let $\delta: X \times U \times \mathbb{X}-\mathbf{Z}$ be the trinsition-stritistics function, and $\mu: X \times U \times$ $\mathbf{X}-\mathbf{R} \times \mathbf{Z}$ be the reurarl-statistics function. Luitially, let $\delta\left(x, u, x^{\prime}\right)=0$. and $\mu\left(x, u, x^{\prime}\right)=\langle 0.0\rangle$ for all $x . x^{\prime} \in X$ and $u \in U$. Every time that the controller performs an artion, $u$. in state. $x$. resulting in next state. $x^{\prime}$. the controller would update the trausition-statistics function by incrementing $\delta\left(x, u, x^{\prime}\right)$ by one. Similarly. every time the controller receives a reward, $r$, in state, $x^{\prime}$, having started in state. $x$, and performed action. $u$, the controller would update the reward-statistics lunction so that $\mu\left(x, u, x^{\prime}\right)=$ $(s+r . n+1)$, where prior to the $\| p d a t e \mu\left(x, u, x^{\prime}\right)=\langle s, n\rangle$. Alter a period of time delermined by liow accurate a model is required. he would compute estimates of the transition probabilities.

$$
\operatorname{Pr}\left(x(t+1)=x^{\prime} \mid x(t)=x \cdot u(t)=u\right)=\frac{\delta\left(x \cdot u \cdot x^{\prime}\right)}{\sum_{x^{\prime \prime} \in X} \delta\left(x, u, x^{\prime \prime}\right)},
$$

and rewards,

$$
R\left(x, u, x^{\prime}\right)=\frac{s}{n} \text { where } \mu\left(x, u . x^{\prime}\right)=\langle s, n\rangle,
$$

and use policy iteration to compute the optimal policy given the estimates for the rewards and transition probabilities.

In theory, the approach outlined above is perfectly reasonable. There are, however, disadvantages. First, it may not be desirable for a robot to perform a random wall during the training period; the robot night become a nuisance or damage itself. Second. the transition probabilities may change gradually over tine; $\ddot{a}$ robot with a fixed training period may construct an initially optimal policy, but that policy unight become significantly sulsoptitual as the transition probabilities change over time. 'Third, policy iteration is computatiunally rather expensive. We consicler each of these three clisaclvantanes in lurn.

Whith regard to performing a randum walk during traituing, the rubot has to explore the space of possible state I ransitions thoroughly enough to obtain reliable statistics. This does not mean. Lowever. that the rubut has to perform actions that are obviously dangerous or socially incorrect, since those actious will, presumably. never be a part of an optimal policy anyway. One obvious method for avoiding dangerous or antisocial behavior is to build the learning system on top of a base controller that only exhibits safe. 
socially correct behavior. In this case. the outputs of the lrarning systcm are the inputs to the base controller. This basic idea of bnilding a learning system on top of an existing cont roller applies to any approach to learning.

With regard to the transition probabilities changing over time. there is no need to have a fixed training period in the scheme outlined above. The controller could continually gather statistics on the rewards and transition probabilities and periodically update its policy. The only problem is that the controller may not obtain adequate statistics if it always follows what it believes to be the optimal pulicy. Hence. in addition to perionlically updating its policy, the controller will have to periodically engage in some exploratory behavior in order to assure that its estimated rewards and transition probabilities are accurate.

The problem in dealing with computational costs is a bit more troubling. Policy iteration is polynomial in the sizes of the state and input spaces. Value determination. which is performed once in each iteration of the policy iteration procedure. requires solving a sustem of $|X|$ simultaneous linear equations. If most of the : ransition probabilities are not zero. simply representing this system of equations takes $O\left(|\mathbf{X}|^{2}\right)$ space. but keep in mind that. in the case of mostly nonzero transition probabilities. it will require $O(|X \times U \times X|)$ space just to store the transition probabilities.

This problem arising from the sizes of the state and input spaces is often called the curse of dimensionality. Generally, the state and input spaces can be viewed as a cross product of subspaces. For example. we might represent the state space. $x$. as an $n$-diniensional product space.

$$
x=\prod_{i=1}^{n} x_{i}
$$

where $\left\{X_{1}, x_{2}, \ldots, X_{n}\right\}$ are the component subspaces. Each subspace. $X_{i}$, might. represent a different property of the environment (c.g.. the robnt's current position. orientation, or amount of remaining fuel). Some of the component subspaces night represent a finite discretization of an infinite space.

Individually, the sizes of the subspares night be modest. but the prospect of quantifying over a product space of size.

$$
|\boldsymbol{X}|=\prod_{i=1}^{n}\left|\mathbf{x}_{i}\right| \text {. }
$$

can be daunting from a computational perspertive. This can he esperially frustrating if large portions of that product space are unreachable (c.g.. if the 
robot's battery is completely dis llarged it cannot have a positive velocity). or uninteresting (f.g.. the robot might be able to detect light. but. for most tasks. the intensity of light has no influence on the robot's choice of action as it. Ilavigates using sonar).

The curse of dimensionality raises a deep issue that will not go away: it is not problem that can be solved. In the following section. we return to this issue. but for the time being we ignore it and consider some approaches that circumvent some of the problems that arise regarding computing optimal policies.

Suppose. for the sake of argument. that the controller has a time- and storage-efficient procedure that, given a state and an action, returns a (next) state according to the dist ributions specified by the dynamical system. (iiven this prccedure. which we refer to as the tmasition omcle, and a reward function. we can now compute an optimal policy by using the following simple stochastic approximation (Monte (arlo) routine for value determination in the standard policy iteration algorithm.

Here is the stochastic value determination routine. For each $x \in X$, compute $\mathrm{V}(x)$ as follows. Use the transition oracle to deternuine $m$ state transition histories of length $k$,

$$
\begin{gathered}
x_{1,1}, u_{1,1}, x_{1,2}, u_{1,2}, \ldots, u_{1, k-1}, x_{1, k} \\
x_{2,1}, u_{2,1}, x_{2,2}, u_{2,2}, \ldots, u_{2, k-1}, x_{2, k} \\
\vdots \\
x_{m, 1}, u_{m, 1}, x_{m, 2}, u_{m, 2}, \ldots, u_{m, k-1}, x_{m, k}
\end{gathered}
$$

where $x_{j, 1}=x$ for $1 \leq j \leq m$, the actions are determined by the current policy.

$$
\eta\left(x_{j, i}, u_{j, i}\right)=x_{j, i+1},
$$

and the state transitione are obtained from the transition oracle. We obtain the approximate value of the state. $x$. given the policy, $\eta$. as

$$
\mathrm{V}(x)=\frac{1}{m} \sum_{j=1}^{m} \frac{1}{k} \sum_{i=1}^{k} \lambda^{i} \mathbf{R}\left(x_{j, i}, u_{j, i}, x_{j, i+1}\right) .
$$

This approximation converges to the true value in the limit as $m$ and $k$ tend to infinity. If in addition to the transition oracle. we are given a time- and storage-efficient means of computing rewards. a reward oracle. then we can compute the optinal policy in a very space efficient manner by some careful programming. 
Or course. the point of this uracle business is that we do indeed have snch oracles. at least in a manner of speaking. The world is our oracle: the rewards and state transitions that it visits upon us are exactly the state transitions and rewards of the physical process that we attempt to capture in our dvnamical models.

In the remainder of this scction. We consider methods for learning optimal policies that rely upon performing experiments in the real world rather than upon explicitly modeling the dinamics and rewards. These methorts emphasize storage efliciency. and. in some cases. were originally conceived of as models of learning in biulugical organisms. In light of the issues that arise with regard to high-dimensional state and input spaces. this focus on storage-efficient methods is likely to have important engineering consequences as well.

In the following approach. we assume that the controller has adequate storage for a value function. $\mid \vec{I}-\mathbf{I}-\mathbf{R}$. In addition, we assume that the controller has storage for a function to be used in computing the policy. This might just be a policy function, $\eta: . \Gamma-U$, or it might be something a bit more complicated, for instance. a function from states and actions to the real numbers providing some expectation of cumulative reward. We assume very little in the way of computation at each state transition. We begin by considering how to learu the value function for a fixed policy. starting with a very simple case.

Consider a finite-state. deterministic dynamical system with a fixed policy. We assume that every state is reachable from every other state. and proceed as we did in the prcvious section on function approximation. Let $x=\{1.2 \ldots, n\}$, and $\mathbf{v} \in \mathbf{R}^{n}$. Since $\mathbf{v}$ changes over time. we provide a temporal index. $v_{i}$, to distinguish between the values at different points in time. Similarly, let $x_{t}$ and $r_{t}$ denote. respectively. the state and the reward at time 1 . Let $V_{t}(i)=v_{t}[i]$, where $v_{t}[i]$ indicates the $i$ th component of the vector $v_{t}$. We define the vector of features.

$$
\phi(x)=\left\langle\Phi_{1}(x) \cdot \phi_{2}(x) \ldots \ldots \phi_{n}(x)\right\rangle .
$$

where

$$
\phi_{i}(x)= \begin{cases}1 & \text { if } x=i \\ 0 & \text { if } x \neq i\end{cases}
$$

C'onsider the following simple update rule.

$$
v_{t+1}=v_{t}+\left[r_{t+1}-v_{t}\left(x_{t}\right)\right] \phi\left(x_{t}\right) .
$$


In this rase. if the system is allowed to run ind initely. the parameter vector will converge to a fixed value given by $Y\left(x_{t}\right)=R\left(x_{t+1}\right)$.

To haudle sequential decision problems of indefinite cluration with discounting rate. $\lambda$. for rewards, we emplor the following variation on the above rule.

$$
v_{t+1}=v_{t}+\left[r_{t+1}+\lambda V_{t}\left(x_{t+1}\right)-V_{t}\left(x_{t}\right)\right] \phi\left(x_{t}\right) .
$$

Here also the parameter vector converges to a fixed value. but. in this case. the value is identical with that obtained using the value determination routine of Cliapter 6.

The above equations should look vaguely familiar. They have the same basic form as the LMS learning rule introduced in the previous section. In the discounting case. the error term is just the difference between the current estimate of the state value. $V\left(x_{t}\right)$. and the rcvised estimate of this value. $r_{t+1}+\lambda V_{t}\left(x_{t+1}\right)$. The abo'e ' 's sinuplifies to just

$$
V_{t+1}\left(x_{t}\right)=r_{t+1}+\lambda V_{t}\left(x_{t+1}\right) \text {. }
$$

The stochastic case is somewhat more complicated. We assume a conpletely ergodic Markov process so thal every stale is visited infinitely often. In this case, the revised estimate of the value of the state, $x_{t}=i$, should be

$$
r_{t+1}+\lambda \sum_{j=1}^{n} \rho_{i j} V_{t}(j), \chi_{j}
$$

where $\rho_{i j}$ is the transition probability defined for the current policy. Of course. we do not have the transition prchai.lities so instead we simply make use of what we do have. The update rule for the stochastic case is exactly the same as the rule for the deterministic case with one variation,

$$
v_{t+1}=v_{t}+1 /\left[r_{t+1}+\lambda V_{t}\left(x_{t+1}\right)-V_{t}\left(x_{t}\right)\right] \phi\left(x_{t}\right) \text {. }
$$

we introduce a learning rate. $0<i \leq 1$. as in the LMS learning rule. In the stochatic case, the values do not converge to the values indicated by value determination. Instead, they Huctuate about the expected valnes according to the most recent state transitions. The variance in these fluctuating values is bounder, and can be made arlitirarily smiall by an appropriate choire of 3. or reduced asymptotically to zero hy choosing an appropriate srhedule for $3\left(\right.$ f.g.,$\left.\beta=\frac{i}{l}\right)$.

Vote the revised value estimates in the above equations are just a special case of estimating long-term returns on the basis of some number of observed 
rewards. In general. We can make use of any number of ohserved rewards using estimates of the form.

$$
V_{t}\left(x_{t}\right)=r_{t+1}+\lambda r_{t+2}+\cdots+\lambda^{n-1} r_{t+n-1}+\lambda^{n} r_{t+n}\left(x_{t+n}\right) .
$$

Estimates with more observations are generally better in that they provide more accurate estiniates and speed learning. but they also require more memory and computation.

Experiment 2 Provide an example illustrating the steady-state performance of an estimation routine using the above update rule. Use the mean of the squared error as an evaluation metric and the robot-courier problem as a test case.

Now that we have a method for conpuning the value function for a given policy. the next step is to develop a method for improving the current policy. To that end. we introduce the idea of learning the expected value of actions. For each state, $x$, and action. $u$. we allocate memory, $W(x, u)$. for storing an estimate, called an action calue, of the expected value of performing that action in that state. Initially all the action values are zero. The update rule uses the value function introduced in the previous paragraphs.

$$
W_{t+1}\left(x_{t}, u_{t}\right)=W_{t}\left(x_{t}, u_{t}\right)+\alpha\left[r_{t+1}+\lambda V_{t}\left(x_{t+1}\right)-V_{t}\left(x_{t}\right)\right] .
$$

where $u_{t}$ is the action taken at time $t$, and all uther actions values. $W_{t+1}(x, u)$ c.rrl that either $x \neq x_{t}$ or $u \neq u_{t}$, remain the same. The intuition behind 1.. . $e$ is as follows.

Recall that $\mathrm{V}$ is the estimated value function with respect to a particular policy. If $u_{t}$ is the action indicated by the current policy in state $x_{t}$, then the error. $\left[r_{t+1}+\lambda V_{t}\left(x_{t+1}\right)-V_{t}\left(x_{t}\right)\right]$, should be zero on average. $O n$ the other hand. if ", is some action other than that recommenderl hy the current policy, then the error will be greater than, less than, or equal to zero on average. depending on whether or not laking that action and then following the rnment policy thereafter results in a higher. Inwer. or identical expected value compared to that for the recommended action.

Now that. assuming the controller sticks to a fixed policy, the values specified by $W$ with the exception of those corresponding to the reconmendations of the fixed policy will not converge: rather. they are likely to increñse or decrease without bound.

These values do. however. provide us with useful information in deciding huw to improve the current policy: the relative values tell us what actions to 
change in the current policy in order to clefine an improved I slicy. Consider the following approach.

1. Following the current policy and updating only the value function, perform a number of steps so that the values for the current policy are good approximations of the actual value function.

2. Set $W_{t}(x, u)=0$ for all $x \in \mathbb{X}$ and $u \in \mathbb{U}$ where $t$ is the current time.

3. Fullowing a randum policy. and. "prlating only the action values. perform a number of steps so that the relative action values are in keeping with the actual cxpected action values with high probability.

4. I'sing the relative action values. choose a new policy.

$$
\eta(x)=\arg \max _{u} W(x, u) .
$$

and set it to be the current policy.

\section{Cio to Step 1.}

The ahove method directly mimics the policy itcration routine introduced in Chapter 6 using stochastic methods instead of exact methods for the value determination and policy improvenent steps. One drawback is that it is likely to take a very long time to converge to an optimal policy. As an alternative to this method, researchers have tried approaches that involve running stochastic value determination and policy inprovemeut continuously. Instead of switching back and forth between a current estimated best policy and a random policy, these approaches generally employ a stochastic policy that, on average, chooses actions from the current estimated best policy, but. according to a fixed distribution. occasionally deviates and experiments with actions other than those recommended by the current policy. It gencrally helps if the value function is only updated if the action selected is the same as the action rccommended by the current policy.

No one has as yet proved that these alternative approaches converge to the op:ininl policy. though they clo appear to converge in practice. However. there is one learning method that has been shown to converge in the limit. This nethod is also interesting because it is a stochastic variant of the value iteration approach described in ('hapter 6 rather than policy iteration approach. 
Recn!l that value iteration is a terhnique that uses successive approximation to compute a value function that converges in the limit to the value function for the optimal policy. The policy at each point in time is determined by the actions that maxinize the current estimate for the optimal value function. lnstead of learning both a value function and a set of action values. the controller learns just the artion values. but, in this approach. the action value are updated by the following learning rule.

$$
W_{1+1}\left(x_{1} \cdot u_{1}\right)=W\left(x_{1} \cdot u_{1}\right)+a_{1}\left(x_{1} \cdot u_{1}\right)\left[r_{1+1}+\lambda \max _{u} W_{t}\left(x_{1+1} \cdot u\right)-W_{1}\left(x_{t}\right)\right] .
$$

where. in order to guarantee convergence. we have to vary the learning rate. $x_{t}$. over time according to a schedule satisfying certain requirenents.

Note that in order to guarantee that the procedure will find the optimal policy in the limit. it is enough to to guarantee that $W$ converges to the optimal value function in the limit. To guarantee that W converges to the optinal value function in the limit. it is sufficient that. for each pair consisting of a state. $x$. and an action. $u$. the following statenents hold.

1. The controller attempts action. $u$, in state. $x$, an unbounded number of times as $t \rightarrow \infty$.

2. The learuing rate $\alpha_{t}(x, u)$ tends to zero as $t-\infty$.

3. The sum $t_{t=0}^{n} t_{t}(x, u)$ increases without bound $\infty$.

Actually. these are very modest requircments. The first statement just requires that the coutroller not permanently ignore portions of the space of states and actions. The second and third restrictions are satisfied by a learuing schedule of the form $\alpha_{t}(x, u)=\frac{1}{t}$.

Experiment 3 Provide an example illust rating the performance of the two learning approaches described above. Once again. use the mean of the squared error with respect to the optimal value function as the performance metric and the robot-courier problem as the test example.

At this point, we can learn an optimal policy. We have a method that is ginaranteed to converge in the limit and that appears to work well in practice for simple problems. The learning methods considered in this section are generally time and space-efficient with the exception of the memory requiterl for storing the requisite functions. Since these functions generally requnire $O(|X|)$ space. it it is worthwhile considering methods to reduce this storage overhead. The next section is concerned with exactly this issue. 


\subsection{Coping With Large Input Spaces}

I.et $x$ be the domain of the function we are interested in learning. Suppose that $|\mathbf{X}|$ is large; so large that it is impractical to allocate storage for each $x \in X$ in the case of a finite $X$ or for each region of a reasonable finite discretization in the case of an infinite $\boldsymbol{X}$. If the function we are trying learn has complex behavior throughout its domain and that behavior does not generalize, then we are in trouble. However, if we are only interested in the behavior of the function in certain regions of $X$ (we assume that we do not know these regions in advance or otherwise we would simply restrict the domain ). or the behavior of the function is only occasionally of sufficient complexity to warrant siguificant amounts of storage for its approximation. the!i we can. at least in certain circumstances. learn a good approximation usine an amount of storage significantly less than that required by $X$.

The basic idea is quite simple: we employ hashing techniques to map a large space into a significantly snaller one. The smaller space is represented by a finite number of storage elenents containing the parameters for the family of candidate functions. Learning proceeds by adjusting these parameters using your favorite learning rule. LMS in the cases that we consider.

The method was originally conceived of as a computational model of motor learning in the cerebellar cortex. It was discovered by James Albus [1] and David Marr [8] independently: but it is generally referred to as the CMAC approach. after the name given to it by Albus, the Cerebellar Model Articulation Controller [2].

As was mentioned. the basic idea is to map a large space onto a smaller one using hashing. As with all hashing techniques, there is always some danger of collisions, the results of mapping different elements of the larger space onto the sanie element of the smaller space. In some cases. this is a good thing (e.g.. when the value of the function is the same for each element of the larger space). but. in others. it degrades performance. To avoid the bad consequences of hashing. C'MAC eniplovs several mapping functions each of which maps each point in the domain into a different storne element as shown in Figure 9.2. The output of CMAC for a given elemans of the domain is the average of the values in the storage eleinents determined by all of the mapping functions. In the following. we introduce notation to describe CMAC: more precisely. 


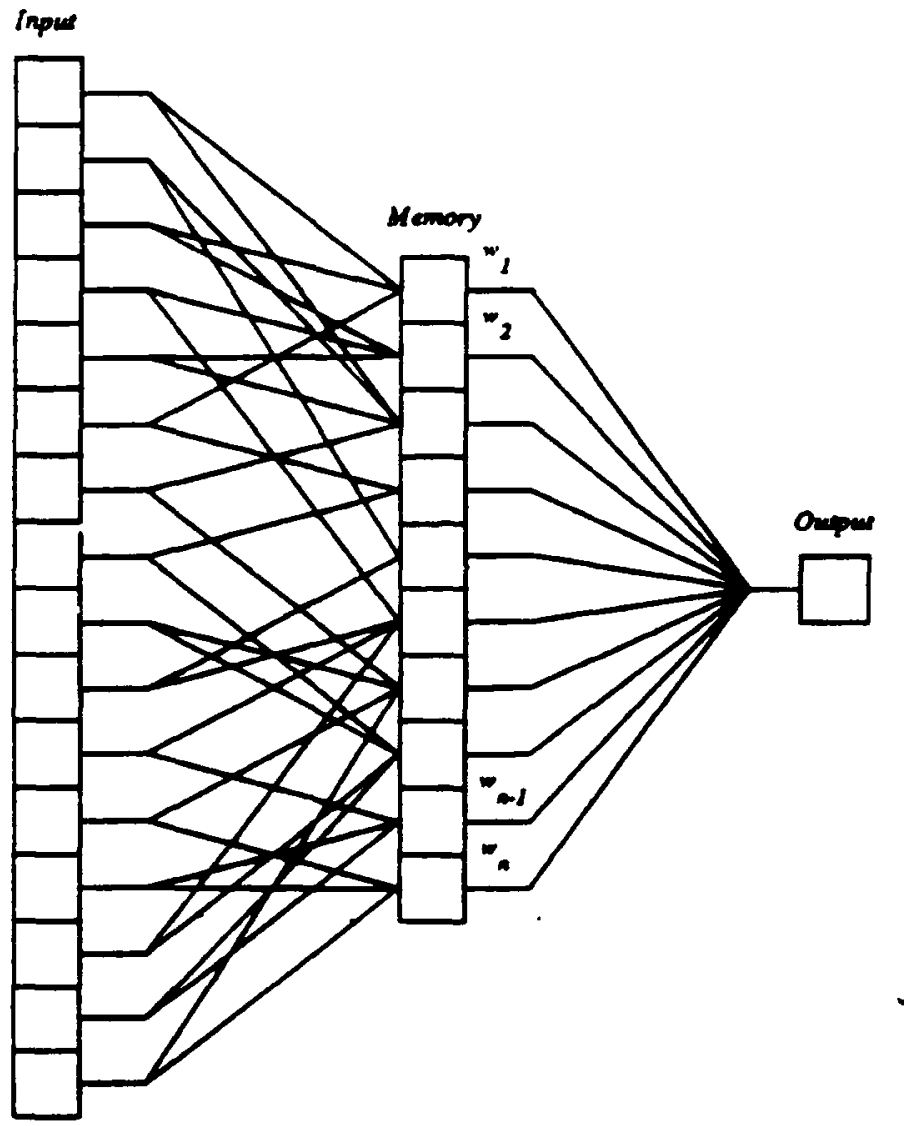

Figure 9.2: Mapping a large domain onto a snaller one 
We begin by defining $m$ partitions of the set $x$.

$$
\begin{gathered}
r_{1.1}, r_{1.2}, r_{1.3} \ldots \\
r_{2.1}, r_{2.2}, r_{2.3} \ldots \\
\ldots \\
r_{m .1} . I_{m .2} . r_{m .3} \ldots
\end{gathered}
$$

A simple and effective methud of generating the $m$ partitions for $X=\mathbf{R}^{d}$ is to create an initial partition. and then modify it to create the $m-1$ remaining partitions. Each of remaining partitions is generated by uniformly displacing the regions of the initial partition by a fixed offset. so that no two partitious have the sante region buundaries.

We need to define a function mapping $x$ to the smaller set $\{1,2 \ldots \ldots n\}$. To provide the redundancy repuired to avojd the problems caused by hashing collisions. we deline $m$ functions. $M_{a} a_{i}: X-\{1,2 \ldots \ldots n\}, 1 \leq i \leq m$, one for each of the $m$ partitions. The ith mapping function is defiued.

$$
\operatorname{Map}_{i}(x)=\operatorname{IIash}\left(\operatorname{Region}_{i}(x)\right) .
$$

where Hash : $\mathbf{Z}-\{1,2 \ldots, n\}$ is the hashing function. and Region $_{i}: \mathrm{X}-$ $\mathbf{Z}$ is defined as

$$
\operatorname{Region}_{i}(x)=j \text { such that } x \in \mathbf{Y}_{i, j} \text {. }
$$

In the case of $\boldsymbol{X}=\mathbf{R}^{d}$, if the regjons of the partitions are isothetic rectangles ( $d$-dimensional rectangular regions aligned with the coordinate axes), then computing the region coutaining $x$ is simple.

In the sinplest case of learning a scalar-valued function. we introduce a parauleter vector.

$$
w=\left\langle w_{1}, w_{2}, \ldots, w_{n}\right)
$$

and a feature vector.

$$
\phi(x)=\left\langle\phi_{1}(x), \phi_{2}(x) \ldots, \phi_{n}(x)\right\rangle .
$$

where

$$
\phi_{i}(x)= \begin{cases}1 & \text { if } \exists j .1 \leq j \leq m \wedge \operatorname{Map},(x)=i \\ 0 & \text { otherwise }\end{cases}
$$

The output of CMAC is defined as the average of the contents of the storage elements deterwined by the m mapping functions. which is just the quantity.

$$
\left.\frac{1}{m} \sum_{i=1}^{m} u_{h}\right|_{k=1} \operatorname{Map}_{i}(x) \text {. }
$$




$$
\frac{1}{m} w \boldsymbol{\phi}(x) \text {. }
$$

in the case that all $m$ mapping functions deternine different storage elements for the input $x$.

The learuing rule for $\mathrm{CMAC}^{\mathrm{M}}$ is just

$$
w_{t+1}=w_{t}+3 c_{t}\left(x_{t}\right) \phi\left(x_{t}\right)
$$

Where the error at time t. $\epsilon\left(x_{1}\right)$. is just the difference betreen the output of the function we are trying to learn given the training example presented at time $t$. and the output of CMAC' given the same training example.

$$
\epsilon_{t}\left(x_{t}\right)=y\left(x_{t}\right)-\frac{1}{m} w_{t} \phi\left(x_{\ell}\right) .
$$

assuming here that all $m$ mapping functions determine different storage elements for the input $x_{1}$.

The intuition behind this rule is fairly strajghtforward. Each element. $x$, of the donain determines $m$ overlapping regions; one from each of the $m$ partitions. Suppose for the sake of argument that these regions map onto $\mathrm{m}$ distinct storage elements. ${ }^{3}$ These $m$ storage elements will be used to encode the approximate value of $y(x)$, as well as the approximate values of $y$ for the nearby neighbors of $x$. Elements of the clomain that are very near $x$ will likely determine the same $m$ regions, and. hence, the same $m$ storage elements. Elements that are further from $x$ will determine few regions in common with those of $x$. and hence will have few storage elements in common.

When updating the approxinate value of $y$ for $x$. we will also disturb the approximate values of $y$ for the ueighbors of $x$, but, at least statistically, this disturbance will be in proportion to how near the neighbors are. Very near neighbors will feel the impact of the updales most strongly: more distant neighborn. becanse they will tend to have fewer storage elements in common with $x$, will feel it less strongly. Implicit in this method is the assumption that the function we are trying to learn is relatively smoorb: if the function rarim 100 much in a given region. then CMAC mav not be able to find a good approximation. because CMAC has only a linited amount of storage available to represent the function over the whole domain.

'If the hashing function is doing its joh correctly, the total number of distinct storase clements determined by the mapping functions for a given inpot should be a significant Iraction of $m$. 
Experiment 4 Apply $(\mathrm{MAC}$ to a simple function approximation problem.

The basic iclea behind ('MAC' 'an he "ssed in a successive refinement. strategy to achieve a nice tradeoff between the speed and the accuracy of leatming. The strategy is described as follows. Suppose that you want to learn a function. call it $y_{1}$. To do so you construct a C'MAC system in which the partitions consist of regions which are rather large. This C'MAC' system will find an approximation to $y_{1}$. call it $f_{1}$, very quickly. but the approximation is likely to be a poor one. given the coarseness of the mapping. To correct for the inaccuracies of $f_{2}$. we build another C'MlAC' system to learn the function. $y_{2}=y_{1}-f_{1}$. but this system makes use of partitions consisting of somewhat smaller regions. 'This second CMAC' svistem will find an approxima! ion to $y_{2}$, call it $f_{2}$, more slowly than the first CMAC'. but it will still represont $y_{2}$ more accurately than $f_{1}$ represented $y_{1}$, and the sum of the two functions. $f_{1}+f_{2}$, will be a better approximation of $y_{1}$ than $f_{1}$ alone. We can continue in this manner to define a sequence of ('MAC' systems each using fiuer partitions than the one before it in the sequence. and each providing a correction for the function corresponding to the sum of functions provided bv the CMAC' systems occurring earlier in the sequence.

One way to impiniment the above strategy is for the learuing system to apply each CMAC systeu in stages, starting with the system using the coarsest partitions and proceeding to those using finer partitions. Each CMIAC is run for a fixed number of steps using a learwing schedule that tends to zero. This sequential implementalion has the disadvantage that it cannot adapt if the function of interest changes slowly over time. An alternative implementation is to run all of the CMAC systems in parallel. using a different fixed learning rate for each ('MAC such that the finer the partition the slower the learuing (smaller the fixed rate). This parallel approach tends to learn somewhat slower than the sequential approach, but the parallel approach is still quite fast and its ability to adapt to haudle time-varying functions makes it useful in a number of applicatious for which the staged approach would not be effective.

Wh nfer to the general approach of building learning systems using severed CMACs employing successively finer partitions as multi-resolution CMAM: It turns out that implenenting multi-resolution CMAC' is actually no more dificult than implementing the version of CMAC described earlier: in some respects it is easier. We describe the basic construction in the followiug paragraplis.

Suppose that we wish to huild a multi-resolution CMLAC' consisting of 
"' IIAC's with successivel, finer partitinns. Becanse there are several C'MAC's, we need nnly one partition per C'MAC' to achieve the redundancy necessary to oftset the consequences of hashing collisions. As in the earlier version of (MAC', we assume $m$ partitions and m mapping functions. In the rase of multi-resolution ('MAC'. We require llat the partitions are arranged in a sequence so that the ith partition represents a finer partition than the $i-1$ partition.

For the ith CMAC. we define the parameter vector.

$$
w_{i}=\left\langle w_{i, 1} \cdot u_{i, 2} \ldots \ldots u_{i, n}\right\rangle,
$$

and the feature vector.

$$
\phi_{i}(x)=\left\langle\phi_{i, 1}(x) \cdot \phi_{i, 2}(x) \ldots . \phi_{i, n}(x)\right\rangle
$$

where

$$
\varphi_{i, j}(x)= \begin{cases}1 & \text { if } \operatorname{Map}_{i}(x)=j \\ 0 & \text { otherkise }\end{cases}
$$

Each of the $m$ CMLACs determines a function.

$$
f_{i}=w_{i} \phi_{i} . \text { for } 1 \leq i \leq m .
$$

intented as an approximation to some ofler function,

$$
f_{i} \approx y_{i}, \text { for } 1 \leq i \leq m .
$$

where $y_{1}$ is just the function we have set out to learn, $y$, and the other $m-1$ functions are defined as follows.

$$
y_{i+1}=y_{i}-f_{i}, \text { for } 1 \leq i \leq m-1 \text {. }
$$

The output of multi-resolution C'MAC' is the approximation.

$$
y \approx \int_{1}+\int_{2}+\cdots+\int_{m} .
$$

Leaming proreedn simullaneously, using the rules.

$$
w_{i, t+1}=w_{i, t}+B_{i}\left(c_{i, l}\left(x_{i}\right) \phi_{i}\left(x_{i}\right) . \text { for } 1 \leq i \leq m .\right.
$$

where $B_{;}$is the learuing rate for the ith CMIC. and the error for the ith CMAC is defined by.

$$
\epsilon_{1, t}\left(x_{1}\right)=y_{i}\left(x_{i}\right)-w_{i, l} \phi_{1}\left(x_{l}\right) . \text { for } 1 \leq i \leq m .
$$


Experiment 5 Apply multi-resolution ( $M A C$ ' to a simple function approximation problem and compare it with the version of $C M I C C^{\prime}$ described earlier.

('MAC' is a simple. fast, and effertive terhuique for approxinating functions. There are more powerful tedhniques hlial can solve more difficult problems. but CMAC is a practical method that should be a part of any engineers repertoire of techuiques. We rank it alongside the halman filter. proportional derivative control. stochastic dymanic programming. and planning by task reduction as useful conponent techniques for building useful plauning and control sustems.

The C $M A C$ : methods described in this section by no means nullify what was referred to as the curse of dimensionality in Section 9.2. If we have a three-dimensional domain. but the output of the function of interest is independent of. say, the third dimension. then C'MAC' still has to allocate the storage necessary to represent all three climensions. ln addition. in urder to construct a good approximation. ( $M A C$ ' has to sample the three-dimensional space instead of the smaller and completely aclequate two-dimensional subspace. An example mentioned earlier illustrates the sort of frustration that can result from this behavior.

Suppose we want a robot to learn a navigation function. The robot has four sensors, a compass or bearing sensor. a position sensor for longitude, a position sensor for latitude, and a light-level sensor. We want the rolsot to learn a function from the resulting four-dimensional input space to some space of actions. Ilaving taken great pains to teach the robot how to uavigate when the light is at one level, we find out that the robot is not able to navigate when the light is at any other level. What we would like is simply to tell the robot to ignore the light level thereby reducing the dimensionality of the learning problem.

What seems easy enough to arroinplish in the above example is quite difficult to achieve in general. It is hardly ever the rase that one sensor is enlirely irrelevant. In most cases. there will be subspaces of the inpul space that can replaced with spaces of reluced dimensionality. Determining theme rubspace reductions in dimensionality can be conplex. however. In building useful learning systems. the curse of dimensionality will probalsly always plague us. In lieu of general-purpose solutions, it is hoped that special-parpose techniques will suffice to achieve satisfactory performance for practical problems. 


\subsection{Rule-Based Learning}

In the beginuing of this chapter. we int roduced learning in terms of approximating functions. The chapter as a whole focusses primarily on learning value functions. In this section. we generalize on this idea of learning value functions to consider a variety of rule-based learning problems.

Value functions are used to derive policies. What we are really interested in learning is optimal policies. All of the techniçnes that we consiclered in Section 9.2 can be thought of as attempting to select an optinal policy from a parameterized class of policies. In each case. the parameterized class is represented as $a$ set of rules of the forn. if the current state is $x$. then perform action "1. where each rule has an associated parameter or rule strength. In Section 9.2. the rule st.rengths were just. the action values.

This parameterized class of policies is quite simple. Each rule represents a condition/action pair, in which the condition corresponds to the rurrent. state of the world and the artion corresponds to some control artion.

In the following, we generalize to allow rules of the form.

$$
\text { If } A_{1} \wedge A_{2} \wedge \cdots \wedge A_{n} \text {, then } C_{1} \wedge C_{2} \wedge \cdots \wedge C_{m} \text {, }
$$

where the antecedents. $\left\{A_{i}\right\}$, and the consequents. $\left\{C_{i}\right\}$, are ground atomic formulae in some appropriate representation language. We associate with each such rule a corresponding weight. We could introduce variables to represent rules with quantifiers. but we will not do so here in order to keep the discussion as simple as possible. Neither will we consider the details of any particulas representation language though there are some interesting issues with regard to the choice of representation language. Instead. we employ a simple database model for our discussion.

We assume a database consisting of ground atomic formulae. The contents of this database change over lime. as determined by the sequence of rules applied and the information provided by the system's sensors. Let Coutenta $(t)$ denote the coutents of the database at time $t$.

For each rulc. $r$. let. Antecedents $(r)$ be the set of antecedeuts of $r$. Conangenta( $r)$ its cousequeuts. and $W(r, t)$ its weight or streugth at tine $t$. We asume an arbitrary threshold. $r \in \mathbf{R}$. used to deternine which rules are applied. A rule. $r$. is applied at time. $t$. just in case the following criterion is satisfied.

$$
\text { Antecedents } s(r) \subset \text { Contents }(t-1) \wedge W(r, t)>r \text {. }
$$


We will consider some alternative criteria for rule application in just a bit. A rule is said to be arfire at time. $t$. denoted Active $(r . t)$. just in case it is applied at $t$. The set of conclusions arailable at time $t$ is just the union of the consequents of all the rules active at $t$.

$$
\text { Conclusions(t) }=\bigcup_{\text {Active(r.t) }} \text { Consecquents }(r) .
$$

Control actions are initialed using proredumil attuchment. Procedural attarhment refers to the practice of associating procellures with the presence or absence of tuples in a relational database or formulae in a predicate-calculus dalabase. In unost procedural attachuent schemes. there is a program desigued to monitor the contents of the database. When a formula is added to or deleted from the database. the monitor progran checks to see if there is a procedure associaled with the addition or deletion of the formula. and. if so. runs the appropriate procedure.

Finally. we define the contents of the database at $t$ as the union of the sensory information and conclusions available at $t$,

$$
\text { Contents }(t)=\text { Sensors }(t) \cup \text { Conclusions }(t) \text {. }
$$

where Sensors $(t)$ is a set of ground atomic formulae summarizing the clata available from the sensors at $t$.

At each point in time, the rule strengths are updated. For earli rule. $r$, applied at time $t$, the system performs the following steps. comprising what is generally called the bucket-brigude algorithm [6].

1. For each rule, $r^{\prime}$. active at time $t-1$ such that

$$
\text { Antecedents }(r) \cap \text { Consequents }\left(r^{\prime}\right) \neq 0
$$

update the strength of $r^{\prime}$ using the following rule.

$$
W\left(r^{\prime} \cdot t+1\right)=\alpha W^{\prime}(r . t) .
$$

where $a \in \mathbf{R}$ is a number between zero and one. sinilar in its use here to the learning rate described in earlier sections.

2. Update the strength of $r$ using the rule.

$$
W(r . t+1)=W(r . t)-a W(r . t)+\mathrm{R}(t) .
$$

where $R(1)$ is the reward at time 1 . 


\begin{tabular}{|c|c|c|c|c|c|c|c|c|c|c|}
\hline$t$ & $u$ & 1 & 2 & 3 & 4 & 5 & tj & 7 & 8 & $y$ \\
\hline$W(\mathrm{~K} 1.1)$ & 100 & 80 & 100 & 110 & 80 & 100 & 100 & 80 & 101.6 & 101.6 \\
\hline$W(R 1 . t)$ & 100 & 100 & 80 & 100 & 100 & 80 & 108 & 108 & 88 & 120.8 \\
\hline$W(R 1 . t)$ & 100 & 100 & 100 & 110 & 110 & 110 & 1,2 & $1: 2$ & 172 & 107.6 \\
\hline Sensorirat & $\{1\}$ & \{\} & \{\} & $\{t\}$ & \{\} & \{\} & $\{A\}$ & \{\} & \{\} & $\{t\}$ \\
\hline Contents $(t)$ & $\{.1\}$ & $\{B\}$ & $\left\{C^{\prime}\right\}$ & $(.1 . D)$ & $\{B\}$ & {$[C]$} & $\{.1, D\}$ & $\{B\}$ & $\{6\}$ & $|-1, D|$ \\
\hline$R(f)$ & 0 & 0 & 0 & in & 11 & 0 & 100 & $n$ & 0 & in) \\
\hline
\end{tabular}

Table 9.1: C'hanges in rule strengt hs over time

For all the rules not applied at $t$. there is no change,

$$
W(r . t+1)=W(r . t) .
$$

To illinstrate the datahase model and the bucket-brigade algorit hm, consider the following simple example.

Let the set of rules be as follows,

$$
\begin{aligned}
& \text { R1: If } A, \text { then } B, 100 \\
& \text { R2: If } B, \text { then } C, 100 \\
& \text { R2: If } C, \text { then } D, 100,
\end{aligned}
$$

where the number on the far right indicates the rule strength at $t=0$ in some arbitrary units. Let $a=0.2$. Suppose that whenever $D$ is added to the database, the systen performs an action that is immediately rewarded at a level of 60 , employing the same units user for rule strengths. Table 9.1 shows the evolution of the rule strengths for 10 time steps. If the sanne cycle of sensor input and rewards found in Table 9.1 is allowed to continne indefinitely, the strengths of all three rules will converge to $\frac{60}{3}=300$. If the rewards are stochastic but average 60 . then the rule strengths will never converge but will average 300 .

Expariment 6 Provide an example showing how the bucket-brigade algorithm might be applied to the problem of learuing to fill tanker trucks. given the flow model described in Chapter 5.

'The bucket-brigade algorithm is often used for classification and prediction problems. In classification problems. the system is given a set of features describing its input and asked to assign the input to one of a finite 
IImuber of calegories. For instance. all assembly-line visnal inspertion sislem unight classify items on a converor bell as ready to ship. defective but repairable. or defective and not worth repairing. For the inspection sistem. the features might correspond to superlicial visual attributes. such as the alignment of external parts, or the number and distribution of flaws on a painted surface. In general. not all of the features given to the system will be relevant to making the classification.

In prediction problems. the system is given a set of features describing the state of the system at the current time and asked to predict the state of the system or some particular aspect of the stale of the systein at some fut ure liwe. For instance. a system designed to regulate the flow of gas through a commercial pipeline might need to predict transient leaks that prevent the instem from delivering gas at the appropriate pressures. In this case. the feat ures might correspond to the current demand for gas. outside temperature. time of day. and pipeline inlet and outlet pressures. The predictions made by the system are used to prevent or reduce the effects of transient leaks by anticipating demand and regulating pipeline inlet pressure.

The simple thresholding method for applying rules descrbed above is unt appropriale for most applicatious. In the case of classification problems where many of the rules correspond to conflicting hypotheses regarding the class of a particular instance. there unay be several rules whose strengths are greater than the threshold. but it would not make sense to apply more than one of them to a given input. A similar case arises in control problems in which there are two or more rules vying to set the same parameter to different values. Nor is it generally appropriate only to apply the rule with the greatest strength: parallel rule invocation is often useful in building effective rule-based control systems.

In most practical applications, the decision as to what rule or fules to apply involves criteria in addition to rule strength. In classification problems. the specificity of the rules antecedents is often taken into account. For instance. using a specificity criterion. given the database. $\{A, B\}$, and the two rules.

R1: If $A \wedge B$. then $C .100$

R2: If $B$. then $D$. 100 ,

only the first rule would be applied. since. though both rules have their antecedent conditions satisfied. the first has a more specific antecedent condition than the second.

Most rule application strategies also involve a component of stochastic 
selection. As we saw in regard to learning optimal policies in stochastic sequential decision problems. the ststem has to experiment with a variety of rules in order to be assured of finding the optimal one. Similarly, for learuing classification and prediction rules. it is necessary to occasionally try rules that are not doing particular well just in case those rules have not as yet had sufficient opportunities to demonstrate their utility.

The bucket-brigade algorithm is often used to select a set of promising rules from a larger set. In rule selection. a set of candidate rnles are applied in a set of experiments. thrir st.rengths adjusted using the burketbrigade algorithn. and the subset of rules with rule strengths above a certain threshold are selected as promising. Rule selection addresses just one issue in designing effective learning systems. There is another issue that we have overlooked up until now. This issue concerns where the rules come from.

In sequential decision problems. we are given a set of rules of the form. if the current state is $x$. then perform $u$. which can be used to specify all possible policies. Even in this case, the number of such rules is often dauntingly large. In some problems, the number of possible rules is infinite or so large that it is unthinkable to generate and store all of thein at once.

There are many techuiques for generating new rales given an existing set of rules. Some of them involve methods for generalizing and specializing antecedents and consequents to form new rules. Other techniques use genetic operators to construct rules by combining parts of two or more existing rules. A detailed discussion of such techuiques is beyond the scope of this chapter. Suffice it to say that cffective generation of new rules is an active arca of learning research with many open problems. In the last section of this chapter. we provide some references for further reading.

Generally, a coniplete learuing systen observes a two-phase cycle of activity. In the first phase, a set of candidate rules is generated using as a basis whatever rules survived the last selection phase. In the second phase. the set of candidate rules is subjected to a series of experiments designed to identify the most useful rules and eliminate the less effective ones. In this chapter, we have focussed primarily on the problem of rule selection. becanen the corresponding area of research is the best developed and most directly relevant to the problems considered in this book. 


\subsection{Learning and Observability}

In this chapler. We forns on the problem of learning an optimal pulicy for a stochastic dymamical system with rewards. In some cases. it may be possible to divide the problem into component problems. For instance. if the dynamical svstem satisfies a separation property. it may make sense to contsider two separate learning problens: one concerned with observation. learn how to determine what state you are in. and one concerned with control. learn what action to take given that yun know what state you are in. You can divide control still further into sustem identification, learn a model of the system dynamics and rewards. and regulation, learn an optimal control law given the dynamics and rewards.

In practice. however. breaking the problem into pieces mav not be the most effective way to proceed. Wiith regard to observation. you probably do not have to kuow exactly what state you are in as knowing the proper equivalence class will sulfice for some appropriate equivalence relation. With regard to control. for the sort of robotics and automation problems that we are uost interested in. observation and contrul are not separable, in which case the optimal policy for an ideal observer will not be of much use. With regard to identification, as pointed out in eartier sections. it may not be necessary to predict the evolution of the state in order to deternine how to act: if we know the value function for a given policy, it is possible to improve that policy without the use of a mode!

In Section 9.2. we considered condition/action rules of the form. if the current state is $x$. then perform action $u$. However, for the techniques involving learning action values that we discussed. we might just as well have considered rules of the form, if our perceptions of the current state are $y$, then perform action $u$. This assumes, of course, that the set of possible actions includes perceptual actions, otherwise there would be 110 way for a robol to infuence its perception of the curreul stale.

As we mentioned earlier, if the dymanical system is separable. we might try to learn an optimal observer and an optimal policy separately. Altermatively, we might proceed as though there was a one-to-one mapping between the robot's perceptual states and the states of the world. If this actually was the cane, then we effectively have an ideal ubserver since there is no need to know or make use of the mapping from perceptual states to world states.

If such a one-to-one mapping does not obtain. then there will be states of the world that the robot cannot distinguish between using its perceptual apparatus. Perceptual states that map to two or more world states are said 
to be ambignons. This ambiguity may not be a problem: there is no need to distinguish between two states if they require the same response. However. if the two states require very differeut responses, then performance could be adversely affected. There are two problems associated with ambiguity leading to adverse performance. First. how do you detect it. and. second. having detected it what can youl do about it.

If you know that the dynamical system is deterministic. then detecting anthiguous perceptual states is rnther easy. For a deterministic system. if the perceptual state is unanbiguous. the action values, assuning a fixed policy, should converge to fixed values (at least in the limit). However, if the perceptual state is ambiguous. then the action values will vary between those for each of the corresponding world states. Detecting ambiguous perceptual states in a deterministic system can be handled by carefully monitoring the variance in the action values. Detecting ambiguous perceptual states in a stochastic system can be managed with more sophisticated statistical tests.

Once you know that a given perceptual state is ambiguous and that the variance is sufficient to warraut doing something about it, you still have to decide how to deal with the ambiguity. You may be able to simply perform appropriate perceptual actions in order to nove to an unambiguous percep-

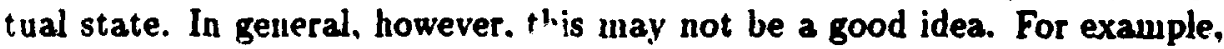
it may be that achieving a goal or maximizing a performance index requires that the system pass through perceptually ambiguous states. In geveral. we recommend simply treating perceptual states as world states, including perceptual actions as possible actions, and using one of the stochastic methods described in Section 9.2. If the dynamical system is deterministic, then it will behave like a stochastic system if there is perceptual ambiguity, but this stochastic behavior will not prevent the system from learning an optimal policy.

Experiment 7 Apply Watkin's stochastic dynanic programning method to learning a navigation function given uncertainty about the robot's position. Acoume a Kalman filtering state estimation front end that provides an estimake of the robut's location to serve as input to the control system.

\subsection{Further Reading}

For more on the update rule of Wirlrow and Hoff. the perceptron learning rulc of Rosenblatt. and discussion of uther learning issues consult the text by Nilsson on learning machines [13] or the first part of the text by Duda and 
Hart or. pattern rlassification and scene analysis [H]. For an introduction in so: re of the issues in function approximation. the paper by Poggio and (iirosi provides a comparison of a variety of techniques [14].

Our treatment of learning in terms of stochastic decision prohlems follows that of Barto, Sutton. and Wat.kins [:3]. For more on solving creclitassignment problems in sequential derision tasks. consider the paper by Sutton [17]. The specific methol of learning action values considered in this chapter is due to Watkins [19]. The theory of learning automata is also relevant to the issues addressed here and the text by Narendra and Thathachar is an excellent introduction to this area of research [12]. Sutton considers some of the issues involved in combining exploration and prediction to speed learning [18]. Whitehead and Ballard [20] discuss some issues regarding ohservalility in learning to solve seppential decision tasks.

Allous CMAC: method is clescriberl in [2]. A multi-resolution (:MAC: method is analyzed in [11], and the variation on this method suitable for learning time-varying functions is rlescribed in [16].

lolland et al describe the hucket-brigrade algorithm for credit. assignment in rule-based systems [6]. For more on the application of rule-based techniques to problens in planning and control, see Laird et al for a general architecture for prohism solving [i] , Minton et al for a perspective that consiclers certain forms of learning as akin to theorem proving [9], and Mitchell al for an approarh to learuing plans by generalizing past experience [10]. Also see Ilammond for a different perspective on learning plans that. deviates from the mure conventional rule-based approaches [5]. Much of the work on learning plans is related to the work on speed up learning discussed in Chapter 8. Many of the techuiques for speedup learning can be characterized in ternss of learning to solve problems efficiently by caching the (generalized) solutions to selected problem instances. 


\section{Bibliography}

[1] Albus. J. S.. A Theory of Cerebellar Functions. Mathematical Biology, $10(19 i 1) 25-61$.

[2] Albus. J. S.. A New Approach to Manipulator Control: The Cerebellar Mlodel Articulation ('outroller (CMAC'). Journal of Dynamic Systems. Measurement. and Control. 97 (19i5) 2i0-2ii.

[3] Barto, Andrew C., Sutton. R. S., and Watkins, C. J. C. II, Lenrning and Sequential Derision Making, 'Technical Report 89-95. Iniversity of Massachusetts at Anulerst. Department of Computer and Information Science, 1989.

[4] Dnda. R. O. and Hart. P. E.. Pattern Classification and Scene Analysis. (John Wiley and Sons. New lork. 19i3).

[5] Hammond. Kris. CIIEF: A Model of Case-based Planning. Proceclings AAAl-86. Philadelphia. Pennsylvania. AAAl. 1986, 26i-2i1.

[6] Holland. John H.; Holyoak. Keith J.. Nisbett, Rjehard E.. and Thagard. Paul R., Indurtion: Prorrases of Inferrnce. Learning, and Dismpnery, (MIT Press, Cambridge, Massachusetts, 1987).

[i] Laird. J. E., Newell, A.. and Rosenbloom. P. S.. SOAR: An Arclitecture for General Intelligence. Artificial Intelligence. 33 (198i) 1-64.

[8] Mar. David. A Theory of C'erelsellar Cortex. Joumal of Physioloyy. $202(1969)+3 i-4 i 0$.

[9] Minton. S.. C'arbonell. I. C... Knolslock. C'. A.. Kunkka. D. R.. Etzioni. O.. and Ciil. Y., Explanation-Based l.earning: A Problem Solving Perspective, Artifirial Intelligenre. 40 (19)(9) (j;3-118. 
[10] Mitchell. T. M. I'tgoff. P.. and Banerji. R.. Learning by Experimentation: Formulating and Cieneralizing Plans from Past Experience. Michalski. R. S.. ('arbonell. J. G.. and Mitchell. T. M.. (Eds.). Machine Lcarning: an Artificial Intelligence Appronch. (Tioga. 198.3).

(11) Moody. John E.. Fast Learning in Multi-Resolution Hierarchies. Touretsky. David. (F.l.). Illrenres in Neuml Information Processing. (Mlorgan-Kaufmanu, Lus Allus. ('alifornia. 1989).

[12] Narendra. Kumpali S. and Thathachar. Mandlayam A. L.. Learning Automata: An Intmdurtion. (Prentice-Hall. Englewood Cliffs. New Jersey, 1989).

[13] Nilsson. Nils, Learning Machines. (McCiraw-Hill. New York. 1965).

[14] Poggio. Tomaso and Cirosi. Federico. A Theory of Veturorks for Approximution and Leaming. Techuical Report Al Mewo No. 1140. MIT Al Laboratory, 1989.

[15] Rosenblatt, F., Principles of Neurodynamics: Perceptrons and the Theory of Bmin Mechanisms, (Spartan Books, Washington, D.C., 1961).

[16] Shewrhuk. John and Dean. Thomas, Towards Learning Time-Varying Functions With High Input Dimensionality, Proccedings of the Fifth IEEE International Symlosium on Intelligent Contml. Philadelphia. Pcnnsyluania, IEEE, 1990, 383-388.

[17] Sutton, Richard S., Learning lo Predict by the Methods of Temporal Differences, Machine Learning. 3 (1988) 9-44.

[18] Sutton, Ilichard S., Integrated Architectures for Learuing, Planning, and Keacting Based ou Approximating Dyuanic Programming, Pro. reedings 7th Intermational C'onference on Machine Learning. Austin. Texus, 1990.

[10] Watkins, C. J. C. H. Lcarning fmm Dclaycd Rewards. PhD thesis, C'ambridge University, 1989.

[20] Whitehead. Steven D. and Ballard. Dana H.. Active Perception and Reinforcennent Learuing. Proceedings 7lh Intemational C'onference on Vachine Learning. A ustin. Texas, 1990. 
[21] Widrow. 13. and Ilolf. M. E.. Adaptive Switchiug ('ircuits. 1960

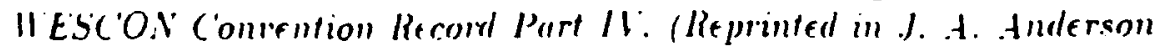
and E. Rosenfrill. Neurocomputing: Fonndations of Research. The $1 / 1 T$ Press. ('mmbridye. .17.4. 1988). 1960. 96-104. 